\title{
VARIATIONAL DERIVATION OF MODAL-NODAL FINITE DIFFERENCE EQUATIONS IN SPATIAL REACTOR PHYSICS
}

\author{
by \\ PATRICK G. BAILEY \\ B.S., University of California at Berkeley \\ (1967) \\ M.S., Massachusetts Institute of Technology \\ (1969)
}
SUBMITTED IN PARTIAL FULFILLMENT
OF THE REQUIREMENTS FOR THE
DEGREE OF DOCTOR OF
PHILOSOPHY

at the
MASSACHUSETTS INSTITUTE OF
TECHNOLOGY

July, 1972

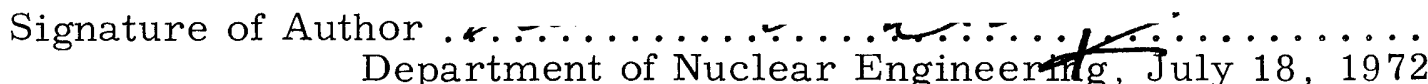

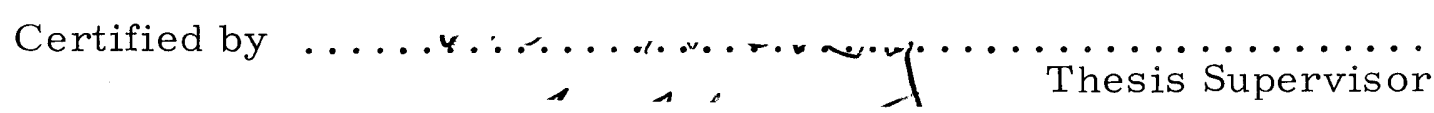

Certified by

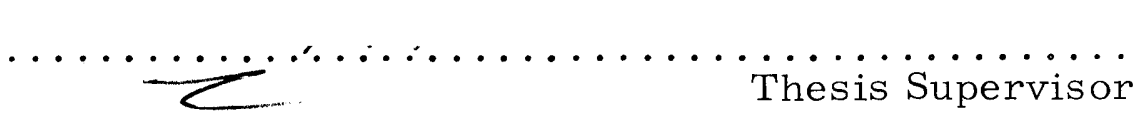

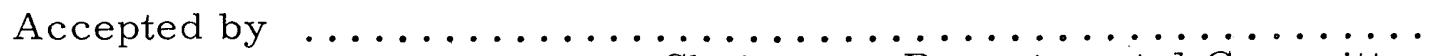
Chairman, Departmental Committee on Graduate Students

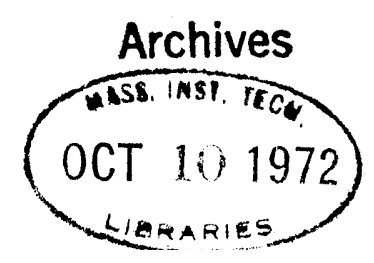




\title{
VARIATIONAL DERIVATION OF MODAL-NODAL FINITE DIFFERENCE EQUATIONS IN
}

SPATIAL REACTOR PHYSICS

by

\author{
PATRICK G. BAILEY
}

Submitted to the Department of Nuclear Engineering on July 18, 1972, in partial fulfillment of the requirements for the degree of Doctor of Philosophy.

\section{ABSTRACT}

A class of consistent coarse mesh modal-nodal approximation methods is presented for the solution of the spatial neutron flux in multigroup diffusion theory. The methods are consistent in that they are systematically derived as an extension of the finite element method by utilizing general modal-nodal variational techniques. Detailed subassembly solutions, found by imposing zero current boundary conditions over the surface of each subassembly, are modified by piecewise continuous Hermite polynomials of the finite element method and used directly in trial function forms. Methods using both linear and cubic Hermite basis functions are presented and discussed.

The proposed methods differ substantially from the finite element methods in which homogeneous nuclear constants, homogenized by flux weighting with detailed subassembly solutions, are used. However, both schemes become equivalent when the subassemblies themselves are homogeneous.

One-dimensional, two-group numerical calculations using representative PWR nuclear material constants and $18-\mathrm{cm}$ subassemblies were performed using entire subassemblies as coarse mesh regions. The results indicate that the proposed methods can yield comparable if not superior criticality measurements, comparable regional power levels, and extremely accurate subassembly fine flux structure with little increase of computational effort in comparison with existing coarse mesh methods.

Thesis Supervisors: Allan F. Henry

Kent F. Hansen

Titles: Professors of Nuclear Engineering 
ACKNOWLEDGMENTS

I would like to express my deep appreciation and sincerest gratitude to Dr. Allan F. Henry whose advice and consultation have been of considerable help during the course of this work. Thanks must also be extended to Dr. Kent F. Hansen, who reviewed the final manuscript and provided many beneficial discussions and programming assistance.

It has also been a privilege to have known and worked with the late Dr. T. J. Thompson, whose untimely death was a great loss to the nation as well as to the department at M.I.T., and Dr. Elias P. Gyftopoulos. Their insistence on high standards of education and quality as well as clarity of expression will continue to remain with me always.

Fellowship support by the U.S. Atomic Energy Commission is also gratefully acknowledged. The work was performed under USAEC Contract AT (30-1) - 3903. All computations were performed on IBM $360 / 65$ and $370 / 155$ computers at the M.I.T. Information Processing Center.

Special appreciation is extended to my wife, Alice, whose patience and understanding have provided immeasurable support throughout the course of this work.

Finally, special thanks are expressed to Mrs. Mary Bosco, who has once again demonstrated her expert ability as a superb technical typist. 
. . Until one is committed there is hesitancy, the chance to draw back, always ineffectiveness. Concerning all acts of initiative (and creation), there is one elementary truth, the ignorance of which kills countless ideas and splendid plans: that the moment one definitely commits oneself, then Providence moves too. All sorts of things occur to help one that would never otherwise have occurred. A whole stream of events issues from the decision, raising in one's favor all manner of unforeseen incidents and meetings and material assistance, which no man could have dreamt would have come his way. I have learned a deep respect for one of Goethe's couplets:

Whatever you can do, or dream you can, begin it. Boldness has genius, power, and magic in it.

- W.H. Murray 
Great importance attaches to the material comforts of life, and equanimity, unconcern, security are all sacrificed to them. The American lives even more for his goals, for the future, than the European. Life for him is always becoming, never being.

- Albert Einstein 1921

\footnotetext{
"When someone is seeking," said Siddhartha, "it happens quite easily that he only sees the thing that he is seeking; that he is unable to absorb anything, because he is only thinking of the thing he is seeking, because he has a goal, because he is obsessed with his goal. Seeking means: to have a goal; but finding means: to be free, to be receptive, to have no goal. You, O worthy one, are perhaps indeed a seeker, for in striving toward your goal, you do not see many things that are under your nose."
}

\section{- Hermann Hesse}


TABLE OF CONTENTS

Abstract 2

Acknowledgments 3

List of Figures $\quad 7$

$\begin{array}{ll}\text { List of Tables } & 10\end{array}$

Chapter 1. Introduction 11

1. 1 Preface 11

1.2 The Time-Independent, Multigroup Diffusion
Theory Equations

1.3 Solution Methods 15

1.3.1 Nodal Methods 16

$\begin{array}{ll}1.3 .2 \text { Modal Methods } & 19\end{array}$

1.3.3 Modal-Nodal Methods 25

Chapter 2. Variational Derivation of Finite Difference Approximations in Time-Independent Multigroup
Diffusion Theory

2.1 Calculus of Variations Applied to Diffusion Theory 28

2.2 Discontinuous Trial Functions 30

2.3 The Finite Element Approximation Methods 34

2.3.1 The Conventional Finite Difference Equations 35

2.3.2 Multichannel Polynomial Synthesis 37

2.3.3 The Linear Basis Function Approximation 46

2.3.4 The Cubic Hermite Basis Function
Approximation 
Chapter 3. Development of a Consistent Coarse Mesh Approximation Method

3.1 Formulation $\quad 52$

3.2 The Proposed Linear Basis Function Approximations 54

3. 3 The Proposed Cubic Hermite Basis Function Approximation

Chapter 4. Numerical Solution Techniques 65

4.1 Solution Methods and Matrix Properties 65

4.2 Calculational and Programming Techniques 77

Chapter 5. Numerical Results 84

5.1 Nuclear Constants and Subassembly Geometry 84

5.2 Subassembly Detailed Solutions and Homogenized
Nuclear Constants

5. 3 Case Studies and Results 95

$\begin{array}{lll}5.3 .1 & \text { Case } 1 & 98\end{array}$

$\begin{array}{lll}5.3 .2 \text { Case } 2 & 113\end{array}$

$\begin{array}{lll}5.3 .3 \text { Case } 3 & 118\end{array}$

$\begin{array}{lll}5.3 .4 \text { Case } 4 & 124\end{array}$

Chapter 6. Conclusions and Recommendations 130

6. 1 Characteristics of the Proposed Approximation Methods 130

6. 2 Applicability and Limitations 132

6.3 Recommendations for Future Work 133

$\begin{array}{ll}\text { References } & 134\end{array}$

$\begin{array}{ll}\text { Biographical Note } & 138\end{array}$ 
Appendix A. Table of Symbols

Appendix B. Difference Equation Coefficients Resulting from Use of the Finite Element Approximation Methods

B. 1 Coefficients of the Conventional Finite Difference Equations

B. 2 Coefficients of the Linear Finite Element Method Equations

B. 3 Coefficients of the Cubic Hermite Finite Element Method Equations

Appendix C. Difference Equation Coefficients Resulting from Use of the Proposed Approximation Methods

C. 1 Coefficient-Integrands of the Proposed Approximation Method Equations Using Linear Basis Functions

C. 2 Coefficient-Integrands of the Proposed Approximation Method Equations Using Cubic Hermite Basis Functions

Appendix D. Description of the Computer Programs

D. 1 Description of Program REF2G

D. 2 Description of Program LINEAR

D. 3 Description of Program CUBIC 163

D. 4 Description of Program ANALYZE

Appendix E. Sample Input and Output Data Blocks for Programs REF2G, LINEAR, CUBIC, and ANALYZE (Included in only the first six copies)

E. 1 REF 2G

E. 2 LINEAR

E. 3 CUBIC

E. 4 ANALYZE

Appendix F. Source Listings of the Programs

(Included in only the first six copies)

F.1 REF 2G

F. 2 LINEAR

F. 3 CUBIC

F. 4 ANALYZE 


\section{LIST OF FIGURES}

No.

1.1 Illustration of One-Dimensional Multichannel Synthesis

1. 2 Illustration of One-Dimensional Overlapping Multichannel Synthesis

2.1 Conventional Nodal Finite Difference Approximation Trial Function Forms

2.2 Matrix Form of the Conventional Finite Difference Equations

2. 3 Basis Functions of Equations 2.23 for $\mathrm{N}=0,1,2$, and 3

2. 4 Cubic B Spline $\Omega_{\mathrm{k}}^{\mathrm{B}}(\mathrm{z})$

2.5 Cubic Hermite Basis Functions $\Omega_{k}{ }^{\mathrm{H}}(\mathrm{z})$ and $\Omega_{\mathrm{k}}{ }^{\mathrm{H}}(\mathrm{z})$

2.6 Matrix Form of the Linear Finite Element Method Approximation

2.7 Matrix Form of the Cubic Hermite Finite Element Method Approximation

4.1 Solution of $\mathbb{A} \underline{F}=\frac{1}{\lambda} \mathbb{B} \underline{F}$ Using the Fission Source Power Iteration Method Without Fission Source Renormalization 66

$\begin{array}{lll}4.2 & \text { Subassembly Notations and Detailed Solutions } & 79\end{array}$

$5.1 \quad$ Subassembly Configuration Geometries $\quad 87$

5.2 Mesh Geometry in Half a Subassembly 88

5.3 Subassembly Detailed Flux Solutions for the One-Group Case

5. 4 Subassembly Detailed Flux Solutions for the Two-Group Case

5. 5 Subassembly Detailed Adjoint Flux Solutions for the Two-Group Case

5. 6 Geometry of the Four Case Studies Composed of Types of Subassemblies 
No.

5.7 Case 1: One-Group Results Using Linear Basis Function Approximations and $18-\mathrm{cm}$ Coarse Mesh Regions

5.8 Case 1: One-Group Results Using Cubic Hermite Basis Function Approximations and $18-\mathrm{cm}$ Coarse Mesh Regions

5.9 Case 1: Two-Group Fast Results Using Linear Basis Function Approximations and $18-\mathrm{cm}$ Coarse Mesh Regions

5.10 Case 1: Two-Group Thermal Results Using Linear Basis Function Approximations and $18-\mathrm{cm}$ Coarse Mesh Regions

5. 11 Case 1: Two-Group Fast Results Using Cubic Hermite Basis Function Approximations and 18-cm Coarse Mesh Regions

5.12 Case 1: Two-Group Thermal Results Using Cubic Hermite Basis Function Approximations and 18- $\mathrm{cm}$ Coarse Mesh Regions

5.13 Case 1: Two-Group Fast Results Using Linear Basis Function Approximations and 9-cm Coarse Mesh Regions

5.14 Case 1: Two-Group Thermal Results Using Linear Basis Function Approximations and 9-cm Coarse Mesh Regions

5.15 Case 1: Two-Group Fast Results Using Cubic Hermite Basis Function Approximations and 9-cm Coarse Mesh Regions

5. 16 Case 1: Two-Group Thermal Results Using Cubic Hermite Basis Function Approximations and $9-\mathrm{cm}$ Coarse Mesh Regions

5.17 Case 1: Two-Group Fast Results Using Cubic Hermite Finite Element Approximations and 18- $\mathrm{cm}$ Coarse Mesh Regions

5.18 Case 1: Two-Group Thermal Results Using Cubic Hermite Finite Element Approximations and $18-\mathrm{cm}$ Coarse Mesh Regions

5. 19 Case 2: Two-Group Fast Results Using Linear Basis Function Approximations and 18- $\mathrm{cm}$ Coarse Mesh Regions 
No.

5.20 Case 2: Two-Group Thermal Results Using Linear Basis Function Approximations and $18-\mathrm{cm}$ Coarse Mesh Regions

5. 21 Case 2: Two-Group Fast Results Using Cubic Hermite Basis Function Approximations and $18-\mathrm{cm}$ Coarse Mesh Regions

5. 22 Case 2: Two-Group Thermal Results Using Cubic Hermite Basis Function Approximations and $18-\mathrm{cm}$ Coarse Mesh Regions

5. 23 Case 3: Two-Group Fast Results Using Linear Basis Function Approximations and 18-cm Coarse Mesh Regions

5.24 Case 3: Two-Group Thermal Results Using Linear Basis Function Approximations and 18-cm Coarse Mesh Regions

5.25 Case 3: Two-Group Fast Results Using Cubic Hermite Basis Function Approximations and $18-\mathrm{cm}$ Coarse Mesh Regions

5.26 Case 3: Two-Group Thermal Results Using Cubic Hermite Basis Function Approximations and $18-\mathrm{cm}$ Coarse Mesh Regions

5.27 Case 4: Two-Group Fast Results Using Linear Basis Function Approximations and 18-cm Coarse Mesh Regions

5.28 Case 4: Two-Group Thermal Results Using Linear Basis Function Approximations and $18-\mathrm{cm}$ Coarse Mesh Regions

5. 29 Case 4: Two-Group Fast Results Using Cubic Hermite Basis Function Approximations and 18-cm Coarse Mesh Regions

5.30 Case 4: Two-Group Thermal Results Using Cubic Hermite Basis Function Approximations and $18-\mathrm{cm}$ Coarse Mesh Regions

F.1 Structure of Program REF2G

F.2 Structure of Program LINEAR 243

F. 3 Structure of Program CUBIC 288

F.4 Structure of Program ANALYZE 


\section{LIST OF TABLES}

No.

3.1 Matrix Order $\mathrm{N}$ of the Proposed Linear Basis Function Approximations as a Function of the Imposed Boundary Conditions

5.1 Representative Two-Group, 18-cm, PWR Subassembly Regional Nuclear Constants

5.2 Representative One-Group, 18-cm, PWR Subassembly Regional Nuclear Constants

5.3 Homogenized Subassembly One-Group Nuclear Constants

5.4 Homogenized Subassembly Two-Group Nuclear Constants

5.5 Test Results Using Three Consecutive Type A Subass emblies

5. 6 Results of Case 1

5. 7 Two-Group Results of Case 2

5. 8 Results of Case 3

5.9 Results of Case 4

D. 1 Sample Storage Requirements and Execution Times of the Programs for Two-Group Results 
Chapter 1

INTRODUCTION

\subsection{Preface}

The large variety of approximation methods and techniques used in computational reactor analysis and simulation has caused the area of numerical reactor physics to become one of the most exciting areas in applied nuclear reactor physics today. The application of numerical analysis is most important in two phases of reactor design; feasibility studies and safety analysis. The primary consideration of the reactor physicist has been and must continue to be the safety of the reactor during and after any foreseeable nuclear accident. A realistic safety analysis can be obtained only if all the physical processes occuring within the reactor can be adequately described and related. Since all of these processes can be shown to be dependent upon the neutron density distribution throughout the reactor core, a detailed solution of the spatial neutron flux is vital. ${ }^{1}$

The dynamic characteristics of a reactor strongly depend upon the spatial approximation and solution of the neutron flux. Approximation methods utilizing gross averaging of the flux near localized strong absorption and production regions, such as cruciform control rods or small water channels, can lead to inaccurate results. Large errors may result from the use of such methods in spatial kinetics problems such as depletion and xenon oscillation calculations. Much attention has therefore been focused upon approximation methods 
which can obtain detailed spatial neutron flux distributions within large reactor cores.

The Boltzmann neutron transport equation ${ }^{2}$ is considered to be a sufficiently detailed description of the physical processes occuring within a nuclear reactor, and naturally is most difficult to solve. The $\mathrm{P}-1$ and diffusion theory approximations ${ }^{3}$ greatly simplify the transport equation into more tractable equations which have been found to approximate adequately the flux distributions for most large-core reactors such as PWR, BWR, and LMFBR core geometries. The advent of high speed digital computers has enabled widespread use of diffusion theory because of its simple mathematical form and straightforward numerical solution techniques inherent with its use.

The treatment of the spatial approximation in diffusion theory is the primary concern of this report. There is in existence an increasingly abundant variety of such approximation methods currently in use. Fine mesh methods, ${ }^{4}$ for example, can yield very accurate results through the use of extremely large numbers of unknowns. However, such methods may well exceed the storage capacity of present day computers, as well as being exceedingly costly. Coarse mesh methods and particularly synthesis techniques, ${ }^{5}$ on the other hand, have recently become attractive as the number of unknowns can be drastically reduced, although the accuracy of many of these methods is in doubt.

The purpose of this report is twofold: first, to present the general development of variational approximation methods used to derive 
difference approximations to the neutron diffusion equation; and second, to extend this development in order to develop systematically a class of consistent coarse mesh approximation methods which can approximate accurately the detailed spatial neutron flux and can also be easily incorporated into present day computer codes. As this report will deal only with the spatial approximation, the inclusion of time dependence will be set aside for future study.

\subsection{The Time-Independent, Multigroup Diffusion Theory Equations}

The energy discretized multigroup $\mathrm{P}-1$ approximation to the Boltzmann neutron transport equation excluding time dependence can be written in standard group notation for each energy group $\mathrm{g}$ as follows: ${ }^{3}$

$$
\begin{aligned}
& \underline{j}_{g}(r)+D_{g}(r) \underline{\nabla}_{g}(r)=0 \\
& \underline{\nabla} \cdot \underline{j}_{g^{\prime}}(r)+\Sigma_{g}(r) \phi_{g}(r)-\sum_{\substack{g^{\prime}=1 \\
g^{\prime} \neq g}}^{G} \Sigma_{g g^{\prime}}(r) \phi_{g^{\prime}}(r)=\frac{1}{\lambda} x_{g} \sum_{g^{\prime=1}}^{G} \nu \Sigma_{f g^{\prime}}(r) \phi_{g^{\prime}}(r)
\end{aligned}
$$

where the group index g runs from the highest energy group, 1 , to the lowest energy group, G. The symbols and notation used throughout this report are summarized in Appendix A. Equations 1.1 are the standard P-1lequations which relate the vector neutron current $j_{g}(r)$ for each energy group $g$ with the scalar neutron flux $\phi_{g}(r)$. The current may be eliminated via Fick's law, Eq. 1.1a, in order to obtain the multigroup diffusion equation:

$$
-\underline{\nabla} \cdot D_{g}(r) \underline{\nabla} \phi_{g}(r)+\Sigma_{g^{\prime}}(r) \phi_{g}(r)-\sum_{\substack{g^{\prime=1} \\ g^{\prime} \neq=}}^{G} \Sigma_{g g^{\prime}}(r) \phi_{g^{\prime}}(r)=\frac{1}{\lambda} \chi_{g} \sum_{g^{\prime=1}}^{G} \nu \Sigma_{f g^{\prime}}(r) \phi_{g^{\prime}}(r)
$$


Equation 1.2 can be written in operator matrix notation as

$$
-\underline{\nabla} \cdot \mathbb{D}(r) \underline{\nabla} \Phi(r)+[\mathbb{M}(r)-\mathbf{T}(r)] \Phi(r)=\frac{1}{\lambda} \mathbb{B}(r)_{\Phi}(r)
$$

where $\mathbb{D}, \mathbb{I}, \mathbf{T}$, and $\mathbb{B}$ are $\mathrm{G} \times \mathrm{G}$ group matrices defined by

$$
\begin{aligned}
& \mathbb{D}(r)=\operatorname{Diag}\left[D_{1}(r) \ldots D_{g}(r) \ldots D_{G}(r)\right] \\
& \mathbb{I M}(r)=\operatorname{Diag}\left[\Sigma_{1}(r) \ldots \Sigma_{g}(r) \ldots \Sigma_{G}(r)\right] \\
& \mathbf{T}(r)=\left[\begin{array}{cccc}
0 & -\Sigma_{12}(r) & \cdots & -\Sigma_{1 G}(r) \\
-\Sigma_{21}(r) & 0 & \cdot & -\Sigma_{2 G}(r) \\
\cdot & \cdot & & \cdot \\
\cdot & \cdot & & \cdot \\
-\Sigma_{G 1}(r) & -\Sigma_{G 2}(r) & \cdots & 0
\end{array}\right] \\
& \mathbb{B}(r)=\left(\begin{array}{c}
x_{1} \\
\cdot \\
\cdot \\
x_{G}
\end{array}\right)\left[\begin{array}{lll}
\nu \Sigma_{f 1}(r) \ldots v \Sigma_{f G}(r)
\end{array}\right.
\end{aligned}
$$

and $\Phi(r)$ is the group flux vector

$$
\Phi(r)=\operatorname{Col}\left[\phi_{1}(r) \ldots \phi_{G}(r)\right]
$$

In problems where no upscattering is present, $\Sigma_{\mathrm{gg}^{\prime}}(\mathrm{r})=0$ for $\mathrm{g}<\mathrm{g}^{\prime}$, and $\mathbb{T}$ becomes $\mathrm{G} \times \mathrm{G}$ lower triangular.

It is also convenient to define the group current vector $\underline{J}(r)$

$$
\underline{J}(r)=\operatorname{Col}\left[\underline{j}_{1}(r) \ldots \underline{j}_{G}(r)\right]
$$

and the $G \times G$ group absorption, scattering and production matrix $\mathbb{\Lambda}(r)$

$$
\Lambda(r)=\mathbb{M}(r)-\mathbb{I}(r)-\frac{1}{\lambda} \mathbb{B}(r)
$$


Equations 1.1 and 1.2 may then be written simply as

$$
\begin{aligned}
& \underline{J}(r)+\mathbb{D}(r) \underline{\nabla} \Phi(r)=0 \\
& \underline{\nabla} \cdot \underline{J}(r)+\Lambda(r) \Phi(r)=0
\end{aligned}
$$

and

$$
-\underline{\nabla} \cdot \mathbb{D}(r) \underline{\nabla} \Phi(r)+\Lambda(r) \Phi(r)=0
$$

respectively. These forms of the group diffusion equations will be used throughout this report. The boundary conditions on $\Phi(\mathrm{r})$ are of the homogeneous Neumann or Dirichlet type, ${ }^{6}$ while the normal component of the current $\underline{J}(r)$ is required to be continuous across all internal interfaces.

\subsection{Solution Methods}

All of the solution methods which can be employed in order to obtain approximate solutions to the time-dependent, multigroup diffusion equations may be conveniently classified as belonging in the area of either nodal analysis or modal analysis, or a combination of the two: modal-nodal analysis. The principal concept in each of these analyses is that the neutron flux, a continuous function of many variables, may be approximated as a set of unknown coefficients and/or functions of possibly fewer variables. The ultimate goals of such approximation methods are to produce easily solvable coupled equations which relate the unknowns to each approximation and yield results of acceptable accuracy at a low cost. Various commonly used methods and their drawbacks are discussed below. 


\subsubsection{Nodal Methods}

Nodal methods involve the local approximation of an average flux at points called nodes, where each node represents a distinct region within the reactor in which the average flux is defined. An ordered set of nodes connected by a grid of mesh lines is then used to approximate the spatial flux behavior. The accuracy of such methods is generally governed by the internodal coupling or neutron current approximation inherent in each method.

\section{A. Conventional Finite Difference Equations}

The common finite difference equations used in diffusion theory can be derived using Taylor series expansion, variational techniques, or box integration methods about each spatial node. 7 The second-order diffusion term at each node is replaced by threepoint difference equations relating consecutive nodes in each spatial direction. The resulting band-structured matrix equations exhibit many advantageous mathematical properties and can be solved with the use of simple solution algorithms.

The attractiveness of these difference equations is further enhanced by the fact that, for properly posed problems (including proper boundary conditions), the approximation can be shown to converge to the solution of the differential equation as the mesh size approaches zero. Also, the accuracy of the approximation can be shown to be in general of order $\theta(h),{ }^{8}$ thus error estimates for the approximation are available. It is for these reasons that these equations are frequently invoked as "exact" solutions to diffusion equation problems. The main disadvantage, however, is that, as the 
number of nodes increases, the amount of labor and cost involved in order to obtain an accurate solution increases geometrically. A point of diminishing returns is then quickly reached where further accuracy is prohibitively expensive. Another disadvantage is that any known physical insight or a priori detailed flux behavior cannot be used with this approximation.

A formal derivation of the conventional difference equations is given in section 2.3 of Chapter 2.

\section{B. Gross Coupling Models}

In gross coupling or coarse mesh nodal techniques an attempt is made to decrease drastically the number of nodes needed for solution without significantly decreasing solution accuracy. Many such methods have been proposed by postulating various forms of neutronic coupling or communication interaction between nodes.

1. Phenomenological Model $1^{9,10,11}$

From a physical viewpoint, the reactor can be divided into several distinct regions, each represented by a node located somewhere in that region. Equations of balance relating state variables of interest (average neutron flux, regional power, etc.) can then be written for each region and between region nodes. Internodal coupling is governed by a set of coefficients, say $p_{i j}$, which may account for the number of neutrons born in region $\mathrm{i}$ which appear in region $\mathrm{j}$. A set of algebraic equations can then be written which describe the coupled core dynamics of the nodal interactions. 
The principal drawback of such methods lies in the definition of the interaction parameters $\mathrm{p}_{i j}$. Although the describing equations of the phenomenological model can be directly formulated from diffusion theory, ${ }^{12}$ the method of calculating the coefficients $p_{i j}$ remains unclear. However, the physical simplicity of this model has made it very appealing in coupled kinetics methods development. Much of the work in this field is based on deriving approximations which reduce to this simple conceptual model:

\section{Effective D/L Coupling 13}

These methods are very similar to finite difference approximations in that the structural forms of the resulting difference equations are identical. In order to compensate for the use of large internodal mesh spacing, the reactor constants, and the diffusion coefficients in particular, may be altered so that they correspond in an average sense to those obtained from a fine mesh calculation. 14 In this way it is hoped that the gross internodal coupling will be sufficiently improved to compensate for the large mesh spacings.

It has been shown that such methods can indeed improve internodal coupling for large mesh regions; however, the results are generally not satisfactory since the coupling constants are dependent in an unpredictable way on changes in the properties of the nodes.

\section{Fission Source Coupling 15}

The assumption that the reactor flux can be separated into partial region fluxes due to nodal fission sources permits a consistent derivation of nodal coupled kinetics equations from multigroup 
diffusion theory. Fission modes can be found from detailed flux solutions which are then used to account for internodal coupling. This method gives reasonably accurate results for fast and thermal reactor transients, although the number of nodes necessary to achieve an accurate solution must increase as the form of the spatial flux becomes more detailed.

4. Multichannel Coupling ${ }^{16}$

By partitioning the reactor into regions called channels and allowing only adjacent channel-to-channel interactions, coupling coefficients $p_{i j}$ can be found which represent the net leakage of neutrons from channel $\mathrm{i}$ into channel $\mathrm{j}$ in terms of the corresponding averaged channel fluxes. The coupling coefficients can be calculated using diffusion theory or variational techniques which yield the diffusion equations as stationary conditions. This model is appealing in that it can be shown to reduce to the conventional difference equations when a regular grid of small channel regions is used.

The above examples of gross coupling models are generally unsatisfactory because they require the use of average fluxes defined within large regions of the reactor. More acceptable results are obtained by utilizing known or a priori detailed spatial flux shapes in the regions in the approximation method.

\subsubsection{Modal Methods ${ }^{17}$}

Modal methods imply an extensive rather than local approximation to the spatial neutron flux. In general the flux is represented by a combination of known functions defined over the regions of interest 
with unknown functions as mixing coefficients. Depending upon the approximation employed, relationships among these coefficients can be derived which are hopefully simpler to solve than the original equation.

A. Helmholtz Modes ${ }^{18}$

The diffusion equation for a completely homogeneous reactor formally has an infinite solution set of eigenvalues and corresponding orthogonal eigenfunctions, called Helmholtz modes, which satisfy the homogeneous boundary conditions. For the general case of a heterogeneous reactor, the spatially dependent flux can be approximated as a linear combination of these modes. The major difficulty with this approach is that a large number of modes is required in order to approximate the solution flux, and thus the appeal for this simplistic modal approach is quickly lost.

B. Lambda ${ }^{19}$ and Omega Modes ${ }^{20}$

Although included in the class of modal approximations, these methods require the use of known spatial solutions for timedependent analysis. Lambda modes belong to the set of detailed flux solutions of the time-independent diffusion equations which correspond to different lambda eigenvalues.

A set of detailed flux solutions can also be found from the timedependent diffusion equations by allowing the time-dependent flux to be separable and given in the form $e^{\omega t}$. The solutions of the resulting equations, called $\omega$ modes, correspond to different omega eigenvalues. 
Both of these methods have successfully been used in the transient analysis of coupled nodal kinetics.

\section{Synthesis Methods ${ }^{5,21}$}

The use of synthesis techniques for the derivation of modal approximations is the most exciting and fastest growing area of reactor analysis methods development. This can be attributed to the fact that all diffusion theory approximation schemes, both modal and nodal, and those including time dependence, can be ultimately derived from one single variational principle. Each approximation scheme is therefore dependent solely upon the form of the trial functions used to represent the flux, current, and weighting functions (or adjoint functions) in the synthesis procedure. The outstanding advantage of the synthesis method is that knowledge of a priori detailed flux shapes or other physical insights can be incorporated directly into the approximation method.

\section{Multichannel Synthesis 22}

This method may be viewed as a modal extension of the multichannel gross coupling method. Assuming the flux to be separable in its variables $(x, y, z)$, the number of unknowns can be reduced by specifying detailed flux shapes in any dimension. A common example assumes that in each channel, $k$, of the reactor the flux trial function, $\mathrm{U}_{\mathrm{k}}(\mathrm{x}, \mathrm{y}, \mathrm{z})$, can be expressed as the product of a known transverse flux, $\psi_{\mathrm{k}}(\mathrm{x}, \mathrm{y})$, with an unknown spatially dependent axial flux, $\rho_{\mathrm{k}}(\mathrm{z})$, as:

$$
\mathrm{U}_{\mathrm{k}}(\mathrm{x}, \mathrm{y}, \mathrm{z})=\rho_{\mathrm{k}}(\mathrm{z}) \psi_{\mathrm{k}}(\mathrm{x}, \mathrm{y})
$$


The specification of the flux in two dimensions reduces the problem to an approximation involving only one dimension. If, however, the flux is approximated by a full spatial solution times an unknown constant,

$$
\mathrm{U}_{\mathrm{k}}(\mathrm{x}, \mathrm{y}, \mathrm{z})=\mathrm{F}_{\mathrm{k}} \psi_{\mathrm{k}}(\mathrm{x}, \mathrm{y}, \mathrm{z})
$$

the method reduces to an approximation similar to the multichannel nodal method. Figure 1.1 illustrates the resulting flux shape characteristics of such a method for the one-dimensional case.

The major disadvantage of these multichannel synthesis methods lies in the fact that in general the flux is discontinuous at channel interfaces. 23,24 Therefore the adjacent channel coupling currents, which then must be continuous across these interfaces, ${ }^{\dagger}$ are defined in terms of averaged channel fluxes. Although these methods can produce detailed flux distributions in each channel, their accuracy appears to be not much better than nodal multichannel methods because of the averaged gross neutronic coupling requirements inherent in these methods. 25

2. Overlapping Multichannel Synthesis 26,27

The interchannel neutronic coupling can be improved by requiring that the flux trial functions be continuous across channel interfaces. This can be accomplished by modulating the known expansion functions, $\psi_{\mathrm{k}}$, by piecewise continuous normalized polynomial functions, $\mathrm{p}_{\mathrm{K}}$, which are nonzero only within coupled channels

†Variational techniques used with diffusion theory in general do not allow the flux and current to be simultaneously discontinuous. Further clarification is given in section 2.2 of Chapter 2. 


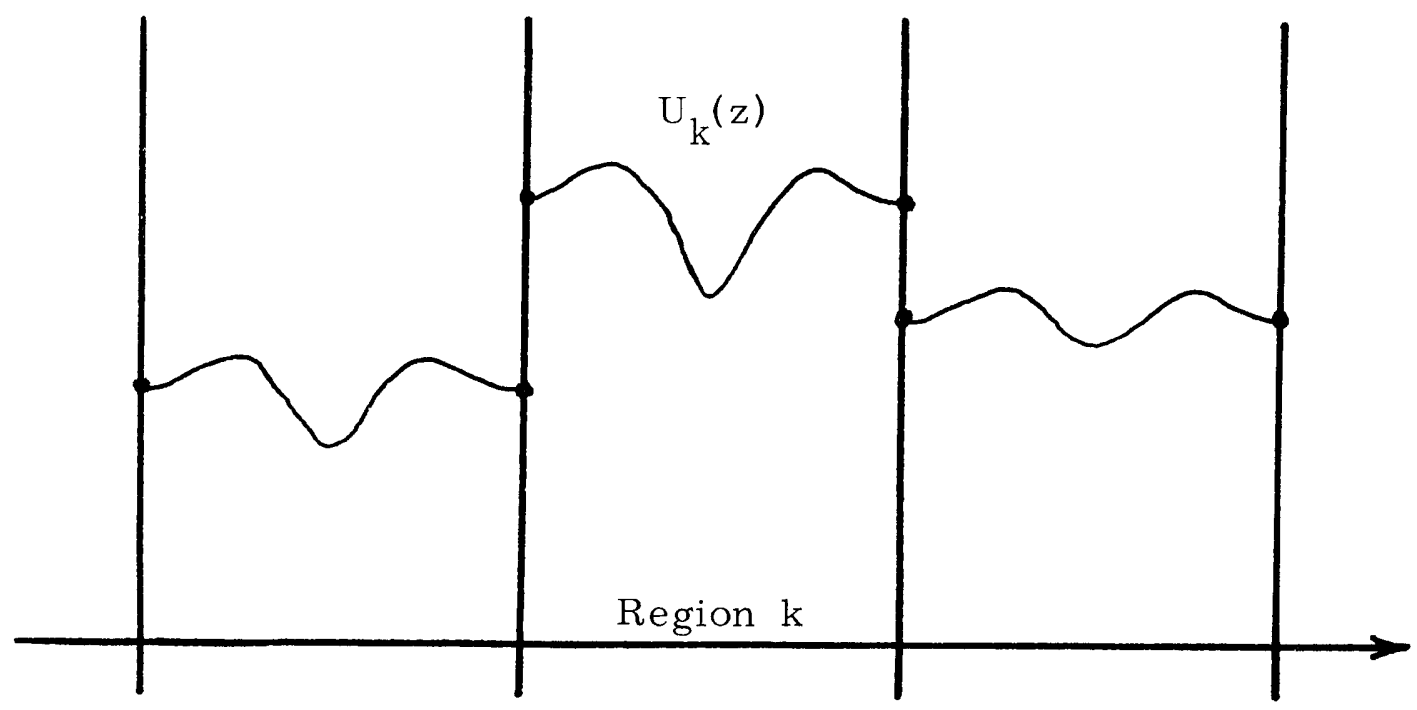

Figure 1.1. Illustration of One-Dimensional Multichannel Synthesis

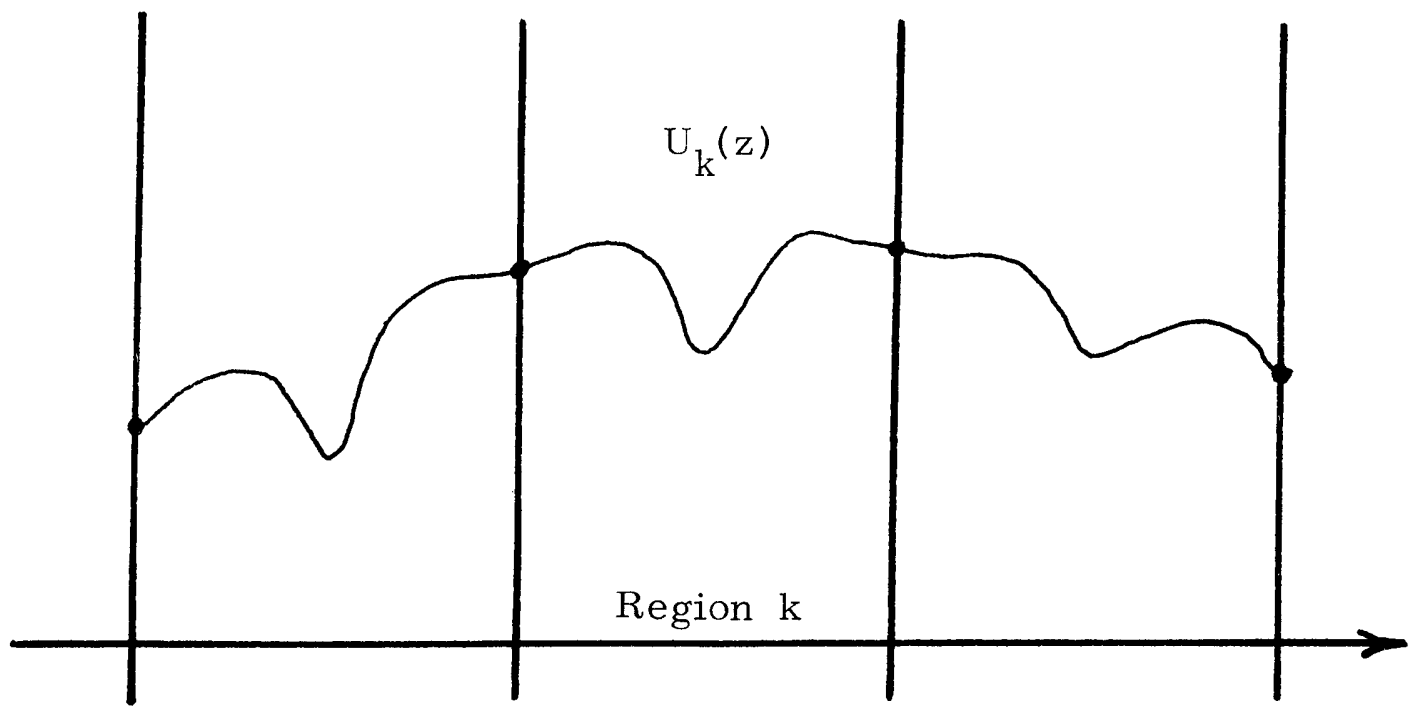

Figure 1.2. Illustration of One-Dimensional Overlapping Multichannel Synthesis 
of interest, providing the expansion functions are continuous over all channels for which the corresponding polynomial functions are nonzero. Such polynomials are required to be normalized to unity at the coupling interface and zero along the external boundary of the channels in order to preserve flux trial function continuity.

In one dimension represented by the continuous variable $\mathrm{z}$ and $\mathrm{K}$ mesh regions bounded by the nodes $\mathrm{z}_{\mathrm{k}}$ where $\mathrm{k}=1$ to $\mathrm{K}+1$, for example, the simple linear functions

$$
p_{k}(z)=\left\{\begin{array}{cl}
\frac{z^{-} z_{k-1}}{z_{k}-z_{k-1}} & z_{k-1} \leqslant z \leqslant z_{k} \\
\frac{z_{k+1}-z}{z_{k+1}-z_{k}} & z_{k} \leqslant z \leqslant z_{k+1} \\
0 & \text { otherwise }
\end{array}\right.
$$

satisfy these conditions. The flux can then be approximated as

$$
U(z)=\sum_{k=1}^{K} F_{k} p_{k}(z) \psi_{k}(z)
$$

where the set of $\mathrm{F}_{\mathrm{k}}$ 's are the unknowns of the method. The resulting flux shape characteristics of this approximation are illustrated in Figure 1.2.

Approximations based on this synthesis method are dependent upon the class of overlapping polynomial functions used as well as the form of the current trial functions employed. The form of the current is extremely important in that it specifies the coupling interaction between regions and in this sense governs the usefulness and accuracy 
of the approximation. Work performed with this method to date has used current trial functions of a form similar to those of the flux trial function. Although the results of these investigations have been encouraging, such methods do not reduce to more simple known approximation methods. In addition, the band-structured matrix equations which arise from the use of such methods do not exhibit mathematical properties desired of such approximation schemes and may be difficult and costly to solve.

\subsubsection{Modal-Nodal Methods}

Approximation methods have also been developed in which the flux has a known extensive definition, or shape, and the unknowns a re local flux values averaged in accordance with their corresponding extensive definition. Such modal-nodal methods retain all of the advantages of modal methods while generally reducing the number of unknowns and producing matrix equations which have desirable mathematical properties for numerical approximation and solution.

The finite element method is the best example of a modal-nodal approximation. Greater accuracy than that of conventional difference techniques can be obtained by allowing the flux in each region of interest to be represented as a polynomial which is continuous at region interfaces. The forms of the flux approximations and the resulting difference equations which arise from the use of the finite element method are described in detail in section 2.3 of Chapter 2. 
The purpose of this report is to present an original and consistent class of modal-nodal coarse mesh approximation methods which retain given or known detailed flux structure within the regions of interest, while providing detailed neutronic coupling between adjacent regions. These methods are consistent in that they are derived from a general variational principle and are a systematic extension of the finite element method as applied to diffusion theory reactor analysis.

For purposes of simplicity, the methods will be developed for the case of one-dimensional, time-dependent, multigroup diffusion theory, although it is expected that these methods can be extended to the general spatially dependent kinetics problem with relative ease.

The remainder of this report is organized as follows. Chapter 2 summarizes the use of variational principles and synthesis techniques in time-independent diffusion theory. The difference equations of the finite element methods applied in one dimension are derived using modal-nodal trial function forms in order to illustrate the use of these techniques. The forms of the proposed approximation methods are given in Chapter 3. The resulting finite difference equations are presented and boundary conditions discussed for approximation methods involving both linear and cubic Hermite basis functions. The numerical properties of the resulting matrix equations, as well as their numerical solution scheme, and useful programming techniques are discussed in Chapter 4. Chapter 5 presents results of the proposed methods for four representative one-dimensional PWR configurations, and compares the results with those of coarse mesh finite element methods. Finally, Chapter 6 presents conclusions and recommendations as well as comments concerning the possibility of extending the proposed methods to multidimensional geometries. 
Chapter 2

\section{VARIATIONAL DERIVATION OF FINITE DIFFERENCE APPROXIMATIONS IN TIME-INDEPENDENT MULTIGROUP DIFFUSION THEORY}

The application of variational calculus to the describing equations of physical systems is perhaps the most general and powerful method of obtaining approximate solutions in mathematical physics. Variational methods seek to combine known "trial functions" into approximate solutions through the use of a variational functional which characterizes the equations of the system.

Essentially, variational methods consist of first finding a characteristic functional whose first-order variation when set to zero yields the describing equations of the system as its Euler equations. A class of trial functions, given in terms of known functions and unknown coefficients (or functions), is then chosen to approximate the solutions of the describing equations. These trial functions are then substituted into the variational functional, and its first variation is set to zero. Allowing arbitrary variations in all of the trial function unknowns results in a set of relationships among the unknowns. These relationships when solved then yield the "best" obtainable approximate solution within the space of trial functions given.

Variational methods can be thought of as a class of weighted residual methods since "weighting functions" appear in the functional and in the equations that result from setting the first variation of the functional to zero. The weighting functions are determined by the 
form of the functional itself; or equivalently, by the set of Euler equations selected to describe the system. In non-self adjoint problems, the adjoint equations a re generally included in the set of Euler equations. The inclusion of corresponding "adjoint trial functions" in the functional results in adjoint weighting in the variation equations and allows greater approximation flexibility of the variational method.

\subsection{Calculus of Variations Applied to Diffusion Theory}

The time-independent multigroup diffusion equations as given by Eq. 1.3 can be written as

$$
\mathrm{IH} \Phi=\frac{1}{\lambda} \mathbb{B} \Phi
$$

where

$$
\mathbb{H}=-\underline{\nabla} \cdot \mathbb{D} \underline{\nabla}+\mathbb{M}-\mathbf{T}
$$

Since the multigroup diffusion equations are not self-adjoint, it is convenient to introduce the adjoint diffusion equations

$$
\mathrm{IH}^{*} \Phi^{*}=\frac{1}{\lambda} \mathbb{B}^{*} \Phi^{*}
$$

where $\mathbb{H}^{*}$ and $\mathbb{B}^{*}$ are the adjoint operators corresponding to $\mathbb{H}$ and $\mathbb{B}$, respectively, and are defined as: ${ }^{3}$

$$
\begin{aligned}
& \mathbb{H}^{*}=\mathbb{H}^{\mathrm{T}}=-\underline{\nabla} \cdot \mathbb{D} \underline{\nabla}+\mathbb{I} \mathbf{M}-\mathbf{T}^{\mathrm{T}} \\
& \mathbb{B}^{*}=\mathbb{B}^{\mathrm{T}}
\end{aligned}
$$

since $\mathbb{D}$ and IM are diagonal. $\Phi^{*}$ is the group adjoint flux vector, or importance vector, which must obey the same boundary conditions as $\Phi .28$ 
The exact solutions $\Phi(r)$ and $\Phi^{* *}(r)$ of the diffusion equations and the adjoint diffusion equations can be approximated by flux and adjoint flux trial functions denoted as $U(r)$ and $U^{*}(r)$ using a variational functional of the form

$$
\mathscr{F}_{1}\left[\mathrm{U}, \mathrm{U}^{*}\right]=\frac{1}{\lambda}=\frac{\int_{\mathrm{R}} \mathrm{U}^{*^{\mathrm{T}}} \mathrm{IHU} \mathrm{dr}}{\int_{\mathrm{R}} \mathrm{U}^{*^{\mathrm{T}}} \mathbb{B} \mathrm{BU} \mathrm{dr}}
$$

where it is assumed that the group-theory flux trial function vectors $\mathrm{U}^{* *}$ and $\mathrm{U}$ as well as the group current vectors $\mathbb{D} \underline{\nabla} \mathrm{U}^{* *}$ and $\mathbb{D} \underline{\nabla} \mathrm{U}$ are everywhere continuous, and that $U^{*}$ and $U$ vanish outside the reactor region $\mathrm{R}$. Allowing arbitrary trial function variations, denoted by $\delta U^{*}$ and $\delta U$, making $\mathscr{F}_{1}$ stationary first with respect to $U^{*}$ and then with respect to $\mathrm{U}$ results ${ }^{29}$ in the following equations:

$$
\begin{aligned}
& \int_{R} \delta U^{* T}\left[\mathbb{H U}-\frac{1}{\lambda} \mathbb{B B U}\right] \mathrm{dr}=0 \\
& \int_{\mathrm{R}}\left[\mathrm{U}^{* \mathrm{~T}} \mathbb{H}-\frac{1}{\lambda} \mathrm{U}^{* \mathrm{~T}} \mathbb{B}\right] \delta \mathrm{Udr}=0
\end{aligned}
$$

The above equations, containing the desired Euler equations, are the equations upon which the approximation method is based.

A significant characteristic of this approximation form is the property of exact solution reproduction. Although general choices of the trial functions $U$ and $U^{*}$ result in approximate eigenvalues which may differ substantially from the exact solution eigenvalue, the exact solutions, when chosen within the given class of trial functions, are yielded as the result of the approximation along with the exact solution eigenvalue. 
The nature of the above approximation depends solely upon the forms of the flux trial functions given. Each trial function can be defined in terms of unknown coefficients (or functions) and known functions. Independent variation of the unknown coefficients of the adjoint trial function in Eq. 2.4a will yield the "best" flux solution obtainable for that class of flux and adjoint flux trial functions given. The corresponding "best" adjoint flux solution can be found in an analogous manner using Eq. 2.4b. These techniques are illustrated in the next section.

Another functional incorporating the flux and adjoint flux diffusion equations can be defined as

$$
\mathscr{F}_{2}\left[\mathrm{U}^{*}, \mathrm{U}\right]=\int_{\mathrm{R}} \mathrm{U}^{*{ }^{*}}\left[\mathrm{HU}-\frac{1}{\lambda} \mathbb{B U}\right] \mathrm{dr}
$$

Although the forms of the above functionals differ, it can be shown that both produce the same variation equations, Eqs. 2.4, when made stationary. The form of $\mathscr{F}_{2}$ and its first variation are much less complex than the form and first variation of $\mathscr{F}_{1}$. For these reasons, functionals of the form of $\mathscr{F}_{2}$ will be used in this report.

\subsection{Discontinuous Trial Functions}

The addition of discontinuous flux trial functions into the class of allowable trial functions for use in diffusion theory variational methods greatly enhances and generalizes the versatility of such methods. 25 However, special provisions must be made in the approximation method itself in order that such trial functions can be properly used. $23,24,30$ In order to account for the discontinuities in the flux (and in general also the current) trial functions, it is necessary to 
include special terms specifying continuity conditions directly within the approximation method. This can be accomplished through the use of a variational functional whose Euler equations include the $\mathrm{P}-1$ equations and continuity conditions for both flux and current. A general functional of this type which allows discontinuous flux, current, and adjoint trial functions can be derived from previous work 30,31 and is given as follows:

$$
\begin{aligned}
\mathscr{F}\left[\mathrm{U}^{*}, \mathrm{U}, \underline{\mathrm{V}}^{*}, \underline{\mathrm{V}}, \alpha, \beta\right]= & \int_{\mathrm{R}}\left\{\mathrm{U}^{*}[\underline{\nabla} \cdot \underline{\mathrm{V}}+\boldsymbol{\Lambda U}]+\underline{\mathrm{V}}^{* T} \cdot\left[\underline{\nabla} \mathrm{U}+\mathbb{D}^{-1} \underline{\mathrm{V}}\right]\right\} \mathrm{dr} \\
& +\int_{\Gamma} \hat{\mathrm{n}} \cdot\left\{\left[\mathrm{U}_{+}^{*} \alpha+\mathrm{U}_{-}^{* T}(\mathrm{I}-\alpha)\right]\left(\underline{\mathrm{V}}_{+}-\underline{\mathrm{V}}_{-}\right)\right. \\
& \left.+\left[\underline{\mathrm{V}}_{+}^{*} \beta+\underline{\mathrm{V}}_{-}^{*}(\mathrm{I}-\beta)\right]\left(\mathrm{U}_{+}-\mathrm{U}_{-}\right)\right\} \mathrm{ds}
\end{aligned}
$$

where $\mathrm{U}^{*}, \mathrm{U}, \underline{\mathrm{V}}^{*}$, and $\underline{\mathrm{V}}$ are the group flux and group current approximations to $\Phi^{*}, \Phi, \underline{J}^{*}$, and $\underline{J}$, respectively, and where the first integral extends over the volume $\mathrm{R}$ of the reactor and the second extends over all interior surfaces $\Gamma$ upon which discontinuities are defined. $\hat{n}$ is the unit vector perpendicular to interior surfaces, and quantities evaluated on sides of surfaces toward which $\hat{\mathrm{n}}$ is pointing are denoted with the subscript (+). Quantities evaluated on sides of surfaces from which $\hat{n}$ is pointing are denoted with the subscript $(-)$. $\alpha$ and $\beta$ are in general $\mathrm{G} \times \mathrm{G}$ undefined variable matrices, and $\mathrm{I}$ is in general a $\mathrm{G} \times \mathrm{G}$ unit matrix, which allow a general treatment of the discontinuities.

The restrictions generally imposed upon trial functions for use in functionals of this type are the following: 
1. The trial functions must be piecewise continuous.

2. The trial functions $U$ and $\underline{V}^{*}$ as well as $U^{*}$ and $\underline{V}$ are not allowed to be discontinuous at the same point.

3. The components of $\mathrm{U}^{\mathrm{T}} \underline{\mathrm{V}}^{*}$ and $\mathrm{U}^{*} \underline{\mathrm{V}}$ normal to the exterior surface of the reactor must vanish.

Due to restriction 2 , the general quantities $\alpha$ and $\beta$ always cancel and are never used within these approximation methods.

The first variation of $\mathscr{F}$ can be found in a straightforward manner, and can be simplified to the following form which indicates the desired $\mathrm{P}-1$ and adjoint $\mathrm{P}-1$ equations and the trial function continuity conditions as Euler equations:

$$
\begin{aligned}
& \delta \mathscr{F}=\int_{R}\left\{\delta U^{* T}[\underline{\nabla} \cdot \underline{V}+\boldsymbol{\Lambda} U]+\delta \underline{V}^{* T} \cdot\left[\underline{\nabla} U+\mathbb{D}^{-1} \underline{V}\right]\right. \\
& \left.+\left[-\underline{\nabla} \mathrm{U}^{* \mathrm{~T}}+\underline{\mathrm{V}}^{* \mathrm{~T}} \mathbb{D}^{-1}\right] \cdot \delta \underline{\mathrm{V}}+\left[-\underline{\nabla} \cdot \underline{\mathrm{V}}^{* \mathrm{~T}}+\mathrm{U}^{* \mathrm{~T}} \boldsymbol{\Lambda}\right] \delta \mathrm{U}\right\} \mathrm{dr} \\
& +\int_{\Gamma} \hat{n} \cdot\left\{\delta U^{* T}\left(\underline{V}_{+}-\underline{V}_{-}\right)+\delta \underline{V}^{* T}\left(U_{+}-U_{-}\right)\right. \\
& \left.+\left(\mathrm{U}_{-}^{*}-\mathrm{U}_{+}^{*}\right)^{\mathrm{T}} \delta \underline{\mathrm{V}}+\left(\underline{\mathrm{V}}_{-}^{*}-\underline{\mathrm{V}}_{+}^{*}\right) \delta \mathrm{U}\right\} \mathrm{ds}
\end{aligned}
$$

In most applications, only approximations to the flux and current solutions are desired. In such instances variations in only the adjoint trial functions need be taken. Setting the first variation of $\mathscr{F}$ equal to zero under these conditions and imposing the above trial function restrictions results in the following variation equation for flux and current approximations: 


$$
\begin{aligned}
& \int_{\mathrm{R}}\left\{\delta \mathrm{U}^{* \mathrm{~T}}[\underline{\nabla} \cdot \underline{\mathrm{V}}+\Delta \mathrm{U}]+\delta \underline{\mathrm{V}}^{*} \cdot\left[\underline{\nabla} \mathrm{U}+\mathbb{D}^{-1} \underline{\mathrm{V}}\right]\right\} \mathrm{dr} \\
& +\int_{\Gamma} \hat{\mathrm{n}} \cdot\left\{\delta \mathrm{U}^{* *}\left(\underline{\mathrm{V}}_{+}-\underline{\mathrm{V}}_{-}\right)+\delta \underline{\mathrm{V}}^{*} \mathrm{~T}\left(\mathrm{U}_{+}-\mathrm{U}_{-}\right)\right\} \mathrm{ds}=0
\end{aligned}
$$

The above approximation can also be expressed independently of adjoint trial functions. If the adjoint trial functions are defined as

$$
\begin{aligned}
& \mathrm{U}^{*}=\mathrm{U} \\
& \underline{\mathrm{V}}^{*}=-\underline{\mathrm{V}}
\end{aligned}
$$

then Eq. 2.8 reduces to the Rayleigh-Ritz Galerkin method, a weighted residual method based upon flux weighting.

Regardless of the choice of weighting, the variation equations can be further simplified for those approximation methods which require the currents to obey explicitly Fick's laws:

$$
\begin{aligned}
& \underline{\mathrm{V}}=-\mathbb{D} \underline{\nabla} \mathrm{U} \\
& \underline{\mathrm{V}}^{*}=+\mathbb{D} \underline{\nabla} \mathrm{U}^{*}
\end{aligned}
$$

Under these conditions the variation equations for discontinuous flux and discontinuous current trial functions reduce to

$$
\begin{aligned}
& \int_{\mathrm{R}}\left\{\delta \mathrm{U}^{* \mathrm{~T}} \Lambda \mathrm{U}-\delta \underline{\mathrm{V}}^{* \mathrm{~T}} \cdot \mathbb{D}^{-1} \underline{\mathrm{V}}\right\} \mathrm{dr} \\
& \quad+\int_{\Gamma} \hat{\mathrm{n}} \cdot\left\{\left(\delta \mathrm{U}_{-}^{*}-\delta \mathrm{U}_{+}^{*}\right)^{\mathrm{T}} \underline{\mathrm{V}}+\delta \underline{\mathrm{V}}^{* \mathrm{~T}}\left(\mathrm{U}_{+}-\mathrm{U}_{-}\right)\right\} \mathrm{ds}=0
\end{aligned}
$$

If in addition the flux is required to be everywhere continuous, the variation equations reduce to the appealing forms

$$
\int_{\mathrm{R}}\left\{\delta \mathrm{U}^{* \mathrm{~T}} \mathbb{\Lambda U}-\delta \underline{\mathrm{V}}^{* \mathrm{~T}} \cdot \mathbb{D}^{-1} \underline{\mathrm{V}}\right\} \mathrm{dr}=0
$$


or equivalently

$$
\int_{R}\left\{\delta U^{* * T} \Lambda U+\left(\underline{\nabla} \delta U^{* *}\right)^{T} \cdot \mathbb{D}(\underline{\nabla} U)\right\} d r=0
$$

Variation equations 2.11 and 2.12 are the approximation equations which are used with the finite element methods and the proposed approximation methods.

\subsection{The Finite Element Approximation Methods 32,33}

This section introduces the notation and techniques used in conjunction with the modal-nodal variational analysis of the finite element method approximations in one-dimensional multigroup diffusion theory. These fundamentals are presented in these simple approximations before applying them to the more general proposed approximation method in the next chapter.

The one-dimensional problem is defined by the continuous variable $\mathrm{z}$ and divided into $\mathrm{K}$ adjoining regions which are in general inhomogeneous. Each region $\mathrm{k}$ is bounded by nodes $\mathrm{z}_{\mathrm{k}}$ and $\mathrm{z}_{\mathrm{k}+1}$ and has width $\mathrm{h}_{\mathrm{k}}=\mathrm{z}_{\mathrm{k}+1}-\mathrm{z}_{\mathrm{k}}$. It is convenient to define the dimensionless variable $\mathrm{x}$ within each region $\mathrm{k}$ as

$$
\mathrm{x}=\frac{\mathrm{z}-\mathrm{z}_{\mathrm{k}}}{\mathrm{h}_{\mathrm{k}}}
$$

so that region $\mathrm{k}$ can be described in terms of $\mathrm{z}$ as

$$
\mathrm{z}_{\mathrm{k}} \leqslant \mathrm{z} \leqslant \mathrm{z}_{\mathrm{k}}+\mathrm{h}_{\mathrm{k}}=\mathrm{z}_{\mathrm{k}+1}
$$

or equivalently in terms of $\mathrm{x}$ as

$$
0 \leqslant x \leqslant 1
$$

for each of the regions $k, k=1$ to $K$. This notation will be used throughout this report. 


\subsubsection{The Conventional Finite Difference Equations}

The conventional nodal flux-averaged, three-point, finite difference equations of one-dimensional diffusion theory can be derived from

Eq. 2.8 using discontinuous flux and current multigroup column vector trial functions of the following form: ${ }^{7,8} \dagger$

$$
\begin{aligned}
& \left.\begin{array}{c}
\mathrm{U}(\mathrm{z})=\mathrm{F}_{\mathrm{k}} \\
\mathrm{U}^{*}(\mathrm{z})=\mathrm{F}_{\mathrm{k}}^{*}
\end{array}\right\} \quad\left\{\begin{array}{c}
\mathrm{z}_{\mathrm{k}-1}+\frac{1}{2} \mathrm{~h}_{\mathrm{k}-1} \\
\mathrm{z}_{1} \text { if } \mathrm{k}=1
\end{array}\right\}<\mathrm{z}<\left\{\begin{array}{c}
\mathrm{z}_{\mathrm{k}}+\frac{1}{2} \mathrm{~h}_{\mathrm{k}} \\
\mathrm{z}_{\mathrm{K}+1} \text { if } \mathrm{k}=\mathrm{K}+1
\end{array}\right\} \mathrm{k=1} \text { to } \mathrm{K}+1 .
\end{aligned}
$$

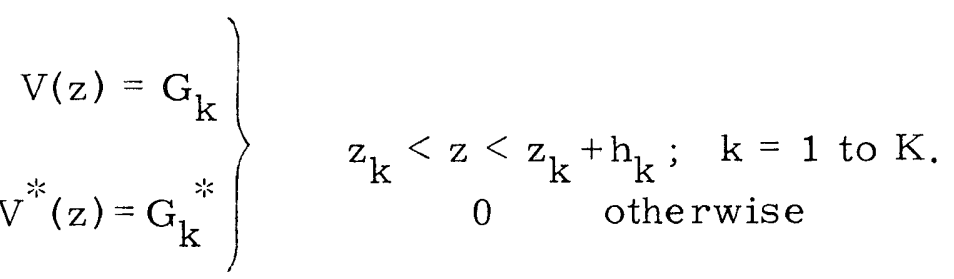

The forms of these trial functions are illustrated in Figure 2.1.

Inserting these trial functions into variation equation 2.8 results in the equation

$$
\begin{aligned}
& \delta \mathrm{F}_{1}^{*}{ }^{\mathrm{T}}\left\{\int_{0}^{\frac{1}{2}} \Lambda_{1} \mathrm{~F}_{1} \mathrm{~h}_{1} \mathrm{dx}+\mathrm{G}_{1}-\mathrm{G}_{0}\right\} \\
& \quad+\sum_{\mathrm{k}=2}^{\mathrm{K}} \delta \mathrm{F}_{\mathrm{k}}^{*}\left\{\int_{\frac{1}{2}}^{1} \Lambda_{\mathrm{k}-1} \mathrm{~F}_{\mathrm{k}} \mathrm{h}_{\mathrm{k}-1} \mathrm{dx}+\int_{0}^{\frac{1}{2}} \Lambda_{\mathrm{k}} \mathrm{F}_{\mathrm{k}} \mathrm{h}_{\mathrm{k}} \mathrm{dx}+\mathrm{G}_{\mathrm{k}}-\mathrm{G}_{\mathrm{k}-1}\right\} \\
& +\delta \mathrm{F}_{\mathrm{K}+1}^{* \mathrm{~T}}\left\{\int_{\frac{1}{2}}^{1} \Lambda_{\mathrm{K}} \mathrm{F}_{\mathrm{K}+1} \mathrm{~h}_{\mathrm{k}} \mathrm{dx}+\mathrm{G}_{\mathrm{K}+1}-\mathrm{G}_{\mathrm{K}}\right\} \\
& \quad+\sum_{\mathrm{k}=1}^{\mathrm{K}} \delta \mathrm{G}_{\mathrm{k}}^{*}\left\{\int_{0}^{1} \mathbb{D}_{\mathrm{k}}^{-1} \mathrm{G}_{\mathrm{k}} \mathrm{h}_{\mathrm{k}} \mathrm{dx}+\mathrm{F}_{\mathrm{k}+1}-\mathrm{F}_{\mathrm{k}}\right\}=0
\end{aligned}
$$

Thifting the domain of definition of the trial functions results in other approximation schemes with equivalently averaged nuclear constants. 34 


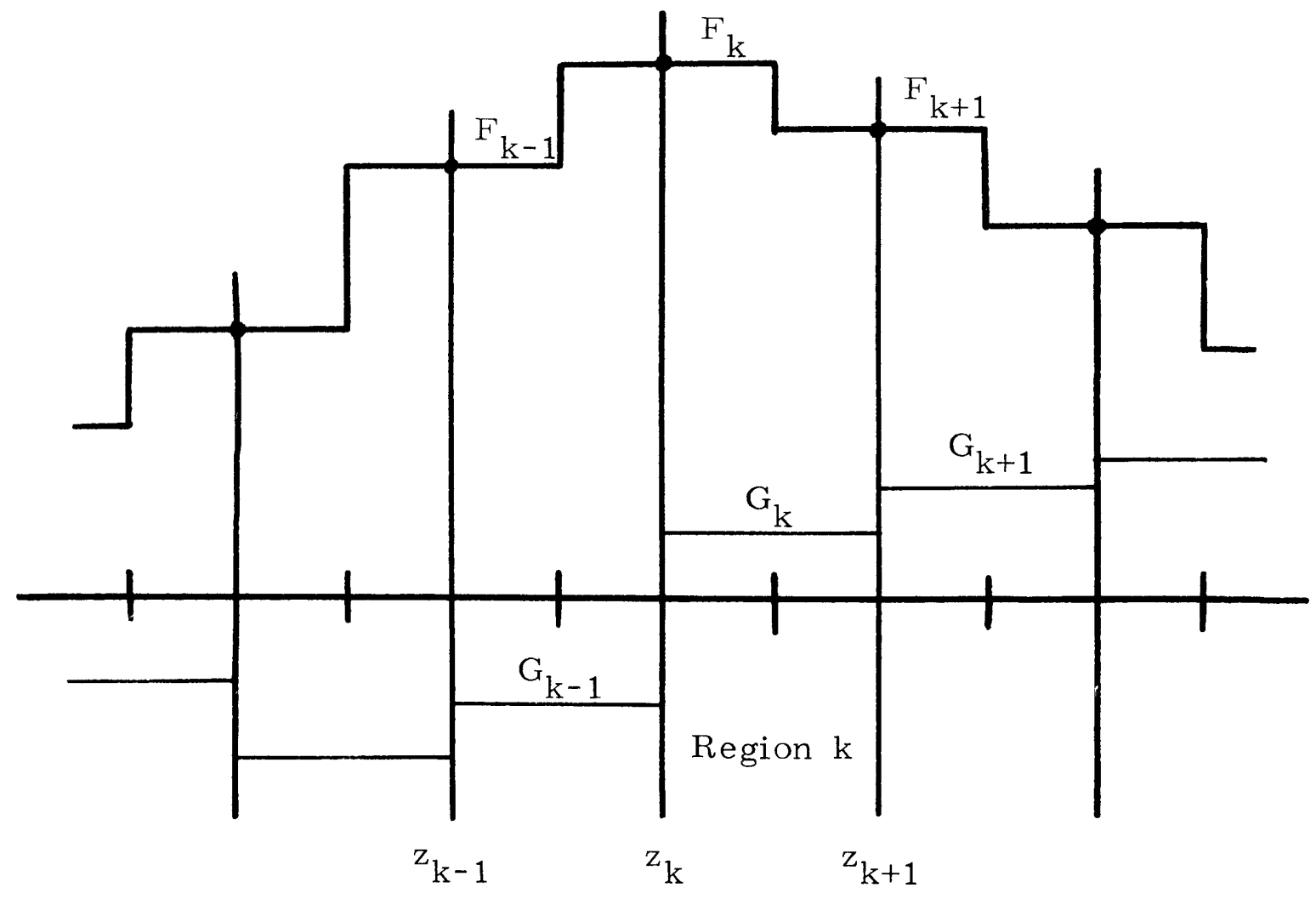

Figure 2.1. Conventional Nodal Finite Difference Approximation Trial Function Forms 
Independent variation of all $\mathrm{F}_{\mathrm{k}}^{*}$ and $\mathrm{G}_{\mathrm{k}}^{*}$ then results in a system of $2 K+1$ equations and $2 K+3$ unknowns (including $G_{0}$ and $G_{K+1}$ ). The choice of boundary conditions supplies the missing equations. Zero flux boundary conditions can be imposed by setting $\mathrm{F}_{1}=\mathrm{F}_{\mathrm{K}+1}=0$, which also requires $\delta \mathrm{F}_{1}^{*}=\delta \mathrm{F}_{\mathrm{K}+1}^{*}=0$ thereby eliminating $\mathrm{G}_{0}$ and $\mathrm{G}_{\mathrm{K}+1}$, and results in a system of $2 \mathrm{~K}-1$ equations and $2 \mathrm{~K}-1$ unknowns. Symmetry boundary conditions can be imposed on the left by $G_{0}=-G_{1}$ and on the right by $G_{K+1}=-G_{K}$, resulting in a system of $2 K+1$ equations and $2 \mathrm{~K}+1$ unknowns.

Elimination of all $\mathrm{G}_{\mathrm{k}}, \mathrm{k}=1$ to $\mathrm{K}$, results in the standard threepoint difference equations

$$
\begin{aligned}
& b_{1} F_{1}+c_{1} F_{2}=0 \\
& a_{k} F_{k-1}+b_{k} F_{k}+c_{k} F_{k+1}=0 ; k=2 \text { to } K \\
& a_{K+1} F_{K}+b_{K+1} F_{K+1}=0
\end{aligned}
$$

where Eqs. 2.16a and 2.16c are used for the cases of symmetry boundary conditions. The $\mathrm{G} \times \mathrm{G}$ matrix coefficients $\left\{\mathrm{a}_{\mathrm{k}}, \mathrm{b}_{\mathrm{k}}, \mathrm{c}_{\mathrm{k}}\right\}$ are of the form $\mathrm{A}-\frac{1}{\lambda} \mathrm{B}$ and are defined assuming homogeneous regional nuclear constants in section 1 of Appendix B. The matrix form of Eqs. 2. 16 for the use of zero flux boundary conditions on the left and symmetry on the right is illustrated in Figure 2.2.

\subsubsection{Multichannel Polynomial Synthesis}

The one-dimensional neutron flux $\Phi_{k}(z)$ defined as nonzero only within each region $\mathrm{k}$ for each region $(\mathrm{k}=1$ to $\mathrm{K}$ ) can be approximated within each region as a polynomial of order $\mathrm{N}$ by the power series 


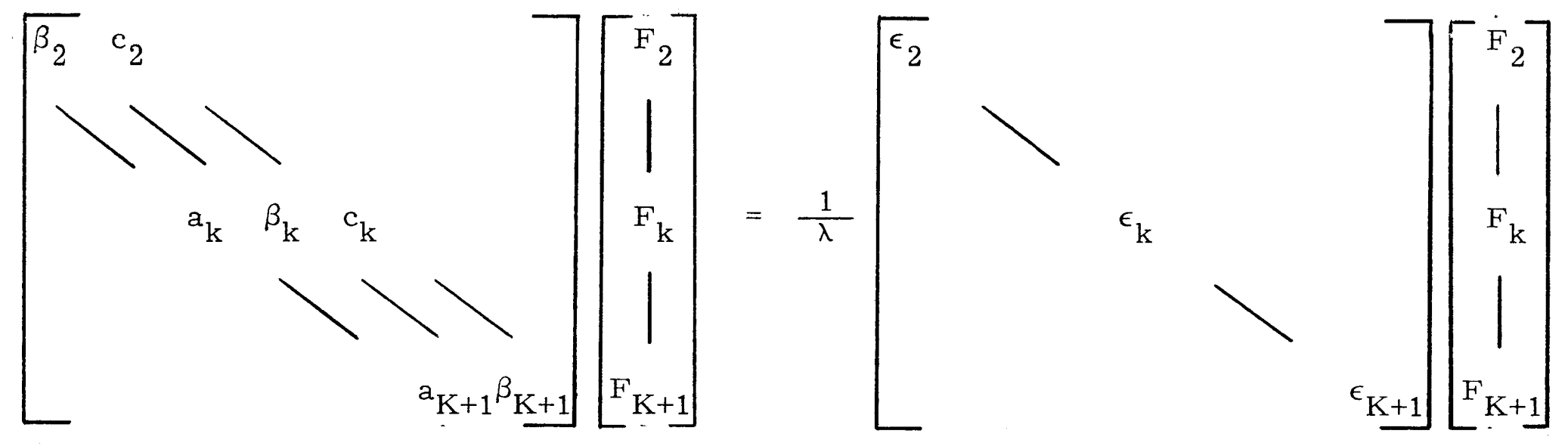

Figure 2.2. Matrix Form of the Conventional Finite Difference Equations. Boundary conditions chosen are zero flux on the left and symmetry "on the right.

$$
\begin{gathered}
\quad a_{k} F_{k-1}+b_{k} F_{k}+c_{k} F_{k+1}=0 ; k=2 \text { to } K . \\
a_{K+1} F_{K}+b_{K+1} F_{K+1}=0 . \\
\text { where: } \quad b_{k}=\beta_{k}-\frac{1}{\lambda} \epsilon_{k} ; k=2 \text { to } K+1 .
\end{gathered}
$$




$$
U_{k}^{(N)}(z)=\sum_{i=0}^{N} a_{k, i} x^{i}
$$

where the distinction between $z$ and $x$ is understood since $0 \leqslant x \leqslant 1$ within each region $\mathrm{k}$. Such approximations are not useful in diffusion theory because: (1) the resulting matrix equations relating the $a_{k, i}$ 's contain full matrices similar to Hibert matrices which may be very difficult to solve; and (2) such matrices a re almost always highly singular and may produce numerical instabilities in the solution method. These difficulties can be eliminated by employing polynomials in the trial functions in the following form:

$$
U_{k}^{(N)}(z) \neq \sum_{i=0}^{N} p_{i}^{(N)}(x) F{ }_{k+\frac{i}{N}}
$$

where the $p_{i}^{(N)}(x)$ are polynomials in $x$ of degree $N$. This form is convenient because for a particular selection of the $p_{i}^{(N)}(x)$ the unknowns $\mathrm{F}_{\mathrm{k}+\frac{\mathrm{i}}{\mathrm{N}}}$ can be defined as the approximate flux solution evaluated at points $z_{i}+\frac{i}{N}$ within region $k$. For high order approximations, $i>0$, the flux can be made continuous by imposing the following restrictions on $p_{i}^{(N)}(x)$ :

$$
p_{i}^{(N)}\left(\frac{\ell}{N}\right)=\left\{\begin{array}{ll}
1 & \ell=i \\
0 & \ell \neq i
\end{array}\right\} \text { for } \ell=0 \text { to } N
$$

The specific polynomial flux approximations of this form through degree $\mathrm{N}=3$ are given below:

$$
\begin{aligned}
& U_{k}^{(0)}(x)=F_{k} \\
& U_{k}^{(1)}(x)=(1-x) F_{k}+x F_{k+1}
\end{aligned}
$$




$$
\begin{aligned}
\mathrm{U}_{\mathrm{k}}^{(2)}(\mathrm{x})= & \left(1-3 \mathrm{x}+2 \mathrm{x}^{2}\right) \mathrm{F}_{\mathrm{k}}+\left(4 \mathrm{x}-4 \mathrm{x}^{2}\right) \mathrm{F}_{\mathrm{k}+\frac{1}{2}}+\left(-\mathrm{x}+2 \mathrm{x}^{2}\right) \mathrm{F}_{\mathrm{k}+1} \\
\mathrm{U}_{\mathrm{k}}^{(3)}(\mathrm{x})= & \left(1-\frac{11}{2} \mathrm{x}+9 \mathrm{x}^{2}-\frac{9}{2} \mathrm{x}^{3}\right) \mathrm{F}_{\mathrm{k}}+\left(9 \mathrm{x}-\frac{45}{2} \mathrm{x}^{2}+\frac{27}{2} \mathrm{x}^{3}\right) \mathrm{F}_{\mathrm{k}+\frac{1}{3}} \\
& +\left(-\frac{9}{2} \mathrm{x}+18 \mathrm{x}^{2}-\frac{27}{2} \mathrm{x}^{3}\right) \mathrm{F}_{\mathrm{k}+\frac{2}{3}}+\left(\mathrm{x}-\frac{9}{2} \mathrm{x}^{2}+\frac{9}{2} \mathrm{x}^{3}\right) \mathrm{F}_{\mathrm{k}+1}
\end{aligned}
$$

An immediate drawback of these approximations lies in the definitions of the corresponding current trial functions. Given a flux polynomial approximation of degree $\mathrm{N}$, polynomial approximations for the current can be of order zero through $\mathrm{N}$, and may even be of higher order than the flux approximation. Each set of chosen trial function pairs ultimately results in a characteristic complex band-structured matrix problem which may or may not have desirable numerical solution properties and is usually very difficult to solve.

Such problems can be eliminated by noting that the use of variational analysis attempts to force the current approximation to obey Fick's law. The obvious solution is direct use of Fick's law in the trial function forms

$$
\mathrm{V}_{\mathrm{k}}(\mathrm{z})=-\mathbb{D}_{\mathrm{k}}(\mathrm{z}) \frac{\mathrm{d}}{\mathrm{dz}} \mathrm{U}_{\mathrm{k}}(\mathrm{z})
$$

which results in simple band-structured matrix equations relating only flux unknowns. The use of current polynomial approximations of order N-1 as given in Eqs. 2.20 with flux approximations of order $\mathrm{N}$, however, does not improve the situation.

The accuracy of these difference equations can be found first by eliminating all non-integer subscripted unknowns, then expanding the resulting three-point difference equations in a Taylor series about 
node $\mathrm{k}$, and comparing results to the exact three-point difference solution known for the one-dimensional case. ${ }^{7,35}$ By comparison of terms containing equal powers of $\mathrm{h}_{\mathrm{k}}$, it can be shown that the $\mathrm{N}=1$ and $\mathrm{N}=2$ polynomial approximations are accurate to order $\theta\left(\mathrm{h}^{2}\right)$ while the $\mathrm{N}=3$ approximation is accurate to order $\theta\left(\mathrm{h}^{3}\right)$.

The approximation of a function by a polynomial of order $\mathrm{N}$ leads immediately to the concept of basis functions. The $\mathrm{N}+1$ polynomial functions which multiply the $\mathrm{N}+1$ unknowns in Eq. 2.18 form a basis for the approximation and can be called basis functions. The simplicity of basis functions becomes apparent in an error analysis of the approximation as follows. An approximate solution $U^{(N)}(z)$ of order $\mathrm{N}$ to the exact one-dimensional solution $\Phi(z)$ can be expressed as

$$
\mathrm{U}^{(\mathrm{N})}(\mathrm{z})=\sum_{\mathrm{k}=1}^{\mathrm{K}} \Phi\left(\mathrm{z}_{\mathrm{k}}\right) \Omega_{\mathrm{k}}^{(\mathrm{N})}(\mathrm{z})
$$

where $\Omega_{\mathrm{k}}^{(\mathrm{N})}(\mathrm{z})$ is a basis function of order N centered about node $\mathrm{z}_{\mathrm{k}}$. By Taylor series expansion about any node, it can be shown ${ }^{36}$ that if $\Omega_{\mathrm{k}}(\mathrm{z})$ satisfies:

$$
\sum_{\mathrm{k}=1}^{\mathrm{K}} \mathrm{z}_{\mathrm{k}}^{\alpha} \Omega_{\mathrm{k}}^{(\mathrm{N})}(\mathrm{z})=\left(\frac{\mathrm{z}}{\mathrm{h}_{\mathrm{k}}}\right)^{\alpha} \text { for }|\alpha| \leqslant \mathrm{N}
$$

then $U^{(N)}(z)$ is an approximation to $\Phi(z)$ accurate to order $\theta\left(h_{k}^{N+1}\right)$.

Basis functions found using Eqs. 2.22 are unique for each $\mathrm{N}$ and generally extend over surrounding regions. The forms of the basis functions for $\mathrm{N} \leqslant 3$ are summarized below and illustrated in Figure 2.3. Since the following basis functions a re symmetric, only the right half, $\mathrm{z} \geqslant \mathrm{z}_{\mathrm{k}}$, is expressly given. 


$$
\begin{aligned}
& N=0: \quad \Omega_{k}^{(0)}(\mathrm{z})= \begin{cases}1 & \mathrm{z}_{\mathrm{k}} \leqslant \mathrm{z}<\mathrm{z}_{\mathrm{k}}+\frac{1}{2} \mathrm{~h}_{\mathrm{k}} \\
0 & \text { otherwise }\end{cases} \\
& N=1: \quad \Omega_{k}^{(1)}(z)= \begin{cases}(1-x) & z_{k} \leqslant z \leqslant z_{k+1} \\
0 & \text { otherwise }\end{cases} \\
& \mathrm{N}=2: \quad \Omega_{\mathrm{k}}^{(2)}(\mathrm{z})=\left\{\begin{array}{cl}
\frac{3}{4}-\mathrm{x}^{2} & \mathrm{z}_{\mathrm{k}} \leqslant \mathrm{z} \leqslant \mathrm{z}_{\mathrm{k}}+\frac{1}{2} \mathrm{~h}_{\mathrm{k}} \\
\frac{1}{2}\left(\frac{3}{2}-\mathrm{x}\right)^{2} & \mathrm{z}_{\mathrm{k}}+\frac{1}{2} \mathrm{~h}_{\mathrm{k}} \leqslant \mathrm{z} \leqslant \mathrm{z}_{\mathrm{k}+1} \\
\frac{1}{2}\left(\frac{1}{2}-\mathrm{x}\right)^{2} & \mathrm{z}_{\mathrm{k}+1} \leqslant \mathrm{z} \leqslant \mathrm{z}_{\mathrm{k}+1}+\frac{1}{2} \mathrm{~h}_{\mathrm{k}+1} \\
0 & \text { otherwise }
\end{array}\right. \\
& \left(\frac{1}{36}\left(30-54 x^{2}+28 x^{3}\right) \quad z_{k} \leqslant z \leqslant z_{k+1}\right. \\
& \mathrm{N}=3: \quad \Omega_{\mathrm{k}}^{(3)}(\mathrm{z})=\left\langle\frac{1}{36}\left(4-24 \mathrm{x}+30 \mathrm{x}^{2}-11 \mathrm{x}^{3}\right) \quad \mathrm{z}_{\mathrm{k}+1} \leqslant \mathrm{z} \leqslant \mathrm{z}_{\mathrm{k}+2}\right. \\
& \frac{1}{36}\left(-1+3 x-3 x^{2}+x^{3}\right) \quad z_{k+2} \leqslant z \leqslant z_{k+3} \\
& \text { otherwise }
\end{aligned}
$$

where $0 \leqslant x \leqslant 1$ within each region $k$ in the above cases.

Use of these basis functions results in approximate solutions which are continuous for $N \geqslant 1$ and whose derivatives $\mathrm{dU}^{(N)}(\mathrm{z}) / \mathrm{dz}$ through $\mathrm{d}^{\mathrm{N}-1} \mathrm{U}^{(\mathrm{N})}(\mathrm{z}) / \mathrm{d} \mathrm{z}^{\mathrm{N}-1}$ are also continuous. In high order approximations in diffusion theory, it is advantageous to retain flux and current continuity and employ basis functions defined over two adjacent regions in order to produce three-point difference equations. This can be accomplished in the $\mathrm{N}=3$ approximations with the cubic Hermite basis functions. 
$\Omega_{\mathrm{k}}^{(0)}(\mathrm{z})$

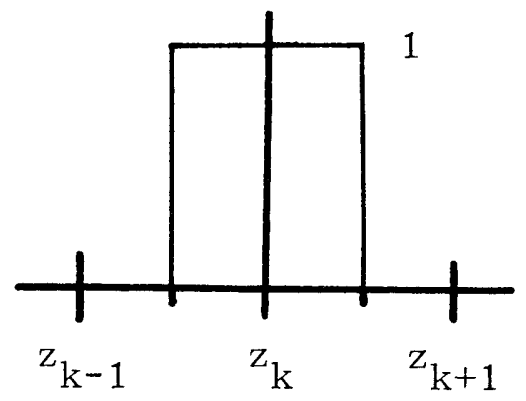

$\Omega_{\mathrm{k}}^{(1)}(\mathrm{z}):$
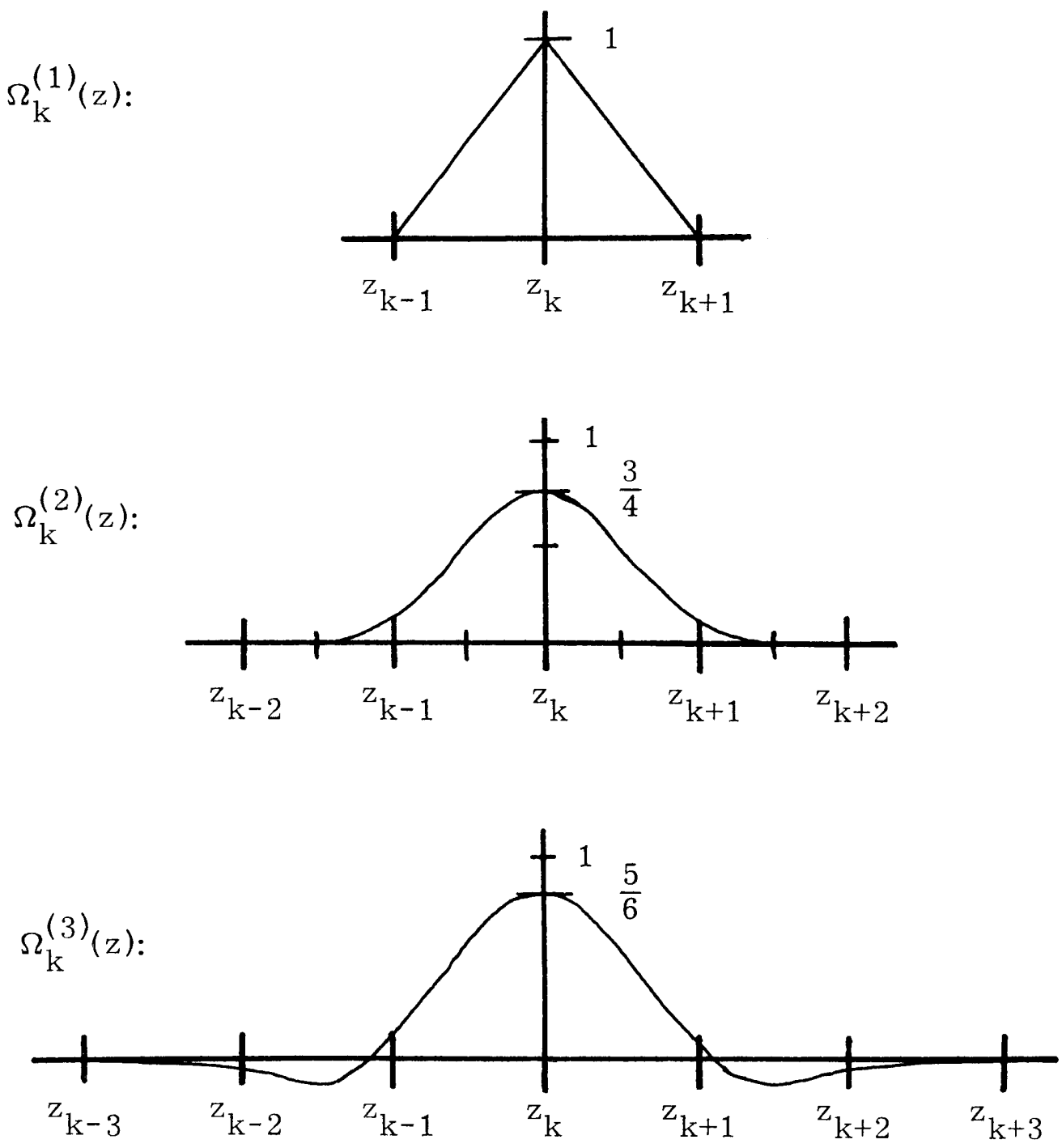

Figure 2.3. Basis Functions of Eqs. 2.23 for $\mathrm{N}=0,1,2$, and 3 
The above cubic basis function $\Omega_{\mathrm{k}}^{(3)}(\mathrm{z})$ can be constructed from a combination of either cubic $B$ splines, $\Omega_{k}^{B}(z)$, or cubic Hermite polynomials, $\Omega_{\mathrm{k}}{ }_{1}(\mathrm{z})$ and $\Omega_{\mathrm{k}}{ }^{\mathrm{H}}(\mathrm{z})$, as follows : ${ }^{37}$

$$
\Omega_{\mathrm{k}}^{(3)}(\mathrm{z})=-\frac{1}{6} \Omega_{\mathrm{k}-1}^{\mathrm{B}}(\mathrm{z})+\frac{4}{3} \Omega_{\mathrm{k}}^{\mathrm{B}}(\mathrm{z})-\frac{1}{6} \Omega_{\mathrm{k}+1}^{\mathrm{B}}(\mathrm{z})
$$

where:

$$
\left.\Omega_{\mathrm{k}}^{\mathrm{B}}(\mathrm{z})=\frac{2}{3} \Omega_{\mathrm{k}}^{\mathrm{H}} 1_{(\mathrm{z})}+\frac{1}{6} \Omega_{\mathrm{k}+1}^{\mathrm{H}} 1 \mathrm{z}\right)-\frac{1}{2} \Omega_{\mathrm{k}+1}^{\mathrm{H}_{2}}(\mathrm{z})
$$

The forms of these cubic B and Hermite polynomials are given below and illustrated in Figures 2.5 and 2.6. Again, only the right half of the functions are expressly given as $\Omega_{k}^{\mathrm{B}}$ and $\Omega_{k}{ }_{1}$ are symmetric, while $\Omega_{\mathrm{k}} \mathrm{H}_{2}$ is antisymmetric.

$$
\begin{aligned}
& \Omega_{\mathrm{k}}^{\mathrm{B}}(\mathrm{z})= \begin{cases}\frac{2}{3}-\mathrm{x}^{2}+\frac{1}{2} \mathrm{x}^{3} & \mathrm{z}_{\mathrm{k}} \leqslant \mathrm{z} \leqslant \mathrm{z}_{\mathrm{k}+1} \\
\frac{1}{6}(1-\mathrm{x})^{3} & \mathrm{z}_{\mathrm{k}+1} \leqslant \mathrm{z} \leqslant \mathrm{z}_{\mathrm{k}+2} \\
0 & \text { otherwise }\end{cases} \\
& \Omega_{\mathrm{k}}^{\mathrm{H}^{1}(\mathrm{z})}= \begin{cases}1-3 \mathrm{x}^{2}+2 \mathrm{x}^{3} & \mathrm{z}_{\mathrm{k}} \leqslant \mathrm{z} \leqslant \mathrm{z}_{\mathrm{k}+1} \\
0 & \text { otherwise }\end{cases} \\
& \Omega_{\mathrm{H}}^{\mathrm{H}}{ }_{2}(\mathrm{z})= \begin{cases}\mathrm{x}-2 \mathrm{x}^{2}+\mathrm{x}^{3} & \mathrm{z}_{\mathrm{k}} \leqslant \mathrm{z} \leqslant \mathrm{z}+1 \\
0 & \text { otherwise }\end{cases}
\end{aligned}
$$

where again $0 \leqslant x \leqslant 1$ in each region $k$.

The fact that the cubic Hermite polynomials form a basis for the cubic basis functions and extend over only two adjacent regions makes them very attractive for use in diffusion theory approximation methods. 


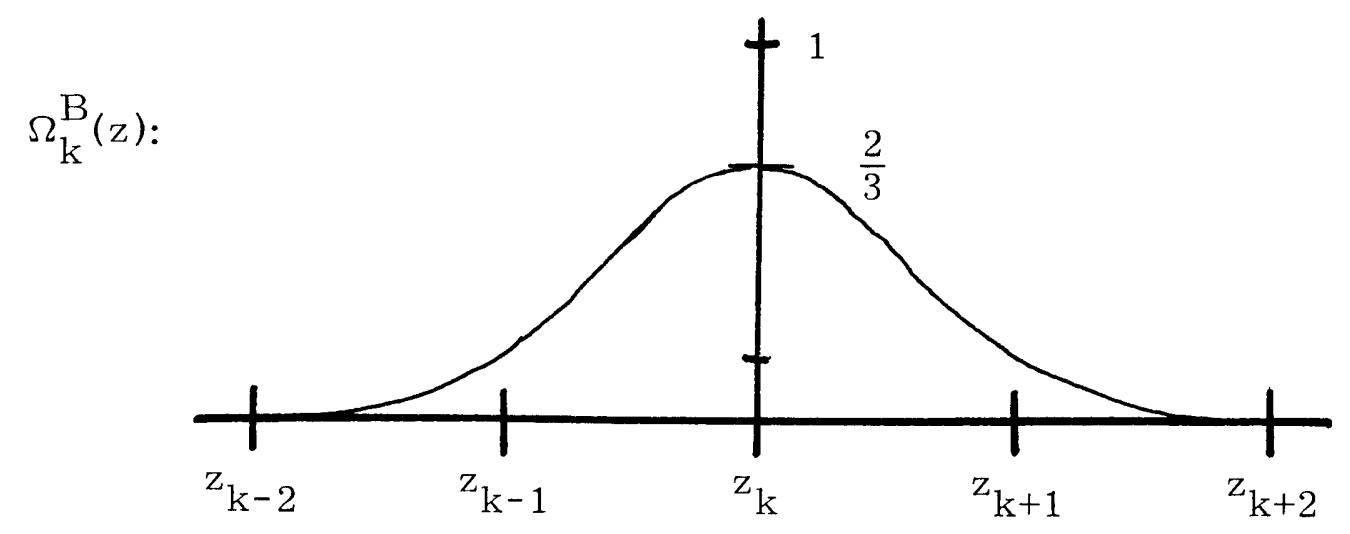

Figure 2.4. Cubic B Spline $\Omega_{\mathrm{k}}^{\mathrm{B}}(\mathrm{z})$ of Eq. 2.26

$\Omega_{\mathrm{k}}^{\mathrm{H}} 1(\mathrm{z})$

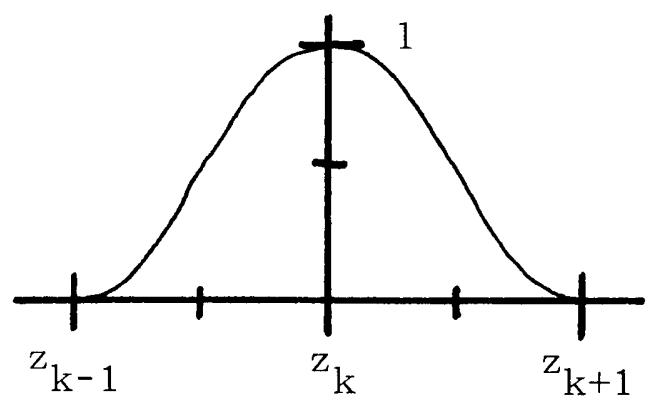

$\Omega_{\mathrm{k}}^{\mathrm{H}}{ }^{2}(\mathrm{z})$

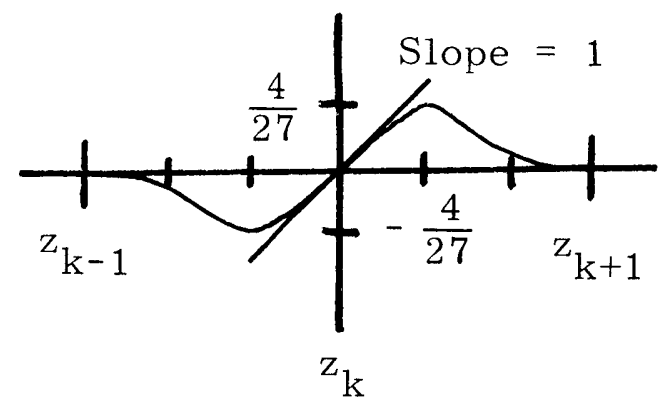

Figure 2.5. Cubic Hermite Basis Functions $\Omega_{k}{ }^{H}(z)$ and $\Omega_{k}{ }^{H}(z)$ of Eqs. 2.27 


\subsubsection{The Linear Basis Function Approximation}

The group flux trial functions defined as nonzero within each region $\mathrm{k}$ can be expressed in modal-nodal form in terms of linear basis functions as

$$
\begin{aligned}
& U_{k}(z)=(1-x) F_{k}+x F_{k+1} \\
& U_{k}^{*}(z)=(1-x) F_{k}^{*}+x F_{k+1}^{*}
\end{aligned}
$$

where $\mathrm{F}_{\mathrm{k}}$ is the approximate group flux column vector at node $\mathrm{z}_{\mathrm{k}}$ and $0 \leqslant x \leqslant 1$ with each region $k$. Although the flux trial functions are continuous, the current trial functions defined within each region by Eqs. 2. 10 are not, and are given by

$$
\begin{aligned}
& \mathrm{V}_{\mathrm{k}}(\mathrm{z})=\frac{1}{\mathrm{~h}_{\mathrm{k}}} \mathbb{D}_{\mathrm{k}}(\mathrm{x})\left[\mathrm{F}_{\mathrm{k}}-\mathrm{F}_{\mathrm{k}+1}\right] \\
& \mathrm{V}_{\mathrm{k}}^{*}(\mathrm{z})=\frac{1}{\mathrm{~h}_{\mathrm{k}}} \mathbb{D}_{\mathrm{k}}(\mathrm{x})\left[\mathrm{F}_{\mathrm{k}+1}^{*}-\mathrm{F}_{\mathrm{k}}^{* k}\right]
\end{aligned}
$$

Insertion of these trial function forms into variation equation $2.12 \mathrm{a}$ results in the equation

$$
\begin{aligned}
& \sum_{\mathrm{k}=1}^{\mathrm{K}} \mathrm{h}_{\mathrm{k}} \int_{0}^{1}\left\{\left[(1-\mathrm{x}) \delta \mathrm{F}_{\mathrm{k}}^{*}+\mathrm{x} \delta \mathrm{F}_{\mathrm{k}+1}^{*}\right]^{\mathrm{T}} \boldsymbol{\Lambda}_{\mathrm{k}}(\mathrm{x})\left[(1-\mathrm{x}) \mathrm{F}_{\mathrm{k}}+\mathrm{x} \mathrm{F}_{\mathrm{k}+1}\right]\right. \\
& \left.+\left[\delta \mathrm{F}_{\mathrm{k}}^{*}-\delta \mathrm{F}_{\mathrm{k}+1}^{*}\right]^{\mathrm{T}} \frac{1}{\mathrm{~h}_{\mathrm{k}}^{2}} \mathbb{D}_{\mathrm{k}}(\mathrm{x})\left[\mathrm{F}_{\mathrm{k}}-\mathrm{F}_{\mathrm{k}+1}\right]\right\} \mathrm{dx}=0
\end{aligned}
$$

Allowing arbitrary variations in all $\mathrm{F}_{\mathrm{k}}^{*}$ results in a system of $\mathrm{K}+1$ equations and $\mathrm{K}+1$ unknowns which can be written as:

$$
\begin{array}{ll}
\mathrm{b}_{1} \mathrm{~F}_{1}+\mathrm{c}_{1} \mathrm{~F}_{2} & =0 \\
\mathrm{a}_{\mathrm{k}} \mathrm{F}_{\mathrm{k}-1}+\mathrm{b}_{\mathrm{k}} \mathrm{F}_{\mathrm{k}}+\mathrm{c}_{\mathrm{k}} \mathrm{F}_{\mathrm{k}+1}=0 ; \mathrm{k}=2, \mathrm{~K} . \\
\mathrm{a}_{\mathrm{K}+1} \mathrm{~F}_{\mathrm{K}}+\mathrm{b}_{\mathrm{K}+1} \mathrm{~F}_{\mathrm{K}+1}=0
\end{array}
$$


where the $\mathrm{G} \times \mathrm{G}$ matrix coefficients $\left\{\mathrm{a}_{\mathrm{k}}, \mathrm{b}_{\mathrm{k}}, \mathrm{c}_{\mathrm{k}}\right\}$ are of the form $A-\frac{1}{\lambda} B$ and are defined assuming homogeneous regional nuclear constants in section 2 of Appendix B. Zero flux boundary conditions can be imposed by use of only Eq. 2.30b with $\mathrm{F}_{1}=\mathrm{F}_{\mathrm{K}+1}=0$, while symmetry boundary conditions require the use of the other equations as well. The matrix form of these equations for the boundary conditions of zero flux on the left and symmetry on the right is given in Figure 2.6.

2.3.4 The Cubic Hermite Basis Function Approximation 38,39

The cubic Hermite polynomials can be incorporated into modalnodal flux trial functions which allow continuous flux and continuous current by defining the flux trial functions within each region $\mathrm{k}$ as

$$
\begin{aligned}
\mathrm{U}_{\mathrm{k}}(\mathrm{z})= & \left(1-3 \mathrm{x}^{2}+2 \mathrm{x}^{3}\right) \mathrm{F}_{\mathrm{k}}+\left(3 \mathrm{x}^{2}-2 \mathrm{x}^{3}\right) \mathrm{F}_{\mathrm{k}+1} \\
& +\left(-\mathrm{x}+2 \mathrm{x}^{2}-\mathrm{x}^{3}\right) \frac{\theta}{\mathrm{h}_{\mathrm{k}}} \mathbb{D}_{\mathrm{k}}^{-1}(\mathrm{x}) \mathrm{G}_{\mathrm{k}}+\left(\mathrm{x}^{2}-\mathrm{x}^{3}\right) \frac{\theta}{\mathrm{h}_{\mathrm{k}}} \mathbb{D}_{\mathrm{k}}^{-1}(\mathrm{x}) \mathrm{G}_{\mathrm{k}+1} \\
\mathrm{U}_{\mathrm{k}}^{*}(\mathrm{z})= & \left(1-3 \mathrm{x}^{2}+2 \mathrm{x}^{3}\right) \mathrm{F}_{\mathrm{k}}^{*}+\left(3 \mathrm{x}^{2}-2 \mathrm{x}^{3}\right) \mathrm{F}_{\mathrm{k}+1}^{*} \\
& +\left(-\mathrm{x}+2 \mathrm{x}^{2}-\mathrm{x}^{3}\right) \frac{\theta}{\mathrm{h}_{\mathrm{k}}} \mathbb{D}_{\mathrm{k}}^{-1}(\mathrm{x}) \mathrm{G}_{\mathrm{k}}^{*}+\left(\mathrm{x}^{2}-\mathrm{x}^{3}\right) \frac{\theta}{\mathrm{h}_{\mathrm{k}}} \mathbb{D}_{\mathrm{k}}^{-1}(\mathrm{x}) \mathrm{G}_{\mathrm{k}+1}^{*}
\end{aligned}
$$

where $\mathrm{k}=1$ to $\mathrm{K}$.

$\mathrm{F}_{\mathrm{k}}$ is again the approximate group flux solution vector at node $\mathrm{z}_{\mathrm{k}}$, and $\mathrm{G}_{\mathrm{k}}$ is proportional to the approximate group current solution vector at node $\mathrm{z}_{\mathrm{k}}$. Application of Fick's law defines the current trial functions for each $\mathrm{k}$ as 


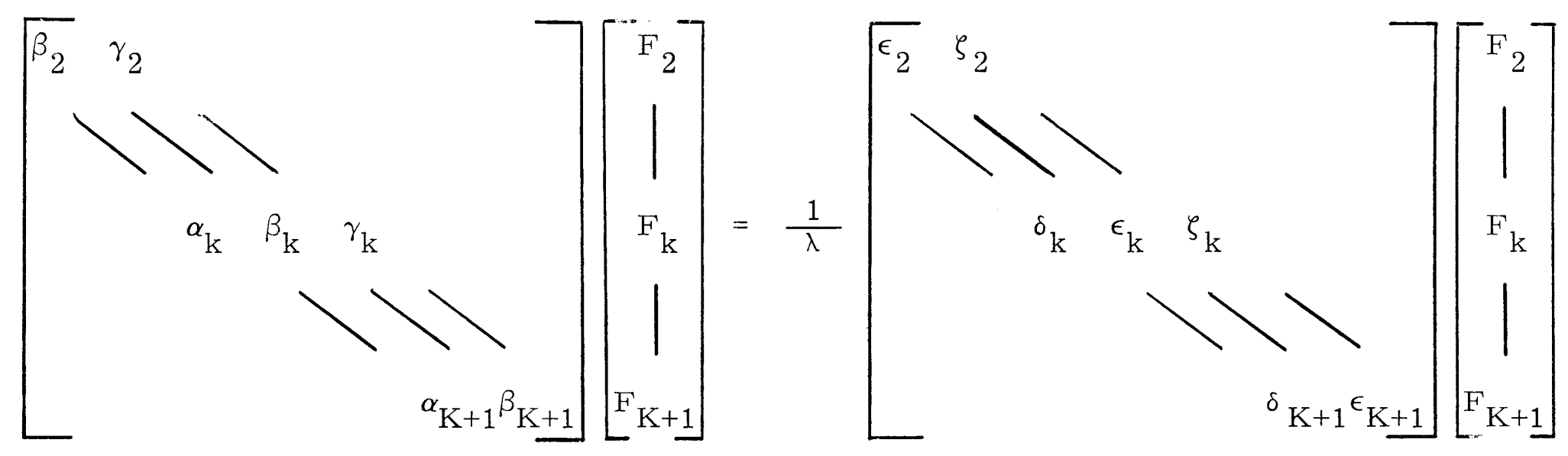

Figure 2.6. Matrix Form of the Linear Finite Element Method Approximation. Eqs. 2.35 for the case of zero flux on the left and symmetry boundary conditions on the right.

$$
\text { where: } \left.\quad \begin{array}{rl}
\mathrm{a}_{\mathrm{k}} & =\alpha_{\mathrm{k}}-\frac{1}{\lambda} \delta_{\mathrm{k}} \\
\mathrm{b}_{\mathrm{k}} & =\beta_{\mathrm{k}}-\frac{1}{\lambda} \epsilon_{\mathrm{k}} \\
\mathrm{c}_{\mathrm{k}} & =\gamma_{\mathrm{k}}-\frac{1}{\lambda} \zeta_{\mathrm{k}}
\end{array}\right\} \quad \mathrm{k}=2 \text { to } \mathrm{K}+1
$$




$$
\begin{aligned}
\mathrm{V}_{\mathrm{k}}(\mathrm{z})= & \frac{1}{\mathrm{~h}_{\mathrm{k}}} \mathbb{D}_{\mathrm{k}}(\mathrm{x})\left(6 \mathrm{x}-6 \mathrm{x}^{2}\right)\left[\mathrm{F}_{\mathrm{k}+1}-\mathrm{F}_{\mathrm{k}}\right] \\
& +\left(1-4 \mathrm{x}+3 \mathrm{x}^{2}\right) \theta \mathrm{G}_{\mathrm{k}}+\left(-2 \mathrm{x}+3 \mathrm{x}^{2}\right) \theta \mathrm{G}_{\mathrm{k}+1} \\
\mathrm{~V}_{\mathrm{k}}^{*}(\mathrm{z})= & \frac{1}{\mathrm{~h}_{\mathrm{k}}} \mathbb{D}_{\mathrm{k}}(\mathrm{x})\left(6 \mathrm{x}-6 \mathrm{x}^{2}\right)\left[\mathrm{F}_{\mathrm{k}}^{*}-\mathrm{F}_{\mathrm{k}+1}^{*}\right] \\
& +\left(-1+4 \mathrm{x}-3 \mathrm{x}^{2}\right) \theta \mathrm{G}_{\mathrm{k}}^{*}+\left(2 \mathrm{x}-3 \mathrm{x}^{2}\right) \theta \mathrm{G}_{\mathrm{k}+1}^{*}
\end{aligned}
$$

Continuity of flux and current are automatically guaranteed since

$$
\begin{aligned}
& \mathrm{U}_{\mathrm{k}}(0)=\mathrm{U}_{\mathrm{k}-1}\left(\mathrm{~h}_{\mathrm{k}-1}\right)=\mathrm{F}_{\mathrm{k}} \\
& \mathrm{U}_{\mathrm{k}}^{*}(0)=\mathrm{U}_{\mathrm{k}-1}^{*}\left(\mathrm{~h}_{\mathrm{k}-1}\right)=\mathrm{F}_{\mathrm{k}}^{*} \\
& \mathrm{~V}_{\mathrm{k}}(0)=\mathrm{V}_{\mathrm{k}-1}\left(\mathrm{~h}_{\mathrm{k}-1}\right)=\theta \mathrm{G}_{\mathrm{k}} \\
& \mathrm{V}_{\mathrm{k}}^{*}(0)=\mathrm{V}_{\mathrm{k}-1}^{*}\left(\mathrm{~h}_{\mathrm{k}-1}\right)=-\theta \mathrm{G}_{\mathrm{k}}^{*} \dagger
\end{aligned}
$$

The normalization constant $\theta$ is introduced in order to produce stiffness matrices having small condition numbers and can be chosen such that $\frac{\theta}{\mathrm{D}_{\mathrm{k}}(0)} \simeq 1$.

Insertion of these trial function forms into variation equation 2. $12 \mathrm{a}$ results in a lengthy equation which can be written as follows:

$\dagger_{\text {Such a choice of }-\mathrm{G}_{\mathrm{k}}^{*}}^{*}$ allows the matrix of coefficients to be positive definite. Cf., Chapter 4. 


$$
\begin{aligned}
& \delta \mathrm{F}_{1}^{* \mathrm{~T}}\left\{\mathrm{~b} 1_{1} \mathrm{~F}_{1}+\mathrm{b} 2_{1} \mathrm{G}_{1}+\mathrm{c} 1_{1} \mathrm{~F}_{2}+\mathrm{c} 2{ }_{1} \mathrm{G}_{2}\right\} \\
& \delta \mathrm{G}_{1}^{* \mathrm{~T}}\left\{\mathrm{~b} 3_{1} \mathrm{~F}_{1}+\mathrm{b} 4_{1} \mathrm{G}_{1}+\mathrm{c} 3{ }_{1} \mathrm{~F}_{2}+\mathrm{c} 4{ }_{1} \mathrm{G}_{2}\right\} \\
& +\sum_{\mathrm{k}=2}^{\mathrm{K}} \delta \mathrm{F}_{\mathrm{k}}^{* \mathrm{~T}}\left\{\mathrm{a} 1_{\mathrm{k}} \mathrm{F}_{\mathrm{k}-1}+\mathrm{a} 2_{\mathrm{k}} \mathrm{G}_{\mathrm{k}-1}+\mathrm{b} 1_{\mathrm{k}} \mathrm{F}_{\mathrm{k}}+\mathrm{b} 2_{\mathrm{k}} \mathrm{G}_{\mathrm{k}}+\mathrm{c} 1_{\mathrm{k}} \mathrm{F}_{\mathrm{k}+1}+\mathrm{c} 2_{\mathrm{k}} \mathrm{G}_{\mathrm{k}+1}\right\} \\
& +\sum_{k=2}^{\mathrm{K}} \delta \mathrm{G}_{\mathrm{k}}^{{ }^{*} \mathrm{~T}}\left\{\mathrm{a} 3_{\mathrm{k}} \mathrm{F}_{\mathrm{k}-1}+\mathrm{a} 4_{\mathrm{k}} \mathrm{G}_{\mathrm{k}-1}+\mathrm{b} 3_{\mathrm{k}} \mathrm{F}_{\mathrm{k}}+\mathrm{b} 4_{\mathrm{k}} \mathrm{G}_{\mathrm{k}}+\mathrm{c} 3_{\mathrm{k}} \mathrm{F}_{\mathrm{k}+1}+\mathrm{c} 4_{\mathrm{k}} \mathrm{G}_{\mathrm{k}+1}\right\} \\
& \delta \mathrm{F}_{\mathrm{K}+1}^{* \mathrm{~T}}\left\{\mathrm{a} 1_{\mathrm{K}+1} \mathrm{~F}_{\mathrm{K}}+\mathrm{a} 2_{\mathrm{K}+1} \mathrm{G}_{\mathrm{K}}+\mathrm{b} 1_{\mathrm{K}+1} \mathrm{~F}_{\mathrm{K}+1}+\mathrm{b} 2_{\mathrm{K}+1} \mathrm{G}_{\mathrm{K}+1}\right\} \\
& \delta \mathrm{G}_{\mathrm{K}+1}^{* \mathrm{~T}}\left\{\mathrm{a}{ }_{\mathrm{K}+1} \mathrm{~F}_{\mathrm{K}}+\mathrm{a}{ }_{\mathrm{K}+1} \mathrm{G}_{\mathrm{K}}+\mathrm{b} 3_{\mathrm{K}+1} \mathrm{~F}_{\mathrm{K}+1}{ }^{+\mathrm{b}} 4_{\mathrm{K}+1} \mathrm{G}_{\mathrm{K}+1}\right\}=0
\end{aligned}
$$

where the $G \times G$ matrix coefficients $\{a 1, \ldots, c 4\}$ are of the form $A-\frac{1}{\lambda} B$ and are defined assuming homogeneous regional nuclear constants in section 3 of Appendix B.

The choice of either zero flux, $\mathrm{F}_{\mathrm{k}}=0$ as well as $\delta \mathrm{F}_{\mathrm{k}}^{*}=0$, or zero current, $\mathrm{G}_{\mathrm{k}}=0$ as well as $\delta \mathrm{G}_{\mathrm{k}}^{*}=0$, boundary conditions for $\mathrm{k}=1$ or $\mathrm{K}+1$ along with arbitrary variations of the remaining $\mathrm{F}_{\mathrm{k}}^{*}$ and $\mathrm{G}_{\mathrm{k}}^{*}$ results in a system of $2 \mathrm{~K}$ equations and $2 \mathrm{~K}$ unknowns. Figure 2.7 illustrates the matrix form of such a system for the case of zero flux on the left and zero current on the right boundary conditions.

The basis functions and approximation techniques presented in this section are applied to the proposed approximation methods in the next chapter. Also, various techniques for treating zero flux and symmetry boundary conditions are discussed. The matrix properties of the equations resulting from the above finite element approximations and their solution methods are discussed in Chapter 4. 


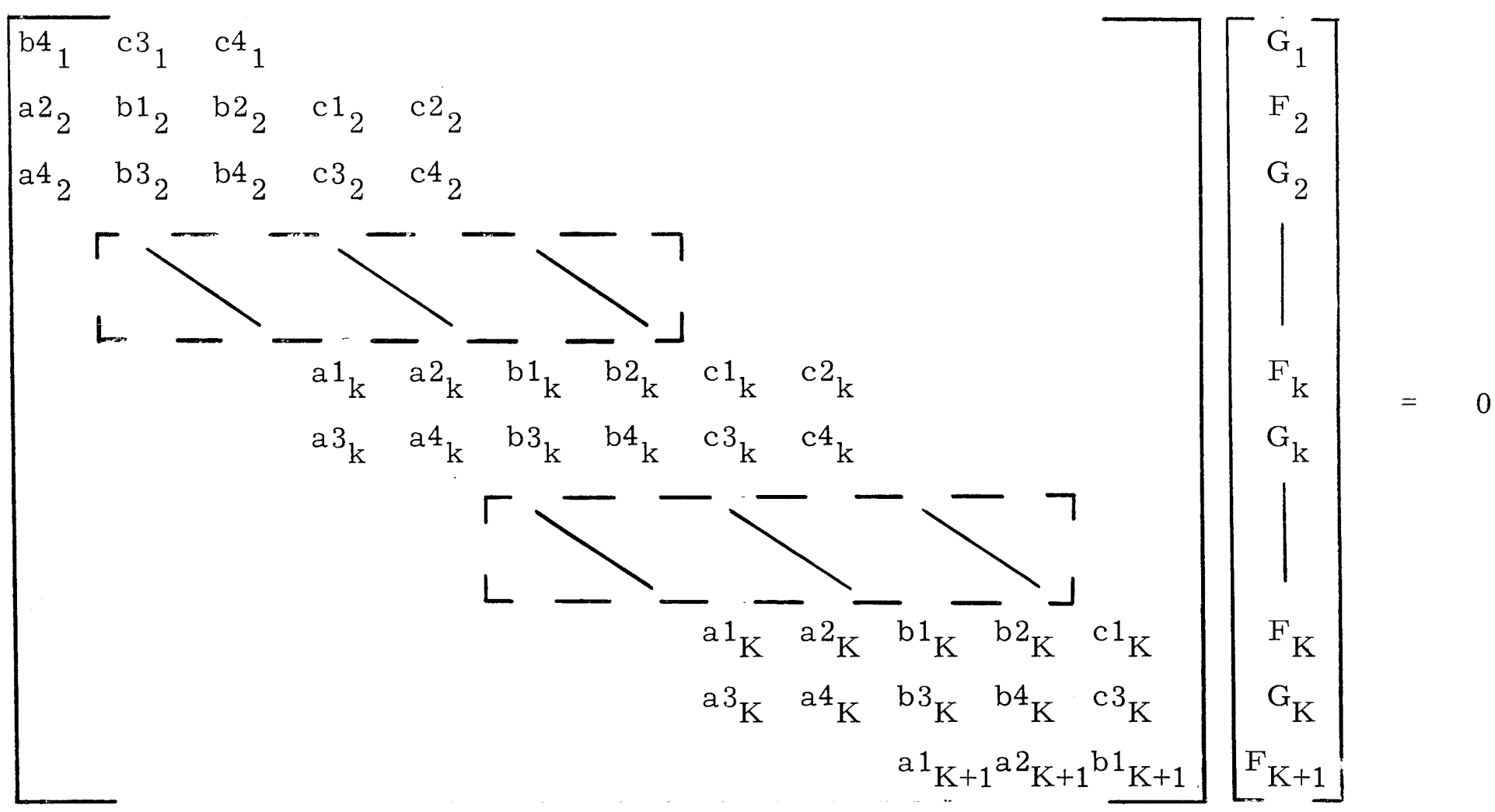

Figure 2.7. Matrix Form of the Cubic Hermite Finite Element Method Approximation. Eqs. 2.39 for the case of zero flux on the left and symmetry boundary conditions on the right.

$$
\begin{aligned}
& \text { where: } \left.\quad a \mathrm{n}_{\mathrm{k}}=\alpha \mathrm{n}_{\mathrm{k}}-\frac{1}{\lambda} \delta \mathrm{n}_{\mathrm{k}}\right) \quad \mathrm{n}=1 \text { to } 4 \text {; } \\
& \left.\mathrm{bn}_{\mathrm{k}}=\beta \mathrm{n}_{\mathrm{k}}-\frac{1}{\lambda} \epsilon \mathrm{n}_{\mathrm{k}}\right\rangle \quad \text { and } \\
& \left.\mathrm{cn}_{\mathrm{k}}=\gamma \mathrm{n}_{\mathrm{k}}-\frac{1}{\lambda} \zeta \mathrm{n}_{\mathrm{k}}\right) \quad \mathrm{k}=1 \text { to } \mathrm{K}+1 \text {. }
\end{aligned}
$$




\section{Chapter 3 \\ DEVELOPMENT OF A CONSISTENT COARSE MESH APPROXIMATION METHOD}

\subsection{Formulation}

The finite element methods have been shown 32,33 to approximate accurately flux solutions and criticality measurements of multigroup diffusion theory when applied to problems allowing homogeneous nuclear material within the mesh regions. Use of such homogeneous material, while simplifying the calculation of the matrix elements (since numerical integrations are not required), may result in limiting the region mesh sizes allowed unless some type of homogenization procedure is used. If the mesh spacing is chosen such that some or all mesh regions are heterogeneous, then direct application of the variational techniques given in Chapter 2 results in weight averaging the nuclear constants with products of the basis functions and their derivatives, as given by the approximation. Although such a procedure is a direct application of the finite element technique, the accuracy of such methods depends upon the placement of the mesh regions and may vary significantly as their placement is altered.

A more useful homogenization procedure which is commonly used in reactor diffusion theory analysis allows the nuclear material within each mesh region to be homogenized by flux weighting with an assumed flux shape determined a priori within that region in order (hopefully) to preserve reaction rates. 
In large reactors the core can be thought of as composed of a lattice of heterogeneous fuel subassemblies containing fuel, clad, coolant channels, and/or absorption control rods. Each subassembly can be divided into several distinct homogeneous regions whose fewgroup microcell macroscopic nuclear constants are found by multigroup energy-dependent calculations. ${ }^{40}$ Detailed subassembly solutions, $\psi_{\mathrm{k}}(\mathrm{r})$, are then found for each subassembly $\mathrm{k}$ by assuming that the current on the boundary of the subassemblies is zero. Flux weighting the nuclear material in each subassembly with the corresponding detailed subassembly solution for each subassembly region then results in regional homogeneous nuclear constants $\left\langle\Sigma_{k}\right\rangle$ which may better approximate the physics of the region.

$$
\left\langle\Sigma_{k}\right\rangle=\frac{\int_{k} \psi_{k}(r) \Sigma_{k}(r) d r}{\int_{k} \psi_{k}(r) d r}
$$

Proper use of detailed flux weighted constants can lead to accurate criticality measurements, but the detailed a priori fine flux structure within each region is lost since it appears only in crosssection homogenization and not in the approximation. Attempts to retain the fine flux structure have only recently been proposed in several multichannel synthesis approximations. $27,41,42,43$ Unfortunately, each of these approximations are approximations in themselves and do not reduce to desirable approximations if the detailed flux solutions are themselves constant, as would be the case in large homogeneous regions. 
Just as the discontinuous multichannel synthesis approximation method can be shown to reduce to low order difference equations (of the type which could result using the finite element method with constant or flat basis functions) when constant trial functions are used, approximation methods are presented below which retain the given detailed flux structure and also reduce to the higher order finite element approximations. The use of linear or cubic Hermite basis functions in the approximation provides flux continuity and results in better approximation accuracy.

The approximations are presented and discussed for the case of one-dimensional, multigroup diffusion theory. Extension to higher dimensions remains a problem that will require some further study. The approximations which are the linear basis functions are considered in the next section, while the approximations using the cubic Hermite basis functions are considered in section 3.3 .

\subsection{The Proposed Linear Basis Function Approximations}

The proposed approximation method utilizing linear basis functions and defined as nonzero within each mesh region $\mathrm{k}, \mathrm{k}=1$ to $\mathrm{K}$, is given by the following modal-nodal trial function forms:

$$
\begin{gathered}
\mathrm{U}_{\mathrm{k}}(\mathrm{z})=\psi_{\mathrm{k}}(\mathrm{x})\left[\psi_{\mathrm{k}}^{-1}(0)(1-\mathrm{x}) \mathrm{F}_{\mathrm{k}}+\psi_{\mathrm{k}}^{-1}(1) \mathrm{x} \mathrm{F}_{\mathrm{k}+1}\right] \\
\mathrm{U}_{\mathrm{k}}^{*}(\mathrm{z})=\psi_{\mathrm{k}}^{*}(\mathrm{x})\left[\psi_{\mathrm{k}}^{*}(0)\left(1-\mathrm{x}_{\mathrm{k}} \mathrm{F}_{\mathrm{k}}^{*}+\psi_{\mathrm{k}}^{*}(1) \mathrm{xF}_{\mathrm{k}+1}^{*}\right]\right. \\
\mathrm{V}_{\mathrm{k}}(\mathrm{z})=\eta_{\mathrm{k}}(\mathrm{x})\left[\psi_{\mathrm{k}}^{-1}(0)(1-\mathrm{x}) \mathrm{F}_{\mathrm{k}}+\psi_{\mathrm{k}}^{-1}(1) \mathrm{xF}_{\mathrm{k}+1}\right] \\
+\frac{1}{\mathrm{~h}_{\mathrm{k}}} \mathbb{D}_{\mathrm{k}}(\mathrm{x}) \psi_{\mathrm{k}}(\mathrm{x})\left[\psi_{\mathrm{k}}^{-1}(0) \mathrm{F}_{\mathrm{k}}-\psi_{\mathrm{k}}^{-1}(1) \mathrm{F}_{\mathrm{k}+1}\right]
\end{gathered}
$$




$$
\begin{aligned}
\mathrm{V}_{\mathrm{k}}^{*}(\mathrm{z})= & \eta_{\mathrm{k}}^{*}(\mathrm{x})\left[\psi_{\mathrm{k}}^{*-1}(0)(1-\mathrm{x}) \mathrm{F}_{\mathrm{k}}^{*}+\psi_{\mathrm{k}}^{*-1}(1) \mathrm{x}_{\mathrm{k}+1}^{*}\right] \\
& +\frac{1}{\mathrm{~h}_{\mathrm{k}}} \mathbb{D}_{\mathrm{k}}(\mathrm{x}) \psi_{\mathrm{k}}^{*}(\mathrm{x})\left[\psi_{\mathrm{k}}^{*-1}(1) \mathrm{F}_{\mathrm{k}+1}^{*}-\psi_{\mathrm{k}}^{*}(0) \mathrm{F}_{\mathrm{k}}^{*}\right]
\end{aligned}
$$

where:

$$
\mathrm{x}=\left(\mathrm{z}-\mathrm{z}_{\mathrm{k}}\right) / \mathrm{h}_{\mathrm{k}}
$$

and $0 \leqslant x \leqslant 1$, as $z_{k} \leqslant z \leqslant z_{k+1}$, for each region $k=1$ to $K$.

$\mathrm{F}_{\mathrm{k}}$ is the unknown approximate group flux column vector at node $\mathrm{z}_{\mathrm{k}}$, and $\psi_{\mathrm{k}}, \psi_{\mathrm{k}}^{*}, \eta_{\mathrm{k}}$, and $\eta_{\mathrm{k}}^{*}$ are $\mathrm{G} \times \mathrm{G}$ diagonal matrices composed of the detailed group flux $\psi_{\mathrm{g}, \mathrm{k}}(\mathrm{z})$ and group current $\eta_{\mathrm{g}, \mathrm{k}}(\mathrm{z})$ solutions, and their adjoints, defined as nonzero only within region k. Because of the variable transformation between $\mathrm{z}$ and $\mathrm{x}, \psi_{\mathrm{k}}(0)$ represents $\psi_{\mathrm{k}}\left(\mathrm{z}_{\mathrm{k}}\right)$, and $\psi_{\mathrm{k}}(1)$ represents $\psi_{\mathrm{k}}\left(\mathrm{z}_{\mathrm{k}+1}\right)$; neither of which, for the moment, is allowed to be zero for any region. The detailed current solutions are given from the detailed flux solutions by Fick's law as

$$
\begin{aligned}
& \eta_{k}(z)=-\mathbb{D}_{k}(z) \frac{d \psi_{k}(z)}{d z} \\
& \eta_{k}^{*}(z)=+\mathbb{D}_{k}(z) \frac{d \psi_{k}^{*}(z)}{d z}
\end{aligned}
$$

As a result, the current trial functions are related to the flux trial functions by analogous expressions.

Continuity of the flux is imposed by the form of the trial functions since

$$
\begin{aligned}
& \mathrm{U}_{\mathrm{k}}(0)=\mathrm{U}_{\mathrm{k}-1}\left(\mathrm{~h}_{\mathrm{k}-1}\right)=\mathrm{F}_{\mathrm{k}} \\
& \mathrm{U}_{\mathrm{k}}^{*}(0)=\mathrm{U}_{\mathrm{k}-1}^{*}\left(\mathrm{~h}_{\mathrm{k}-1}\right)=\mathrm{F}_{\mathrm{k}}^{*}
\end{aligned}
$$

The current trial functions, however, are discontinuous. It is evident 
by comparison to Eqs. 2.28, that this approximation reduces to the linear basis function finite element method if the detailed flux solutions for each group are taken to be constant.

Insertion of these trial function forms in $\mathrm{Eq} .2 .12 \mathrm{a}$ results in the following variation equation:

$$
\begin{aligned}
& \sum_{\mathrm{k}=1}^{\mathrm{K}} \mathrm{h}_{\mathrm{k}} \int_{0}^{1}\left\{\psi_{\mathrm{k}}^{*^{\mathrm{T}}}(\mathrm{x}) \psi_{\mathrm{k}}^{* \mathrm{~T}^{-1}}(0)(1-\mathrm{x}) \delta \mathrm{F}_{\mathrm{k}}^{*} \boldsymbol{\Lambda}_{\mathrm{k}}(\mathrm{x}) \mathrm{U}_{\mathrm{k}}(\mathrm{x})\right. \\
& +\psi_{\mathrm{k}}^{*}{ }^{\mathrm{T}}(\mathrm{x}) \psi_{\mathrm{k}}^{* \mathrm{~T}^{-1}}(1) \mathrm{x} \delta \mathrm{F}_{\mathrm{k}+1}^{*} \boldsymbol{\mathrm { T }}_{\mathrm{k}}(\mathrm{x}) \mathrm{U}_{\mathrm{k}}(\mathrm{x}) \\
& +\left[\eta_{\mathrm{k}}^{* \mathrm{~T}}(\mathrm{x}) \psi_{\mathrm{k}}^{*^{\mathrm{T}^{-1}}}(0)(1-\mathrm{x})-\frac{1}{\mathrm{~h}_{\mathrm{k}}} \mathrm{D}_{\mathrm{k}}(\mathrm{x}) \psi_{\mathrm{k}}^{* \mathrm{~T}^{\mathrm{T}}}(\mathrm{x}) \psi_{\mathrm{k}}^{* \mathrm{~T}^{-1}}(0)\right] \delta \mathrm{F}_{\mathrm{k}}^{* \mathrm{~T}^{\mathrm{T}}} \mathrm{DD}_{\mathrm{k}}^{-1}(\mathrm{x}) \mathrm{V}_{\mathrm{k}}(\mathrm{x}) \\
& \left.+\left[\eta_{\mathrm{k}}^{* \mathrm{~T}}(\mathrm{x}) \psi_{\mathrm{k}}^{* * \mathrm{~T}^{-1}}(1) \mathrm{x}+\frac{1}{\mathrm{~h}_{\mathrm{k}}} \mathbb{D}_{\mathrm{k}}(\mathrm{x}) \psi_{\mathrm{k}}^{* \mathrm{~T}}(\mathrm{x}) \psi_{\mathrm{k}}^{* \mathrm{~T}^{-1}}(1)\right] \delta \mathrm{F}_{\mathrm{k}+1}^{* \mathrm{~T}} \mathbb{D}_{\mathrm{k}}^{-1}(\mathrm{x}) \mathrm{V}_{\mathrm{k}}(\mathrm{x})\right] \mathrm{dx}=0
\end{aligned}
$$

This equation can be written in the form

$$
\begin{gathered}
\delta \mathrm{F}_{1}^{*}\left[\mathrm{~b}_{1} \mathrm{~F}_{1}+\mathrm{c}_{1} \mathrm{~F}_{2}\right] \\
+\sum_{\mathrm{k}=2}^{\mathrm{K}} \delta \mathrm{F}_{\mathrm{k}}^{*}\left[\mathrm{a}_{\mathrm{k}} \mathrm{F}_{\mathrm{k}-1}+\mathrm{b}_{\mathrm{k}} \mathrm{F}_{\mathrm{k}}+\mathrm{c}_{\mathrm{k}} \mathrm{F}_{\mathrm{k}+1}\right] \\
+\delta \mathrm{F}_{\mathrm{K}+1}^{*}\left[\mathrm{a}_{\mathrm{K}+1} \mathrm{~F}_{\mathrm{K}}+\mathrm{b}_{\mathrm{K}+1} \mathrm{~F}_{\mathrm{K}+1}\right]=0
\end{gathered}
$$

where the $G \times G$ matrix coefficients $\left\{a_{k}, b_{k}, c_{k}\right\}$ are integral quantities of the form $A-\frac{1}{\lambda} B$ and are defined in detail in section 1 of Appendix $C$.

External zero flux boundary conditions are easily imposed by setting $\mathrm{F}_{1}=\mathrm{F}_{\mathrm{K}+1}=0$. This requires that $\mathrm{F}_{1}^{*}$ and $\mathrm{F}_{\mathrm{K}+1}^{*}$ must then also be zero, which in turn requires the $\delta \mathrm{F}_{1}^{*}$ and $\delta \mathrm{F}_{\mathrm{K}+1}^{*}$ coefficients in 
Eqs. 3. 5 and 3.6 to vanish. Allowing independent variations in the remaining $\mathrm{F}_{\mathrm{k}}^{*}, \mathrm{k}=2$ to $\mathrm{K}$, results in a matrix problem of the form illustrated in Figure 2.6 which would contain $\mathrm{K}-1$ equations and $\mathrm{K}-1$ unknowns.

Zero current boundary equations are found using symmetry considerations. If, for example, a zero current or symmetry boundary condition is imposed on the right at $\mathrm{z}_{\mathrm{K}+1}$, then a "boundary condition equation" can be derived by assuming a pseudo-region $\mathrm{k}=\mathrm{K}+1$ of width $\mathrm{h}_{\mathrm{K}}$ having mirror image properties of region $\mathrm{K}$ about $\mathrm{z}_{\mathrm{K}+1}$ with corresponding symmetric flux and antisymmetric current properties of the detailed flux and current solutions. These properties in pseudo-region $K+1$ can be related to properties of region $K$ as a function of $x$ in each region as

$$
\begin{aligned}
& \text { DI })_{K+1}(x)=\mathrm{ID}_{K}(1-x) \\
& \triangle_{K+1}(x)=\Lambda_{K}(1-x)
\end{aligned}
$$

and

$$
\begin{aligned}
& U_{K+1}(x)=U_{K}(1-x) \\
& U_{K+1}^{* *}(x)=U_{K}^{*}(1-x) \\
& V_{K+1}(x)=-V_{K}(1-x) \\
& V_{K+1}^{*}(x)=-V_{K}^{*}(1-x)
\end{aligned}
$$

The addition of pseudo-region $\mathrm{K}+1$ to the summation in Eq. 3.5 results in the calculation of coefficients $\mathrm{a}_{\mathrm{K}+1}$ and $\mathrm{b}_{\mathrm{K}+1}$ in $\mathrm{Eq}$. 3.6. Detailed definitions of the $G \times G$ zero current coefficient matrices $b_{1}, c_{1}$, $a_{K+1}$, and $b_{K+1}$, all of which vanish for the case of zero flux boundary conditions, are also given in Appendix C.1. If symmetry is imposed 
on both sides of the problem, independent variations in $\mathrm{F}_{\mathrm{k}}^{*}$ for $\mathrm{k}=1$ to $K+1$ result in a matrix problem of $K+1$ equations and $K+1$ unknowns of similar form as illustrated in Figure 2.6.

Other boundary conditions may be imposed on the approximation, including albedo and reflector boundary conditions, which specify the flux to current ratio at the boundaries. Such conditions will always lead to a variation equation of the form of $\mathrm{Eq} .3 .6$, where in general the matrix coefficients $a_{2}, b_{2}, b_{K}$, and $c_{K}$ as well as the boundary coefficients $b_{1}, c_{1}, a_{K+1}$, and $b_{K+1}$ will have modified definitions.

A serious drawback of the approximation given by Eqs. 3.2 is that it does not allow the use of detailed flux solutions containing explicit zero flux boundary conditions. For this reason the exact solution, $\psi_{\mathrm{k}}(\mathrm{z})=\Phi_{\mathrm{k}}(\mathrm{z})$ for all $\mathrm{k}$, is excluded from the class of admissible trial function forms. However, such detailed solutions can be allowed by modifying the trial function forms in the boundary regions. If a detailed solution $\psi_{1}(\mathrm{z})$ is given in the first region with the zero flux condition $\psi_{1}\left(z_{1}\right)=0$, for example, the trial functions of Eqs. 3.2 could be modified for region $\mathrm{k}=1$ as

$$
\begin{aligned}
& \mathrm{U}_{1}(\mathrm{z})=\psi_{1}(\mathrm{x}) \psi_{1}^{-1}(1) \mathrm{F}_{2} \\
& \mathrm{U}_{1}^{*}(\mathrm{z})=\psi_{1}^{*}(\mathrm{x}) \psi_{1}^{*-1}(1) \mathrm{F}_{2} \\
& \mathrm{~V}_{1}(\mathrm{z})=\eta_{1}(\mathrm{x}) \psi_{1}^{-1}(1) \mathrm{F}_{2} \\
& \mathrm{~V}_{1}^{*}(\mathrm{z})=\eta_{1}^{*}(\mathrm{x}) \psi_{1}^{*-1}(1) \mathrm{F}_{2}^{*}
\end{aligned}
$$

In this way, the imposed zero flux boundary condition is explicitly given by $\psi_{1}(z)$ rather than in the form of the trial function. Similar 
trial functions can be given for an explicit zero flux boundary condition in the last region, $\mathrm{k}=\mathrm{K}$.

The use of these special trial functions in the boundary regions alters the definitions of the matrix coefficients $b_{2}$ and $b_{K}$ as given in Eq. 3.6. Detailed definitions of these coefficients when these special trial functions are used are also included in Appendix C.

Regardless of the types of boundary conditions imposed, Eq. 3.6 results in an $\mathrm{N} \times \mathrm{N}$ matrix problem of the form

$$
\underline{A} \underline{F}=\frac{1}{\lambda} \mathrm{IBF}
$$

where $\mathbb{A}$ and $\mathbb{B}$ are independent of $\lambda$. The order $N$ of the matrix equations is dependent upon the chosen boundary conditions, and is given for various choices in Table 3.1 .

Table 3.1. Matrix Order $\mathrm{N}$ of the Proposed Linear Basis Function Approximations as a Function of the Imposed Boundary Conditions.

$$
\begin{aligned}
& 1 \text { - Explicit or Implicit Zero Flux } \\
& 2 \text { - Symmetry }
\end{aligned}
$$

\begin{tabular}{ccc}
\hline Boundary Condition Type & Matrix Order \\
\hline on Left & on Right & \multicolumn{1}{c}{$\mathrm{N}$} \\
\hline 1 & 1 & $\mathrm{G} \times(\mathrm{K}-1)$ \\
1 & 2 & $\mathrm{G} \times \mathrm{K}$ \\
2 & 1 & $\mathrm{G} \times \mathrm{K}$ \\
2 & 2 & $\mathrm{G} \times(\mathrm{K}+1)$ \\
\hline
\end{tabular}


3.3 The Proposed Cubic Hermite Basis Function Approximation

The proposed modal-nodal approximation method utilizing the cubic Hermite polynomials

$$
\begin{aligned}
& p_{1}(x)=1-3 x^{2}+2 x^{3} \\
& p_{2}(x)=3 x^{2}-2 x^{3} \\
& p_{3}(x)=-x+2 x^{2}-x^{3} \\
& p_{4}(x)=x^{2}-x^{3}
\end{aligned}
$$

and their negative derivatives

$$
\begin{aligned}
& q_{1}(x)=6 x-6 x^{2} \\
& q_{2}(x)=-6 x+6 x^{2} \\
& q_{3}(x)=1-4 x+3 x^{2} \\
& q_{4}(x)=-2 x+3 x^{2}
\end{aligned}
$$

and defined as nonzero only within each mesh region $\mathrm{k}$, is given by the below regional trial function forms. $F_{k}$ and $G_{k}$ are again the unknown group column approximate flux and current solutions at $\mathrm{z}_{\mathrm{k}}$ respectively, and the remaining symbols have been previously defined. As in the cubic Hermite finite element method described in section 2.3.4 of Chapter $2, \theta$ is an optional normalization parameter.

$$
\begin{aligned}
\mathrm{U}_{\mathrm{k}}(\mathrm{z})= & \psi_{\mathrm{k}}(\mathrm{x})\left[\psi_{\mathrm{k}}^{-1}(0) \mathrm{p}_{1}(\mathrm{x}) \mathrm{F}_{\mathrm{k}}+\psi_{\mathrm{k}}^{-1}(1) \mathrm{p}_{2}(\mathrm{x}) \mathrm{F}_{\mathrm{k}+1}\right. \\
& \left.+\mathrm{h}_{\mathrm{k}} \theta \mathrm{ID}_{\mathrm{k}}^{-1}(0) \psi_{\mathrm{k}}^{-1}(0) \mathrm{p}_{3}(\mathrm{x}) \mathrm{G}_{\mathrm{k}}+\mathrm{h}_{\mathrm{k}} \theta \mathbb{D}_{\mathrm{k}}^{-1}(1) \psi_{\mathrm{k}}^{-1}(1) \mathrm{p}_{4}(\mathrm{x}) \mathrm{G}_{\mathrm{k}+1}\right]
\end{aligned}
$$




$$
\begin{aligned}
& \mathrm{U}_{\mathrm{k}}^{*}(\mathrm{z})=\psi_{\mathrm{k}}^{*}(\mathrm{x})\left[\psi_{\mathrm{k}}^{*^{-1}}(0) \mathrm{p}_{1}(\mathrm{x}) \mathrm{F}_{\mathrm{k}}^{*}+\psi_{\mathrm{k}}^{*^{-1}}(1) \mathrm{p}_{2}(\mathrm{x}) \mathrm{F}_{\mathrm{k}+1}^{*}\right. \\
& \left.+\mathrm{h}_{\mathrm{k}} \theta \mathrm{D}_{\mathrm{k}}^{-1}(0) \psi_{\mathrm{k}}^{*-1}(0) \mathrm{p}_{3}(\mathrm{x}) \mathrm{G}_{\mathrm{k}}^{*}+\mathrm{h}_{\mathrm{k}} \theta \mathrm{D}_{\mathrm{k}}^{-1}(1) \psi_{\mathrm{k}}^{*-1}(1) \mathrm{p}_{4}(\mathrm{x}) \mathrm{G}_{\mathrm{k}+1}^{*}\right] \\
& \mathrm{V}_{\mathrm{k}}(\mathrm{z})=\eta_{\mathrm{k}}(\mathrm{x})\left[\psi_{\mathrm{k}}^{-1}(0) \mathrm{p}_{1}(\mathrm{x}) \mathrm{F}_{\mathrm{k}}+\psi_{\mathrm{k}}^{-1}(1) \mathrm{p}_{2}(\mathrm{x}) \mathrm{F}_{\mathrm{k}+1}\right. \\
& \left.+\mathrm{h}_{\mathrm{k}} \theta \mathrm{D}_{\mathrm{k}}^{-1}(0) \psi_{\mathrm{k}}^{-1}(0) \mathrm{p}_{3}(\mathrm{x}) \mathrm{G}_{\mathrm{k}}+\mathrm{h}_{\mathrm{k}} \theta \mathrm{D}_{\mathrm{k}}^{-1}(1) \psi_{\mathrm{k}^{-1}}^{-1}(1) \mathrm{p}_{4}(\mathrm{x}) \mathrm{G}_{\mathrm{k}+1}\right] \\
& +\mathbb{D}_{\mathrm{k}}(\mathrm{x}) \psi_{\mathrm{k}}(\mathrm{x})\left[\frac{1}{\mathrm{~h}_{\mathrm{k}}} \psi_{\mathrm{k}}^{-1}(0) \mathrm{q}_{1}(\mathrm{x}) \mathrm{F}_{\mathrm{k}}+\frac{1}{\mathrm{~h}_{\mathrm{k}}} \psi_{\mathrm{k}}^{-1}(1) \mathrm{q}_{2}(\mathrm{x}) \mathrm{F}_{\mathrm{k}+1}\right. \\
& \left.+\theta \mathbb{D}_{\mathrm{k}}^{-1}(0) \psi_{\mathrm{k}}^{-1}(0) \mathrm{q}_{3}(\mathrm{x}) \mathrm{G}_{\mathrm{k}}+\theta \mathbb{D}_{\mathrm{k}}^{-1}(1) \psi_{\mathrm{k}}^{-1}(1) \mathrm{q}_{4}(\mathrm{x}) \mathrm{G}_{\mathrm{k}+1}\right] \\
& \mathrm{V}_{\mathrm{k}}^{*}(\mathrm{z})=\eta_{\mathrm{k}}^{*}(\mathrm{x})\left[\psi_{\mathrm{k}}^{*-1}(0) \mathrm{p}_{1}(\mathrm{x}) \mathrm{F}_{\mathrm{k}}^{*}+\psi_{\mathrm{k}}^{*-1}(1) \mathrm{p}_{2}(\mathrm{x}) \mathrm{F}_{\mathrm{k}+1}^{*}\right. \\
& \left.+\mathrm{h}_{\mathrm{k}} \theta \mathbb{D}_{\mathrm{k}}^{-1}(0) \psi_{\mathrm{k}}^{*-1}(0) \mathrm{p}_{3}(\mathrm{x}) \mathrm{G}_{\mathrm{k}}^{*}+\mathrm{h}_{\mathrm{k}} \theta \mathrm{D}_{\mathrm{k}}^{-1}(1) \psi_{\mathrm{k}}^{*^{-1}}(1) \mathrm{p}_{4}(\mathrm{x}) \mathrm{G}_{\mathrm{k}+1}^{*}\right] \\
& -\mathbb{D}_{\mathrm{k}}(\mathrm{x}) \psi_{\mathrm{k}}^{*}(\mathrm{x})\left[\frac{1}{\mathrm{~h}_{\mathrm{k}}} \psi_{\mathrm{k}}^{*-1}(0) \mathrm{q}_{1}(\mathrm{x}) \mathrm{F}_{\mathrm{k}}^{*}+\frac{1}{\mathrm{~h}_{\mathrm{k}}} \psi_{\mathrm{k}}^{*^{-1}}(1) \mathrm{q}_{2}(\mathrm{x}) \mathrm{F}_{\mathrm{k}+1}^{*}\right. \\
& \left.+\theta \mathbb{D}_{\mathrm{k}}^{-1}(0) \psi_{\mathrm{k}}^{*-1}(0) \mathrm{q}_{3}(\mathrm{x}) \mathrm{G}_{\mathrm{k}}^{*}+\theta \mathbb{D}_{\mathrm{k}}^{-1}(1) \psi_{\mathrm{k}}^{*-1}(1) \mathrm{q}_{4}(\mathrm{x}) \mathrm{G}_{\mathrm{k}+1}^{*}\right]
\end{aligned}
$$

Again, for the moment, $\psi_{\mathrm{k}}(0)$ and $\psi_{\mathrm{k}}(1)$ and their adjoints are not allowed to be zero in any region.

The forms of these trial functions impose both flux and current continuity since:

$$
\begin{aligned}
& \mathrm{U}_{\mathrm{k}}\left(\mathrm{z}_{\mathrm{k}}\right)=\mathrm{U}_{\mathrm{k}-1}\left(\mathrm{z}_{\mathrm{k}-1}+\mathrm{h}_{\mathrm{k}-1}\right)=\mathrm{F}_{\mathrm{k}} \\
& \mathrm{U}_{\mathrm{k}}^{*}\left(\mathrm{z}_{\mathrm{k}}\right)=\mathrm{U}_{\mathrm{k}-1}^{*}\left(\mathrm{z}_{\mathrm{k}-1}+\mathrm{h}_{\mathrm{k}-1}\right)=\mathrm{F}_{\mathrm{k}}^{*} \\
& \mathrm{~V}_{\mathrm{k}}\left(\mathrm{z}_{\mathrm{k}}\right)=\mathrm{V}_{\mathrm{k}-1}\left(\mathrm{z}_{\mathrm{k}-1}+\mathrm{h}_{\mathrm{k}-1}\right)=\mathrm{G}_{\mathrm{k}} \\
& \mathrm{V}_{\mathrm{k}}^{*}\left(\mathrm{z}_{\mathrm{k}}\right)=\mathrm{V}_{\mathrm{k}-1}^{*}\left(\mathrm{z}_{\mathrm{k}-1}+\mathrm{h}_{\mathrm{k}-1}\right)=-\mathrm{G}_{\mathrm{k}}^{*}
\end{aligned}
$$


where it is assumed that at region mesh points the detailed current solutions are zero:

$$
\begin{aligned}
& \eta_{\mathrm{k}}\left(\mathrm{z}_{\mathrm{k}}\right)=\eta_{\mathrm{k}}\left(\mathrm{z}_{\mathrm{k}}+\mathrm{h}_{\mathrm{k}}\right)=0 \\
& \eta_{\mathrm{k}}^{*}\left(\mathrm{z}_{\mathrm{k}}\right)=\eta_{\mathrm{k}}^{*}\left(\mathrm{z}_{\mathrm{k}}+\mathrm{h}_{\mathrm{k}}\right)=0
\end{aligned}
$$

for all regions $\mathrm{k}=1$ to $\mathrm{K}$.

Also, by comparison to Eqs. 2.31, it is evident that this approximation reduces to the cubic Hermite finite element method when the $\psi_{\mathrm{k}}{ }^{\prime}$ 's are constant, and the $\eta_{\mathrm{k}}{ }^{\prime} \mathrm{s}$ are correspondingly zero.

The insertion of the above trial function forms into variation equation 2.12 a results in a lengthy equation which can be simplified to the following form:

$$
\begin{aligned}
& \delta \mathrm{F}_{1}^{* \mathrm{~T}}\left[\mathrm{~b} 1_{1} \mathrm{~F}_{1}+\mathrm{b} 2{ }_{1} \mathrm{G}_{1}+\mathrm{c} 1_{1} \mathrm{~F}_{2}+\mathrm{c} 2{ }_{1} \mathrm{G}_{2}\right] \\
& +\delta \mathrm{G}_{1}^{* \mathrm{~T}}\left[\mathrm{~b} 3{ }_{1} \mathrm{~F}_{1}+\mathrm{b} 4_{1} \mathrm{G}_{1}+\mathrm{c} 3{ }_{1} \mathrm{~F}_{2}+\mathrm{c} 4_{1} \mathrm{G}_{2}\right] \\
& +\sum_{k=2}^{K}\left\{\delta F_{k}^{* T}\left[a 1_{k} F_{k-1}+a 2_{k} G_{k-1}+b 1_{k} F_{k}+b 2_{k} G_{k}+c 1_{k} F_{k+1}+c 2_{k} G_{k+1}\right]\right. \\
& +\delta G_{k}^{* T}\left[a 3_{k} F_{k-1}+a 4_{k} G_{k-1}+b 3 k_{k} F_{k}+b 4_{k} G_{k}+c 3{ }_{k} F_{k+1}+c 4_{k} G_{k+1}\right] ; \\
& +\delta F_{K+1}^{* T}\left[a 1_{K+1} F_{K}+a 2_{K+1} G_{K}+b 1_{K+1} F_{K+1}+b 2_{K+1} G_{K+1}\right] \\
& +\delta \mathrm{G}_{\mathrm{K}+1}^{*^{\mathrm{T}}}\left[\mathrm{a}{ }^{\mathrm{T}}{ }_{\mathrm{K}+1} \mathrm{~F}_{\mathrm{K}}{ }^{+\mathrm{a}} 4_{\mathrm{K}+1} \mathrm{G}_{\mathrm{K}}+\mathrm{b}{ }_{\mathrm{K}+1} \mathrm{~F}_{\mathrm{K}+1}+\mathrm{b} 4_{\mathrm{K}+1} \mathrm{G}_{\mathrm{K}+1}\right]=0
\end{aligned}
$$

where the detailed definitions of the twelve integral $G \times G$ matrix coefficients $\left\{a 1_{k}, \ldots, c 4_{k}\right\}$ of the form $A-\frac{1}{\lambda} B$ for all $k$ are given in section 2 of Appendix C. 
Boundary conditions for either zero flux or symmetry are easily imposed by setting either $\mathrm{F}_{\mathrm{k}}$ or $\mathrm{G}_{\mathrm{k}}$, respectively, to zero with $\mathrm{k}=1$ for the conditions on the left at $z_{1}$ or $k=K+1$ for conditions on the right at $\mathrm{z}_{\mathrm{K}+1}$ 。 The corresponding variations for $\mathrm{k}=1$ and $\mathrm{k}=\mathrm{K}+1$ then vanish. Allowing a rbitrary variations in the remaining $\mathrm{F}_{\mathrm{k}}^{*}$ and $\mathrm{G}_{\mathrm{k}}^{*}$ in Eq. 3.16 results in a system of $2 \mathrm{~K} \mathrm{G} \times \mathrm{G}$ matrix equations relating $2 \mathrm{~K} \mathrm{G}$ column vector unknowns, as illustrated in Figure 2. 7 .

Explicit zero flux boundary conditions imposed by $\psi_{1}\left(z_{1}\right)=0$ or $\psi_{\mathrm{K}}\left(\mathrm{z}_{\mathrm{K}+1}\right)=0$ can be incorporated into the approximation by modifying the trial function definitions in the boundary regions. The modified flux trial functions in the first region, for example, are

$$
\begin{aligned}
& \mathrm{U}_{1}(\mathrm{x})=\psi_{1}(\mathrm{x})\left[\psi_{1}^{-1}(1) \mathrm{F}_{2}+\mathrm{h}_{1} \theta \mathbb{D}_{1}^{-1}(0) \psi_{1}^{-1}(0) \mathrm{p}_{3}(\mathrm{x}) \mathrm{G}_{1}+\mathrm{h}_{1} \theta \mathbb{D}_{1}^{-1}(1) \psi_{1}^{-1}(1) \mathrm{p}_{4}(\mathrm{x}) \mathrm{G}_{2}\right] \\
& \mathrm{U}_{1}^{*}(\mathrm{x})=\psi_{1}^{*}(\mathrm{x})\left[\psi_{1}^{*-1}(1) \mathrm{F}_{2}^{*}+\mathrm{h}_{1} \theta \mathbb{D}_{1}^{-1}(0) \psi_{1}^{*-1}(0) \mathrm{p}_{3}(\mathrm{x}) \mathrm{G}_{1}^{*}+\mathrm{h}_{1} \theta \mathbb{D}_{1}^{-1}(1) \psi_{1}^{*-1}(1) \mathrm{p}_{4}(\mathrm{x}) \mathrm{G}_{2}\right]
\end{aligned}
$$

where the current trial functions are again given by Fick's laws. Use of modified trial function forms of this type in the boundary regions results in $2 \mathrm{~K}$ equations with different definitions of $\mathrm{c} 3_{1}, \mathrm{a} 2{ }_{2}, \mathrm{~b} 1_{2}$, $\mathrm{b} 2_{2}$, and $\mathrm{b} 3_{2}$ as well as $\mathrm{b} 1_{\mathrm{K}}, \mathrm{b} 2_{\mathrm{K}}, \mathrm{c} 2_{\mathrm{K}}$, and $\mathrm{a} 3_{\mathrm{K}+1}$, which are also included in Appendix C.

Other boundary condition restrictions may be imposed on this approximation, but the matrix form of the resulting difference equations will remain unchanged. Only the coefficients defined for $\mathrm{k}=1,2, \mathrm{~K}$, and $\mathrm{K}+1$ will in general be altered. 
The matrix equations resulting from this approximation can always be written as

$$
\mathbb{A} \underline{F}=\frac{1}{\lambda} \mathbb{I B} \underline{F}
$$

where $\mathbb{A}$ and $\mathbb{B}$ are $(\mathrm{G} \times 2 \mathrm{~K})$ by $(\mathrm{G} \times 2 \mathrm{~K})$ matrices, independent of $\lambda$ 。 and $F$ is the $\mathrm{G} \times \mathrm{K}$ column vector of unknowns containing both $\mathrm{F}_{\mathrm{k}}$ and $\mathrm{G}_{\mathrm{k}}$ column vectors for each $\mathrm{k}$. The matrix properties and solution methods of the matrix equations derived in these proposed approximation methods are discussed in the next chapter. 
Chapter 4

NUMERICAL SOLUTION TECHNIQUES

The matrix properties of the difference equations resulting from both the proposed approximations and the finite element methods in one dimension, as well as the solution schemes used to solve these equations, are summarized in the following section. Various calculational and programming techniques used in conjunction with these approximation methods and their solution schemes are presented and discussed in section 4.2 .

\section{1 Solution Methods and Matrix Properties}

The matrix equations which result from the approximations given in this report are of the form

$$
\mathbb{A} \underline{F}=\frac{1}{\lambda} \mathbb{B} \underline{F}
$$

and are solved using the fission source power iteration method without fission source renormalization. 7,44 The method of solution is illustrated schematically in Figure 4.1. Other definitions of the iteration eigenvalue $\lambda^{(i)}$ can be found elsewhere. 45

Figure 4.1 illustrates that an outer iteration solution scheme 46 is used, and that the geometry and nuclear properties of the reactor are not altered. Since the fission source is not normalized by the iteration eigenvalue during the iterations, $\lambda^{(i)}$ converges to the effective multiplication factor, $\mathrm{k}_{\mathrm{eff}}$, of the problem. Had fission source renormalization been included, by $\underline{S}^{(i)}=\mathbb{B} \underline{F}^{(i)} / \lambda^{(i-1)}$ for example, then $\lambda^{(i)}$ 
$i=0$

Guess $\underline{F}^{(1)}>0$

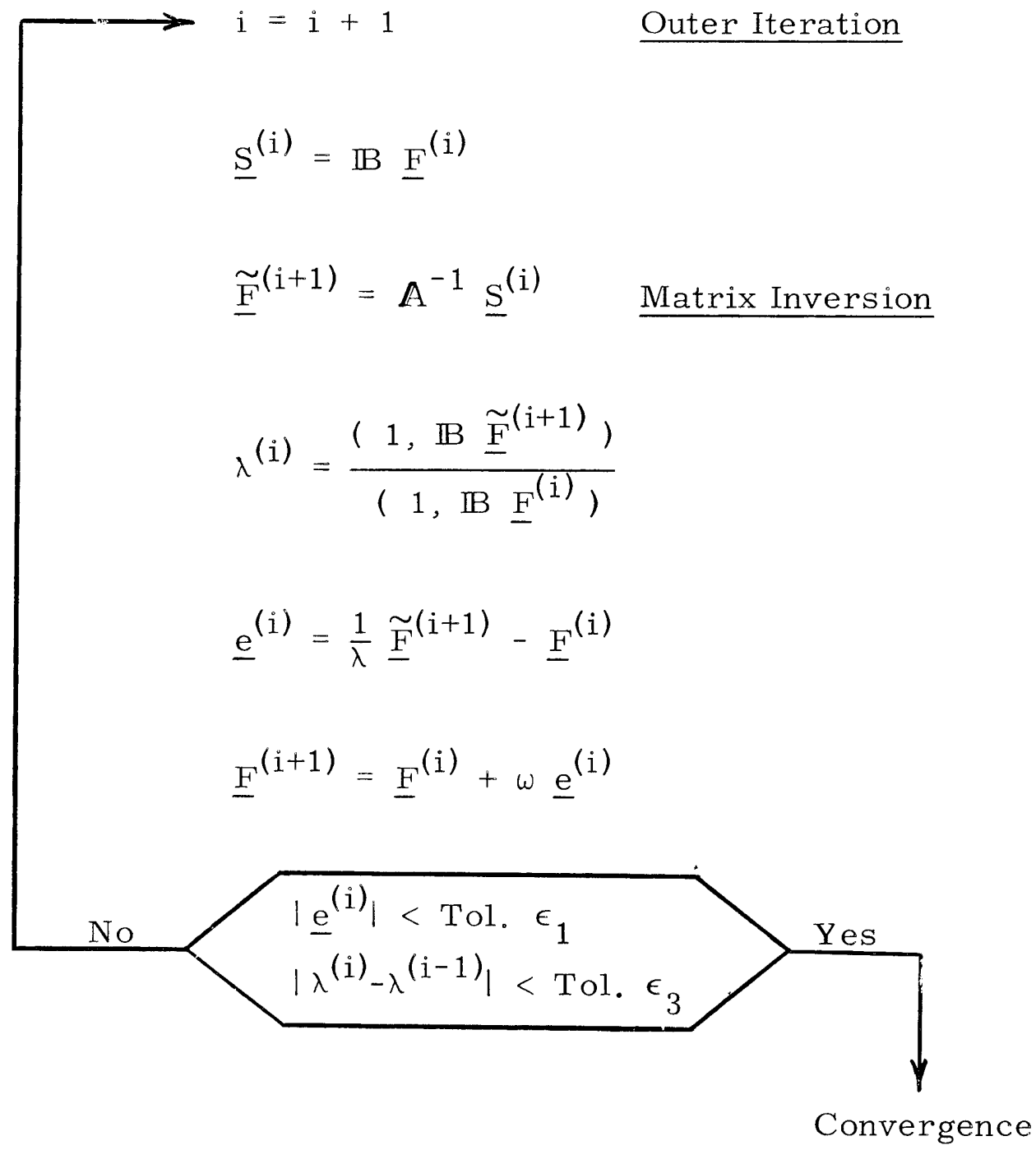

Figure 4.1. Solution of $\mathbb{A} \underline{F}=\frac{1}{\lambda} \mathbb{B} \underline{F}$ Using the Fission Source Power Iteration Method Without Fission Source Renormalization. 
would converge to unity. The $\mathrm{k}_{\text {eff }}$ of the problem would then be simply the product of all of the iteration eigenvalues. $46,47,48$

The matrix inversions required within the iteration scheme were performed directly. Although overrelaxation methods are usually employed only in iterative matrix inversion schemes (or inner iterations), ${ }^{49}$ an overrelaxation parameter $\mathrm{w}, 1 \leqslant \mathrm{w} \leqslant 2$, is available in the outer iteration in order to hasten the convergence of the solution vector.

The power method is very appealing to neutron diffusion flux calculations because it converges to the largest or fundamental eigenvalue $\left|\lambda_{0}\right|>\left|\lambda_{i}\right|, i \neq 0$, and the corresponding eigenvector $\underline{F}_{O}$ of the given matrix problem. The convergence rate is governed by the dominance ratio, defined as $\max _{i \neq 0}\left|\lambda_{i} / \lambda_{0}\right|$, in such a way that smaller ratios result in faster convergence. Although the power method will always converge when $\lambda_{\mathrm{O}}$ is positive and unique, specific matrix properties of $\mathbb{A}$ and $\mathrm{IB}$ are sufficient but not always necessary to insure convergence to a positive $\mathrm{k}_{\text {eff }}$ and everywhere positive neutron flux approximation. 50

In many problems the order of $\mathbb{A}$ may be quite large, and solution methods which require the direct inversion of $\mathbb{A}$ may not be practical. For the purposes of this report, as in most multigroup calculational schemes, neutron up-scattering will not be permitted. The inversion of $\mathbb{A}$ is then performed by successive group-iteration techniques.

The equations given in Eq. 4.1 have been defined as ordered first by spatial indexing followed by group indexing within each spatial index. It is convenient to reorder these equations so that they are ordered first by group indexing followed by spatial indexing within each group. 
After reordering, Eq. 4.1 can be written as

$$
(\mathbb{I}+\mathbb{I N}) \underline{F}=\mathbf{T} \underline{F}+\frac{1}{\lambda} \mathbb{B} \underline{F}
$$

where

$$
\mathbb{A}=\mathbb{I}+\mathbb{I M}-\mathbb{T}
$$

and: $\mathbb{L}$, the stiffness matrix, results from leakage; $\mathbb{I M}$, the mass matrix, results from absorption; $\boldsymbol{T}$ is the group-to-group scattering transfer matrix; and $\mathbb{B}$ is the fission source production matrix. Assuming $\mathrm{K}$ spatial unknowns in each of the $\mathrm{G}$ groups, $\mathbb{I}$ and $\mathbb{M}$ are $\mathrm{G} \times \mathrm{K}$ block diagonal matrices composed of $\mathrm{G} \mathrm{K} \times \mathrm{K}$ matrices $\mathbb{L}_{\mathrm{g}}$ and $\mathrm{IM}_{\mathrm{g}}$ of the form

$$
\begin{aligned}
& \mathbb{L}=\operatorname{Diag}\left[\mathbb{L}_{1}, \ldots, \mathbb{L}_{\mathrm{G}}\right] \\
& \mathbb{I M}=\operatorname{Diag}\left[\mathbb{M}_{1}, \ldots, \mathbb{M}_{\mathrm{G}}\right]
\end{aligned}
$$

and $\mathbb{T}$ and $\mathbb{B}$ are in general full block matrices composed of $G^{2} \mathrm{~K} \times \mathrm{K}$ matrices $\mathbf{T}_{\text {gg' }}$ and $\mathbb{B}_{\text {gg' }}$, respectively. Since only downscattering is permitted, $\mathbf{T}$ becomes lower block triangular; $\mathbf{T}_{\mathrm{gg}^{\prime}}=0$ whenever $g^{\prime} \geqslant g$. The matrix inversion, $\underline{\widetilde{F}}^{(i+1)}=\mathrm{A}^{-1} \underline{S}^{(i)}$, can then be solved for the GK unknowns

$$
\underline{\widetilde{F}}^{(i+1)}=\operatorname{Col}\left[\underline{\underline{F}}_{1}^{(i+1)} \ldots \widetilde{\underline{F}}_{G}^{(i+1)}\right]
$$

by solving successively the following system of group equations:

$$
\begin{aligned}
& \text { Do for } g=1 \text { to } G: \\
& \quad \underline{S}_{g}^{(i)}=\sum_{g^{\prime}=1}^{G}\left(\mathbf{T}_{g g^{\prime}}+\mathbb{B}_{g g^{\prime}} \underline{F}_{g^{\prime}}^{(k)}\right. \\
& \quad \text { where: } k=\left\{\begin{array}{c}
i+1 ; g^{\prime}<g \\
i ; g^{\prime} \geqslant g
\end{array}\right. \\
& L \quad \underline{\widetilde{F}}_{g}^{(i+1)}=\left(\mathbb{L} g_{g}+\mathbb{M}_{g}\right)^{-1} \underline{S}_{g}^{(i)}
\end{aligned}
$$


where the updating of the group fission source by the iteration index $k=i+1$ for $g^{\prime}<g$ generally enables a faster rate of convergence of the outer iteration than $\mathrm{k}=\mathrm{i}$.

The desirable convergence properties of a positive eigenvalue and everywhere positive flux solution when using the above group iteration method depend upon the properties of the $K \times K$ spatial matrices for each group $\mathrm{g}$ : $\mathbb{I}_{\mathrm{g}} ; \mathbb{I}_{\mathrm{g}}$; and $\mathbf{T}_{\mathrm{gg}}$ and $\mathbb{B}_{\mathrm{gg}}$, for $\mathrm{g}^{\prime}=1$ to $\mathrm{G}$. Using the Perron-Frohenius theorem, ${ }^{50}$ it can be shown that if $\mathbb{T}_{g g^{\prime}}$ and $\mathbb{B}_{\mathrm{gg}^{\prime}}$ are all nonnegative for each group $\mathrm{g}$ and $\mathbb{I} \mathrm{L}_{\mathrm{g}}$ and $\mathbb{I M}$ g are both Stieltjes or $\mathrm{S}$-type matrices ${ }^{\dagger}$ for each group $\mathrm{g}$, then the power method will converge to a positive eigenvalue, $\lambda_{\mathrm{O}}>0$, and a corresponding positive eigenvector, $\underline{F}_{\mathrm{O}}>0$. These matrix properties naturally depend upon the form of the spatial approximations employed and generally differ for different approximation schemes.

The conventional finite difference approximation has become popular because the spatial matrices which arise from its use exhibit these desirable properties regardless of the size of the mesh regions chosen. The spatial matrices resulting from the linear finite element method, however, are known to exhibit these properties only if the mesh size is restricted by

$$
\mathrm{h}_{\mathrm{k}} \leqslant \max _{\mathrm{g}=1 \text { to } \mathrm{G}}\left\{\sqrt{6} \ell_{\mathrm{g}, \mathrm{k}}\right\}
$$

where $\ell_{\mathrm{g}, \mathrm{k}}$ is the diffusion length, $\ell_{\mathrm{g}, \mathrm{k}}^{2}=\mathrm{D}_{\mathrm{g}, \mathrm{k}} / \Sigma_{\mathrm{g}, \mathrm{k}}$, for group $\mathrm{g}$ in mesh region $k$. The spatial matrices resulting from the cubic Hermite $\dagger_{\text {A Stieltjes matrix is a real, irreducible, positive definite matrix }}$
with nonpositive off-diagonal elements. 
finite element method do not exhibit these "desirable" characteristics. Since the eigenvector $\underline{F}$ contains current as well as flux unknowns, convergence to an all-positive solution vector is not desirable.

The properties of the spatial matrices for each group g resulting from the proposed approximation methods can be found by generalizing the proposed trial function forms in each group as

$$
\begin{aligned}
& U_{g}(z)=\sum_{k=1}^{K}\left[\underline{P}_{g, k}^{-T}(x) \underline{F}_{g, k}+\underline{P}_{g, k}^{+T} \underline{F}, k+1\right] \\
& U_{g}^{* *}(z)=\sum_{k=1}^{K}\left[\underline{P}_{g, k}^{-* T}(x) \underline{F}_{g, k}^{*}+\underline{P}_{g, k}^{+*} \underline{F}_{g, k}^{*}{ }^{*}\right]
\end{aligned}
$$

where the $\underline{F}_{g, k}$ are in general column vectors of length $\mathrm{N}$ given by:

$$
\underline{\mathrm{F}}_{\mathrm{g}, \mathrm{k}}=\mathrm{F}_{\mathrm{g}, \mathrm{k}} \quad(\mathrm{N}=1)
$$

for the linear basis function approximations, and

$$
\underline{F}_{g, k}=\operatorname{Col}\left[F_{g, k}, G_{g, k}\right] \quad(N=2)
$$

for the cubic Hermite basis function approximations. Similar definitions hold for the $\underline{F}_{\mathrm{g}, \mathrm{k}^{*}}^{*}$ The $\underline{\mathrm{P}}_{\mathrm{g}, \mathrm{k}}^{ \pm}(\mathrm{x})$ are column vectors of length $\mathrm{N}$ whose elements are functions of $\mathrm{z}$ ( or $\mathrm{x}$ ) defined as nonzero only within region $\mathrm{k}$ which provide the basis for the approximations. The definitions of the $\underline{\mathrm{P}}_{\mathrm{g}, \mathrm{k}}^{ \pm}(\mathrm{x})$ for the proposed approximations are given as follows:

$\mathrm{N}=1$; Linear Basis Functions:

$$
\begin{aligned}
& \underline{\mathrm{P}}_{\mathrm{g}, \mathrm{k}}^{-}(\mathrm{x})=(1-\mathrm{x}) \psi_{\mathrm{g}, \mathrm{k}}^{-1}(0) \psi_{\mathrm{g}, \mathrm{k}}(\mathrm{x}) \\
& \underline{\mathrm{P}}_{\mathrm{g}, \mathrm{k}}^{+}(\mathrm{x})=\mathrm{x} \psi_{\mathrm{g}, \mathrm{k}}^{-1}(1) \psi_{\mathrm{g}, \mathrm{k}}(\mathrm{x})
\end{aligned}
$$


$\mathrm{N}=2$; Cubic Hermite Basis Functions:

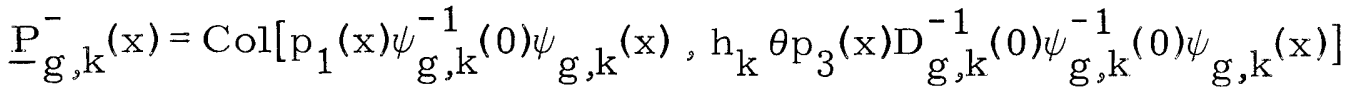

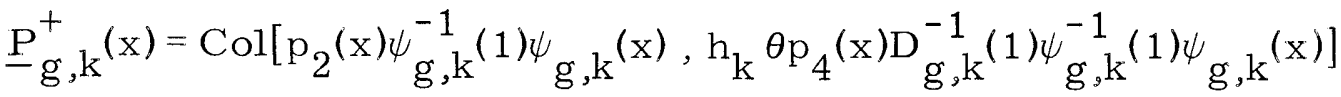

where $\psi_{\mathrm{g}, \mathrm{k}}(\mathrm{x})$ and $\mathrm{D}_{\mathrm{g}, \mathrm{k}}(\mathrm{x})$ are the detailed flux solutions and diffusion coefficients of group $g$, the polynomials $p_{1}(x)$ through $p_{4}(x)$ are defined in Eqs. 3.11, and $0 \leqslant x \leqslant 1$ within each region $k$. Similar definitions hold for the $\underline{\mathrm{P}}_{\mathrm{g}, \mathrm{k}}^{ \pm *}(\mathrm{x})$.

Equations 4.7 can be written in matrix form as

$$
\begin{aligned}
& U_{g}(z)=\mathbb{P}_{g}(x) \underline{F}_{g} \\
& U_{g}^{*}(z)=\mathbb{P}_{g}^{*}(x) \underline{F}_{g}^{*}
\end{aligned}
$$

where:

$$
\underline{F}_{g}=\operatorname{Col}\left(F_{g, 1}, \ldots, F_{g, K+1}\right)
$$

and $\operatorname{IP}_{\mathrm{g}}(\mathrm{x})$ is the $\mathrm{K}$ by $\mathrm{N}(\mathrm{K}+1)$ matrix defined by

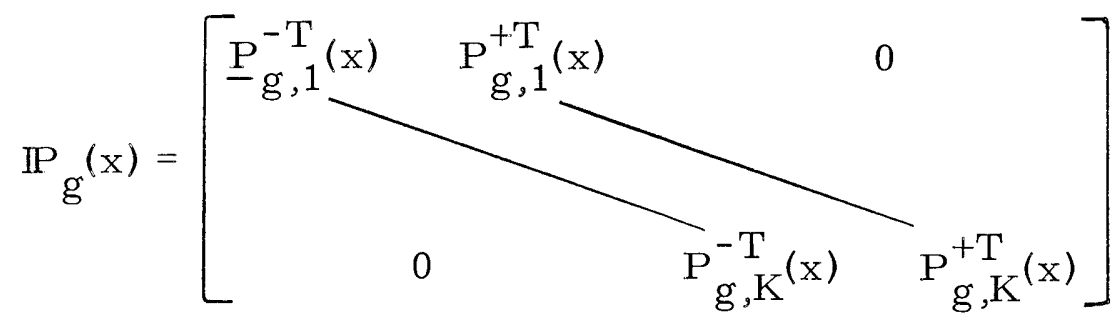

IP $_{\mathrm{g}}^{*}(\mathrm{x})$ is defined similarly. Insertion of these trial function forms into variation equation $2.12 \mathrm{~b}$ for each group $\mathrm{g}$ results in 


$$
\delta \underline{\mathrm{F}}_{\mathrm{g}}^{*^{\mathrm{T}}} \int_{\mathrm{K}} \dot{\mathbb{P}}_{\mathrm{g}}^{{ }^{*} \mathrm{~T}} \mathrm{D} \dot{\mathrm{I}}_{\mathrm{g}} \underline{\mathrm{F}}_{\mathrm{g}}+\mathbb{P}_{\mathrm{g}}^{*^{\mathrm{T}}} \sum_{\mathrm{g}^{\prime}=1}^{\mathrm{G}} \Lambda_{\mathrm{gg}^{\prime}} \mathbb{P}_{\mathrm{g}^{\prime}} \underline{\mathrm{F}}_{\mathrm{g}^{\prime}} \mathrm{d}_{\mathrm{f}}=0
$$

where $\mathbb{D}_{\mathrm{g}}$ and $\mathbb{M}_{\mathrm{gg}}$ are $\mathrm{K} \times \mathrm{K}$ diagonal matrices of the form

$$
\mathbb{D}_{g}=\mathbb{D}_{g}(x)=\operatorname{Diag}\left[D_{g, 1}(x), \ldots, D_{g, K}(x)\right]
$$

and

$$
\Lambda_{\mathrm{gg}^{\prime}}=\Lambda_{\mathrm{gg}^{\prime}}(\mathrm{x})=\operatorname{Diag}\left[\Lambda_{\mathrm{gg}^{\prime}, 1}(\mathrm{x}), \ldots, \Lambda_{\mathrm{gg}^{\prime}, \mathrm{K}}(\mathrm{x})\right]
$$

The quantity $\dot{\mathbb{P}}_{\mathrm{g}}$ represents the derivative of $\mathbb{P}_{\mathrm{g}}(\mathrm{z})$ with respect to $\mathrm{z}$, and the integration over $\mathrm{K}$ denotes integration over the entire range of $z ; z_{1} \leqslant z \leqslant z_{K+1}$.

$\mathrm{D}_{\mathrm{g}, \mathrm{k}}$ and $\Lambda_{\mathrm{gg}^{\prime}, \mathrm{k}}$

$$
\Lambda_{\mathrm{gg}^{\prime}, \mathrm{k}}=\left(\Sigma_{\mathrm{tg}}-\Sigma_{\mathrm{gg}^{\prime}}-\frac{1}{\lambda} x_{\mathrm{g}} \nu \Sigma_{\mathrm{fg}^{\prime}}\right)_{\text {in region } \mathrm{k}}
$$

are the group material constants in mesh region $\mathrm{k}$, and are usually dependent on $\mathrm{x} . \mathbb{\Lambda}_{\mathrm{gg}}$ can thus be conveniently expanded as

$$
\Lambda_{g g^{\prime}}=\mathbb{\Lambda}_{g}^{\mathrm{A}}-\Lambda_{\mathrm{gg}^{\prime}}^{\mathrm{S}}-\frac{1}{\lambda} \mathbb{\Lambda}_{\mathrm{gg}^{\prime}}^{\mathrm{F}}
$$

Allowing arbitrary variations in each element of $\underline{F}_{\mathrm{g}}^{* *}$ for each group $\mathrm{g}$ in Eq. 4.13 results in the matrix equations

$$
\text { (II. } \left.{ }_{g}+\mathbb{M}_{g^{\prime}}\right)_{g}=\sum_{g^{\prime=1}}^{G}\left(\mathbb{T}_{g^{\prime} g^{\prime}}+\frac{1}{\lambda} \mathbb{B}_{g^{\prime} g^{\prime}}\right) \underline{F}_{g^{\prime}} ; g=1 \text { to } G
$$

as described in Eqs. 4.1 through 4.5, where:

$$
\begin{aligned}
& \mathbb{L}_{\mathrm{g}}=\int_{\mathrm{K}} \dot{\mathbb{P}}_{\mathrm{g}}^{*} \mathbb{D}_{\mathrm{g}} \dot{\mathbb{P}}_{\mathrm{g}} \mathrm{dz} \\
& \mathbb{M}_{\mathrm{g}}=\int_{\mathrm{K}} \mathbb{P}_{\mathrm{g}}^{* \mathrm{~T}} \mathbb{\Lambda}_{\mathrm{g}}^{\mathrm{A}} \mathbb{P}_{\mathrm{g}} \mathrm{dz}
\end{aligned}
$$




$$
\begin{aligned}
& \mathrm{T}_{\mathrm{gg}^{\prime}}=\int_{\mathrm{K}} \mathbb{P}_{\mathrm{g}}^{* \mathrm{~T}^{\mathrm{T}}} \mathbb{\Lambda}_{\mathrm{gg}}^{\mathrm{S}} \mathbb{P}_{\mathrm{g}^{\prime}} \mathrm{dz} \\
& \mathbb{B}_{\mathrm{gg}^{\prime}}=\int_{\mathrm{K}} \mathbb{P}_{\mathrm{g}}^{*} \mathbb{\Lambda}_{\mathrm{gg}}^{\mathrm{T}} \mathbb{P}_{\mathrm{g}^{\prime}} \mathrm{dz}
\end{aligned}
$$

These matrices are $\mathrm{N}(\mathrm{K}+1)$ by $\mathrm{N}(\mathrm{K}+1)$ block tridiagonal of similar form whose $\mathrm{N} \times \mathrm{N}$ submatrices are integrals of $\mathrm{N} \times \mathrm{N}$ dyads. The $\mathrm{k}^{\text {th }}$ row of the product $\Lambda_{\mathrm{gg}^{\prime}} \underline{\mathrm{F}}_{\mathrm{g}^{\prime}}$ is, for example:

$$
\begin{aligned}
& {\left[\Lambda_{\mathrm{gg}^{\prime}} \underline{\mathrm{F}}_{\mathrm{g}^{\prime}}\right]_{\mathrm{k}}=\left\{\int_{0}^{1} \mathrm{~h}_{\mathrm{k}-1} \Lambda_{\mathrm{gg}^{\prime}, \mathrm{k}-1}(\mathrm{x}) \underline{\mathrm{P}}_{\mathrm{g}, \mathrm{k}-1}^{+^{*}}(\mathrm{x}) \underline{\mathrm{P}}_{\mathrm{g}^{\prime}, \mathrm{k}-1}^{-\mathrm{T}}(\mathrm{x}) \mathrm{dx}\right\} \mathrm{F}_{\mathrm{g}^{\prime}, \mathrm{k}-1}} \\
& +\left\{\int_{0}^{1} h_{k-1} \Lambda_{g g^{\prime}, k-1}(x) \underline{P}_{g, k-1}^{+^{*}}(x) \underline{P}_{g^{\prime}, k-1}^{+T}(x) d x\right. \\
& \left.+\int_{0}^{1} h_{k} \Lambda_{g g^{\prime}, k}(x) \underline{P}_{g, k}^{-*}(x) \underline{P}_{g^{\prime}, k}^{-T}(x) d x\right\} F_{g^{\prime}, k} \\
& +\left\{\int_{0}^{1} h_{k} \Lambda_{g g^{\prime}, k}(x) \underline{P}_{g, k}^{-*}(x) \underline{P}_{g^{\prime}, k}^{+T}(x) d x\right\} F_{g^{\prime}, k+1}
\end{aligned}
$$

These matrix relationships allow presentation of the following matrix properties.

Theorem 1: $\mathbb{I}_{\mathrm{g}}$ and $\mathrm{IM}_{\mathrm{g}}$ are guaranteed to be positive definite whenever the detailed weighting functions $\psi_{\mathrm{g}, \mathrm{k}}^{*}(\mathrm{z})$ have a similar shape to that of the detailed flux solutions $\psi_{\mathrm{g}, \mathrm{k}}(\mathrm{z})$, as given by:

$$
\psi_{\mathrm{g}, \mathrm{k}}^{*}(\mathrm{z})=\mathrm{C}_{\mathrm{g}, \mathrm{k}} \psi_{\mathrm{g}, \mathrm{k}}(\mathrm{z})
$$

where $\mathrm{C}_{\mathrm{g}, \mathrm{k}}$ is a positive constant for each energy group $\mathrm{g}$ and each region $\mathrm{k}$. 
Proof: Under these conditions, $\underline{P}_{g, k}^{*^{ \pm}}=C_{g, k} \underline{P}_{g, k}^{ \pm}$

hence,

$$
\mathbb{P}_{\mathrm{g}}^{*}=\mathbb{C}_{\mathrm{g}} \mathrm{IP}_{\mathrm{g}}
$$

where

$$
\mathbb{C}_{\mathrm{g}}=\operatorname{Diag}\left(\mathrm{C}_{\mathrm{g}, 1}, \ldots, \mathrm{C}_{\mathrm{g}, \mathrm{K}}\right)
$$

First consider $\mathbb{I M}_{\mathrm{g}}$. Given any arbitrary constant nonzero vector $q$,

$$
\underline{q}^{\mathrm{T}} \mathbb{I M} q=\int_{K}\left(\mathbb{P}_{g} q\right)^{\mathrm{T}} \mathbb{C}_{g}^{\mathrm{T}} \Lambda_{g}^{\mathrm{A}} \mathbb{P}_{g} q d z
$$

and since $\mathbb{C}_{\mathrm{g}}$ and $\Lambda_{\mathrm{g}}^{\mathrm{A}}$ are both positive block diagonal matrices with diagonal submatrices, their product can be factored into

$$
\mathbb{C}_{\mathrm{g}}^{\mathrm{T}} \Lambda_{\mathrm{g}}^{\mathrm{A}}=\left[\left(\boldsymbol{\Lambda}_{\mathrm{g}}^{\mathrm{A}} \mathbb{C}_{\mathrm{g}}\right)^{\frac{1}{2}}\right]^{\mathrm{T}}\left(\mathbb{C}_{\mathrm{g}} \Lambda_{\mathrm{g}}^{\mathrm{A}}\right)^{\frac{1}{2}}
$$

Therefore,

$$
\begin{aligned}
\underline{q}^{\mathrm{T}} \mathbb{I M}_{\mathrm{g}} q & =\int_{K}\left[\left(\mathbb{C}_{\mathrm{g}} \Lambda_{\mathrm{g}}^{\mathrm{A}}\right)^{\frac{1}{2}} \mathbb{P} \mathrm{g} q\right]^{\mathrm{T}}\left[\left(\mathbb{C}_{\mathrm{g}} \Lambda_{\mathrm{g}}^{\mathrm{A}}\right)^{\frac{1}{2}} \mathbb{P} \mathrm{g}_{\mathrm{g}} \mathrm{q}\right] \mathrm{dz} \\
& =\int_{K} \mathbb{R}^{\mathrm{T}} \mathbb{R} \mathrm{dz}
\end{aligned}
$$

which is always greater than zero for arbitrary nonzero q. Hence, by definition, $\mathbb{I M}_{\mathrm{g}}$ is positive definite. A similar proof holds for $\mathbb{L}_{\mathrm{g}}$ using Eq. 4.16a.

The following corollaries immediately result.

Corollary 1: If Rayleigh-Ritz Galerkin weighting, $\underline{U}^{*}=\underline{U}$, is used in the approximation, then $\mathbb{I}_{\mathrm{g}}$ and $\mathbb{I M}_{\mathrm{g}}$ are positive definite. 
Corollary 2: $\quad \mathbb{L}_{\mathrm{g}}$ and $\mathbb{I M}$ g resulting from the finite element methods using linear and cubic Hermite basis functions are both positive definite.

Corollary 3: If $\mathbb{L}_{\mathrm{g}}$ and $\mathbb{M}_{\mathrm{g}}$ are positive definite, then so is the matrix $\left(\mathrm{IL}+\mathrm{IM} \mathrm{g}_{\mathrm{g}}\right)$. These three matrices are then also symmetric.

It is also interesting to note the properties of $\mathrm{IL}_{\mathrm{g}}$ and $\mathrm{IM}_{\mathrm{g}}$ for cases of symmetry; that is, when the material properties and detailed flux solutions are symmetric about the center of each coarse mesh region k. Such symmetry occurs in regular repeating reactor geometries, and is denoted by:

$$
\mathrm{D}_{\mathrm{g}, \mathrm{k}}(\mathrm{x})=\mathrm{D}_{\mathrm{g}, \mathrm{k}}(1-\mathrm{x})
$$

and

$$
\Lambda_{\mathrm{g}, \mathrm{k}}(\mathrm{x})=\Lambda_{\mathrm{g}, \mathrm{k}}(1-\mathrm{x})
$$

Hence,

$$
\psi_{\mathrm{g}, \mathrm{k}}(\mathrm{x})=\psi_{\mathrm{g}, \mathrm{k}}(1-\mathrm{x})
$$

and

$$
\eta_{g, k}(x)=-\eta_{g, k}(1-x)
$$

and similarly for the weighting fluxes and currents. Under such conditions, the $\underline{\mathrm{P}}_{\mathrm{g}, \mathrm{k}}^{+}(\mathrm{x})$ and $\underline{\mathrm{P}}_{\mathrm{g}, \mathrm{k}}^{-}(\mathrm{x})$ support functions can be found by inspection of Eqs. 4.9 and 4.10 to obey the following symmetries:

For $\mathrm{N}=1$ :

$$
\begin{aligned}
& \underline{\mathrm{P}}_{\mathrm{g}, \mathrm{k}}^{\mp}(\mathrm{x})=\underline{\mathrm{P}}_{\mathrm{g}, \mathrm{k}}^{ \pm}(1-\mathrm{x}) \\
& \dot{\mathrm{P}}_{\mathrm{g}, \mathrm{k}}^{\mp}(\mathrm{x})=-\underline{\dot{P}}_{\mathrm{g}, \mathrm{k}}^{ \pm}(1-\mathrm{x})
\end{aligned}
$$


For $N=2$ :

$$
\begin{aligned}
& \underline{\mathrm{P}}_{\mathrm{g}, \mathrm{k}}^{\mp}(\mathrm{x})=\left[\begin{array}{rr}
1 & 0 \\
0 & -1
\end{array}\right] \underline{\mathrm{P}}_{\mathrm{g}, \mathrm{k}}^{ \pm}(1-\mathrm{x}) \\
& \dot{\mathrm{P}}_{\mathrm{g}, \mathrm{k}}^{\mp}(\mathrm{x})=-\left[\begin{array}{rr}
1 & 0 \\
0 & -1
\end{array}\right] \dot{\mathrm{P}}_{\mathrm{g}, \mathrm{k}}^{ \pm}(1-\mathrm{x})
\end{aligned}
$$

where the following symmetries of the polynomials defined in Eqs. 3.11 have been used:

$$
\begin{aligned}
& p_{1}(x)=p_{2}(1-x) \\
& p_{3}(x)=-p_{4}(1-x) \\
& q_{1}(x)=-q_{2}(1-x) \\
& q_{3}(x)=q_{4}(1-x)
\end{aligned}
$$

Similar identities with identical signs hold for the weighting quantities $\underline{P}_{g, k}^{ \pm^{*}}(\mathrm{x})$

Theorem 2: For cases of symmetry, as given above, the matrices $\mathbb{I L}_{\mathrm{g}}, \mathbb{M} \mathrm{I}_{\mathrm{g}}, \mathbb{T}_{\mathrm{gg}}$, and $\mathbb{B}_{\mathrm{gg}^{\prime}}$ are all symmetric regardless of the relation of $\psi_{\mathrm{g}, \mathrm{k}}^{(\mathrm{x})}$ to $\psi_{\mathrm{g}, \mathrm{k}}(\mathrm{x})$.

Proof: Referring to Eq. 4.17, $\mathrm{I} \mathrm{g}_{\mathrm{g}}$, for example, is symmetric only if

$$
\int_{0}^{1} h_{k} D_{g, k}(x) \dot{P}_{g, k}^{+*}(x) \dot{P}_{g, k}^{-T}(x) d x=\int_{0}^{1} h_{k} D_{g, k}(x) \dot{P}_{g, k}^{-{ }^{*}}(x) \dot{P}_{g, k}^{+{ }^{T}}(x) d x
$$

This can be shown for any $\mathrm{N}$ by changing variables in one of the integrals from $x$ to $1-x^{\prime}$ and using the symmetry properties of Eqs. 4.23 and 4.24. Similar proofs hold for the other matrices. 
It is unfortunate that the above symmetry conditions do not allow direct proof that $\mathbb{L}_{\mathrm{g}}$ and $\mathbb{I M}_{\mathrm{g}}$ have positive diagonal elements and are also diagonally dominant (for at least one row) for arbitrary positive and symmetric detailed flux solutions. Under such conditions, $\mathrm{IL}_{\mathrm{g}}$ and II ${ }_{\mathrm{g}}$ would then be positive definite, since they are block tridiagonal with nonzero diagonal elements and hence irreducible. Instead, these conditions can be used to obtain a set of algebraic equations which, for completely arbitrary detailed flux solutions, must be satisfied in order that $I_{\mathrm{g}}$ and $\mathrm{MI}_{\mathrm{g}}$ be positive definite.

The requirement that $\mathbb{L}_{\mathrm{g}}$ and $\mathbb{I M}_{\mathrm{g}}$ be positive definite is useful only in the inversion of $\left(\mathbb{L}_{g}+\mathbb{M}_{g}\right)$. Although the inversion can always be performed using Gaussian elimination techniques, the property of positive definiteness allows the use of Cholesky's method, discussed in the next section, which is faster and requires less computer storage.

\subsection{Calculational and Programming Techniques}

Calculation of the one-dimensional subassembly detailed fluxes, currents, and adjoint solutions, as well as detailed "exact" or reference solutions, were performed using the program REF2G described in section 1 of Appendix D. Assuming subassembly $\mathrm{k}$ to be divided into $\mathrm{N}$ homogeneous intervals at nodes $t_{i}$ and of width hs $s_{i}$, the program uses fine mesh linear finite element approximations to calculate the detailed flux solutions for each group. Omitting group subscripts, the detailed flux solution for each group in subassembly $k$ is represented by a set of $\mathrm{N}+1$ points

$$
\psi_{\mathrm{k}}(\mathrm{x})=\left\{\psi_{\mathrm{k}, \mathrm{i}}: \mathrm{i}=1, \mathrm{~N}+1\right\}
$$


where $\psi_{k}(x)$ is linear between points. Detailed group current solutions $\eta_{\mathrm{k}}(\mathrm{x})$ are represented by a set of $\mathrm{N}$ points

$$
\eta_{\mathrm{k}}(\mathrm{x})=\left\{\eta_{\mathrm{k}, \mathrm{i}}: \mathrm{i}=1, \mathrm{~N}\right\}
$$

which are found from the converged flux solutions by Fick's law

$$
\eta_{k, i}=\frac{1}{h s_{i}} D_{k, i}\left[\psi_{k, i}-\psi_{k, i+1}\right] ; i=1, N
$$

where $D_{k, i}$ is the diffusion constant homogeneous in interval $i$. $\eta_{k}(x)$ is of constant value, $\eta_{k, i}$, within each interval. The forms of these solutions are illustrated in Figure 4.2.

In order to approximate the symmetry boundary conditions imposed on the detailed subassembly flux solutions, small intervals hs ${ }_{1}$ and hs ${ }_{N}$ are defined at the edges of each subassembly. The detailed current solutions can then be made to have zero boundary values by setting $\eta_{\mathrm{k}, 1}$ and $\eta_{\mathrm{k}, \mathrm{N}}$ to zero. However, since the currents in each interval are defined as inversely proportional to the mesh size, the calculated boundary currents using this scheme may not be small enough to be negligible.

Explicit zero current boundary conditions can be imposed on the detailed current solutions by transforming the above discontinuous current $\eta_{\mathrm{K}}(\mathrm{x})$ into a continuous current solution $\tilde{\eta}_{\mathrm{k}}(\mathrm{x})$ represented by a set of $\mathrm{N}+1$ points

$$
\tilde{\eta}_{\mathrm{k}}(\mathrm{x})=\left\{\tilde{\eta}_{\mathrm{k}, \mathrm{i}}: \mathrm{i}=1 \text { to } \mathrm{N}+1\right\}
$$

where $\tilde{\eta}_{\mathrm{k}}(\mathrm{x})$ is linear between points, as also illustrated in Figure 4.2. By seeking to minimize the mean square error between $\eta_{k}(x)$ and $\tilde{\eta}_{k}(x)$ within each interval $i$, variational techniques yield the following set of N-1 equations for each group: 


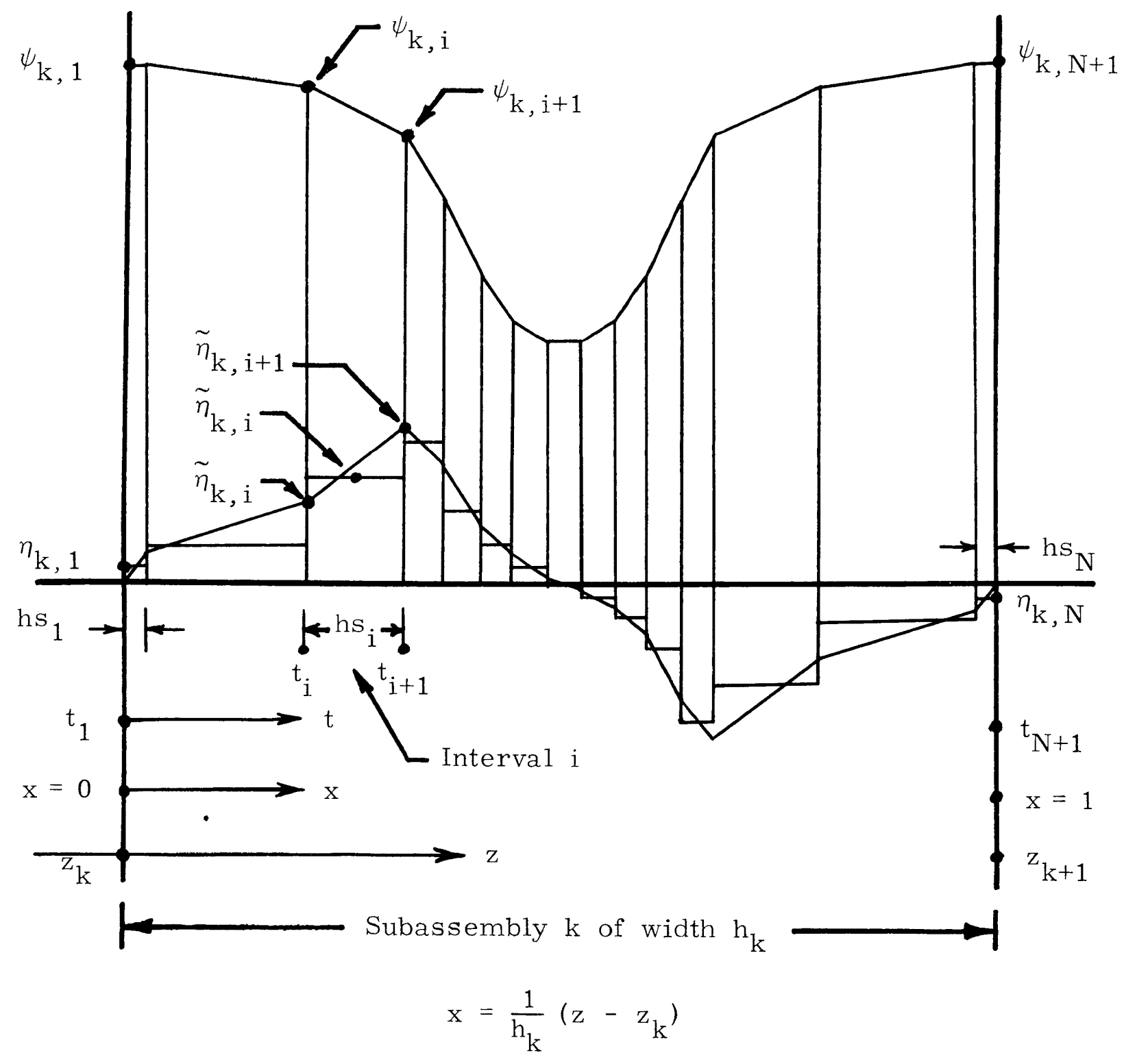

$0 \leqslant x \leqslant 1 ;$ for each subassembly $k ; k=1$ to $K$.

$$
y=\frac{1}{h s_{i}}\left(t-t_{i}\right)
$$

$0 \leqslant y \leqslant 1$; for each interval $i$ in subassembly $k ; i=1$ to $\mathrm{N}$.

Figure 4.2. Subassembly Notations and Detailed Solutions 


$$
\begin{gathered}
\left(\frac{1}{6} h s_{i-1}\right) \tilde{\eta}_{k, i-1}+\frac{1}{3}\left(h s_{i-1}+h s_{i}\right) \tilde{\eta}_{k, i}+\left(\frac{1}{6} h s_{i}\right) \tilde{\eta}_{k, i+1} \\
=\left(\frac{1}{2} h s_{i-1}\right) \eta_{k, i-1}+\left(\frac{1}{2} h s_{i}\right) \eta_{k, i} ; i=2 \text { to } \mathrm{N}
\end{gathered}
$$

These equations, given the $\eta_{\mathrm{k}, \mathrm{i}}$ from Eq. 4.29, are easily solved for the $\tilde{\eta}_{\mathrm{k}, \mathrm{i}}, \mathrm{i}=2$ to $\mathrm{N}$, where $\tilde{\eta}_{\mathrm{k}, 1}$ and $\tilde{\eta}_{\mathrm{k}, \mathrm{N}+1}$ are set to zero. Both forms of the detailed currents, $\eta_{\mathrm{k}}(\mathrm{x})$ and $\tilde{\eta}_{\mathrm{k}}(\mathrm{x})$, are allowed for use in the proposed approximation methods.

The proposed methods using linear and cubic Hermite basis functions have been programmed into computer codes LINEAR and CUBIC which are described respectively in sections 2 and 3 of Appendix D.

The matrix elements required for use in the approximation methods are integrals of products of subassembly detailed solutions and polynomial functions. These integrals are calculated, for each jndex $\mathrm{k}$, from the basic integral unit

$$
B I U_{k}=\int_{0}^{1} f_{k}(x) g_{k}(x) C_{k}(x) x^{n} h_{k} d x
$$

where the functions $f_{k}(x)$ and $g_{k}(x)$ represent flux and/or current solutions for same or different groups. These functions may be either constant within each interval

$$
f_{k}(x)=\left\{f_{k, i}: i=1 \text { to } N\right\}
$$

or of linear form within each interval

$$
f_{k}(x)=\left\{(1-y) f_{k, i}+y f_{k, i+1}: i=1 \text { to } N\right\}
$$

where $y=\frac{1}{h s_{i}}\left(t-t_{i}\right)$, as defined in Figure 4.2. $C_{k}(x)$ represents a 
group nuclear constant which is homogeneous in each interval

$$
C_{k}(x)=\left\{C_{k, i}: i=1 \text { to } N\right\}
$$

and $\mathrm{n}$ is a positive integer exponent in the range $0 \leqslant n \leqslant 6$. Since the following remarks concern only subassembly $\mathrm{k}$, the index $\mathrm{k}$ is dropped for simplicity.

The basic integral unit can be broken into integrals over each interval by transforming variables from $\mathrm{x}$ to $\mathrm{y}$. The result

$$
B I U_{k}=\frac{1}{h_{k}^{n}} \sum_{i=1}^{N} h s_{i} \int_{0}^{1} f_{i}(y) g_{i}(y) C_{i}\left(t_{i}+h s_{i} y\right)^{n} d y
$$

can be integrated analytically by expanding $\left(t_{i}+h s_{i} y\right)^{n}$ into a binomial series. The results of these integrations for any $\mathrm{n}$ depend only on the given forms of $f(x)$ and $g(x)$, and are summarized in Table 4.1 .

The coarse mesh flux-weighting homogenization calculations were performed using the above basic integral unit with $\mathrm{n}=0$. In these calculations a linear form of $\mathrm{f}(\mathrm{x})$, representing the detailed subassembly flux solutions from REF $2 \mathrm{G}$, and a constant value of $\mathrm{g}(\mathrm{x})=1$ were used.

Once the elements of the matrices of the approximation methods have been formed, considerable computer storage can be saved by collapsing the sparse band-structured matrices into full matrix form using row index transformations. In this way, a $\mathrm{N} \times \mathrm{N}$ tridiagonal matrix $\mathbb{L}$ resulting from the use of linear basis functions can be stored as the $\mathrm{N} \times 3$ matrix $\mathbb{I}^{\prime}$ by

$$
\left(\mathrm{IL}^{\prime}\right)_{\mathrm{ik}}=(\mathrm{IL})_{\mathrm{ij}}
$$

where $k=j+2-i$, and $k$ values outside $1 \leqslant k \leqslant 3$ are omitted. 
Table. 4.1. Calculation of the Basic Integral Unit in Subassembly $k$.

1. Constant $f(x)$ and constant $g(x)$ in each interval $i$ :

$$
\operatorname{BIU}_{k}=\frac{1}{h_{k}^{n}} \sum_{i=1}^{N}\left\{h s_{i} f_{i} g_{i} C_{i} \sum_{\ell=0}^{n} b_{\ell}^{n} t_{i}^{n-\ell} h s_{i}^{\ell} \frac{1}{(\ell+1)}\right\}
$$

2. Linear $f(x)$ and constant $g(x)$ in each interval $i$ :

$$
\mathrm{BIU}_{k}=\frac{1}{h_{k}^{n}} \sum_{i=1}^{N}\left\{h s_{i} g_{i} C_{i} \sum_{\ell=0}^{n} b_{\ell}^{n} t_{i}^{n-\ell} h s_{i}^{\ell}\left[\frac{f_{i}}{(\ell+1)(\ell+2)}+\frac{f_{i+1}}{(\ell+2)}\right]\right\}
$$

3. Linear $f(x)$ and linear $g(x)$ in each interval $i$ :

$$
\begin{aligned}
\mathrm{BIU}_{\mathrm{k}}= & \frac{1}{\mathrm{~h}_{\mathrm{k}}^{\mathrm{n}}} \sum_{i=1}^{\mathrm{N}}\left\{\mathrm { hs } _ { i } \mathrm { C } _ { i } \sum _ { \ell = 0 } ^ { \mathrm { n } } \mathrm { b } _ { \ell } ^ { \mathrm { n } } \mathrm { t } _ { \mathrm { i } } ^ { \mathrm { n } - \ell } \mathrm { hs } \mathrm { i } _ { i } ^ { \ell } \left[\frac{2 \mathrm{f}_{\mathrm{i}} \mathrm{g}_{\mathrm{i}}}{(\ell+1)(\ell+2)(\ell+3)}\right.\right. \\
& \left.\left.+\frac{\mathrm{f}_{\mathrm{i}} \mathrm{g}_{\mathrm{i}+1}+\mathrm{f}_{\mathrm{i}+1} \mathrm{~g}_{\mathrm{i}}}{(\ell+2)(\ell+3)}+\frac{\mathrm{f}_{\mathrm{i}+1} \mathrm{~g}_{\mathrm{i}+1}}{(\ell+3)}\right]\right\}
\end{aligned}
$$

where

$$
\mathrm{b}_{\ell}^{\mathrm{n}}=\frac{\mathrm{n} !}{\ell !(\mathrm{n}-\ell) !}
$$

is the binomial series coefficient. 
Similarly, a $N \times N$ matrix $\mathbb{L}$ of half-band width equal to three, which results from the use of cubic Hermite basis functions, can be stored as the $\mathrm{N} \times 6$ matrix $\mathbb{L}^{\prime}$, as given above, where in this case:

$$
k=j-i+3+ \begin{cases}1 & \text { for } i \text { odd } \\ 0 & \text { for } i \text { even }\end{cases}
$$

and $\mathrm{k}$ values outside $1 \leqslant \mathrm{k} \leqslant 6$ are omitted.

In those cases where the mass and stiffness matrices are both positive definite, Cholesky's method of matrix factorization,

$$
\mathbb{L}=\mathbb{G} \mathbb{G}^{\mathrm{T}}
$$

where $\mathbb{L}$ is positive definite and $\mathbb{G}$ is lower triangular, can be used to solve the matrix inversion for each group in the power method. The matrix elements $g_{i j}=(\mathbb{G})_{i j}$ are calculated from the elements $\ell_{i j}=(\mathbb{L}){ }_{i j}$ by the following algorithm: ${ }^{51}$

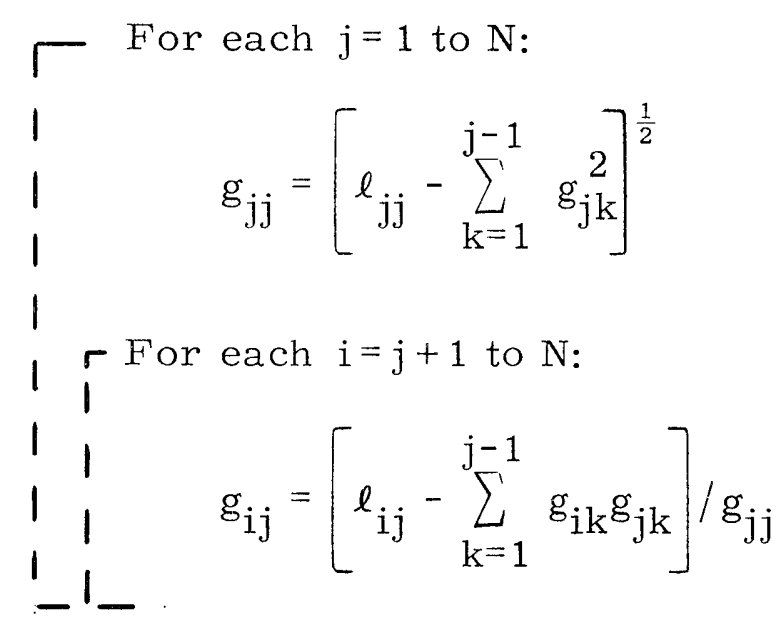

Similar algorithms of a more complex form are used in the computer codes in conjunction with the matrix collapsing schemes given above. 
Chapter 5

\section{NUMERICAL RESULTS}

\subsection{Nuclear Constants and Subassembly Geometry}

The effectiveness and accuracy of the proposed approximation methods were examined using one-dimensional, one- and two-group reactor configurations composed of representative PWR fuel subassemblies. Four separate subassemblies with identical geometry but different number constants are considered. Each subassembly is represented as an $18-\mathrm{cm}$, homogeneous fuel region of low, medium, or high enrichment, surrounding a $1-\mathrm{cm}$ centrally located absorption rod or water channel. Two-group regional nuclear constants used to represent such PWR subassembly geometries are given in Table 5.1, ${ }^{52}$ where all fission neutrons are assumed to be born in the fast group. These constants were collapsed into representative one-group constants using the standard infinite medium group reduction procedure for two groups:

$$
\langle\Sigma\rangle_{1 \mathrm{G}}=\frac{1}{(1+\alpha)}\left(\Sigma_{1}+\alpha \Sigma_{2}\right)
$$

where $\Sigma_{1}$ and $\Sigma_{2}$ are macroscopic cross sections for the fast and thermal groups, respectively, and $\alpha$ is the infinite medium thermal to fast flux ratio. ${ }^{53}$ The resulting one-group regional constants for the fuel and rod regions are given in Table 5.2, where the flux ratios of the three fuel regions have been averaged in order to collapse the absorption rod constants. 
Table 5.1. Representative Two-Group, 18-cm, PWR Subassembly Regional Nuclear Constants. $x_{1}=1.0 ; x_{2}=0.0$.

\begin{tabular}{lllll}
\hline Region Material & $\Sigma_{\mathrm{T}}$ & $\nu \Sigma_{\mathrm{f}}$ & $\mathrm{D}$ & $\Sigma_{21}$ \\
\hline Fuel A: Low w/o & .0259 & .00485 & 1.396 & .0179 \\
& .0532 & .0636 & .388 & \\
Fuel B: Medium w/o & .0260 & .00553 & 1.397 & .0172 \\
& .0710 & .102 & .389 & \\
Fuel C: High w/o & .0261 & .00659 & 1.399 & .0168 \\
& .0832 & .129 & .387 & \\
Absorption Rod & .0452 & 0.0 & 1.0 & 0.0 \\
& .959 & 0.0 & 1.0 & .0380 \\
Water & .0383 & 0.0 & 1.63 & \\
\hline
\end{tabular}

Fast group constants appear first for each region material, followed by thermal group constants. Fission neutrons are assumed to be born in the fast group only.

Table 5.2. Representative One-Group, 18-cm, PWR Subassembly Regional Nuclear Constants.

\begin{tabular}{lccc}
\hline Region Material & $\Sigma_{\mathrm{T}}$ & $\nu_{\mathrm{f}}$ & $\mathrm{D}$ \\
\hline Fuel A: Low w/o & .0329 & .0199 & 1.14 \\
Fuel B: Medium w/o & .0348 & .0244 & 1.20 \\
Fuel C: High w/o & .0357 & .0272 & 1.23 \\
Absorption Rod & .235 & 0.0 & 1.0 \\
Water & .0136 & 0.0 & .414 \\
\hline
\end{tabular}


Four subassembly configurations, labeled A through D, were used in the one- and two-group test configurations, and a re illustrated in Figure 5.1. Subassemblies labeled A, B, and C contain homogeneous fuel of low, medium, and high enrichment, respectively, surrounding the 1 - $\mathrm{cm}$ absorption rod while subassembly D contains low enriched homogeneous fuel surrounding a 1-cm water channel.

\subsection{Subassembly Detailed Solutions and Homogenized Nuclear Constants}

The detailed flux and current solutions for each subassembly were found using the computer code REF2G with symmetry boundary conditions and a 68-mesh region per subassembly geometry as indicated in Figure 5.2. The resulting one-group detailed flux solutions for each subassembly are shown in Figure 5.3. The resulting twogroup detailed flux and adjoint flux solutions for each subassembly are shown in Figures 5.4 and 5.5 , respectively.

Homogeneous subassembly group constants for use in the finite element approximations were found by flux weighting the group cross sections in each subassembly by the corresponding subassembly detailed group flux solutions. The resulting homogenized one-group constants for each subassembly are given in Table 5.3 , and the resulting homogenized two-group constants are given in Table 5.4 . The results of homogenizing the diffusion coefficient as the transport cross section, $1 /\langle 1 / D\rangle$, as well as by direct homogenization, $\langle D\rangle$, are included in the tables. The results of both schemes were found to differ at most by only $2 \%$. The directly homogenized diffusion coefficients, $\langle D\rangle$, were used in the finite element approximations. 
Subassembly Type A:

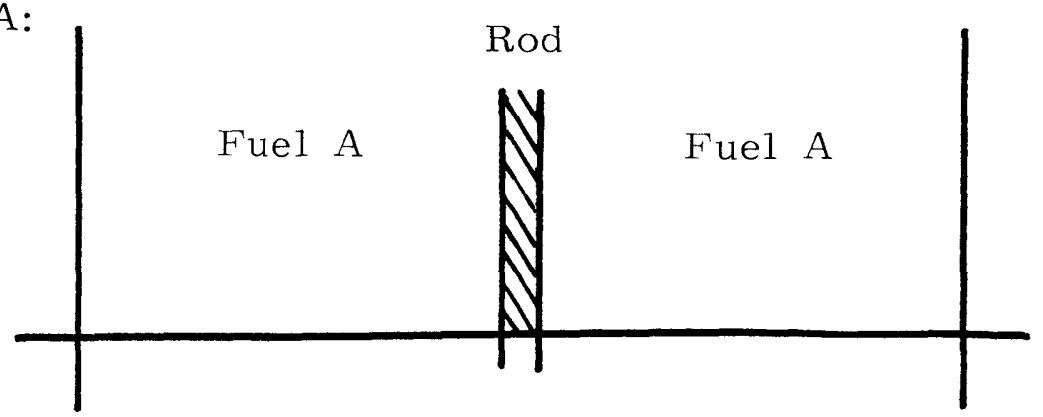

Subassembly Type B:

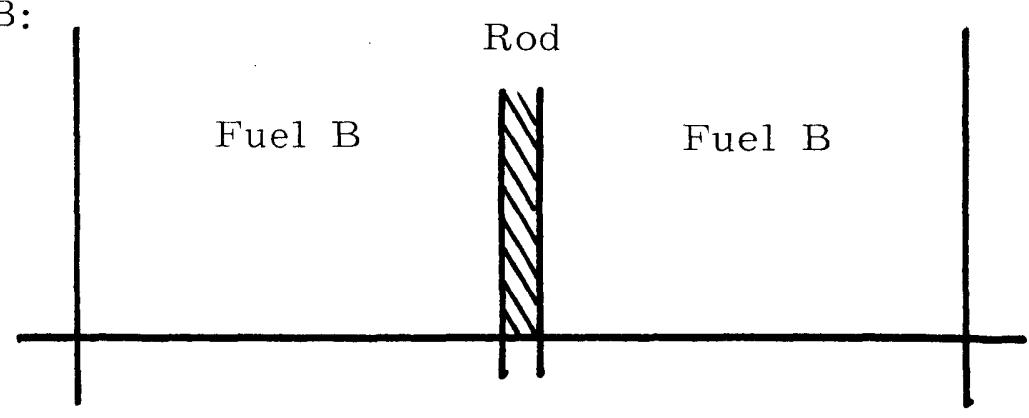

Subassembly Type C:

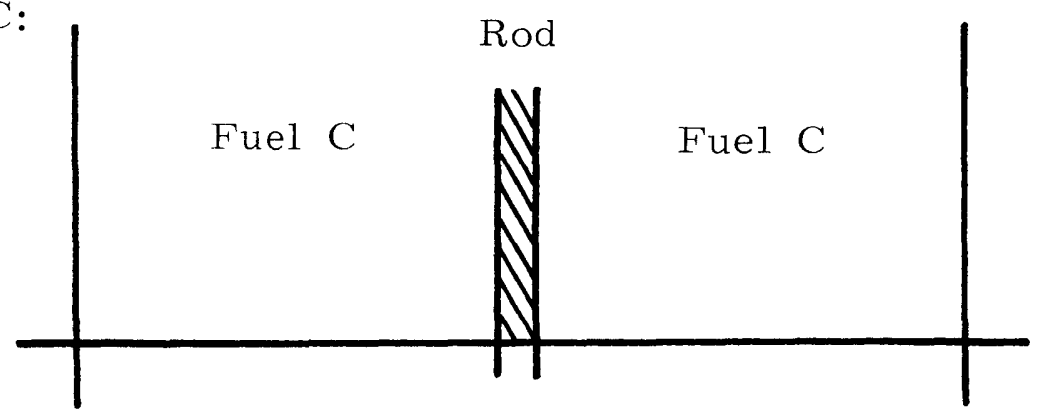

Subassembly Type D:

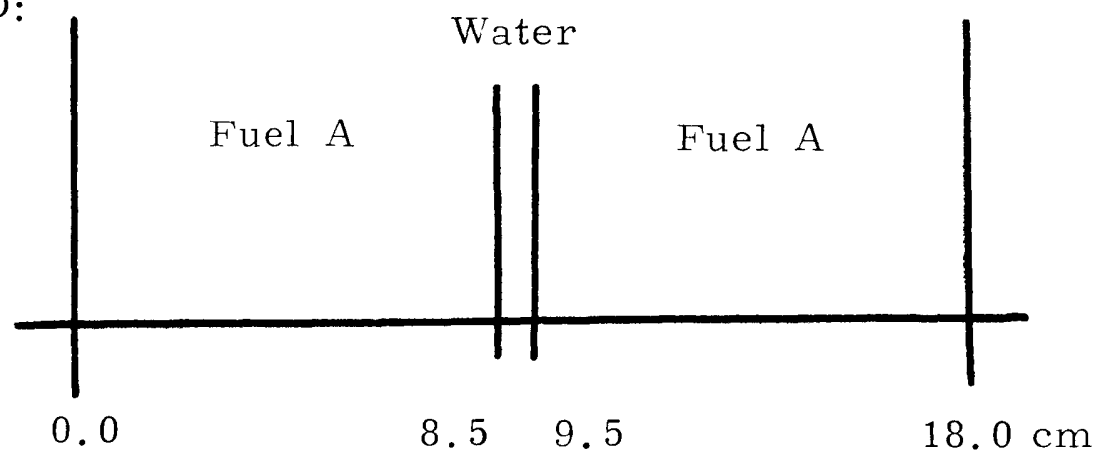

Figure 5.1. Subassembly Configuration Geometries 


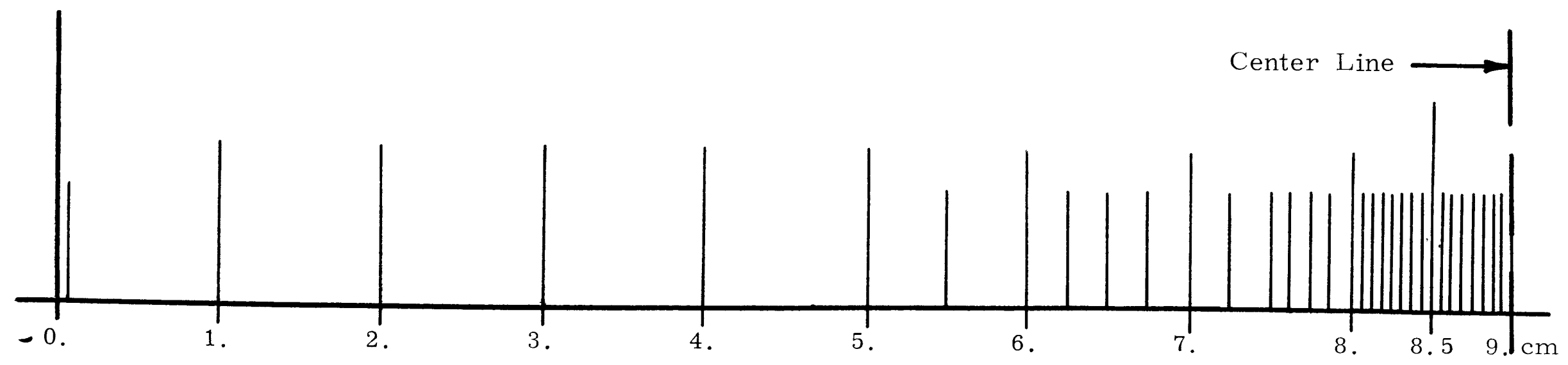

Symmetric Partitioning:

$1(1 / 16 \mathrm{~cm})+1(15 / 16)+4(1)+2(1 / 2)+6(1 / 4)+4(1 / 8)+8(1 / 16)+8(1 / 16)$

Figure 5.2. Mesh Geometry in Half a Subassembly.

Detailed flux and current solution calculations use

this 68 intervals/subassembly geometry in each

subassembly type. 


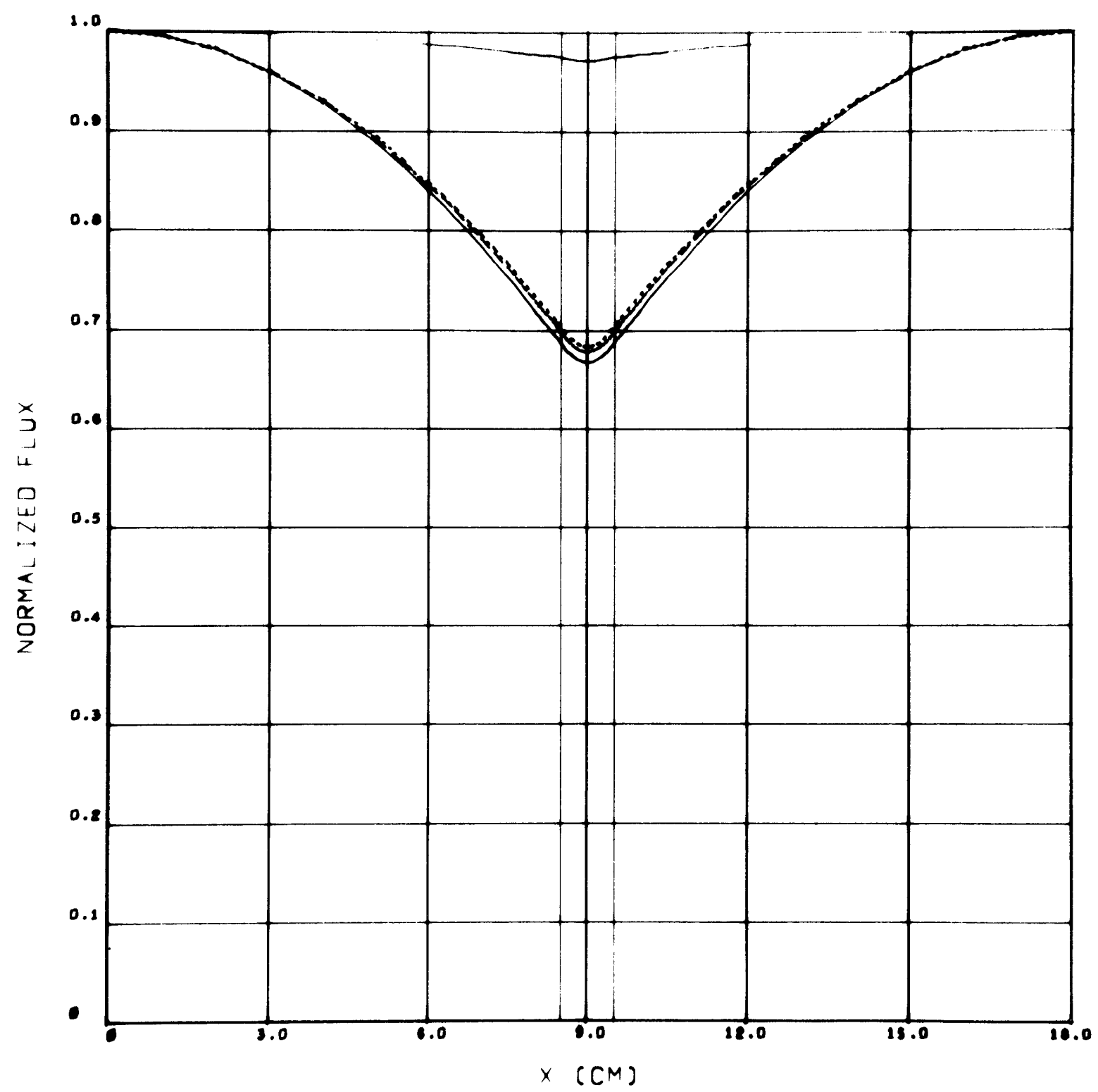

Figure 5.3. Subassembly Detailed Flux Solutions for the One-Group Case

Subassembly Type A (lower curve)

Subassembly Type B - - - -

Subassembly Type C $\ldots \ldots \ldots$

Subassembly Type D (upper curve) 


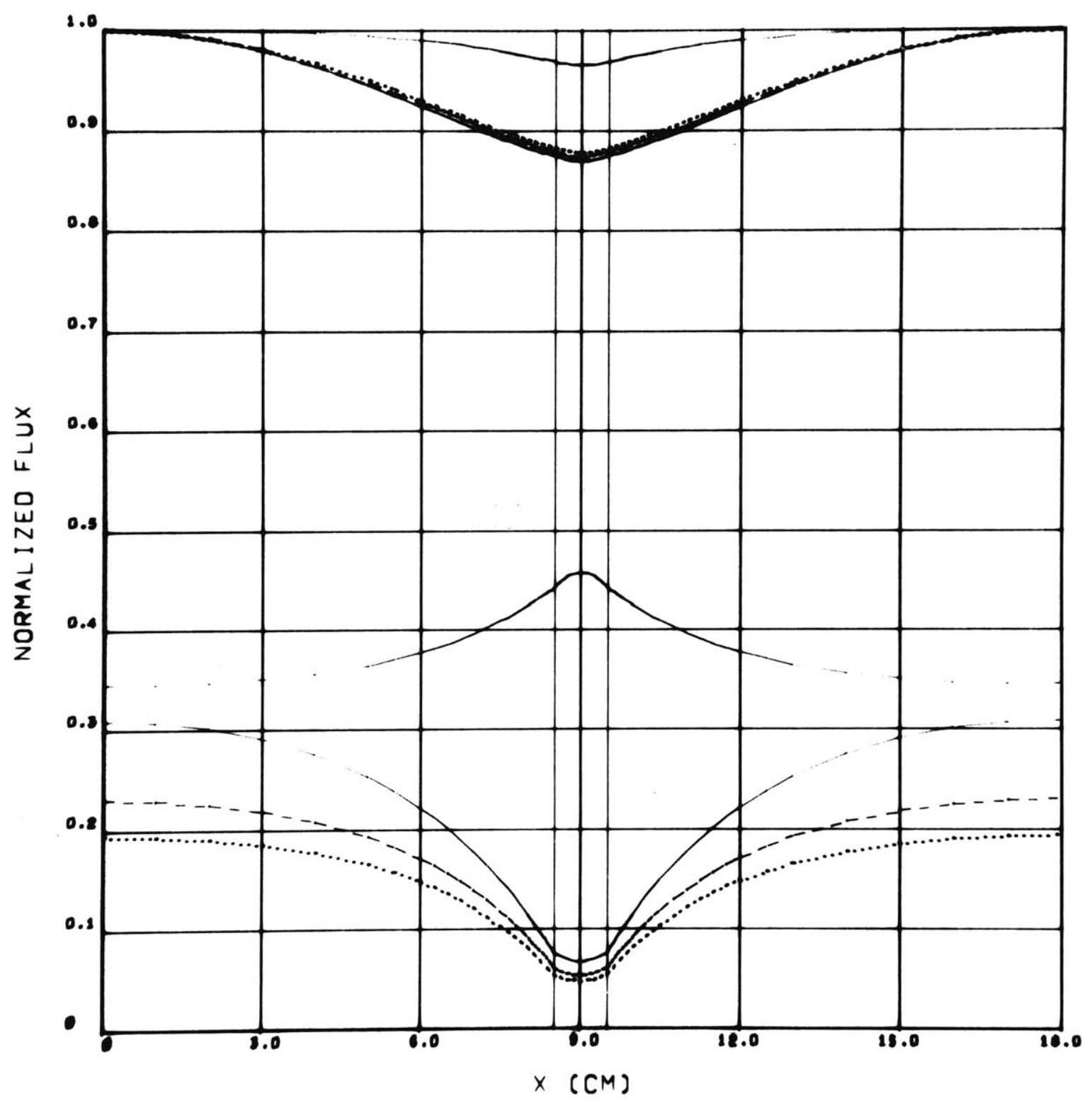

Figure 5.4. Subassembly Detailed Flux Solutions for the Two-Group Case

The fluxes are normalized by fast flux values so that the thermal fluxes appear in the lower portion of the figure.

Subassembly Type A (lower curves)

Subassembly Type B - - - -

Subassembly Type C $\ldots \ldots \ldots$

Subassembly Type D (upper curves) 


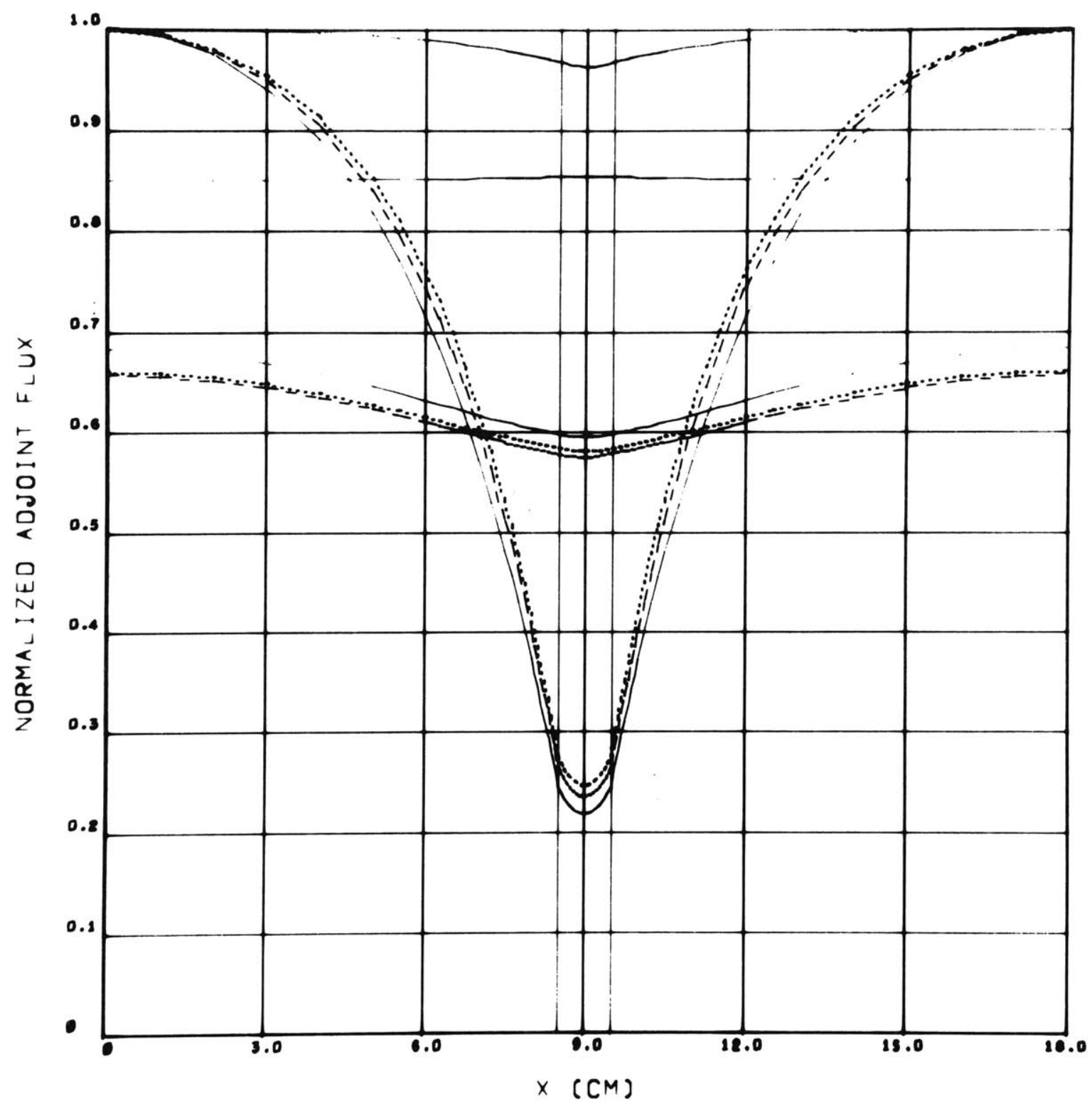

Figure 5.5. Subassembly Detailed Adjoint Flux Solutions for the Two-Group Case

Subassembly Type A (lower curves)

Subassembly Type B - - - -

Subassembly Type C

Subassembly Type D (upper curves) 
Table 5.3. Homogenized Subassembly One-Group Nuclear Constants.

\begin{tabular}{ccccc}
\hline Subassembly & $\left\langle\Sigma_{\mathrm{T}}\right\rangle$ & $\left\langle\nu \Sigma_{\mathrm{f}}\right\rangle$ & $\langle\mathrm{D}\rangle$ & $1 /\langle 1 / \mathrm{D}\rangle$ \\
\hline $\mathrm{A}$ & .04149392 & .01905379 & 1.134047 & 1.133253 \\
$\mathrm{~B}$ & .04341140 & .02335046 & 1.191397 & 1.189765 \\
$\mathrm{C}$ & .04431869 & .02602374 & 1.220054 & 1.217887 \\
$\mathrm{D}$ & .03184731 & .01881458 & 1.100401 & 1.040479 \\
\hline
\end{tabular}

Table 5.4. Homogenized Subassembly Two-Group Nuclear Constants. $x_{1}=1.0 ; x_{2}=0.0$.

\begin{tabular}{cccccc}
\hline $\begin{array}{c}\text { Sub- } \\
\text { assembly }\end{array}$ & $\left\langle\Sigma_{\mathrm{T}}\right\rangle$ & \multicolumn{1}{c}{$\left\langle\nu \Sigma_{\mathrm{f}}\right\rangle$} & $\langle\mathrm{D}\rangle$ & $\left\langle\Sigma_{21}\right\rangle$ & $1 /\langle 1 / \mathrm{D}\rangle$ \\
\hline $\mathrm{A}$ & .02688787 & .004601752 & 1.379526 & .01698379 & 1.371911 \\
& .06812834 & .06255182 & .3980863 & & .3919533 \\
$\mathrm{~B}$ & .02698495 & .005246314 & 1.379480 & .01631765 & 1.37185 \\
& .08647802 & .1002221 & .3996499 & & .3931874 \\
$\mathrm{C}$ & .02708207 & .006251160 & 1.379433 & .01593619 & 1.371787 \\
& .09893614 & .1266822 & .3980142 & & .391310 \\
$\mathrm{D}$ & .02657213 & .004587110 & 1.412467 & .0189895 & 1.410790 \\
& .05034835 & .05932253 & .3804001 & & .3775656 \\
\hline
\end{tabular}

Fast group constants appear first for each subassembly, followed by thermal group constants. 
Before applying the proposed approximation methods to complex reactor geometries, test runs were performed in order to evaluate the differences between using either flux or adjoint flux weighting, and using current solutions of either constant or linear form in each subassembly interval, as described in section 4.2. The test problem consisted of three consecutive Type A subassemblies with symmetry boundary conditions imposed on each end so that the converged eigenvalue $\lambda\left(\mathrm{k}_{\text {eff }}\right)$ should be identical to that of the detailed flux solution of subassembly A. Entire subassemblies were chosen as the mesh regions so that the proposed synthesis methods should converge to flux values of unity, and current values of zero. The numerical results of these tests for the one- and two-group cases are summarized in Table 5.5. Although the choice of weighting function did not influence the results for either approximation, use of current solutions of the linear form enables better eigenvalue accuracy. In addition, the results when using currents of linear form converged to flux values of unity and current values of zero, as expected, while results using the constant current form produced errors of about $0.5 \%$ in the converged flux and $0.01 \%$ in the converged current at interior points. Although the difference in accuracy between the use of these different current forms is small, the small flux and current errors resulting from the use of the constant current form may lead to larger errors in larger and more complex problems. For the above reasons, the linear current form was used in the following case studies. Adjoint weighting was also used. Although the use of adjoint weighting has not been shown to guarantee the success of Cholesky's method in the numerical solution scheme, no difficulties with its use were ever encountered. 
Table 5.5. Test Results Using Three Consecutive Type A Subassemblies. $\% \lambda=\left[\left(\lambda_{\text {Sub. }} \mathrm{A}^{-\lambda}\right.\right.$ Conv. $\left.) / \lambda_{\text {Sub. }} \mathrm{A}\right] \times 100 \%$.

\begin{tabular}{|c|c|c|c|c|}
\hline $\begin{array}{c}\text { Synthesis } \\
\text { Approximation }\end{array}$ & $\begin{array}{l}\text { Weighting } \\
\text { Function }\end{array}$ & $\begin{array}{l}\text { Form of } \\
\text { Currents }\end{array}$ & $\begin{array}{c}\text { Converged } \\
\lambda\end{array}$ & $\% \lambda$ \\
\hline \multicolumn{5}{|c|}{ ONE GROUP: $\lambda_{\text {Sub. } A}=0.459194$} \\
\hline Linear & FLUX & Constant & .459363 & $-.036 \%$ \\
\hline Linear & FLUX & Linear & .459254 & $-.013 \%$ \\
\hline Cubic & FLUX & Constant & .459363 & $-.036 \%$ \\
\hline Cubic & FLUX & Linear & .459254 & $-.013 \%$ \\
\hline
\end{tabular}

TWO GROUPS: $\lambda_{\text {Sub. } A}=0.751095$

\begin{tabular}{ccccc}
\hline Linear & FLUX & Linear & .751284 & $-.025 \%$ \\
Linear & ADJOINT & Constant & .7513818 & $-.038 \%$ \\
Linear & ADJOINT & Linear & .751284 & $-.025 \%$ \\
Cubic & FLUX & Linear & .751284 & $-.025 \%$ \\
Cubic & ADJOINT & Constant & .7513818 & $-.038 \%$ \\
Cubic & ADJOINT & Linear & .751284 & $-.025 \%$ \\
\hline
\end{tabular}




\section{3 Case Studies and Results}

Four one-dimensional reactor configurations, each made up of different combinations of types of subassemblies, are considered in the case studies below. One-group calculations were performed only for the first case, and two-group calculations were performed in all cases. Entire 18-cm subassemblies were used as coarse mesh regions in each case, while the effect of using half-subassembly mesh regions was also included in Case 1 . The geometry and subassembly configurations of the case studies are shown in Figure 5.6.

Three separate approximation methods were used to calculate converged detailed flux solutions for comparison in each case. They are:

1. The proposed approximation methods using heterogeneous nuclear constants and subassembly detailed flux solutions for coarse mesh solutions.

2. The finite element methods using subassembly homogenized nuclear constants for coarse mesh solutions.

3. The linear finite element method for fine mesh reference solutions.

Calculations of both the proposed approximation and the coarse mesh finite element method using linear basis functions were performed using program LINEAR, while the corresponding cubic Hermite basis function approximations were performed using program CUBIC. The fine mesh reference solutions were calculated using program REF2G, and the results of these approximations were compared and analyzed by program ANALYZE. Descriptions of these programs are given in Appendix D. 
Case 1:

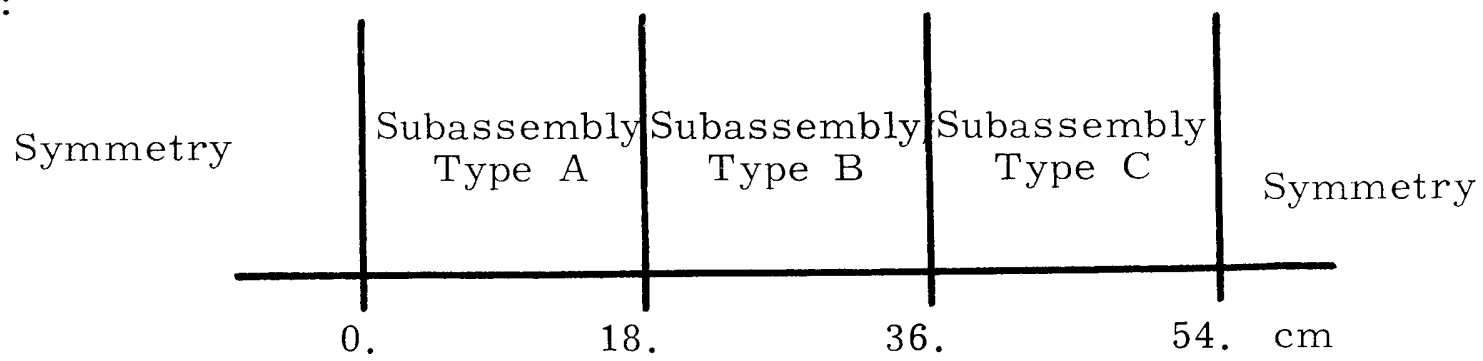

Case 2:

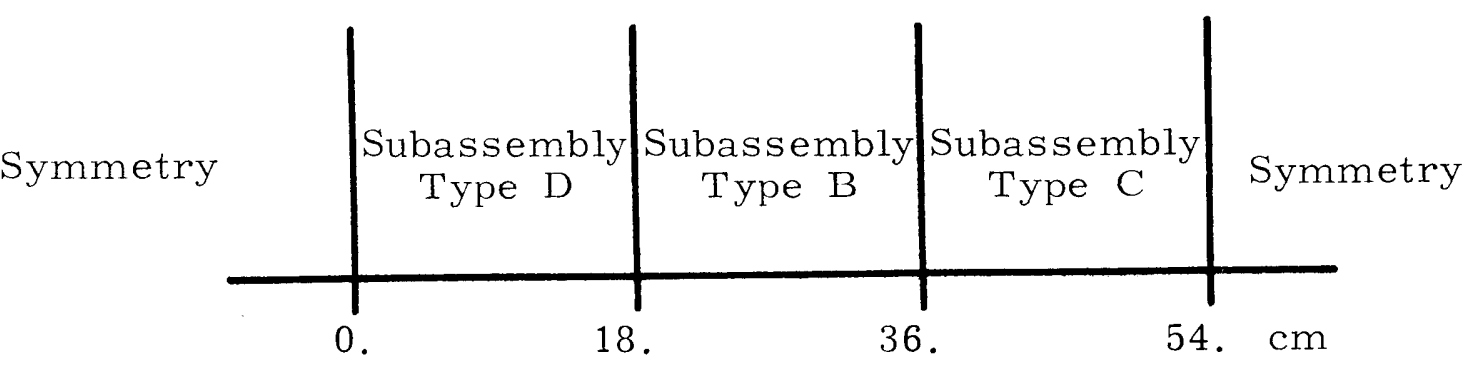

Case 3:

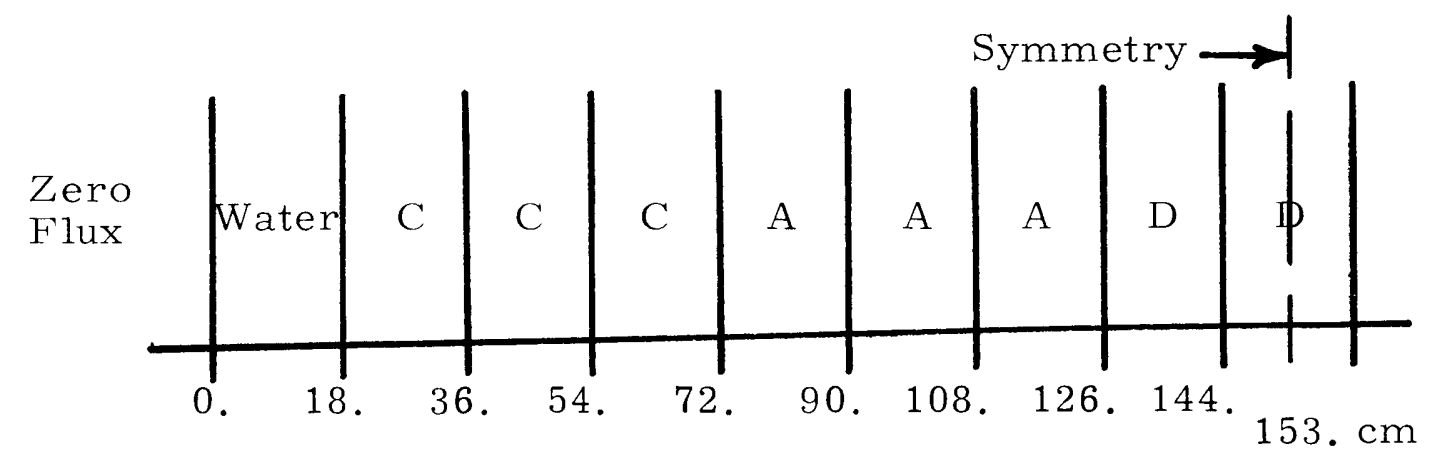

Case 4 :

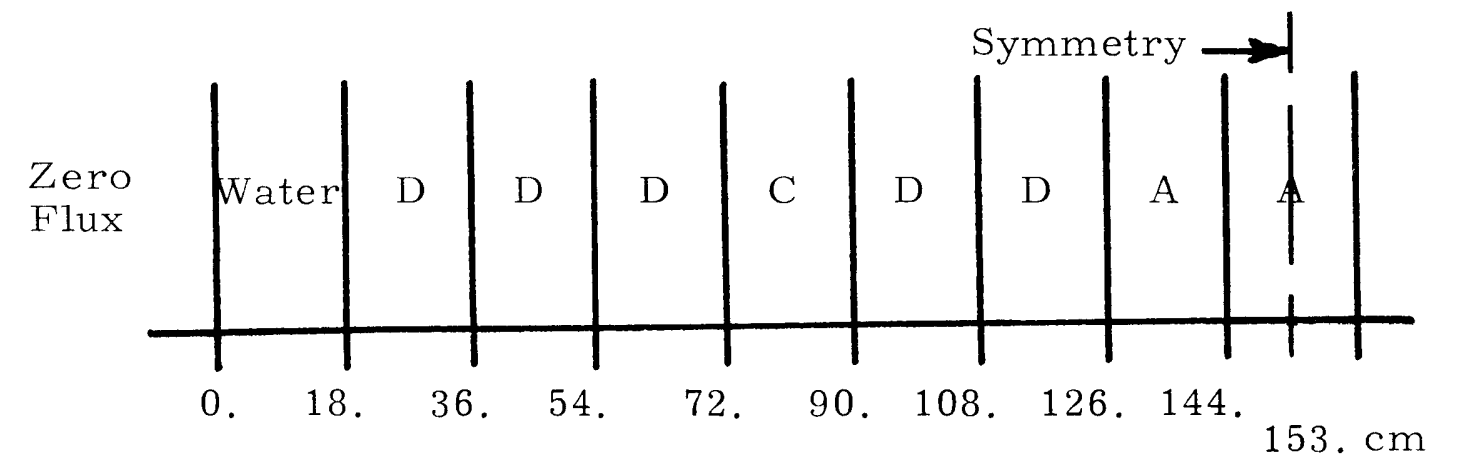

Figure 5.6. Geometry of the Four Case Studies

Composed of Types of Subassemblies 
The results of each case study are divided into the two approximation method categories as defined below.

1. The linear basis function approximation

A. Linear FEM:

(The linear finite element method using homogenized coarse mesh nuclear constants)

B. Linear Synth:

(The proposed approximation method using heterogeneous coarse mesh nuclear constants and detailed coarse mesh solutions)

2. The cubic Hermite basis function approximations
A. Cubic FEM
B. Cubic Synth

The results of the approximations in each category are compared to the reference solution by examining:

1. The converged eigenvalues $\lambda\left(\mathrm{k}_{\text {eff }}\right)$ and their percent normalized eigenvalue error,

$$
\% \lambda=\left(\lambda_{\text {Ref }}-\lambda_{\text {Conv }}\right) / \lambda_{\text {Ref }} \times 100 \%
$$

2. Composite graphs of the converged detailed group flux solutions $U_{g}(z)$ normalized to equivalent power levels

3. The fractional normalized power levels $P(k)$ calculated for each $18-\mathrm{cm}$ subassembly $\mathrm{k}$ by 


$$
P(k)=\frac{\int_{\text {Sub. } k} \sum_{g=1}^{G} \nu \Sigma_{f g}(z) U_{g}(z) d z}{\int_{\text {All Subs }} \sum_{g=1}^{G} \nu \Sigma_{f g}(z) U_{g}(z) d z}
$$

and their percent normalized errors

$$
{ }_{0} \mathrm{P}(\mathrm{k})=\left(\mathrm{P}(\mathrm{k})_{\text {Ref }}-\mathrm{P}(\mathrm{k})_{\mathrm{Conv}}\right) / \mathrm{P}(\mathrm{k})_{\operatorname{Ref}} \times 100 \%
$$

5.3.1. Case 1: Three different subassemblies of Types A, B, and C with symmetry boundary conditions.

The graphical results of the one-group approximation methods for this case are shown in Figures 5.7 and 5.8 , while the results of the two-group approximation methods are presented in Figures 5.9-5.12. Only the coarse mesh boundaries are labeled in the figures, which indicates that entire $18-\mathrm{cm}$ subassemblies were used as the coarse mesh regions. Two-group results using only half-subassemblies as the coarse mesh regions are shown in Figures 5.13-5.16. The reference solutions were calculated using 150-mesh regions, as defined by the symmetric partitioning

$$
5(1 \mathrm{~cm})+4(.5 \mathrm{~cm})+4(.25 \mathrm{~cm})+4(.125 \mathrm{~cm})+8(.0625 \mathrm{~cm})
$$

in each of the three subassemblies. The converged approximation eigenvalues and fractional normalized subassembly power levels for the one- and two-group calculations are summarized in Table 5.6. The fractional powers, $\mathrm{P}(\mathrm{k})$, for each subassembly are listed in the reference solution column, while the percent errors, $\% \mathrm{P}(\mathrm{k})$ are listed in the approximation columns. 


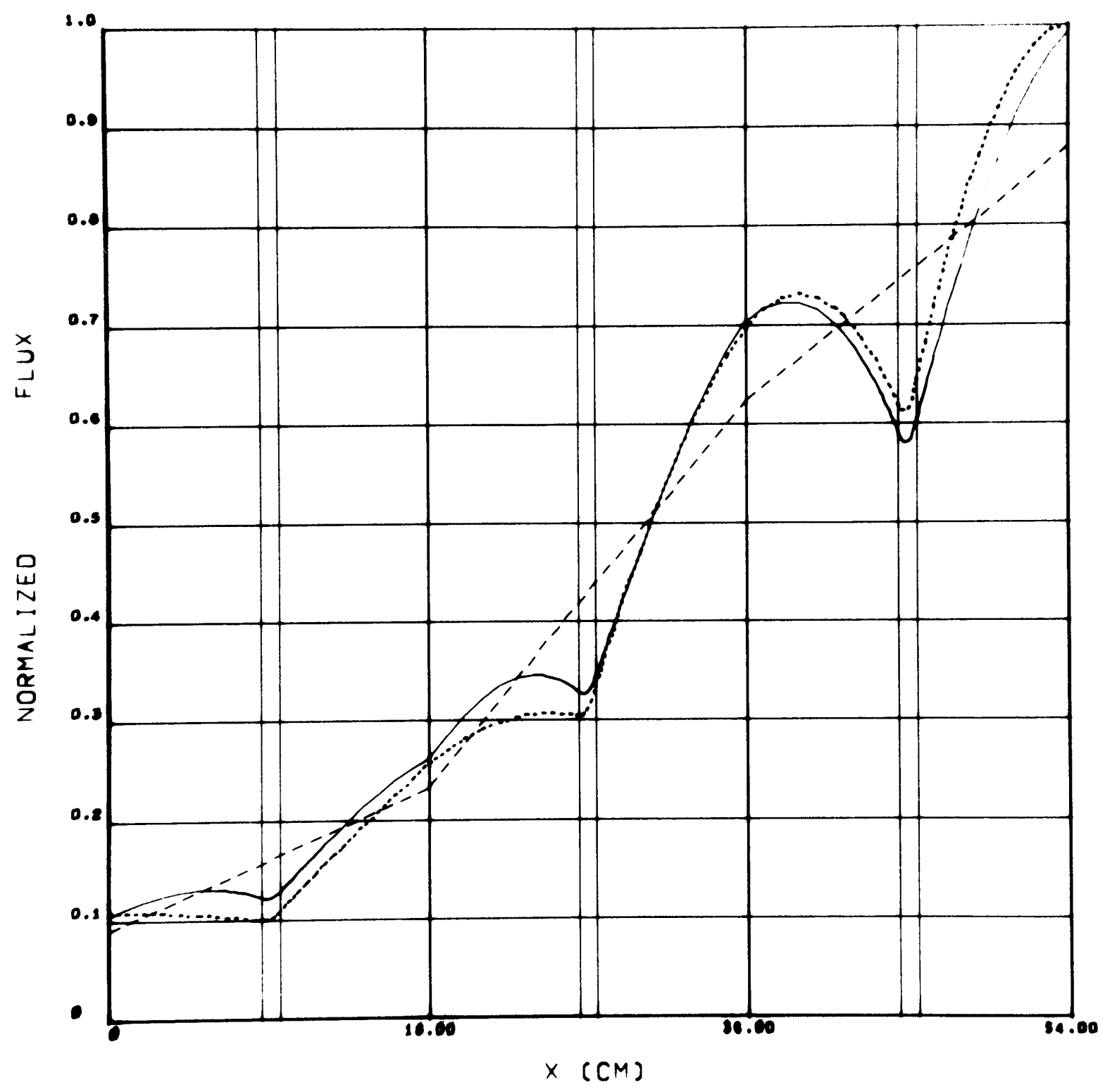

Figure 5.7. Case 1: One-Group Results Using Linear Basis Function Approximations and 18-cm Coarse Mesh Regions

\begin{tabular}{lll}
\multicolumn{1}{c}{ Method } & & $\frac{\lambda}{1}$ \\
Reference & $\ldots \ldots \ldots$ & .559045 \\
Linear FEM & ----- & .556943 \\
Linear Synth & & .557154
\end{tabular}




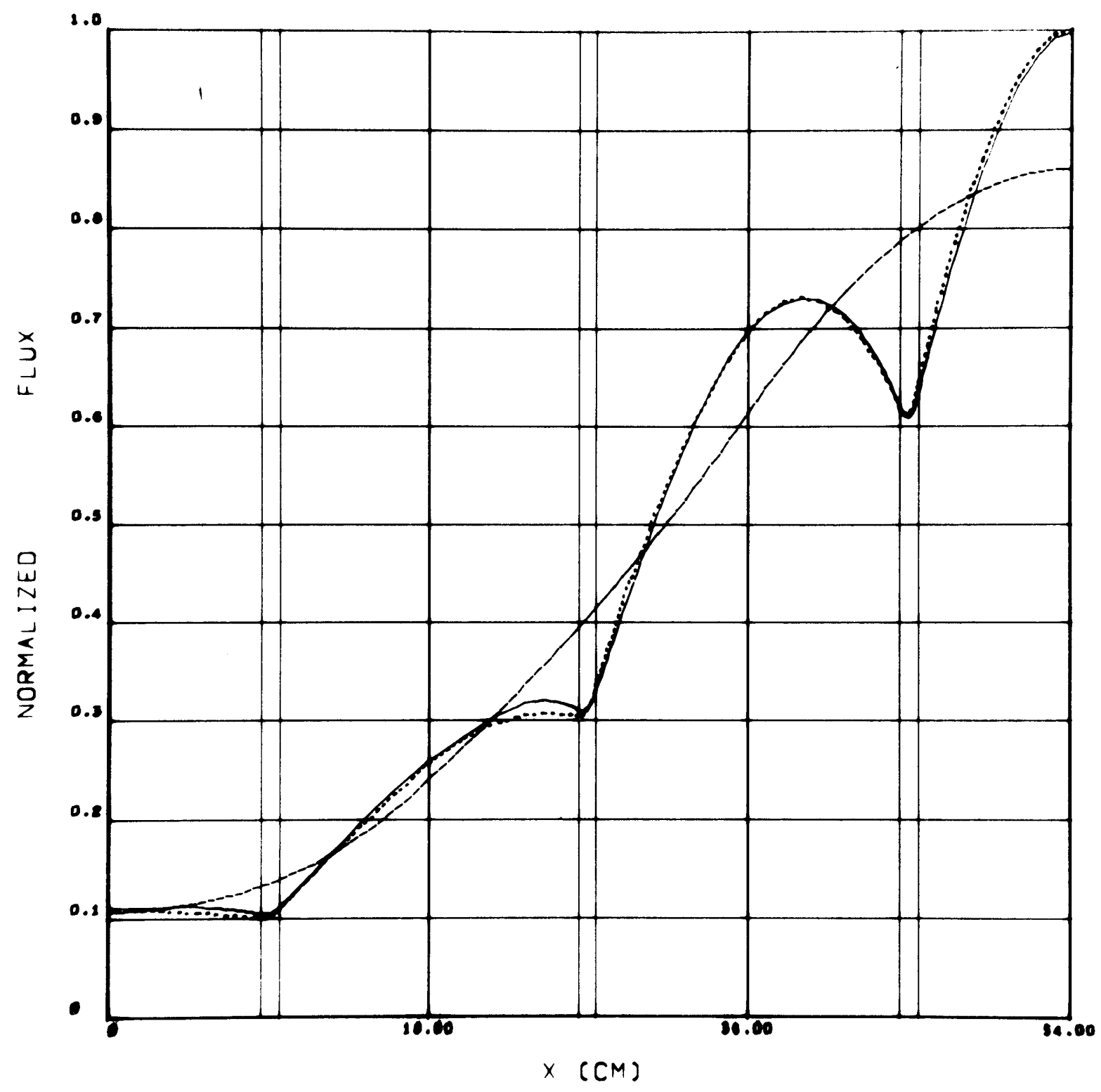

Figure 5.8. Case 1: One-Group Results Using Cubic Hermite Basis Function Approximations and 18-cm Coarse Mesh Regions

\begin{tabular}{lcc}
\multicolumn{1}{c}{ Method } & & $\lambda$ \\
Reference & $\ldots \ldots \ldots$ & .559045 \\
Cubic FEM & ----- & .558647 \\
Cubic Synth & & .558761
\end{tabular}




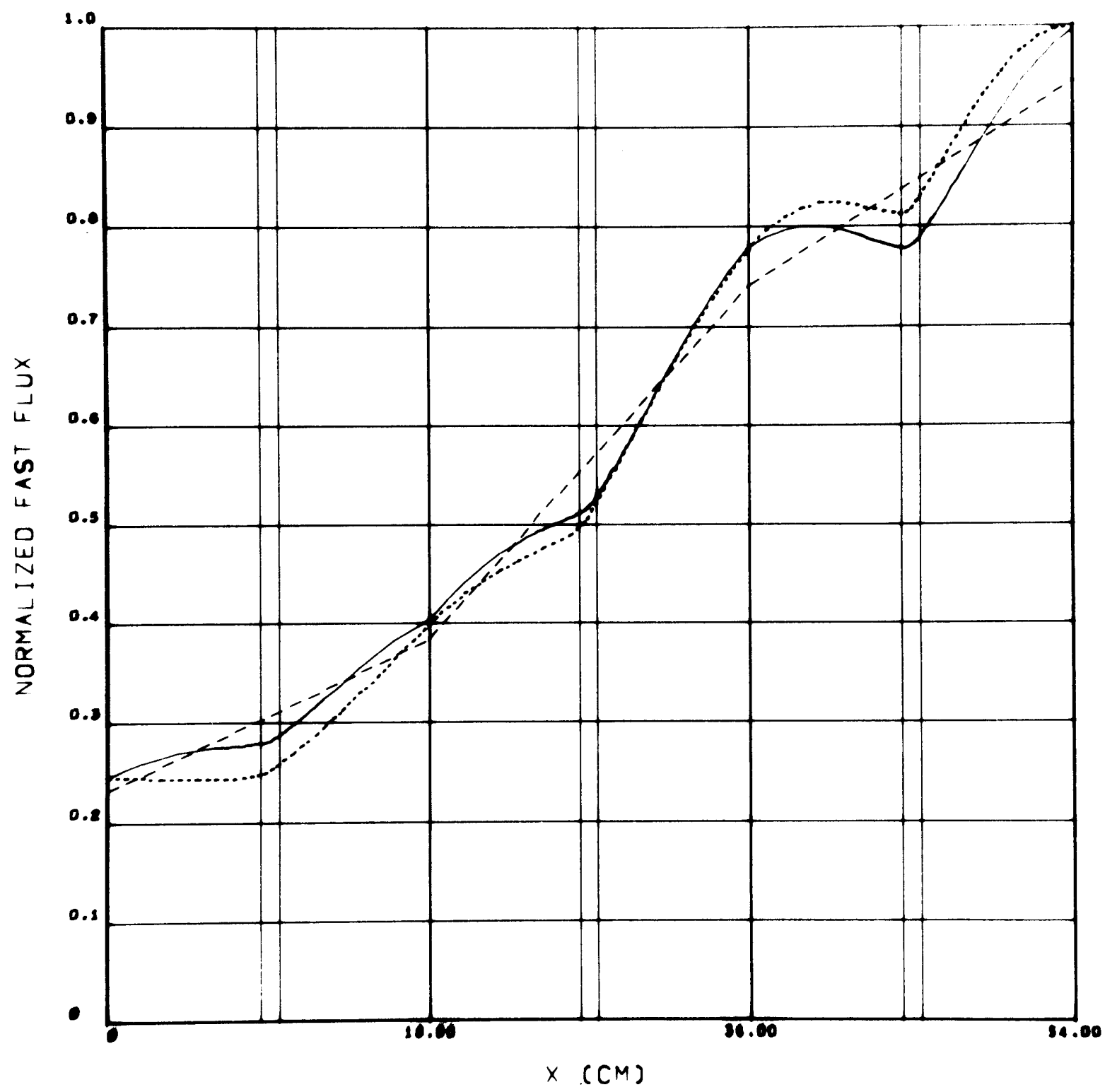

Figure 5.9. Case 1: Two-Group Fast Results Using Linear Basis Function Approximations and $18-\mathrm{cm}$ Coarse Mesh Regions

\begin{tabular}{llc}
\multicolumn{1}{c}{ Method } & & $\frac{\lambda}{1 .}$ \\
Reference & $\ldots \ldots$ & .917267 \\
Linear FEM & $-\ldots---$ & .914489 \\
Linear Synth & - & .915221
\end{tabular}




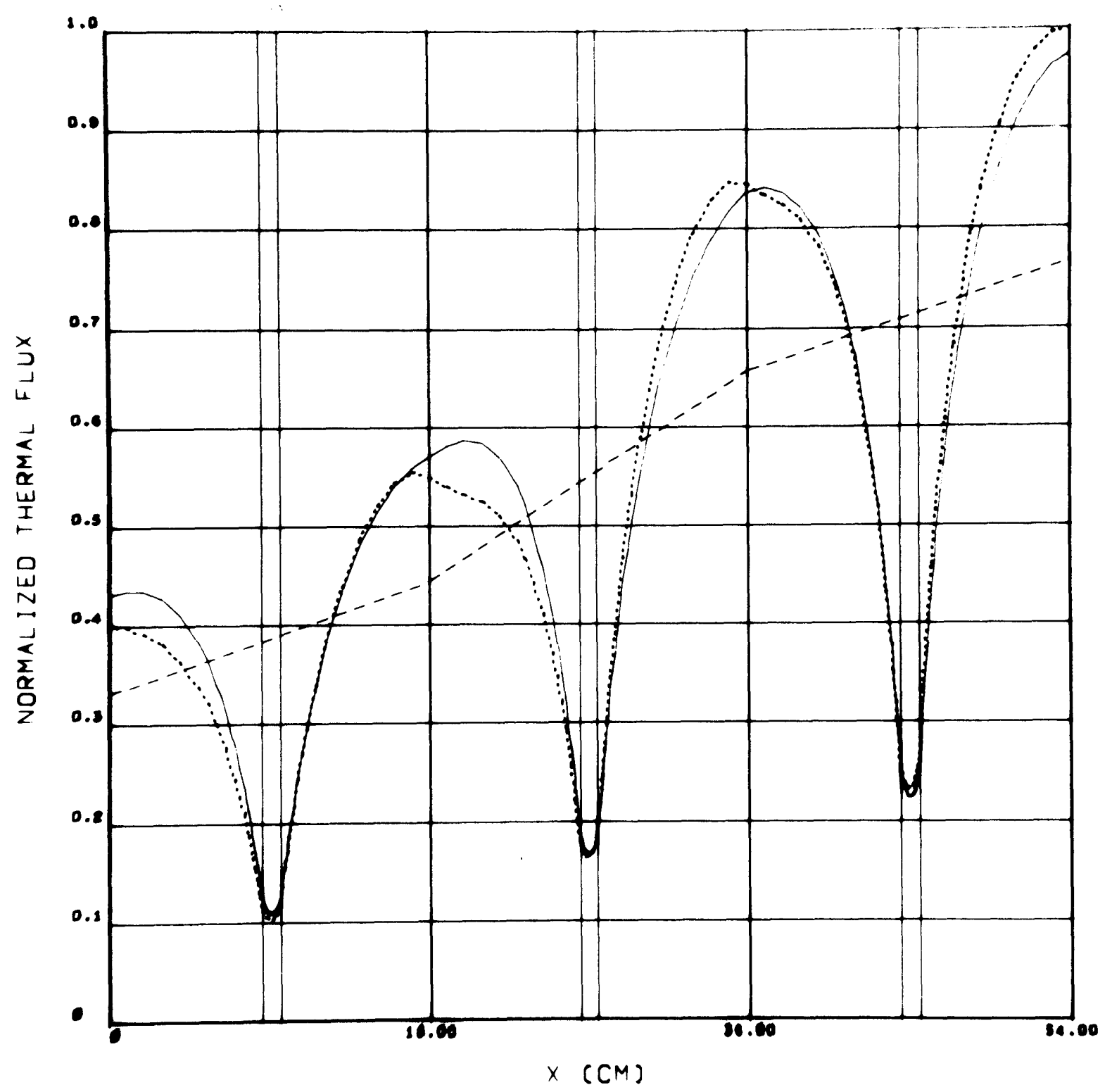

Figure 5.10. Case 1: Two-Group Thermal Results Using Linear Basis Function Approximations and 18-cm Coarse Mesh Regions

\begin{tabular}{lcc}
\multicolumn{1}{c}{ Method } & & $\lambda$ \\
Reference & $\ldots \ldots \ldots \ldots$ & .917267 \\
Linear FEM & ----- & .914489 \\
Linear Synth & & .915221
\end{tabular}




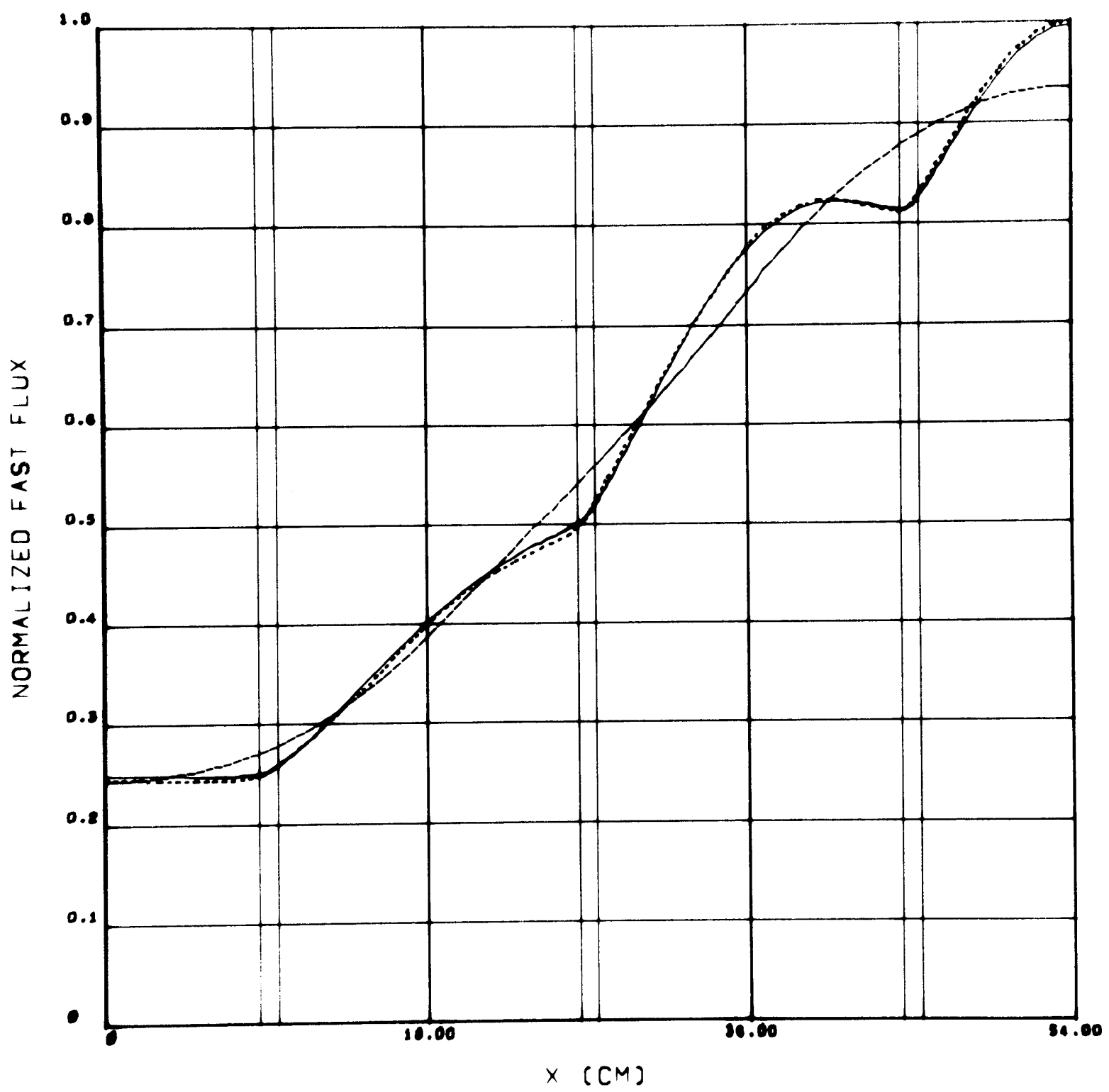

Figure 5.11. Case 1: Two-Group Fast Results Using Cubic Hermite Basis Function Approximations and $18-\mathrm{cm}$ Coarse Mesh Regions

\begin{tabular}{llc}
\multicolumn{1}{c}{ Method } & & $\frac{\lambda}{1 .}$ \\
Reference & $\ldots \ldots \ldots$ & .917267 \\
Cubic FEM & $-\ldots-\ldots-$ & .916717 \\
Cubic Synth & & .917059
\end{tabular}




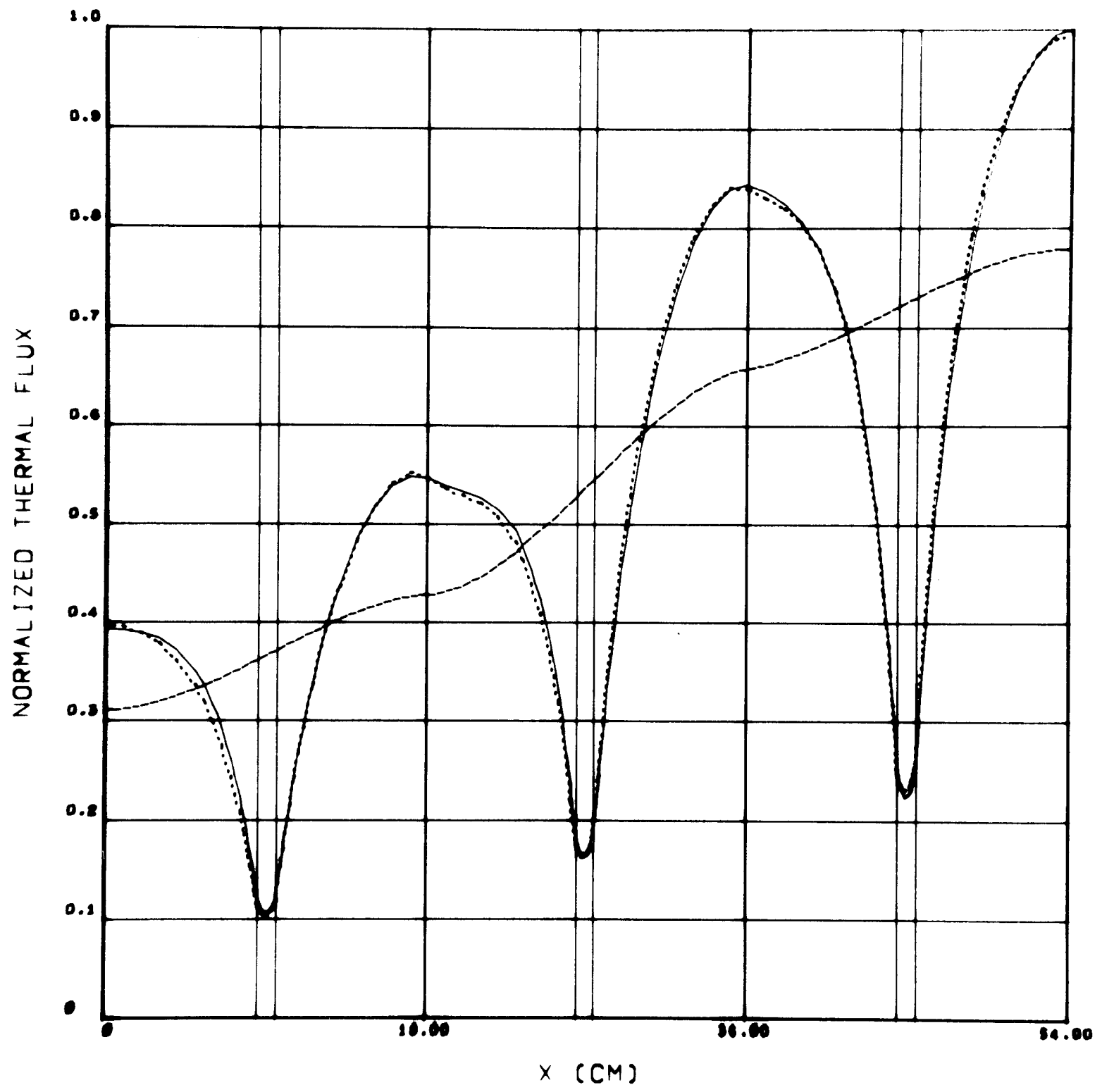

Figure 5.12. Case 1: Two-Group Thermal Results Using Cubic Hermite Basis Function Approximations and $18-\mathrm{cm}$ Coarse Mesh Regions

\begin{tabular}{lll}
\multicolumn{1}{c}{ Method } & & $\lambda$ \\
Reference & $\ldots \ldots \ldots$ & .917267 \\
Cubic FEM & ----- & .916717 \\
Cubic Synth & & .917059
\end{tabular}




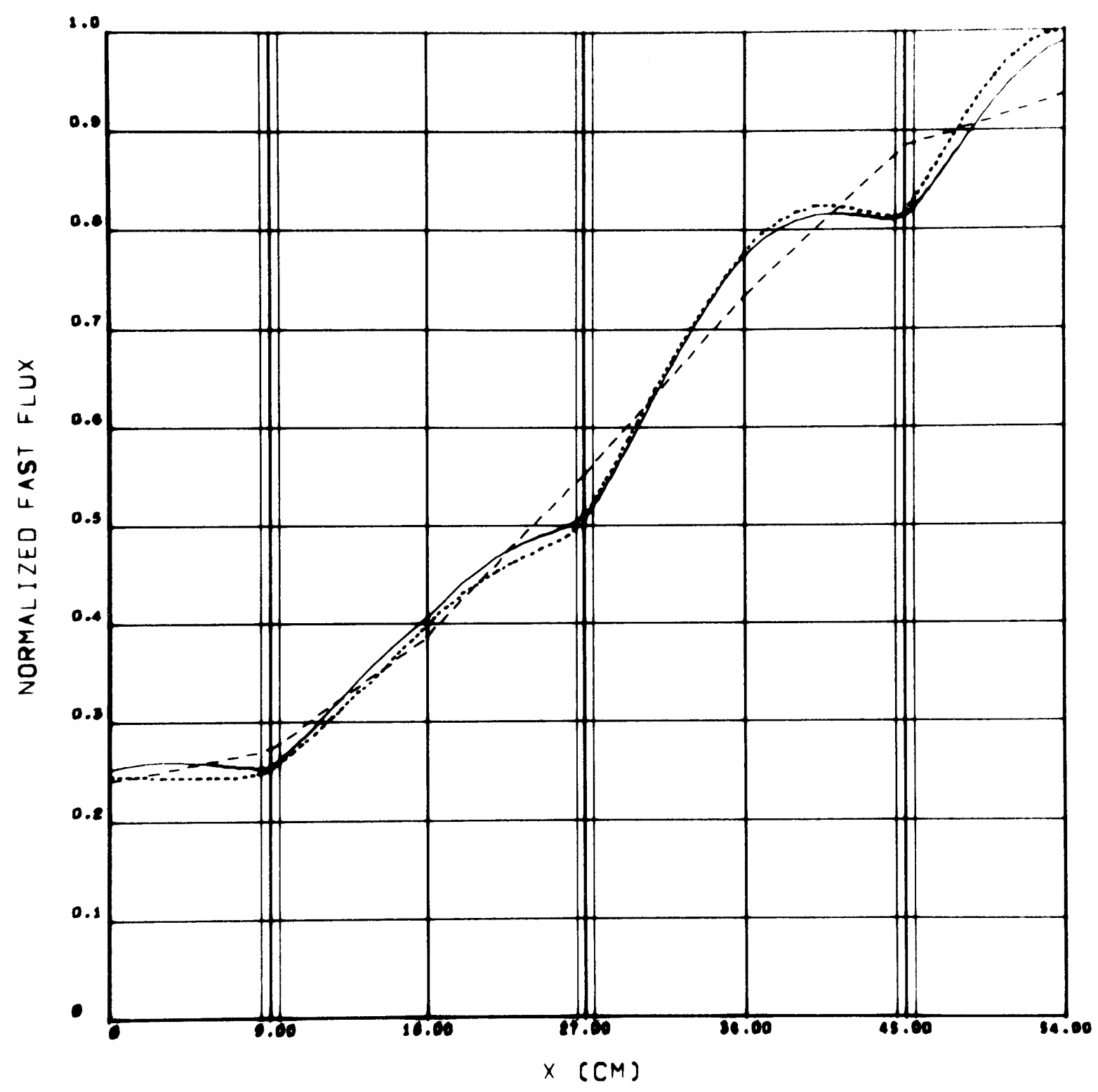

Figure 5.13. Case 1: Two-Group Fast Results Using Linear Basis Function Approximations and $9-\mathrm{cm}$ Coarse Mesh Regions

\begin{tabular}{llc}
\multicolumn{1}{c}{ Method } & & $\frac{\lambda}{1 .}$ \\
Reference & $\ldots \ldots \ldots$ & .917267 \\
Linear FEM & ----- & .916356 \\
Linear Synth & & .916427
\end{tabular}




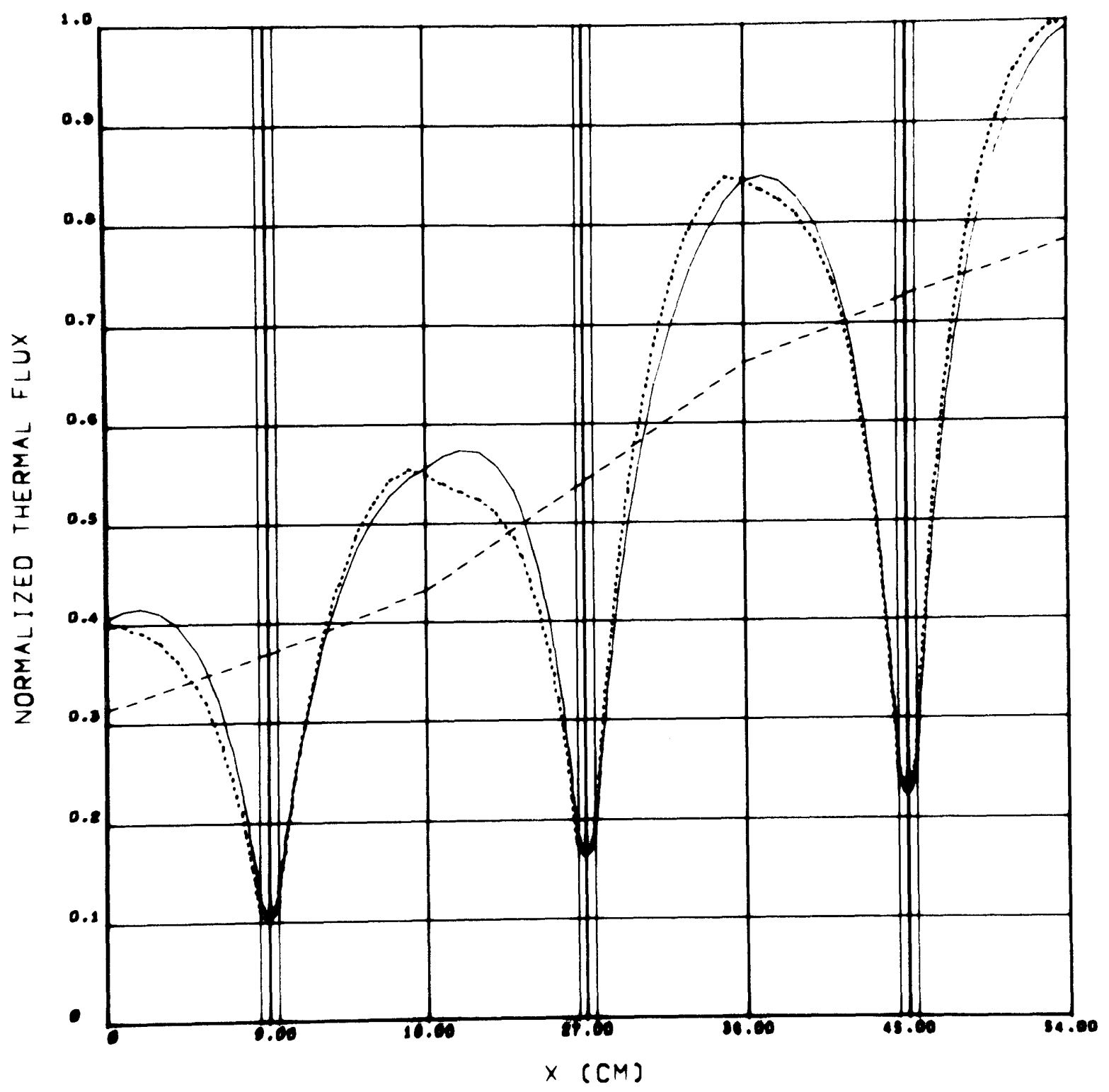

Figure 5.14. Case 1: Two-Group Thermal Results Using Linear Basis Function Approximations and 9-cm Coarse Mesh Regions

\begin{tabular}{lll}
\multicolumn{1}{c}{ Method } & & $\frac{\lambda}{1 .}$ \\
Reference & $\ldots \ldots \ldots$ & .917267 \\
Linear FEM & $-\ldots---$ & .916356 \\
Linear Synth & & .916427
\end{tabular}




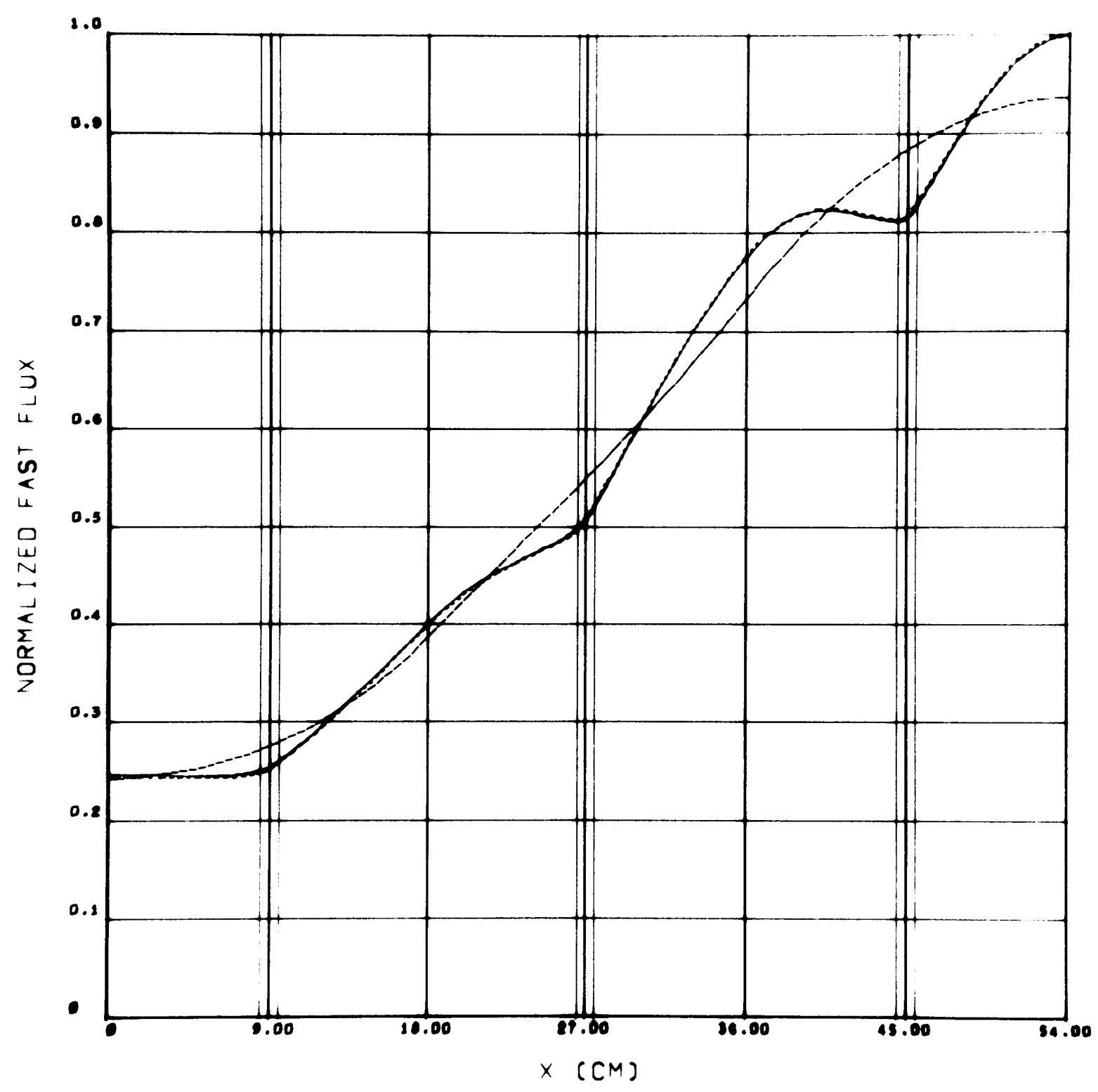

Figure 5.15. Case 1: Two-Group Fast Results Using Cubic Hermite Basis Function Approximations and 9-cm Coarse Mesh Regions

\begin{tabular}{llc}
\multicolumn{1}{c}{ Method } & & \multicolumn{1}{c}{$\lambda$} \\
Reference & $\ldots \ldots \ldots$ & .917267 \\
Cubic FEM & ----- & .916669 \\
Cubic Synth & & .917294
\end{tabular}




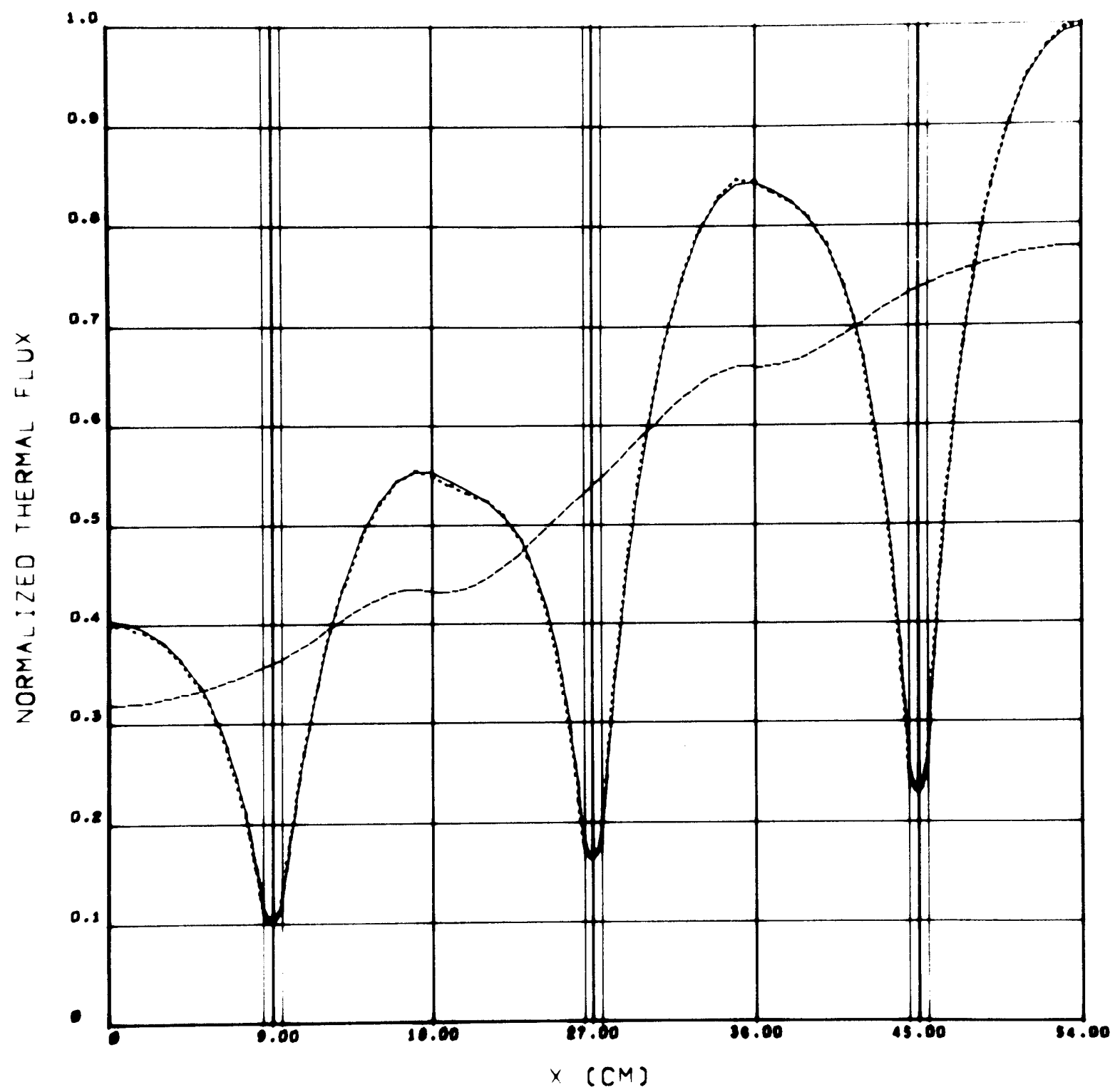

Figure 5.16. Case 1: Two-Group Thermal Results Using Cubic Hermite Basis Function Approximations and 9-cm Coarse Mesh Regions

\begin{tabular}{lcc}
\multicolumn{1}{c}{ Method } & & \multicolumn{1}{c}{$\lambda$} \\
Reference & $\ldots \ldots \ldots$ & .917267 \\
Cubic FEM & $---\ldots-$ & .916669 \\
Cubic Synth & & .917294
\end{tabular}


Table 5.6. Results of Case 1.

\begin{tabular}{lccccc}
\hline Method & Reference & $\begin{array}{c}\text { Linear } \\
\text { FEM }\end{array}$ & $\begin{array}{c}\text { Linear } \\
\text { Synth }\end{array}$ & $\begin{array}{c}\text { Cubic } \\
\text { FEM }\end{array}$ & $\begin{array}{c}\text { Cubic } \\
\text { Synth }\end{array}$ \\
\hline
\end{tabular}

ONE-GROUP RESULTS:

\begin{tabular}{cccccc}
\hline$\lambda$ & .559045 & .556943 & .557154 & .558647 & .558761 \\
\hline$\% \lambda$ & - & $.376 \%$ & $.338 \%$ & $.072 \%$ & $.051 \%$ \\
\hline$P(1)$ & .084 & $-12.1 \%$ & $-11.9 \%$ & $-3.07 \%$ & $-2.44 \%$ \\
$P(2)$ & .294 & $-4.29 \%$ & $-3.93 \%$ & $-.241 \%$ & $-.434 \%$ \\
$P(3)$ & .622 & $+3.67 \%$ & $+3.47 \%$ & $+.528 \%$ & $+.534 \%$ \\
\hline
\end{tabular}

TWO-GROUP RESULTS:

\begin{tabular}{cccccc}
\hline$\lambda$ & .917267 & .914489 & .915221 & .916717 & .917059 \\
\hline$\% \lambda$ & -- & $.302 \%$ & $.223 \%$ & $.060 \%$ & $.023 \%$ \\
\hline$P(1)$ & .134 & $-6.93 \%$ & $-5.63 \%$ & $-1.43 \%$ & $-1.13 \%$ \\
$P(2)$ & .315 & $-1.63 \%$ & $-1.39 \%$ & $-.072 \%$ & $-.120 \%$ \\
$P(3)$ & .549 & $+2.63 \%$ & $+2.17 \%$ & $+.391 \%$ & $+.347 \%$ \\
\hline
\end{tabular}

Two-Group Results Using Half-Subassembly Mesh Regions

\begin{tabular}{cccccc}
\hline$\lambda$ & .917267 & .916356 & .916427 & .916669 & .917294 \\
\hline$\% \lambda$ & -- & $.093 \%$ & $.092 \%$ & $.065 \%$ & $.003 \%$ \\
\hline$P(1)$ & .134 & $-2.38 \%$ & $-2.69 \%$ & $-1.51 \%$ & $-.461 \%$ \\
$P(2)$ & .315 & $-.475 \%$ & $-.602 \%$ & $-.063 \%$ & $-.047 \%$ \\
$P(3)$ & .549 & $+.851 \%$ & $-2.63 \%$ & $-3.22 \%$ & $+1.40 \%$ \\
\hline
\end{tabular}


It is apparent from these results that the proposed approximation methods, and in particular the method utilizing the cubic Hermite basis functions, approximate to a high degree of accuracy the detailed reference spatial flux. Comparison of the eigenvalue and fractional power results in Table 5.6 indicates that comparable if not superior measurements are obtained using the proposed methods in this case.

It is interesting to note the effects of employing the given subassembly heterogeneous nuclear constants rather than subassembly homogenized nuclear constants for use in the finite element method calculations. Under such conditions, the finite element method becomes identical to the proposed methods in which the heterogeneous nuclear constants and constant or flat subassembly solutions are used. Two-group calculations using the cubic Hermite approximation method were performed for Case 1 and are presented in Figures 5.17 and 5.18. This scheme was found to give very poor detailed flux results, converge to an eigenvalue $21 \%$ in error, and yield an average of $20 \%$ error in the fractional normalized power levels in each subassembly. This example clearly illustrates the necessity for the use of homogenized constants in the finite element method, or equivalently, the importance of the subassembly detailed solutions in the proposed approximations. 


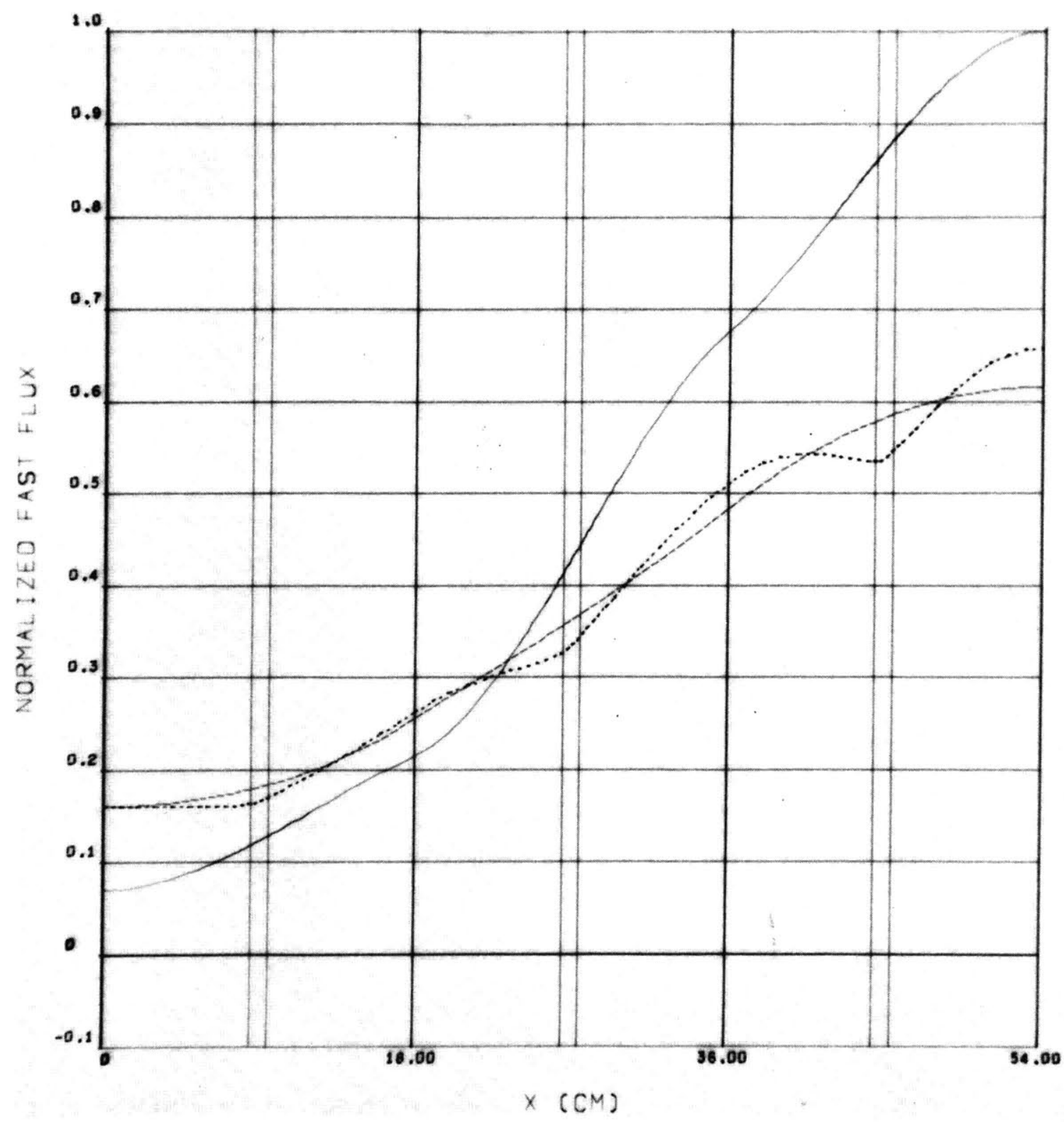

Figure 5.17. Case 1: Two-Group Fast Results Using Cubic Hermite Finite Element Approximations and $18-\mathrm{cm}$ Coarse Mesh Regions

Method

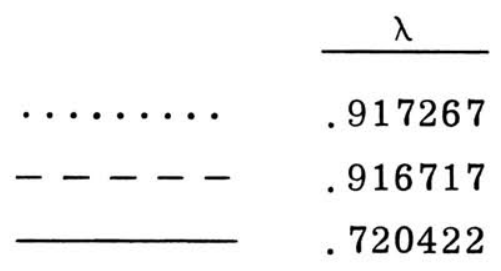

Reference

Cubic FEM + Homogenized Consts. .720422 


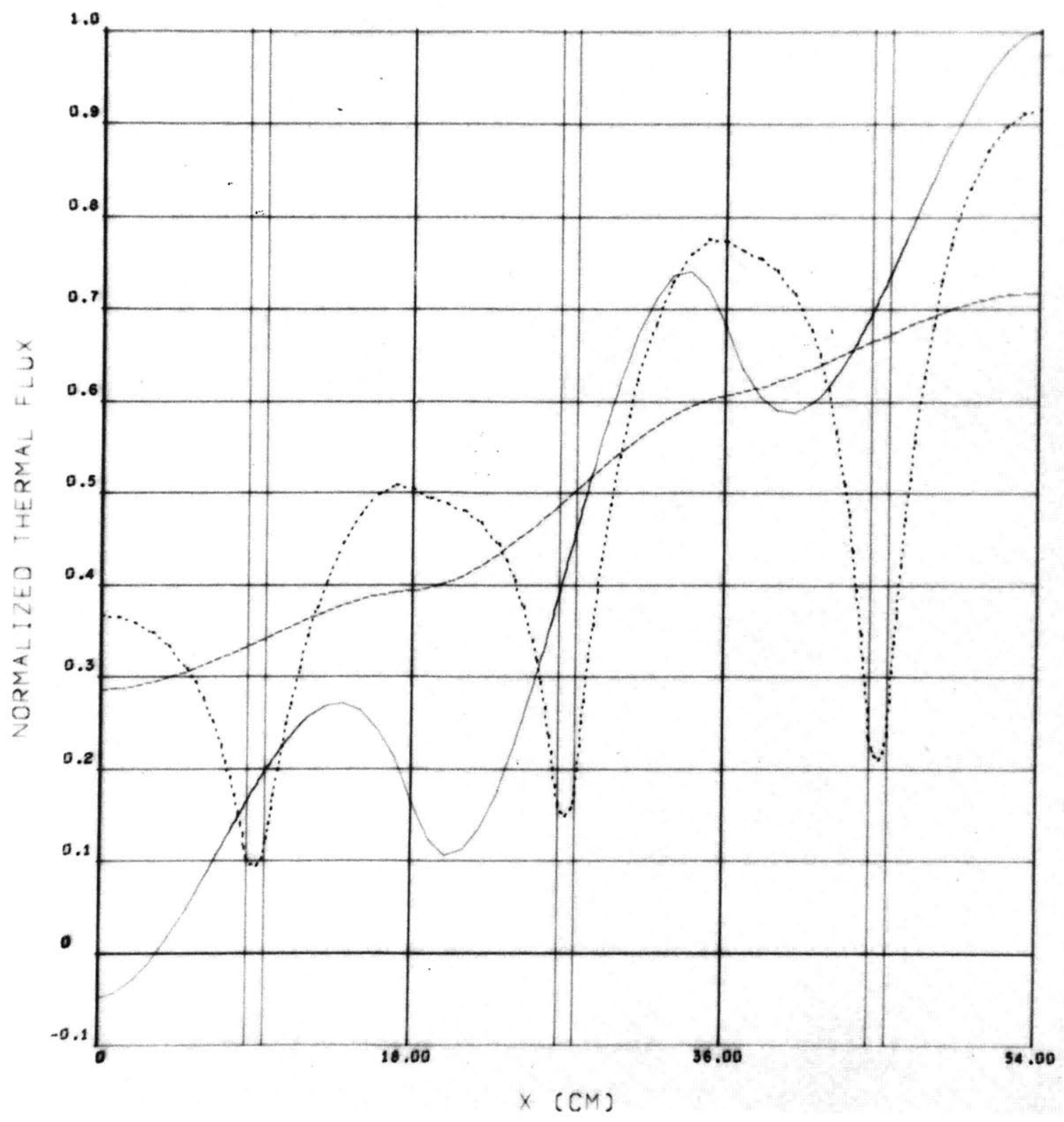

Figure 5.18. Case 1: Two-Group Thermal Results Using Cubic Hermite Finite Element Approximations and $18-\mathrm{cm}$ Coarse Mesh Regions

Method

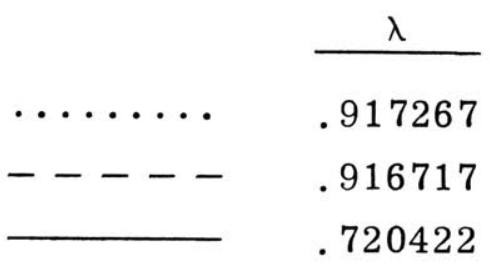


5.3.2. Case 2: Three different subassemblies of Types D, B, and C with symmetric boundary conditions.

The results of the two-group approximations for Case 2 are presented in Figures 5.19-5.22, where entire subassemblies were taken as the coarse mesh regions. The reference solutions were calculated using the same reference mesh geometry as in Case 1. The converged eigenvalues and fractional normalized power levels in each subassembly are summarized in Table 5.7. These results better illustrate the superiority of the cubic Hermite basis function approximations over the linear basis function approximations, and the superiority of the proposed approximations over the finite element method in all aspects.

Table 5.7. Two-Group Results of Case 2 .

\begin{tabular}{cccccc}
\hline Method & Reference & $\begin{array}{c}\text { Linear } \\
\text { FEM }\end{array}$ & $\begin{array}{c}\text { Linear } \\
\text { Synth }\end{array}$ & $\begin{array}{c}\text { Cubic } \\
\text { FEM }\end{array}$ & $\begin{array}{c}\text { Cubic } \\
\text { Synth }\end{array}$ \\
\hline$\lambda$ & .969986 & .965260 & .970236 & .966816 & .969578 \\
\hline$\% \lambda$ & -- & $.487 \%$ & $-.026 \%$ & $.326 \%$ & $.042 \%$ \\
\hline $\mathrm{P}(1)$ & .381 & $+11.26 \%$ & $+3.46 \%$ & $+6.07 \%$ & $+.643 \%$ \\
$\mathrm{P}(2)$ & .296 & $-11.59 \%$ & $-5.59 \%$ & $-3.08 \%$ & $-.157 \%$ \\
$\mathrm{P}(3)$ & .322 & $-2.66 \%$ & $+1.03 \%$ & $-4.34 \%$ & $-.616 \%$ \\
\hline
\end{tabular}




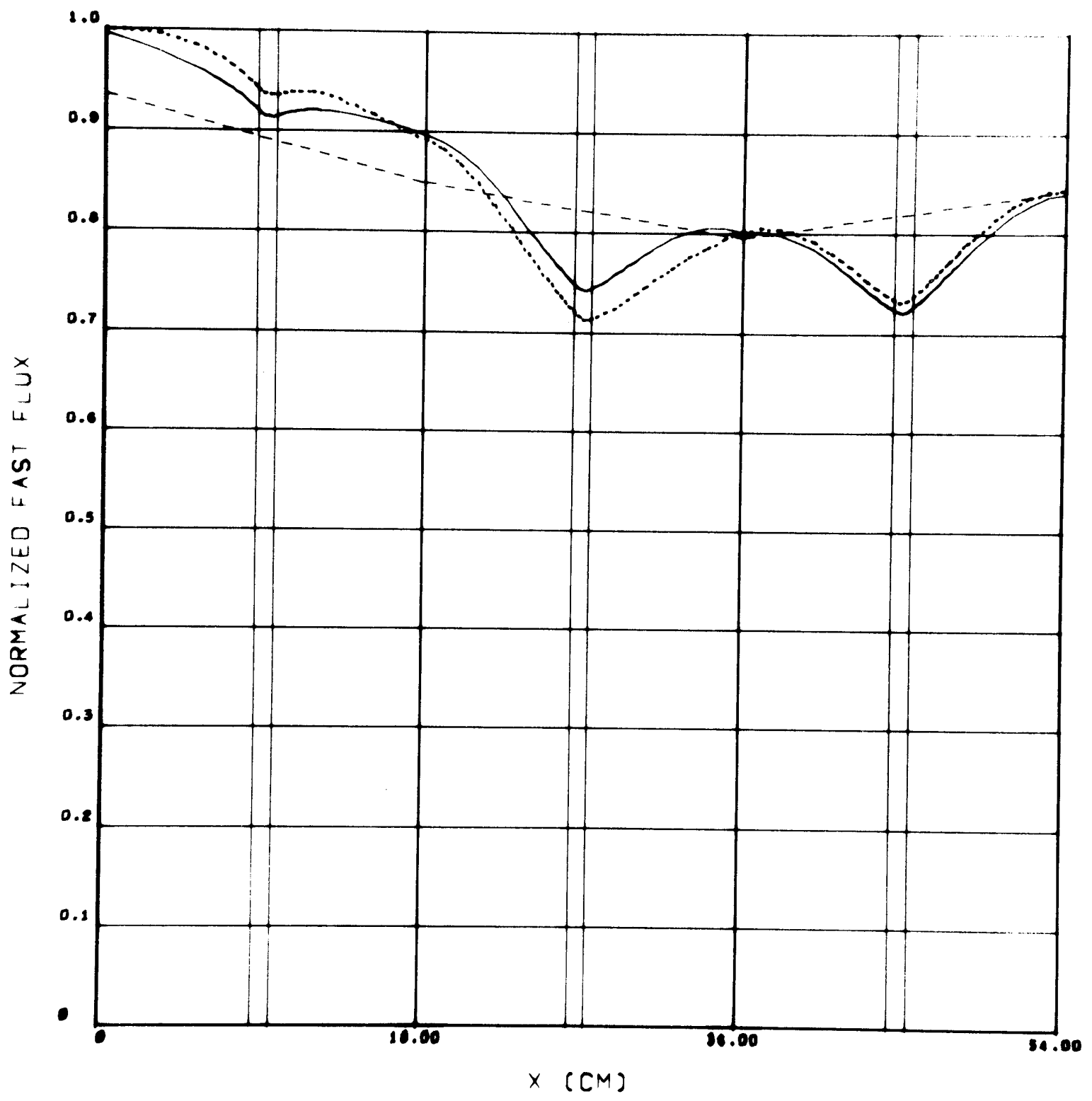

Figure 5.19. Case 2: Two-Group Fast Results Using Linear Basis Function Approximations and $18-\mathrm{cm}$ Coarse Mesh Regions

\begin{tabular}{llc}
\multicolumn{1}{c}{ Method } & & $\lambda$ \\
Reference & $\ldots \ldots \ldots \ldots$ & .969986 \\
Linear FEM & ----- & .965260 \\
Linear Synth & & .970236
\end{tabular}




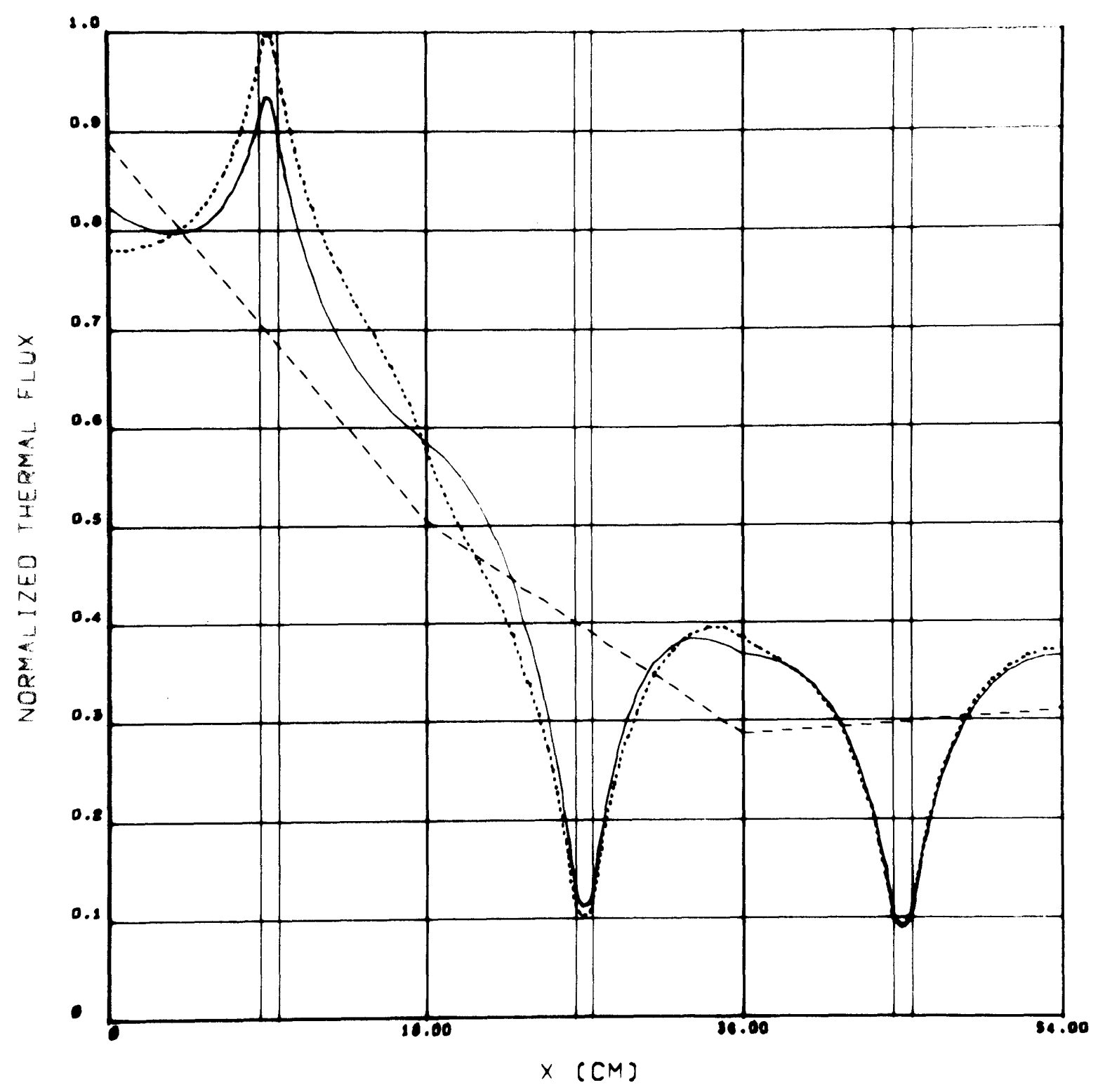

Figure 5.20. Case 2: Two-Group Thermal Results Using Linear Basis Function Approximations and 18-cm Coarse Mesh Regions

\begin{tabular}{lcc}
\multicolumn{1}{c}{ Method } & & $\frac{\lambda}{1969986}$ \\
Reference & $\ldots \ldots \ldots$ & .96996 \\
Linear FEM & ----- & .965260 \\
Linear Synth & & .970236
\end{tabular}




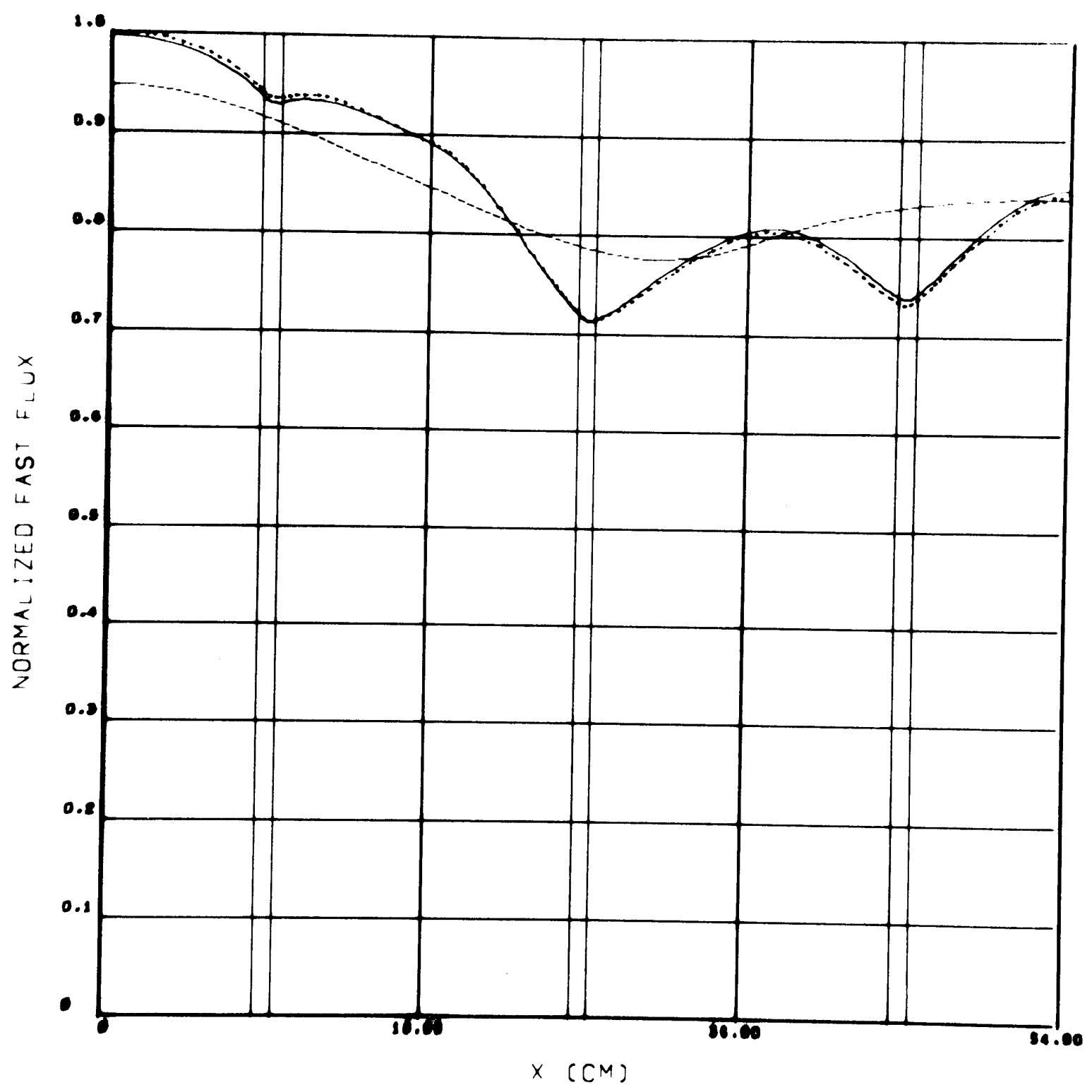

Figure 5.21. Case 2: Two-Group Fast Results Using Cubic Hermite Basis Function Approximations and 18-cm Coarse Mesh Regions

\begin{tabular}{lll}
\multicolumn{1}{c}{ Method } & & \multicolumn{1}{c}{$\lambda$} \\
Reference & $\ldots \ldots \ldots$ & .969986 \\
Cubic FEM & $-\ldots---$ & .966816 \\
Cubic Synth & - & .969578
\end{tabular}




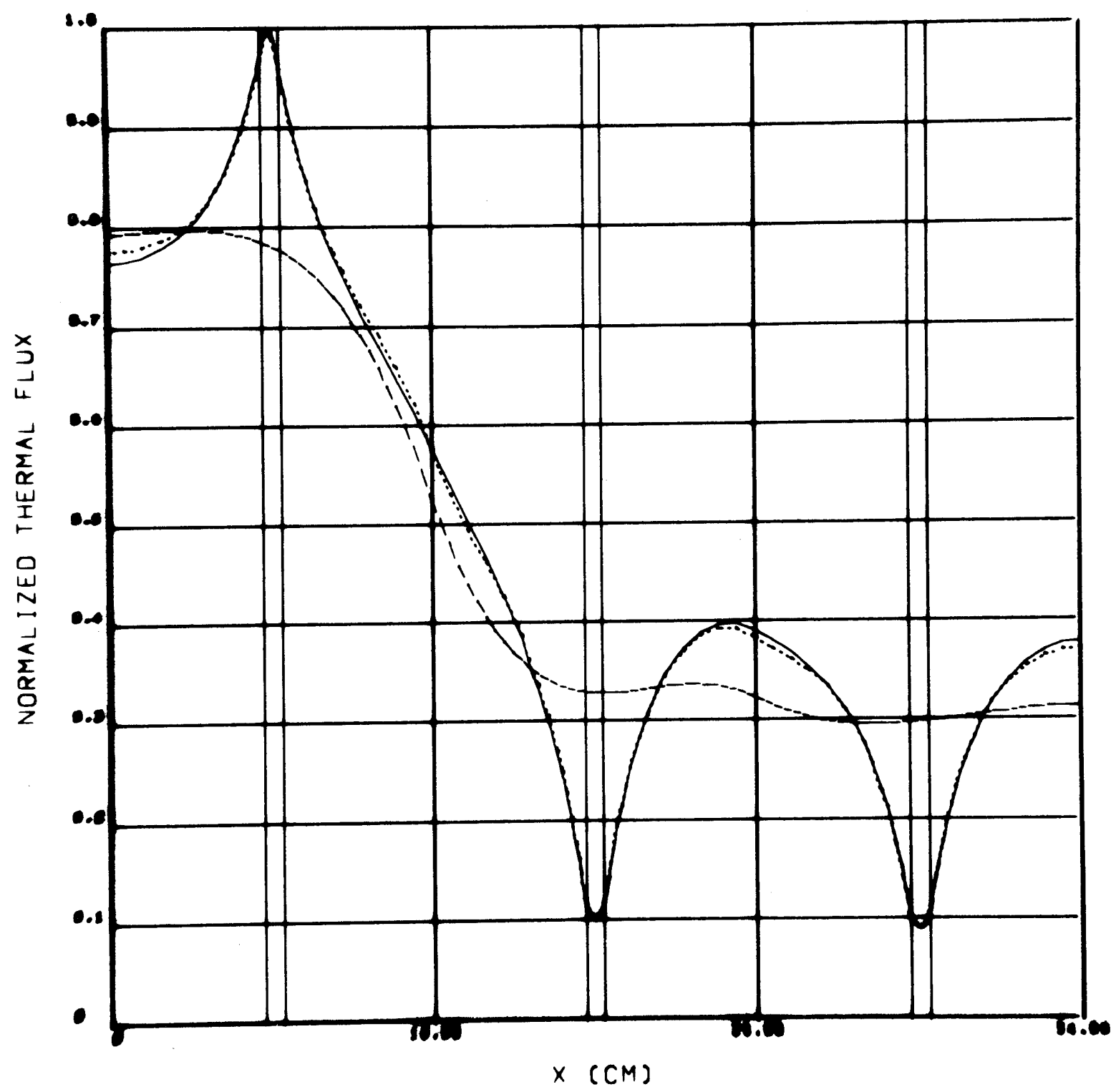

Figure 5.22. Case 2: Two-Group Thermal Results Using Cubic Hermite Basis Function Approximations and $18-\mathrm{cm}$ Coarse Mesh Regions

\begin{tabular}{lll}
\multicolumn{1}{c}{ Method } & & $\frac{\lambda}{1 .}$ \\
Reference & $\ldots \ldots \ldots 9986$ \\
Cubic FEM & $-\ldots---$ & .966816 \\
Cubic Synth & - & .969578
\end{tabular}


5.3.3. Case 3: Half-core reflected PWR composed of an 18-cm water reflector, the seven subassemblies C, C, C, A, A, A, D, and half of subassembly D. Zero flux boundary conditions are imposed outside the reflector, and symmetry is imposed in the center of the last D-type subass embly.

The Case 3 results of the two-group approximations using full 18-cm coarse mesh regions in all but the last $9-\mathrm{cm}$ region are presented in Figures 5.23-5.26, and summarized in Table 5.8. The reference solutions were obtained using 198 mesh regions given by the symmetric partitioning

$$
2(2 \mathrm{~cm})+2(1 \mathrm{~cm})+4(.5 \mathrm{~cm})+2(.25 \mathrm{~cm})+2(.25 \mathrm{~cm})
$$

in each of the subassemblies, and $18(1 \mathrm{~cm})$ regions in the reflector.

The use of many subassemblies containing absorption rods throughout the reactor, except in the center subassemblies where water channels are present, results in central peaked fluxes with large gradients and, by comparison, a relatively small thermal neutron peak in the reflector.

Both coarse mesh methods were found to overestimate the flux in the subassemblies near the reflector, and underestimate the flux in the central subassembly regions regardless of the type of basis function approximations used. The larger inaccuracies of the linear basis function methods can be in part attributed to the fact that these methods cannot approximate the peaked thermal flux in the reflector, and result in large flux values in the subassemblies nearest the reflector. The cubic Hermite basis function approximations, however, are better able to approximate both the thermal flux reflector peak and the complex 


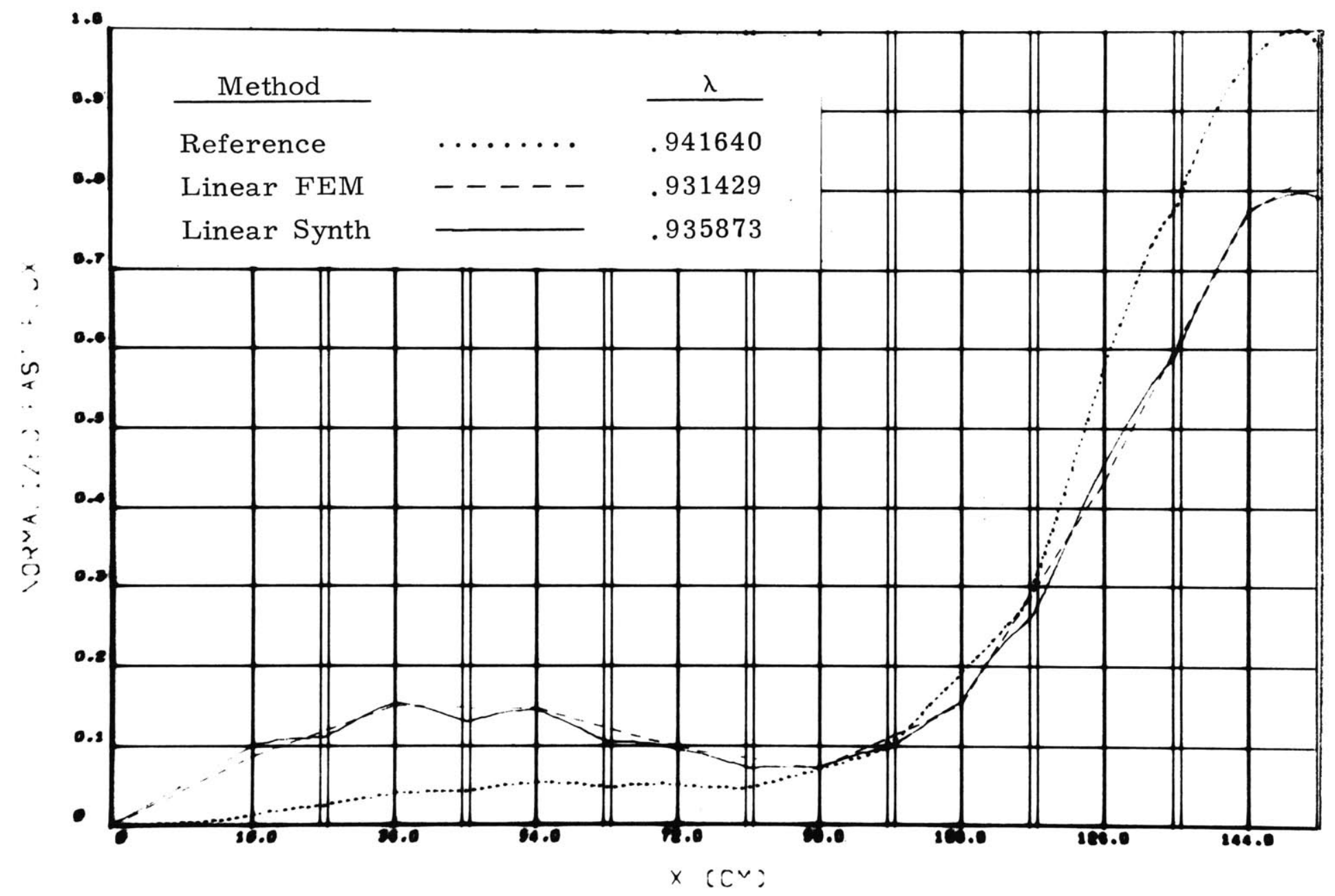

Figure 5.23. Case 3: Two-Group Fast Results Using Linear Basis Function Approximations and $18-\mathrm{cm}$ Coarse Mesh Regions 


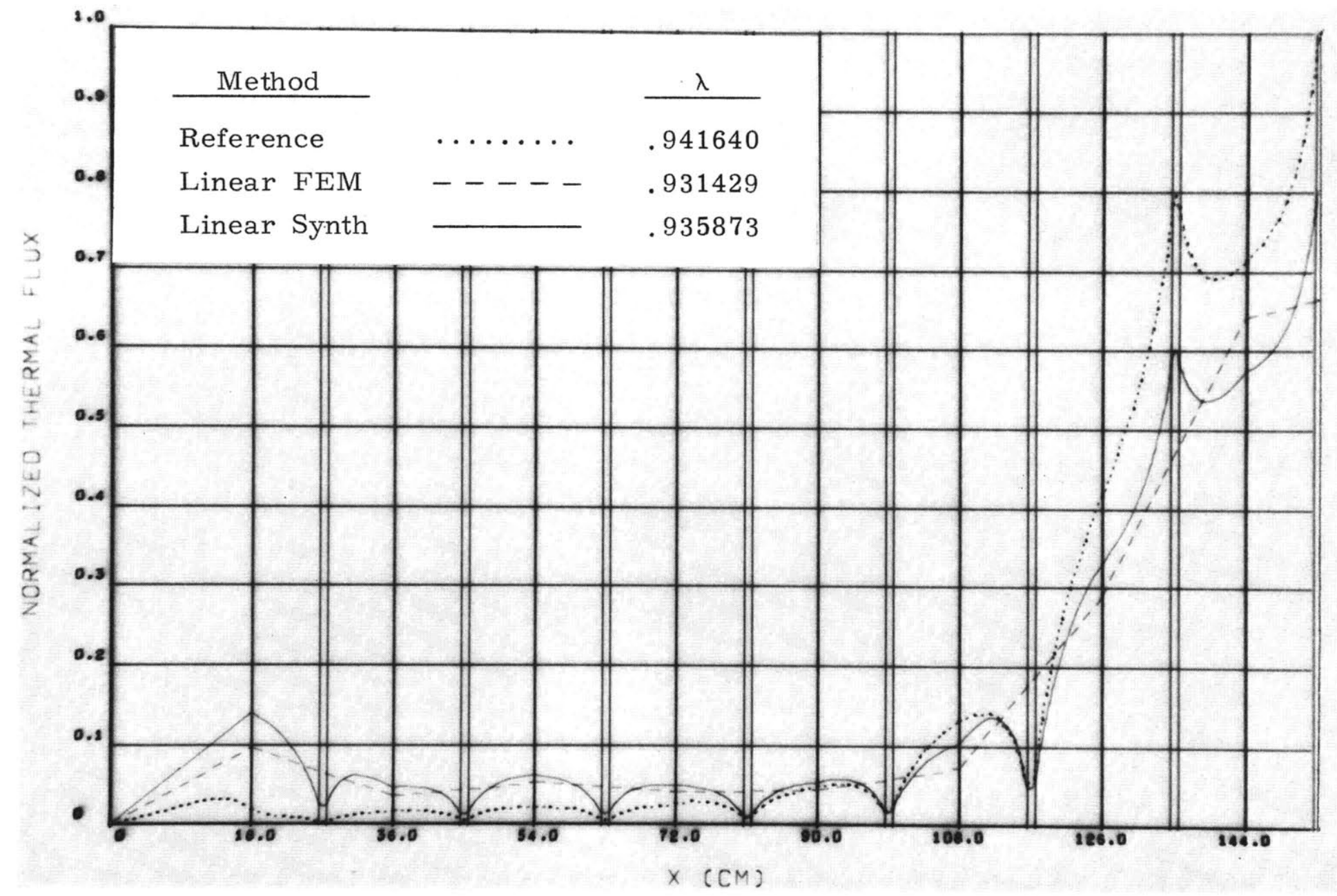

Figure 5.24. Case 3: Two-Group Thermal Results Using Linear Basis Function Approximations and $18-\mathrm{cm}$ Coarse Mesh Regions 


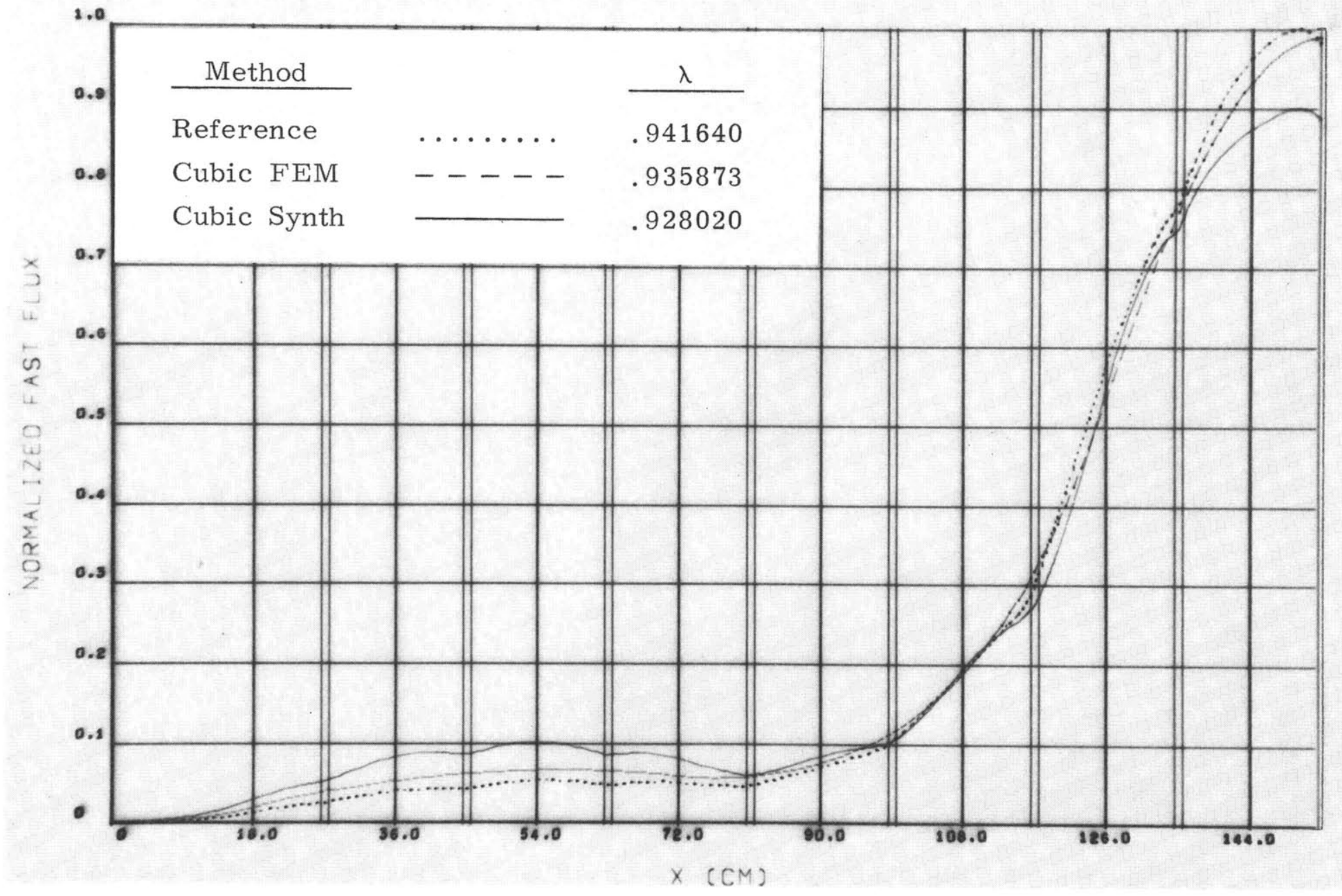

Figure 5.25. Case 3: Two-Group Fast Results Using Cubic Hermite Basis Function Approximations and $18-\mathrm{cm}$ Coarse Mesh Regions 


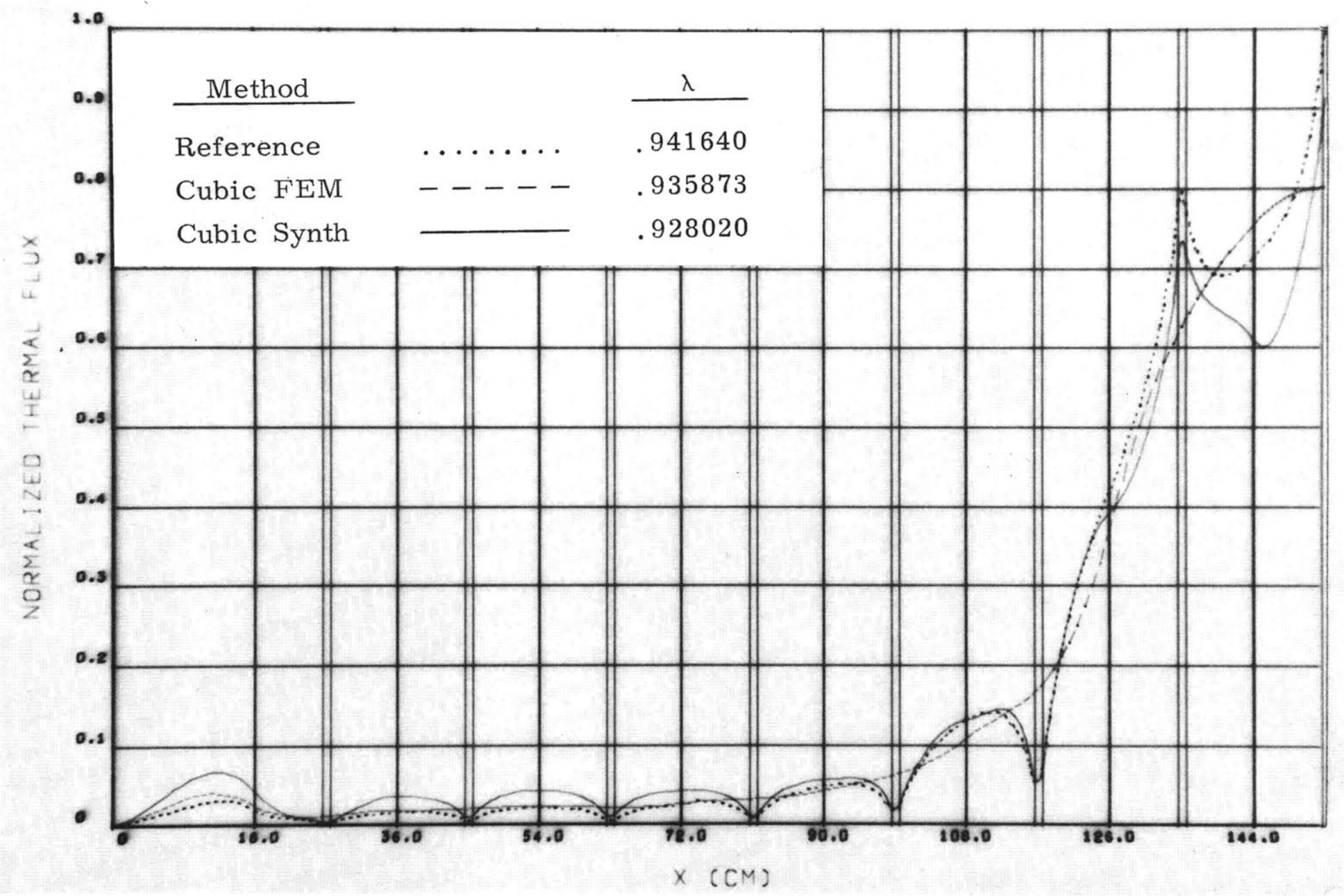

Figure 5.26. Case 3: Two-Group Thermal Results Using Cubic Hermite Basis Function Approximations and $18-\mathrm{cm}$ Coarse Mesh Regions 
Table 5.8. Results of Case 3 .

\begin{tabular}{cccccc}
\hline Method & Reference & $\begin{array}{c}\text { Linear } \\
\text { FEM }\end{array}$ & $\begin{array}{c}\text { Linear } \\
\text { Synth }\end{array}$ & $\begin{array}{c}\text { Cubic } \\
\text { FEM }\end{array}$ & $\begin{array}{c}\text { Cubic } \\
\text { Synth }\end{array}$ \\
\hline$\lambda$ & .941640 & .931429 & .938561 & .935873 & .928020 \\
\hline$\% \lambda$ &.- & $1.08 \%$ & $.32 \%$ & $.61 \%$ & $1.44 \%$ \\
\hline $\mathrm{P}(1)$ & .01699 & $-461 . \%$ & $-514 . \%$ & $-37.4 \%$ & $-85.1 \%$ \\
$\mathrm{P}(2)$ & .02513 & $-192 . \%$ & $-179 . \%$ & $-32.8 \%$ & $-98.6 \%$ \\
$\mathrm{P}(3)$ & .02890 & $-149 . \%$ & $-133 . \%$ & $-26.0 \%$ & $-79.6 \%$ \\
$\mathrm{P}(4)$ & .0224 & $-50.2 \%$ & $-36.9 \%$ & $-13.5 \%$ & $-31.5 \%$ \\
$\mathrm{P}(5)$ & .04969 & $.8 .52 \%$ & $8.24 \%$ & $-2.78 \%$ & $-7.63 \%$ \\
$\mathrm{P}(6)$ & .1465 & $7.47 \%$ & $13.7 \%$ & $-.494 \%$ & $.259 \%$ \\
$\mathrm{P}(7)$ & .4362 & $26.4 \%$ & $22.9 \%$ & $5.01 \%$ & $8.40 \%$ \\
$\mathrm{P}(8)$ & .2740 & $18.6 \%$ & $20.1 \%$ & $1.99 \%$ & $13.1 \%$ \\
\hline
\end{tabular}

neutron leakage across the core, and give better results. Table 5.8 indicates that the cubic Hermite basis function approximations better approximate the detailed reference solutions, and that results obtained using the cubic Hermite finite element method were for this case better than those obtained using either of the proposed approximations. The ability of these methods to approximate large thermal flux peaks in the reflector regions is considered in the next case. 
5.3.4. Case 4: Half-core reflected PWR composed of an 18-cm water reflector, the seven subassemblies D, D, D, C, D, D, A, and half of subassembly Type A. Zero flux boundary conditions are imposed in the center of the last Type A subassembly.

The Case 4 geometry produces a large but detailed thermal flux in the half-core region and a large thermal peak in the reflector region, as seen from the results in Figures 5.27-5.30. The reference solutions were calculated using the reference mesh geometry as given in Case 3 . The results of the approximations are summarized in Table 5.9.

The results show that the linear basis function approximations cannot approximate accurately the thermal flux reflector peak and result in large flux and fractional power errors in the subassemblies near the reflector. The cubic Hermite basis function approximations, on the other hand, are better able to approximate this thermal peak and result in much more accurate power levels, especially in the first subassembly region.

The Case 4 results typify the approximation accuracy of both the finite element method and the proposed approximation method. In general, the cubic Hermite basis function approximations are superior to the linear basis function approximations, and the proposed methods give comparable or superior results as compared to those obtained from the finite element method using the same class of basis functions. In this case, the proposed method using cubic Hermite basis functions was able to estimate the reference eigenvalue within $0.04 \%$, closely approximate the detailed reference flux solution to within a few percent. at all spatial points, and result in fractional normalized power levels in each subassembly with less than $5 \%$ error. 


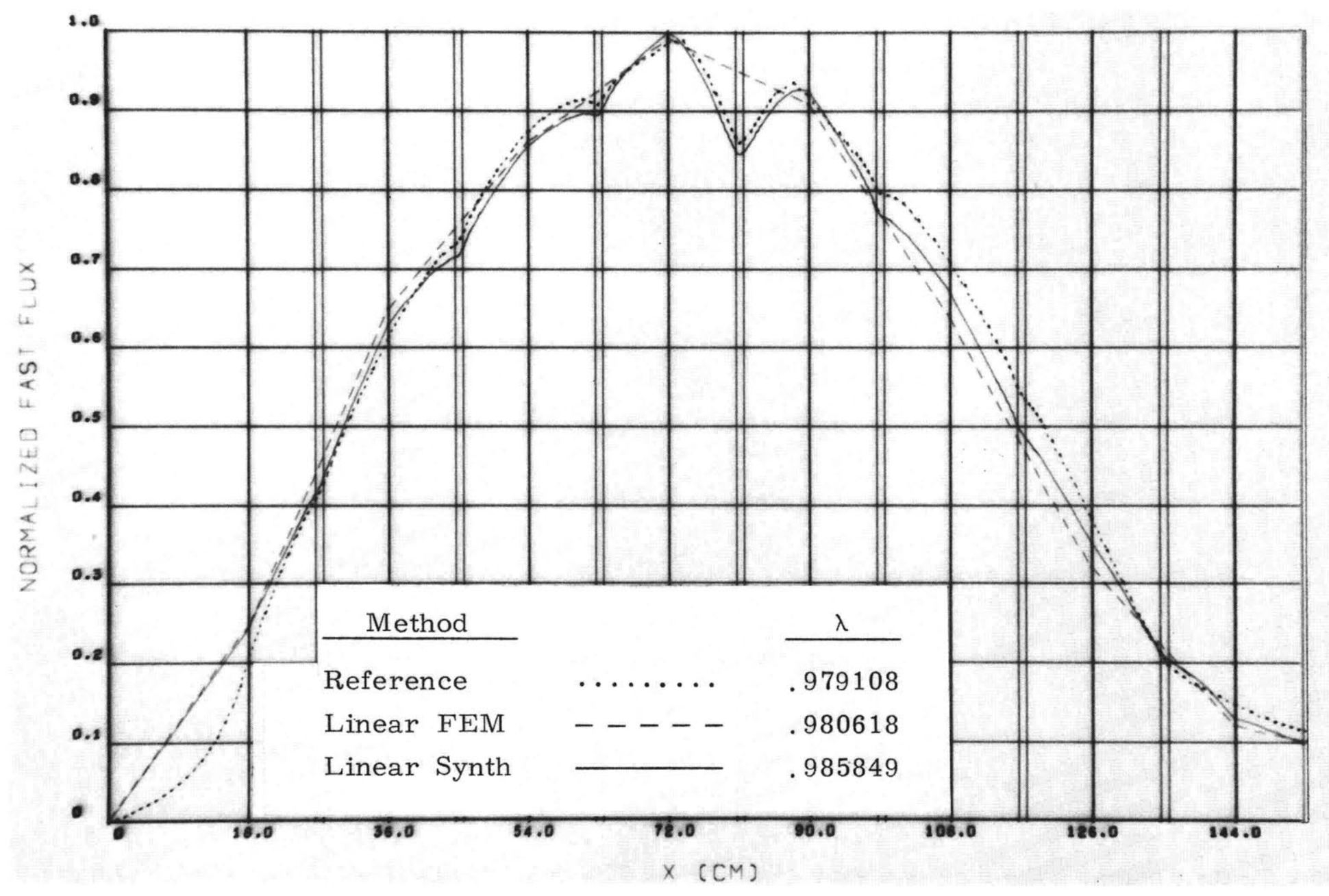

Figure 5.27. Case 4: Two-Group Fast Results Using Linear Basis Function Approximations and $18-\mathrm{cm}$ Coarse Mesh Regions 


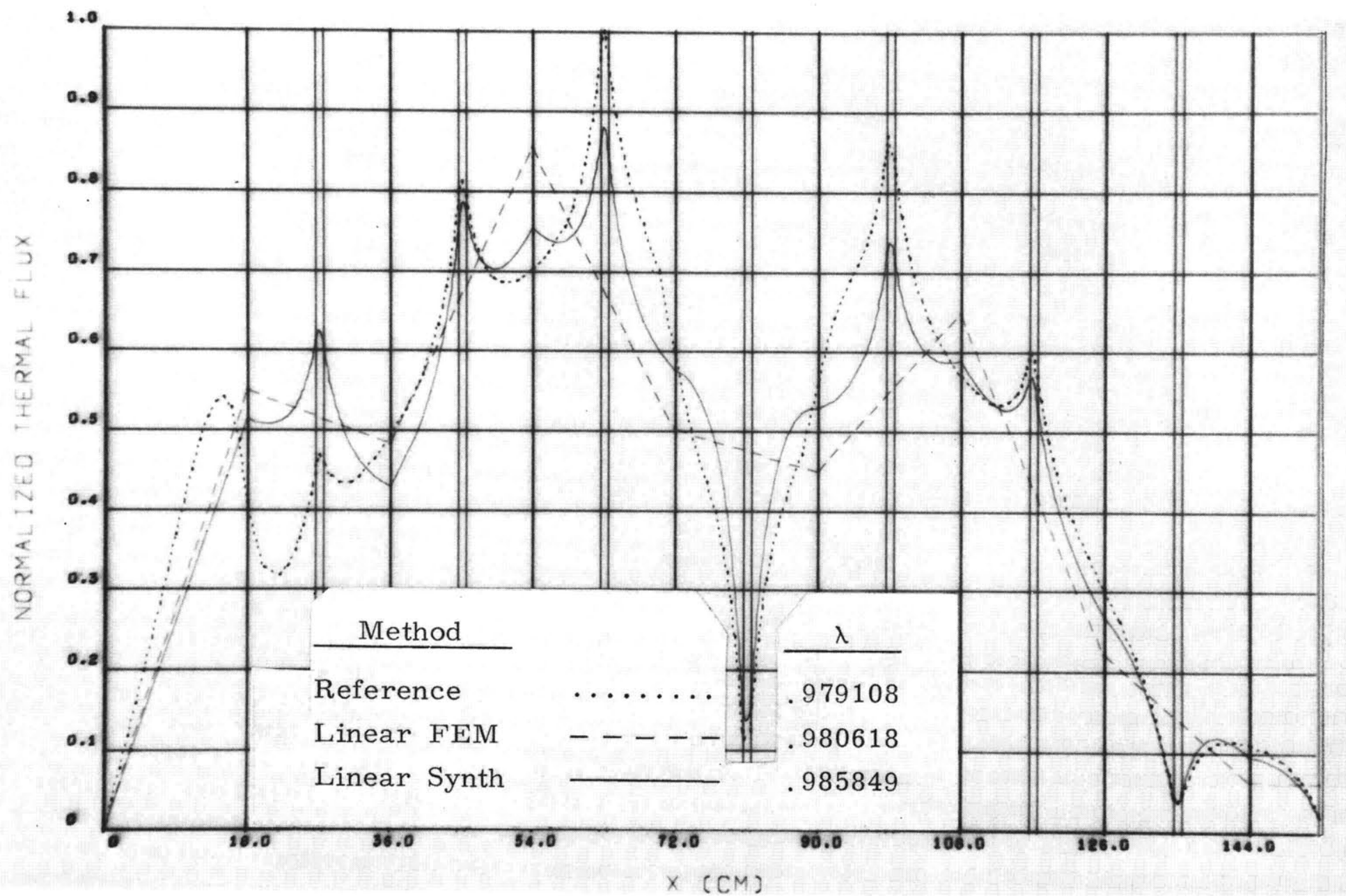

Figure 5.28. Case 4: Two-Group Thermal Results Using Linear Basis Function Approximations and $18-\mathrm{cm}$ Coarse Mesh Regions 


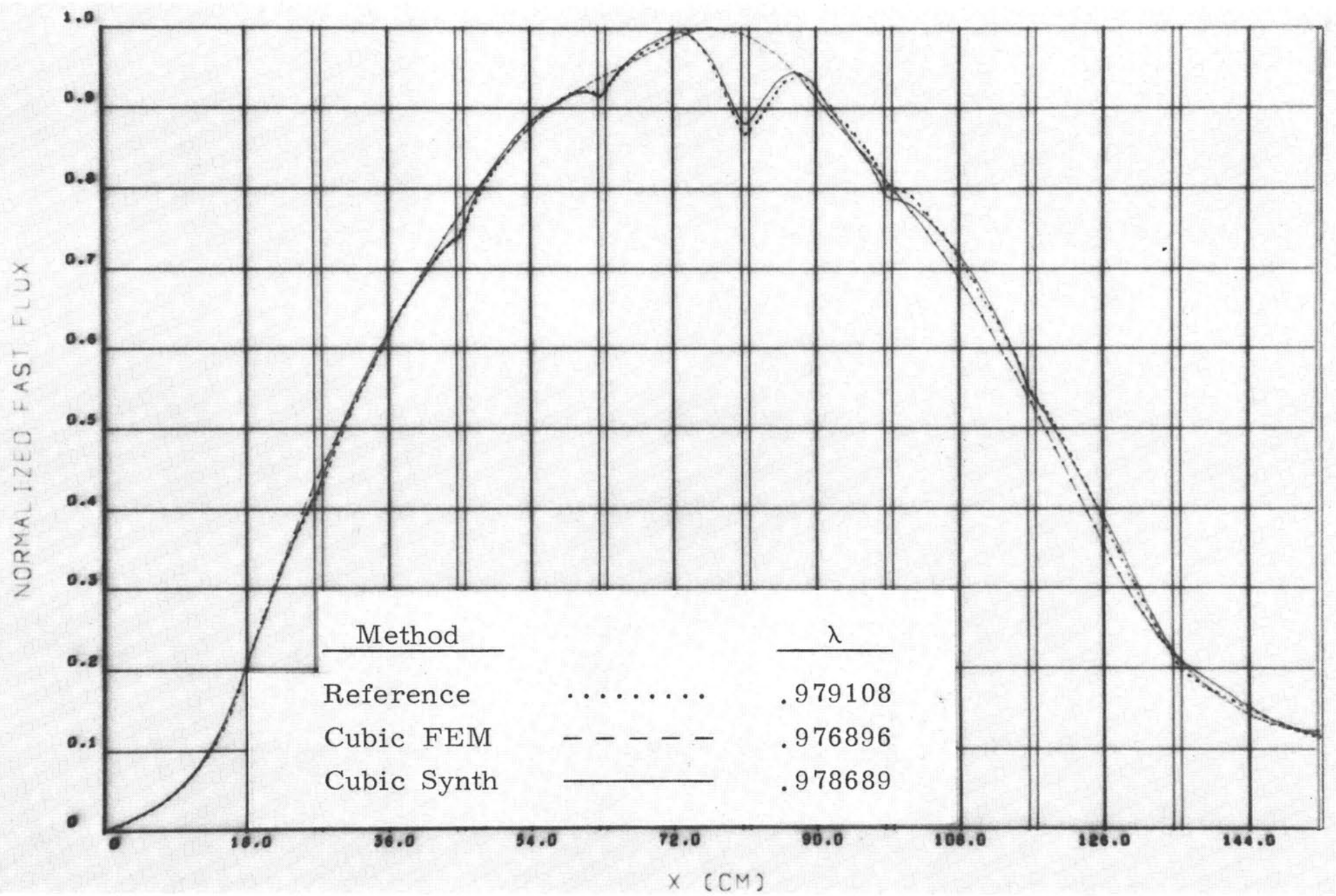

Figure 5.29. Case 4: Two-Group Fast Results Using Cubic Hermite Basis Function Approximations and $18-\mathrm{cm}$ Coarse Mesh Regions 


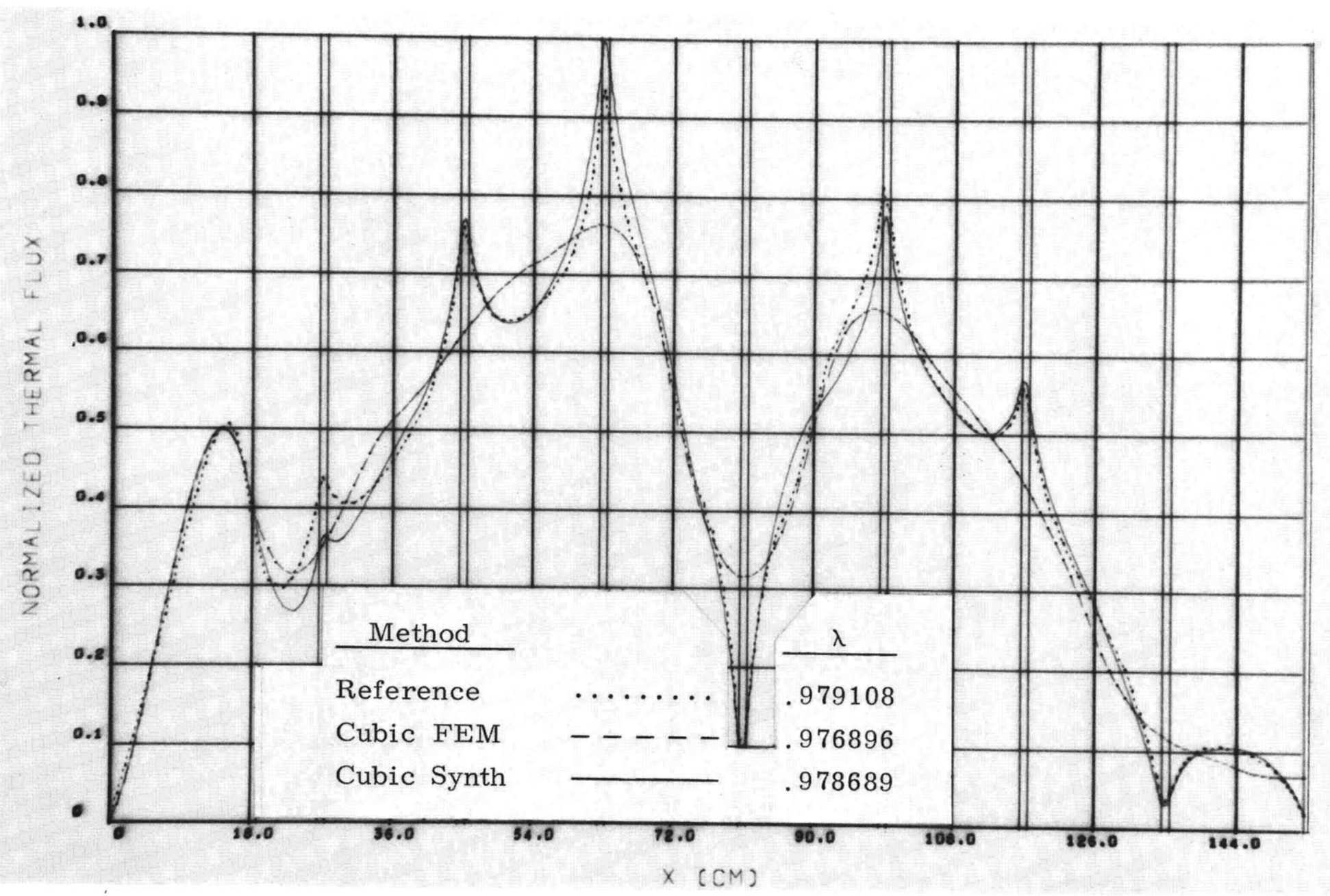

Figure 5.30. Case 4: Two-Group Thermal Results Using Cubic Hermite Basis Function Approximations and 18-cm Coarse Mesh Regions 
Table 5.9. Results of Case 4.

\begin{tabular}{cccccc}
\hline Method & Reference & $\begin{array}{c}\text { Linear } \\
\text { FEM }\end{array}$ & $\begin{array}{c}\text { Linear } \\
\text { Synth }\end{array}$ & $\begin{array}{c}\text { Cubic } \\
\text { FEM }\end{array}$ & $\begin{array}{c}\text { Cubic } \\
\text { Synth }\end{array}$ \\
\hline$\lambda$ & .979108 & .980618 & .985849 & .976896 & .978689 \\
\hline$\% \lambda$ & -- & $-.15 \%$ & $-.69 \%$ & $.22 \%$ & $.04 \%$ \\
\hline $\mathrm{P}(1)$ & .098 & $-23.4 \%$ & $-21.4 \%$ & $-13.3 \%$ & $5.54 \%$ \\
$\mathrm{P}(2)$ & .160 & $-1.53 \%$ & $2.39 \%$ & $-.893 \%$ & $-1.08 \%$ \\
$\mathrm{P}(3)$ & .193 & $11.5 \%$ & $7.11 \%$ & $1.83 \%$ & $-3.35 \%$ \\
$\mathrm{P}(4)$ & .211 & $-19.5 \%$ & $-10.6 \%$ & $-7.23 \%$ & $-1.60 \%$ \\
$\mathrm{P}(5)$ & .168 & $17.4 \%$ & $10.3 \%$ & $3.86 \%$ & $3.79 \%$ \\
$\mathrm{P}(6)$ & .118 & $10.6 \%$ & $4.96 \%$ & $5.97 \%$ & $-1.01 \%$ \\
$\mathrm{P}(7)$ & .039 & $1.45 \%$ & $3.44 \%$ & $1.65 \%$ & $3.25 \%$ \\
$\mathrm{P}(8)$ & & & & & \\
\end{tabular}




\section{Chapter 6 \\ CONCLUSIONS AND RECOMIMENDATIONS}

\subsection{Characteristics of the Proposed Approximation Methods}

The use of detailed subassembly flux solutions or other a priori flux shapes directly in the spatial shape or trial function form of flux approximations in reactor physics has resulted in many coarse mesh approximation schemes which are classified in the broad area of overlapping multichannel synthesis. The proposed approximation methods are similar to existing synthesis methods of this kind, but are unique in that they reduce to conventional and well understood approximation methods in regions where little or no spatial flux information is given, or in completely homogeneous regions. In contrast, the overlapping synthesis methods proposed to date do not. This characteristic is especially important in calculations involving homogeneous regions, of which reflector regions are a prime example.

The proposed approximations are very similar to coarse mesh finite element method approximations in which detailed flux behavior has been used to flux-weight the nuclear constants in each region. The methods are conceptually different and become equivalent only when all of the coarse mesh regions are homogeneous.

The matrix equations resulting from the use of the proposed methods are identical in form to those resulting from the finite element method utilizing similar basis functions. In addition, the matrix 
elements of the proposed methods are curiously different from those of the finite element methods using detailed flux-weighted nuclear constants. Although the spatial mass and stiffness matrices of the proposed methods for each group have been proven to be positive definite only for the case of Galerkin flux weighting, the lise of adjoint weighting in all of the cases considered did not alter these properties. In addition, the proposed methods were found always to converge to a positive eigenvalue and to flux shapes which were everywhere positive.

The numerical results indicate that the proposed methods are able to predict accurate criticality or $\mathrm{k}_{\text {eff }}$ measurements and regional power levels as well as to approximate the reference detailed flux shapes for each group with a high degree of accuracy. The results indicate that in general, use of the proposed methods results in superior criticality estimates over those obtained by the use of the finite element method with flux-weighted constants; this behavior was observed for each type of basis function approximation. Moreover,. each of the proposed methods is in general vastly superior to its finite element method counterparts in approximating the actual detailed flux behavior and regional as well as total power levels.

Detailed flux behavior could be reintroduced into the results of the homogenized finite element methods by normalizing the detailed subassembly solutions in each coarse mesh region to match the power levels of the converged results in each region. The detailed solutions resulting from such a procedure would be discontinuous at the region boundaries and may, to some extent, exhibit the fine flux structure present in the results of the proposed methods. However, the results 
are not expected to be as good as an approximation as those of the proposed methods, since the current coupling or diffusion approximation is not made until after the coarse mesh homogenization procedure.

\subsection{Applicability and Limitations}

Because the matrix forms of the equations which result from the use of the proposed methods are identical to those which result from the use of the finite element methods, the proposed approximations can be incorporated into existing finite element approximation schemes. Although additional integrations must be performed in the proposed methods, they can be reduced to sums of known products so that little additional computation time is required.

As in any coarse mesh approximation method, inaccurate results can occur when the coarse mesh region sizes chosen are too large. For a given region size, the accuracy of the results for any approximation scheme is unknown. The accuracy of the finite element methods is known to improve geometrically as the mesh size is decreased, resulting in a useful exror criterion for the method. A disadvantage of the proposed methods is that no such error criterion has been developed. The inability to predict error estimates has always been a major drawback of synthesis techniques. However, the use of such methods, and use of the proposed methods, has been shown to be justified through proper physical insight and experience. 


\subsection{Recommendations for Future Work}

Obviously the next step is the application of these proposed methods to two-dimensional diffusion problems. However, the onedimensional problem still contains areas which may deserve closer attention. One such area is the examination of the matrix properties of both the finite element method and the proposed approximation methods which are necessary in order to guarantee convergence to a positive eigenvalue and an everywhere positive flux solution. Another area is the development of error criteria for the proposed methods. The close similarity between the proposed methods and the finite element methods may allow an extension or generalization of characteristics which hitherto have belonged only to the finite element methods.

The usefulness of the proposed methods depends on their applicability and accuracy in two- and three-dimensional diffusion problems. Just as the finite element approximations can be derived in two- or three-dimensions using variational modal-nodal techniques, so can the proposed methods for multidimensional problems. The proposed trial functions could be defined as continuous at mesh nodes, but may in general be discontinuous along mesh line interfaces. In order that: the flux and current trial functions not be allowed to be discontimous at identical spatial points, the current trial functions would then have to be defined as continuous across these interfaces. The use of the proposed class of trial function forms in the two-dimensional problem will raise the challenge of extending the spatial overlapping synthesis methods of this type to multidimensional reactor problems. 


\section{REFERENCES}

1. T. J. Thompson and J. G. Beckerley, The Technology of Nuclear Reactor Safety, Volume 1, M.I.T.Press (1964).

2. A. M. Weinberg and E. P. Wigner, The Physical Theory of Neutron Chain Reactors, University of Chicago Press (1958).

3. J. R. Lamarsh, Introduction to Nuclear Reactor Theory, Addison-Wesley (1966).

4. F. B. Hildebrand, Finite Difference Equations and Simulations, Prentice-Hall (1968).

5. S. Kaplan, "Synthesis Methods in Reactor Analysis," in Advances in Nuclear Science and Technology, Volume 3. Academic Press, 233(1966).

6. P. M. Morse and H. Feshbach, Methods of Theoretical Physics, Chap. 6, McGraw-Hill (1953).

7. E. L. Wachspress, Iterative Solution of Elliptic Systems and Applications to the Neutron Diffusion Equations of Reactor Physics, Prentice-Hall (1966).

8. M. Clark, Jr. and K. F. Hansen, Numerical Methods of Reactor Analysis, Academic Press (1964).

9. R. Avery, "Theory of Coupled Reactors," Proc. 2nd U.N. Intern. Conf. Peaceful Uses of Atomic Energy, Geneva, 12, $182(1958)$.

10. W. H. Köhler, "Summary of Derivations of Coupled Point Reactor Kinetics Equations," Proc. National Topical Meeting on Coupled Reactor Kinetics, Texas A and M Univ., 192 (1967).

11. M. W. Dyes and K. F. Hansen, "Approximate Methods for Analyzing Coupled Core Kinetics, WARD-35 (1968).

12. M. Komata, "On the Derivation of Avery's Coupled Reactor Kinetics Equations," Nucl. Sci. Eng. , 38, 193 (1969).

13. J. B. Yasinsky and A. F. Henry, "Some Numerical Experiments Concerning Space-Time Reactor Kinetics Behavior," Nucl. Sci. Eng., 22, 171 (1965).

14. P. T. Antonopoulos, "Large Mesh Model Development Study," Sc.D. Thesis, M.I.T., April, 1972. 
15. M. G. Steveson, "Investigations on the Macroscopic Nodal Approach to Space-Dependent Nuclear Reactor Kinetics,' Ph.D. Thesis, University of Texas at Austin (1968).

16. E. L. Wachspress, "Digital Computations of Space-Time Variations of Neutron Fluxes," KAPL-2090 (1960).

17. W. M. Stacey, Jr., Modal Approximations: Theory and an Application to Reactor Physics, Research Monograph No. 41. M.I.T. Press (1967).

18. A. Foderaco and H. L. Garabedian, "Two-Group Reactor Kinetics," Nucl. Sci. Eng., 14, 22 (1962).

19. D. R. Harris, S. Kaplan, and S. G. Marbolis, "Modal Analysis of Flux Tilt: Transients in a Nonuniform Reactor," Trans. Am. Nucl. Soc., 2 (2), 178 (1959).

20. A. F. Henry, "The Application of Inhour Modes to the Description of Nonseparable Reactor Transients," Nucl. Sci. Eng. , 13, 22 (1964).

21. E. L. Wachspress, "Variational Methods and Neutron Flux Synthesis," CONF-690401, 271 (1969).

22. E. L. Wachspress, R. D. Burgess, and S. Baron, "Multichannel Flux Synthesis," Nucl. Sci. Eng., 12, 381 (1962).

23. A. J. Buslik, "Interface Conditions for Few Group Neutron Diffusion Problems with Flux-Adjoint Weighted Constants, Nucl. Sci. Eng., 32, 233 (1968).

24. W. B. Terney, "Interface Conditions for Discontinuous Flux Synthesis Methods," Nucl. Sci. Eng. , 41, 303 (1970).

25. J.B. Yasinsky and S. Kaplan, "Synthesis of ThreeDimensional F'lux Shapes Using Discontinuous Sets of Trial Functions," Nucl. Sci. Eng., 28, 426 (1967).

26. J. B. Yasinsky and L. R. Foulke, "Improved Spatial Differencing for the Solution of Space-Time Problems Arising from the Movement of Reactor Materials," Nucl. Sci. Eng., $\underline{4}$. 72 (1971).

27. W. M. Stacey, Jr., "Variational Flux Synthesis Methods for Multigroup Neutron Diffusion Theory," Nucl. Sci. Eng. , 47. 449 (1972).

28. M. Becker, The Principles and Applications of Variational Methods, Research Monograph No. 27, M.I.T. Press (1964). 
29. A. F. Henry, "Variational Techniques as a Means of Deriving Approximation Methods," in M.I.T. Course 22.25 Notes, Advanced Reactor Physics, Chap. 15, p. 104 (Spring, 1969).

30. V. Luco and P. Lambropoulos," "Functionals for Discontinuous Trial Function Flux Synthesis, J. Nucl. Energy A/B, 24, p. 551 (1970).

31. G. C. Pomraning, "A Variational Description of Dissipative Processes," J. of Nuclear Energy A/B, Vol. 20, p. 617 (1966).

32. C. M. Kang and K. F. Hansen, "Finite Element Methods for the Neutron Diffusion Equations," Trans. Am. Nucl. Soc., Vol. 14, No. 1, p. 199 (1971).

33. C. M. Kang and K. F. Hansen," "Finite Element Methods for Space-Time Reactor Analysis," Sc.D. Thesis, Dept. of Nucl. Eng., M.I.T., MITNE-135 (1971).

34. B. W. Roos and W. C. Sangren, "Some Aspects of the Use of Digital Computers in Nuclear Reactor Design," in Advances in Nuclear Science and Technology, Vol. 2, p. 303 (1964).

35. A. F. Henry, "Demonstration That Homogenized Constants for a Cell Depend on Properties of the Surrounding Medium," in M.I.T. Course 22. 25 Notes, Advanced Reactor Physics, Chap. 12, p. 63 (Spring, 1969).

36. G. Strang, "The Finite Element Method and Approximation Theory," Numerical Solution of Partial Differential Equations - II, SYNSPADE 1970, Ed. B. Hubbard, p. 547, Academic Press (1971).

37. G. Fix and G. Strang, "Fourier Analysis of the Finite Element Method in Ritz-Galerkin Theory," Studies in Applied Math., $\underline{48}$, 265 (1969).

38. G. Birkhoff, C. deBoor, B. Swartz, and B. Wendroff, "Rayleigh-Ritz Approximation by Piecewise Cubic Polynomials." J. SIAM Numer. Anal., Vol. 3, No. 2, p. 188 (1966).

39. J. J. Göel, "Construction of Basic Functions for Numerical Utilization of Ritz's Method," Numer. Math., 12, p. 435 (1968).

40. A. Radkowsky, Editor, Naval Reactors Physics Handbook, Vol. 1, Selected Basic Techniques, "Homogenization Techniques," Chap. 4.3, USAEC, p. 620 (1964).

41. R. E. Alcouffe and R. W. Albrecht, "A Generalization of the Finite Difference Approximation Method with an Application to Space-Time Nuclear Reactor Kinetics," Nucl. Sci. Eng., 39, 1 (1970). 
42. S. Nakamura, "A Variational Rebalancing Method for Linear Iterative Convergence Schemes of Neutron Diffusion and Transport Equations," Nucl. Sci. Eng. , 39, 278 (1970).

43. S. Nakamura, "Coarse Mesh Acceleration of Iterative Solution of Neutron Diffusion Equations," Nucl. Sci. Eng., 43, 116 (1971).

44. A. Radkowsky, Editor, Naval Reactors Physics Handbook, Vol. 1, Selected Basic Techniques, "Reactor Physics Computations," Chap. 7.2, USAEC, p. 1414 (1964).

45. G. Bilodeau and L. A. Hageman, "A Survey of Numerical Methods in the Solution of Diffusion Problems,"WAPD-TM-64, (July, 1957).

46. G. I. Bell and S. Glasstone, Nuclear Reactor Theory, Van Nostrand Reinhold Company (1960).

47. W. W. Little, Jr. and R. W. Hardie, "2 DB User's Manual Revision 1," BNWL-831 REV 1, UC-32: Mathematics and Computers, Battelle Memorial Institute, Pacific Northwest Laboratories, Richland, Washington (August, 1969).

48. L. A. Hageman, "Numerical Methods and Techniques Used in the Two-Dimensional Neutron-Diffusion Program PDQ-5," WAPD-TM-364, UC-32: Mathematics and Computers, Bettis Atomic Power Company, Pittsburg, Pennsylvania (February, 1963).

49. E. Isaacson and H. B. Keller, Analysis of Numerical Methods, Wiley (1966).

50. R. S. Varga, Matrix Iterative Analysis, Prentice-Hall (1962).

51. G. Forsythe and C. B. Moler, Computer Solution of Linear Algebraic Systems, Prentice-Hall (1967).

52. L. A. Hageman and J. B. Yasinsky, "Comparison of Alternating-Direction Time-Differencing Methods with Other Implicit Methods for the Solution of the Neutron Group-Diffusion Equations," Nucl. Sci. Eng., 38, p. 8 (1969).

53. A. Radkowsky, Editor, Naval Reactors Physics Handbook, Vol. 1, Selected Basic Techniques, "Few-Group Approximations," Chap. 2.8, USAEC, p. 197 (1964).

54. M.I. T. Information Processing Center, "Stromberg-Carlson 4020," Application Program Series, AP-36 Revision 2, November 30, 1971. 
BIOGRAPHICAL NOTE

The author is a native Californian. Born in San Francisco August 19, 1945, he was raised in Orange, and upon graduation from Orange High School in 1963, he received the Bank of America Award in Mathematics and the Orange County Industrial Education Association Award in Electronics.

He then attended the University of California at Berkeley, and supported by special as well as Regents' Scholarship awards, graduated in 1967 from the School of Engineering with a Bachelor of Science degree in Physics. He was elected to Tau Beta Pi, Phi Beta Kappa, and received a four-year letter in varsity gymnastics.

The author then enrolled at the Massachusetts Institute of Technology. Supported by an Atomic Energy Commission Special Fellowship in Nuclear Engineering, he received the Master of Science degree from the Department of Nuclear Engineering in 1969.

As a research associate in the Space-Time Kinetics Project in the same department, the author completed this work and obtained the degree of Doctor of Philosophy in 1972.

While at M.I.T. the author was active in many organizations, most notable of which was the M.I. T. Rugby Football Club. 
A P P E N D C ES 


\section{Appendix A}

\section{TABLE OF SYMBOLS}

$$
\begin{aligned}
& \text { g Energy group index which runs from the highest } \\
& \text { to the lowest energy group as } \mathrm{g}=1 \text { to } \mathrm{G} \text {. } \\
& \phi_{g}(r) \quad \text { Scalar neutron flux in energy group } g \\
& \text { (neutrons } / \mathrm{cm}^{2} \cdot \mathrm{sec} \text { ). } \\
& \underline{J}_{g}(r) \quad \text { Vector neutron current in energy group } g \\
& \text { (neutrons } / \mathrm{cm}^{2} \cdot \mathrm{sec} \text { ). } \\
& \mathrm{D}_{\mathrm{g}}(\mathrm{r}) \quad \text { Diffusion coefficient for neutrons in energy } \\
& \text { group } \mathrm{g}(\mathrm{cm}) \text {. } \\
& \Sigma_{\mathrm{g}}(\mathrm{r}) \quad \text { Macroscopic total removal cross section in } \\
& \text { energy group } \mathrm{g}\left(\mathrm{cm}^{-1}\right) \text {. } \\
& \nu \Sigma_{f g}(r) \quad \text { Macroscopic fission-production cross section in } \\
& \text { energy group } \mathrm{g}\left(\mathrm{cm}^{-1}\right) \text {. } \\
& \Sigma_{\text {gg }}(r) \quad \text { Macroscopic transfer cross section from energy } \\
& \text { group } \mathrm{g}^{\prime} \text { to energy group } \mathrm{g}\left(\mathrm{cm}^{-1}\right) \text {. } \\
& x_{\mathrm{g}} \quad \text { Fission spectrum yield in energy group } \mathrm{g} \text {. } \\
& \lambda \quad \text { The eigenvalue or criticality of the diffusion } \\
& \text { problem. } \\
& \Phi(r), \Phi^{*}(r) \quad \text { Scalar group flux column vector of length } G \text { and } \\
& \text { its adjoint. } \\
& \underline{J}(r), \underline{J}^{*}(r) \quad \text { Vector group current column vector of length } G \\
& \text { and its adjoint. }
\end{aligned}
$$




$$
\begin{aligned}
& \mathbb{A}(r) \quad G \times G \text { group material removal, scattering, and } \\
& \text { production matrix. } \\
& \mathbb{D}(r) \quad G \times G \text { diagonal group diffusion coefficient matrix. } \\
& \mathrm{U}(\mathrm{r}), \mathrm{U}^{*}(\mathrm{r}) \quad \text { Scalar group flux and weighting flux trial function } \\
& \text { column vectors of length } G \text {. } \\
& \underline{\mathrm{V}}(\mathrm{r}), \underline{\mathrm{V}}^{*}(\mathrm{r}) \quad \text { Vector group current and weighting current } \\
& \text { trial function column vectors of length } G \text {. } \\
& \mathrm{k} \quad \text { One-dimensional spatial index which runs from } \\
& \text { the leftmost first region to the rightmost } \mathrm{K} \text {-th } \\
& \text { region, as } \mathrm{k}=1 \text { to } \mathrm{K} \text {. } \\
& \mathrm{z} \quad \text { The one-dimensional axis variable divided into } \\
& \mathrm{K} \text { regions such that each region } \mathrm{k} \text { is bounded } \\
& \text { by nodes } z_{k} \text { and } z_{k+1} \text {. } \\
& \mathrm{x} \quad \text { A dimensionless variable defined in each region } \\
& \mathrm{k} \text { as } \mathrm{x}=\left(\mathrm{z}-\mathrm{z}_{\mathrm{k}}\right) /\left(\mathrm{z}_{\mathrm{k}+1}-\mathrm{z}_{\mathrm{k}}\right) \text {, such that } 0 \leqslant \mathrm{x} \leqslant 1 \\
& \text { as } z_{k} \leqslant z \leqslant z_{k+1} \text {. } \\
& \mathrm{F}_{\mathrm{k}} \quad \text { Approximate one-dimensional group flux solution } \\
& \text { at node } \mathrm{z}_{\mathrm{k}} \text {. } \\
& \mathrm{G}_{\mathrm{k}} \quad \text { Approximate one-dimensional group current } \\
& \text { solution at node } \mathrm{z}_{\mathrm{k}} \text {. } \\
& \psi_{\mathrm{k}}(\mathrm{z}), \psi_{\mathrm{k}}^{*}(\mathrm{z}) \quad \text { Detailed one-dimensional subassembly flux and } \\
& \text { weighting flux solutions in coarse mesh region } \mathrm{k} \\
& \text { whose form is linear within each homogeneous } \\
& \text { subassembly interval. } \\
& \eta_{\mathrm{k}}(\mathrm{z}), \eta_{\mathrm{k}}^{*}(\mathrm{z}) \quad \text { Detailed one-dimensional subassembly current } \\
& \text { and weighting current solutions in coarse mesh } \\
& \text { region } \mathrm{k} \text { whose form is constant within each } \\
& \text { homogeneous subassembly interval. }
\end{aligned}
$$




$$
\begin{aligned}
& \tilde{\eta}_{k}(z), \tilde{n}_{k}^{*}(z) \quad \text { Detailed one-dimensional subassembly current } \\
& \text { and weighting current solutions in coarse mesh } \\
& \text { region } \mathrm{k} \text { whose form is linear within each } \\
& \text { homogeneous subassembly interval. } \\
& \text { A Discretized matrix form of the } \mathrm{G} \times \mathrm{G} \text { group dif- } \\
& \text { fusion, absorption, and scattering matrices. } \\
& \mathbb{B} \text { Discretized matrix form of the } \mathrm{G} \times \mathrm{G} \text { group } \\
& \text { fission-production matrix. } \\
& \text { F The unknown approximate group flux solution } \\
& \text { vector which may contain group current } \\
& \text { unknowns. } \\
& \% \lambda \quad \text { Normalized eigenvalue percent error: } \\
& \% \lambda=\left(\lambda_{\text {Reference }}{ }^{-\lambda} \text { Method }\right) / \lambda_{\text {Reference }} \times 100 \% \text {. } \\
& \mathrm{P}(\mathrm{k}) \quad \text { Fractional power produced in coarse mesh } \\
& \text { region } \mathrm{k} \text { when the total power produced has } \\
& \text { been normalized to unity. } \\
& \% \mathrm{P}(\mathrm{k}) \quad \text { Normalized fractional power percent error: } \\
& { }_{0} \mathrm{P}(\mathrm{k})=\left[\mathrm{P}(\mathrm{k})_{\text {Reference }}{ }^{\left.-\mathrm{P}(\mathrm{k})_{\text {Method }}\right] / \mathrm{P}(\mathrm{k})_{\text {Reference }}}\right. \\
& \times 100 \% \text {. }
\end{aligned}
$$




\section{Appendix B \\ DIFFERENCE EQUATION COEFFICIENTS RESULTING FROM USE OF THE FINITE ELEMENT \\ APPROXIMATION METHODS}

The $\mathrm{G} \times \mathrm{G}$ matrix coefficients resulting from the conventional finite difference approximation, the linear finite element approximation, and the cubic Hermite finite element approximation in onedimensional multigroup diffusion theory are defined below in sections B.1, B.2, and B. 3, respectively. The coefficients are given in terms of assumed homogeneous regional nuclear constants through the use of the $G \times G$ group matrices $\mathbb{D}_{\mathrm{k}}$ and $\Lambda_{\mathrm{k}}$, where $\Lambda_{\mathrm{k}}=$ $\mathbb{I M}_{\mathrm{k}}-\mathbf{T}_{\mathrm{k}}-\frac{1}{\lambda} \mathbb{B}_{\mathrm{k}}$, which are defined in Chapter 2 and are constant for each region $\mathrm{k}$, where $\mathrm{k}=1$ to $\mathrm{K}$.

More general definitions of these coefficients may be found from the coefficients resulting from the use of the proposed approximations, given in Appendix $C$, by requiring that $\psi_{k}(z)$ be constant and $\eta_{k}(z)$ be zero in each region $\mathrm{k}$.

B.1. Coefficients of the Conventional Finite Difference Equations (as defined by Eqs. 2.16)

Interior Coefficients; $\mathrm{k}=2$ to $\mathrm{K}$ :

$$
\begin{aligned}
& \mathrm{a}_{\mathrm{k}}=-\mathbb{D}_{\mathrm{k}-1} / \mathrm{h}_{\mathrm{k}-1} \\
& \mathrm{~b}_{\mathrm{k}}=\frac{1}{2}\left(\boldsymbol{\Lambda}_{\mathrm{k}-1} \mathrm{~h}_{\mathrm{k}-1}+\boldsymbol{\Lambda}_{\mathrm{k}} \mathrm{h}_{\mathrm{k}}\right)+\mathbb{D}_{\mathrm{k}-1} / \mathrm{h}_{\mathrm{k}-1}+\mathbb{D}_{\mathrm{k}} / \mathrm{h}_{\mathrm{k}} \\
& \mathrm{c}_{\mathrm{k}}=-\mathbb{D}_{\mathrm{k}} / \mathrm{h}_{\mathrm{k}}
\end{aligned}
$$


Symmetry Boundary Condition Coefficients:

$$
\begin{aligned}
& \mathrm{b}_{1}=\frac{1}{2} \bigwedge_{1} \mathrm{~h}_{1}+\mathbb{D}_{1} / \mathrm{h}_{1} \\
& \mathrm{c}_{1}=-\mathbb{D}_{1} / \mathrm{h}_{1} \\
& \mathrm{a}_{\mathrm{K}+1}=-\mathbb{D}_{\mathrm{K}} / \mathrm{h}_{\mathrm{K}} \\
& \mathrm{b}_{\mathrm{K}+1}=\frac{1}{2} \boldsymbol{\Lambda}_{\mathrm{K}} \mathrm{h}_{\mathrm{K}}+\mathbb{D}_{\mathrm{K}} / \mathrm{h}_{\mathrm{K}}
\end{aligned}
$$

B.2. Coefficients of the Linear Finite Element Method Equations (as defined by Eqs. 2.30)

Interior Coefficients; $\mathrm{k}=2$ to $\mathrm{K}$ :

$$
\begin{aligned}
& \mathrm{a}_{\mathrm{k}}=\frac{1}{6} \mathbb{\Lambda}_{\mathrm{k}-1} \mathrm{~h}_{\mathrm{k}-1}-\mathbb{D}_{\mathrm{k}-1} / \mathrm{h}_{\mathrm{k}-1} \\
& \mathrm{~b}_{\mathrm{k}}=\frac{1}{3}\left[\mathbb{\Lambda}_{\mathrm{k}-1} \mathrm{~h}_{\mathrm{k}-1}+\mathbb{\Lambda}_{\mathrm{k}} \mathrm{h}_{\mathrm{k}}\right]+\mathbb{D}_{\mathrm{k}-1} / \mathrm{h}_{\mathrm{k}-1}+\mathbb{D}_{\mathrm{k}} / \mathrm{h}_{\mathrm{k}} \\
& \mathrm{c}_{\mathrm{k}}=\frac{1}{6} \mathbb{\Lambda}_{\mathrm{k}} \mathrm{h}_{\mathrm{k}}-\mathbb{D}_{\mathrm{k}} / \mathrm{h}_{\mathrm{k}}
\end{aligned}
$$

Symmetry Boundary Condition Coefficients:

$$
\begin{aligned}
& \mathrm{b}_{1}=\frac{1}{3} \Lambda_{1} \mathrm{~h}_{1}+\mathbb{D}_{1} / \mathrm{h}_{1} \\
& \mathrm{c}_{1}=\frac{1}{6} \bigwedge_{1} \mathrm{~h}_{1}-\mathbb{D}_{1} / \mathrm{h}_{1} \\
& \mathrm{a}_{\mathrm{K}+1}=\frac{1}{6} \Lambda_{\mathrm{K}} \mathrm{h}_{\mathrm{K}}-\mathbb{D}_{\mathrm{K}} / \mathrm{h}_{\mathrm{K}} \\
& \mathrm{b}_{\mathrm{K}+1}=\frac{1}{3} \Lambda_{\mathrm{K}} \mathrm{h}_{\mathrm{K}}+\mathbb{D}_{\mathrm{K}} / \mathrm{h}_{\mathrm{K}}
\end{aligned}
$$


B.3. Coefficients of the Cubic Hermite Finite Element Method Equations (as defined by Eq. 2.33)

Interior Coefficients; $\mathrm{k}=2$ to $\mathrm{K}$ :

$$
\begin{aligned}
& \mathrm{a}_{\mathrm{k}}=\frac{9}{70} \boldsymbol{\Lambda}_{\mathrm{k}-1} \mathrm{~h}_{\mathrm{k}-1}-\frac{6}{5} \mathbb{D}_{\mathrm{k}-1} / \mathrm{h}_{\mathrm{k}-1} \\
& \mathrm{a} 2_{\mathrm{k}}=\left(-\frac{13}{420} \Lambda_{\mathrm{k}-1} \mathrm{~h}_{\mathrm{k}-1}^{2} \mathbb{D}_{\mathrm{k}-1}^{-1}+\frac{1}{10}\right) \theta \\
& \mathrm{b} 1_{\mathrm{k}}=\frac{13}{35}\left(\bigwedge_{\mathrm{k}-1} \mathrm{~h}_{\mathrm{k}-1}+\mathbb{\Lambda}_{\mathrm{k}} \mathrm{h}_{\mathrm{k}}\right)+\frac{6}{5}\left(\mathbb{D}_{\mathrm{k}-1} / \mathrm{h}_{\mathrm{k}-1}+\mathbb{D}_{\mathrm{k}} / \mathrm{h}_{\mathrm{k}}\right) \\
& \mathrm{b} 2_{\mathrm{k}}=\frac{11}{210}\left(\Lambda_{\mathrm{k}-1} \mathrm{~h}_{\mathrm{k}-1}^{2} \mathbb{D}_{\mathrm{k}-1}^{-1}-\boldsymbol{\Lambda}_{\mathrm{k}} \mathrm{h}_{\mathrm{k}}^{2} \mathbb{D}_{\mathrm{k}}^{-1}\right) \theta \\
& \mathrm{c} 1_{\mathrm{k}}=\frac{9}{70} \boldsymbol{\Lambda}_{\mathrm{k}} \mathrm{h}_{\mathrm{k}}-\frac{6}{5} \mathbb{D}_{\mathrm{k}} / \mathrm{h}_{\mathrm{k}} \\
& \mathrm{c} 2_{\mathrm{k}}=\left(\frac{13}{420} \Lambda_{\mathrm{k}} \mathrm{h}_{\mathrm{k}}^{2} \mathrm{D}_{\mathrm{k}}^{-1}-\frac{1}{10}\right) \theta \\
& \text { a } 3_{k}=\left(\frac{13}{420} \mathbb{D}_{k-1}^{-1} h_{k-1}^{2} \Lambda_{k-1}-\frac{1}{10}\right) \theta \\
& { }^{2} 4_{k}=\left(-\frac{1}{140} \mathbb{D}_{k-1}^{-1} h_{k-1}^{3} \Lambda_{k-1} \mathbb{D}_{k-1}^{-1}-\frac{1}{30} h_{k-1} \mathbb{D}_{k-1}^{-1}\right) \theta^{2} \\
& \mathrm{~b} 3_{\mathrm{k}}=\frac{11}{210}\left(\mathbb{D}_{\mathrm{k}-1}^{-1} \mathrm{~h}_{\mathrm{k}-1}^{2} \mathbb{\Lambda}_{\mathrm{k}-1}-\mathbb{D}_{\mathrm{k}}^{-1} \mathrm{~h}_{\mathrm{k}}^{2} \mathbb{\Lambda}_{\mathrm{k}}\right) \theta \\
& \mathrm{b} 4_{\mathrm{k}}=\left[\frac{1}{105}\left(\mathbb{D}_{\mathrm{k}-1}^{-1} \mathrm{~h}_{\mathrm{k}-1}^{3} \Lambda_{\mathrm{k}-1} \mathbb{D}_{\mathrm{k}-1}^{-1}+\mathbb{D}_{\mathrm{k}}^{-1} \mathrm{~h}_{\mathrm{k}}^{3} \Lambda_{\mathrm{k}} \mathbb{D}_{\mathrm{k}}^{-1}\right)\right. \\
& \left.+\frac{2}{15}\left(\mathrm{~h}_{\mathrm{k}-1} \mathbb{D}_{\mathrm{k}-1}^{-1}+\mathrm{h}_{\mathrm{k}} \mathbb{D}_{\mathrm{k}}^{-1}\right)\right] \theta^{2} \\
& \mathrm{c} 3_{\mathrm{k}}=\left(-\frac{13}{420} \mathbb{D}_{\mathrm{k}}^{-1} \mathrm{~h}_{\mathrm{k}}^{2} \bigwedge_{\mathrm{k}}+\frac{1}{10}\right) \theta \\
& \mathrm{c}_{\mathrm{k}}=\left(-\frac{1}{140} \mathbb{D}_{\mathrm{k}}^{-1} \mathrm{~h}_{\mathrm{k}}^{3} \Lambda_{\mathrm{k}} \mathbb{D}_{\mathrm{k}}^{-1}-\frac{1}{30} \mathrm{~h}_{\mathrm{k}} \mathbb{D}_{\mathrm{k}}^{-1}\right) \theta^{2}
\end{aligned}
$$


Zero Flux Boundary Condition Coefficients:

$$
\begin{aligned}
& \mathrm{b}_{1}=\left(\frac{1}{105} \mathbb{D}_{1}^{-1} \mathrm{~h}_{1}^{3} \Lambda_{1} \mathbb{D}_{1}^{-1}+\frac{2}{15} \mathrm{~h}_{1} \mathbb{D}_{1}^{-1}\right) \theta^{2} \\
& \mathrm{c} 3_{1}=\left(-\frac{13}{420} \mathbb{D}_{1}^{-1} \mathrm{~h}_{1}^{2} \Lambda_{1}+\frac{1}{10}\right) \theta \\
& \mathrm{c}_{1}=\left(-\frac{1}{140} \mathbb{D}_{1}^{-1} \mathrm{~h}_{1}^{3} \Lambda_{1} \mathbb{D}_{1}^{-1}-\frac{1}{30} \mathrm{~h}_{1} \mathbb{D}_{1}^{-1}\right) \theta^{2} \\
& \mathrm{a}_{\mathrm{K}+1}=\left(\frac{13}{420} \mathbb{D}_{\mathrm{K}}^{-1} \mathrm{~h}_{\mathrm{K}}^{2} \Lambda_{\mathrm{K}}-\frac{1}{10}\right) \theta \\
& \mathrm{a}_{\mathrm{K}+1}=\left(-\frac{1}{140} \mathbb{D}_{\mathrm{K}}^{-1} \mathrm{~h}_{\mathrm{K}}^{3} \mathbb{\Lambda}_{\mathrm{K}} \mathbb{D}_{\mathrm{K}}^{-1}-\frac{1}{30} \mathrm{~h}_{\mathrm{K}} \mathbb{D}_{\mathrm{K}}^{-1}\right) \theta^{2} \\
& \mathrm{~b} 4_{\mathrm{K}+1}=\left(\frac{1}{105} \mathbb{D}_{\mathrm{K}}^{-1} \mathrm{~h}_{\mathrm{K}}^{3} \Lambda_{\mathrm{K}} \mathbb{D}_{\mathrm{K}}^{-1}+\frac{2}{15} \mathrm{~h}_{\mathrm{K}} \mathbb{D}_{\mathrm{K}}^{-1}\right) \theta^{2}
\end{aligned}
$$

Symmetry Boundary Condition Coefficients:

$$
\begin{aligned}
& \mathrm{b}_{1}=\frac{13}{35} \Lambda_{1} \mathrm{~h}_{1}+\frac{6}{5} \mathbb{D}_{1} / \mathrm{h}_{1} \\
& \mathrm{c} 1_{1}=\frac{9}{70} \Lambda_{1} \mathrm{~h}_{1}-\frac{6}{5} \mathbb{D}_{1} / \mathrm{h}_{1} \\
& \mathrm{c}{ }_{1}=\left(\frac{13}{420} \Lambda_{1} \mathrm{~h}_{1}^{2} \mathbb{D}_{1}^{-1}-\frac{1}{10}\right) \theta \\
& \mathrm{a}_{\mathrm{K}+1}=\frac{9}{70} \Lambda_{\mathrm{K}} \mathrm{h}_{\mathrm{K}}-\frac{6}{5} \mathbb{D}_{\mathrm{K}} / \mathrm{h}_{\mathrm{K}} \\
& \mathrm{a} 2_{\mathrm{K}+1}=\left(-\frac{13}{420} \Lambda_{\mathrm{K}} \mathrm{h}_{\mathrm{K}}^{2} \mathbb{D}_{\mathrm{K}}^{-1}+\frac{1}{10}\right) \theta \\
& \mathrm{b} 1_{\mathrm{K}+1}=\frac{13}{35} \Lambda_{\mathrm{K}} \mathrm{h}_{\mathrm{K}}+\frac{6}{5} \mathbb{D}_{\mathrm{K}} / \mathrm{h}_{\mathrm{K}}
\end{aligned}
$$


Appendix C

DIFFERENCE EQUATION COEFFICIENTS RESULTING FROM USE OF THE PROPOSED APPROXIMATION METHODS

The $\mathrm{G} \times \mathrm{G}$ matrix coefficients resulting from the proposed approximation methods using (1) linear basis functions, and (2) cubic Hermite basis functions, in one-dimensional multigroup diffusion theory are defined below in sections C. 1 and C.2, respectively.

The coefficients are given as integrands of functions of $\mathrm{x}$ where the integration of every coefficient-integrand over a region

$$
\text { Coefficient }=\int_{0}^{1}[\text { Coefficient-Integrand }(x)] d x
$$

is understood.

In order to simplify the forms of the coefficient-integrands, it is convenient to define the following $\mathrm{G} \times \mathrm{G}$ matrices:

$$
\begin{aligned}
& \mathbb{K}_{\mathrm{k}}(\mathrm{x})=\psi_{\mathrm{k}}^{*} \mathrm{~T}_{\mathrm{k}}^{\mathrm{T}}(\mathrm{x}) \mathbb{A}_{\mathrm{k}}(\mathrm{x}) \mathrm{h}_{\mathrm{k}} \psi_{\mathrm{k}}(\mathrm{x}) \\
& \mathbb{L}_{\mathrm{k}}(\mathrm{x})=\eta_{\mathrm{k}}^{* \mathrm{~T}^{\mathrm{T}}}(\mathrm{x}) \mathbb{D}_{\mathrm{k}}^{-1}(\mathrm{x}) \mathrm{h}_{\mathrm{k}} \eta_{\mathrm{k}}(\mathrm{x}) \\
& \mathbb{P}_{\mathrm{k}}(\mathrm{x})=\psi_{\mathrm{k}}^{*}(\mathrm{x}) \eta_{\mathrm{k}}(\mathrm{x}) \\
& \mathbb{Q}_{\mathrm{k}}(\mathrm{x})=\eta_{\mathrm{k}}^{*^{\mathrm{T}}}(\mathrm{x}) \psi_{\mathrm{k}}(\mathrm{x}) \\
& \mathbb{R}_{\mathrm{k}}(\mathrm{x})=\frac{1}{\mathrm{~h}_{\mathrm{k}}} \psi_{\mathrm{k}}^{*}(\mathrm{x}) \mathbb{D}_{\mathrm{k}}(\mathrm{x}) \psi_{\mathrm{k}}(\mathrm{x})
\end{aligned}
$$

for each region $\mathrm{k}$. In each approximation below, two sets of polynomial functions $\mathrm{p}_{1}(\mathrm{x}) \ldots \mathrm{p}_{2 \mathrm{~N}}(\mathrm{x})$ and $\mathrm{q}_{1}(\mathrm{x}) \ldots \mathrm{q}_{2 \mathrm{~N}}(\mathrm{x})$ are given 
which represent the basis functions of the approximation and their negative derivatives, where $\mathrm{N}=1$ for the linear basis function approximations and $\mathrm{N}=2$ for the cubic Hermite basis function approximations.

The $G \times G$ coefficient-integrands are then listed in terms of these matrices and polynomials by the $G \times G$ collapsed matrices $\mathbb{E}_{k}^{i, j}(x)$ defined as

$$
\begin{aligned}
\mathbb{E}_{k}^{i_{j} j}(x)= & p_{i}(x) p_{j}(x) \mathbb{K}_{k}(x)-p_{i}(x) p_{j}(x) \mathbb{L}_{k}(x)+q_{i}(x) p_{j}(x) \mathbb{P}_{k}(x) \\
& -p_{i}(x) q_{j}(x) \mathbb{Q}_{k}(x)+q_{i}(x) q_{j}(x) \mathbb{R}_{k}(x)
\end{aligned}
$$

for given values of $i$ and $j$ for each region $k$, where $k=1$ to $K$. It should be noted that $\mathbb{E}_{k}^{i, j}(x)$ is not symmetric about $i$ and $j$; i. e.:

$$
\mathbb{E}_{k}^{i, j}(x) \neq \mathbb{E}_{k}^{j, i}(x), \quad \text { for } i \neq j
$$

C.1. Coefficient-Integrands of the Proposed Approximation Method Equations Using Linear Basis Functions (as defined by Eq. 3.6)

These coefficients are given in terms of the polynomial functions

$$
\begin{aligned}
& p_{1}(x)=(1-x) \\
& p_{2}(x)=x \\
& q_{1}(x)=1 \\
& q_{2}(x)=-1
\end{aligned}
$$

for use in the $\mathbb{E}_{k}^{i, j}(x)$ below. 
Interior Coefficient-Integrands; $\mathrm{k}=2$ to $\mathrm{K}$ :

$$
\begin{aligned}
& \mathrm{a}_{\mathrm{k}}(\mathrm{x})=\psi_{\mathrm{k}-1}^{* \mathrm{~T}}(1) \mathbb{E}_{\mathrm{k}-1}^{2,1}(\mathrm{x}) \psi_{\mathrm{k}-1}^{-1}(0) \\
& \mathrm{b}_{\mathrm{k}}(\mathrm{x})=\psi_{\mathrm{k}-1}^{* \mathrm{~T}}(1) \mathbb{E}_{\mathrm{k}-1}^{2,2}(\mathrm{x}) \psi_{\mathrm{k}-1}^{-1}(1)+\psi_{\mathrm{k}}^{*} \mathrm{~T}^{-1}(0) \mathbb{E}_{\mathrm{k}}^{1,1}(\mathrm{x}) \psi_{\mathrm{k}}^{-1}(0) \\
& \quad-1 \\
& \mathrm{c}_{\mathrm{k}}(\mathrm{x})=\psi_{\mathrm{k}}^{* \mathrm{~T}}(0) \mathbb{E}_{\mathrm{k}}^{1,2}(\mathrm{x}) \psi_{\mathrm{k}}^{-1}(1)
\end{aligned}
$$

Symmetry Coefficient-Integrands:

$$
\begin{aligned}
& \mathrm{b}_{1}(\mathrm{x})=\psi_{1}^{-1} \mathrm{~T}^{*}(0) \mathrm{E}_{1}^{1,1}(\mathrm{x}) \psi_{1}^{-1}(0) \\
& \mathrm{c}_{1}(\mathrm{x})=\mathrm{c}_{\mathrm{k}}(\mathrm{x}) ; \text { where } \mathrm{k}=1 \\
& \mathrm{a}_{\mathrm{K}+1}(\mathrm{x})=\mathrm{a}_{\mathrm{K}}(\mathrm{x}) ; \text { where } \mathrm{k}=\mathrm{K}+1 \\
& \mathrm{~b}_{\mathrm{K}+1}(\mathrm{x})=\psi_{\mathrm{K}}^{\text {* } \mathrm{T}}(1) \mathbb{E}_{\mathrm{K}}^{2,2}(\mathrm{x}) \psi_{\mathrm{K}}^{-1}(1)
\end{aligned}
$$

Implied Zero Flux Boundary Condition Coefficient-Integrands (Corresponding with the modified trial functions of the type in Eqs. 3.9)

$$
\begin{aligned}
& \left.\mathrm{b}_{2}(\mathrm{x})=\psi_{1}^{-1} \mathrm{~T}_{(1)} \mathrm{T} \mathbb{K}_{1}(\mathrm{x})-\mathbb{L}_{1}(\mathrm{x})\right] \psi_{1}^{-1}(1)+\psi_{2}^{* \mathrm{~T}}(0) \mathbb{E}_{2}^{1,1}(\mathrm{x}) \psi_{2}^{-1}(0) \\
& \mathrm{b}_{\mathrm{K}}(\mathrm{x})=\psi_{\mathrm{K}-1}^{-1}(1) \mathbb{E}_{\mathrm{K}-1}^{2,2}(\mathrm{x}) \psi_{\mathrm{K}-1}^{-1}(1)+\psi_{\mathrm{K}}^{* \mathrm{~T}}(0)\left[\mathbb{K}_{\mathrm{K}}-\mathbb{L}_{\mathrm{K}}\right] \psi_{\mathrm{K}}^{-1}(0)
\end{aligned}
$$


C.2. Coefficient-Integrands of the Proposed Approximation Method Equations Using Cubic Hermite Basis Functions (as defined in $\mathrm{Eq}$. 3.16)

These coefficients are given in terms of the polynomials $p_{1}(x)$ through $\mathrm{p}_{4}(\mathrm{x})$ and $\mathrm{q}_{1}(\mathrm{x})$ through $\mathrm{q}_{4}(\mathrm{x})$, previously defined in Eqs. 3.11 and 3.12 , for use in the $\mathbb{E}_{\mathrm{k}}^{\mathrm{i}, \mathrm{j}}(\mathrm{x})$ below.

Interior Coefficient-Integrands; $\mathrm{k}=2$ to $\mathrm{K}$ :

$$
\begin{aligned}
\mathrm{a} 1_{\mathrm{k}}(\mathrm{x})= & \psi_{\mathrm{k}-1}^{* \mathrm{~T}}(1) \mathbb{E}_{\mathrm{k}-1}^{2,1}(\mathrm{x}) \psi_{\mathrm{k}-1}^{-1}(0) \\
& -1 \\
\mathrm{a} 2_{\mathrm{k}}(\mathrm{x})= & \psi_{\mathrm{k}-1}^{* \mathrm{~T}}(1) \mathbb{E}_{\mathrm{k}-1}^{2,3}(\mathrm{x}) \psi_{\mathrm{k}-1}^{-1}(0) \mathbb{D}_{\mathrm{k}-1}^{-1}(0) \theta \\
& -1 \\
\mathrm{~b} 1_{\mathrm{k}}(\mathrm{x})= & \psi_{\mathrm{k}-1}^{* \mathrm{~T}}(1) \mathbb{E}_{\mathrm{k}-1}^{2,2}(\mathrm{x}) \psi_{\mathrm{k}-1}^{-1}(1)+\psi_{\mathrm{k}}^{*} \mathrm{~T}(0) \mathbb{E}_{\mathrm{k}}^{1,1}(\mathrm{x}) \psi_{\mathrm{k}}^{-1}(0) \\
\mathrm{b} 2_{\mathrm{k}}(\mathrm{x})= & \psi_{\mathrm{k}-1}^{* \mathrm{~T}}(1) \mathbb{E}_{\mathrm{k}-1}^{2,4}(\mathrm{x}) \psi_{\mathrm{k}-1}^{-1}(1) \mathbb{D}_{\mathrm{k}-1}^{-1}(1) \theta \\
& +\psi_{\mathrm{k}}^{* \mathrm{~T}}(0) \mathbb{E}_{\mathrm{k}}^{1,3}(\mathrm{x}) \psi_{\mathrm{k}}^{-1}(0) \mathbb{D}_{\mathrm{k}}^{-1}(0) \theta \\
& -1 \\
\mathrm{c} 1_{\mathrm{k}}(\mathrm{x})= & \psi_{\mathrm{k}}^{* \mathrm{~T}}(0) \mathbb{E}_{\mathrm{k}}^{1,2}(\mathrm{x}) \psi_{\mathrm{k}}^{-1}(1) \\
& -1 \\
\mathrm{c} 2_{\mathrm{k}}(\mathrm{x})= & \psi_{\mathrm{k}}^{* 1}(0) \mathbb{E}_{\mathrm{k}}^{1,4}(\mathrm{x}) \psi_{\mathrm{k}}^{-1}(1) \mathbb{D}_{\mathrm{k}}^{-1}(1) \theta \\
& -1 \\
\mathrm{a} 33_{\mathrm{k}}(\mathrm{x})= & \psi_{\mathrm{k}-1}^{* \mathrm{~T}}(1) \mathbb{D}_{\mathrm{k}-1}^{-1}(1) \mathbb{E}_{\mathrm{k}-1}^{4,1}(\mathrm{x}) \psi_{\mathrm{k}-1}^{-1}(0) \theta \\
& -1 \\
\mathrm{a} 4 \mathrm{k}_{\mathrm{k}}(\mathrm{x})= & \psi_{\mathrm{k}-1}^{* \mathrm{~T}}(1) \mathbb{D}_{\mathrm{k}-1}^{-1}(1) \mathbb{E}_{\mathrm{k}-1}^{4,3}(\mathrm{x}) \psi_{\mathrm{k}-1}^{-1}(0) \mathbb{D}_{\mathrm{k}-1}^{-1}(0) \theta^{2}
\end{aligned}
$$




$$
\begin{aligned}
& \mathrm{b} 3_{\mathrm{k}}(\mathrm{x})=\psi_{\mathrm{k}-1}^{* \mathrm{~T}}(1) \mathbb{D}_{\mathrm{k}-1}^{-1}(1) \mathbb{E}_{\mathrm{k}-1}^{4,2}(\mathrm{x}) \psi_{\mathrm{k}-1}^{-1}(1) \theta \\
& +\psi_{\mathrm{k}}^{* \mathrm{~T}}(0) \mathbb{D}_{\mathrm{k}}^{-1}(0) \mathbb{E}_{\mathrm{k}}^{3,1}(\mathrm{x}) \psi_{\mathrm{k}}^{-1}(0) \theta \\
& \mathrm{b} 4_{\mathrm{k}}(\mathrm{x})=\psi_{\mathrm{k}-1}^{-1}(1) \mathbb{D}_{\mathrm{k}-1}^{-1}(1) \mathbb{E}_{\mathrm{k}-1}^{4,4}(\mathrm{x}) \psi_{\mathrm{k}-1}^{-1}(1) \mathbb{D}_{\mathrm{k}-1}^{-1}(1) \theta^{2} \\
& +\psi_{\mathrm{k}}^{* \mathrm{~T}}(0) \mathbb{D}_{\mathrm{k}}^{-1}(0) \mathbb{E}_{\mathrm{k}}^{3,3}(\mathrm{x}) \psi_{\mathrm{k}}^{-1}(0) \mathbb{D}_{\mathrm{k}}^{-1}(0) \theta^{2} \\
& \mathrm{c} 3{ }_{\mathrm{k}}(\mathrm{x})=\psi_{\mathrm{k}}^{-1} \mathrm{~T}(0) \mathbb{D}_{\mathrm{k}}^{-1}(0) \mathbb{E}_{\mathrm{k}}^{3,2}(\mathrm{x}) \psi_{\mathrm{k}}^{-1}(1) \theta \\
& \mathrm{c} 4_{\mathrm{k}}(\mathrm{x})=\psi_{\mathrm{k}}^{-1} \mathrm{~T}^{-1}(0) \mathbb{D}_{\mathrm{k}}^{-1}(0) \mathbb{E}_{\mathrm{k}}^{3,4}(\mathrm{x}) \psi_{\mathrm{k}}^{-1}(1) \mathbb{D}_{\mathrm{k}}^{-1}(1) \theta^{2}
\end{aligned}
$$

Zero Flux Boundary Condition Coefficient-Integrands:

$$
\begin{aligned}
& \mathrm{b} 4_{1}(\mathrm{x})=\psi_{1}^{* \mathrm{~T}}(0) \mathbb{D}_{1}^{-1}(0) \mathbb{E}_{1}^{3,3}(\mathrm{x}) \psi_{1}^{-1}(0) \mathbb{D}_{1}^{-1}(0) \theta^{2} \\
& \mathrm{c} 3_{1}(\mathrm{x})=\mathrm{c} 3_{\mathrm{k}}(\mathrm{x}) ; \text { where } \mathrm{k}=1 \\
& \mathrm{c} 4_{1}(\mathrm{x})=\mathrm{c} 4_{\mathrm{k}}(\mathrm{x}) ; \text { where } \mathrm{k}=1 \\
& \mathrm{a} 3_{\mathrm{K}+1}(\mathrm{x})=\mathrm{a}{ }_{\mathrm{k}}(\mathrm{x}) ; \text { where } \mathrm{k}=\mathrm{K}+1 \\
& \mathrm{a} 4_{\mathrm{K}+1}(\mathrm{x})=\mathrm{a} 4_{\mathrm{K}+1}(\mathrm{x}) ; \text { where } \mathrm{k}=\mathrm{K}+1 \\
& \quad-1 \\
& \mathrm{~b} 4_{\mathrm{K}+1}(\mathrm{x})=\psi_{\mathrm{K}}^{* \mathrm{~T}}(1) \mathbb{D}_{\mathrm{K}}^{-1}(1) \mathbb{E}_{\mathrm{K}}^{4}, 4(\mathrm{x}) \psi_{\mathrm{K}}^{-1}(1) \mathbb{D}_{\mathrm{K}}^{-1}(1) \theta^{2}
\end{aligned}
$$


Symmetry Boundary Condition Coefficient-Integrands:

$$
\begin{aligned}
& \mathrm{b} 1_{1}(\mathrm{x})=\psi_{1}^{* \mathrm{~T}}(0) \mathbb{E}_{1}^{1,1}(\mathrm{x}) \psi_{1}^{-1}(0) \\
& \mathrm{c} 1_{1}(\mathrm{x})=\mathrm{c} 1_{\mathrm{k}}(\mathrm{x}) ; \text { where } \mathrm{k}=1 \\
& \mathrm{c} 2_{1}(\mathrm{x})=\mathrm{c} 2_{\mathrm{k}}(\mathrm{x}) ; \text { where } \mathrm{k}=1 \\
& \mathrm{a} 1_{\mathrm{K}+1}(\mathrm{x})=\mathrm{a} 1_{\mathrm{k}}(\mathrm{x}) ; \text { where } \mathrm{k}=\mathrm{K}+1 \\
& \mathrm{a} 2_{\mathrm{K}+1}(\mathrm{x})=\mathrm{a} 2_{\mathrm{k}}(\mathrm{x}) ; \text { where } \mathrm{k}=\mathrm{K}+1 \\
& \mathrm{~b} 1_{\mathrm{K}+1}(\mathrm{x})=\psi_{\mathrm{K}}^{* \mathrm{~T}}(1) \mathbb{E}_{\mathrm{K}}^{2,2}(\mathrm{x}) \psi_{\mathrm{K}}^{-1}(1)
\end{aligned}
$$

Implied Zero Flux Boundary Condition Coefficient-Integrands (corresponding with the modified trial functions of the type in Eq. 3.17):

$$
\left.\begin{array}{l}
\mathrm{c} 3{ }_{1}(\mathrm{x})=\mathrm{c} 3_{\mathrm{k}}(\mathrm{x}) ; \text { where } \mathrm{k}=1 \\
\mathrm{a} 2_{2}(\mathrm{x})=\mathrm{a} 2_{\mathrm{k}}(\mathrm{x}) ; \text { where } \mathrm{k}=2 \\
\mathrm{~b} 1_{2}(\mathrm{x})=\mathrm{b} 1_{\mathrm{k}}(\mathrm{x}) ; \text { where } \mathrm{k}=2 \\
\mathrm{~b} 2_{2}(\mathrm{x})=\mathrm{b} 2_{\mathrm{k}}(\mathrm{x}) ; \text { where } \mathrm{k}=2 \\
\mathrm{~b} 3{ }_{2}(\mathrm{x})=\mathrm{b} 3_{\mathrm{k}}(\mathrm{x}) ; \text { where } \mathrm{k}=2
\end{array}\right\} \text { and where }\left\{\begin{array}{l}
\mathrm{p}_{2}(\mathrm{x})=1 \\
\mathrm{q}_{1}(\mathrm{x})=0 \\
\mathrm{q}_{2}(\mathrm{x})=0
\end{array}\right.
$$




\section{Appendix D \\ DESCRIPTION OF THE COMPUTER PROGRAMS}

The computer programs REF2G, LINEAR, CUBIC, and ANALYZE are described respectively in the following four sections. The programs are written in FORTRAN IV, allow double precision calculations, and were used with the I.B.M. 360/65 and 370/155 FORTRAN G compilers at the M.I.T. Information Processing Center. Sample storage requirements and execution times of the programs are summarized in Table D. 1.

The power method employed in the first three programs allows a maximum of 300 iterations to converge, and program execution continues after this limit. Initial group flux shapes are sinusoidal or flat, depending upon the boundary conditions chosen.

The input and output data of each program are divided into data blocks for ease of representation as described below.

\section{D.1. Description of Program REF 2G}

REF2G finds the reference solutions of the one-dimensional, twogroup diffusion equations of each case study, or the detailed subassembly solutions of each subassembly, using the linear finite element approximation method. The program allows up to a total of two hundred homogeneous fine mesh regions and employs combinations of both zero flux and symmetry boundary conditions. Identical material regions can be automatically repeated with no additional input. 
Options for plotting graphically the history of the converging spatial flux as well as the converging eigenvalue are also available. In addition, the program allows the calculation of the adjoint flux and current solutions.

The approximate current solutions are linear within each mesh region and are calculated from the converged flux solutions using Eqs.4.29 and 4.31. The converged flux and current solutions and the converged adjoint solutions can be punched out for future use as described below.

A. Reference Solution Input Block

Card Type 1: Format (20A4)

An Appropriate Problem Title

Card Type 2: Format (2I5, 3E10.3, 5I5)

KR Total number of homogeneous fine mesh regions. $\mathrm{KR} \leqslant 200$.

IBC Boundary Condition Option

1. Zero flux on both boundaries

2. Zero flux on the left, symmetry on the right

3. Symmetry on the left, zero flux on the right

4. Symmetry on both boundaries

EPS1 Iteration tolerance to be met by differences between elements of successive iteration solution vectors:

$$
\left|F_{j}^{(i)}-F_{j}^{(i-1)}\right|<\epsilon_{1} ; \text { for all } j
$$

EPS2 Iteration tolerance to be met by the mean square error between successive iteration solution vectors:

$$
\left\{\sum_{j}\left[F_{j}^{(i)}-F_{j}^{(i-1)}\right]^{2}\right\}^{\frac{1}{2}}<\epsilon_{2}
$$


Table D.1. Sample Storage Requirements and Execution Times of the Programs for Two-Group Results. Obtained using the M.I.T. I.B. M. 360/155.

Storage Requirements in Bytes (without overlays):

REF2G: $\quad 260 \mathrm{~K}$

LINEAR: $\quad 200 \mathrm{~K}$

CUBIC: $\quad 250 \mathrm{~K}$

ANALYZE: $205 \mathrm{~K}$

\section{C.P.U. Execution Times in Minutes:}

REF2G: $\quad$ Detailed Subassembly Solutions (68 regions) ${ }^{\mathrm{a}}$ : $\quad .120$

Case 1 Reference Solution (150 regions) ${ }^{\mathrm{b}}$ : $\quad .238$

Case 4 Reference Solution (198 regions) $)^{\mathrm{b}}$ : $\quad .644$

LINEAR: $\quad$ Case 1 Synthesis (Homogenized ${ }^{c}$ ) Method (3 regions): $\quad .284$

Case 4 Synthesis (Homogenized ${ }^{\mathrm{C}}$ ) Method (9 regions): .296

$(.157)$

$.296 \quad(.209)$

CUBIC: $\quad$ Case 1 Synthesis (Homogenized ${ }^{C}$ ) Method (3 regions): 1.227

(.183)

Case 4 Synthesis (Homogenized ${ }^{c}$ ) Method (9 regions): $\quad 1.464$

(.328)

ANALYZE: Case 1 Linear (Cubic Hermite) Basis Functions: 087

Case 4 Linear (Cubic Hermite) Basis Functions:

a. Including adjoint flux and current calculations.

b. Not including adjoint calculations.

c. Including . 126 minutes for calculation of the two-group homogenized constants. 
EPS3 Iteration tolerance to be met by the difference between successive iteration eigenvalues:

$$
\left|\lambda^{(i)}-\lambda^{(i-1)}\right|<\epsilon_{3}
$$

IPLOT Allows printed graphical display of the converging flux solution:

$0 \quad$ No display

1 Plot only the resultant normalized flux

2 Plot a normalized history of the converging flux

JPLOT Allows printed graphical display of the history of the converging eigenvalue when $\mathrm{JPLOT}=1$.

IPUNCH Allows punched output when IPUNCH $=1$.

ISEE Allows printing of storage information:

0 No information printed

1 Input regional properties are printed

2 Input regional properties as well as the Common/B5/ storage arrays and the Common/B3/ power method matrices are printed.

NOADJ Adjoint calculations are performed when NOADJ $=0$, and bypassed if NOADJ $=1$.

Card Type 3: Format (25I2)

ITF(k) The consecutive type-number of each region from left to right as $\mathrm{k}=1$ to $\mathrm{KR}$. Allows for repeating identical regions with no additional input.

Card Type 3 is repeated KR/25 times (rounded off to the next highest integer).

Card Type 4: Format (2F10.5)

CHI(1), CHI(2) The fission yields $x_{1}$ and $\chi_{2}$ for the fast and thermal groups, respectively. 
* An Input Region Data Block:

Repeated for each different material region; $\max _{\mathrm{k}}[\operatorname{ITF}(\mathrm{k})]$ times.

Card Type 5: Format (I5)

$\mathrm{k}$ The consecutive mesh region number (counting from from left to right) for identification purposes.

Card Types 6, 7: Format (3F10.5, 4E10.3, /, 30X, 3E10.3)

The geometry and nuclear constants for region $\mathrm{k}$ :

$\mathrm{z}(1)$ Beginning spatial coordinate of region $\mathrm{k}(\mathrm{cm})$

$\mathrm{z}(2) \quad$ Ending spatial coordinate of region $\mathrm{k}(\mathrm{cm})$

$\mathrm{H} \quad$ Width of region $\mathrm{k}(\mathrm{cm})$

A(1) Fast-group macroscopic total cross section in region $\mathrm{k}\left(\mathrm{cm}^{-1}\right)$

F(1) Fast-group macroscopic production cross section, $\nu \Sigma_{f}$, in region $\mathrm{k}\left(\mathrm{cm}^{-1}\right)$

$\mathrm{D}(1) \quad$ Fast-group diffusion coefficient in region $\mathrm{k}(\mathrm{cm})$

$\mathrm{S} \quad$ Fast-to-thermal macroscopic scattering cross section in region $\mathrm{k}\left(\mathrm{cm}^{-1}\right)$

A(2) Thermal-group macroscopic total cross section in region $\mathrm{k}\left(\mathrm{cm}^{-1}\right)$

$\mathrm{F}$ (2) Thermal-group macroscopic production cross section, $\nu \Sigma_{f}$, in region $\mathrm{k}\left(\mathrm{cm}^{-1}\right)$

$\mathrm{D}(2)$ Thermal-group diffusion coefficient in region $\mathrm{k}(\mathrm{cm})$

* End of an Input Region Data Block.

** Power Method Input Block: Optional

Card Type 8: Format (F10.5)

$\omega \quad$ Outer iteration overrelaxation parameter $1 \leqslant \omega \leqslant 2$. Default is $\omega=1.25$.

Card Type 9: Format (D25.14)

$\lambda^{(0)}$ Initial eigenvalue guess. Default is $\lambda^{(0)}=1.0$. 
Card Type 10: Format (4E20.10)

$((F(g, i), i=1$ to $N), g=1$ to 2$) \quad$ Initial group flux solution guess without zero flux boundary values. Default is $\underline{F}=1.0$.

** End of Power Method Input Block.

B. Reference Solution Output Block

When $\operatorname{IPUNCH}=1, \mathrm{REF} 2 \mathrm{G}$ punches out the number of fine mesh regions, KR, under Format (I5) followed by the converged flux solutions $\psi(\mathrm{g}, \mathrm{k})$ and corresponding current solutions $\tilde{\eta}(\mathrm{g}, \mathrm{k})$ for each group $\mathrm{g}$ and spatial node $\mathrm{k}$ including boundary conditions. When adjoint calculations are included, the results are punched out under Format (4D20.10) as

$$
\left(\left(\psi(\mathrm{g}, \mathrm{k}), \tilde{\eta}(\mathrm{g}, \mathrm{k}), \psi^{*}(\mathrm{~g}, \mathrm{k}), \tilde{\eta}^{*}(\mathrm{~g}, \mathrm{k}), \mathrm{k}=1 \text { to } \mathrm{KR}+1\right), \mathrm{g}=1 \text { to } 2\right)
$$

where the notation denotes case reference solutions as well as detailed subassembly solutions. When the adjoint calculations have been bypassed, the results are punched out under Format (2D20.10) as

$$
((\psi(\mathrm{g}, \mathrm{k}), \tilde{\eta}(\mathrm{g}, \mathrm{k}), \mathrm{k}=1 \text { to } \mathrm{KR}+1), \mathrm{g}=1 \text { to } 2)
$$

A total of $2 \mathrm{KR}+3$ cards are punched out.

\section{D.2. Description of Program LINEAR}

Program LINEAR forms and solves the difference equations resulting from the proposed approximation method using the linear basis functions. The program allows up to twenty-five coarse mesh regions, each of which is allowed to be broken into not more than one hundred homogeneous intervals. Combinations of both zero flux and 
symmetry boundary conditions as well as use of the modified trial function forms in the boundary regions are allowed. Spatial flux and eigenvalue iteration history plots are also available.

The program allows a choice of the type of weighting, Galerkin or adjoint, to be used in the approximation. Also, either form of the detailed subassembly current solutions $\eta_{\mathrm{k}}(\mathrm{x})$ or $\tilde{\eta}_{\mathrm{k}}(\mathrm{x})$ is allowed. In addition, identical coarse mesh regions with identical detailed subassembly solutions can be repeated implicitly.

LINEAR also calculates results of the linear finite element method when suitable input is used. Such results can be obtained by using homogenized coarse mesh region nuclear constants and defining the detailed group flux solutions to be constant and the detailed currents to be zero (or by setting ITC $=0$ ).

Punched results using detailed subassembly solutions constitute a Synthesis Method Output Block, while punched output resulting from the reduction to the finite element method with homogenized regional constants constitutes a Homogenized Method Output Block.

A. Homogenized or Synthesis Method Input Block

Undefined input parameters are identical to those previously defined in the REF $2 \mathrm{G}$ input.

Card Type 1: Format (20A4)

An Appropriate Problem Title

Card Type 2: Format (2I5, 3E10.3,6I5)

KR Total number of coarse mesh regions. $K R \leqslant 25$. 
IBC $\quad 1-4$ As previously defined

5 Modified trial function (no tilting) in the first region, symmetry on the right

6 Zero flux on the left, modified trial function in the last region

$7 \quad$ Modified trial functions in both boundary regions

EPS1

EPS2

EPS 3

IPLOT

JPLOT

IPUNCH

ISEE

ITW Type of approximation weighting desired:

0 Flux (Galerkin)

1 Adjoint

ITC Form of the detailed current solutions in all subassemblies:

$0 \quad \eta_{\mathrm{k}}(\mathrm{x}), \eta_{\mathrm{k}}^{*}(\mathrm{x})$ as calculated by Fick's laws $1 \tilde{\eta}_{k}(\mathrm{x}), \tilde{\eta}_{\mathrm{k}}^{*}(\mathrm{x})$ as given from REF $2 \mathrm{G}$ output

\section{Card Type 3: Format (25I2)}

ITF(k) The consecutive type-number of each coarse mesh region from left to right as $\mathrm{k}=1$ to KR. Allows for repeating identical subassemblies with no additional input.

Card Type 3 is repeated KR/25 times (rounded off to the next highest integer)。

Card Type 4: Format (2F10.5)

CHI(1), CHI(2) 
* An Input Subassembly Region Data Block:

Repeated for each different coarse mesh region; $\underset{\mathrm{k}}{\max }[\operatorname{ITF}(\mathrm{k})]$ times.

Card Type 5: Format (2I5)

$\mathrm{k} \quad$ The consecutive coarse mesh region number (from left to right).

$\mathrm{N}$ The number of homogeneous intervals in subassembly k. $\mathrm{N} \leqslant 100$.

Card Types 6, 7: Format (3F10.5, 4E10.3, /, 30X, 3E10.3)

The subassembly geometry and nuclear constants within each interval corresponding to the detailed subassembly solutions.

Repeated for each interval as $\mathrm{i}=1$ to $\mathrm{N}$ :

$z(i)$ Beginning spatial coordinate of interval i $(\mathrm{cm})$

$z(i+1) \quad$ Ending spatial coordinate of interval i $(\mathrm{cm})$

$\mathrm{H}(\mathrm{i})$ Width of interval i $(\mathrm{cm})$

A(1,i) Fast-group macroscopic total cross section in interval i $\left(\mathrm{cm}^{-1}\right)$

$F(1, i) \quad$ Fast-group macroscopic production cross section, $\nu \Sigma_{\mathrm{f}}$, in interval i $\left(\mathrm{cm}^{-1}\right)$

$\mathrm{D}(1, \mathrm{i}) \quad$ Fast-group diffusion coefficient in interval $\mathrm{i}(\mathrm{cm})$

S(i) Fast-to-thermal macroscopic scattering cross section in interval $\mathrm{i}\left(\mathrm{cm}^{-1}\right)$

A(2,i) Thermal-group macroscopic total cross section in interval $\mathrm{i}\left(\mathrm{cm}^{-1}\right)$

F(2,i) Thermal-group macroscopic production cross section, $\nu \Sigma_{\mathrm{f}}$, in interval $\mathrm{i}\left(\mathrm{cm}^{-1}\right)$

$\mathrm{D}(2, \mathrm{i}) \quad$ Thermal-group diffusion coefficient in interval i $(\mathrm{cm})$ 
Card Type 8: Format (4D20.10)

The detailed subassembly solutions.

$\left(\left(\psi(\mathrm{g}, \mathrm{k}), \tilde{\eta}(\mathrm{g}, \mathrm{k}), \psi^{*}(\mathrm{~g}, \mathrm{k}), \tilde{\eta}^{*}(\mathrm{~g}, \mathrm{k}), \mathrm{k}=1\right.\right.$ to $\left.\mathrm{KR}+1\right), \mathrm{g}=1$ to 2$)$

A subassembly's Reference Solution Output Block without the first card.

* END of an Input Subassembly Region Data Block.

** Expected Solution Input Block: Optional

Card Type 9: Format (D25.14)

$\lambda_{R E F}$ Expected eigenvalue solution. Default is $\lambda_{R E F}=1.0$.

Card Type 10: Format (4E20.10)

( $F(i, g), i=1$ to $N), g=1$ to 2$) \quad$ Expected group flux solution without zero flux boundary values. Default is $\underline{F}=1.0$.

** END of the Expected Solution Input Block.

**** Power Method Input Block: Optional

As previously defined in the REF $2 \mathrm{G}$ input.

*** END of the Power Method Input Block.

B. Homogenized or Synthesis Method Output Block

When IPUNCH $=1$, LINEAR punches out the total number of coarse mesh regions, KR, under Format (I5) followed by the resultant flux solutions including boundary conditions. The flux solutions are punched out under Format (2E20.7) as

$$
(\mathrm{F}(1, \mathrm{k}), \mathrm{F}(2, \mathrm{k}), \mathrm{k}=1 \text { to } \mathrm{KR}+1)
$$

These cards represent either a Homogenized or Synthesis Method Output Block, depending upon the type and form of input data used. 
D. 3 Description of Program CUBIC

Program CUBIC forms and solves the difference equations resulting from the proposed approximation method using the cubic Hermite basis functions. The program is very similar in form to program LINEAR and uses similar input.

A. Homogenized or Synthesis Method Input Block

The input to CUBIC is identical to that of LINEAR except for the following:

1. The boundary condition options are restricted by $1 \leqslant \mathrm{IBC} \leqslant 4$.

2. The normalization constant $\theta$ can be included on Card Type 4 after CHI (2) under Format (3F10.5). Default is $\theta=1.0$.

3. Both the expected group solutions and the initial group solutions of the Expected Solution and Power Method Input Blocks, respectively, are of the form $((\mathrm{F}(\mathrm{g}, \mathrm{i}), \mathrm{i}=1$ to $\mathrm{N}), \mathrm{g}=1$ to 2$)$ without either zero flux or zero current (or symmetry) boundary conditions. The solution vector is made up of alternating flux and current values as described in section 3.3 of Chapter 3. Default values are flux values of unity and current values of zero.

B. Homogenized or Synthesis Method Output Block

When IPUNCH $=1$, CUBIC punches out the total number of coarse mesh regions, KR, under Format (I5) followed by the resultant flux and current solutions including boundary conditions. The solutions are punched out under Format (4E20.7) as

$$
(F(1, k), F(2, k), G(1, k), G(2, k), k=1 \text { to } K R+1)
$$


where $F(g, k)$ represents the flux, and $G(g, k)$ the current solution of group $\mathrm{g}$ at node $\mathrm{k}$.

As in the case of LINEAR, these $K R+2$ output cards represent either a Synthesis or Homogenized Method Output Block, depending upon the type and form of input data used.

\section{4 Description of Program ANALYZE}

ANALYZE compares the results of the reference solution, homogenized finite element method, and the proposed synthesis method for each case study where either linear or cubic Hermite basis functions have been used in the latter methods. For each of these three methods, the program first forms the complete detailed flux solution and then normalizes the flux distributions for each method such that their total power levels are unity. The fractional (normalized) power levels produced in each coarse mesh region are then calculated, compareci, and listed. Finally, the detailed group fluxes of each method are plotted graphically relative to one another using the Stromberg-Carlson

Computer Recorder, SC-4020, facility at M.I.T. ${ }^{54}$ The graphic results for each group are normalized by the largest group-flux value such that the equivalent total power levels are preserved.

\section{A. ANALYZE Input}

The input to ANALYZE is read from five device units: $1,2,3,11$, 12. 13, and 5. Input and output data of the reference and approximation programs are read from the former six units while the standard input unit, 5, is reserved for SC-4020 plotting information. 
The input is described by "Header Cards" and previously defined Input and Output Blocks. Header cards consist of one or more cards defined as follows:

Header Card 1: Format (4I5)

Method Indicates the type of basis function approximation:

1 Linear

2 Cubic Hermite

NK Total number of coarse mesh regions involved.

NR Total number of fine mesh regions involved. $\quad N R=N K$ except for reference solution calculations.

NAP Number of additional points to be plotted within each coarse mesh region. Used with the homogenized finite element method calculations. NAP $<0$ denotes that the additional points are to be used in the first region (reflector) only.

Header Card 2: For use in device unit 3 input when $\mathrm{NR} \neq \mathrm{NK}$. Format (16I5).

NRNK(k) The number of fine mesh regions which make up each coarse mesh region $\mathrm{k}$, as $\mathrm{k}=1$ to $\mathrm{NK}$.

The program is dimensioned to accept up to 200 fine mesh regions (or intervals) per coarse mesh region, up to 25 coarse mesh regions, and up to a grand total of 1000 fine mesh regions in each case study. The form of the ANALYZE input is given as follows: 
Input Data for Unit 1:

Header Cards

[Homogenized Method Input Block $]$

Input Data for Unit 2:

Header Cards

[Synthesis Method Input Block]

Input Data for Unit 3:

Header Cards

[Reference Solution Input Block $]$

Input Data for Unit 11:

[Homogenized Method Output Block $]$

Input Data for Unit 12:

[Synthesis Method Output Block]

Input Data for Unit 13:

[Reference Solution Output Block $]$

Input Data for Unit 5:

No SC-4020 plots are generated if this data is omitted.

Card 1: Format (20A4)

An appropriate title written above each plotted graph. 
Card 2: Format (2F10.5)

XINCH Total width of the graph in inches including labels (limited to $\left.7.45^{\prime \prime}\right)$.

YINCH Total height of the graph in inches including labels (limited to $7.45^{\prime \prime}$ ).

Card 3: Format (I10, F10.5)

NCELL Total number of coarse mesh regions. NCELL $\leqslant 25$. (NCELL $<0$ indicates that the last region is of width $\frac{1}{2}$ WCELL .)

WCELL Width of each coarse mesh region in $\mathrm{cm}$.

Card 4: Optional. Format (I10, 7F10.5)

NLL Number of vertical light lines to be added to the plotted graphs. NLL $\leqslant 100$.

$\mathrm{XL}(\mathrm{i}) \quad$ Spatial location $(\mathrm{cm})$ of the light lines; $i=1$ to 7 .

Card 5: Format (8F10.5)

XL(i) As above when NLL $>7$. 


\begin{abstract}
Appendix E
SAMPLE INPUT AND OUTPUT DATA BLOCKS

FOR PROGRAMS REF2G, LINEAR, CUBIC, AND ANALYZE
\end{abstract}

(Included in only the first six copies of this report.) 


\begin{tabular}{|c|c|c|c|c|c|c|c|c|c|c|}
\hline $\begin{array}{l}60 \\
0.0^{\circ}\end{array}$ & $\begin{array}{ll}1 & \\
& 0.25\end{array}$ & 0.25 & $\begin{array}{l}2.60 \\
7.10\end{array}$ & $\begin{array}{l}D-2 \\
D-2\end{array}$ & $\begin{array}{l}5.53 \\
1.02\end{array}$ & $\begin{array}{l}D-3 \\
D-1\end{array}$ & $\begin{array}{l}1.397 \\
3.89\end{array}$ & $\begin{array}{ll}D & 0 \\
D-1\end{array}$ & 1.72 & $D-2$ \\
\hline 64 & 1 & & & & & & & & & \\
\hline 0.0 & 0.125 & 0.125 & $\begin{array}{l}2.60 \\
7.10\end{array}$ & $\begin{array}{l}D-2 \\
D-2\end{array}$ & $\begin{array}{l}5.53 \\
1.02\end{array}$ & $\begin{array}{l}D-3 \\
D-1\end{array}$ & $\begin{array}{l}1.397 \\
3.89\end{array}$ & $\begin{array}{l}D \\
D-1\end{array}$ & 1.72 & $D-2$ \\
\hline 68 & 1 & & & & & & & & & \\
\hline 0.0 & 0.0625 & 0.0625 & $\begin{array}{l}4.52 \\
9.59\end{array}$ & $\begin{array}{l}D-2 \\
D-1\end{array}$ & $\begin{array}{l}0.0 \\
0.0\end{array}$ & $\begin{array}{ll}\text { D } & 0 \\
\text { D } & 0\end{array}$ & $\begin{array}{l}1.0 \\
1.0\end{array}$ & $\begin{array}{ll}D & 0 \\
D & 0\end{array}$ & 0.0 & D 0 \\
\hline 101 & 1 & & & & & & & & & \\
\hline 0.0 & 1.0 & 1.0 & $\begin{array}{l}2.61 \\
8.32\end{array}$ & $\begin{array}{l}D-2 \\
D-2\end{array}$ & $\begin{array}{l}6.59 \\
1.29\end{array}$ & $\begin{array}{l}D-3 \\
D-1\end{array}$ & $\begin{array}{l}1.399 \\
3.87\end{array}$ & $\begin{array}{ll}D & 0 \\
D & -1\end{array}$ & 1.68 & $D-2$ \\
\hline $\begin{array}{l}106 \\
0.0\end{array}$ & 10.5 & 0.5 & 2.61 & $D-2$ & 6.59 & $D-3$ & $1 \cdot 399$ & D 0 & 1.68 & $D-2$ \\
\hline 110 & 1 & & 32 & $D-2$ & 1.29 & $D-1$ & 37 & $D-1$ & & \\
\hline 0.0 & 0.25 & 0.25 & $\begin{array}{l}2.61 \\
8.32\end{array}$ & $\begin{array}{l}D-2 \\
D-2\end{array}$ & $\begin{array}{l}6.59 \\
1.29\end{array}$ & $\begin{array}{l}D-3 \\
D-1\end{array}$ & $\begin{array}{l}1.399 \\
3.87\end{array}$ & $\begin{array}{ll}D & 0 \\
D-1\end{array}$ & 1.68 & $D-2$ \\
\hline 114 & 1 & & & & & & & & & \\
\hline 0.0 & 0.125 & 0.125 & $\begin{array}{l}2.61 \\
8.32\end{array}$ & $\begin{array}{l}D-2 \\
D-2\end{array}$ & $\begin{array}{l}6.59 \\
1.29\end{array}$ & $\begin{array}{l}D-3 \\
D-1\end{array}$ & $\begin{array}{l}1.399 \\
3.87\end{array}$ & $\begin{array}{l}D \\
D-1 \\
D-1\end{array}$ & 1.68 & $D-2$ \\
\hline 118 & 1 & & & & & & & & & \\
\hline 0.0 & 0.0625 & 0.0625 & $\begin{array}{l}4.52 \\
9.59\end{array}$ & $\begin{array}{l}D-2 \\
D-1\end{array}$ & $\begin{array}{l}0.0 \\
0.0\end{array}$ & $\begin{array}{ll}\text { D } & 0 \\
\text { D } & 0\end{array}$ & $\begin{array}{l}1.0 \\
1.0\end{array}$ & $\begin{array}{ll}D & 0 \\
D & C\end{array}$ & 0.0 & D 0 \\
\hline
\end{tabular}


CELL A TWO GRJJP CELL SOLUTION: FUEL A + CRUCIFORM ROD. TWO GROUP CONSTANTS.

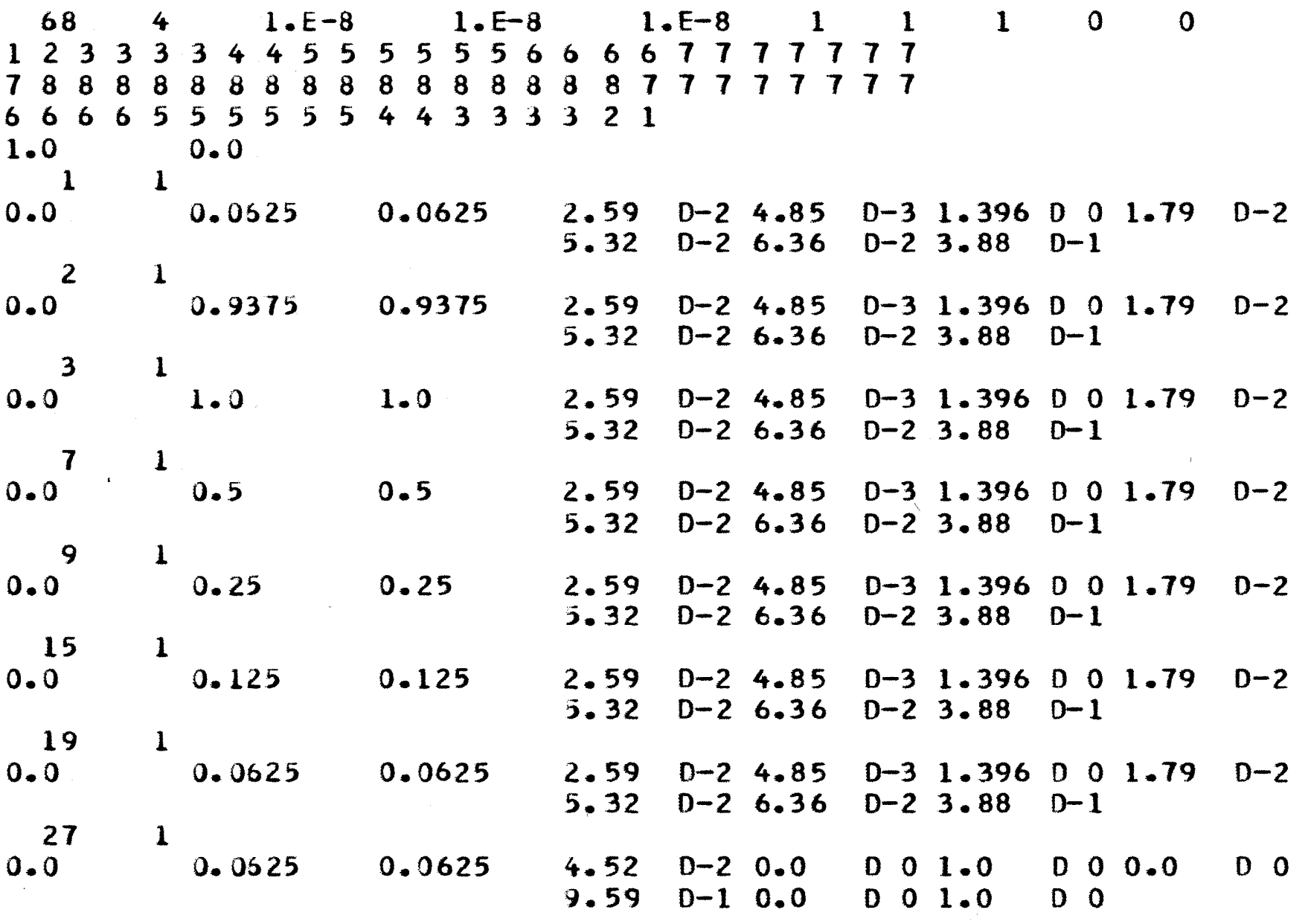


SAMPLE REF2G REFERENCE SOLUTION JUTPUT BLOCK (SAMPLE SUBASSEMBLY SOLUTION):

68

\begin{tabular}{|c|c|c|c|c|c|c|}
\hline $\begin{array}{l}0.1000000 J \\
0.9999906 J \\
0.99760400\end{array}$ & $\begin{array}{l}01 \\
00 \\
00\end{array}$ & 0.0 & $\begin{array}{r}D 0 \\
0.20623190-02 \\
0.63514340-02\end{array}$ & $\begin{array}{l}0.6840883 D \\
0.68408190 \\
0.6824493 D\end{array}$ & $\begin{array}{l}00 \\
00 \\
00\end{array}$ & $\begin{array}{r}0.0 \\
-0.1410808 D-0 \\
-0.4344942 D-0\end{array}$ \\
\hline
\end{tabular}

63 ADDITIOVAL FAST GROUP DATA CARDS

\begin{tabular}{|c|c|c|c|c|c|c|c|}
\hline $\begin{array}{l}0.9976 J 40 J \\
0.9999906)\end{array}$ & $\begin{array}{l}00 \\
00\end{array}$ & \multicolumn{2}{|r|}{$\begin{array}{l}-0.6351434 \mathrm{D}-02 \\
-0.2062319 \mathrm{D}-02\end{array}$} & $\begin{array}{l}0.68244930 \\
0.68408190\end{array}$ & $\begin{array}{l}00 \\
00\end{array}$ & \multicolumn{2}{|r|}{$\begin{array}{l}0.4344942 \mathrm{D}-02 \\
0.1410808 \mathrm{D}-02\end{array}$} \\
\hline 0 & 0 & 0.0 & D 0 & 30 & 00 & 0.0 & D 0 \\
\hline 450 & 00 & 0.0 & D 0 & 0.10000000 & 01 & 0.0 & D 0 \\
\hline $090072 J$ & 00 & & $0.44843120-03$ & $762 D$ & 00 & & $-0.1451165 \mathrm{D}-02$ \\
\hline 0711030 & 00 & & $0.14180280-02$ & 0.99383770 & 00 & & $588871 D-02$ \\
\hline
\end{tabular}

63 ADDITIJYAL THERMAL GROUP DATA CARDS

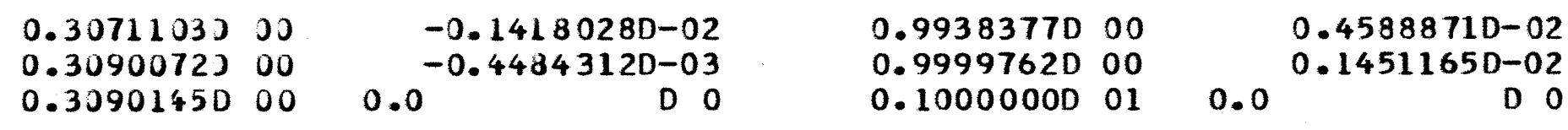




\section{E.2. LINEAR SAMPLE INPUT AND OUTPUT DATA BLOCKS}

SAMPLE LINEAR JR CUBIC HOMOGENIZED METHOD INPUT BLOCK:

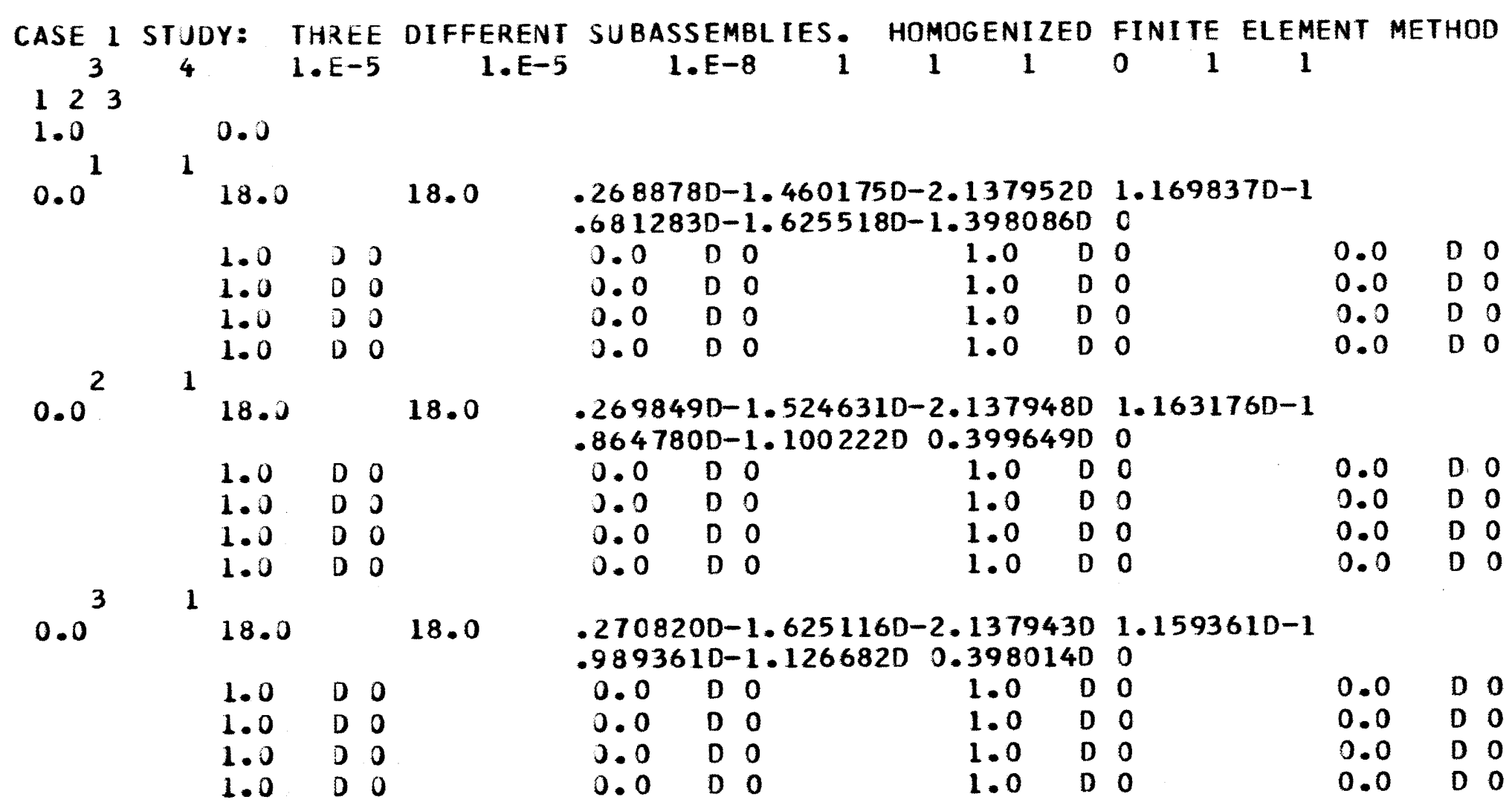


SAMPLE LINEAR METHOD OUTPUT BLOCK:

3

0.2444087000

$0.4060855 \mathrm{D} 0 \mathrm{~J}$

0.7838067300

$67580410-01$

$0.9039887 D-01$

0.1339626000

0.1000000001

0.1563839000 


\section{E.3. CUBIC SAMPLE INPUT AND OUTPUT DATA BLOCKS}

SAMPLE LINEAR JR CUBIC PROPOSED SYNTHESIS METHOD INPUT BLOCK:

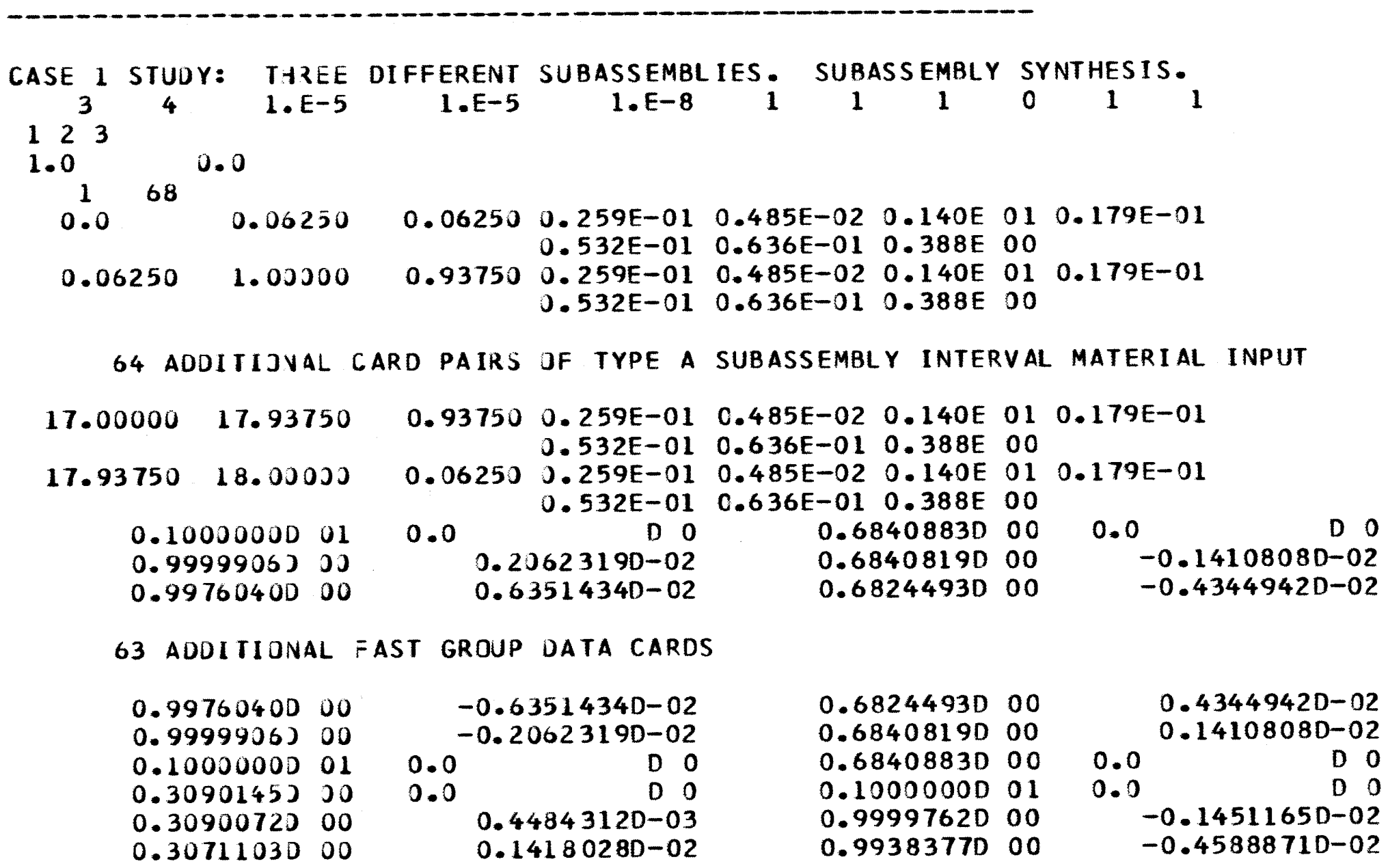

63 ADDITIOVAL THERMAL GROUP DATA CARDS 


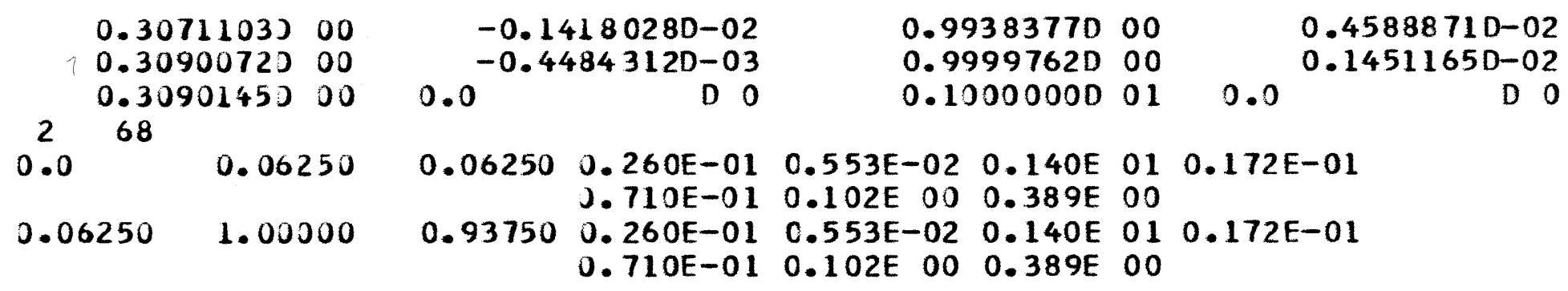

64 ADDITIJVAL CARD PAIRS JF TYPE B SUBASSEMBLY INTERVAL MATERIAL INPUT

\begin{tabular}{|c|c|c|c|c|c|c|c|}
\hline 17.00000 & 17.93750 & $\begin{array}{l}0.93750 \\
0.06250\end{array}$ & $\begin{array}{l}0.260 E-01 \\
0.710 E-01 \\
0.260 E-01 \\
0.710 E-01\end{array}$ & $\begin{array}{l}0.553 E-02 \\
0.102 E \quad 00 \\
0.553 E-02 \\
0.102 E 00\end{array}$ & $\begin{array}{l}0.140 E \\
0.389 E \\
0.140 E \\
0.389 E\end{array}$ & $\begin{array}{ll}0 & 1 \\
0 & 0 \\
0 & 1 \\
0 & 0\end{array}$ & $0.172 E-01$ \\
\hline $\begin{array}{l}0.10 \\
0.99 \\
0.99\end{array}$ & $\begin{array}{ll}000000 & 01 \\
99912 J & 00 \\
774740 & 00\end{array}$ & $\begin{array}{ll}0.0 & \\
& 0.1 \\
& 0.5\end{array}$ & $\begin{array}{r}D 0 \\
86980-02 \\
8934 D-02\end{array}$ & $\begin{array}{l}0.65 \\
0.65 \\
0.65\end{array}$ & $\begin{array}{l}5791580 \\
5791000 \\
5643380\end{array}$ & $\begin{array}{l}00 \\
00 \\
00\end{array}$ & $\begin{array}{r}0.0 \\
-0.1275500 \mathrm{D}-02 \\
-0.39336350-02\end{array}$ \\
\hline
\end{tabular}

63 ADDITIUNAL FAST GROUP DATA CARDS

\begin{tabular}{|c|c|c|c|c|c|c|c|}
\hline $\begin{array}{l}0.9977474 J \\
0.9999912 J\end{array}$ & $\begin{array}{l}00 \\
j 0\end{array}$ & \multicolumn{2}{|r|}{$\begin{array}{l}-0.5978934 \mathrm{D}-02 \\
-0.1938698 \mathrm{D}-02\end{array}$} & $\begin{array}{l}0.6564338 D \\
0.6579100 D\end{array}$ & $\begin{array}{l}00 \\
00\end{array}$ & \multicolumn{2}{|r|}{$\begin{array}{l}0.39336350-02 \\
0.12755000-02\end{array}$} \\
\hline 10000 & 01 & 0.0 & D 0 & $0.6579158 \mathrm{D}$ & 00 & 0.0 & D 0 \\
\hline 22944880 & 00 & 0.0 & D 0 & 0.10000000 & 01 & 0.0 & D 0 \\
\hline .22944420 & 00 & & $0.27926490-03$ & $0.9999801 D$ & 00 & & $-0.1217112 \mathrm{D}-02$ \\
\hline
\end{tabular}

63 ADDI TIOVAL THERMAL GROJP DATA CARDS

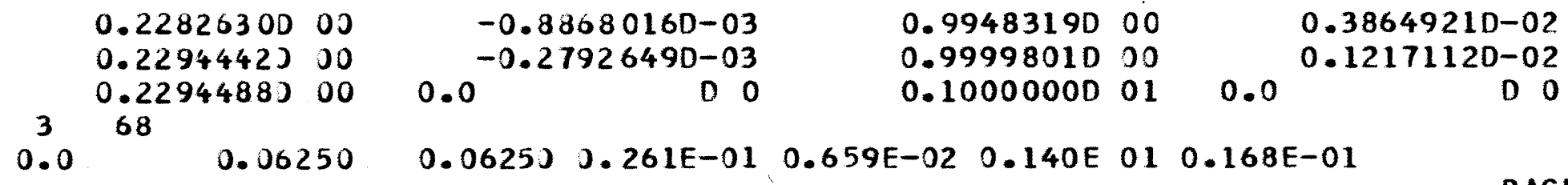




$\begin{array}{rrrrrrr}0.832 E-01 & 0.129 E & 00 & 0.387 E & 00 \\ 0.06250 \quad 1.00000 \quad 0.93750 & 0.261 E-01 & 0.659 E-02 & 0.140 E & 01 & 0.168 E-01 \\ 0.832 E-01 & 0.129 E & 00 & 0.387 E & 00\end{array}$

64 ADDITIJNAL CARD PAIRS OF TYPE C SUBASSEMBLY INTERVAL MATERIAL INPUT

\begin{tabular}{|c|c|c|c|c|c|c|c|}
\hline $\begin{array}{l}17.00000 \\
17.93750\end{array}$ & $\begin{array}{l}17.93750 \\
18.00000\end{array}$ & 0.93750 & $\begin{array}{l}0.261 E-01 \\
0.832 E-01 \\
0.261 E-01 \\
0.832 E-01\end{array}$ & $\begin{array}{l}0.659 E-02 \\
0.129 E \quad 00 \\
0.659 E-02 \\
0.129 E \quad 00\end{array}$ & $\begin{array}{l}0.140 E \\
0.387 E \\
0.140 E \\
0.387 E\end{array}$ & $\begin{array}{ll}01 \\
00 \\
01 \\
00\end{array}$ & $0.168 E-01$ \\
\hline $\begin{array}{l}0.1 \\
0.9 \\
0.9\end{array}$ & $\begin{array}{ll}00000 & 01 \\
99163 & 00 \\
86653 & 00\end{array}$ & $\begin{array}{cc}0.0 & \\
& 0 . \\
& 0 .\end{array}$ & $\begin{array}{r}\text { D } 0 \\
78990-02 \\
3004 D-02\end{array}$ & $\begin{array}{l}0.6 \\
0.6 \\
0.6\end{array}$ & $\begin{array}{l}6150580 \\
615002 D \\
6009440\end{array}$ & $\begin{array}{l}00 \\
00 \\
00\end{array}$ & $\begin{array}{r}0.0 \\
-0.12157810-02 \\
-0.37527250-02\end{array}$ \\
\hline
\end{tabular}

63 ADDITIOVAL FAST GROUP DATA CARDS

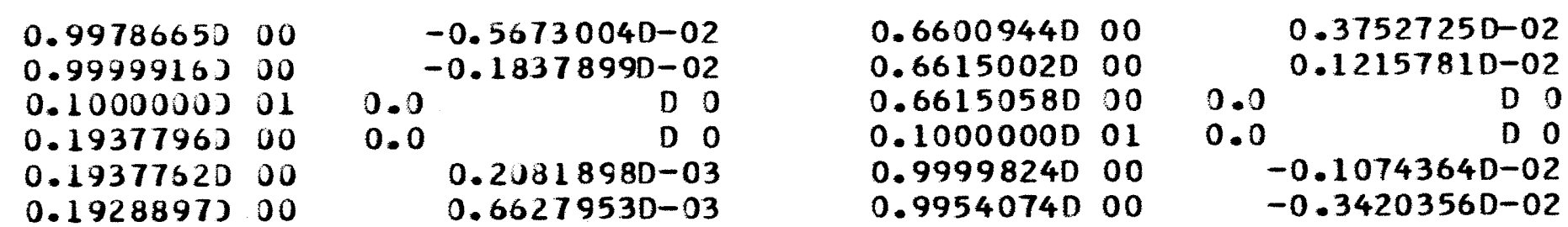

63 ADDITIOVAL' THERMAL GROJP DATA CARDS
$0.19288970 \quad 00$
$0.1937752 \mathrm{D} 00$
0.1937796000
$-0.66279530-03$
$-0.2081898 D-03$
$0.9954074 \mathrm{D} 00$
$0.9999824 \mathrm{D} \quad 00$
0.1000000001
$0.3420356 \mathrm{D}-02$ $0.1074364 \mathrm{D}-02$
0.0
D 0

PAGE 177 
SAMPLE CUBIO METHOO OUTPUT BLOCK:

$0.25031910 \quad 00$

0.4035480000

0.7785649300

0.1000000001
0.0

$-0.2202874 D-01$ $-0.27310310-01$

0.0
$0.7648905 \mathrm{D}-01$

0.1062932000

0.1642353000

0.1946404000
0.0

$0.5636188 \mathrm{D}-03$

$0.35792100-03$

0.0 
SAMPLE ANALYZE INPUT (CASE 1 CUBIC METHCDS RESULTS):

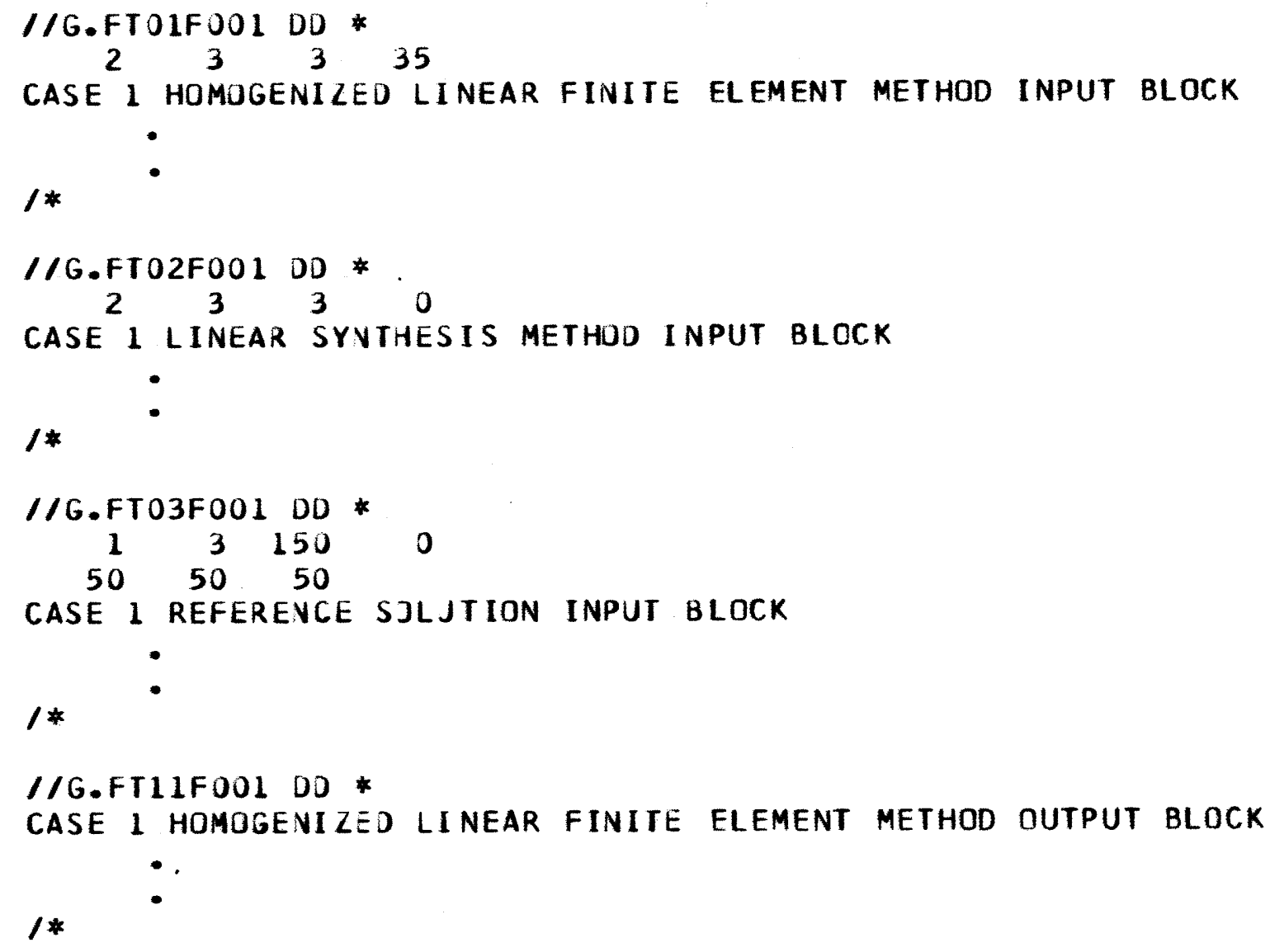




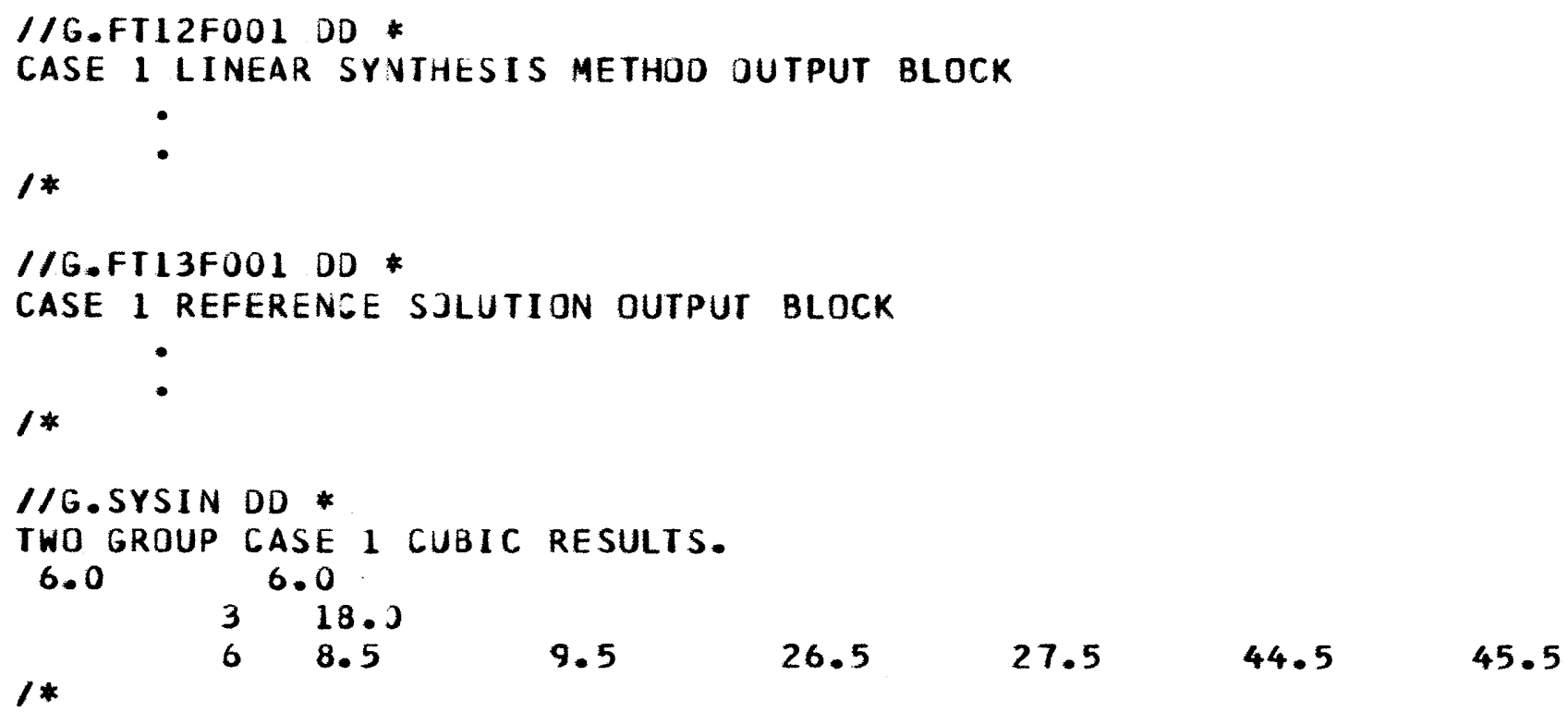




\section{ANALYZE PRINTED OUTPUT: Case 1 with Linear Basis Functions.}

RESULTS OF THE INTEGRATED POWER IN EACH OF THF 3 REGIONS:

CALCULATED POWER LEVELS, AND NUMBER OF SUBREGIONS PER REGION:

REGION :

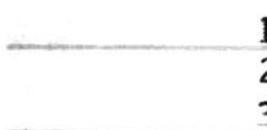

TOTALS:
HOMOGENI ZED RESULTS:

$\begin{array}{lll}1 & 0.1158776 E & 00 \\ 1 & 0.2585564 E & 00 \\ 1 & 0.4313925 E & 00 \\ 3 & 0.8058265 E & 00\end{array}$

SYNTHESIZED RESULTS:

$\begin{array}{lll}68 & 0.1086056 E & 00 \\ 68 & 0.2447225 E & 00\end{array}$

$68 \quad 0.4112111 \mathrm{~F} 00$

$2040.7645392 E \quad 00$
RFFERENCE RESULTS:

$$
\begin{array}{ccc}
50 & 0.1024175 E & 00 \\
50 & 0.2404295 E & 00 \\
50 & 0.4187305 E & 00 \\
150 & 0.7615775 E & 00
\end{array}
$$

FRACT IONAL POWER LEVELS:

REG ION :

HOMOGENEOUS RESULTS:

$$
\begin{array}{ll}
0.1437997 E & 00 \\
0.3208586 E & 00 \\
0.5353416 E & 00 \\
0.9999999 E & 00
\end{array}
$$

SYNTHESIZED RESULTS:

$0.1420537 F \quad 00$

$0.3200914 E \quad 00$

$0.537854 R E$ OO

0.9909999500
REFERENCE RESULTS:

$$
\begin{aligned}
& 0.1344807 \text { E } 00 \\
& 0.3156993 \text { F } 00 \\
& 0.5408199 \text { E OO }
\end{aligned}
$$

$0.9099999 E_{00}$

FRACTIONAL POWER NORMALIZED PERCENT ERRORS:

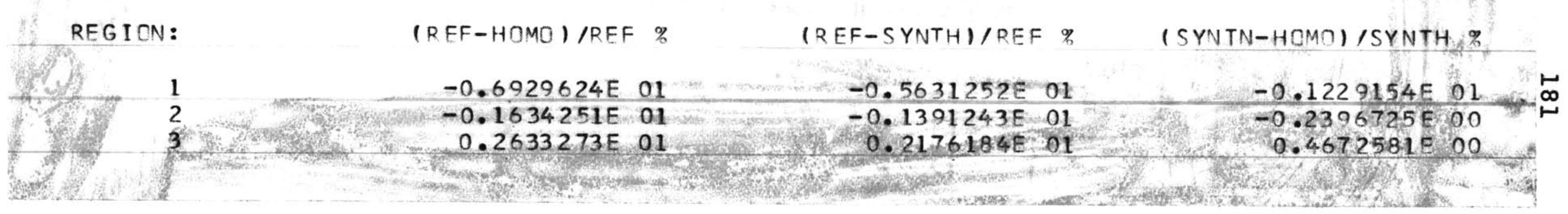


ANALYZE PRINTED OUTPUT: Case 1 with Linear Basis Functions.

EXECUTING GENERAL ANALYSIS AND FLUX PLITT ING PROGRAM:

TITLE OF PLOTTING RUN IS: I THREF DIFFERENT SUBASSFMBLYS PROBLEM. I

REACTOR GEOMETRY PARANETERS:

NCELL $=3$

WCELL $=18.00000$

$X M I N=0.0$

$X$ MAX $=54.00000$

YNIN $=0.0$

YMAX $=1.00000$

NLL $=6$

$(X L(I), I=1, N L L)=$

8.50000

9.50000

26.50000

27.50000

4.4 .50000

45.50000 


\section{Appendix F \\ SOURCE LISTINGS OF THE PROGRAMS}

FORTRAN source listings of programs REF 2G, LINEAR, CUBIC, and ANALYZE are listed in only the first six copies of this report in the following four sections.

A figure of a subroutine overlay structure precedes each listing in order to indicate the construction of each program. 
F.1. SOURCE LISTING of Program REF2G 


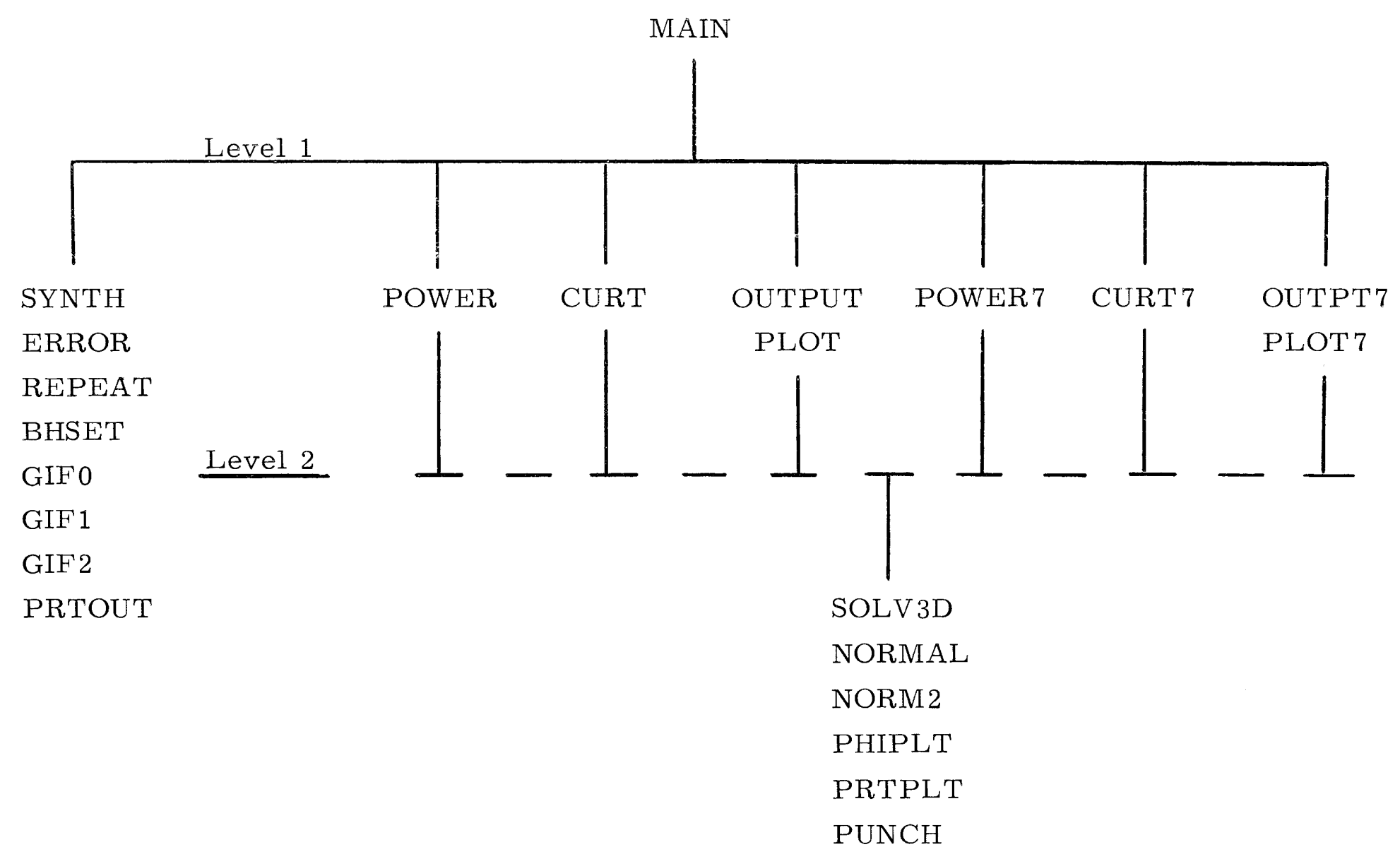

Figure F.1. Structure of Program REF2G. 
C PROGRAM REF2G:

REF 20001

REF 20002

REF 20003

REF 20004

CALL TIMING (I1)

CALL SYNTH

CALL TIMING (I2)

CALL PUWER

CALL TIMING (I3)

CALL CURT

CALL TIMINO (I4)

CALL OJTPUT

CALL TIMING (I5)

CALL PUWER7

CALL TIMING(I6)

CALL CURT7

CALL TIMING (17)

CALL OUTPI 7

CALL TIMINO (I 8)

C

$$
\text { TIMING EXECUTION }
$$

$$
\text { WRITE }(6,30)
$$

30 FORMAT (IHI, 'TIMING PROGRAM EXECUTION:,$/)$

$J=I 2-I I$

WRITE $(6,701) \mathrm{J}$

$J=13-12$

WRITE $(6,702) \mathrm{J}$

$J=I 4-I 3$

WRITE $(6,703) \mathrm{J}$

$J=I 5-I 4$

WRITE $(6,704) \mathrm{J}$

$J=16-15$

WRITE $(6,705) \mathrm{J}$

$J=I 7-16$

WRITE $(0,706) \mathrm{J}$

$J=[8-I]$

WRITE $(6,707) \mathrm{J}$

701 FORMAT (1H, SYNTH HAS TAKEN, I6," $/ 100$ SECONDS, $"$ )

REF20005

REF 20006

REF 20007

REF 20008

REF20009

REF20010

REF20011

REF 20012

REF 20013

REF 20014

REF 20015

REF 20016

REF 20017

REF 20018

REF20019

REF 20020

REF20021

REF 20022

REF 20023

REF20024

REF 20025

REF20026

REF20027

REF 20028

REF20029

REF20030

REF 20031

REF 20032

REF 20033

REF 20034

REF 20035

REF 20036

PAGE 186 
702 FORMAT (1H, ' POWER HAS TAKEN',16,"/100 SECONDS $\left.{ }^{\circ}\right)$

REF20037

REF 20038

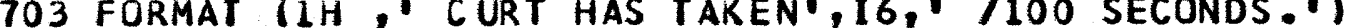

704 FORMAT (IH," OUTPUT HAS TAKEN $, 15, " / 100$ SECONDS.')

REF20039

705 FORMAT $11 \mathrm{H}$, POWER7 HAS TAKEN',16, 1100 SECONDS.'

706 FORMAT (11," CURT7 HAS TAKEN',16,'/100 SECONDS.')

707 FORMAT (1H,' JUTPUTT HAS TAKEN",I5," 1100 SECONDS.')

CALL TIMING $(120)$

$J=I 20-I 1$

WRITE $(6,720) \mathrm{J}$

720 FORMAT (LHO," THIS RUN HAS TAKEN $, 16, " 1100$ SECONDS TO RUN.') STOP

END

REF 20041

REF20042

REF 20043

REF20044

REF 20045

REF20046

REF20047

REF20048 
SUBRUUT INE SYNTH LINEAR FINITE ELEMENT METHOD:

SYNTO0O1

SYNT 0002

C * N

C ADJOINT QUANTITIES OF VARIBLES ARE DENOTED BY 7 RATHER THAN *.

SYNT 0003

SYNT 0004 THUS: PHIT (RATHER THAN PHI*) IS THE ADJOINT OF PHI. ETC.

SYNTO005

SYNTO006 IMPLICIT REAL*B (A-H,K-L)

COMMON /BI/ IBC, I PLOT, JPLOT, I PUNCH, ISEE, NOADJ

COMMON /B2/ KR, NN

COMMON /B3/ L1 $(201,3), L 2(201,3), F 1(201,3), F 2(201,3), T(201,3)$

COMMON /B5/ KAO $(2,200), \operatorname{KAL}(2,200), \operatorname{KA} 2(2,200), \operatorname{KBO}(2,200)$,

$X \quad K B 1(2,200), K B 2(2,200), L A O(2,200), \operatorname{LA} 1(2,200), L A 2(2,200)$,

$x \quad S R O(2,200), S R 1(2,20)), S R 2(2,200), P(2,200), P 1(2,200)$,

$X \quad Q(2,200), Q 1(2,200), R(2,200), P O(2,200), P 07(2,200), P H(2,200)$,

$X \quad P H 7(2,200), A L(2,200), B L(2,200), C L(2,200), A F(2,200), B F(2,200)$, $X \quad C F(2,200), A T(200), B T(200), C T(200)$,

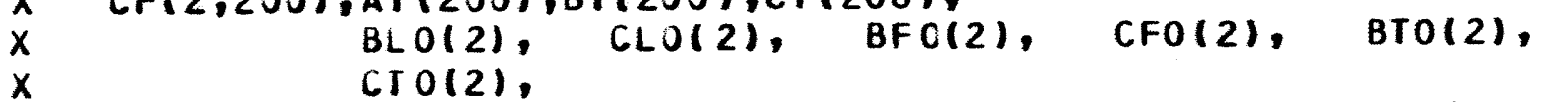

$x \quad A L K(2) ; \quad B L K(2), A F K(2), B F K(2), A T K(2)$,

$x$

COMMON /B7/ HH(200),DD(2,200)

COMMON /:HIF/ CHI(2)

COMMON / BH/ $X(2), H(1)$

COMMON /ER/ EPS1,EPS2,EPS3

DIMENSIOV PAI $(2,2), \operatorname{PHI} 7(2,2), \operatorname{CUR}(2,2), \operatorname{CUR} 7(2,2)$,

$\mathrm{X}$

$A(2,1), F(2,1), D(2,1), S(2,1), D I(2,1), X U(2,2)$

DIMENSION ITF $(200), \operatorname{KTF}(200)$

REAL TITLE(20)

INTEGER KR,K,KS,KS1,KRO, NN

INTEGER NUMITF, KTF, NOADJ

READ $(5,200)$ TITLE

200 FORMAT (20A4)

WR ITE $(6,201)$ TITLE

C

201 FORMAT $(1 \mathrm{H} 1,20 A 4, / /)$

C

READ IN THE NUMBER OF REGION TRIAL FUNCTIONS AND TYPE OF B.C.S.

AS WELL AS THE TOLERANCES AND THE OUTPUT TYPES DESIRED.

READ $(5,1) K R, I B C, E P S 1, E P S 2$, EPS 3, I PLOT, JPLOT, I PUNCH, I SEE, NOADJ

SYNT 0007

SYNT 0008

SYNT 0009

SYNT 0010

SYNT 0011

SYNTO012

SYNT 0013

SYNT 0014

SYNTO015

SYNT 0016

SYNTO017

SYNT0018

SYNT 0019

SYNT 0020

SYNT 0021

SYNT 0022

SYNT 0023

SYNT0024

SYNT 0025

SYNT0026

SYNT 0027

SYNT 0028

SYNT 0029

SYNTO030

SYNT 0031

SYNT 0032

SYNT 0033

SYNT 0034

SYNT 0035

SYNT0036

PAGE 188 
2 FORMAT ('OVARIATIONAL SYNTHESIS PROGRAM \#2G(200): ,5X, 'USING ', 13 , $X$ - SUBREACTJTR REgIONS, OR TRIAL FUNCTIONS.",

$X$ 'OBUUNBry CONDITION NUMBER (IBC) IS $, 11, \cdots, 1 /$,

$X$ - OMATERIAL PROPERTIES AND TRIAL FUNCTIONS FOR EACH SUBREgION FO XLLOW: $\bullet, /$,

$X$ - UMATERIAL PROPERTIES ARE hOMOGENEOUS IN THE INDICATED REgIONS. $x \cdot, 1$,

$x$ OFLUX TRIAL FUNCTIONS ARE LINEAR IN EACH SEgMENT OF THE SUBREg

SYNT 0040

SYNT0041

SYNT0042

SYNT 0043

SYNT 0044

SYNT 0045

SYNT 0046

SYNT 0047

SYNT 0048

SYNT 0049

SYNT 0050

SYNT 0051

SYNT 0052

SYNT 0053

SYNT 0054

XIONS., 1,

$x$ ocurrent trial functions are flat in each of the,

$X$ 'SUBREGIUNS.'I

WRI TE 16,201 EP S1, EPS 2, EPS 3 , I PLCT, JPLOT, I PUNCH, ISEE, NOADJ

20 FORMAT $1 / 1, \circ$ OTJLERANCES TO POWER ARE : EPS $1=1,1$ PDI $10.3,1$,

$x \quad 28 X,{ }^{\circ}$ EPS $2=1,1$ PDI $0.3,1,28 X, \cdot$ EPS $3=1,1$ PDI $0.3,1$,

$x$ OOUTPUT PARAMETERS TO POWER ARE: IPLOT $=1,11,1$,

$X \quad 34 X, \cdot J P L J T=1, I 1,1,34 X, 1$ PUNCH $=1,11,1$,

$X \quad 34 X, \cdot I$ SEE $=1,11,1$,

$X \quad 34 X, \cdot$ NOADJ $=1,11,1.1)$

WRITE $(6,22) \mathrm{CHI}(1), \mathrm{CHI}(2)$

22 FORMAT $(1$, OFISSION YIELOS ARE: CHI(1)= , F10.5, , $X \quad 22 X, \cdot C H I(2)=\bullet, F 10.5)$

IF ((KR.LE.2).AND. (IBC.EQ.1) CALL ERROR(1,KR)

IF (KR.GT.200) CALL ERROR (2,KR)

IF IEPS1.LT.1.0E-16) CALL ERROR $(6,1)$

IF (EPS2.LT.1.0E-16) CALL ERROR $(6.2)$

IF (EPS3.LT.1.0E-16) CALL ERROR(6,3)

IF ( IBC.LT.1).OR. (IBC.GT.4)) CALL ERROR(7, IBC)

SYNT 0055

SYNT 0056

SYNT 0057

SYNT 0058

SYNT0059

SYNT0060

SYNTO061

SYNT0062

SYNT 0063

SYNT0064

SYNT0065

SYNT 0066

SYNT0067

SYNT 0068

SYNT 0069

SYNT C070

SYNT 0071

SYNTO072

PAGE 189 
c

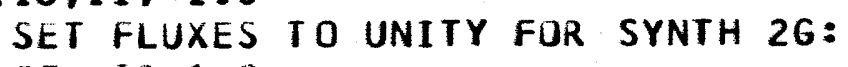

C

25 PHIT(IG,II) $=1.0$ NUMI TF $=1$

COUNTER OF THE NUMBER OF TYPE-NUMBERS OF EACH TF REGION:

WRITE $(6,9)$

9 FORMAT $(\cdot 1 \cdot)$

C BEGIN TJ READ IN THE TF REGION DATA AND FILL THE ARRAYS,

C DEPENDIVG JN THE TYPE-NUMBER OF EACH TF REGION. DO $50 \quad I=1, K R$ $J=I \operatorname{IF}(I)$ 
IF (ISEE,EQ.O) GO TO 14

WRITE $(6,10) \quad K, K R, K S,(J, X(J), X(J+1), H(J), A(1, J), F(1, J), D(1, J)$,

$X \quad S(1, J), A(2, J), F(2, J), D(2, J), J=1, K S)$

10 FORMAT 1 OINPUT MATERIAL PROPERTIES FOR SUBREGION NUMBER, 13,

$X$ ' OF THE ', I3,' USED.', //,

$X$ $5 X, \cdot$ THIS SUBREGION IS DIVIDED INTO $, 13, \cdot$ HOMOGENEOUS SEGMENTS

XAS FOLLONS:, $1 /$,

$X$ 5X, 'FAST GROUP CONSTANTS APPEAR FIRST: $1,1 /$,

$X$ - REGION \#",5X, 'INTERNAL BOUNDARIES $, 13 X, 1 H I D T H^{\circ}, 3 X$,

$X$ 'ABSORB. CX $(1 / C M) \cdot, 3 X,{ }^{\circ} F I S S I O N$ CX $(1 / C M) \cdot, 6 X,{ }^{\circ}$ IFFUSION (CM)',

$X \quad 4 X, \cdot S C A T T . C X(1 / C M) \cdot, /$,

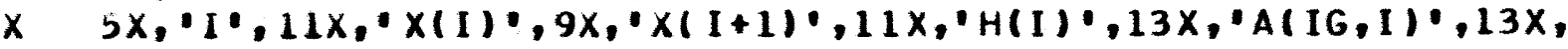

$x \quad f(1 G, I) \cdot, 13 x, 0(I G, I) \cdot, 14 x, \cdot S(1, I) \cdot, 11$,

C

14 CONT INUE

$(16,3 F 15.4,4020.8,1,51 X, 3020.8))$

END OF IHE IN-OUT SECTION.

C

C

DEFINING MISC. ARRAYS FOR THE INTEGRATION FUNCTIONS:

LEGNTH JF THE SUBREGION: HT

$H T=X(K S 1)-X(1)$

$H H(K)=H T$

$\mathrm{DD}(1, K)=\mathrm{D}(1,1)$

$D D(2, K)=D(2,1)$

INVERSE OF D ARRAYS:

DO $13 \mathrm{~J}=1, \mathrm{KS}$

$D I(1, J)=1, / D(1, J)$

$13 D I(2, J)=1 . / D(2, J)$

FORMATION OF THE INTEGRATION FUNCTIONS:

CALL BHSETIKS)

DO FOR ALL ENERGY GROUPS:

DO $50 \quad$ IS $=1,2$

$K A O(I G, K)=G I F O(I G, P H I 7, P H I, A, K S)$

$K A 1(I G, K)=G I F I(I G, P H I 7, P H I, A, K S)$

$K A 2(I G, K)=G I F 2(I G, P H I 7, P H I, A, K S)$

$K B O(I G, K)=G I F O(I G, P H I 7, P H I, F, K S)$

$K B I(I G, K)=G I F 1(I G, P H I 7, P H I, F, K S)$

$K B 2(I G, K)=\sigma I F 2(I G, P H I 7, P H I, F, K S)$
SYNTO 109

SYNTO110

SYNTO111

SYNTO112

SYNTO113

SYNT 0114

SYNTO115

SYNT 0116

SYNTO117

SYNTO118

SYNTO119

SYNT 0120

SYNT 0121

SYNT 0122

SYNT 0123

SYNT 0124

SYNTO 125

SYNT 0126

SYNTO127

SYNT0128

SYNT 0129

SYNTO130

SYNT 0131

SYNT 0132

SYNTO133

SYNTO134

SYNT0135

SYNT0136

SYNT 0137

SYNTO138

SYNTO 139

SYNT 0140

SYNTO141

SYNTO142

SYNTO143

SYNTO144

PAGE 191 
50 CONTINUE

NUMI TF $=$ NUMI TF -1

WRITE $(6,51)$ NUMITF

51 FORMAT ("ITHERE ARE ONLY', I3," DIFFERENT TRIAL FUNCTICN REgIONS.") WRITE $(6,52)$ (I, I TF $(I), I=1, K R)$

52 FORMAT $1 \%$, OTABLE OF THE TRIAL FUNCTION REGION TYPES: $1, / /$,

$X \quad 3 X, \cdot$ IF REGION',4X,'REGION TYPE-NUMBER',//,

$X \quad(17,12 X, 17))$

C DETERMINATION OF THE B.C. OPTION PARAMETERS: NN IS THE MM AND FF MATRIX BLOCK SIZE.

IF (IBC.EQ.1) NN=KR-1

If $((I B C \cdot E Q \cdot 2) \cdot O R \cdot(I B C \cdot E Q \cdot 3)) \quad N N=K R$

If (IBC.EQ.4) NN=KR+1

$\mathbf{C}$ FORMATION OF THE COEFFICIENT VECTORS:

C THE INTERIOR COEFFS:

DO $60 \quad I G=1,2$

DO $60 \mathrm{~K}=2, K R$

$J=K-1$

$A L(I G, K)=K A 1(I G, J)-K A 2(I G, J)-R(I G, J)$

$B L(I G, K)=K A 2(I G, J)+R(I G, J)+K A O(I G, K)-2$.*KAI $(I G, K)+K A 2(I G, K)$

$X$

$$
+R(I G, K)
$$

$C L(I G, K)=K A I(I G, K)-K A 2(I G, K)-R(I G, K)$

$A F(I G, K)=K B 1(I G, J)-K B 2(I G, J)$

$B F(I G, K)=K B 2(I G, J)+K B O(I G, K)-2$. *KB1(IG,K)+KB2(IG,K)

$C F(I G, K)=K B 1(I G, K)-K B 2(I G, K)$

$A T(K)=S R 1(1, J)-\operatorname{SR} 2(1, J)$

$\operatorname{BT}(K)=\operatorname{SR} 2(1, J)+\operatorname{SRO}(1, K)-2 . * \operatorname{SR} 1(1, K)+\operatorname{SR} 2(1, K)$

$C T(K)=S R 1(1, K)-S R 2(1, K)$

60 CONIINUE

THE LERO FLUX COEFFS:

SYNT 0147

SYNTO148

SYNT 0149

SYNTO150

SYNT 0151

SYNT0152

SYNTO153

SYNT 0154

SYNT 0155

SYNTO156

SYNT0157

SYNTO158

SYNTO159

SYNT 0160

SYNT 0161

SYNTO162

SYNTO163

SYNTO164

SYNT 0165

SYNTO166

SYNT0167

SYNT 0168

SYNT 0169

SYNT 0170

SYNT 0171

SYNT 0172

SYNT 0173

SYNT 0174

SYNT 0175

SYNT0176

SYNTO177

SYNT0178

SYNTO179

SYNTO 180 
$D O 61 I G=1,2$

$B L O(I G)=K A O(I G, 1)-2 . * K A 1(I G, 1)+K A 2(I G, 1)+R(I G, 1)$

SYNTO181

$B F O(I G)=K B O(I G, 1)-2 . * K B 1(I G, 1)+K B 2(I G, 1)$

$C L O(I G)=K A 1(I G, 1)-K A 2(I G, 1)-R(I G, 1)$

$61 C F O(I G)=K B 1(I G, 1)-K B 2(I G, 1)$

$B T O(1)=\operatorname{SRO}(1,1)-2 . * \operatorname{SR} 1(1,1)+\operatorname{SR} 2(1,1)$

CTO $(1)=\operatorname{SR} 1(1,1)-\operatorname{SR} 2(1,1)$

C

THE ZERO CURRENT COEFFS: $K=K R$

$0062 \quad I G=1,2$

$A L K(I G)=K A 1(I G, K)-K A 2(I G, K)-R(I G, K)$

$B L K(I G)=K A 2(I G, K)+R(I G, K)$

$A F K(I G)=K B I(I G, K)-K B 2(I G, K)$

$62 B F K(I G)=K B 2(1 G, K)$

$A T K(1)=S R 1(1, K)-S R 2(1, K)$

$\operatorname{BTK}(1)=S R 2(1, K)$

C

ZERO MATRICES:

$L 1(1,1)=0$.

$L 2(1,1)=0$.

$F 1(1,1)=0$.

$F 2(1,1)=0$.

T $(1,1)=0$.

$L 1(N N, 3)=0$.

$L 2(N N, 3)=0$.

$F_{1}(N N, 3)=0$.

$F 2(N N, 3)=0$.

$T(N N, 3)=0$.

C

FILL ALL THE MATRICES FOR POWER: $\mathrm{J}=1$

C DETERMIVE THE LEFT BUUNDARY CONDITIONS:

IF (IBC.LT.3) GO TO 67

$L 1(J, 2)=B L O(1)$

$L 2(J, 2)=B L O(2)$

$F 1(J, 2)=B F O(1)$

$F 2(J, 2)=B F O(2)$

$T(J, 2)=B T O(1)$

SYNTO182

SYNTO183

SYNTO184

SYNTO185

SYNTO186

SYNTO187

SYNTO188

SYNT 0189

SYNTO190

SYNTO191

SYNT 0192

SYNTO193

SYNTO194

SYNT0195

SYNTO196

SYNT 0197

SYNTO198

SYNTO199

SYNT 0200

SYNT0201

SYNT0202

SYNT0203

SYNT 0204

SYNT0205

SYNT 0206

SYNT 0207

SYNT0208

SYNT0209

SYNT0210

SYNTO211

SYNT0212

SYNT 0213

SYNTO214

SYNTO215

SYNT 0216

PAGE 193 
L1 (J, 3)=CLO(1)

L2 (J, 3$)=C L O(2)$

F1 (J, 3)= GFO(1)

$F 2(J, 3)=C F O(2)$

T(J, 3$)=C T O(1)$

$J=J+1$

C

FOR ALL THE INTERIOR EQUATIONS:

67 DO $70 \quad K=2, K R$

IF $(J \cdot E U .1)$ GU TO 69

$L 1(J, 1)=A L(1, K)$

$L 2(J, \quad L)=A L(2, K)$

$F 1(J, \quad 1)=A F(1, K)$

$F 2(J, L)=A F(2, K)$

$T(J, 1)=A T(K)$

$69 L 1(J, 2)=B L(1, K)$

$L 2(J, 2)=B L(2, K)$

$F 1(J, 2)=B F(1, K)$

$F 2(J, 2)=B F(2, K)$

$T(J, 2)=B T(K)$

$L 1(J, \quad 3)=C L(1, K)$

$L 2(J, \quad 3)=C L(2, K)$

$F 1(J, \quad 3)=C F(1, K)$

$F 2(J, 3)=C F(2, K)$

$T(J, \quad 3)=C T(K)$

$J=J+1$

C

70 CONTINUE

DETERMINE THE RIGHT BUUNDARY CONDITIONS:

IF (IBC.EQ.1). OR. (IBC.EQ.3)) GO TO 80

LIIJ, 1)=ALK(1)

L2 $(J, 1)=A L K(2)$

$F 1(J, 1)=A F K(1)$

$F 2(J, 1)=A F K(2)$

T(J, 1)=ATK(1)

$L 1(J, 2)=B L K(1)$

$L 2(J, 2)=B L K(2)$

$F 1(J, 2)=B F K(1)$

SYNTO217

SYNT0218

SYNT 0219

SYNT 0220

SYNT 0221

SYNT 0222

SYNT 0223

SYNT0224

SYNT 0225

SYNT0226

SYNTO227

SYNT 0228

SYNT0229

SYNT 0230

SYNT 0231

SYNT0232

SYNT 0233

SYNTO234

SYNT 0235

SYNT 0236

SYNT 0237

SYNT 0238

SYNT 0239

SYNT 0240

SYNT 0241

SYNT 0242

SYNT 0243

SYNT0244

SYNT0245

SYNT0246

SYNT0247

SYNT 0248

SYNT 0249

SYNT0250

SYNTO251

SYNT 0252

PAGE 194 
$F 2(J, 2)=B F K(2)$

SYNT 0253

$T(J, 2)=B T K(1)$

SYNT 0254

SYNT 0255

SYNT0256

$\begin{array}{ll}C & \text { PRINTS OUT THE SYNTH K ARRAYS, AND THE MATRICES GIVEN TO POWER } \\ C & \text { FOR ISEE }=2 .\end{array}$

IF (ISEE.EQ.2) CALL PRTOUT

RETURN

SYNT 0257

SYNTO258

SYNT 0259

END

SYNT 0260 


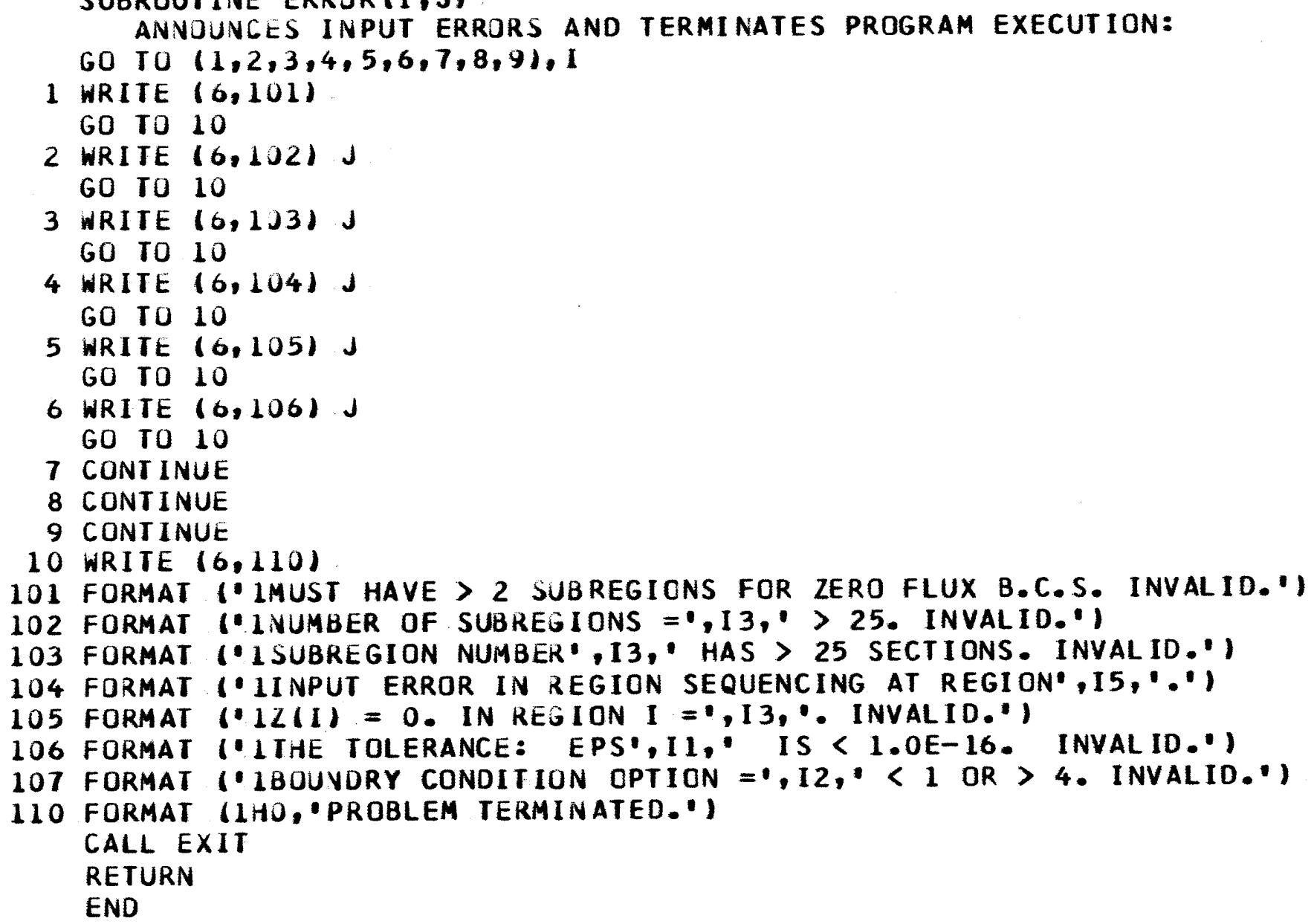

ERRO0001

ERRO0002

ERR00003

ERRO0004

ERR00005

ERR00006

ERR00007

ERR00008

ERRO0009

ERRO0010

ERRO0011

ERRO0012

ERROO013

ERRO0014

ERRO0015

ERRO0016

ERRO0017

ERRO0018

ERRO0019

ERRO0020

ERRO0021

ERRO0022

ERRO0023

ERRO0024

ERRO0025

ERRO0026

ERRO0027

ERRO0028

ERR00029

ERRO0030 
SUBROUTINE REPEAT $(K, L)$

C SETS THE /BS/ ARRAYS (K) EQUAL TO PAST STORED ARRAYS (L):

REPEO001 IMPLICIT REAL*8 (A-Z)

COMMON $/ B 5 / K A O(2,200), K A 1(2,200), K A 2(2,200), K B O(2,200)$,

REPE0002

REPEO003

REPE0004

REPE0005

REPE0006

REPE0007

REPE0008

REPE0009

REPE0010

REPEO011

REPE0012

REPEO013

REPE0014

REPE0015

REPE0016

REPE0017

REPE0018

REPE0019

REPE0020

REPE0021

REPE 0022

REPE0023

REPE 0024

REPE 0025

REPE0026

REPE0027

REPE0028

REPE0029

REPE0030

REPE0031

REPE0032

REPE0033

REPE0034

REPE 0035

REPE0036

10 CONTINUE

$H H(K)=H H(L)$ 
$\operatorname{DD}(1, K)=0 D(1, L)$

$D D(2, K)=D D(2, L)$

RETURN

REPE0037

REPE0038

REPE0039

END

REPE 0040 
FIRST OF 4 ANALYTICAL INTEGRATICN RCUTINES. IMPL ICIT REAL $* 8 \quad(A-H, L-L)$

COMMON /BH/ $X(2), H(1), H 2(L), H 3(1), H 4(1), H 5(1)$

DO $1, I=1, K$

$H 2(I)=X(I+1) * * 2-X(I) * * 2$

$H 3(I)=X(I+1) * * 3-X(I) * * 3$

$H 4(I)=X(I+1) * * 4-X(I) * * 4$

$H 5(I)=X(I+1) * * 5-X(I) * * 5$

1 CONTINUE

RETURN

END

BHSEOOO1

BHSE 0002

BHSEOOO3

BHSE0004

BHSE0005

BHSE0006

BHSE0007

BHSE0008

BHSEOOOO

BHSE0010

BHSE0011

BHSE0012 
DOUBLE PRECISION FUNCTION GIFO(IG,Y,Z,C,K)

GIF00001

IMPLICIJ REAL $* 8$ (A-H,L-Z)

COMMON /BH/ X(2),H(1),H2(1),H3(1),H4(1),H5(1)

GIF00002

GIF00003

DIMENSION $Y(2,2), Z(2,2), C(2,1)$

SUM $=0.0$

DO $1 \quad I=1, K$

SUM $=C(I G, I) *(Y(I G, I) * Z(I G, I) * H(I)+H(I) *(Z(I G, I) *$

$X \quad(Y(I G, I+1)-Y(I G, I))+Y(I G, I) *(Z(I G, I+1)-Z(I G, I))) / 2$.

$X+H(I) *(Y(I G, I+I)-Y(I G, I)) *(Z(I G, I+I)-Z(I G, I)) / 3)$.+ SUM

1 CONT INUE

GIFO = SUM

RETURN

END

GIF00004

GIF00005

GIF00006

GIF00007

GIF00008

GIF00009

GIF00010

GIF00011

GIF00012

GI F00013 
DOUBLE PRECISION FUNCTION GIFI(IG,Y,Z,C,K)

GIF 10001

IMPLICIT REAL *8 (A-H,L-Z)

COMMON /BH/ X(2), H(1),H2(1), H3(1),H4(1), H5(1)

GIF10002

DIMENSION $Y(2,2), Z(2,2), C(2,1)$

SUM $=0.0$

DO $1 \quad I=1, K$

SUM $=C(I G, I) *((H 2(I) / 2,-X(1) * H(I)) * Y(I G, I) * Z(I G, I)$

$X \quad+(Z(I G, I) *(Y(I G, I+I)-Y(I G, I))+Y(I G, I) *$

$X \quad(Z(I G, I+I)-Z(I G, I)) *(1, I H(I)) *(H 3(I) / 3,-H 2(I) *(X(I)+X(1)) / 2$.

$+X(I) * X(1) * H(I))+(Y(I G, I+1)-Y(I G, I) *(Z(I G, I+1)-Z(I G, I))$

$*\left(H 4(I) / 4 \cdot-H 3(I) *(2, * X(I)+X(1)) / 3_{\bullet}+H 2(I) *(X(I) * X(I)+2 * * X(I)\right.$

$* X(1)) / 2 .-X(1) * X(I) * X(I) * H(I)) /(H(I) * H(I)))+$ SUM

1 CONTINUE

GIFI $=$ SUM $/(X(K+1)-x(1))$

RETURN

END

GIF 10003

GIF 10004

GIF10005

GIF 10006

GIFI 0007

GIF10008

GIF10009

GIF 10010

GIF10011

GIF 10012

GIF10013

GIF 10014

GIF10015

GIF10016 
DOUBLE PRECISION FUNCTION GIF $2(I G, Y, Z, C, K)$

IMPL ICI T REAL $* 8 \quad(A-H, L-Z)$

COMMON /BH/ X(2),H(1),H2(1), H3(1), H4(1), H5 (1)

DIMENSION $Y(2,2), Z(2,2), C(2,1)$

SUM $=0.0$

DO $1 \quad I=1, K$

SUM $=C(I G, I) *(Y(I G, I) * Z(I G, I) *(H 3(I) / 3,-X(1) * H 2(I)+X(1) * X(1) * H(I))$

$X+(1, I H(I)) *(Z(I G, I) *(Y(I G, I+I)-Y(I G, I))+Y(I G, I) *(Z(I G, I+1)$

$X \quad-Z(I G, I))) *(H 4(I) / 4 .-H 3(I) *(2 . * X(1)+X(I)) / 3 .+H 2(I) *(X(1) * X(1)$

$x+2 * * x(1) * x(I)) / 2 .-X(1) * X(1) * X(I) * H(I))+(1 . /(H(I) * * 2))$

$X \quad *(Y(I G, I+I)-Y(I G, I)) *(L(I G, I+I)-Z(I G, I) *(H 5(I) / 5$.

$X \quad-H 4(I) *(X(1)+X(I)) / 2 *+H 3(I) *(X(1) * X(1)+4 * * X(1) * X(I)+X(I) * X(I))$

$X \quad 13 .-H 2(I) *(X(1) * X(I) * X(I)+X(1) * X(1) * X(I))$

$X+X(1) * X(1) * X(1) * X(1) * H(1))+$ SUM

1 CONT INUE

GIF2 $=$ SUM $/((X(K+1)-X(1)) * * 2)$

RETURN

END

GIF20001

GIF 20002

GIF 20003

GIF 20004

GIF 20005

GIF20006

GIF 20007

GIF 20008

GIF20009

GIF 20010

GIF 20011

GI F20012

GIF20013

GIF20014

GIF20015

GIF20016

GI F 20017

GIF2001 8 
C SUBROUTINE PRTUUT

PRINTS UUT THE /B5/ ARRAYS AND THE MATRICES GIVEN TO POWER:

I MPLICI T REAL*8 (A-H,K-Z)

COMMON /B2/ KR, N

COMMUN/B3/LI(201,3), L2(201,3), F $1(201,3), F 2(201,3), T(201,3)$

COMMON /B5/ KAO $(2,200), K A 1(2,200), K A 2(2,200), K B O(2,200)$,

$X \quad K B 1(2,200), K B 2(2,200), L A O(2,200), L A 1(2,200), L A 2(2,200)$,

$X \quad S R O(2,200), S R 1(2,200), S R 2(2,200), P(2,200), P 1(2,200)$,

$\mathrm{X} \quad \mathrm{Q}(2,200), Q 1(2,200), R(2,200), \mathrm{PO}(2,200), \mathrm{PO}(2,200), \mathrm{PH}(2,200)$,

$X \quad P H 7(2,200)$

C

INTEGER KR, G, N

KA AND KB ARRAYS:

WRITE $(6,10)$

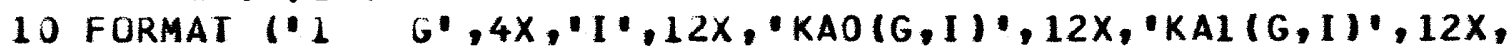

$X \quad K A 2(G, I), 12 X, \cdot K B O(G, I) \cdot, 12 X, \cdot K B 1(G, I), 12 X, \cdot K B 2(G, I) \cdot)$

DO $11 G=1,2$

WR ITE $(6,12)$

11 WRITE $(6,15)(6, I, K A O(G, I), K A 1(G, I), K A 2(G, I), K B O(G, I), K B 1(G, I)$, $X$ $K B 2(G, I), I=1, K R)$

12 FURMAT (')

15 FORMAT (2I5,6020.7)

C

LA AND SR ARRAYS: WRITE $(6,20)$

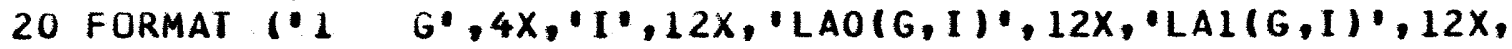

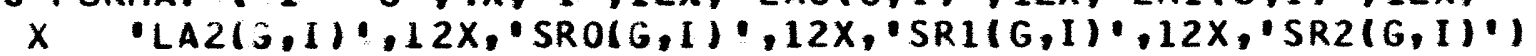
$G=1$ WRITE $(6,12)$

WRITE $(6,15)(0, I, L A O(G, I), L A 1(G, I), L A 2(G, I), S R O(G, I), S R 1(G, I)$, $X$ $G=2$ $S R 2(G, I), I=I, K R)$

\section{WR ITE $(6,12)$}

WRITE $(6,25),(6, I, L A O(G, I), L A 1(G, I), L A 2(G, I), I=1, K R)$

C

25 FURMAT $(215,3020.7)$

P, Q. AND R ARRAYS:

WR I TE $(6,30)$

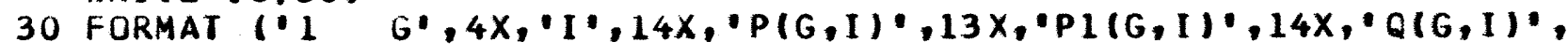

PRTO0001

PRT00002

PRT00003

PRTO0004

PRT00005

PRT00006

PRTO0007

PRTO0008

PRT00009

PRTO0010

PRTO0011

PRTO0012

PRT00013

PRTO0014

PRTO0015

PRTO0016

PRTO0017

PRTO0018

PRTO0019

PRTO0020

PRTO0021

PRT00022

PRTO0023

PR TO0024

PRTO0025

PRT00026

PR TO0027

PRTO0028

PRT00029

PRT00030

PRT00031

PRTO0032

PRTO0033

PRT00034

PRTO0035

PRTO0036

PAGE 203 
$X \quad 13 X, \cdot Q 1(G, I) \cdot, 14 X, \cdot R(G, I) \cdot 1)$

PRT00037 DD $31 G=1,2$

WRITE $(6,12)$

31 WRITE $(6,35)(0, I, P(G, I), P I(G, I), Q(G, I), Q I(G, I), R(G, I), I=1, K R)$

35 FORMAT (2I5,5020.7)

C PO AND PH ARRAYS: WRITE $(6,40)$

40 FORMAT (' 1 G',4X,'I',13X, PPO(G,I)',12X,'POT(G,I)',13X,'PH(G,I)', $X \quad 12 X, \cdot P H 7(G, I) \cdot)$

DO $41 G=1,2$

WRITE $(6,12)$

41 WRITE $(6,45)(6, I, P O(G, I), P O 7(G, I), P H(G, I), P H 7(G, I), I=1, K R)$

45 FORMAT (2I5,4020.7)

PRINT OUT THE /B3/ MATRICES:

WRITE $(6,50)$

50 FORMAT ('IMATRIX LI: ',/)

WRITE $(6,55)((L 1(I, J), J=1,3), I=1, N)$

55 FORMAT (3E12.3,7X,3E12,3,7X,3E12.3)

WRITE $(6,60)$

60 FORMAT $($ LMATRIX L2:,$/ 1)$

WRITE $(6,55)$ ( $(L 2(I, J), J=1,3), I=1, N)$

WRITE $(6,70)$

70 FORMAT ('IMATRIX F1:,$/ 1)$

WRITE $(6,55) \quad((F 1(I, J), J=1,3), I=1, N)$

WRITE $(6,80)$

80 FORMAT ('IMATRIX F2:,,$/)$

WRITE $(6,55) \quad((F 2(I, J), J=1,3), I=1, N)$

WRITE $(6,90)$

90 FORMAT ('IMATRIX $T:=, /)$

WRITE $(6,55)$ ( $T(I, J), J=1,3), I=1, N)$

RETURN

END

PRT00038

PRT00039

PRT00040

PRT00041

PRTO0042

PRT T00043

PRT00044

PRT00045

PRTO0046

PRT00047

PRT00048

PRTO0049

PRT T00050

PRTO0051

PRT00052

PR TO0053

PRT00054

PRTO0055

PRT00056

PRTO0057

PRTO0058

PRTO0059

PRTO0060

PRTO0061

PRTO0062

PRT 00063

PRTO0064

PRTO0065

PR T00066

PRT00067

PRT00068 
SUBROUTINE POWER $\begin{array}{ll}C & \text { SOLVES THE } 2 * N \text { MULTIGROUP EQUATICNS: } M * P H I=(1 / L A M D A) * F * P H I \\ C & \text { BY THE FISSION SOURCE POWER METHOD }\end{array}$

USING SIMULTANEOUS OVERRELAXATION.

WHERE: M AND F ARE DUUBLE PRECISION 2N BY 2N BLOCK MATRICES;

AND: PHI IS THE 2N FLUX (FAST AND THERMAL) VECTCR.

$L 1 * P H I 1=C H I 1 *(F 1 * P H I 1+F 2 * P H I 2)$

$-T * P H I 1+L 2 * P H I 2=$ CHI $2 *(F 1 * P H I 1+F 2 * P H I 2)$

METHOD FOLLOWS WACHPRESS, PAGE 83. SOLUTION BY GROUP ITERATION. IMPLICIT REAL $* 8$ (A-H,L-Z)

COMMUN /BI/ IBC, IPLOT, JPLOT, I PUNCH, ISEE

COMMON $/ B 2 / K R, N$

COMMON /B3/L1(201,3), L2(201,3), F1 $(201,3), F 2(201,3), T(201,3)$

COMMON /B4/ PHI $(2,201)$, PSI $(2,201)$, LAMDA, ICOUT

COMMON /B5/ S(201), ERROR $(2,201), Z(201)$

COMMUN /B6/ TE1 $(2,5), T E 2(2,5), T E 3(5)$, IN (5)

COMMON /B7/ HH( 200)

COMMON /CHIF/ CHI (2)

COMMON /ER/ EPS1, EPS2,EPS3

COMMON /T/ I 1,14

COMMON /FSTR/ PHISTR $(2,201,6)$

COMMON /ESTR/ LAMSTR(300), EFSTR(2,300), EFMSTR(2,300), ERLAM(300)

COMMON /READS/ R5

DIMENSION PSI $1(201), \operatorname{PS} I 2(201), \operatorname{SQ}(2), \operatorname{DPHI}(2), \operatorname{ERRMAX}(2)$

INTEGER N

C

R5 $=1$.

DEFAULT OPTIONS FOR POWER PARAMETERS:

$A L P H A=1.25$

LAMDA $=1.0$

$H X=0.0$

DO $505 \quad I=1, K R$

$505 H X=H X+H H(1)$

DO 555 I $G=1,2$

IF (IBC.NE.4) GO TO 551

DO $550 \quad I=1, N$

$550 \operatorname{PHI}(I G, I)=1.0$

POW 0001

POW 0002

POW 0003

POW 0004

POW 0005

POW 0006

POW 0007

POW 0008

POH 0009

POW 0010

POW 0011

POW 0012

POW 0013

POW 0014

POW 0015

POW 0016

POW 0017

POW 0018

POW 0019

POW 0020

POW 0021

POW 0022

POW 0023

POW 0024

POW 0025

POW 0026

POW 0027

POW 0028

POW 0029

POW 0030

POW 0031

POW 0032

POW 0033

POW 0034

POW 0035

POW 0036

PAGE 205 
GO TO 555

$551 X=3.1415926 / H X$

IF ( IBC.NE.1) $X=X / 2.0$

SUM $1=0.0$

DO $552 K=1, K R$

SUM $1=S U M 1+H H(K)$

552 PHI $(I G, K)=$ DSIN $($ SUM $1 * X)$

555 CONT I NUE

C

c READ IN:

OVERRELAXATION PARAMETERS

INITIAL GUESS AT EIGENVALUE;

INITI AL NORMAL IZED FLUX

READ $(5,506, E N D=510)$ ALPHA

READ $(5,502$, END $=510)$ LAMDA

READ $(5,503) \quad(P H I(1, I), I=I, N)$

READ $(5,503)$ (PHI $(2, I), I=1, N)$

506 FORMAT (F 10.5)

502 FORMAT (E25.14)

503 FORMAT $((4 E 20.10))$

GO TO 511

510 R5 $=0$.

511 CONTINUE

C

STORINJ FOR PRINTING THE MULTIGRCUP FLUX SHAPE.

DO $11 \quad I G=1,2$

DO $10 . I=1, N$

10 PHISTRI $16,1,2)=\operatorname{PHI}(I G, I)$

C

FILL RUNNING COORD IN PHISTR

$K R 1=K R+1$

DO $11 I=1, K R 1$

$11 \operatorname{PHISTR}(I G, 1,1)=$ DFLOAT (I)

C I $K=1$

IK IS THE FLUX PLOTTING CCUNTER.

C

STORES THE ITERATION NUMBER FOR FLUX HISTORY PLOTTING: IN $(1)=0$

C
STORES TEMPJRARY ERRORS FOR FLUX HI STORY PLOTTING:

TE $1(1,1)=0$.

$\operatorname{TE} 1(2,1)=0$.
POW 0037

POW 0038

POW 0039

POW 0040

POW 0041

POW 0042

POW 0043

POW 0044

POW 0045

POW 0046

POH 0047

POW 0048

POW 0049

POW 0050

POW 0051

POW 0052

POW 0053

POW 0054

POW 0055

POW 0056

POW 0057

POW 0058

POW 0059

POW 0060

POW 0061

POW 0062

POW 0063

POW 0064

POW 0065

POW 0066

POW 0067

POW 0068

POW 0069

POW 0070

POW 0071

POW 0072

PAGE 206 
$\operatorname{TE} 2(1,1)=0$.

$\operatorname{TE} 2(2,1)=0$.

TE3 $(1)=0.0$

C EIGENVALUE OF THE PREVIOUS ITERATION: LAMB $4=\angle A M D A$

C THE MAXIMUM NUMBER OF ALLOWED ITERATIONS: ICMAX ICMAX $=300$

C PRINT OUT THE POHER METHOD PARAMETER INFORMATION:

WRITE $(6,700)$ ICMAX, ALPHA,LAMDA, (PHI I I I),I=1,N)

WRITE $(6,701)$ ( $\operatorname{PHI}(2, I), I=1, N)$

700 FORMAT 'IEXECUTING MULTIOROUP FISSION SOURCE POWER ITERATION METH XOD., $1 / 1$,

$X$ 5X, MAXIMUM NUMBER OF ALLOWABLE ITERATIONS:,$/$

$X \quad 10 X, 1$ ICMAX $=\bullet, 14,1 / 1$.

$X$ 5X, OUTER ITERATION RELAXATICN PARAMETER:, 1,

$X \quad 10 X, \cdot A L P H A=\bullet, F 7,3,1 \%$

$x$ 5X, INITIAL GUESS AT EIGENVALUE:, 1,

$X \quad 10 X$, 'LAMBDA $=\circ, E 22.14,1 \%$,

$X$ $5 X$, INITIAL GUESS AT THE GROUP FLUX SHAPE CONNECTION POINTS:

$X \quad 1 /, 8 \times$, FAST GROUP:०,,

$X \quad 10 X, \cdot F(K) \cdot S=\cdot, 4 E 25,14,1,(18 X, 4 E 25,14))$

701 FORMAT $10^{\circ}, 7 \times, 0^{\circ}$ THERMAL GROUP:, 1.

$X \quad 10 X, \cdot F(K) \cdot S=0,4 E 25.14,1,(18 X, 4 E 25.14))$ BEGIN ITERATION LOOP. I COUT $=0$

C ICUUT IS THE OUTER ITERATION COUNTER.

$20 I$ ICOUT $=I$ COUT +1

IF (ICOUT.GT.ICMAX) GO TO 100

C FORM THE ITERATION SOURCE VECTOR, S; AND ITS L-2 NORM, SUMI: $S U M 1=0$

DO $15 \quad I=1, N$

$S(I)=0$.

I $0=1$

$I 1=3$

IF $(1 . E Q \cdot 1) \quad(10=2$

IF $(I \cdot E Q \cdot N) \quad I I=2$

POW 0078

POW 0079

POW 0080

POW 0081

POW 0082

POW 0083

POW 0084

POW 0085

POW 0086

POW 0087

POW 0088

POW 0089

POW 0090

POW 0091

POW 0092

POW 0093

POW 0094

POW 0095

POW 0096

POW 0097

POW 0098

POW 0099

POW 0100

POW 0101

POW 0102

POW 0103

POW 0104

POH 0105

POW 0106

POW 0107

POW 0108

PAGE 207 


$$
\prod_{K=I-2+J} 14 J=10,11
$$

$14 S(I)=S(I)+F I(I, J) * \operatorname{PHI}(1, K)+F 2(I, J) * \operatorname{PHI}(2, K)$

15 SUM $1=S U M 1+S(I) * * 2$

SUM $1=D S Q R T(S U M 1)$

SUM $1=\operatorname{SUML} *(\mathrm{CHI}(1)+\mathrm{CHI}(2))$

C SULVE FUR THE NEW GROUP FLUX VECTORS: PSI:

FAST GROUP: SOURCE VECTOR:

DO $25 \quad I=1, N$

$25 Z(I)=C H I(1) * S(I)$

C

FAST FLUX:

CALL SOLV $30(N, L 1, P S I 1, Z)$

C THERMAL GROUP; SOURCE VECTOR:

DO $27 \quad I=1, N$

$Z(I)=0$.

I $0=1$

I $1=3$

IF $(1 \cdot E Q \cdot 1): \quad 10=2$

IF $(I, E Q \cdot V) \quad I I=2$

DO $26 \mathrm{~J}=10, \mathrm{I} 1$

$K=I-2+J$

$26 Z(I)=Z(I)+T(I, J) * P S I I(K)$

$27 Z(I)=Z(I)+C H I(2) * S(I)$

C

$$
\text { THERMAL FLUX: }
$$

CALL SOLV3O(N,L2,PSI2,Z)

C

FORM NEW SOURCE VECTOR FROM THE NEW UNNORMALIZED FLUXES; PSI: SUM2 $=0$.

DO $29 I=1, N$

$S(I)=0$.

I $0=1$

I $1=3$

If $(I . E Q \cdot 1) \quad I 0=2$

IF $(I \bullet E Q \cdot V), \quad I 1=2$

DO $28 \mathrm{~J}=10,11$

$K=I-2+J$

$28 S(I)=S(I)+F 1(I, J) * P S I 1(K)+F 2(I, J) * P S I 2(K)$

POW 0109

POW 0110

POW 0111

POW 0112

POW 0113

POW 0114

POW 0115

POW 0116

POW 0117

POW 0118

POW 0119

POW 0120

POW 0121

POW 0122

POW 0123

POW 0124

POW 0125

POW 0126

POW 0127

POW 0128

POW 0129

POW 0130

POW 0131

POW 0132

POW 0133

POW 0134

POW 0135

POW 0136

POW 0137

POW 0138

POW 0139

POW 0140

POW 0141

POW 0142

POW 0143

POW 0144

PAGE 208 
29 SUMZ $=S U M Z+S(I) * * 2$

SUML $=$ DSQRT $($ SUMZ)

SUM $2=\operatorname{SUM} 2 *(C H I(1)+C H I(2))$

C

CALCULATION OF THE EIGENVALUE:

LAMDA $=$ SUM2/ SUMI

LAMSTR (I COUT) = $\triangle A M D A$

$E R R L A M=D A B S$ (LAM DA-LAMB4)

C

PUT PSI 1 ANO PSI 2 INTO BIGGER PSI:

DO $30 \quad I=I, N$

PSI $(1, I)=P S I 1(1)$

$30 \operatorname{PSI}(2, I)=\operatorname{PSI} 2(I)$

PUINT BY POINT SIMULTANEOUS RELAXATION FLUX ITERATION: $X=A L P H A$

$C$ DO NOT RELAX DURING THE FIRST THREE ITERATIONS:

IF (I COUT.LE.3) $X=1.0$

CALCULATE THE NEW GROUP FLUX ITERATES AND GROUP ERRORS:

DO $40 \quad I G=1,2$

$S Q(I G)=0$.

DO $40 \quad I=1, N$

ERROR (IG,I) $=P S I(I G, I) / L A M D A-P H I(I G, I)$

$S Q(I G)=S Q(I G)+E R R O R(I G, I) * * 2$

PHI I IG, I $)=P H I(I G, I)+X * E R R O R(I G, I)$

C AND FOR PLOTTING PURPOSES:

$P S I(I G, I)=P H I(I G, I)$

40 CONTINUE

DO $34 \quad 1 G=1,2$

C

$34 S Q(I G)=D S Q R T(S Q(I G))$

NORMAL ILE PSI:

NORMALILES BOTH ARRAY GROUPS TO 1.0:

CALL NORM2 (PSI, N)

DO 36 IG $=1,2$

C ERRMAX(IG) = THE MAX ERROR BETWEEN THE GROUP ITERATION FLUXES: ERRMAX $(I G)=E R R O R(I G, 1)$

DO $36 I=2, N$

IF (DABSIERROR(IG,I)).GT.ERRMAX (IG)) ERRMAX(IG)=DABS (ERROR(IG,I)) 36 CONTINUE

POW 0145

POW 0146

POW 0147

POW 0148

POW 0149

POW 0150

POW 0151

POW 0152

POW 0153

POW 0154

POW 0155

POW 0156

POW 0157

POW 0158

POW 0159

POW 0160

POW 0161

POW 0162

POW 0163

POW 0164

POW 0165

POW 0166

POW 0167

POW 0168

POW 0169

POW 0170

POW 0171

POW 0172

POW 0173

POW 0174

POW 0175

POW 0176

POW 0177

POW 0178

POW 0179

POW 0180

PAGE 209 
C

IF (IPLOT.NE.2) GO TO 45

IHE FOLLJWING IS FOR NICELY PLOTTING THE GROUP FLUX HISTORY.

DO $41 \quad I G=1,2$

DO $41 \quad I=1, N$

$41 \operatorname{ERROR}(16, I)=\operatorname{PSI}(16, I)$

C

ERRUR NOW CONTAINS THE NEW NORMALIZED FLUX ITERATE PHI. $\mathrm{JK}=\mathrm{I} K$

If $(I K \cdot E Q \cdot 0) \quad J K=5$

vO $42 \quad I S=1,2$

DO $42 \quad I=1, N$

IF (UABSIERROR(IG,I)-PHISTR(IG,I,JK+1)).GE.0.01) GO TO 43

42 CONTINUE

C

C

43

$$
\text { FLJX HA }
$$

GO TO 45

SAVE THE NORMALIZED FLUX FOR PLOTTING:

$$
I K=I K+1
$$

$I N(I K)=I$ COUT

TE3 (IK) = ERRLAM

DO $44 \quad I G=1,2$

TEI I IG,IK) $=$ ERRMAX $(I G)$

TE2 $(I G, I K)=S Q(I G)$

DO $44 \quad I=1, N$

$44 \operatorname{PHISTR}(1 G, I, I K+1)=E R R O R(I G, I)$

C

IF (IK.NE.5) GO TO 45

PLOT THE LAST FIVE SAVED FLUXES:

CALL PHIPLT(5)

I $K=0$

45 CONT INUE

C

ERROR CRITER IA FOR ACCEPTANCE OF CONVERGENCE.

I $F L A G 1=0$

IFLAG2 $=0$

I $F L A G 3=0$

C STURE THE ERRORS FCR COMPARISON:

C ERROR BETWEEN ITERATION EIGENVALUES:

ERLAM ( I COUT ) =ERRL AM

DO $46 \quad 1 \mathrm{G}=1,2$
POW 0181

POW 0182

POW 0183

POW 0184

POW 0185

POW 0186

POW 0187

POW 0188

POW 0189

POW 0190

POW 0191

POW 0192

POW 0193

POW 0194

POH 0195

POW 0196

POW 0197

POW 0198

POW 0199

POW 0200

POW 0201

POW 0202

POW 0203

POW 0204

POW 0205

POW 0206

POW 0207

POW 0208

POW 0209

POW 0210

POW 0211

POW 0212

POW 0213

POW 0214

POW 0215

POW 0216

PAGE 210 

EFMSTR ( IG, I COUT ) $=$ SQ (IG)

46 CONT INUE

IF ((ERRMAX (1),LT .EPS1).AND. (ERRMAX (2) .LT EEPS 1)) IFLAGI=1

POW 0220

POW 0221

IF ( (SQ (1).LT.EPS2).AND. (SQ (2),LT.EPS2))

IFLAG I $=1$
$I F L A G 2=1$

IF (ERRLAM.LT.EPS3) IFLAG $3=1$

IFLAG4=IFLAG I*IFLAG2*IFLAG3

IF (IFLAG4.EQ.1) GO TO 50

OTHERWISE CONTINUE THE ITERATION.

LAMB $4=$ LAMDA

GO TO 20

50 CONI I NUE

C CONVERGENCE ACCOMPLISHED.

C NORMALIZE THE CONVERGEO FLUX VECTOR:

CALL NORMAL ( PHI, N)

C PLOT ANY LEFT OVER FLUX HISTORY PLOTS:
IF (IPLJT.EQ.2).AND.(IK.NE.0) CALL PHIPLT(IK)

C BOUNDRY CONDITION INSERTICNS. $I E R=0$

IER ALLOWS B.C. INSERTIONS FOR YES AND NO CONVERGENCE:

C

55 IF (IBC.EQ.4) GO TO 90

IF (IBC.NE.3) GO TO 60

PHI $(1, K R+1)=0$.

$P H I(2, K R+1)=0$.

GO TO 90

$600070 \quad I=1, N$

$J=N+1-I$

$\operatorname{PHI}(1, J+1)=\operatorname{PHI}(1, J)$

$70 \operatorname{PHI}(2, J+1)=\operatorname{PHI}(2, J)$

$\operatorname{PHI}(1,1)=0$.

$\operatorname{PHI}(2,1)=0$.

IF (IBC.NE.1) GO TO 90

PHI $(1, K R+1)=0$.

PHI $(2, K R+1)=0$.

POW 0222

POW 0223

POW 0224

POW 0225

POW 0226

POW 0227

POW 0228

POW 0229

POW 0230

POW 0231

POW 0232

POW 0233

POW 0234

POW 0235

POW 0236

POW 0237

POW 0238

POW 0239

POW 0240

POW 0241

POW 0242

POW 0243

POW 0244

POW 0245

POW 0246

POW 0247

POW 0248

POW 0249

POW 0250

POW 0251

POW 0252

PAGE 211 
90 IF (IER.EQ.1) GO TO 102 RE TURN

C

$$
\text { NO SONVERGENCE ACCOMPLISHED: }
$$

100 CONT INUE

C NORMALILE THE UNCONVERGED FLUX:

$$
\begin{aligned}
& \text { NORMALI LE THE UNC } \\
& \text { NORMAL (PHI,N) }
\end{aligned}
$$

I COUT $=$ I COUT -1

WRITE $(6,101)$ I COUT

101 FORMAT IIHI, 'POWER METHOD DID NOT CCNVERGE FOR THIS CASE AFTER',

$$
\text { I4," ITERATI ONS. }, / /, 1 \mathrm{X} \text {, 'EXECUT ICN TERMINATED ') }
$$

IER $=1$

GO TO 55

102 CONTINUE

C

FOR PRIVTING OUT THE EIGENVALUE HISTORY AND THE FINAL FLUX SHAPE:

IF (IPLOT.EQ.O) IPLOT $=1$

IF (JPLOT.EQ.0) JPLOT=1

RETURN

END

POW 0256

POW 0257

POW 0258

POW 0259

POW 0260

POW 0261

POW 0262

POW 0263

POW 0264

POW 0265

POW 0266

POW 0267

POW 0268

POW 0269

POW 0270 
SUBROUTINE CURT

SOLVES FOR THE CURRENT FROM THE INPUT H(K)'S AND D(K)'S

USING F(K) $S$ FROM POWER:

CURRENT IS LINEAR (LEAST SQUARES - VARIATIONAL) AND PUT INTO ARRAY C. IMPLICIT REAL*8 (A-H,O-L)

COMMON /B2/ KR

COMMON /B4/F(2,201), C(2,201)

COMMON /B5/T(201,3), S1(201), S2(201), C1(201), C2(201)

COMMON /B7/ H(200), D $(2,200)$

C FROM THE MATRIX PROBLEM FOR LINEAR FIT OF STEP DATA: $M=K R$

$N=K R+1$

$T(1,1)=0$.

$T(N, 3)=0$.

$T(1,2)=H(1) / 3$.

$T(1,3)=H(1) / 6$.

$T(N, 1)=H(M) / 6$.

$T(N, 2)=H(M) / 3$

$S 1(1)=D(1,1) *(F(1,1)-F(1,2)) / 2$.

$S 2(1)=D(2,1) *(F(2,1)-F(2,2)) / 2$.

$S 1(N)=D(1, M) *(F(1, M)-F(1, M+1)) / 2$.

$S 2(N)=D(2, Y) *(F(2, M)-F(2, M+1)) / 2$.

DO $20 \quad I=2, M$

$\mathrm{J}=\mathrm{I}-1$

$T(1,1)=H(J) / 6$.

$T(I, 2)=(H(J)+H(I)) / 3$.

$T(I, \quad 3)=H(I) / 6$.

$S 1(I)=(D(1, J) *(F(1, J)-F(1, I))+D(1, I) *(F(1, I)-F(1, I+1))) / 2$. $S 2(I)=(D(2, J) *(F(2, J)-F(2, I))+D(2, I) *(F(2, I)-F(2, I+1))) / 2$.

20 CONTINUE

CALL SOLVBD(N,T,C1,S1)

CALL SOLV $3 D(N, T, C 2, S 2)$

DO $30 \quad I=1, N$

$C(1, I)=C I(I)$

$30 C(2, I)=C 2(I)$

RETURN

CUR 0005

CUR 0006

CUR 0007

CUR 0008

CUR 0009

CUR 0010

CUR 0011

CUR 0012

CUR 0013

CUR 0014

CUR 0015

CUR 0016

CUR 0017

CUR 0018

CUR 0019

CUR 0020

CUR 0021

CUR 0022

CUR 0023

CUR 0024

CUR 0025

CUR 0026

CUR 0027

CUR 0028

CUR 0029

CUR 0030

CUR 0031

CUR 0032

CUR 0033

CUR 0034

CUR 0035

CUR 0036

PAGE 213 
SUBROUTINE OUTPUT

PRINTS THE RESULTS OF THE METHOD.

IMPLICIT REAL*8 (A-H,L-Z)

COMMON /BI/ IBC,IPLOT,JPLOT, IPUNCH, ISEE, NOADJ

COMMON /B2/ KR,N

COMMUN /B4/ PHI $(2,201), \operatorname{CUR}(2,201)$, LAMDA, ICOUT

COMMON /ER/ EPS 1,EPS2,EPS3

COMMON /ESTR/ LAMSTR(300), EFSTR(2,300), EFMSTR(2,300), ERLAM(300)

INTEGER N, NOADJ

$K R O=K R-1$

$K R 1=K R+1$

WRITE $(6,1)$

1 FORYAT ('1RESULTS OF THE MULTIGROUP METHOD:') WRITE $(6,10)$ ICOUT

10 FORMAT $1 \%,{ }^{\circ}$ PROBLEM TERMINATED AFTER , 15,

$X$

WRITE $(6,20)$ LAMDA

C

20 FORMAT $(1,10 X$, 'LAMDA $=$, IPE21.14) PRINT OUT EI GENVALUES.

CALL PLOT

WRITE $(6,30)$

30 FORMAT 1 'IRESULTS AFTER PROBLEM TERMINATION:',/,

$X$ 'ONUMBER',9X, 'THERMAL FLUX',4X, 'THERMAL CURRENT', $12 \mathrm{X}$,

$X$ 'FAST FLUX', 7X,'FAST CURRENT', 11

WRITE $(6,50)$ (K,PHI $(2, K), \operatorname{CUR}(2, K), \operatorname{PHI}(1, K), \operatorname{CUR}(1, K), K=1, K R 1)$

50 FORMAT (17,1PE2 1.7, OPE19.7, 1PE21.7, OPE 19.7)

PRINT OUT THE STORED ITERATION ERRORS:

WRITE $(6,110)$ EPS 1, (EFSTR $(2,1), I=1$, ICOUT)

WRITE $(6,111)$ EPS 1, (EFSTR $(1,1), I=1$, ICOUT)

WRITE $(6,112)$ EPS2, (EFMSIR $(2,1), I=1$, ICOUT)

WRITE $(6,113)$ EPS3, (EFMSTR $(1,1), I=1, I C O U T)$

WRITE $(6,114)$ EPS 3, (ERL AM (1), I =1, ICOUT)

110 FORMAT ('IMAXIMUM ERRORS BETWEEN THE THERMAL FLUX ITERATIONS:",

$X$ 25X,'TOLERANCE USED $=1,1$ PE12.4, $/ 1,(1 P 5 E 20.5))$

111 FORMAT (IMAXIMUM ERRORS BETWEEN THE FAST FLUX ITERATIONS:",

$X \quad 25 X, \cdot T O L E R A N C E$ USED $=1.1$ PE12.4, $/ 1,(1$ P5E20.5)

OUT 0001

DUT 0002

OUT 0003

OUT 0004

OUT 0005

OUT 0006

OUT 0007

OUT 0008

OUT 0009

DUT 0010

OUT 0011

DUT 0012

OUT 0013

OUT 0014

OUT 0015

OUT 0016

DUT 0017

DUT 0018

DUT 0019

OUT 0020

DUT 0021

OUT 0022

OUT 0023

OUT 0024

OUT 0025

OUT 0026

OUT 0027

OUT 0028

OUT 0029

OUT 0030

OUT 0031

OUT 0032

OUT 0033

OUT 0034

OUT 0035

OUT 0036

PAGE 215 
112 FORMAT 1 'IMEAN SQUARE ERROR BETWEEN THE THERMAL FLUX ITERATIONS:', $X$ 18X, TOLERANCE USED $=1$, 1PE12.4, $/ 1,(1 P 5 E 20.51)$

113 FORMAT ('IMEAN SQUARE ERROR BETWEEN THE FAST FLUX ITERATIONS:", $X \quad 18 X$, TOLERANCE USED $=1,1$ PE12.4, $/,(1 P 5 E 20.5))$

114 FORMAT 1 'IERROR BETWEEN THE ITERATICN EIGENVALUES: ',

$X \quad 28 X, \cdot T O L E R A N C E$ USED $=1,1$ PE12.4, $/ 1,(1$ P5E 20.5) $)$

C

IF (NOADJ.EQ.O) RETURN

OTHERWISE ADJOINT CALCULATIONS ARE NOT EXECUTED: WRITE $(6,120)$

120 FORMAT ('IADJOINT CALCULATIONS HAVE BEEN BYPASSED.', $/ /$,

$X$ ' PROGRAM TERMINATED.'I

C IPUNCH $=1$ PUNCHES OUT THE FAST FLUX FOR SYNTH 1 G INPUTS: IF (IPUNCH.EQ.1) WRITE (7, 124) KR

IF (IPUNCH.EQ.1) WRITE $(7,125)$ (PHI $(1, I), C U R(1, I), I=1, K R 1)$

IF (IPUNCH.EQ.1) WRITE $(7,125) \quad(\operatorname{PHI}(2, I), \operatorname{CUR}(2, I), I=1, K R 1)$

124 FORMAT (15)

125 FORMAT (2020.10)

CALL EXIT

RETURN

C

END

OUT 0037

OUT 0038

OUT 0039

OUT 0040

OUT 0041

OUT 0042

OUT 0043

OUT 0044

OUT 0045

OUT 0046

OUT 0047

OUT 0048

OUT 0049

OUT 0050

OUT 0051

OUT 0052

OUT 0053

OUT 0054

OUT 0055

OUT 0056

OUT 0057 
SUBROUTINE PLQT

PLOTS UUT THE EIGENVALUE HISTORY AS A TABLE AND A GRAPH,

C AS WELL AS PLOTTING OUT THE FINAL MULTIGROUP FLUX SHAPES. IMPLICIT REAL $* 8 \quad(A-H, L-L)$

COMMON /BI/ IBC, IPLOT, JPLOT, IPUNCH

COMMON /B2/ KR

COMMON /B4/ PHI $(2,201)$, PSI $(2,201)$, LAMDA, ICOUT

COMMON /B5/ B $(300.2)$

COMMON /ESTR/ LAMSTR(300)

DIMENSI ON C $(201,3)$

IN URDER TO SAVE SOME SPACE:

EQUIVALEVCE $(B(1), C(1))$

INTEGER ND

$N D=201$

WRITE $(6,1)$ (LAMSTR ( I), I =1, I COUT)

1 FORMAT ( OTABLE OF EIGENVALUES DURING THE POWER ITERATION: ", $X$ $1 /,(1 P 5 E 25.14))$

IF (JPLOT.EQ.0) GO TO 20

DO $10 \quad I=1, I$ COUT

$B(1,1)=I$

$10 \mathrm{~B}(I, 2)=$ LAMSTR $(1)$

CALL PRTPLT (1,B, I COUT, 2, ICOUT, 0,300,2,1)

WRITE $(6,11)$

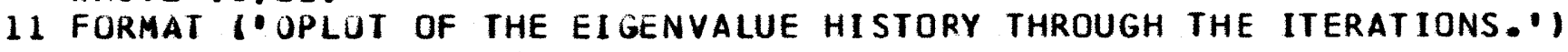

20 IF IIPLJT.EQ.0) RETURN

$K R 1=K R+1$

DO $30 \quad I=1, K R 1$

$C(1,1)=1$

$C(1,2)=P H I(1,1)$

$30 \mathrm{C}(1,3)=\mathrm{PHI}(2,1)$

CALL PRTPLT (2,C,KR 1,3,KR 1, 0,ND, 3,2)

WRITE $(0,31)$

31 FORMAT ('OFINAL CONVERGED CONNECTING FLUX POINTS: F(K)., $1 /$,

$X$ 5X, 'FAST FLUX: $\bullet, /, 5 X, \cdot$ THERMAL FLUX: -1$)$

RETURN

END

PLT 0001

PLT 0002

PLT 0003

PLT 0004

PLT 0005

PLT 0006

PLT 0007

PLT 0008

PLT 0009

PLT 0010

PLT 0011

PLT 0012

PLT 0013

PLT 0014

PLT 0015

PLT 0016

PLT 0017

PLT 0018

PLT 0019

PLT 0020

PLT 0021

PLT 0022

PLT 0023

PLT 0024

PLT 0025

PLT 0026

PLT 0027

PLT 0028

PLT 0029

PLT 0030

PLT 0031

PLT 0032

PLT 0033

PLT 0034

PLT 0035

PLT 0036

PAGE 217 
SUBROUTINE POWERT

P0W70001

*** ADJOINT PROBLEM ***

SOLVES IHE 2*N MULTIGROUP ADJOINT EGUATIONS:

$M * P H I=(1 /$ LAMDA $) * F * P H I$

BY THE FISSION SOURCE POWER METHOD

USING SIMULTANE OUS OVERRELAXATICN.

WHERE: M AND F ARE DUUBLE PRECISION 2N BY 2N BLOCK MATRICES;

ANO: PHI IS THE 2N ADJOINT (FAST AND THERMAL) VECTOR.

$L 1 *$ PHI $1-T * P H I 2=$ CHI $1 * F 1 * P H I 1 *$ CHI $2 * F 1 * P H I 2$

$$
\text { L } 2 * P H I 2=C H I 1 * F 2 * P H I 1+C H I 2 * F 2 * P H I 2
$$

IMPLICIT REAL*8 (A-H,L-L)

COMMON /BI/ IBC, IPLOT,JPLOT, I PUNCH, ISEE

COMMON /B2/ KR,N

COMMUN /B3/L1(201,3), L2(201,3), F1(201,3), F2 $(201,3), T(201,3)$

COMMON /B47/ PHI $(2,201)$, PSI $(2,201)$, LAMDA, ICOUT

COMMON /B5/ S(201), ERROR $(2,201), Z(201)$

COMMON /BO/ TE1 $(2,5), \operatorname{TE} 2(2,5), \operatorname{TE} 3(5), \operatorname{IN}(5)$

COMMON /B7/ HH( 200)

COMMON /CHIF/ CHI(2)

COMMON /ER/ EPS 1, EPS2, EPS3

COMMON /T/ I 1, I4

COMMON /FSTRI PHISTR $(2,201,6)$

COMMON /ESTR/ LAMSTR(300), EFSTR(2,300), EFMSTR $(2,300), \operatorname{ERLAM(300)}$

COMMON /READ5/ R5

DIMENSION PSI $1(201)$, PSI $2(201)$, SQ $(2)$, DPHI $(2)$, ERRMAX $(2)$

INTEGER N

C

DEFAULT OPTIONS FOR POWER PARAMETERS:

$A L P H A=1.25$

$\angle A M D A=1.0$

$H X=0.0$

DO $505 \quad 1=1, K R$

$505 \mathrm{HX}=\mathrm{HX}+\mathrm{HH}(\mathrm{I})$

DO 555 I $G=1,2$

IF (IBC.NE.4) GO TO 551

DO $550 \quad I=1, N$

550 PHI (IG,I $)=1.0$

POW70002

POW70003

POW70004

P0W70005

POW70006

POW70007

POW70008

P0W70009

POW70010

P0W70011

POW70012

POW70013

POW70014

POW70015

POW70016

POW70017

POW70018

POW70019

POW70020

POW70021

POW70022

POW70023

POW70024

POW70025

POW70026

P0W70027

POW70028

POW70029

POW 70030

P0W70031

P0W70032

POW70033

POW70034

POW70035

P0W70036

PAGE 218 
GO TO 555

POW70037

P0W70038

$551 X=3.1415926 / A X$

IF (IBC.NE.1) $X=X / 2.0$

POW70039

SUM $1=0.0$

DO $552 K=1, K R$

SUMI $=S U M L+H H(K)$

552 PHI $(I G, K)=$ DSIN $(\operatorname{SUM} I * X)$

555 CONTINUE

IF (R5.EQ.O.): GO TO 510

C
C
C

READ IN: OVERRELAXAT IN ITIAL GUESS AT EIGENVALUE:

ALPHA (OUTER ITERATION)

READ $(5,506, E N D=510)$ ALPHA INITIAL NORMALIZED FLUX ; PHI (1-N)

READ $(5,502, E N D=510)$ LAMDA

READ (5,5)3) (PHI $(1, I), I=1, N)$

READ $(5,503) \quad(P H I(2, I), I=1, N)$

506 FURMAT (F 10.5 )

502 FORMAT (E2).14)

503 FORMAT ((4E20.10))

510 CONTINUE

c

STORING FOR PRINTING THE MULTIGROUP FLUX SHAPE.

DO $11 \quad I G=1,2$

DO $10 \quad I=1, N$

10 PHISTR $(I G, 1,2)=P H I(I G, I)$

C

FILL RUNNING COORD IN PHISTR

$K R 1=K R+1$

DO $11 \quad I=1, K R 1$

$11 \operatorname{PHISTR}(I G, I, 1)=$ DFLOAT (I)

C IK IS THE FLUX PLOTTING COUNTER. I $K=1$

STORES THE ITERATION NUMBER FOR FLUX HISTORY PLOTTING: IN $(1)=0$

STURES TEMPORARY ERRORS FOR FLUX HISTORY PLOTTING:

C

TE $1(1,1)=0$.

$\operatorname{TE} 1(2,1)=0$.

$\operatorname{TE} 2(1,1)=0$.

POW70040

P0W70041

POW70042

POW70043

POW70044

P0W70045

POW70046

POW70047

POW70048

POW70049

POW70050

P0W70051

POW70052

P0W70053

POW70054

POW70055

POW70056

P0W70057

P0W70058

P0W70059

POW70060

POW70061

POW70062

P0W70063

POW70064

POW70065

POW70066

P0W70067

POW70068

P0W70069

POW70070

P0W70071

P0W70072

PAGE 219 
$\operatorname{TE} 2(2,1)=0$.

TE3 $(1)=0.0$

C EIGENVALUE OF THE PREVIOUS ITERATION:

LAMB $4=$ = AMDA

C THE MAXIMUM NUMBER OF ALLOWED ITERATIONS: ICMAX I CMAX $=300$

C PRINT OUT THE POWER METHOC PARAMETER INFORMATION:

WRITE $(0,700)$ ICMAX, ALPHA,LAMOA, $(P H I(1, I), I=1, N)$

WRITE $(6,701)$ (PHI $(2, I), I=1, N)$

700 FORMAT 1 IEXECUTING MUTIGROUP ADJOINT FISSION SOURCE POWER ITERATI XON METHOD.,$/ / /$,

$X \quad 5 X, \cdot$ MAXIMUM NUMBer OF allowable ITERATIONS:, 1,

$X \quad 10 X, \cdot 1$ CMAX $=0,14,1 / 1$,

$x$ 5X, OUTER ITERATION RELAXATION PARAMETER:,, 1,

$X \quad 10 X, \cdot A L P H A=\circ, F 7,3,1 /$,

$x \quad 5 X, \cdot$ INITIAL GUESS AT ADJOINT EIGENVALUE:,,$/$,

$X \quad 10 X$, LAMBDA $=$ "E22.14,1/,

$x$ 5x, INITIAL gUESS at THE gRCUP FlUX SHAPE CONNECTION POINTS:,

$X \quad / /, 8 X$, IFAST ADJOINT GROUP:,$/$,

$X \quad 10 X, \cdot F(K) \cdot S=\cdot, 4 E 25.14, /,(18 X, 4 E 25.14))$

701 FORMAT $10^{\circ}, 7 X$, 'THERMAL ADJOINT GROUP:",,

$X \quad 10 X, \cdot F(K) \cdot S=0,4 E 25.14,1,(18 X, 4 E 25.14))$

BEGIN I TERATION LOOP.

I COUT $=0$

ICOUT IS THE DUTER ITERATION COUNTER.

C 20 ICOUT $=$ I COUT +1

IF (ICOUT.GT.ICMAX) GO TO 100

FORM THE GROUP TOTAL SOURCE S, AND ITS L-2 NORM SUM1:

C

ANO THE THERMAL ADJOINT SOURCE VECTOR $Z$ : SUM $1=0$.

DO $15 \quad I=1, N$

$Z(I)=0$.

$S(I)=0$.

I $0=1$

$11=3$

IF $(1 . E Q .1) \quad I 0=2$

POW70073

POW70074

POW70075

P0W70076

POW70077

POW70078

POW70079

POW70080

POW70081

POW 70082

POW70083

POW70084

POW70085

POW7C086

POW70087

POW70088

POW70C89

POW70090

POW70091

POW70092

POW70093

POW70094

P0W70095

POW70096

P0W70097

POW70098

POW70099

P0W70100

P0W70101

POW70102

POW70103

POW70104

POW70105

P0W70106

POW70107

P0W70108

PAGE 220 
IF $(I \cdot E Q \cdot N) \quad I 1=2$

DO $14 J=I 0,11$

$K=I-2+J$

$Z(I)=Z(I)+F 2(I, J) *(C H I(1) * P H I(1, K)+C H I(2) * P H I(2, K))$

$14 S(I)=S(I)+F I(I, J) *(C H I(1) * P H I(1, K)+C H I(2) * \operatorname{PHI}(2, K))$

$S(I)=S(I)+Z(I)$

15 SUM $1=\operatorname{SUM} 1+S(I) * * 2$

SUMI $=$ DSQR T ( SUM 1$)$

C

SOLVE FOR THE NEW GROUP ADJOINT FLUX VECTORS; PSI:

THERMAL ADJUINT FLUX:

CALL SOLV3O $(N, L 2, P S I 2, Z)$

C

FAST ADJOINT GROUP; SOURCE VECTOR:

DO $27 \quad I=1, N$

$S(I)=0$.

$Z(I)=0$.

I $0=1$

$I 1=3$

IF $(I . E Q \cdot 1) \quad I 0=2$

IF $(I \cdot E Q \cdot N), I 1=2$

DO $26 \quad J=I 0, I 1$

$K=I-2+J$

$S(I)=S(I)+C H I(1) * F I(I, J) * P H I(1, K)+C H I(2) * F I(I, J) * P S I 2(K)$

$26 Z(I)=Z(I)+T(I, J) * P S I 2(K)$

$27 Z(I)=Z(I)+S(I)$

FAST ADJOINT FLUX:
SOLV3O(N,LI,PSI 1,2$)$

C

C

FORM NEW GROUP TOTAL SOURCE S FROM PSI'S, AND ITS L-2 NORM SUMZ: SUM2 $=0$.

DO $29 \quad I=1, N$

$S(I)=0$.

$10=1$

$11=3$

IF $(I \cdot E Q \cdot 1) \quad 10=2$

IF (I.EQ.N) $\quad I 1=2$

DO $28 J=I 0, I 1$

$K=I-2+J$

POW70109

POW70110

POW70111

POW70112

POW70113

POW70114

POW70115

POW70116

POW70117

POW70118

POW70119

POW70120

POW70121

POW70122

POW70123

POW70124

POW70125

P0W70126

POW70127

POW70128

POW70129

POW70130

POW70131

POW70132

POW70133

POW70134

POW70135

POW70136

POW70137

POW70138

POW70139

POW70140

POW70141

POW70142

POW70143

POW70144

PAGE 221 
$28 S(I)=S(I)+(F 1(I, J)+F 2(I, J)) *(C H I(I) * P S I I(K)+C H I(2) * P S I 2(K))$

P0W70145

POW70146

POW70147

POW70148

POW70149

POW70150

POW70151

P0W70152

POW70153

POW70154

P0W70155

POW70156

POW70157

POW70158

POW70159

P0170160

P0W70161

POW70162

POW70163

POW70164

POW70165

P0W70166

POW70167

POW70168

POW70169

P0W70170

POW70171

POW70172

P0W70173

POW70174

POW70175

POW70176

P0W70177

POW70178

POW70179

DO $36 \quad I=2, N$

IF (UABS(ERRUR (IG,I)).GT.ERRMAX (IG)) $\operatorname{ERRMAX(IG)=DABS(ERROR(IG,I))~}$

POW70180 36 CONTINUE 
IF (IPLOT.NE.2) GO TO 45

THE FOLLOWING IS FOR NICELY PLOTTING THE GROUP FLUX HISTORY.

DO 41 I $G=1,2$

DO $41 \quad I=1, N$

C

ERROR $(I J, I)=P S I(I G, I)$

ERROR NOW CONTAINS THE NEW NORMALIZED FLUX ITERATE PHI. $J K=I K$

IF IIK.EQ.0) JK=5

DO 42 I $G=1,2$

DO $42 \quad I=1, N$

IF (DABS(ERROR(IG, I)-PHISTR(IG,I,JK+1)).GE.0.01) GO TO 43

42 CONTINUE

C FLUX HAS NOT CHANGED ENOUGH FCR PLOTTING.

GO TO 45 SAVE THE NORMALIZEO FLUX FOR PLOTTING:

\section{$I K=I K+1$}

IN $(I K)=I$ COUT

TE3 $3(K)=E R R L A M$

DO 44 I $G=1,2$

TE $1(I G, I K)=E R R M A X(I G)$

$\operatorname{TE} 2(I G, I K)=S Q(I G)$

DO $44 \quad I=1$, iN

$44 \operatorname{PHISTR}(I G, I, I K+1)=E R R O R(I G, I)$

IF (IK.NE.5) GO TO 45

C

$$
\text { PLUT THE LAST FIVE SAVED fluXES: }
$$

\section{CALL PHIPLT (5)}

I $K=0$

C

45 CONT INUE

ERROR CRITERIA FOR ACCEPTANCE OF CONVERGENCE.

IFLAGI $=0$

I $F L A G 2=0$

IFLAG $3=0$

C

STORE THE ERRORS FOR CUMPARISCN:

ERROR BETWEEN I TERATION EIGENVALUES:

$E R L A M(I C D J T)=E R R L A M$

DO $46 \quad I G=1,2$

P0W70181

POW70182

POW70183

POW70184

POW70185

POW70186

POW70187

POW70188

POW70189

POW70190

P0W70191

POW70192

POW70193

POW70194

POW70195

POW70196

P0W70197

POW70198

POW70199

POW 70200

POW70201

POW70202

P0W70203

POW70204

P0W70205

POW70206

POW70207

POW70208

P0W70209

POW70210

POW70211

POW70212

P0W70213

POW70214

P0W70215

POW70216

PAGE 223 

EFSTR (IG,ICOUT) =ERRMAX (IG) EFMSTR ( IG, I COUT $)=S Q(I G)$

POW70219

46 CONTINUE

IF (IERRYAX (1) -LT •EPS 1).AND - (ERRMAX (2) -LT.EPS 1)) IFLAGI=1

POW70220

POW70221

IF ( $(S Q(1), L T \cdot E P S 2)$.AND. $(S Q(2), L T \cdot E P S 2))$

IFLAG2 $=1$

IF (ERRLAM.LT.EPS3) IFLAG $3=1$

POW70222

POW70223

POW70224

POW70225

IFLAG4=IFLAG1*IFLAG2*IFLAG 3

C

IF (IFLAG4.EQ.1) GO TO 50

POW70226

P0W70227 LAMB $4=$ LAMOA

GO TO 20

50 CONTINUE

C CONVERGENCE ACCOMPLISHED.

C NURMALILE THE CONVERGED FLUX VECTOR:

CALL NURMAL (PHI,N)

PLOT ANY LEFT OVER FLUX HISTORY FLOTS:

IF ( (IPLDT . EQ.2) . AND. (IK.NE • O))

CALL PHIPLT(IK) BOUNDRY CONUITION INSERTICNS. IER $=0$

IER ALLJWS B.C. INSERTIONS FOR YES AND NO CONVERGENCE:

POW70228

POW70229

POW70230

POW70231

POW70232

POW70233

POW70234

P0W70235

P0W70236

POW70237

POW70238

POW70239

POW70240

POW70241

POW70242

PHI $(1, K R+1)=0$.

$\operatorname{PHI}(2, K R+1)=0$.

GO TO 90

$600070 \quad I=1, N$

$J=N+1-I$

$\operatorname{PHI}(1, J+1)=\operatorname{PHI}(1, J)$

POW70243

POW70244

POW70245

POW70246

POW70247

POW70248

POW70249

POW70250

POW70251

POW70252

PHI $(1, K R+1)=0$.

PAGE 224 
90 IF (IER.EQ.1) GO TO $102 \quad$ POW70253 $\begin{array}{lr}\text { RETURN } & \text { P0W70254 }\end{array}$

C NO CONVERGENCE ACCOMPLISHED:

POW70255

POW70256

100 CONT INUE

POW70257

POW70258

CALL NORMAL (PHI,N)

I COUT $=I$ COUT -1

WRITE $(6,1) 1)$ ICOUT

101 FORMAT (IHL, 'POWER METHOD DID NOT CCNVERGE FOR THIS CASE AFTER',

$X \quad I 4, \cdot$ ITERATIONS $\cdot, / /, 1 X$, 'EXECUTICN TERMINATED $' 1$

IER $=1$

GO TO 55

102 CONTINUE

C

FOR PRINIING OUT THE EIGENVALUE HISTORY AND THE FINAL FLUX SHAPE:

IF (IPLJT.EQ.0) IPLOT $=1$

IF (JPLOT.EQ.0) JPLOT $=1$

RETURN

POW70259

POW70260

P0W70261

POW70262

POW70263

POW70264

POW70265

POW70266

POW70267

POW70268

POW70269

END

P0W70270 
SUBROUTINE CURT 7

SOLVES FJR THE ADJOINT CURRENT FRCM THE INPUT H(K)'S AND D(K)'S

CUR70001 USING $F(K)$ 'S FROM POWER 7:

CURRENT IS LINEAR (LEAST SQUARES - VARIATIONAL) AND PUT INTO ARRAY C. IMPLICIT REAL*8 (A-H,O-Z)

COMMON /B2/ KR

COMMON $1847 / F(2,201), C(2,201)$

COMMON /85/T(201,3),S1(201),S2(201), C1(201), C2(201)

COMMON /B7/ H(200), D $(2,200)$

C

FROM THE MATRIX PROBLEM FOR LINEAR FIT OF STEP DATA: $M=K R$

$N=K R+1$

$T(1,1)=0$.

$T(N, 3)=0$.

$T(1,2)=H(1) / 3$.

$T(1,3)=H(1) / 6$.

$T(N, 1)=H(M) / 6$.

$T(N, 2)=H(M) / 3$.

$S 1(1)=D(1,1) *(F(1,1)-F(1,2)) / 2$.

$S 2(1)=U(2,1) *(F(2,1)-F(2,2)) / 2$.

$S 1(N)=D(1, M) *(F(1, M)-F(1, M+1)) / 2$.

$S 2(N)=D(2, M) *(F(2, M)-F(2, M+1)) / 2$.

DO $20 \quad I=2, M$

$\mathrm{J}=\mathrm{I}-1$

$T(I, 1)=H(J) / 6$.

$T(I, 2)=(H(J)+H(I)) / 3$.

$T(I, 3)=H(I) / 6$.

$S 1(I)=(D(1, J) *(F(1, J)-F(1, I))+D(1, I) *(F(1, I)-F(1, I+1))) / 2$. $S 2(I)=(D(2, J) *(F(2, J)-F(2, I))+D(2, I) *(F(2, I)-F(2, I+1))) / 2$.

20 CONTINUE

CALL SOLV3O(N,T,C1,S1)

CALL SOLV $30(N, T, C 2, S 2)$

DO $30 \quad I=1, N$

$C(1,1)=-C 1(I)$

$30 C(2, I)=-C 2(I)$

RETURN

CUR70002

CUR70003

CUR70004

CUR70005

CUR70006

CUR70007

CUR70008

CUR70009

CUR70010

CUR70011

CUR70012

CUR70013

CUR70014

CUR70015

CUR70016

CUR70017

CUR70018

CUR70019

CUR 70020

CUR70021

CUR70022

CUR70023

CUR70024

CUR70025

CUR70026

CURT0027

CUR70028

CUR70029

CUR70030

CUR70031

CUR70032

CUR70033

CUR70034

CUR70035

CUR70036

PAGE 226 
SUBROUTINE DUTPTT

OUT70001

PRINTS THE RESULTS OF THE ADJOINT METHOD:

OUT70002

OUT 70003

IMPLICIT REAL*8 (A-H,L-L)

COMMON /BI/ IBC, IPLOT, JPLOT, I PUNCH

COMMON /B2/ KR,N

COMMON /B47/ PHI $(2,201)$, CUR $(2,201)$, LAMDA, ICOUT

COMMON /ER/ EPS 1,EPS2,EPS3

COMMON /ESTR/ LAMSTR(300), EFSTR (2,300), EFMSTR(2,300), ERLAM(300)

INTEGER N

$K R O=K R-1$

$K R 1=K R+1$

WR ITE $(6,1)$

1 FORMAT ('IRESULTS OF THE MULTIGROUP ADJOINT METHOD:')

WRITE $(6,10)$ ICOUT

10 FORMAT $(1 /$, PROBLEM TERMINATED AFTER', I5,

WRITE $(6,20)$ LAMDA

20 FORMAT $(1,10 X, \cdot$ ADJOINT LAMBDA $=1,1$ PE21.14) PRINT OUT EI GENVALUES.

CALL PLOT7

WRITE $(6,30)$

30 FORMAT 1 IRESULTS AFTER PROBLEM TERMINATION:,$/$,

$X \quad 5 X$, 'ADJOINTS:', $/$,

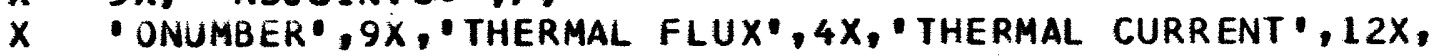

'FAST FLUX',7X,'FAST CURRENT',/

WRITE $(6,50)(K, P H I(2, K), C U R(2, K), P H I(1, K), C U R(1, K), K=1, K R 1)$

50 FORMAT (17, 1PE2 1.7,OPE19.7, 1PE21.7, OPE 19.7)

PRINT JUT THE STORED ITERATION ERRORS:

WRITE $(6,110)$. EPS 1 , (EFSTR $(2, I), I=1$, ICOUT)

WRITE $(6,111)$ EPS $1,(E F S T R(1,1), I=1$, ICOUT)

WRITE $(6,112)$ EPS2, (EFMSTR $(2, I), I=1$, ICOUT)

WRITE $(6,113)$ EPS3, (EFMSTR $(1, I), I=1$, ICCUT)

WRITE $(6,114)$ EPS3, (ERLAM(I),I=1, ICOUT)

110 FORMAT (IMAXIMUM ERRORS BETWEEN THE THERMAL FLUX ITERATIONS:"

$X$ 25X. TULERANCE USED $=1,1$ PE12.4.1/, (1P5E20.5))

111 FORMAT 1 MAXIMUM ERRORS BETWEEN THE FAST FLUX ITERATIONS:",

OUT70004

OUT70005

OUT70006

OUT70007

OUT70008

OUT70009

OUT70010

OUT70011

OUT70012

OUT70013

DUT70014

OUT70015

OUT70016

OUT70017

OUT70018

OUT70019

OUT70020

OUT70021

OUT70022

DUT70023

OUT70024

DUT70025

OUT70026

DUT70027

OUT 70028

DUT70029

OUT70030

OUT70031

OUT70032

OUT70033

OUT70034

DUT70035

DUT 70036

PAGE 228 
X 25X, TOLERANCE USED $=1,1$ PE12.4.1/, (1P5E20.5))

112 FORMAT "IMEAN SQUARE ERROR BETWEEN THE THERMAL FLUX ITERATIONS: "

$X \quad 18 X, 1$ TULERANCE USED $=\because, 1$ PE12.4, 1, (1P5E20.5))

113 FORMAT "IMEAN SQUARE ERROR BETWEEN THE FAST FLUX ITERATIONS:",

X 18X, TOLERANCE USED $=1,1$ PE 12.4, $1,(1 P 5 E 20.5)$ )

114 FORMAT "IERROR BETWEEN THE ITERATICN EIGENVALUES:"

$X \quad 28 X, 1$ TOLERANCE USED $=1,1$ PE12.4, $1,(1$ P5E20.5)

C

CHECK FUR CALL TO PUNCH:

IF (IPUNCH.EQ.1) CALL PUNCH

RETURN

END

OUT70037

OUT 70038

OUT70039

OUT70040

OUT70041

OUT70042

OUT70043

OUT70044

OUT 70045

OUT70046

OUT70047 
PLUTS DUT THE

Pluts DUt THE eigenvalue history as a table and a graph,

AS WELL AS PLOTTING OUT THE FINAL MULTIGROUP FLUX SHAPES.

FOR THE ADJOINTS:

IMPLICIT REAL*8 (A-H,L-Z)

COMMON /BI/ IBC, I PLOT, JPLOT, I PUNCH

COMMON /B2/ KR

COMMON /B47/ PHI $(2,201)$, CUR $(2,201)$, LAMDA, ICOUT

COMMON /B5/ B $(300,2)$

COMMON /ESTR/ LAMSTR(300)

DIMENSIUN C(201,3)

IN ORDER TO SAVE SOME SPACE:

EQUIVALENCE $(B(1), C(1))$

INTEGER ND

$N D=201$

WRITE (6,1) (LAMSTR(I), I=1, I COUT)

1 FORMAT ('OTABLE OF EIGENVALUES DURING THE POWER ITERATION:",

$\mathrm{X}$ $1 / .(1 P 5 E 25.14))$

C

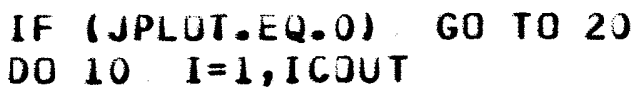

IF (JPLUT.EQ.0) GO TO 20

DO $10 \quad I=1, I$ COUT

$B(1,1)=1$

$10 B(I, 2)=$ LAMSTR $(I)$

CALL PRTPLTI $1, B$, ICOUT, 2, ICUUT, $0,300,2,1)$

WRITE $(6,11)$

11 FORMAT ('OPLOT OF THE EIGENVALUE HISTORY THROUgh THE ITERATIONS.")

20 IF (IPLJT.EQ.0) RETURIV

$K R L=K R+1$

DO $30 \quad I=1, K R I$

$C(I, 1)=I$

$C(I, 2)=\operatorname{PHI}(1,1)$

$30 \mathrm{C}(I, 3)=\mathrm{PHI}(2,1)$

CALL PRTPLT $(2, C, K R 1,3, K R 1,0, N D, 3,2)$

WRITE $(6,31)$

31 FORMAT (OOFINAL CONVERGED CCNNECTING FLUX POINTS; $F(K), \cdots / 1$,

$X$ 5X,'FAST FLUX: $\because, 1,5 X$, THERMAL FLUX: $-")$

PLT70001

PLT70002

PLT70003

PLT70004

PLT 70005

PLT70006

PLT70007

PLT70008

PLT70009

PLT70010

PLT70011

PLT70012

PLT70013

PLT70014

PLT70015

PLT70016

PLT70017

PLT70018

PLT70019

PLT70020

PLT70021

PLT70022

PL.T70023

PLT70024

PLT70025

PLT70026

PLT70027

PLT70028

PLT70029

PLI70030

PLT70031

PLT70032

PLT70033

PLT70034

PLT70035

PLT70036

PAGE 230 
RETURN

PLT70037

PLT 70038

END

PAGE 231 
SUBROUTINE SOLV $3 D(N, A, X, Y)$

$C$ SOLVES THE N DUUBLE PRECISION MATRIX EQUATIONS: $A * X=Y$,

C FOR $X$ - GIVEN THE $N$ BY N TRIDIAGCNAL MATRIX A

AND THE SOURCE VECTOR $Y$. METHOD IS FJRWARD ELIMINATION FOLLOWED BY BACKWARD SUBSTITUTION. CF - WACHPRESS, PAGE 23.

REAL $* 8$ A, $X, Y, H, P, D$

DIMENSIOV A(201,3), X(201), Y(201), H(201), P(201)

IF $(A(1,2) . E Q .0 .0)$ GO TO 10

$H(1)=-A(1,3) / A(1,2)$

$P(1)=Y(1) / A(1,2)$

DO $1 M=2, N$

$D=A(M, 2)+A(M, \quad 1) * H(M-1)$

IF $(0 . E Q .0 .0)$ GO TO 20

$P(M)=(Y(M)-A(M, \quad 1) * P(M-1)) / D$

IF (M.EQ.V) GU TO 1

$H(M)=-A(M, \quad 3) / D$

1 CONT INUE

$X(N)=P(N)$

DO $2 \quad I=2, N$

$M=N+1-I$

$2 X(M)=P(M)+H(M) * X(M+1)$

RETURN

C

IN CASE OF ANY IMPENDING ZERO DIVISORS:

10 WRITE $(6,11)$

11 FORMAT ('OFIRST ELEMENT OF $A, A(1,1)$, IS ZERO., 1 ,

$X$ 5X, BETTER FIX IT BOSS.')

GO TO 30

20 WRITE $(6,21) M$

21 FORMAT 1 OZZERO DIVISOR ENCOUNTERED IN EQUATION $M=\bullet, 13, \cdots, 1,1$,

$X$ SX, BETTER FIX IT BOSS.'I

30 WRITE $(6,31)$

31 FORMAT ('OEXECUTION TERMINATED.')

CALL EXIT

RETURN

END

SOLV0001

SOLV0002

SOLV0003

SOLV0004

SOLV0005

SOLV0006

SOLV0007

SOLV 0008

SOLV0009

SOLV0010

SCLV0011

SOLV0012

SOLV 0013

SOLVOO 14

SOLV0015

SOLV0016

SOLV0017

SOLV0018

SOLV0019

SOLV0020

SOLV 0021

SOLV0022

SOLV 0023

SOLV0024

SOLV 0025

SOLV0026

SOLV0027

SOLV0028

SOLV0029

SOLV0030

SOLV0031

SOLV0032

SOLV0033

SOLV0034

SOLV0035

SOLV 0036

PAGE 232 
SUBROUT INE NORMAL (PHI,N)

$A=D A B S(P H I(1,1))$

NORL0003

NORL 0004

DO 1 I $G=1,2$

DO $1 \quad I=1, N$

IF (DABS(PHI(IG,I)),GT.A) A=DABS(PHI(IG,I))

NORL 0005

NORLO006

NORLO007

NORL 0008

NORL 0009

NORL 0010

NORL 0011

NORL 0012

PHI (IG,I)=PHI I IG,I)/A

RETURN

END

NORL 0013 
SUBRUUT INE NORM2 (PSI, N)

\section{NORMALI ZES BOTH ENERGY}

REAL*8 PSI $(2,201), A(2)$

DO 1 I $G=1,2$

$A(I G)=D A B S(P S I(I G, 1))$

DO $1 \quad I=1, N$

IF (DABS(PSI(IG,I)).GT.A(IG)) A(IG)=DABS(PSI (IG,I))

1 CONTINUE

DO $2 \quad I G=1,2$

DO $2 \quad I=1, N$

$2 \operatorname{PSI}(I G, I)=P S I(I G, I) / A(I G)$

RETURN

END

NOR 20001

NOR20002

NOR20003

NOR20004

NOR 20005

NOR 20006

NOR20007

NOR2 0008

NOR20009

NOR20010

NOR20011

NOR20012

NOR20013 
SUBROUTINE PHIPLT (L)

PLOTS THE GROUP FLU

FAST ANO THERMAL GROUP FLUXES ARE PLOTTED SEPERATELY.

$L$ IS THE NUMBER OF FLUXES TO BE PLOTTED.

$L$ IS BETWEEN 1 AND 5 .

IMPLICIT REAL 8 8 (A-H,O-Z)

COMMON /BL/ IBC

COMMUN /B2/ KR,N

COMMON /B5/S(201), $A(201,6), B(201,6)$

COMMON /B6/ TEL $(2,5), \operatorname{TE} 2(2,5), \operatorname{TE} 3(5), \operatorname{IN}(5)$

COMMON /ER/ EPS 1, EPS $2, E P S 3$

COMMON /FSTR/ PHISTR $(2,201,6)$

DIMENSI ON SYMBOL (5)

INTEGER SYMBOL $1, \cdot, \cdot-1,1+1, \cdot *^{\prime}, 0 * 0 /$

$N D=201$

C

$K R 1=K R+1$

SET UP B.C. CONDITIONS

IF $(I B C . E 2.4)$ GO TO 5

IF $(I B C \cdot E Q \cdot 3)$ GO TO 3

DO 2 I $G=1,2$

$D O 2 K=1, L$

DO $1 \quad I=1, N$

$\mathrm{J}=\mathrm{N}+\mathrm{I}-\mathrm{I}$

1 PHISTR(IG, J $+1, K+1)=\operatorname{PHISTR}(I G, J, K+1)$

$2 \operatorname{PHISTR}(I G, 1, K+1)=0$.

3 IF (IBC.EQ.2) GO TO 5

DO $4 \quad I G=1,2$

DO $4 \quad K=1, L$

4 PHISTR(IG,KRI,K+1)=0.

5 CONTINUE

FLUXES IN PHISTR HAVE BEEN NORMALIZED IN POWER.

PUT THE FAST FLUX IN A, AND THE THERMAL FLUX IN B: $L 1=L+1$

DO $10 K=1, L 1$

DO $10 \quad I=1, K R I$

$A(I, K)=\operatorname{PHISTR}(1, I, K)$

PHIPOOOI

PHIP0002

PHIP0003

PHIP 0004

PHIP 0005

PHIP 0006

PHIP0007

PHIP0008

PHIP0009

PHIP0010

PHIP0011

PHIP0012

PHIP0013

PHIP0014

PHIP0O15

PHIP0016

PHIP0017

PHIP0018

PHIPOO19

PHIP0020

PHIP 0021

PHIP 0022

PHI P 0023

PHIP0024

PHIP0025

PHIP0026

PHIP0027

PHIP 0028

PHIP 0029

PHIP 0030

PHIP0031

PHIP 0032

PHIP 0033

PHIP0034

PHIP0035

PHIP 0036

PAGE 235 
$10 B(I, K)=P H I S T R(2, I, K)$ CALL PRTPLT $(0, A, K R 1, L 1, K R 1,0, N D, 6,2)$

PHIP0038 WRITE $(0,20)$

20 FORMAT $(1$, OFAST FLUX ITERATION HISTCRY PLOT.",/) WRITE $(6,30)$

30 FORMAT

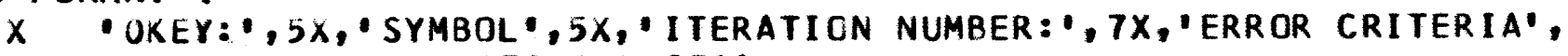
$X \quad 11 X$, 'ERROR', 13X,"TOLERANCE') DO $35 I=1, L$

35 WRITE $(6,40)$ SYMBOL (I),IN(I),TE1 (1, I), EPS $1, T E 2(1, I), E P S 2$, $X$ TE $3(1), E P S 3$

40 FORMAT $(/, 12 X, A 1,15 X, 13,16 X$, 'FLUX',14X,1PD15.5,5X,1PD15.5,/,

$x \quad 47 x$, MEAN SQ. FLUX $, 5 X, 1$ PD15.5,5X,1 PD15.5,,

$X \quad 47 X,{ }^{\circ} I G E N V A L U E \cdot, 8 X, 1 P D 15.5,5 X, 1 P D 15.51$

PLOT THE L THERMAL FLUX SHAPES CN THE OTHER GRAPH: CALL PRTPLT $(0,8, K R 1, L 1, K R 1,0, N D, 6,2)$ WRITE $(6,50)$

50 FORMAT (\%, OTHERMAL FLUX I TERATICN PLOT.,$/)$ WRITE $(6,30)$ DO $55 \quad I=1, L$

55 WRITE $(6,40)$ SYMBOL $(I), I N(I), T E 1(2, I), \operatorname{EPS} 1, \operatorname{TE2}(2, I), E P S 2$, $X$ IE $3(I), E P S 3$

RETURN

END

PHIP 0040

PHIP 0041

PHIP0042

PHIP 0043

PHIP0044

PHIP 0045

PHIP0046

PHIP 0047

PHIP 0048

PHIP0049

PHIP0050

PHIP0051

PHIP 0052

PHIP0053

PHIP 0054

PHIP0055

PHIP0056

PHIP0057

PHIP0058

PHIP0059

PHIP0060

PHIP0061

PHIP0062 
SUBROUTINE PRTPLT (NO, B,N,M,NL,NS, KX,JX, ISP)

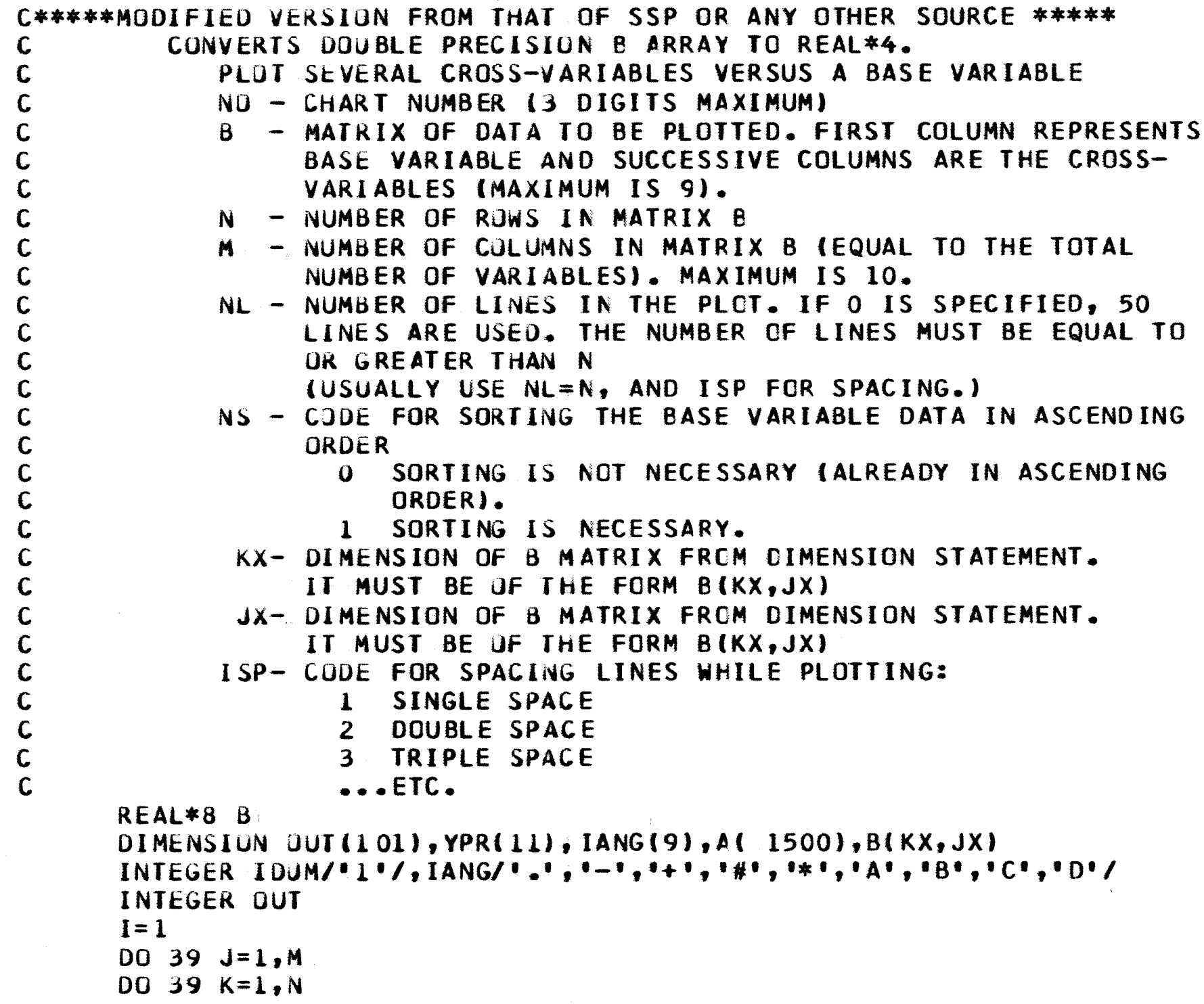

PRTPOOO 1 PRTPO002 PRTP 0003 PRTPO004 PRTP0005 PR TP 0006 PRTP 0007 PRTP0008

PRTP0009

PRTP0010

PR TP0011

PRTP 0012

PRTP 0013

PRTP0014

PR TP 0015

PRTP0016

PRTP0017

PRTP0018

PR IP0019

PRTP0020

PRTP0021

PRTP 0022

PRTP0023

PRTP0024

PRTP0025

PRTP0026

PR TP0027

PR TP 0028

PRTP0029

PR TP0030

PRTP0031

PRTP0032

PRTP0033

PRTP0034

PRTP0035

PRTP0036

PAGE 237 


$$
A(I)=B(K, J)
$$

$$
I=I+I
$$

1 FORMAT ( $1 \mathrm{HL}, 60 \mathrm{X}, 7 \mathrm{TH}$ CHART $, 13,1 / 1$

2 FORMAT ( $1 H$, F $11,4,5 X, 101 A 1)$

3 FORMAT ( $1 \mathrm{H}$ )

7 FORMAT $1 \mathrm{H}, 16 \mathrm{X}, 101 \mathrm{Ht}$

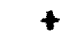

8 FORMAT $(1 H, 9 X, 11 F 10.4)$

NLL $=N L$

IF(NS) 16, 16, 10

C

10 DO $15 \mathrm{I}=1, \mathrm{~N}$

DO $14 \mathrm{~J}=\mathrm{I}, \mathrm{N}$

IF $(A(I)-A(J)) 14,14,11$

$11 \mathrm{~L}=\mathrm{I}-\mathrm{N}$

$L L=J-N$

DO $12 K=1, M$

$L=L+N$

$L L=L L+N$

$F=A(L)$

$A(L)=A(L L)$

$12 \quad A(L L)=F$

14 CONTINUE

15 CONTINUE

C

$$
\text { TEST NLL }
$$

16 IF (NLL) $20,18,20$

$18 \mathrm{NLL}=5 \mathrm{O}$

C

PRINT TITLE

20 WRITE $(6,1) N O$

C DEVELOP BLANK AND DIGITS FOR PRINTING $B L A N K=0$

C FIND SCALE FOR BASE VAR I ABLE $X S C A L=(A(N)-A(1)) /(F L O A T(N L L-1))$

C FIND SCALE FOR CROSS-VARIABLES YMIN $=1.0 E 75$
PRTP0037

PRTP0038

PRTP 0039

PRTP0040

PRTP0041

PRTP0042

PR TP0043

PRTP0044

PRTP0045

PRTP0046

PRTP 0047

PR TP 0048

PRTP 0049

PRTP0050

PRTP 0051

PRTP 0052

PRTP 0053

PRTP0054

PRTP 0055

PRTP0056

PRTP0057

PRTP0058

PRTP0059

PRTP0060

PRTP0061

PRTP0062

PRTP 0063

PRTP0064

PRTP 0065

PRTP0066

PR TP0067

PRTP0068

PRTP0069

PRTP0070

PRTP0071

PRTPO072

PAGE 238 
YMAX $=-1.0 E 75$

PRTP0073

$M 1=N+1$

$M 2=M * N$

DO $40 \mathrm{~J}=\mathrm{M1}, \mathrm{M2}$

IF $(A(J) \cdot G T \cdot Y M A X) \quad Y M A X=A(J)$

IF $(A(J), L T \cdot Y M I N) \quad Y M I N=A(J)$

PRTPJ074

PRTP 0075

PRTP0076

PR TP 0077

PRTP0078

PRTP0079

PRTP0080

YSCAL $=(Y M A X-Y M I N) / 100.0$

CHECK TJ SEE IF THE SPREAD IN Y IS TOO SMALL FOR PLOTTING:

PRTP0081

IF (YSCAL.EQ.0.0) GO TO 100

OTHERWISE, A DIVIDE CHECK WILL OCCUR AFTER STATEMENT 56. FIND BASE VARIABLE PRINT POSITION

C $X B=A(1)$

$L=1$

$M Y=M-1$

$I T=I S P-1$

DO $80 \quad I=1, N L L$

$F=I-1$

$X P R=X B+F * X S C A L$

C

IF (A(L)-XPR-XSCAL *0.5) $50,50,70$

FIND CROSS-VARI ABLES

50 DO 55 I $X=1,101$

55 OUT $(I X)=B L A N K$

57 CONTINUE

DO $60 \mathrm{~J}=1$, MY

$56 L L=L+J * N$

$J P=((A(L L)-Y M I N) / Y S C A L)+1.0$

OUT (JP) $=I$ ANG $(J)$

60 CONT INUE

c

PRINT LINE AND CLEAR, OR SKIP

IF (L.EQ.N) GO TO 61

$L=L+1$

IF $(A(L)-X P R-X S C A L * 0.5) \quad 57,57,61$

61 CONT INUE

WRITE $(6,2)$ XPR, ( OUT $(I Z), I Z=1,101)$

IF (IT.EQ.O) GO TO 65

PRTP 0082

PRTP0083

PRTP0084

PRTP0085

PRTP0086

PRTP0087

PRTP0088

PRTP0089

PR TP0090

PRTP0091

PRTP0092

PRTP0093

PRTP0094

PRTP0095

PRTP0096

PRTP0097

PRTP0098

PRTP0099

PRTP0100

PRTP0101

PRTP0102

PRTP0103

PRTP0104

PRTP0105

PRTP0106

PRTP0107

PRTP0108

PAGE 239 
DO 64 I $V=1, I T$

PRTP0109

PRTPO110

PRTPO111

65 GO TO 80

70 WRITE $(6,3)$

80 CONT INUE

C

PRINT CROSS-VARIABLES NUMBERS

PRTP0112

PRTP0113

PRTPO114

WRITE $(6,7)$

$Y P R(1)=Y M I N$

DO $90 \quad K N=1,9$

$90 \quad Y P R(K N+1)=Y P R(K N)+Y S C A L * 10.0$

$Y P R(11)=Y M A X$

WRITE $(6,8)(Y P R(I P), I P=1,11)$

RETURN

100 WRITE $(6,101)$

101 FORMAT ('ONO PLOT IS GENERATED BECAUSE THE SPREAD IN THE Y VARIBLE

$x$ IS TOU SMALL. $, 1,10 X, 1$ (I.E. - EQUALS ZERO UNDER REAL*4.1, 11,

$X$ 5X, 'EXECUTION CONTINUING.")

RETURN

END

RTPOI15

PRTP0116

PRTP0117

PRTP0118

PRTP0119

PRTP0120

PRTP0121

PRTP0122

PRTP0123

PRTP 0124

PR TP0125

PRTP0126

PRTP0127 


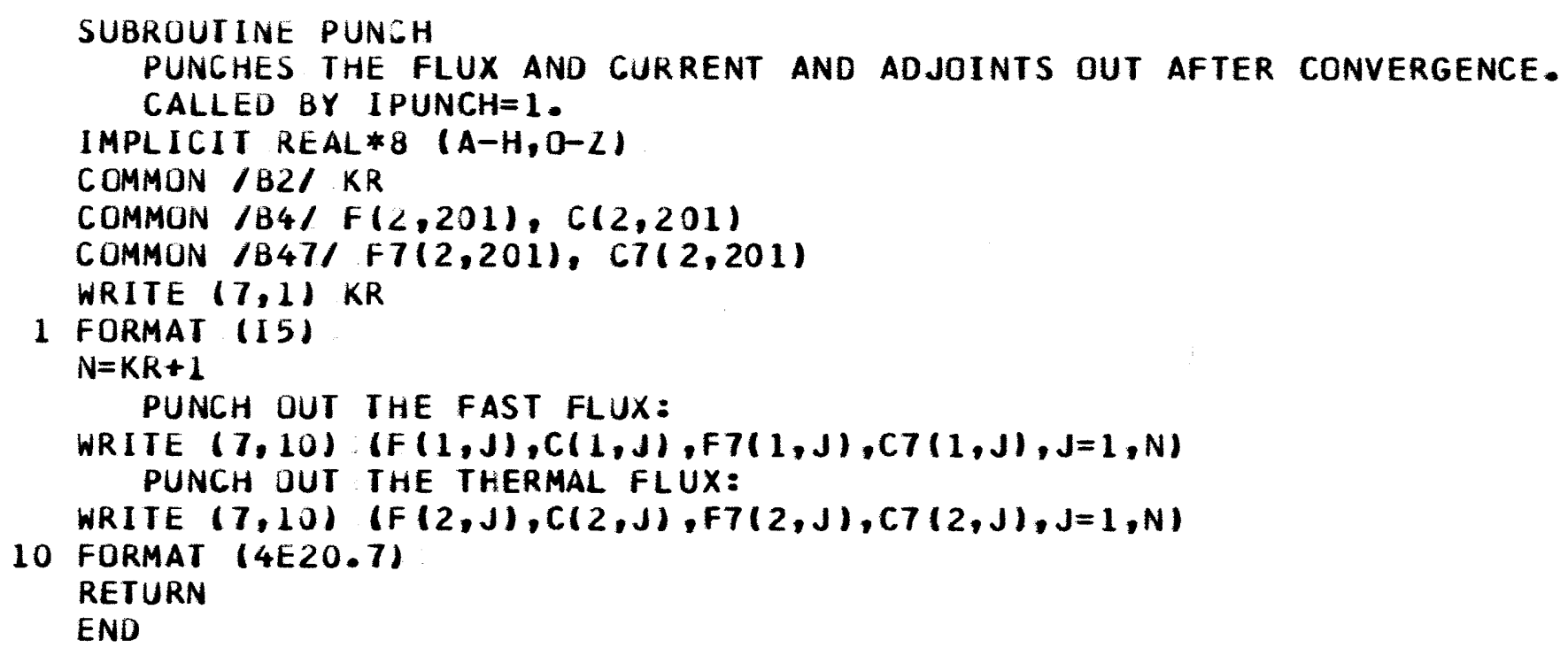

PNCHOOO1

PNCHOOO2

PNCHOOO3

PNCHOOO4

PNCH0O05

PNCHOOO6

PNCHOOOT

PNCHODO 8

PNCHOOO9

PNCHOOIO

PNCHOO11

PNCHOO12

PNCHOO13

PNCHOO14

PNCH0015

PNCHOOL 6

PNCHOO 17 
F.2. SOURCE LISTING of Program LINEAR 


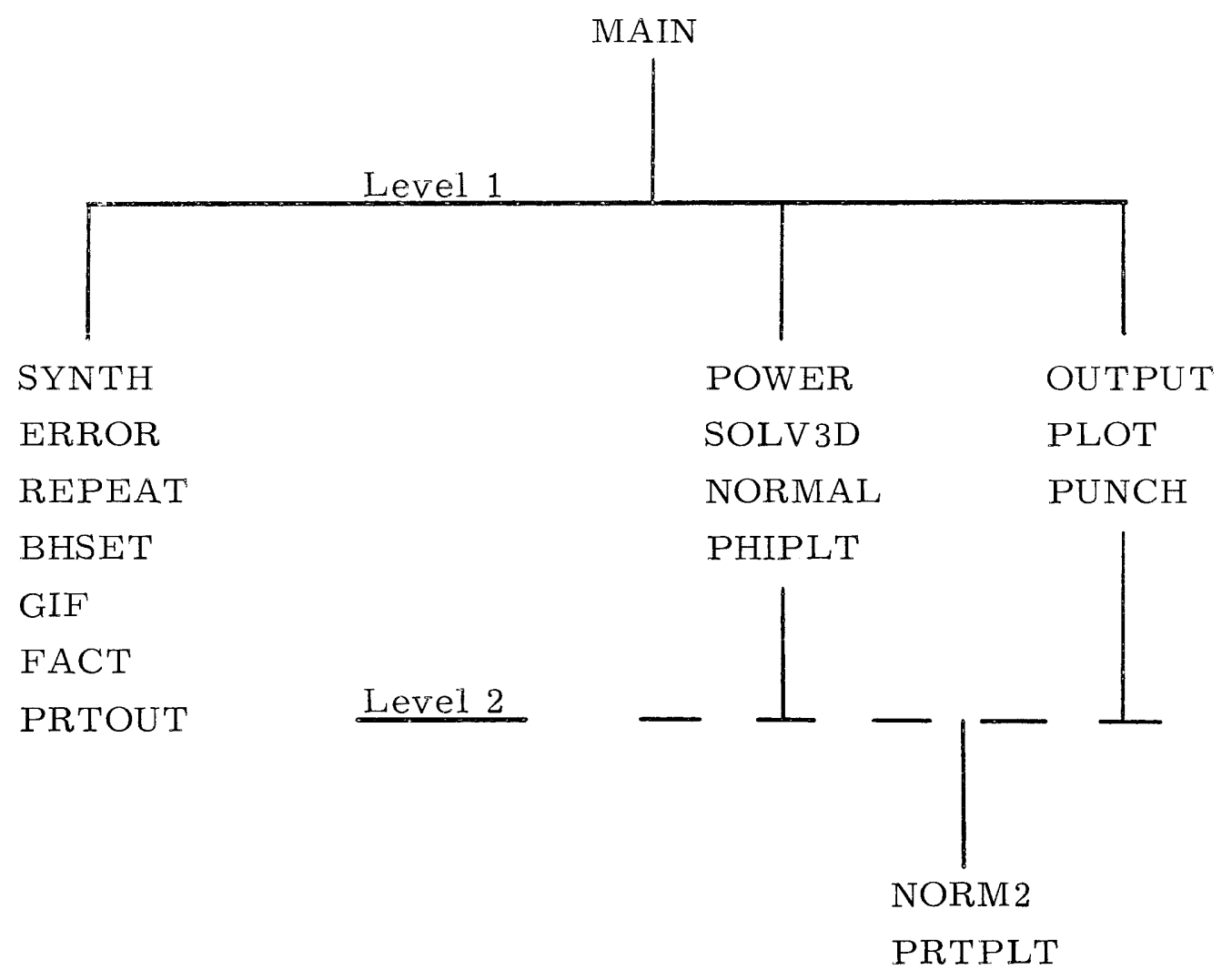

Figure F.2. Structure of Program LINEAR. 
C PROGRAM LINEAR:

LI NE 0001

TWO GROUP PROPOSED METHOD USING LINEAR BASIS FUNCTIONS.

LINE0002

CALL TIAING (II)

CALL SYNTH

CALL TIMING (I 4)

CALL POWER

CALL TIMINS (I 6)

CALL TIMING(I7)

CALL UUTPUT

CALL TIMING (18)

TIMING EXECUTION

WRITE $(6,30)$

30 FORMAT (1H1, TIMING PROGRAM EXECUTICN:, 1$)$

$J=14-11$

WRI TE $(6,701)$ J

$J=16-14$

WRITE $(0,704) \mathrm{J}$

$\mathrm{J}=\mathrm{I} 7 \mathrm{~T}-1 \mathrm{I}$

WRITE $(6,706) \mathrm{J}$

$\mathrm{J}=\mathrm{I}$ 8-I 7

WRITE $(6,707)$ J

701 FORMAT (1H," SYNTH HAS TAKEN", 16," 1100 SECONDS.")

704 FORMAT (IH: POWER HAS TAKEN $, 16,0^{\circ} / 100$ SECONDS.')

706 FORMAT (IH," CURENT HAS TAKEN', I5,'/100 SECONDS.')

707 FORMAT (1H " OUTPUT HAS TAKEN", 15," $/ 100$ SECONDS $\cdot 1$

CALL TIMING (I 20$)$

$J=I 20-I I$

WR I TE $(6,720) \mathrm{J}$

720 FORMAT (1HO,' THIS RUN HAS TAKEN $, 16,,^{\circ} / 100$ SECONDS TO RUN.') STOP

END

LINE0003

LINE0004

LINE0005

L INE 0006

LINE0007

LI NE 0008

LI NE0009

L INE0010

LI NE 0011

LINE0012

LINE0013

LINE0014

LI INE0015

LINE 0016

LI NE0017

LINE0018

LINE0O19

LINE0020

LINE0021

LINE0022

LINE0023

LINE0024

LI NE 0025

LINE0026

LINE0027

LINE 0028

LINE0029

LINE0030

LINE0031 
SUBROUTINE SYNTH

PROPOSED LINEAR SYNTHESIS METHOD:

SYNTO001

SYNT 0002

C * ********************************************

C

ADJUINT QUANTITIES OF VARIBLES ARE DENOTED BY 7 RATHER THAN *

THUS: PHIT (RATHER THAN PHI*) IS THE ADJOINT OF PHI. ETC.

IMPLICI T REAL*8 (A-H,K-Z)

COMMON/BI/IBC, IPLOT, JPLOT, IPUNCH, ISEE

COMMON /B2/ KR, NN

COMMON /B3/L1(26,26),L2(26,26),F1(26,26),F2(26,26),

$\mathrm{X}$

$F 3(26,26), F 4(26,26), T(26,26)$

COMMON /B5, KAO $(2,25), K A 1(2,25), K A 2(2,25), K B O(2,25), K B 1(2,25)$,

$\mathrm{x}$

$\mathbf{X}$

$\mathbf{x}$

$x$

$x$

$x$

$x$

$x$

$x$

$\mathrm{X}$

COMMON /CHIF/ CHI (2)

$K B 2(2,25), \operatorname{LAO}(2,25), \operatorname{LA} 1(2,25), \operatorname{LA} 2(2,25), \operatorname{SRO}(1,25)$,

$\operatorname{SR} 1(1,25), \operatorname{SR} 2(1,25), K C 0(1,25), K C 1(1,25), K C 2(1,25)$,

$K O 0(1,25), K D 1(1,25), K D 2(1,25)$,

$P(2,25), P 1(2,25), Q(2,25)$,

$Q 1(2,25), R(2,25), P O(2,25), P 07(2,25), P H(2,25)$,

PH $7(2,25), A L(2,25), B L(2,25), C L(2,25), A F(4,25)$,

BF $(4,25), C F(4,25), A T(25), B T(25), C T(25)$,

$A L K(2), B L K(2), A F K(4), \operatorname{BFK}(4), \operatorname{ATK}(2)$,

$B T K(2), B L O(2), C L O(2), B F O(4), C F O(4), B T O(2), C T O(2)$,

$\mathrm{CO}(2), \mathrm{CH}(2)$

COMMUN/XAXIS/ HX, HR (25)

COMMON/BH/ X(101), H(101)

COMMON /ER/ EPS 1, EPS2,EPS3

DIMENSION PHI(2,101),PHI7(2,101),CUR(2,101),CUR7(2,101), $\mathrm{X}$

$\mathrm{x}$

DIMENSION $V(2), V 1(2), V 2(2), V 3(2)$

$A(2,100), F(2,100), D(2,100), S(2,100), D I(2,100)$, $X \cup(2,100)$

DIMENSION I TF (25), KTF(25)
EQUIVALENCE (PHI(1),L1) 1$)$,

$x$
$X$
$x$
$X$

(CUR (1),L2(1)),

(XU(1),F1(1))。

$(F(1), F 2(1))$,

(S $(1), T(1))$.
(PHI7 (1),L1(301)),

(CUR7 (1), L2(301)),

(A) (1),F1(301)).

(D(1), F2 (301)).

(DI (1), T(301))
SYNT 0003

SYNT 0004

SYNT 0005

SYNTO006

SYNT 0007

SYNT 0008

SYNT0009

SYNT 0010

SYNTOO11

SYNT 0012

SYNT 0013

SYNT 0014

SYNTOO15

SYNTOO16

SYNTO017

SYNT 0018

SYNT 0019

SYNT 0020

SYNT 0021

SYNTO022

SYNT 0023

SYNTO024

SYNT 0025

SYNT 0026

SYNT 0027

SYNT0028

SYNT0029

SYNT 0030

SYNT0031

SYNTO032

SYNT 0033

SYNT 0034

SYNTO0 35

SYNT 0036

PAGE 245 
REAL TITLE(20)

SYNTO037

SYNT 0038

INTEGER KR, $K, K S, K S L, K R O, N N, N U M I T F, K T F$

SYNTOO39

READ $(5,200)$ TITLE

200 FORMAT (20A4)

WR ITE $(6,201)$ TITLE

C

201 FORMAT (1H1,20A4, //)

READ IN THE NUMBER OF REGICN TRIAL FUNCTIONS AND TYPE OF B.C.S.

AS WELL AS THE TOLERANCES AND THE OUTPUT TYPES DESIRED:

REAU $(5,1) \mathrm{KR}, \mathrm{I} B C$, EPS 1,EPS 2, EPS 3, IPLCT, JPLOT, I PUNCH, ISEE, ITH, ITC

1 FORMAT $(215,3010.3,615)$

IF (IBC.EQ.3) IBC=2

C REAO IN THE TYPE-NUMBER OF EACH TF REGION: $\operatorname{READ}(5,100)$ (I $\operatorname{TF}(I), I=1, K R$ )

100 FORMAT (2512)

C READ IN THE FISSION YEILDS FOR EACH GROUP: READ $(5,101) \mathrm{CHI}(1), \mathrm{CHI}(2)$

101 FORMAT (2F 10.5$)$ $K R O=K R-1$

WRITE $(6,2) \mathrm{KR}$, IBC, ISEE, ITW, ITC

2 FORMAT ('OUNE DIMENSICNAL TWO GROUP LINEAR SYNTHESIS PROGRAM: $1 / 1 /$, $X$ 5X, NUMBER OF COARSE MESH REGIONS: $K R=1,12,1$

$X \quad 5 X, \cdot B O U N D A R Y$ CONDITION NUMBER: $\quad I B C=\cdots, 12,1$,

$X$ SX, AMOUNT UF OUTPUT REQUESTED: ISEE $=1,12,11$,

$X$ 5X, TYPE OF WEIGHTING FUNCTICNS: ITW $=1,12,1$,

$X$ 5X, TYPE OF CURRENT FUNCTICNS: $\quad$ ITC $=1,12,11$,

$x$. $5 x, \cdot R E g I$ 'NAL INPUT MATER IAL PROPERTIES AND FLUX SHAPES FOLLOW',

$X \quad /, 5 x_{0} \cdot$ IF ISEE $>0: \cdot, / /$,

$X \quad 5 X, \cdot F L U X$ SHAPES ARE LINEAR IN EACH INDICATED SUBREGION.'I

IF (ITC.EQ.0) WRITE $(6,16)$

IF (ITC.EQ.1) WRITE $(6,17)$

16 FORMAT (5X, 'CURRENTS ARE CONSTANT IN EACH INDICATED SUBREGION. $'$ )

17 FORMAT ( $5 X$, "CURRENTS ARE LINEAR IN EACH INDICATED SUBREGICN.")

IF (ITW.EQ.0) WRITE $(6,116)$

IF (ITW.EQ.1) WRITE $(6,117)$

116 FORMAT $1 /, 5 X, "$ WIGHTING FLUX = FLUX;, $1 /, 5 X$, 'WEIGHTING CURRENT = XCURRENT.'j

SYNT CO40

SYNTO041

SYNT 0042

SYNT 0043

SYNT 0044

SYNT0045

SYNT 0046

SYNT 0047

SYNT 0048

SYNT 0049

SYNT 0050

SYNT 0051

SYNT0052

SYNT0053

SYNT 0054

SYNT 0055

SYNT 0056

SYNT 0057

SYNT 0058

SYNT 0059

SYNT0060

SYNT 0061

SYNT0062

SYNT 0063

SYNT 0064

SYNT 0065

SYNT0066

SYNT 0067

SYNT 0068

SYNTO069

SYNT 0070

SYNT 0071

SYNT 0072

PAGE 246 
117 FORMAT $1 /, 5 \mathrm{X}, \cdot$ WIGHTING FLUX = ADJOINT FLUX; $, 1,5 X, \cdot$ WEIGHTING CURR

SYNT 0073 XENT = ADJOINT CURRENT $" 1$

WRITE 10,20$)$ EPS 1, EPS2, EPS 3 , IPLOT, JPLOT, I PUNCH

20 FORMAT $1 / 1, \cdot$ UTULERANCES TO POWER ARE : EPS $1=1,1$ PD $10.3,1$,

$X \quad 28 X,{ }^{\circ}$ EPS $2=1,1$ PD1 $0.3,1,28 X,{ }^{\circ}$ EPS $3=1,1$ PD10.3, 1,

$X$ 'OOUTPUT PAZAMETERS TO POWER ARE: IPLOT $=1,11,1$,

$X \quad 34 X, \cdot J P L O T=1, I 1,1,34 X, \cdot I P U N C H=1,111$

WRITE $(6,22) \mathrm{CHI}(1), \mathrm{CHI}(2)$

22 FORMAT $(1$, OFISSICN YIELDS ARE: CHII) $=\bullet, F 10.5,1$, $\left.X \quad 22 X,{ }^{\circ} C H I(2)=,, F 10.5\right)$

IF ( (KR.LE.2).AND. (IBC.EQ.1)) CALL ERROR(1,KR)

IF (KR.GT.25) CALL ERROR(2,KR)

IF (EPS 1.LT.1. OE-16) CALL ERROR $(6,1)$

IF (EPS2.LT.1.0E-16) CALL ERROR $(6,2)$

IF (EPS3.LT.1.0E-16) CALL ERROR $(6,3)$

IF (IBC.LT.1).OR, (IBC.GT.7)) CALL ERROR(7,IBC)

C DUMMY NURMAL VECTOR XU = UNITY. (FOR THE INTEGRATION FUNCTIONS)

DO $21 \quad I G=1,2$

DO $21 \quad I I=1,100$

$21 \times U(I G, I I)=1.0$

I T $\mathrm{CO}=2$

I TCL $=2$

IF (ITC.EQ.1) GO TO 23

I TCO $=0$

I T $C_{1}=1$

C

COUNTER OF THE NUMBER OF TYPE-NUMBERS OF EACH TF REGION:

23 NUMI IF $=1$

$H X=0.0$

C

BEGIN TO READ IN THE TF REGICN DATA AND FILL THE ARRAYS, DEPENDING ON THE TYPE-NUMBER OF EACH TF REGION.

DO $50 \quad I=1, K R$

IF (ITF(I).EQ.NUMITF) GO TO 110

C

FILL THE ARRAYS FROM OLD TF REGICN TYPES: $J=I T F(I)$

CALL REPEAT (I,KTF(J))

GO TU 50

SYNT 0074

SYNT 0075

SYNT 0076

SYNT 0077

SYNTOC78

SYNT0079

SYNT 0080

SYNT 0081

SYNT 0082

SYNT 0083

SYNT 0084

SYNT 0085

SYNT 0086

SYNTOC87

SYNT0088

SYNTOC89

SYNT0090

SYNT0091

SYNT0092

SYNT 0093

SYNT 0094

SYNT0095

SYNT 0096

SYNT 0097

SYNT CO98

SYNT 0099

SYNTO100

SYNTO101

SYNT 0102

SYNTO103

SYNTO104

SYNT 0105

SYNTO 106

SYNT 0107

SYNTO108

PAGE 247 
READ THE SUBREgION NUMBER AND THE NUMBER OF REgIONS IN THE SUBREgION: READ (5,1) K, KS

IF (KS.GT.100) CALL ERROR (3,I)

CHECK FUR IMPROPER SEQUENCING OF INPUT DATA:

C

IF (I.NE.K) CALL ERROR $(4, I)$

READ IN THE GEOMETRY AND THE MATERIAL PROPERTIES:

C READ $(5,3)(X(J), X(J+1), H(J), A(1, J), F(1, J), D(1, J), S(1, J)$, $X$ $A(2, J), F(2, J), D(2, J), J=1, K S)$

3 FORMAT (3F10.5,4010.3,/,30X,3010.3) READ IN THE REGIONAL GROUP TRIAL FUNCTIONS: KS $1=K S+1$

$\operatorname{READ}(5,4)(\operatorname{PHI}(1, \mathrm{~J}), \operatorname{CUR}(1, \mathrm{~J}), \operatorname{PHI} 7(1, \mathrm{~J}), \operatorname{CUR} 7(1, \mathrm{~J}), \mathrm{J}=1, K S 1)$

$\operatorname{READ}(5,4)(\operatorname{PHI}(2, J), \operatorname{CUR}(2, J), \operatorname{PHI} 7(2, \mathrm{~J}), \operatorname{CUR} 7(2, J), J=1, K S 1)$

4 FORMAT (4020.7)

IF (ITW.EQ.1) GO TO 120

C

FORM WEIGHTING FUNCTIONS FROM THE GIVEN FUNCTIONS:

DO 119 IG $=1,2$

DO $119 \mathrm{~J}=1, \mathrm{KS} 1$

PHI $I(I G, J)=P H I(I G, J)$

$119 \operatorname{CURT}(I G, J)=-\operatorname{CUR}(I G, J)$

C

120 IF (ITC.EQ. L) GO TO 5 FORM THE REGION CONSTANT CURRENTS:

DO $7 \quad I G=1,2$

DO $6 \mathrm{~J}=1, \mathrm{KS}$

CUR $(I G, J)=-D(I G, J) *(-P H I(I G, J)+P H I(I G, J+1)) / H(J)$

$6 \operatorname{CUR7}(I G, J)=+D(I G, J) *(-P H I 7(I G, J)+P H I 7(I G, J+1)) / H(J)$

CUR $(I G, K S I)=0.0$

$7 \operatorname{CURT}(I G, K S \perp)=0.0$

C

WRITING OUT THE INPUT INFCRNATICA:

5 IF (ISEE.EQ.0) GO TO 14

WRITE $(6,10) \quad K, K R, K S,(J, X(J), X(J+1), H(J), A(1, J), F(1, J), D(1, J)$,

$X \quad S(1, J), A(2, J), F(2, J), D(2, J), J=1, K S)$

10 FORMAT (IINPUT MATERIAL PROPERTIES FOR REGION NUMBER ", I3,

SYNTO109

SYNTO110

SYNTO111

SYNT 0112

SYNTO 113

SYNT 0114

SYNTO115

SYNTO116

SYNTO117

SYNT 0118

SYNTO119

SYNTO120

SYNTO121

SYNT 0122

SYNT 0123

SYNT 0124

SYNT 0125

SYNT0126

SYNTO127

SYNT 0128

SYNTO129

SYNT 0130

SYNT0131

SYNTO 132

SYNT 0133

SYNTO134

SYNT 0135

SYNTO136

SYNT 0137

SYNT0138

SYNT 0139

SYNT 0140

SYNT 0141

SYNTO142

SYNT 0143

SYNTO144

PAGE 248 
$\mathrm{C}$

$X \quad \because$ OF THE $, 13,1$ USED.', $/ 1$,

$X$ $5 X, \cdot T H I S$ REGION IS DIVIDED INTO $, 13, \cdot$ HOMOGENEOUS SUBREGIONS $A$ XS FOLLOWS:, $1 /$,

$X$ $5 X$, 'FAST GROUP CONSTANTS APPEAR FIRST:, $1 /$,

$X$. SUBREGION \#",5X,'INTERNAL BOUNDARIES , 10X, 'WIDTH',3X,

$X$ TOTAL CX $(1 / C M) \cdot, 3 X,{ }^{\prime}$ FISSICN CX $(1 / C M), 6 X,{ }^{\circ}$ IFFUSION $(C M)^{\prime}$,

$x \quad 4 X, ' S C A T T . C X(1 / C M) \cdot, 1$,

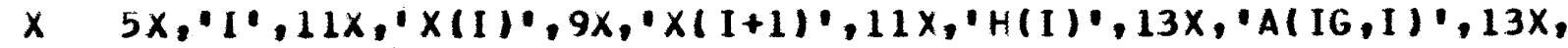

$X \quad \cdot F(I G, I) \cdot, 13 x, \cdot D(I G, I) \cdot, 14 x, \cdot S(1, I) \cdot, / /$,

C

$(16,3 F 15.4,4020.8,1,51 X, 3020.8))$

DO $15 \quad$ IG $=1,2$

15 WRITE $(6,11)$ IG,K,KR, $(J, X(J), P H I(I G, J), C U R(I G, J), P H I T(I G, J)$, $X \quad \operatorname{CUR7}(16, J), J=1, K S 1)$

11 FORMAT 1 'IINPUT TRIAL FUNCTIONS FOR GRCUP',12,' FOR REGION', I3,

$X$ - OUT OF THE , I3,' USED:,$/ /$,

$X$ ' INDEX',5X, 'COORD',16X, 'FLUX',13X, 'CURRENT',8X,' WEIGHT FLUX',

$X$ 5X, 'EIGHT CURRENT', //, (16,F 10.5,4020.7))

14 CONT INUE

C

END OF THE IN-OUT SECTION:

DEFINING MISC. ARRAYS FOR THE INTEGRATION FUNCTIONS:

LEGNTH OF THE SUBREGION: HT

$H T=X(K S 1)-X(1)$

$H R(K)=H T$

$H X=H X+H R(K)$

C

INVERSE JF THE D ARRAYS:

DO $13 \mathrm{~J}=1, \mathrm{KS}$

$D I(1, J)=1 . / D(1, J)$

C

$13 D I(2, J)=1.10(2, J)$

FORMATION OF THE INTEGRATICN FUNCTICNS:

CALL BHSET (KS)

C DO FOR ALL ENERGY GROUPS:

DO $50 \quad I G=1,2$

$K A O(I G, K)=G I F(0, I G, P H I 7, I G, A, P H I, K S, 2)$

$K A I(I G, K)=G I F(1, I G, P H I 7, I G, A, P H I, K S, 2)$

$K A 2(I G, K)=G I F(2, I G, P H I 7, I G, A, P H I, K S, 2)$
SYNT 0145

SYNTO146

SYNTO147

SYNTO 148

SYNTO149

SYNT0150

SYNTO151

SYNT0152

SYNTO153

SYNT 0154

SYNTO 155

SYNTO156

SYNTO157

SYNT 0158

SYNTO 159

SYNT 0160

SYNTO161

SYNT 0162

SYNTO 163

SYNT 0164

SYNT 0165

SYNT 0166

SYNT 0167

SYNT0168

SYNT0169

SYNT 0170

SYNTO171

SYNT 0172

SYNT 0173

SYNTO 174

SYNT 0175

SYNT 0176

SYNTO177

SYNTO178

SYNTO179

SYNTO 180

PAGE 249 
$K B O(I G, K)=G I F(0, I G, P H I 7, I G, F, P H I, K S, 2)$

SYNTO181

$K B I(I G, K)=G I F(1, I G, P H I 7, I G, F, P H I, K S, 2)$

SYNTO 182

$K B 2(I G, K)=G I F(2, I G, P H I 7, I G, F, P H I, K S, 2)$

SYNTO183

$L A O(I G, K)=G I F(O, I G, C U R 7, I G, D I, C U R, K S, I T C O)$

$L A I(I G, K)=G I F(1, I G, C U R 7, I G, D I, C U R, K S, I T C O)$

$L A 2(I G, K)=G I F(2, I G, C U R 7, I G, D I, C U R, K S, I T C O)$

$P(I G, K)=G I F(0, I G, P H I 7, I G, X U, C U R, K S, I T C I) / H T$

$P(1(G, K)=G I F(1, I G, P H I 7, I G, X U, C U R, K S, I T C 1) / H T$

$Q(I G, K)=G I F(0, I G, P H I, I G, X U, C U R 7, K S, I T C 1) / H T$

$Q 1(I G, K)=G I F(I, I G, P H I, I G, X U, C U R 7, K S, I T C 1) / H T$

$R(I G, K)=G I F(0, I G, P H I T, I G, D, P H I, K S, 2) / H T * * 2$

STORE THE TERMINAL POINTS FOR LATER USE:

$P O(I G, K)=P H I(I G, 1)$

POT $(I G, K)=P H I T(I G, 1)$

$P H(I G, K)=P H I(I G, K S I)$

$\operatorname{PH} 7(I G, K)=P H I 7(1 G, K S 1)$

IF (K.EQ.1) $\quad \operatorname{CO}(I G)=\operatorname{CUR}(I G, 1)$

IF (NUMITF-1.EQ.ITF(KR).AND.ITC.EQ.C) CH(IG)=CUR(IG,KS)

IF (NUM ITF-I,EQ.ITF(KR). AND. ITC.EQ.1) CH(IG)=CUR(IG,KSI) FOR THE OFF DIAGCNAL MATRIX ELEMENTS:

IF $(I G . E Q .2)$. GO TO 50

$S R O(I G, K)=G I F(0,2, P H I 7,1, S, P H I, K S, 2)$

$S R 1(16, K)=G I F(1,2, P H I 7,1, S, P H I, K S, 2)$

$S R 2(I G, K)=G I F(2,2, P H I 7,1, S, P H I, K S, 2)$

$K C O(I G, K)=G I F(0,1, P H I 7,2, F, P H I, K S, 2)$

$K C 1(I G, K)=G I F(1,1, P H I 7,2, F, P H I, K S, 2)$

$K C 2(I G, K)=G I F(2,1, P H I 7,2, F, P H I, K S, 2)$

$K D O(I G, K)=G I F(0,2, P H I 7,1, F, P H I, K S, 2)$

$K D \perp(I G, K)=G I F(1,2, P H I 7,1, F, P H I, K S, 2)$

$K D 2(I G, K)=G I F(2,2, P H I 7,1, F, P H I, K S, 2)$

SYNTO184

SYNT 0185

SYNTO186

SYNTO187

SYNT 0188

SYNTO189

SYNT 0190

SYNT 0191

SYNT 0192

SYNT 0193

SYNTO194

SYNT 0195

SYNT 0196

SYNTO197

SYNT0198

SYNT 0199

SYNT 0200

SYNT0201

SYNTO202

SYNT 0203

SYNT0204

SYNT0205

SYNT0206

SYNT0207

SYNTO208

SYNT0209

SYNTO210

SYNT0211

SYNTO212

NUMI TF $=$ NUMI TF -1

WRI TE $(0,51)$ NUMITF

51 FORMAT ('1THERE ARE ONLY', I3," DIFFERENT TRIAL FUNCTION REGIONS.') WRITE $(6,52)$ (I, ITF $(I), I=1, K R)$

52 FORMAT $/ /$, OTABLE OF THE TRIAL FUNCTICN NUMBER TYPES:,$/ /$,

SYNT0213

SYNTO2 14

SYNTO215

SYNT0216 
$X$ 3X, TF REGION',4X, 'REGION TYPE-NUMBER', $/$,

IF (ISEE.GE.2) CALL PRTOUT (1) $N N=K R$ NN IS THE MATRIX BLOCK SIZE:

IF $(I B C \cdot E Q \cdot 1, O R, I B C, G E \cdot 6), N N=K R-1$

IF $(1 B C \cdot E Q .4) \quad N N=K R+1$

SYNT0218

SYNT 0219

SYNT 0220

SYNT 0221

SYNT 0222

SYNT 0223

SYNT 0224

SYNT 0225

SYNT 0226

SYNT 0227

SYNT 0228

SYNT 0229

SYNT 0230

SYNT0231

SYNTO232

SYNT 0233

SYNT 0234

SYNT 0235

SYNT0236

SYNT0237

SYNT 0238

SYNT 0239

SYNT 0240

SYNT 0241

SYNT 0242

SYNT 0243

SYNT 0244

SYNT 0245

SYNT 0246

SYNT 0247

SYNT 0248

SYNT 0249

SYNT 0250

SYNT 0251

SYNT 0252

PAGE 251 
$B T(K)=S R 2(1, J) /(P H 7(2, J) * P H(1, J))$

$x+(S R O(1, K)-2, * S R 1(1, K)+S R 2(1, K)) /(P O 7(2, K) * P O(1, K))$

SYNTO253

$B F(3, K)=K C 2(I G, J) /(P H 7(1, J) * P H(2, J))$

$X+(K C O(I G, K)-2 . * K C 1(I G, K)+K C 2(I G, K)) /(P O 7(1, K) * P O(2, K))$

$B F(4, K)=K D 2(I G, J) /(P H 7(2, J) * P H(1, J))$

$X+(K D O(I G, K)-2, * K D 1(I G, K)+K D 2(I G, K)) /(P O 7(2, K) * P O(1, K))$

$C T(K)=(S R 1(1, K)-S R 2(1, K)) /(P 07(2, K) * P H(1, K))$

$C F(3, K)=(K C 1(I G, K)-K C 2(I G, K)) /(P 07(1, K) * P H(2, K))$

$C F(4, K)=(K D 1(I G, K)-K D 2(I G, K)) /(P 07(2, K) * P H(1, K))$

60 CONTINUE

C
THE LERO FLUX COEFFS:

NONE NEEDED AS $F(1,1)$ AND $F(2,1)$ BOTH $=0.0$.

IF (IBC.EQ.1), GO TO 64

IF (.NOT. (IBC.EQ.5.OR.IBC.EQ.7)) GO TO 66

LERO FLUX COEFFICIENTS FOR NO TILTING ON THE LEFT:

DO $61 \quad I G=1,2$

$V(I G)=1 . /(P H 7(I G, 1) * P H(I G, 1))$

$V I(I G)=1 . /(P 07(I G, 2) * P O(I G, 2))$

$V 2(I G)=1 . /(P 07(I G, 2) * P H(1 G, 2))$

$B L O(I G)=(K A O(I G, 1)-L A O(I G, 1) * V(I G)+(K A O(I G, 2)-2$ **KAI $(I G, 2)$

$x+K A 2(I G, 2)-L A O(I G, 2)+2 * * L A 1(I G, 2)-L A 2(1 G, 2)+P(I G, 2)-P 1(I G, 2)$

$X \quad-Q(I G, 2)+Q 1(I G, 2)+R(I G, 2)) * V I(I G)$

$C L O(I G)=(K A 1(I G, 2)-K A 2(I G, 2)+L A 2(I G, 2)-L A 1(I G, 2)+P 1(I G, 2)$

$X \quad+Q(I G, 2)-Q 1(I G, 2)-R(I G, 2)) * V 2(I G)$

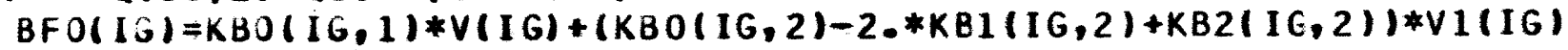

$61 C F O(I G)=(K B 1(1 G, 2)-K B 2(I G, 2)) * V 2(I G)$

BTO $(1)=\operatorname{SRO}(1,1) /(\operatorname{PH} 7(2,1) * P H(1,1))$

$X \quad+(S R O(1,2)-2, * S R 1(1,2)+S R 2(1,2)) /(P 07(2,2) * P 0(1,2))$

$B F O(3)=K C O(1,1) /(P H 7(1,1) * P H(2,1))$

$X+(K C O(1,2)-2, * K C 1(1,2)+K C 2(1,2)) /(P 07(1,2) * P O(2,2))$

$B F O(4)=K D O(1,1) /(P H 7(2,1) * P H(1,1))$

$X+(K O O(1,2)-2, * K D 1(1,2)+K C 2(1,2)) /(P 07(2,2) * P O(1,2))$

CTO 1$)=(\operatorname{SR} 1(1,2)-\operatorname{SR} 2(1,2)) /(\operatorname{PO}(2,2) * P H(1,2))$

$C F O(3)=(K C L(1,2)-K C 2(1,2)) /(P 07(1,2) * P H(2,2))$

$C F O(4)=(K D 1(1,2)-K D 2(1,2)) /(P O T(2,2) * P H(1,2))$
SYNTO 254

SYNT 0255

SYNT 0256

SYNT 0257

SYNT 0258

SYNT 0259

SYNT 0260

SYNT 0261

SYNTO262

SYNT0263

SYNT 0264

SYNTO265

SYNT 0266

SYNTO 267

SYNT 0268

SYNT 0269

SYNTO270

SYNT 0271

SYNT0272

SYNT 0273

SYNT 0274

SYNT0275

SYNT 0276

SYNT 0277

SYNT 0278

SYNT 0279

SYNT 0280

SYNT0281

SYNT 0282

SYNT 0283

SYNT 0284

SYNT 0285

SYNT 0286

SYNT 0287

SYNT 0288

PAGE 252 

$K=K R-1$

DO $67 \quad I G=1,2$

$V(I G)=1 . /(P H T(I G, K) * P O(I G, K))$

$V 1(I G)=1 . /(P O 7(I G, K R) * P O(I G, K R))$

$V 2(I G)=1 . /(P H 7(I G, K) * P H(I G, K))$

$A L(I G, K K)=(K A I(I G, K)-K A 2(I G, K)-L A I(I G, K)+L A 2(I G, K)-P(I G, K)$

$X$ $+P I(I G, K)-Q \perp(I G, K)-R(I G, K)) * V(I G)$

$B L(I G, K R)=(K A O(I G, K R)-L A O(I G, K R)) * V 1(I G)+(K A 2(I G, K)-L A 2(I G, K)$

SYNTO291

SYNT 0292

SYNTO293

SYNTO294

SYNTO295

SYNTO296

SYNT 0297

SYNT 0298

SYNT 0299

$A F(I G, K R)=(K B 1(I G, K)-K B 2(I G, K)) * V(I G)$

$B F(I G, K R)=K B O(I G, K R) * V 1(I G)+K B 2(I G, K) * V 2(I G)$

IF $(I G . E Q .2)$ GO TO 67

AT $(K R)=(S R 1(I G, K)-S R 2(I G, K)) /(P H 7(2, K) * P O(1, K))$

$A F(3, K K)=(K C 1(I G, K)-K C 2(I G, K)) /(P H 7(1, K) * P O(2, K))$

$A F(4, K R)=(K D 1(I G, K)-K D 2(1 G, K)) /(P H 7(2, K) * P O(1, K))$

$B T(K R)=S R O(I G, K R) /(P O 7(2, K R) * P O(1, K R))$

$X$ $+S R 2(I G, K) /(P H 7(2, K) * P H(1, K))$

$B F(3, K R)=K C O(I G, K R) /(P O 7(1, K R) * P O(2, K R))$

$X \quad+K C 2(I G, K) /(P H 7(1, K) * P H(2, K))$

$B F(4, K R)=K D O(I G, K R) /(P O 7(2, K R) * P O(1, K R))$

$X \quad+K D 2(I G, K) /(P H 7(2, K) * P H(1, K))$

67 CONTINUE

GO TO O4

C THE ZERO CURRENT COEFFS:

$69 K=K R$

DO $62 \quad I G=1,2$

$V(I G)=1 . /(P H 7(I G, K) * P O(I G, K))$

$V 1(I G)=1 . /(P H 7(I G, K) * P H(I G, K))$

$A L K(I G)=(K A I(I G, K)-K A 2(I G, K)-R(I G, K)+P I(I G, K)-P(I G, K)+L A 2(I G, K)$

$-L A I(I G, K)-Q I(I G, K)) * V(I G)$

$B L K(I G)=(K A 2(I G, K)+R(I G, K)-P 1(I G, K)-L A 2(I G, K)+Q 1(I G, K)) * V 1(I G)$

$X \quad+C H(I G) / P H(I G, K R)$

$A F K(I G)=(K B 1(1 G, K)-K B 2(I G, K)) * V(I G)$

$62 B F K(I G)=K B 2(1 G, K) * V 1(I G)$

SYNT 0300

SYNTO301

SYNT 0302

SYNT 0303

SYNT 0304

SYNT 0305

SYNT0306

SYNT0307

SYNT 0308

SYNT0309

SYNT 0310

SYNT0311

SYNT 0312

SYNT0313

SYNT 0314

SYNT 0315

SYNT 0316

SYNT0317

SYNT0318

SYNT 0319

SYNTO 320

SYNT 0321

SYNT 0322

SYNT 0323

SYNT 0324

PAGE 253 
$\operatorname{ATK}(1)=(\operatorname{SR} 1(1, K)-\operatorname{SR} 2(1, K)) /(\operatorname{PH} 7(2, K) * \operatorname{PO}(1, K))$

SYNT 0330

IF (IBC.NE.4) GO TO 64

$$
K=1 \text { ZERU CURRENT ON THE LEFT COEFFS: }
$$$$
\text { DO } 63 \quad I G=1,2
$$

$V 2(I G)=1 . /(P 07(I G, K) * P O(I G, K))$

$V 3(I G)=1.1(P 07(I G, K) * P H(I G, K))$

$B L O(I G)=$

$X+(K A O(I G, K)-2 . * K A 1(I G, K)+K A 2(I G, K)-L A O(I G, K)+2$ * LA I (IG,K)

$X \quad-L A 2(I G, K)+Q 1(I G, K)-Q(I G, K)+P(I G, K)-P 1(I G, K)+R(I G, K)) * V 2(I G)$

$X \quad-\operatorname{CO}(1 G) / P O(1 G, 1)$

$C L O(I G)=(K A I(I G, K)-K A 2(I G, K)-R(I G, K)+L A 2(I G, K)-L A I(I G, K)+Q(I G, K)$ $-Q 1(I G, K)+P I(I G, K)) * V 3(I G)$ $B F O(I G)=$ $V 2(I G) *(K B O(I G, K)-2 . * K B 1(I G, K)+K B 2(I G, K))$

$63 C F O(I G)=(K B I(I G, K)-K B 2(I G, K)) * V 3(I G)$

BTO $(1)=(\operatorname{SRO}(1, K)-2, * \operatorname{SR} 1(1, K)+\operatorname{SR} 2(1, K)) /(P 07(2, K) * P O(1, K))$

$B F O(3)=(K C O(1, K)-2 * * K C 1(1, K)+K C 2(1, K)) /(P O 7(1, K) * P O(2, K))$ $B F O(4)=(K D O(1, K)-2, * K D 1(1, K)+K D 2(1, K)) /(P O 7(2, K) * P 0(1, K))$ CTO $(1)=(S R 1(1, K)-S R 2(1, K)) /(P O 7(2, K) * P H(1, K))$ $C F O(3)=(K C 1(1, K)-K C 2(1, K)) /(P 07(1, K) * P H(2, K))$

$640065 \quad I=1, N N$

DO $65 \mathrm{~J}=1, \mathrm{NN}$

$L 1(1, J)=0.0$

$L 2(I, J)=0.0$

$F 1(I, J)=0.0$

$F 2(I, J)=0.0$

$F 3(I, J)=0.0$

$F(I, J)=0.0$

SYNT0331

SYNT0332

SYNT0333

SYNTO 0334

SYNT 0335

SYNT0336

SYNT 0337

SYNT 0338

SYNT 0339

SYNTO340

SYNT 0341

SYNT 0342

SYNTO343

SYNTO344

SYNT 0345

SYNTO346

SYNT 0347

SYNT 0348

SYNT 0349

SYNT 0350

SYNT 0351

SYNTO 352

SYNTO353

SYNTO 354

SYNT 0355

SYNTO356

SYNT 0357

SYNT0358

SYNT0359

$65 \quad T(I, J)=0.0$ 
C FILLING THE MATRICES FOR POWER:

SYNT 0361

SYNT 0362 $J=1$

DETERMINE THE LEFT BC:

SYNT 0363

If $(I B C \cdot L E \cdot 2 \cdot O R \cdot I B C \cdot E Q \cdot 6)$ GC TC 75

SYNT0364

$L 1(J, 1)=B L O(1)$

$L 2(J, 1)=B L O(2)$

$F 1(J, 1)=B F O(1)$

$F 2(J, 1)=B F O(2)$

$F 3(J, 1)=B F J(3)$

$F 4(J, 1)=B F O(4)$

$T(J, 1)=B T O(1)$

$\operatorname{LI}(J, 2)=C L O(1)$

$L 2(J, 2)=C L O(2)$

$F 1(\mathrm{~J}, 2)=C F O(1)$

$F 2(J, 2)=C F O(2)$

$F 3(J, 2)=C F O(3)$

$\mathrm{F} 4(\mathrm{~J}, 2)=\mathrm{CFO}(4)$

$T(J, 2)=C T O(1)$

$\mathrm{J}=\mathrm{J}+1$

C

FOR ALL INTERNAL EQUATIONS:

75 DO $70 K=I X, I Y$

IF (J.EQ.1) GO TO 76

$L 1(J, J-1)=A L(1, K)$

$L 2(J, J-1)=A L(2, K)$

$F(1, J-1)=A F(1, K)$

$F 2(J, J-1)=A F(2, K)$

$F 3(J, J-1)=A F(3, K)$

$F 4(J, J-1)=A F(4, K)$

$T(J, J-1)=A T(K)$

$76 L(1(J, J)=B L(1, K)$

$L I(J, J+1)=C L(1, K)$

$L 2(J, J)=B L(2, K)$

$L 2(J, J+1)=C L(2, K)$

$F(J, J)=B F(1, K)$

$F 1(J, J+1)=C F(1, K)$

$F 2(J, J)=B F(2, K)$

SYNT0365

SYNT0366

SYNT 0367

SYNT 0368

SYNT0369

SYNT 0370

SYNT 0371

SYNT 0372

SYNT 0373

SYNT0374

SYNT0375

SYNT 0376

SYNT0377

SYNT0378

SYNT0379

SYNT 0380

SYNT0381

SYNT 0382

SYNT 0383

SYNT 0384

SYNT 0385

SYNT0386

SYNT 0387

SYNT 0388

SYNT 0389

SYNT 0390

SYNT 0391

SYNT 0392

SYNT0393

SYNT 0394

SYNT 0395

SYNT 0396

PAGE 255 
$F 2(J, J+1)=C F(2, K)$

SYNT0397

$F 3(J, J)=B F(3, K)$

$F 3(J, J+1)=C F(3, K)$

SYNT 0398

SYNTO399

$F 4(J, J)=B F(4, K)$

SYNT 0400

$F 4(J, J+1)=C F(4, K)$

SYNT 0401

$T(J, J)=B T(K)$

$T(J, J+1)=C T(K)$

SYNT 0402

SYNT 0403

$\mathrm{J}=\mathbf{J}+\mathbf{1}$

70 CONT INUE

IF (IBC.EQ.1.OR.IBC.EQ.5) GO TO 500

SYNT 0404

SYNT 0405

SYNT0406

SYNT 0407

SYNT 0408

SYNT 0409

$=N N$

$L 1(K, K-1)=A L K(1)$

$L 1(K, K)=B \operatorname{LK}(1)$

$L 2(K, K-1)=A L K(2)$

$L 2(K, K)=B L K(2)$

$F 1(K, K-1)=\operatorname{AFK}(1)$

$F 1(K, K)=B F K(1)$

$F 2(K, K-1)=\operatorname{AFK}(2)$

$F 2(K, K)=B F K(2)$

$F 3(K, K-1)=A F K(3)$

$F 3(K, K)=B F K(3)$

$F 4(K, K-1)=A F K(4)$

$F 4(K, K)=B F K(4)$

$T(K, K-1)=A T K(1)$

$(K=N N)$ :

SYNT 0410

SYNT 0411

SYNT 0412

SYNT0413

SYNT0414

SYNT0415

SYNT0416

SYNT0417

SYNT0418

SYNT0419

SYNT0420

SYNT 0421

SYNT 0422

SYNT 0423

SYNT 0424

SYNT 0425

SYNT 0426

SYNT 0427 
1 WRITE $(6,101)$

$$
\text { GO TO } 10
$$

2 WRITE $(6,102) \mathrm{J}$ GO TO 10

3 WRITE $(6,103) \mathrm{J}$ GO TO 10

4 WRITE $(6,104) \mathrm{J}$ GO TO 10

5 WRITE $(6,105) \mathrm{J}$ GO TO 10

6 WRITE $(6,106) \mathrm{J}$ GO TO 10

7 CONTINUE

8 CONTINUE

9 CONT INUE

10 WRITE $(6,110)$

101 FORMAT ('IMUST HAVE $>2$ SUBREGICNS FCR ZERO FLUX B.C.S. INVALID.")

102 FORMAT ('INUMBER OF SUBREGIONS $=\cdot, 13,{ }^{\prime}>25$. INVALID.")

103 FORMAT ('ISUBREGICN NUMBER', 13," HAS > 25 SECTIONS. INVALID.')

104 FORMAT $1 \cdot 1$ INPUT ERROR IN REGICN SEQUENCING AT REGION',I5,', )

105 FORMAT $(\circ 12(1)=0$. IN REGICN I $=1,13, \circ$ INVALID.')

106 FORMAT (1THE TOLERANCE: EPS , 11, IS $<1.0$ I 16. INVAL I0.")

107 FORMAT $\left(\cdot 18\right.$ OUNDRY CONDITION OPTICN $=1,12,1^{\prime}<1$ OR > 7 . INVALID.')

110 FORMAT (IHO,'PROBLEM TERMINATEC.')

\section{CALL EXIT}

RETURN

END

ERR00006

ERRO0007

ERRO0008

ERROO009

ERRO0010

ERRO0011

ERROOO 12

ERRO0013

ERRO0014

ERRO0015

ERRO0016

ERRO0017

ERRO0018

ERRO0019

ERRO0020

ERRO0021

ERRO0022

ERRO0023

ERRO0024

ERRO0025

ERRO 0026

ERRO0027

ERRO0028

ERRO0029

ERRO0030 
SUBROUTINE REPEAT $(K, L)$

SETS THE /BS/ ARRAYS (K) EQUAL TO PAST STORED ARRAYS (L): IMPLICIT REAL*8 (A-Z)

COMMON /B5/ KAO $(2,25), \operatorname{KAl}(2,25), \operatorname{KA} 2(2,25), K B O(2,25), K B 1(2,25)$,

$x$

$x$
$x$

$x$
$x$

$x$

$x$

$\mathrm{X}$

$K B 2(2,25), \operatorname{LAU}(2,25), \operatorname{LA} 1(2,25), \operatorname{LA} 2(2,25), \operatorname{SRO}(1,25)$, $\operatorname{SR} 1(1,25), \operatorname{SR} 2(1,25), K C O(1,25), K C 1(1,25), K C 2(1,25)$, KDO $(1,25), K D 1(1,25), K D 2(1,25)$,

$P(2,25), P 1(2,25), Q(2,25)$,

$Q 1(2,25), R(2,25), P O(2,25), P 07(2,25), P H(2,25)$, PH7 $(2,25)$

COMMON /XAXIS/ HX,HR(25)

INTEGER $K, L, G$

$H R(K)=H R(L)$

$H X=H X+H R(K)$

DO $10 \quad G=1,2$

$K A O(G, K)=K A O(G, L)$

$K A I(G, K)=K A I(G, L)$

$K A 2(G, K)=K A 2(G, L)$

$K B O(G, K)=K B O(G, L)$

$K B 1(G, K)=K B 1(G, L)$

$K B 2(G, K)=K B 2(G, L)$

$L A O(G, K)=L A O(G, L)$

$L A 1(G, K)=L A 1(G, L)$

$L A 2(G, K)=L A 2(G, L)$

IF (G.EQ.2) GO TO 5

$S R O(G, K)=\operatorname{SRO}(G, L)$

$S R 1(G, K)=S R I(G, L)$

$S R 2(G, K)=S R 2(G, L)$

$K C O(G, K)=K C O(G, L)$

$K C \perp(G, K)=K C 1(G, L)$

$K C 2(G, K)=K C 2(G, L)$

$K D O(G, K)=K D O(G, L)$

$K D I(G, K)=K D 1(G, L)$

$K D 2(G, K)=K D 2(G, L)$

5 CONTINUE

$P(G, K)=P(G, L)$

REPE0001

REPE0002

REPE 0003

REPE0004

REPE0005

REPE0006

REPE0007

REPE0008

REPE0009

REPE0010

REPE0011

REPE0012

REPE0013

REPE0014

REPE0015

REPEO016

REPE0017

REPE0018

REPE 0019

REPE0020

REPE0021

REPE0022

REPE 0023

REPE0024

REPE 0025

REPE0026

REPE 0027

REPE 0028

REPE0029

REPE0030

REPE0031

REPE0032

REPE 0033

REPE0034

REPE 0035

REPE0036

PAGE 258 
$P 1(G, K)=P 1(G, L)$

$Q(G, K)=Q(G, L)$

$Q 1(G, K)=Q 1(G, L)$

$R(G, K)=R(G, L)$

$P O(G, K)=P O(G, L)$

$P 07(G, K)=P 07(G, L)$

$P H(G, K)=P H(G, L)$

$P H 7(G, K)=P H 7(G, L)$

10 CONT INUE

RETURN

END

REPE0039

REPE0040

REPE0041

REPE0042

REPE 0043

REPE0044

REPE 0045

REPE0046

REPE 0047 
SUBROUTINE BHSET(K)
C SETS UP THE /BH/ ARRAYS FOR GIF:

SETS UP THE /BH/ ARRAYS FCR GIF:
CPLICII REAL $* 8(A-H, L-Z)$

COMMON /BH/ X(101), H(101), Z(101)

DO $1 \quad I=1, K$

$1 Z(I)=X(I)-X(1)$

RETURN

END

BHSE 0001

BHSE 0002

BHSE0003

BHSE 0004

BHSE0005

BHSE0006

BHSE 0007

BHSE0008 
DOUBLE PRECISION FUNCTION GIF(N,G1,F,G2,C,G,K, ITC)

GIF 0001

INTEGRATES: $F(G 1, J) * C(G 2, J) * G(G 2, J) *(Z / H) * * N$

OVER ALL K SUBREGIONS J

WHERE $Z$ RUNS FRCM 0 TO $X(K+1)-X(1)$ IN THIS REGION.

WHERE THE FORM OF $F$ AND $G$ IN REGICN J IS GIVEN BY ITC:

ITC $=0:$ F AND G ARE BOTH CONSTANT.

ITC $=1:$ F IS LINEAR AND G IS CCNSTANT.

ITC $=2:$ F AND G ARE BOTH LINEAR.

IMPLICIT REAL*8 (A-H,O-Z)

COMMON /BH/ X(101),H(101),Z(101)

DIMENSION $F(2,101), G(2,101), C(2,100)$

INTEGER G1,G2

$N 1=N+1$

SUMJ $=0.000$

IF (ITC.EQ.O) GO TO 40

IF (ITC.EQ.1) GO TO 20

C

LINEAR F AND G IN REGIONS J:

DO $10 \quad J=1, K$

$A=F(G 1, J) * G(G 2, J)$

$B=F(G 1, J) * G(G 2, J+1)+F(G 1, J+1) * G(G 2, J)$

$D=F(G 1, J+1) * G(G 2, J+1)$

SUML $=0.000$

DO $5 \quad I=1, N 1$

$L=I-1$

$M=N-L$

IF (H(J).EQ.0.0.AND.L.EQ.O) GO TO 1

$E H=H(J) * * L$

GO TO 2

$1 E H=1.0$

2 IF $(Z(J) \cdot E Q \cdot 0.0 . A N D \cdot M . E Q .0)$ GO TO 3

$E Z=Z(J) * * M$

GO TO 4

$3 E Z=1.0$

4 SUML $=S U M L+F A C T(N) /(F A C T(M) * F A C T(L)) * E H * E Z *$

$X \quad(2 . D O * A / O F L J A T((L+3) *(L+2) *(L+1))+B / D F L O A T((L+3) *(L+2))$

$X \quad+D / D F L O A T(L+3))$

GIF 0002

GIF 0003

GIF 0004

GIF 0005

GIF 0006

GIF 0007

GIF 0008

GIF 0009

GIF 0010

GIF 0011

GIF 0012

GIF 0013

GIF 0014

GIF 0015

GIF 0016

GIF 0017

GIF 0018

GIF 0019

GIF 0020

GIF 0021

GIF 0022

GIF 0023

GIF 0024

GIF 0025

GIF 0026

GIF 0027

GIF 0028

GIF 0029

GIF 0030

GIF 0031

GIF 0032

GIF 0033

GIF 0034

GIF 0035

GIF 0036

PAGE 261 
5 CONT INUE

SUMJ $=S U M J+H(J) * C(G 2, J) * S U M L$

GIF 0037

GIF 0038

GIF 0039

GIF 0040

GO TO 100

C

LINEAR F AND CONSTANT G IN REGICAS J:

GIF 0041

GIF 0042

GIF 0043

GIF 0044

GIF 0045

GIF 0046

GIF 0047

GIF 0048

GIF 0049

GIF 0050

GIF 0051

GIF 0052

GIF 0053

GIF 0054

GIF 0055

GIF 0056

GIF 0057

GIF 0058

GIF 0059

GIF 0060

GIF 0061

GIF 0062

GIF 0063

GIF 0064

GIF 0065

GIF 0066

GIF 0067

GIF 0068

GIF 0069

GIF 0070

GIF 0071

52 IF $(Z(J) \cdot E Q \cdot 0 \cdot 0 . A N D . M . E Q .0)$ GO TO 53

GIF 0072

PAGE 262 
60 TO 54

$53 E Z=1.0$

54 SUML = SUML+FACT $(N) /(F A C T(M) * F A C T(L)) * E H * E Z$ *

$X$ (1./DFLOAT $(L+1))$

55 CONTINUE

SUMJ $=S U M J * H(J) * F(G 1, J) * C(G 2, J) * G(G 2, J) * S U M L$

50 CONTINUE

100 GIF $=$ SUMJ $/(X(K+1)-X(1)) * *$ iN

RETURN

END

GIF 0073

GIF 0074

GIF 0075

GIF 0076

GIF 0077

GIF 0078

GIF 0079

GIF 0080

GIF 0081

GIF 0082 
DOUBLE PRECISION FUNCTION FACT(N)

FACT 0001 COMPUTES N FACTORIAL:

FACT0002

$F A C T=1.0 D 0$

IF (N.LE.1) RETURN

DO $1 \quad I=2, N$

FACT 0003

FACT0004

1 FACT $=F A C T * D F L O A T(I)$

RETURN

FACT 0005

FACT0006

END

FACT 0007

FACT 0008 


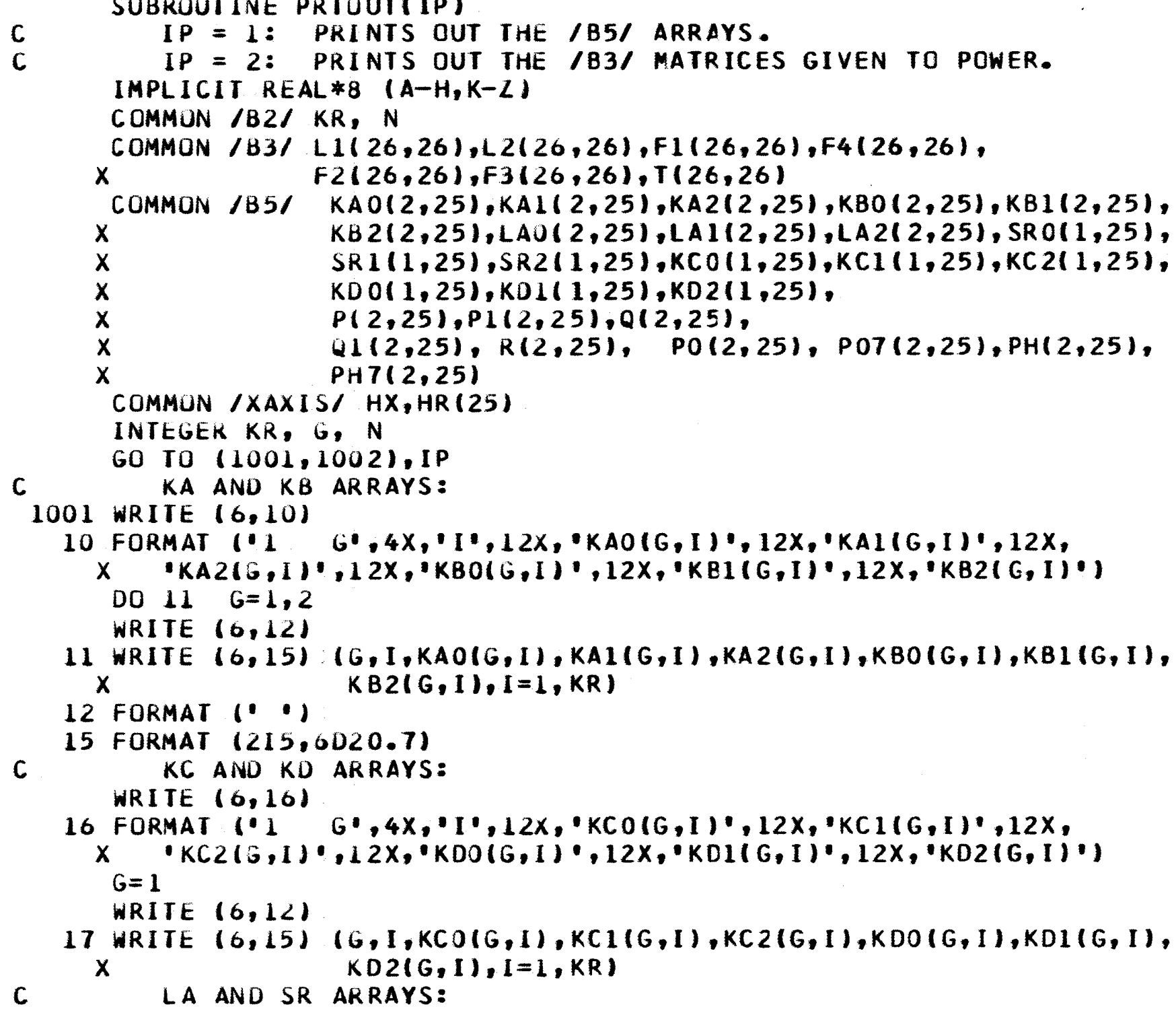

PRT00001

PRTO0002

PRTO0003

PRTO0004

PRTO0005

PRTO0006

PRTO0007

PRT00008

PRTO0009

PRTO0010

PRTO0011

PRTO0012

PRTO0013

PRT00014

PRTO0015

PRTO0016

PRTO0017

PRTO0018

PRTO0019

PRTO0020

PRTO0021

PRT00022

PRT T00023

PRTO0024

PRTO0025

PRTO0026

PRTO0027

PRTO0028

PRTO0029

PRTO0030

PRTO0031

PRTO0032

PRT00033

PRTO0034

PRTO0035

PRT00036

PAGE 265 
WR ITE $(6,20)$

20 FORMAT $(\cdot 1$ G',4X,'I',12X,'LAO(G,I)',12X,'LAI(G,I)',12X,

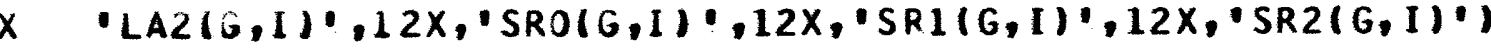
$G=1$ WRITE $(6,12)$ WRITE $(6,15)$ (G,I,LAO(G,I),LA1(G,I),LA2(G,I),SRO(G,I),SRI(G,I), $X$ $S R 2(G, I), I=1, K R)$

$G=2$

WRITE $(6,12)$

WRITE $(6,25)(G, I, L A O(G, I), L A 1(G, I), L A 2(G, I), I=1, K R)$

25 FORMAT $(215,3020.7)$

C

$P, Q, A N D R$ ARRAYS:

WRITE $(6,30)$

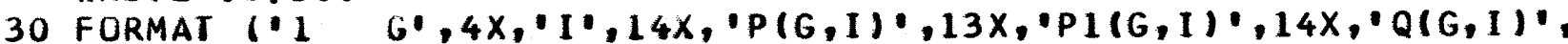
$X \quad 13 X, \cdot Q 1(G, I) \cdot, 14 X, \cdot R(G, I) \cdot)$

DO $31 G=1,2$

WRITE $(6,12)$

31 WRITE $(6,35)(G, I, P(G, I), P 1(G, I), Q(G, I), Q 1(G, I), R(G, I), I=1, K R)$

35 FORMAT $(215,5020.7)$

PO, PH, AND HR ARRAYS:

WRITE $(6,40)$

40 FORMAT $(\cdot 1$ G',4X,'I',13X,'PO(G,I)!,12X,'P07(G,I)!,13X,'PH(G,I)', $X \quad 12 X, \cdot P H 7(G, 1) \cdot, 15 X, " H R(I) \cdot)$

DO $41 G=1,2$

WRITE $(6,12)$

41. WRITE $(6,45)(G, I, P O(G, I), P O 7(G, I), P H(G, I), P H 7(G, I), H R(I), I=1, K R)$

45 FORMAT (2I5,5020.7)

GO TO 100

PRINT OUT THE /B3/ MATRICES:

1002 WRITE $(6,50)$

50 FORMAT ('IMATRIX L1:,$/)$

DO $51 \quad I=1, N$

51 WRIJE $(6,55)$ (L $1(I, J), J=1, N)$

55 FORMAT (1OD12.3, , (2X,10012.3))

WRITE $(0,60)$

60 FORMAT ('IMATRIX L2:,$/ 1)$
PRTO0037

PRTO0038

PRT00039

PRTO0040

PRTO0041

PRT00042

PRT00043

PRTO0044

PRTO0045

PRT00046

PRT00047

PRTO0048

PRT00049

PRT T00050

PRTO0051

PRTO0052

PRT00053

PR T00054

PRT00055

PRT00056

PR TO0057

PRT00058

PRT00059

PRT00060

PRTD0061

PRT00062

PRTO0063

PRT00064

PRTT00065

PRT00066

PRT00067

PRTO0068

PRT00069

PRTO007J

PRT00071

PRTO0072

PAGE 266 
DO $61 \quad I=1, N$

61 WRITE $(6,55)$ (L2(I,J),J=1,N)

PRT00073

PRTO0074

WRITE $(6,70)$

70 FORMAT ('IMATRIX FI:',/) DO $71 \quad I=1, N$

71 WRITE $(6,55)$ (F $1(I, J), J=1, N)$ WRITE $(6,80)$

80 FORMAT ('IMATRIX F2:,$/ /)$ DO $81 \quad I=1, N$

81 WRITE $(6,55) \cdot(F 2(I, J), J=1, N)$ WRITE $(6,82)$

82 FORMAT ('IMATRIX F3: ',/) DO $83 \quad I=1, N$

83 WRITE $(6,55) \quad(F 3(I, J), J=1, N)$ WR ITE $(6,84)$

84 FORMAT ('IMARTIX F4:",/) DO $85 \quad I=1, N$

85 WRITE $(6,55)$ (F4 (I,J),J=1,N) WRITE $(6,90)$

90 FORMAT ('IMATRIX T:',/) DO $91 \quad I=1, N$

91 WRITE $(6,55)$ (T (I,J), J=I,N)

100 RETURN

END

PRTO0075

PRTO0076

PRTO0077

PR T00078

PRTO0079

PRT00080

PRTO0081

PRTO0082

PRTO0083

PRTO0084

PRT 00085

PRTO0086

PRTO0087

PRT 00088

PRTO0089

PRTO0090

PRTO0091

PRT00092

PRT00093

PR T00094

PRTOC095

PRT00096 
SUBROUTINE POWER

C SOLVES THE 2*N MULTIGROUP EQUATICNS: M*PHI = (1/LAMDA)*F*PHI

BY THE FISSION SOURCE POWER METHOD

USING SIMULTANEOUS OVERRELAXATICN.

WHERE: M AND F ARE DOUBLE PRECISION 2N BY 2N BLOCK MATRICES;

AND:

PHI IS THE 2N FLUX (FAST ANC THERMAL) VECTCR.

$L 1 * P H I 1=C H I 1 *(F 1 * P H I 1+F 2 * P H I 2)$

$-T * P H I 1+L 2 * P H I 2=C H I 2 *(F 3 * P H I 1+F 4 * P H I 2)$

METHOD FOLLOWS WACHPRESS, PAGE 83. SOLUTION BY GROUP ITERATION.

IMPLICIT REAL*8 (A-H,L-Z)

COMMON /BI/ IBC, IPLOT,JPLOT, IPUNCH, ISEE

COMMUN /B2/ KR, N

COMMUN /B3/ L $1(26,26), L 2(26,26), F 1(26,26), F 4(26,26)$,

$x$ $F 2(26,26), F 3(26,26), T(26,26)$

COMMON /B4/ PHI $(2,26)$, PSI $(2,26)$, LAMDA, ICOUT

COMMON /B5/S(26), ERROR $(2,26), 2(26)$

COMMON /B6/ TEL $(2,5), \operatorname{TE} 2(2,5), \operatorname{TE} 3(5), \operatorname{IN}(5)$

COMMON /CHIFI CHI (2)

COMMON /XAXIS/ HX,HR (25)

COMMON /ER/ EPS 1,EPS2,EPS3

COMMON /FSTR/ PHISTR $(2,26,6)$

COMMON IESTR/ LAMSTR $(300)$, EFSTR $(2,300)$, EFMSTR $(2,300)$, ERLAM(300)

COMMON /TRUEJ TRULAM, TRUPHI $(2,26)$, PHICON(2,300), LAMCON(300), IFT

DIMENSION PSI $1(26)$, PSI $2(26)$, SQ $(2)$, DPHI $(2)$, ERRMAX $(2)$

INTEGER N

DEFAULT OPTIONS FOR THE TRUE EIGENVALUE AND FLUXES:

TRULAM $=1.0$

DO $5 \quad I G=1,2$

DO $5 \quad I=1, N$

5 IRUPHI $(I G, I)=1.0$

DEFAULT OPTIONS FOR POHER PARAMETERS:

ALPHA $=1.25$

LAMDA $=1.0$

DO 555 IG $=1,2$

IF (IBC.NE.4) GO TO 551

DO $550 \quad I=1, N$

POWE0001

POWE 0002

POWE0003

POWE0004

POWE 0005

POWE0006

POWE0007

POWE0008

POWE 0009

POWE0010

POWE0011

POWE 0012

POWE0013

POWE0014

POWEOO15

POWE 0016

POWEO017

POWE 0018

POWE0019

POWE 0020

POWE0021

POWE0022

POWE0023

POWE0024

POWE0025

POWE0026

POWE0027

POWE 0028

POWE0029

POWE0030

POWE 0031

POWE0032

POWE0033

POWE 0034

POWE0035

POWE 0036 


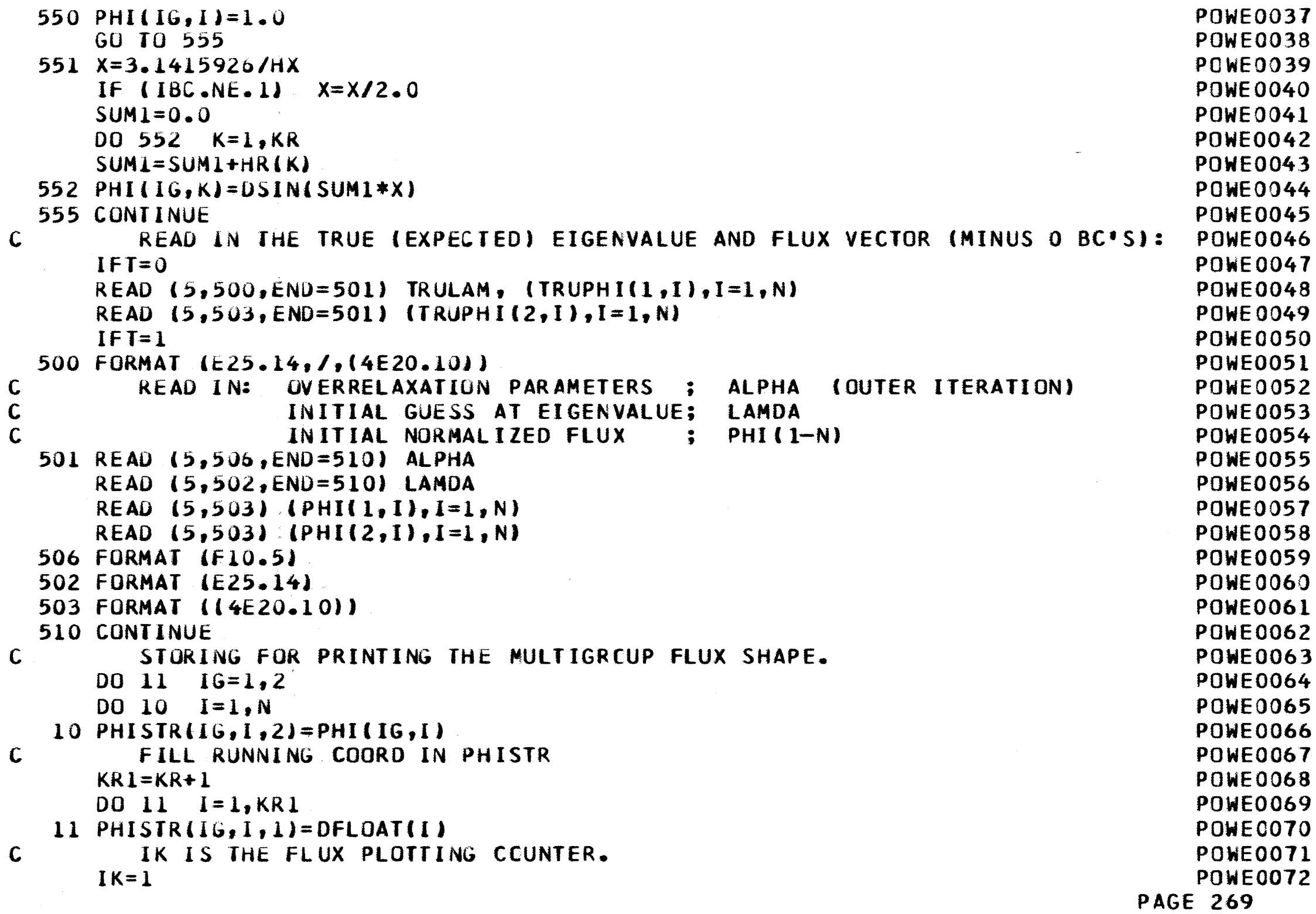

POWE0046

POWE 0047

POWE0048

POWE 0049

POWE0050

POWE0051

POWE0052

POWE 0053

POWE 0054

POWE 0055

POWE0056

POWE0057

POWE0058

POWE0059

POWE 0060

POWE0061

POWE0062

POWE0063

POWE0064

POWE0065

POWE0066

POWE0067

POWE0068

POWE0069

POWEC070

POWE0071

POWE0072 
$\operatorname{TE} 2(2,1)=0$.

$T E 3(1)=0.0$

C

EIGENVALUE OF THE PREVIOUS ITERATION:

LAMB 4= LAMDA

C THE MAXIMUM NUMBER OF ALLOWED ITERATIONS: ICMAX ICMAX $=300$

C PRINT OUT THE POWER METHOD PARAMETER INFORMATION:

WRITE $(6,700)$ I CMAX, ALPHA, LAMCA, (PHI(1,I), I=1,N)

WRITE $(6,701)(P H I(2, I), I=1, N)$

700 FORMAT ('IEXECUTING MULTIGROUP FISSION SOURCE POWER ITERATION METH $X O D \cdot,, / 1 /$,

$X \quad 5 X, \cdot M A X I M U M$ NUMBER OF ALLCWABLE ITERATIONS:,$/$,

$X \quad 10 X, 1$ ICMAX $=1,14,1 / 1$,

$X \quad 5 X, \circ$ OUTER ITERATION RELAXATICN PARAMETER:,$/$,

$X \quad 10 X, \cdot A L P H A=1, F 7.3,1 /$,

$X \quad 5 X$, INITIAL guess at EIGENVALUE:,$/$,

$X \quad$ LOX, "LAMBDA $=1, E 22.14,1 /$,

$\hat{x}$ 5X, INITIAL GUESS AT THE GROUP FLUX SHAPE CONNECTICN POINTS:",

$X \quad / /, 8 X, \cdot F A S T$ GROUP:,$/$,

$X \quad 10 X, \circ F(K) \cdot S=:, 4 E 25,14, /,(18 X, 4 E 25.14))$

701 FORMAT $(\cdot 0 \cdot, 7 X, \cdot$ THERMAL GROUP:, 1,

$X \quad 10 X, \cdot F(K) \cdot \because S=0,4 E 25.14, /,(18 X, 4 E 25.14))$

C BEGIN ITERATION LOOP. I COUT $=0$

C ICOUT IS THE OUTER ITERATICN COUNTER.

20 I COUT $=I$ COUT +1 IF (ICOUT.GT ICMAX) GO TO 100
SOLVE FOR THE NEW GROUP FLUX VECTORS: PSI:

C FAST GRJUP; SOURCE VECTOR:

DO $25 \quad I=1, N$

POWE0074

POWE 0075

POWE0076

POWE0077

POWE 0078

POWE0079

POWE0080

POWE0081

POWE0082

POWE0083

POWE0084

POWE0085

POWEO086

POWEO087

POWE0088

POWE0089

POWEO090

POWEOJ91

P0WE0092

POWE0093

POWE 0094

POWE 0095

POWE 0096

POWE 0097

POWE 0098

POWE 0099

POWEO 100

POWE0101

POWE 0102

POWEO103

POWE0104

POWE0105

POWE0106

POWE0107

POWE0108 
$S(I)=0.0$

DO $24 J=1, N$

$24 S(I)=S(I)+F I(I, J) * \operatorname{PHI}(I, J)+F 2(I, J) * \operatorname{PHI}(2, J)$

$25 \mathrm{~S}(I)=\mathrm{CHI}(1) * \mathrm{~S}(I)$

FAST FLUX:

C

CALL SOLV3D(N,LI,PSI1,S)

C

THERMAL GROUP; SOURCE VECTOR:

DO $27 \quad I=1, N$

$S(I)=0.0$

$Z(I)=0.0$

DO $26 \mathrm{~J}=1, \mathrm{~N}$

$S(I)=S(I)+F 3(I, J) * P S I I(J)+F 4(I, J) * P H I(2, J)$

$26 Z(I)=Z(I)+T(I, J) * P S I I(J)$

$27 Z(I)=Z(I)+C H I(2) * S(I)$

C THERMAL FLUX :

CALL SOLV3O(N,L2,PSI2,Z)

C

CALCULATION OF THE EIGENVALUE:

SUM $1=0.000$

SUM $2=0.000$

DO $28 \quad I=1, N$

SUM $2=S U M 2+P S I 1(I) * P S I 1(I)+P S I 2(I) * P S I 2$ (I)

$28 \operatorname{SUM} 1=\operatorname{SUM} 1+\operatorname{PSI} 1(I) * \operatorname{PHI}(1, I)+\operatorname{PSI} 2(I) * \operatorname{PHI}(2, I)$

LAMDA $=$ SUM $2 /$ SUM 1

LAMSTR (ICOUT $)=$ LAMCA

$E R R L A M=D A B S(L A M D A-L A M B 4)$

PUT PSI 1 AND PSI 2 INTO BIGGER PSI:

DO $30 \quad I=1, N$

$\operatorname{PSI}(1, I)=\operatorname{PSI} I(1)$

C

30 PSI $(2, I)=P S I 2(I)$

POINT BY POINT SIMULTANEQUS RELAXATION FLUX ITERATION: $X=A L P H A$

C DO NOT RELAX DURING THE FIRST THREE ITERATIONS:

IF (ICOUT . LE. 3) $X=1.0$

C CALCULATE THE NEW GROUP FLUX ITERATES AND GRCUP ERRORS:

DO $40 \quad I G=1,2$

DO $40 \quad I=1, N$

POWEOIO9

POWE0110

POWEOI11

POWE0112

POWE 0113

POWE0114

POWEO115

POWE 0116

POWE0117

POWE 0118

POWE0119

POWE 0120

POWE0121

POWE 0122

POVE0123

POWE0124

POWE0125

POWE 0126

POWEO127

POWE 0128

POWE0129

POWE 0130

POWE 0131

POWE0132

POWE0133

POWE 0134

POWE0135

POWE 0136

POWE0137

POWE 0138

POWE 0139

POWE0140

POWE 0141

POWE0142

POWE0143

POWE0144

PAGE 271 
40 PSI I IG,I $)=P H I(I G, I)+X *(P S I(I G, I) / L A M D A-P H I(I G, I))$

CALL NORMAL (PSI, N)

DO $39 \quad I G=1,2$

ERRMAX $(I G)=0.0$

$S Q(I G)=0.0$

DO $38 \quad I=1, N$

ERROR(IG,I) = DABS( (PSI(IG,I)-PHI (IG,I) /PSI (IG,I))

IF (ERRUR $(I G, I), G T \cdot E R R M A X(I G)) \quad \operatorname{ERRMAX}(I G)=\operatorname{ERROR}(I G, I)$

$S Q(I G)=S Q(I G)+E R R O R(I G, I) * * 2$

C UPUATE THE FLUX ITERATE:

38 PHI $(I G, I)=P S I(I G, I)$

$39 S Q(I G)=D S Q R T(S Q(I G))$

NORMALI LE PSI GROUPS TO UNITY:

C

CALL NORM2 (PSI, TRUPHI, N)

IF (IFT.EQ.O) GO TO 37

DLAM=LAMDA-TRUL AM

DO $36 \quad I G=1.2$

DPHI (IG) $=0.0$

DO $35 I=1, N$

35 DPHI (IG) $=$ DPHI (I G) +(PSI(IG,I )-TRUPHI $(I G, I)) * * 2$

36 DPHI (IG)=DSQRT (DPHI (IG))

37 IF (IPLOT.NE.2) GO TO 45

C THE FOLLJWING IS FOR NICELY PLOTTING THE GROUP FLUX HISTORY. $0041 \quad I G=1,2$

DO $41 \quad I=1, N$

C

41 ERROR (IG, I) = PS I (IG,I) ERROR NOW CONTAINS THE NEW NORMALIZED FLUX ITERATE PHI. $J K=I K$

IF (IK.EQ.O) JK=5

DO $42 \quad I G=1,2$

DO $42 \quad I=1, N$

IF (DA3S(ERROR(IG,I)-PHISTR(IG,I,JK+1)).GE.0.01) GO TO 43

42 CONTINUE

C FLUX HAS NOT CHANGED ENOUGH FOR PLOTTING.

GO TO 45

C SAVE THE NORMALIZED FLUX FOR PLOTTING:

POWE0145

POWE 0146

POWE0147

POWE0148

POWE0149

POWE 0150

POWE0151

POWE 0152

POWE0153

POWE 0154

POWE 0155

POWE0156

POWE 0157

POWE 0158

POWE 0159

POWE0 160

POWE0161

POWEO 162

POWE 0163

POWE0164

POWE0165

POWE0166

POWE 0167

POWE 0168

POWE0169

POWE 0170

POWE0171

POWE0172

POWE 0173

POWE 0174

POWE 0175

POWE 0176

POWE0177

POWE0178

POWE0179

POWEO180

PAGE 272 
44 PHISTR $(I G, I ; I K+1)=E R R U R(I G, I)$

POWE0187

IF (IK.NE.5) GO TO 45

CALL PHIPLTI5)

POWE 0189

POWEO 190

I $K=0$

45 CONT INUE

I $F L A G 2=0$

IFLAG $3=0$

STORE THE ERRORS FOR COMPARISCN: ERROR BETWEEN ITERATICN EIGENVALUES:

POWE 0194

POWE 0195

POWE 0196

POWE 0197

POWE0198

POWE 0199

$E R L A M(I$ COUT $)=E R R L A M$

POWE 0200

DO $46 \quad I G=1,2$

C EFSTR $(1 G, I$ COUT $)=\operatorname{ERRMAX}(I G)$

MAXIMUM ERROR BETWEEN ITERATION FLUXES:

POWE 0201

POWE 0.202

POWE0203 MEAN SQUARE ERROR BETWEEN ITERATION FLUXES:

POWE 0204

POWE0205 EFMSTR (IG, I COUT $)=S Q(I G)$ PHI CUN ( IG, I COUT ) =DPH I (IG)

POWE0 207

POWE0208

ERRJR BETWEEN THE ITERATION EIGENVALUE AND GIVEN TRUE EIGENVALUE:

POWE 0209 LAMCON $($ I COUT $)=$ DLAM

IF ((ERRMAX (1).LT.EPS 1).AND. (ERRMAX (2),LT.EPS 1)) IFLAG1=1

POWE 0210

IF ( (SQ 1 (1).LT.EPS 2$)$. AND. $(S Q(2) \cdot L T \cdot E P S 2)) \quad$ IFLAG $2=1$

POWE0211

IF (ERRLAM.LT.EPS3) IFLAG3 $=1$

POWE0212

POWE0213

IFLAG4=IFLAG 1*I FLAG2* IFLAG 3

POWE0214

IF (IFLAG4.EQ.1) GO TO 50

POWE0215

C OTHERWISE CONTINUE THE I TERATION. 
LAMB $4=$ LAMDA

POWE 0217

GO TO 20

50 CONT INUE

C

CONVERGENCE ACCOMPLISHED.

C NORMALIZE THE CONVERGED FLUX VECTOR:

CALL NORMAL (PHI, N)

C PLOT ANY LEFT OVER FLUX HISTORY PLOTS:

IF (IPLJT.EQ.2).AND.(IK.NE.0)) CALL PHIPLTIK) BOUNDRY CONDITION INSERT ICNS.

C I $E R=0$

C IER ALLONS B.C. INSERTIONS FOR YES AND NO CONVERGENCE:

POWE0218

POWEO 219

POWE 0220

POWE0221

POWE 0222

POWE0223

POWE0224

POWE0225

POWE 0226

POWE 0227

POWE 0228

POWE0229

POWE0230

POWE0231

POWE0232

POWE0233

POWE0234

POWE0235

POWE0236

POWE 0237

POWE 0238

POWE0239

POWE0240

POWE 0241

POWE 0242

POWE 0243

POWE0244

POWE0245

POWE 0246

POWE 0247

POWE0248

POWE0249

POWE0250

POWE0251

POWE0252

90 IF (IER.EQ.1) GO TO 102 RETURN
NO CONVERGENCE ACCOMPLISHED: 
I COUT $=I$ COUT -1

WRITE $(6,101)$ I COUT

101 FORMAT (IHI, POWER METHOD DID NOT CCNVERGE FOR THIS CASE AFTER',

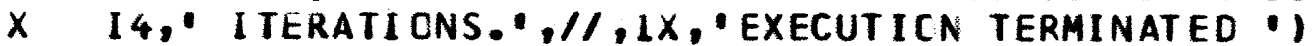

POWE0 254

POWE0255

POWE 0256

POWE 0257

POWE 0258

POWE0259

POWE0260

POWE0261

GO TO 55

102 CONTINUE

C

FOR PRINTING OUT THE EIGENVALUE HISTORY AND THE FINAL FLUX SHAPE:

POWE0262

POWE0263 I PLOT $=1$ JPLOT $=1$

POWE 0264

POWE0265

RETURN

POWE 0266

END

POWE0267 
SUBROUTINE SOLV $30(N, A, X, Y)$

C SOLVES THE N DOUBLE PRECISICN MATRIX EGUATIONS: $A * X=Y$,

C FOR $X$ - GIVEN THE $N$ BY $N$ TRIDIAGONAL MATRIX A

C AND THE SOURCE VECTOR $Y$.

C

METHUD IS FORWARD ELIMINATION FOLLOWED BY BACKHARD SUBSTITUTION. CF - WACHPRESS, PAGE 23.

REAL* 8 A, $X, Y, H, P, D$

DIMENSION $A(26,26), X(26), Y(26), H(26), P(26)$

IF $(A(1,1), E Q .0 .0)$ GO TO 10

$H(1)=-A(1,2) / A(1,1)$

$P(1)=Y(1) / A(1,1)$

DO $1 M=2, N$

$D=A(M, M)+A(M, M-1) * H(M-1)$

IF $(D . E Q .0 .0)$ GO TO 20

$P(M)=(Y(M)-A(M, M-1) * P(M-1)) / D$

IF $(M \cdot E Q \cdot N)$. GO TO 1

$H(M)=-A(M, M+1) / D$

1 CONTINUE

$X(N)=P(N)$

DO $2 \quad I=2, N$

$M=N+1-I$

$2 X(M)=P(M)+H(M) * X(M+1)$

RETURN

C

IN CASE OF ANY IMPENDING ZERO DIVISCRS:

10 WRITE $(6,11)$

11 FORMAT ("OFIRST ELEMENT OF $A, A(1,1)$, IS $2 E R O, \cdots$,

$X$ 5X, BETTER FIX IT BOSS." I

GO TO 30

20 WRITE $(6,21) M$

21 FORMAT 1 OZERO DIVISOR ENCOUNTERED IN EQUATION $M=1,13,1,1,1$,

$X \quad 5 X$, 'BETTER FIX IT BOSS.' '

30 WRITE $(6,31)$

31 FORMAT ('OEXECUTION TERMINATED.')

CALL EXIT

RETURN

END

SCLV0001

SOLVOOO2

SOLV 0003

SOLV0004

SOLV0005

SOLV0006

SOLV0007

SOLV0008

SOLV0009

SOLV0010

SOLV0011

SOLV0012

SOLV0013

SOLV0014

SOLV0015

SOLV0016

SOLV0017

SOLV0018

SOLV0019

SOLVOO2O

SOLV0021

SOLV 0022

SOLV 0023

SOLV 0024

SOLV 0025

SOLV0026

SOLV 0027

SCLV0028

SOLV0029

SOLV0030

SOLV0031

SOLV0032

SOLV0033

SOLV0034

SOLV 0035

SOLV0036

PAGE 276 
SUBRUUT INE NORMAL (PHI,N)

NORMALI LES THE GROUP FLUXES TO ONE. NOT BOTH GROUPS.

REAL*8 PHI $(2,26), A$

$A=D A B S(P H I(1,1))$

DO 1 I G $=1,2$

DO $1 \quad I=1, N$

IF (DABS(PHIIIG,I)).GT.A) A=DABS(PHI(IG,I))

1 CONTINUE

DO 2 I $G=1,2$

DO $2 \quad I=1, N$

$2 \operatorname{PHI}(I G, I)=$ PHI (IG,I) /A RETURN

END

NORL 0001

NORLOOO2

NORL 0003

NORL0004

NORL 0005

NORL 0006

NORL0007

NORL 0008

NORL 0009

NORL 0010

NORL 0011

NORLO012

NORL 0013 
SUBRUUT INE PHIPLTIL)

PLOTS THE GROUP FLUX HISTORY, WITH UP TO 5 GROUP FLUXES PER PLOT.

PHI P0001

FAST AND THERMAL GROUP FLUXES ARE PLOTTED SEPERATELY.

$L$ IS THE NUMBER OF FLUXES TO BE PLOTTED.

$L$ IS BET WEEN 1 AND 5.

IMPLICIT REAL *8 (A-H,O-Z)

COMMON /BL/ IBC

COMMON /B2/ KR,N

COMMON /B5/ S(26), $A(26,6), B(26,6)$

COMMUN /B6/ TE1 $(2,5), T E 2(2,5), \operatorname{TE} 3(5), \operatorname{IN}(5)$

COMMON /ER/ EPS 1, EPS 2,EPS3

COMMON /FSTR/ PHISTR $(2,26,6)$

DIMENSION SYMBOL (5)

INTEGER SYMBOL $, \cdot, \cdot, \cdot \cdot, \cdot+\cdot, \cdot * \cdot, \cdot * \cdot /$

$K R 1=K R+1$

C

SET UP B.C. CONDITIONS

IF $(I B C . E Q \cdot 4)$ GO TO 5

IF $(I B C . E Q .3)$ GO TO 3

DO 2 I $G=1,2$

DO $2 K=1, L$

DO $1 \quad I=1, N$ $J=N+1-I$

$1 \operatorname{PHISTR}(I G, J+1, K+1)=\operatorname{PHISTR}(I G, J, K+1)$

$2 \operatorname{PHISTR}(I G, 1, K+1)=0$.

3 IF (IBC.EQ.2) GO TO 5

DO 4 I $G=1,2$

DO $4 \quad K=1, L$

4 PHISTR(IG,KRI,K+1)=0.

5 CONTINUE

FLUXES IN PHISTR hAVE BEEN NORMALIZED IN POWER.

PUT THE FAST FLUX IN A, AND THE THERMAL FLUX IN B: $L 1=L+1$

DO $10 \quad K=1, L 1$

DO $10 \quad I=1, K R 1$

$A(I, K)=P H I S T R(1, I, K)$

$10 B(I, K)=\operatorname{PHISTR}(2, I, K)$

PHIP0002

PHIP0003

PHIP0O04

PHIP0005

PHIP0006

PHIP 0007

PHIP 0008

PHIP0009

PHIP0010

PHIP0011

PHIP0012

PHIP0013

PHIP0014

PHIP0O 15

PHIP 0016

PHIP0017

PHIP0018

PHIP0019

PHIP 0020

PHIP0021

PHIP0022

PHIP0023

PHIP0024

PHIP0025

PHIP0026

PHIP 0027

PHIP0028

PHIP 0029

PHIP 0030

PHIP0031

PHIP 0032

PHI P0033

PHIP 0034

PHIP0035

PHIPO036

PAGE 278 
20 FORMAT $(/$, 'OFAST FLUX ITERATION HISTCRY PLOT.',/)

PHIP 0039

WRI TE $(6,30)$

30 FORMAT

$X$ 'OKEY:',5X,'SYMBOL',5X,' ITERATION NUMBER: ', 7X,'ERRCR CRITERIA',

$X \quad 11 X, \cdot E R R O R ', 13 X$, 'TCLERANCE')

DO $35 \quad I=1, L$

35 WRITE $(6,40)$ SYMBOL (I), IN(I),TEI (1, I), EPS1,TE2(1,I), EPS 2, $\mathrm{X}$

TE $3(1), E P S 3$

40 FORMAT $(/, 12 X, A 1,15 X, 13,16 x$, 'FLUX',14X,1PD 15.5,5X,1 PD 15,5,/,

$X \quad 47 X, \cdot M E A N$ SQ. FLUX $, 5 X, 1$ PD15.5,5X,1 PD15.5,1,

$X \quad 47 X, \cdot E I G E N V A L U E \cdot, 8 X, 1 P O 15.5,5 X, 1 P D 15.51$

PLOT THE L THERMAL FLUX SHAPES ON THE OTHER GRAPH:

CALL PRTPLT $(0, B, K R 1, L 1, K R L, 0,26,6,2)$

WRITE $(6,50)$

50 FORMAT ( $/$, OTHERMAL FLUX ITERATICN PLOT.',/)

WRITE $(6,30)$

DO $55 \quad I=1, L$

55 WRITE $(6,40)$ SYMBOL (I), INI I ),TE1 (2, I), EPS 1,TE2 (2,I), EPS 2,

$X$

TE $3(I), E P S 3$

RETURN

END

PHIP0040

PHIP 0041

PHIP0042

PHIP0043

PHIP 0044

PHIP0045

PHIP 0046

PHIP0047

PHIP 0048

PHI P 0049

PHIP0050

PHIP0051

PHIP 0052

PHIP0053

PHIP 0054

PHIP0055

PHIP0056

PHIP0057

PHIP0058

PHIP0059

PHIP0060 
1 FORMAT ('IRESULTS OF THE MULTIGROUP METHOD: ') WRITE $(6,10)$ IC OUT

10 FORMAT $1 / \%$, PROBLEM TERMINATED AFTER',I5,

30 FORMAT 1 IRESULTS AFTER PROBLEM TERMINATION:', /

$X$ 'ONUMBER',5X, "THERMAL FLUX POINTS',5X, "FAST FLUX POINTS') WRITE $(6,50)$ (K, PHI $(2, K), P H I(1, K), K=1, K R 1)$

50 FORMAT (I 5, 1PE26.7, 1PE21.7)

IF IIPUNCH.EQ.1) CALL PUNCH

CALCULATE THE FINAL TO EXPECTED FLUX RATIOS:

NORMALIZE BOTH PHI GROUP FLUXES FOR TRUPHI COMPARISON: CALL NORM2 (PHI, TRUPHI, KRI)

$K 1=1$

$K 2=K R 1$

IF $(I B C, L E \cdot 2) \quad K 1=2$

IF $((I B C \cdot E Q \cdot 1) \cdot Q R \cdot(I B C \cdot E Q .3)) \quad K 2=K R$

DO $60 \quad I G=1,2$

If $(I B C \cdot L E \cdot 2) \quad P S I(I G, 1)=1.0$

DUTP 0005

OUTP0006

OUTP 0007

OUTP0008

OUTP0009

OUTP0010

OUTP0011

OUTP 0012

OUTP0013

OUTPOO14

OUTP0015

OUTP0O16

QUTP 0017

OUTPOO 18

OUTP 0019

OUTP0020

OUTP 0021

OUTP0022

DUTP 0023

OUTP0024

OUTP 0025

OUTP0026

OUTP0027

DUTP0028

DUTP 0029

OUTP0030

OUTP0031

OUTP0032

DUTP0033

OUTP0034

DUTP0035

OUTP0036

PAGE 280 


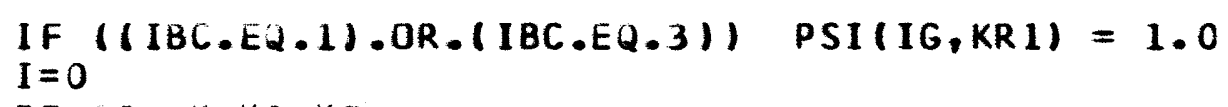

70 FORMAT 1 IRATIOS OF THE TERMINATED GROUP FLUX TO THE EXPECTED GROU XP FLUX:,$/ /$,

$X$ IOX, - AN INDICATION OF THE ACCURACY OF THE CONVERGENCE $-1,1 /$, PRINT OUT THE STORED ITERATICN ERRORS: WRITE $(6,110)$ EPS 1 , (EFSTR $(2, I), I=1$, ICOUT) WRITE $(6,111)$ EPS $1,(E F S T R(1, I), I=1$, ICOUT) WRITE $(0,112)$ EPS2, (EFMSTR $(2,1), 1=1$, ICOUT) WRITE $(6,113)$ EPS 3, (EFMSTR $(1,1), I=1$, ICOUT) WR ITE $(6,114)$ EPS3, (ERLAM( I), I = 1, ICCUT)

110 FORMAT (IMAXIMUM NORMALIZED ERRORS BETWEEN THE THERMAL FLUX ITERA XTIONS: :

$X$ 25X, TOLERANCE USED $=1,1$ PE12.4, $11,(1 P 5 E 20.5)$ )

111 FORMAT (IMAXIMUM NORMALIZEC ERRORS BETWEEN THE FAST FLUX ITERATIO $X N S: \cdot$,

$X \quad 25 X, \cdot T$ TLERANCE USED $=1,1$ PE 12.4, $11,($ IP5E20.5)

112 FORMAT 1 IMEAN SQUARE NORMALIZED ERRCR BETHEEN THE THERMAL FLUX IT XERATIONS: ',

$X \quad 18 X$, 'TOLERANCE USED $=1,1$ 1PE12.4, $11,(1 P 5 E 20.51)$

113 FORMAT 1 IMEAN SQUARE NORMALIZED ERRCR BETHEEN THE FAST FLUX ITERA XIIONS:",

$X$ 18X,'TOLERANCE USED $=1,1$ PE12.4, $11,($ IP5E 20.5)

114 FORMAT ('IERROR BETHEEN THE ITERATICN EIGENVALUES: ',

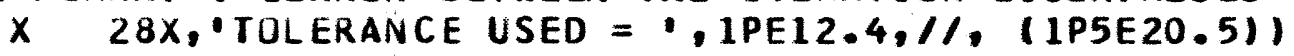

IF (IFT.EQ.0) RETURN

WRITE $(6,115)$ IRULAM, ( $T R U P H I(3-J, I), J=1,2), I=1, N)$

115 FORMAT 1 1THE GIVEN TRUE EI GENVALUE:, $1 /, 15 X$,

$X$ - TRULAM $=\bullet, E 22.14, / / 1$,

$X$ 'OTHE GIVEN MULTIGROUP FLUXES: $1,1 /$,

OUTP0037

DUTP 0038

OUTP 0039

OUTP 0040

DUTP0041

OUTP0042

OU TP 0043

OUTP0044

DUTP 0045

OUTP 0046

OUTP 0047

DUTP0048

OUTP 0049

OUTP0050

OU TP 0051

OUTP0052

OUTP0053

DUTP 0054

DUTP0055

OUTP 0056

OUTP0057

OUTP0058

OUTP0059

OUTP 0060

OUTP0061

OUTP0062

QUTP 0063

OUTP 0064

OUTP 0065

OUTP0066

OUTP0067

OUTP0068

OUTP0069

OUTP 0070

OUTP0071

OUTP 0072

PAGE 281 

WRITE $(6,120)$ (PHICON $(2, I), I=1, I C O U T)$ WRITE $(6,121)$ (PHICON $(1, I), I=1, I$ COUT) WRITE $(6,122)$ (LAMCON(I),I=1, ICCUT)

120 FORMAT ('IMEAN SQUARE ERROR BETWEEN THE THERMAL ITERATION FLUX AND $X$ THE GIVEN TRUE THERMAL FLUX:",//, (1P5E20.5))

121 FORMAT (IIMEAN SQUARE ERROR BETWEEN THE FAST ITERATION FLUX AND TH XE GIVEN TRUE FAST FLUX:,$/ / /,(1 P 5 E 20.5))$

122 FORMAT ('IERROR BETWEEN THE ITERATICN EIGENVALUES AND THE GIVEN TR XUE EIGENVALUE: $, / 1,(1$ P5E20.5)

RETURN

END 
SUBROUTINE PLOT

C AS WELL AS PLOTTING OUT THE FINAL MULTIGROUP FLUX SHAPES. AS WELL AS PLOTTING OUT
PLICIT REAL*8 (A-H,L-Z)

COMMON /BI/ IBC, IPLOT,JPLOT, IPUNCH

COMMON /B2/ KR

COMMUN /B4/ PHI $(2,26)$, PSI $(2,26)$, LAMDA, ICOUT

COMMON /B5/ B $(300,2)$

COMMON /ESTR/ LAMSTR(300)

DIMENSI UN $C(26,3)$

IN ORDER TO SAVE SCME SPACE:

EQUIVALENCE (B (1),C(1))

WRITE $(6,1)$ (LAMSTR $(I), I=1, I$ ICOUT)

1 FORMAT ('OTABLE OF EIGENVALUES DURING THE POWER ITERATION:" $1 /,(1 P 5 E 25.14))$

IF (JPLOT.EQ.0) GO TO 20

DO $10 \quad I=1, I$ COUT

$B(1,1)=I$

$10 \mathrm{~B}(1,2)=$ LAMSTR(I)

CALL PRTPLT $(1,8$, I COUT, 2, ICOUT, $0,300,2,1)$

WRITE $(6,11)$

11 FORMAT ('OPLJT OF THE EIGENVALUE HISTORY THROUgh THE ITERATIONS.")

20 IF (IPLOT.EQ.0) RETURN

$K R 1=K R+1$

DO $30 \quad I=1, K R 1$

$C(1,1)=1$

$C(1,2)=P H I(1,1)$

$30 C(I, 3)=P H I(2, I)$

CALL PRTPLT $(2, C, K R 1,3, K R 1,0,26,3,2)$

WRITE $(6,31)$

31 FORMAT ('OFINAL CONVERGEO CCNNECTING FLUX POINTS; $F(K), 1,1 /$,

$X$ 5X,'FAST FLUX: $\because, 1,5 X$, 'THERMAL FLUX: -1$)$

RETURN

PLOT 0001

PLOT0002

PLOT 0003

PLOT 0004

PLOT 0005

PLOT0006

PLOT0007

PLOT0008

PLOT 0009

PLOTO010

PLOT 0011

PLOT 0012

PLOT 0013

PLOT 0014

PLOT0015

PLOT0016

PLOT 0017

PLOT0018

PLOT 0019

PLOT 0020

PLOT 0021

PLOT 0022

PLOT 0023

PLOT 0024

PLOT 0025

PLOT0026

PLOT 0027

PLOT 0028

PLOT 0029

PLOT 0030

PLOT 0031

PLOT0032

PLOT 0033

END

PLOT 0034 
SUBROUTINE PUNCH

PNCHOOOI

PUNCHES OUT INPUT AND OUTPUT DATA.

PNCHOOO2

COMMON /B2/ KR

COMMON /B4/ $F(2,26)$

REAL * $8 F$

$K R 1=K R+1$

WRITE $(7,1) \quad K R,(F(1, I), F(2, I), I=1, K R 1)$

1 FORMAT (I $5,1,(2 E 20.7))$

WRITE $(6,100)$

100 FORMAT $(\% / \%$, THE OUTPUT HAS BEEN FUNCHED OUT ONTO CARDS 1 RETURN

END

PNCHOOO3

PNCHOOO4

PNCH0005

PNCHOOO6

PNCHOOOT

PNCHOOO 8

PNCHOOO9

PNCHOO 10

PNCHOOL1

PNCHOOLI 
SUBROUTINE NORM 2 (PSI, TRUPHI, N)

NOR20001

NOR 20002

NOR20003

NOR20004

NOR20005

NOR20006

NOR20007

NOR20008

NOR 20009

$A(I G)=D A B S(P S I(I G, 1))$

DO $1 \quad I=1, N$

IF (DABS(PSI (IG,I)),GT.A(IG)) A(IG)=DABS(PSI (IG,I))

NOR20010

NOR2 0011

1 CONTINUE

DO 2 IG $=1,2$

DO $2 \quad I=1, N$

$2 \operatorname{PSI}(I G, I)=P S I(I G, I) / A(I G)$

IF (K.NE・1) RETURN

DO 5 I $G=1,2$

$A(I G)=0$.

DO $5 \quad I=1, N$

IF (TRUPHI $(I G, I), G T \cdot A(I G)) . \quad A(I G)=T R U P H I(I G, I)$

NOR 20012

NOR20013

NOR20014

NOR20015

NOR20016

NOR20017

NOR20018

NOR20019

NOR20020

NOR20021

NOR20022

NOR 20023

NOR20024

NOR20025

NOR20026 
PRTP0001

PRTP 0002

* identical to subroutine prtplt previously listed in program refzg.

PRTP0003

C * IDENTICAL TO SUBROUTINE PRTPLT PREVIOUSLY LISTED IN PROGRAM REF2G. PRTPOO04

RETURN

END

(1)

PRTP0006 
F.3. SOURCE LISTING of Program CUBIC 


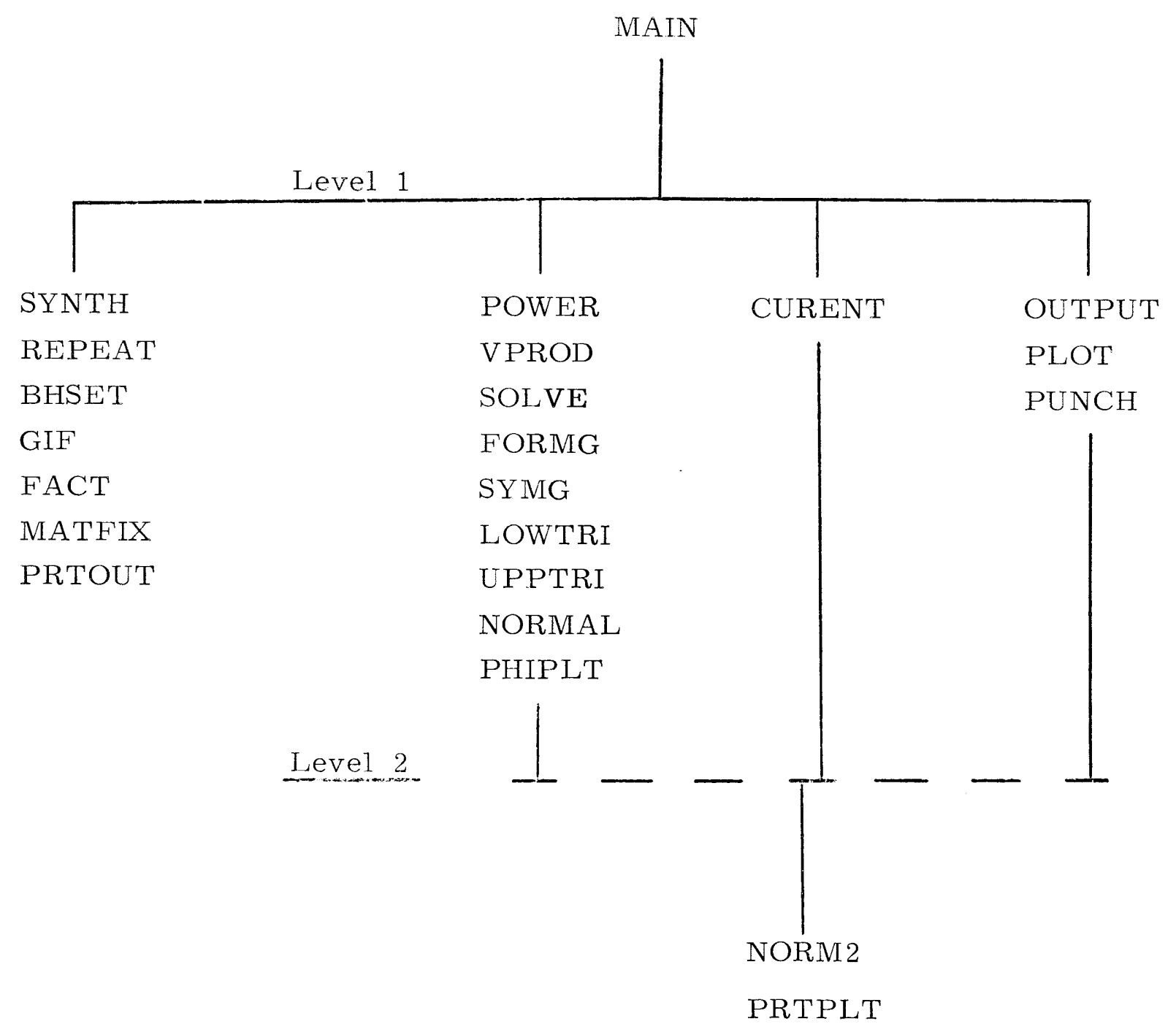

Figure F.3. Structure of Program CUBIC. 
C PROGRAM CUBIC:

CUBI 0001

CUBI 0002

CUBI 0003

CALL TIMING (II)

CALL SYNTH

CALL TIMINS (14)

CALL POWER

CALL TIMINS (16)

CALL CURENT

CALL TIMING (17)

CALL OUTPUT

CALL TIMING (I8)

TIMING EXECUTION

WRITE $(6,30)$

30 FORMAT (1H1, 'TIMING PROGRAM EXECUTION: ',/)

$J=I 4-I]$

WRITE $(6,701) \mathrm{J}$

$J=I 6-I 4$

WRITE $(6,704) \mathrm{J}$

$J=I 7-16$

WRI TE $(6,706) \mathrm{J}$

$J=18-[7$

WRITE $(6,707) \mathrm{J}$

701 FORMAT (1H : SYNTH HAS TAKEN, 16," 1100 SECONDS $\because)$

704 FORMAT (1H, POWER HAS TAKEN, I6," 1100 SECONDS.')

706 FORMAT (1H," CURENT HAS TAKEN', 15,'/100 SECONDS.')

707 FORMAT (IH ,' OUTPUT HAS TAKEN', 15," $/ 100$ SECONDS.')

CALL TIMING $(120)$

$J=I 20-I 1$

WR I TE $(6,720) J$

720 FORMAT (1HO," THIS RUN HAS TAKEN $, 16, \cdots / 100$ SECONDS TO RUN.") STOP

END

CUBI 0004

CUBI 0005

CUBI 0006

CUBI 0007

CUBI 0008

CUBI 0009

CUBI 0010

CUBIO011

CUBI 0012

CUBI 0013

CUBI 0014

CUBI 0015

CUBI 0016

CUBI 0017

CUBI0018

CUBI 0019

CUBI 0020

CUBI 0021

CUBI 0022

CUBI 0023

CUBI 0024

CUBI 0025

CUBI 0026

CUBI 0027

CUBI 0028

CUBI 0029

CUBI 0030

CUBI 0031

CUBI 0032 
SUBROUTINE SYNTH

PRUPUSED CUBIC HERMITE SYNTHESIS METHOD:
$C * * * * * * * * * * * * * * * * * * * * * * * *$

SYNTOOO1

SYNT 0002

ADJOINT QUANTITIES OF VARIBLES ARE DENOTED BY 7 RATHER THAN * *

THUS: PHIT (RATHER THAN PHI*) IS THE ADJOINT OF PHI. ETC.

SYNT 0003

SYNT 0004

SYNT 0005

IMPLICII REAL*8 (A-H,K-L)

COMMUN /BI/ IBC, IPLOT, JPLOT, IPUNCH, ISEE

COMMON /B2/ KR, NN

COMMON /B3/LT(50,6,2), FT $(50,6,4), T(50,6)$

COMMON /B5/

$X \quad K A O(2,25), K A 1(2,25), K A 2(2,25), K A 3(2,25), K A 4(2,25), K A 5(2,25)$, $K A 6(2,25), K B O(2,25), K B 1(2,25), K B 2(2,25), K B 3(2,25), K B 4(2,25)$, $K B 5(2,25), K B 6(2,25), \operatorname{LAO}(2,25), \operatorname{LA} 1(2,25), \operatorname{LA} 2(2,25), \operatorname{LA} 3(2,25)$, $\operatorname{LA4}(2,25), \operatorname{LA} 5(2,25), \operatorname{LA}(2,25), P 0(2,25), P 1(2,25), P 2(2,25)$, $P 3(2,25), P 4(2,25), P 5(2,25), P 6(2,25), Q 0(2,25), Q 1(2,25)$, $Q 2(2,25), Q 3(2,25), Q 4(2,25), Q 5(2,25), Q 6(2,25), R 0(2,25)$, $R 1(2,25), R 2(2,25), R 3(2,25), R 4(2,25), S R O(1,25), S R 1(1,25)$, $\operatorname{SR2}(1,25), \operatorname{SR} 3(1,25), \operatorname{SR} 4(1,25), \operatorname{SR} 5(1,25), \operatorname{SR} 6(1,25), \operatorname{KC} 0(1,25)$, $K C 1(1,25), K C 2(1,25), K C 3(1,25), K C 4(1,25), K C 5(1,25), K C 6(1,25)$, $\operatorname{KDO}(1,25), K D 1(1,25), K D 2(1,25), K D 3(1,25), K D 4(1,25), K D 5(1,25)$, $K D 6(1,25)$,

$P O(2,25), P H(2,25), P O 7(2,25), P H 7(2,25), D O(2,25), D H(2,25)$, CO(2), CH(2), TITLE(20), ITF(25), KTF(25)

COMMON /CHIF/ CHI(2)

COMMON /XAXIS/ HX, HR(25)

COMMON /BH/ X(101), H(101)

COMMON /ER/ EPS 1, EPS2,EPS3

DIMENSION PHI $(2,101), P H I 7(2,101), \operatorname{CUR}(2,101), \operatorname{CUR} 7(2,101)$

DIMENSION A $(2,100), F(2,100), D(2,100), S(2,100), D I(2,100), X U(2,100)$ IN ORDER TO SAVE SPACE:

C

EQUIVALENCE (XU(1),LT(1)), $X$

(A(1),LT(201)), (F(1),LT(401)),

REAL TITLE

(D(1),FT(201)), (S(1),FT(401))

SYNT 0006

SYNTOOOT

SYNT 0008

SYNT 0009

SYNTOO 10

SYNT 0011

SYNT 0012

SYNT 0013

SYNTO014

SYNT 0015

SYNT 0016

SYNT 0017

SYNT 0018

SYNTO019

SYNT 0020

SYNT 0021

SYNT 0022

SYNT 0023

SYNT 0024

SYNTO025

SYNTO0 26

SYNT 0027

SYNT0028

SYNT 0029

SYNT 0030

SYNT 0031

SYNT 0032

SYNT 0033

SYNT 0034

INTEGER KR, K, KS, KS $1, K R O, N V, N U M I T F, K T F, N$

READ $(5,200)$ TI TLE

200 FORMAT (20A4)

SYNT 0035

SYNT 0036

PAGE 290 
WRITE $(6,201)$ TITLE

201 FORMAT (IH1,20A4,//)

C

READ IN THE NUMBER OF REGICN TRIAL FUNCTIONS AND TYPE OF B.C.S.

AS WELL AS THE TOLERANCES AND THE OUTPUT TYPES DESIRED:

READ (5,1) KR, I BC, EPS1, EPS 2, EPS 3, IPLCT, JPLOT, I PUNCH, I SEE, ITW, ITC

1 FORMAT $(215,3010.3,615)$

IF $(I B C \cdot E Q \cdot 3) \quad I B C=2$

C READ IN THE TYPE-NUMBER OF EACH TF REGION: READ (5,100) (I TF(I), I=I,KR)

100 FORMAT (2512)

C READ IN THE FISSION YEILDS FOR EACH GROUP:

C AND THE MATRIX NORMALILATION PARAMETER: THETA (DEFAULT = 1.0 ): READ $(5,101)$ CHI(1), CHI (2), THETA

101 FORMAT (3F 10.5$)$

IF (THETA.EQ.0.0) THETA=1.0

$K R O=K R-1$

WR ITE $(6,2) \mathrm{KR}$, IBC, ISEE, ITW, ITC

2 FORMAT 1 OONE DIMENSIONAL TWO GROUP CUBIC SYNTHESIS PROGRAM:, $1 /$,

$X \quad 5 X$, NUMBER OF COARSE MESH REGIONS: $K R=1,12,1$,

$5 X, \cdot B O U V D A R Y$ CONDITION NUMBER: IBC $=1,12,1$,

$5 X, \circ$ AMOUNT OF OUTPUT REQUESTED: ISEE $=1,12,11$,

$5 X, \cdot$ TYPE OF MEIGHTING FUNCTICNS: ITW $=1,12,1$,

$5 X, "$ TYPE OF CURRENT FUNCTIONS: ITC $=1,12,11$

$5 X, \cdot R E G I O N A L$ INPUT MATER IAL PROPERTIES AND FLUX SHAPES FOLLOW', $1.5 X, \cdot$ IF ISEE > $0: 1,1 /$.

$5 X, '$ FLUX SHAPES ARE LINEAR IN EACH INDICATED SUBREgION.')

IF (ITC.EQ.0) WRITE $(6,16)$

IF (ITC.EQ.1) WRITE $(6,17)$

16 FORMAT (5X, CURRENTS ARE CONSTANT IN EACH INDICATED SUBREGION.")

17 FORMAT (5X, "CURRENTS ARE LINEAR IN EACH INDICATED SUBREGION.")

IF (ITW.EQ.0) WRITE $(6,116)$

IF $(I T W . E Q .1)$ WRITE $(6,117)$

116 FORMAT $1 /, 5 X, \cdot$ WEIGHTING FLUX = FLUX; $, /, 5 X, \cdot$ WEIGHTING CURRENT = XCURRENT

117 FORMAT $1 /, 5 X,{ }^{\circ}$ WEIGHTING FLUX = ADJOINT FLUX; $, /, 5 X, 1$ WEIGHTING CURR XENT = ADJOINT CURRENT $\cdot 1$

SYNT 0037

SYNTO038

SYNT 0039

SYNT 0040

SYNT0041

SYNT 0042

SYNT 0043

SYNT 0044

SYNT 0045

SYNT 0046

SYNT 0047

SYNT 0048

SYNT0049

SYNT 0050

SYNT 0051

SYNT 0052

SYNT0053

SYNT 0054

SYNT 0055

SYNT 0056

SYNT 0057

SYNT0058

SYNT 0059

SYNT 0060

SYNT0061

SYNT0062

SYNT 0063

SYNT0064

SYNT0065

SYNT 0066

SYNT0067

SYNT0068

SYNT 0069

SYNT 0070

SYNT 0071

SYNT 0072

PAGE 291 
WRITE $(6,20)$ EP S1,EPS2,EPS 3 , IPLOT, JPLOT, I PUNCH

SYNT 0073

20 FORMAT $(/ 1$, OTOLERANCES TO POWER ARE : EPS $1=1,1$ PD1 $0.3,1$,

SYNT 0074

SYNT0075

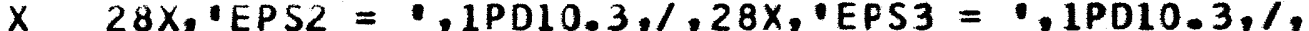

$x$ OOUTPUT PARAMETERS TO POWER ARE: IPLOT $=1,11.1$,

$X \quad 34 X, \cdot J P L O T=1, I 1,1,34 X, \cdot I P U N C H=1, I 11$

WRITE $(6,22) \mathrm{CHI}(1), \mathrm{CHI}(2)$, THETA

22 FORMAT $(1, \cdot$ OFISSION YIELDS ARE: CHIII) $=\bullet, F 10.5,1$,

$X \quad 22 X,{ }^{\circ} \mathrm{CHI}(2)={ }^{\prime}, \mathrm{F} 10.5,1$,

$X$ OINPUT THETA PARAMETER (FCR MATRICES) $=0,015.7$ )

IF ((KR.LE.2).AND. (IBC.EQ.1)) CALL ERROR(1,KR)

IF (KR.GT.25) CALL ERROR(2,KR)

IF (EPSL.LT.1.OE-16) CALL ERROR $(6,1)$

IF (EPS2.LT $1.0 E-16)$ CALL ERROR $(6,2)$

IF (EPS3.LT.1.OE-16) CALL ERROR $(6,3)$

IF ((IBC.LT.1).OR.(IBC.GT.4)) CALL ERROR(7, IBC)

C DUMMY NORMAL VECTOR XU = UNITY. (FOR THE INTEGRATION FUNCTIONS)

SYNT 0076

SYNT 0077

SYNT0078

SYNT 0079

SYNT0080

SYNT0081

SYNT 0082

SYNT0083

SYNT 0084

SYNT0085

SYNTO086

SYNTO087

SYNTO088

SYNT0089

DO $21 \quad I G=1,2$

DO $21 \quad I I=1,100$

$21 X U(I G, I I)=1.0$

I T $C O=2$

I T $C 1=2$

IF (ITC.EQ.1) GO TO 23

I I C $0=0$

I $T C I=1$

COUNTER OF THE NUMBER OF TYPE-NUMBERS OF EACH TF REGION:

23 NUMITF=1

$H X=0.0$

C

BEGIN TJ READ IN THE TF REGION DATA AND FILL THE ARRAYS, DEPENDING ON THE TYPE-NUMBER OF EACH TF REGION.

DO $50 \quad I=1, K R$

IF (ITF (I).EQ.NUMITF) GO TO 110

FILL THE ARRAYS FROM OLD TF REGION TYPES:

$J=I T F(I)$

CALL REPEAT (I,KTF (J))

GO TO 50

C READ IN THE TF REGION'S CATA FOR NEW TF REGICN TYPE-NUMBERS:

SYNT 0090

SYNT0091

SYNT 0092

SYNT 0093

SYNT0094

SYNT 0095

SYNT0096

SYNT 0097

SYNT 0098

SYNT 0099

SYNTO100

SYNTO101

SYNTO102

SYNT 0103

SYNTO104

SYNT 0105

SYNTO106

SYNT 0107

SYNT 0108

PAGE 292 
110 NUMI IF $=$ NUMI $T F+1$ KTF (NUMITF-1) $=1$

C READ THE SUBREgION NUMBER AND THE NUMBER OF REGIONS IN THE SUBREgION: READ $(5,1) K, K S$

IF (KS.GT.100) CALL ERRUR (3,I)

$K S 1=K S+1$

C

CHECK FOR IMPROPER SEQUENCING OF INPUT DATA:

IF (I.NE.K): CALL ERROR (4,I)

C READ IN THE GECMETRY AND THE MATERIAL PROPERTIES: $\operatorname{READ}(5,3)(X(J), X(J+1), H(J), A(1, J), F(1, J), D(1, J), S(1, J)$, $X$ $A(2, J), F(2, J), D(2, J), J=1, K S)$

C

3 FORMAT $(3 F 10,5,4010.3,1,30 X, 3010.3)$

READ IN THE REGIONAL GROUP TRIAL FUNCTIONS:

$\operatorname{READ}(5,4)(\operatorname{PHI}(1, J), \operatorname{CUR}(1, J), \operatorname{PHI} 7(1, J), \operatorname{CUR} 7(1, J), J=1, \operatorname{KS} 1)$

$\operatorname{REAO}(5,4)(\operatorname{PHI}(2, J), \operatorname{CUR}(2, J), \operatorname{PHI} 7(2, J), \operatorname{CUR} 7(2, J), J=1, K S 1)$

4 FORMAT (4D20.7)

IF (ITW.EQ.1) GO TO 120

C FURM WEIGHTING FUNCTIONS FROM THE GIVEN FUNCTIONS:

DO $1191 \mathrm{G}=1,2$

DO $119 \quad J=1, K S 1$

PHI T IIG,J) $=$ PHI (IG,J)

$119 \operatorname{CUR} 7(I G, J)=-\operatorname{CUR}(I G, J)$

120 IF (ITC.EQ. 1 ) GO TO 5 FORM THE REGION CONSTANT CURRENTS FROM THE FLUXES:

DO $7 \quad 1 \mathrm{G}=1,2$

DO $6 J J=1, K S$

CUR $(I G, J)=-D(I G, J) *(-P H I(I G, J)+P H I(I G, J+I)) / H(J)$

$6 \operatorname{CURT}(I G, J)=+D(I G, J) *(-P H I T(I G, J)+P H I T(I G, J+1)) / H(J)$

CUR $(I G, K S \perp)=0.0$

$7 \operatorname{CURT}(1 G, K S 1)=0.0$

C

WRITE OUT THE INPUT INFORMATION IF ISEE - GE.2:

5 IF (ISEE.LE. I) GO TO 14

WRITE $(6,10) \quad K, K R, K S,(J, X(J), X(J+1), H(J), A(1, J), F(1, J), D(1, J)$,

$X \quad S(1, J), A(2, J), F(2, J), O(2, J), J=1, K S)$

10 FORMAT ('IINPUT MATERIAL PROPERTIES FOR REGION NUMBER "I3,

$X$, OF THE $, 13,1$ USED.', /1,

SYNT0109

SYNT 0110

SYNTO111

SYNTO112

SYNT 0113

SYNTO114

SYNTO115

SYNTO116

SYNTO117

SYNTO118

SYNT 0119

SYNTO 120

SYNT 0121

SYNTO122

SYNTO123

SYNTO124

SYNT 0125

SYNT 0126

SYNT 0127

SYNTO128

SYNT 0129

SYNTO130

SYNT 0131

SYNT 0132

SYNTO133

SYNT 0134

SYNT 0135

SYNTO136

SYNT 0137

SYNT0138

SYNT 0139

SYNT 0140

SYNTO141

SYNT 0142

SYNT 0143

SYNT 0144

PAGE 293 
$X$ 5X, THIS REGION IS DIVIDED INTO $, 13, \cdot$ HOMOGENEOUS SUBREgIONS A

SYNT 0145

$X S$ FOLLOWS:, $1 / 1$,

$X$ 5X, $\mathrm{X}$ FST GROUP CCNSTANTS APPEAR FIRST:, $1 /$,

- SUBREgION \#',5X,'INTERNAL BOUNCAR IES', 10X, 'WIDTh',3X,

- TOTAL $C X(1 / C M)^{\prime}, 3 X, \cdot F I S S I O N C X(1 / C M) \cdot, 6 X, \cdot D I F F U S I C N$ (CM)', $4 X, \cdot S C A T T \cdot C X \quad(1 / C M) \cdot, /$,

$5 x, I^{\prime}, 11 X, x^{\prime}(I) \cdot, 9 x, \cdot X(I+1) \cdot, 11 x, \cdot H(I) \cdot, 13 x, \cdot A(I G, I) \cdot, 13 X$,

$\cdot F(16,1) \cdot, 13 x, \cdot D(1 G, 1) \cdot, 14 x, \cdot S(1,1) \cdot, 1 /$,

$(16,3 F 15.4,4020.8,1,51 X, 3020.8))$

DO $15 \quad I G=1,2$

15 WRITE $(6,11) I G, K, K R,(J, X(J), P H I(I G, J), C U R(I G, J), P H I 7(I G, J)$,

$X \quad C U R 7(I G, J), J=1, K S 1)$

11 FORMAT (IINPUT TRIAL FUNCTIONS FCR GROUP',I2,' FOR REGICN',I3,

$X$ - OUT OF THE', I3,' USED:, $1 /$,

$X$ ' INDEX',5X, 'COORD', 16X, 'FLUX',13X,'CURRENT', 8X," WEIGHT FLUX',

$X$ 5X, WEIGHT CURRENT',//, (16,F10.5,4020.7)

14 CONT INUE

END OF THE IN-OUT SECTION:

DEFINING MISC. ARRAYS FOR THE INTEGRATION FUNCTIONS:

LEGNTH OF THE SUBREGION: HT

$H T=X(K S 1)-X(1)$

$H R(K)=H T$

$H X=H X+H R(K)$

INVERSE OF THE D ARRAYS:

DO $13 \quad J=1, K S$

$01(1, J)=1 . / 0(1, J)$

$13 D I(2, J)=1.10(2, J)$

FORMATION OF THE INTEGRATION FUNCTIONS:

CALL BHSETIKS)

DO FOR ALL ENERGY GROUPS:

DO $50 \quad I G=1,2$

$K A O(I G, K)=G I F(O, I G, P H I 7, I G, A, P H I, K S, 2)$

$K A I(I G, K)=G I F(1, I G, P H I 7, I G, A, P H I, K S, 2)$

$K A 2(I G, K)=G I F(2, I G, P H I 7, I G, A, P H I, K S, 2)$

$K A 3(1 G, K)=G I F(3, I G, P H I 7, I G, A, P H I, K S, 2)$

$K A 4(I G, K)=G I F(4, I G, P H I 7, I G, A, P H I, K S, 2)$

SYNTO146

SYNTO147

SYNTO148

SYNTO149

SYNT 0150

SYNTO151

SYNT 0152

SYNTO153

SYNT 0154

SYNT 0155

SYNT 0156

SYNTO157

SYNT0158

SYNTO159

SYNTO160

SYNT 0161

SYNTO 162

SYNT0163

SYNTO164

SYNT 0165

SYNTO166

SYNTO 167

SYNT0168

SYNTO169

SYNT 0170

SYNTO171

SYNTO172

SYNTO173

SYNT 0174

SYNTO 175

SYNT 0176

SYNTO177

SYNTO178

SYNT 0179

SYNTO180

PAGE 294 
$K A 5(I G, K)=G I F(5, I G, P H I 7, I G, A, P H I, K S, 2)$ $K A G(I G, K)=G I F(6, I G, P H I 7, I G, A, P H I, K S, 2)$ $K B O(I G, K)=G I F(0, I G, P H I 7,1 G, F, P H I, K S, 2)$ $K B 1(1 G, K)=G I F(1,1 G, P H I 7,1 G, F, P H I, K S, 2)$ $K B 2(I G, K)=G I F(2, I G, P H I 7, I G, F, P H I, K S, 2)$ $K B 3(I G, K)=G I F(3, I G, P H I 7, I G, F, P H I, K S, 2)$ $K B 4(1 G, K)=G I F(4, I G, P H I 7, I G, F, P H I, K S, 2)$ $K B 5(I G, K)=G I F(5, I G, P H I 7, I G, F, P H I, K S, 2)$ $K B G(I G, K)=G I F(6, I G, P H I 7, I G, F, P H I, K S, 2)$ $L A O(I G, K)=G I F(0, I G, C U R 7, I G, D I, C U R, K S, I T C O)$ $L A 1(I G, K)=G I F(1, I G, C U R], I G, D I, C U R, K S, I T C O)$ $L A 2(I G, K)=G I F(2, I G, C U R 7, I G, D I, C U R, K S, I T C 0)$ $L A 3(I G, K)=G I F(3, I G, C U R 7, I G, D I, C U R, K S, I T C O)$ $L A 4(I G, K)=G I F(4, I G, C U R 7, I G, D I, C U R, K S, I T C O)$ $L A 5(I G, K)=G I F(5, I G, C U R 7, I G, D I, C U R, K S, I T C O)$ LA $6(I G, K)=G I F(6, I G, C U R 7, I G, D I, C U R, K S, I T C O)$ $P O(I G, K)=G I F(0, I G, P H I 7, I G, X U, C U R, K S, I T C 1) / H T$ $P I(I G, K)=G I F(1, I G, P H I 7, I G, X U, C U R, K S, I T C I) / H T$ $P 2(I G, K)=G I F(2, I G, P H I 7, I G, X U, C U R, K S, I T C 1) / H T$ $P 3(I G, K)=G I F(3, I G, P H I 7, I G, X U, C U R, K S, I T C 1) / H T$ $P 4(I G, K)=G I F(4, I G, P H I 7, I G, X U, C U R, K S, I T C 1) / H T$ $P 5(I G, K)=G I F(5, I G, P H I 7, I G, X U, C U R, K S, I T C 1) / H T$ P6 $(I G, K)=G I F(6, I G, P H I 7, I G, X U, C U R, K S, I T C 1) / H T$ $Q O(I G, K)=G I F(0, I G, P H I, I G, X U, C U R 7, K S, I T C 1) / H T$ $Q 1(I G, K)=G I F(I, I G, P H I, I G, X U, C U R 7, K S, I T C I) / H T$ $Q 2(I G, K)=G I F(2, I G, P H I, I G, X U, C U R 7, K S, I T C 1) / H T$ $Q 3(I G, K)=G I F(3, I G, P H I, I G, X U, C U R 7, K S, I T C I) / H T$ $Q 4(I G, K)=G I F(4, I G, P H I, I G, X U, C U R 7, K S, I T C I) / H T$ $Q 5(I G, K)=G I F(5, I G, P H I, I G, X U, C U R 7, K S, I T C 1) / H T$ $Q 6(I G, K)=G I F(6, I G, P H I, I G, X U, C U R 7, K S, I T C 1) / H T$ $R O(I G, K)=G I F(0, I G, P H I 7, I G, D, P H I, K S, 2) / H T * * 2$ $R 1(1 G, K)=G I F(1, I G, P H I 7, I G, D, P H I, K S, 2) / H T * * 2$ $R 2(I G, K)=G I F(2, I G, P H I 7, I G, D, P H I, K S, 2) / H T * * 2$ $R 3(I G, K)=G I F(3, I G, P H I 7, I G, D, P H I, K S, 2) / H T * * 2$

SYNTO181

SYNTO182

SYNTO183

SYNT 0184

SYNT 0185

SYNTO186

SYNTO187

SYNTO188

SYNT0189

SYNTO190

SYNTO191

SYNT 0192

SYNTO193

SYNTO194

SYNTO195

SYNT 0196

SYNTO197

SYNTO198

SYNTO199

SYNT 0200

SYNTO201

SYNT 0202

SYNT 0203

SYNT 0204

SYNT 0205

SYNT 0206

SYNT 0207

SYNT 0208

SYNT0209

SYNT 0210

SYNTO211

SYNT 0212

SYNTO213

SYNT 0214

SYNTO215

SYNT 0216 PAGE 295 
$P O(I G, K)=P H I(I G, 1)$

SYNTO217

$P 07(I G, K)=P H I 7(I G, 1)$

SYNT 0218

$P H(I G, K)=P H I(I G, K S I)$

SYNT 0219

PHT $(I G, K)=P H I 7(I G, K S 1)$

SYNT 0220

$D O(I G, K)=D(I G, 1)$

$D H(I G, K)=D(I G, K S)$

IF $(K, E Q \cdot 1): \operatorname{CO}(I G)=\operatorname{CUR}(I G, 1)$

IF (NUMITF-1.EQ.ITF (KR).AND.ITC.EQ.0) CH(IG)=CUR $(I G, K S)$

IF (NUMITF-1.EQ - ITF (KR). AND I ITC.EQ.1) CH(IG)=CUR (IG,KSI) (NUM ITF-1.EQ.ITF(KR) AND I ITC EQ - I) CH
FOR THE OFF DIAGONAL MATRIX ELEMENTS:

IF (IG.EQ.2) GO TO 50

SROI $1, K)=6 I F(0,2, P H I 7,1, S, P H I, K S, 2)$

$S R 1(1, K)=G I F(1,2, P H I 7,1, S, P H I, K S, 2)$

$\operatorname{SR2}(1, K)=G I F(2,2, P H I 7,1, S, P H I, K S, 2)$

$S R 3(1, K)=G I F(3,2, P H I 7,1, S, P H I, K S, 2)$

$S R 4(1, K)=G I F(4,2, P H I 7,1, S, P H I, K S, 2)$

SR5 $1, K)=G I F(5,2, P H I 7,1, S, P H I, K S, 2)$

SR6 $(1, K)=G I F(6,2, P H I 7,1, S, P H I, K S, 2)$

$K C O(1, K)=G I F(0,1, P H I 7,2, F, P H I, K S, 2)$

$K C 111, K)=G I F(1,1, P H I 7,2, F, P H I, K S, 2)$

$K C 2(1, K)=G I F(2,1, P H I 7,2, F, P H I, K S, 2)$

$K C 3(1, K)=G I F(3,1, P H I 7,2, F, P H I, K S, 2)$

$K C 4(1, K)=G I F(4,1, P H I 7,2, F, P H I, K S, 2)$

$K C 5(1, K)=G I F(5,1, P H I 7,2, F, P H I, K S, 2)$

$K C 61,1, K)=G I F(6,1, P H I 7,2, F, P H I, K S, 2)$

$K D O(1, K)=G I F(0,2, P H I 7,1, F, P H I, K S, 2)$

$K D 1(1, K)=G I F(1,2, P H I 7,1, F, P H I, K S, 2)$

$K D 2(1, K)=G I F(2,2, P H I 7,1, F, P H I, K S, 2)$

$K D 3(1, K)=G I F(3,2, P H I 7,1, F, P H I, K S, 2)$

$K D 4(1, K)=G I F(4,2, P H I 7,1, F, P H I, K S, 2)$

$\operatorname{KD5}(1, K)=G I F(5,2, P H I 7,1, F, P H I, K S, 2)$

$K D 6(1, K)=G I F(6,2, P H I 7,1, F, P H I, K S, 2)$

SYNT 0221

SYNT 0222

SYNT 0223

SYNT 0224

SYNT 0225

SYNT 0226

SYNTO227

SYNTO229

SYNT 0229

SYNTO230

SYNTO231

SYNT 0232

SYNT0233

SYNT 0234

SYNTO235

SYNT 0236

SYNT 0237

SYNT 0238

SYNT 0239

SYNT 0240

SYNTO241

SYNT 0242

SYNTO243

SYNT0244

SYNTO245

SYNTO246

SYNT 0247

SYNT 0248

SYNT 0249

SYNT 0250

NUMI TF $=$ NUMII IF -1

WRI TE $(6,51)$ NUMITF

51 FORMAT ('ITHERE ARE ONLY', I3," DIFFERENT TRIAL FUNCTION REgIONS.')

SYNT 0251

SYNTO 252 
WRITE $(6,52) \quad(1, \operatorname{ITF}(1), I=1, K R)$

SYNTO253

52 FORMAT $1 /$, OTABLE OF THE TRIAL FUNCTION NUMBER TYPES: $1, / /$,

SYNTO254

SYNT 0255

$X \quad 3 X$, IF REGION', 4X, 'REGION TYPE-NUMBER',//,

$X \quad(17,12 X, 17))$

C

TO PRINT OUT THE /B5/ ARRAYS:

IF (ISEE.GE.2) CALL PRTOUT (1)

DETERMINATIONS OF THE B.C. CPTION PARAMETERS:

C

C

C

C

C

C

C NN IS

$N N=2 * K R$

TON OF THE COEFFICIENT VECTORS:

FILLING THE MATRICES FOR POWER:

FOR BOTH ENERGY GROUPS:

DO 60 IG $=1,2$

$$
I=1
$$

THE MATRIX ROW INDEX:

FOR ALL THE INTERIOR COEFFICIENTS:

$D O 60 \quad K=2, K R$

$I=I+1$

$56 \mathrm{~J}=\mathrm{K}-1$

$V=1, /(P H 7(I G, J) * P O(I G, J))$

$V 1=1 . /(P H 7(I G, J) * P H(I G, J))$

$V 2=1 . /(P O T(I G, K) * P O(I G, K))$

$V 3=1 . /(P O Z(I G, K) * P H(I G, K))$

$L T(I, 1, I G)=(3, * K A 2(I G, J)-2, * K A 3(I G, J)-9 . * K A 4(I G, J)+12$ *KA5(IG,J)

$X \quad-4 . * K A G(I G, J)-(3 . * L A 2(I G, J)-2 * * L A 3(I G, J)-9 . * L A 4(I G, J)+12 . *$

$X \quad L A 5(I G, J)-4, * L A G(I G, J))-(6 . * P 1(I G, J)-6 . * P 2(I G, J)-18 . * P 3(I G, J)$

$X+30 . * P 4(I G, J)-12 . * P 5(I G, J))-(18, * Q 3(I G, J)-30 * * Q 4(I G, J)+12 . *$

$x \quad Q 5(I G, J) i-(36 . * R 2(I G, J)-72 . * R 3(I G, J)+36 . * R 4(I G, J))) * V$

$L T(I, 2, I G)=(-3 . * K A 3(I G, J)+8 . * K A 4(I G, J)-7 . * K A 5(I G, J)+2 . * K A 6(I G, J)$

$x-(-3, * L A 3(I G, J)+8 . * L A 4(I G, J)-7 . * L A 5(I G, J)+2, * L A 6(I G, J))$

$x \quad-(-6 . * P 2(I G, J)+18 . * P 3(I G, J)-18 . * P 4(I G, J)+6 . * P 5(I G, J))$

$x-(3 . * Q 2(I G, J)-14 . * Q 3(I G, J)+17 . * 04(I G, J)-6 . * 05(I G, J))$

$x \quad-(6 . * R I(I G, J)-30 . *$

$x \quad R 2(I G, J)+42 . * R 3(I G, J)-18 . * R 4(I G, J))) * V * H R(J) / D O(I G, J)$

$\operatorname{LT}(I, 3, I G)=(9 . * K A 4(I G, J)-12$ * *KA5(I G, J) +4**KA6(I G,J)-(9.*LA4 (I G,J)

$x-12 . * L A 5(I G, J)+4 . * L A G(I G, J))-(18 . * P 3(I G, J)-30 * * P 4(I G, J)+12$ * *

SYNT 0256

SYNT 0257

SYNT 0258

SYNT 0259

SYNT0260

SYNT 0261

SYNT0262

SYNT 0263

SYNTO264

SYNT 0265

SYNTO266

SYNT 0267

SYNT 0268

SYNT0269

SYNT 0270

SYNT 0271

SYNT 0272

SYNT 0273

SYNT 0274

SYNT 0275

SYNT 0276

SYNT0277

SYNT 0278

SYNT 0279

SYNT 0280

SYNT 0281

SYNT 0282

SYNT 0283

SYNT 0284

SYNT0285

SYNT 0286

SYNT0287

SYNT 0288 
$x \quad P 5(I G, J))+(18, * Q 3(I G, J)-30 . * Q 4(I G, J)+12, * Q 5(I G, J))$

$+36 . * R 2(I G, J)-72 . * R 3(I G, J)+36 . * R 4(I G, J)) * V 1+$

$(K A O(I G, K)-6 . * K A 2(I G, K)+4 . * K A 3(I G, K)+9 . * K A 4(I G, K)-12 . * K A 5(I G, K)$

+4.*KAG $(I G, K)-(L A O(I G, K)-6 . * L A 2(I G, K)+4 . * L A 3(I G, K)+9 . * L A 4(I G, K)$

$-12 . * L A 5(I G, K)+4 . * L A G(I G, K))+6 . * P 1(I G, K)-6 . * P 2(I G, K)-18$.*

$P 3(I G, K)+30 . * P 4(I G, K)-12 * P 5(I G, K)-(6, * Q 1(I G, K)-6, * Q 2(I G, K)$

$-18 . * Q 3(I G, K)+30 . * 04(I G, K)-12 . * 05(I G, K))+36 . * R 2(I G, K)-72$ * $R 3(I G, K)+36, * R 4(I G, K)) * V 2$

$\operatorname{LT}(I, 4, I G)=(3, * K A 4(I G, J)-5 . * K A 5(I G, J)+2 . * K A 6(I G, J)-(3 . * L A 4(I G, J)$ $-5 . * L A 5(I G, J)+2 . * L A 6(I G, J))-(6 . * F 3(I G, J)-12 . * P 4(I G, J)+6 . *$ $P 5(I G, J))-(-6 . * Q 3(I G, J)+13 . * 04(1 G, J)-6 . * Q 5(I G, J))-($

$-12 . * R 2(I G, J)$

$+30 . * R 3(I G, J)-18 . * R 4(I G, J))) * V 1 * H R(J) / D H(I G, J)$

$+(-K A L(I G, K)+2 . * K A 2(I G, K)+2 . * K A 3(I G, K)-8 . * K A 4(I G, K)+7 . *$ $K A 5(I G, K)-2 . * K A 6(I G, K)-(-L A 1(I G, K)+2 . * L A 2(I G, K)+2 . * L A 3(I G, K)$ $-8 . * L A 4(I G, K)+7 . * L A 5(I G, K)-2 . * L A G(I G, K))-6 . * P 2(I G, K)+18$.* $P 3(I G, K)-18 . * P 4(I G, K)+6 . * P 5(I G, K)-(C O(I G, K)-4 . * Q 1(I G, K)+14$ * $Q 3(I G, K)-17, * Q 4(I G, K)+6 . * 05(I G, K))+6, * R 1(I G, K)-30 . * R 2(I G, K)$ $+42 . * R 3(I G, K)-18 . * R 4(I G, K)) * V 2 * H R(K) / D O(I G, K)$

$L T(I, 5, I G)=(3, * K A 2(I G, K)-2, * K A 3(I G, K)-9 . * K A 4(I G, K)+12$.*KA5(IG,K) $-4 . * K A 6(I G, K)-(3, * L A 2(I G, K)-2$ * $L A 3(I G, K)-9 . * L A 4(I G, K)+12$ ** $L A 5(I G, K)-4 . * L A 6(I G, K))+18, * P 3(I G, K)-30 * * P 4(I G, K)+12 * * P 5(I G, K)$ $+(6 . * Q 1(I G, K)-6 . * Q 2(I G, K)-18 . * 03(I G, K)+30 . * Q 4(I G, K)-12$.* $Q 5(I G, K))-(36 . * R 2(I G, K)-72 . * R 3(I G, K)+36 . * R 4(I G, K)) * V 3$

$L T(1,6, I G)=(K A 2(I G, K)-K A 3(I G, K)-3$ **KA4 $(I G, K)+5$.*KA5 $(I G, K)-2$ ** $K A 6(1 G, K)-(L A 2(I G, K)-L A 3(I G, K)-3$ * *LA4 (IG,K)+5.*LA5 (IG,K)-2.* $L A 6(I G, K))+6 . * P 3(I G, K)-12 . * P 4(I G, K)+6 . * P 5(I G, K)-(-2 . * Q 1(I G, K)+$ $3 . * Q 2(I G, K)+6, * Q 3(I G, K)-13, * 04(I G, K)+6 . * 05(I G, K))-12, * R 2(I G, K)$ $+30 . * R 3(I G, K)-18 * * R 4(I G, K)) * V 3 * H R(K) / D H(I G, K)$

$F T(I, 1, I G)=(3 . * K B 2(I G, J)-2 . * K B 3(I G, J)-9 . * K B 4(I G, J)+12 . * K B 5(I G, J)$ $X \quad-4 . * K B 6(I G, J)) * V$

$F T(I, 2, I G)=(-3 . * K B 3(I G, J)+8 . * K B 4(I G, J)-7 . * K B 5(I G, J)+2 . * K B 6(I G, J))$ $* V * H R(J) / D O(I G, J)$

$F T(I, 3, I G)=(9 . * K B 4(I G, J)-12 . * K B 5(I G, J)+4 . * K B 6(I G, J)) * V 1$

$x+(K B O(I G, K)-6, * K B 2(I G, K)+4 . * K B 3(I G, K)+9 . * K B 4(I G, K)$

$X \quad-12 \cdot * K B 5(I G, K)+4 . * K B 6(I G, K)) * V 2$

SYNT0289

SYNT 0290

SYNT 0291

SYNT 0292

SYNTO293

SYNT 0294

SYNT 0295

SYNT 0296

SYNT 0297

SYNT 0298

SYNT 0299

SYNT 0300

SYNT 0301

SYNT 0302

SYNT 0303

SYNT 0304

SYNT 0305

SYNT 0306

SYNT 0307

SYNT 0308

SYNT0309

SYNT 0310

SYNT 0311

SYNT 0312

SYNT0313

SYNT 0314

SYNT 0315

SYNT0316

SYNT0317

SYNT 0318

SYNTOZ19

SYNT0320

SYNT0321

SYNT 0322

SYNT 0323

SYNTO324

PAGE 298 
$F T(I, 4, I G)=(3, * K B 4(I G, J)-5, * K B 5(I G, J)+2, * K B 6(I G, J))$

$X \quad * V 1 * H R(J) / D H(I G, J)+1-K B I(I G, K)+2 . * K B 2(I G, K)+2 . * K B 3(I G, K)$

$X \quad-8 . * K B 4(I G, K)+7 . * K B 5(I G, K)-2 * K B 6(I G, K)) * V 2 * H R(K) / D 0(I G, K)$

$F T(I, 5, I G)=(3, * K B 2(I G, K)-2 * * K B 3(I G, K)-9, * K B 4(I G, K)+12, * K B 5(I G, K)$

$X \quad-4 * * K B 6(1 G, K)) * V 3$

$F T(I, 6, I G)=(K B 2(I G, K)-K B 3(I G, K)-3 . * K B 4(I G, K)+5 . * K B 5(I G, K)$

$X$ $-2 . * K B 6(I G, K)) * V 3 * H R(K) / D H(1 G, K)$

IF $(1 G . E Q .2) \quad 60$ TO 57

$T(1,1)=(3 . * S R 2(I G, J)-2 . * S R 3(I G, J)-9 . * S R 4(I G, J)+12 . * S R 5(I G, J)$

$x \quad-4 . * S R 6(I G, J) / /(P H 7(2, J) * P C(1, J))$

$T(I, 2)=(-3 . * \operatorname{SR} 3(I G, J)+8 . * \operatorname{SR} 4(I G, J)-7 . \operatorname{SR} 5(I G, J)+2, * S R 6(I G, J))$

$X \quad$ *HR $(J) /(P H 7(2, J) * P 0(1, J) * D O(1, J))$

$T(I, 3)=(9 . * S R 4(16, J)-12 *$ SR5 $(I G, J) * 4 . * S R 6(I G, J))$

$X \quad /(P H 7(2, J) * P H(1, J))$

$X+(S R O(I G, K)-6, * S R 2(I G, K) * 4 . * S R 3(I G, K)+9 . * S R 4(I G, K)$

$X \quad-12 . * S R 5(I G, K) * 4 . * S R 6(I G, K)) /(P 07(2, K) * P O(1, K))$

$T(I, 4)=(3, * S R 4(I G, J)-5 . * S R 5(I G, J)+2 . * S R 6(I G, J))$

$X \quad * H R(J) /(P H 7(2, J) * P H(1, J) * D H(1, J))$

$X+(-S R 1(I G, K)+2 . * S R 2(I G, K)+2 * * S R 3(I G, K)$

$X \quad-8 . * S R 4(I G, K)+7 . * S R 5(I G, K)-2, * S R 6(I G, K)$ *HR $(K)$

$X \quad /(P 07(2, K) * P O(1, K) * D O(1, K))$

$T(I, 5)=(3 . * S R 2(I G, K)-2 *$ SR3 $(I G, K)-9, * S R 4(I G, K)+12, * S R 5(I G, K)$

$X \quad-40 * 5 R 6(I G, K)) /(P O 7(2, K) * P H(1, K))$

$T(I, 6)=(S R 2(I G, K)-S R 3(I G, K)-3, * S R 4(I G, K)+5, * S R 5(I G, K)$

$X \quad-2 . * S R 6(1 G, K)) * H R(K) /(P O 7(2, K) * P H(1, K) * D H(1, K))$

$F T(I, 1,3)=(3, * K D 2(I G, J)-2 * k D 3(I G, J)-9 . * K D 4(I G, J)+12, * K D 5(I G, J)$

$X \quad-4 . * K D 6(16, J)) /(P H 7(2, J) * P O(1, J))$

FT $(I, 2,3)=(-3, * K D 3(I G, J)+8, * K C 4(I G, J)-7, * K D 5(I G, J)+2, * K D 6(I G, J))$ *HR $(J) /(P A 7(2, J) * P O(1, J) * D O(1, J))$

$F T(I, 3,3)=(9, * K D 4(I G, J)-12, * K D 5(I G, J)+4 . * K D 6(I G, J))$

$X \quad /(P H 7(2, J) * P H(1, J))$

$x+(K D O(I G, K)-6, * K D 2(I G, K)+4, * K D 3(I G, K)+9, * K D 4(I G, K)$

$X \quad-12 . * 0 D(I G, K)+4 . * K D 6(I G, K)) /(P 07(2, K) * P 0(1, K))$

$F T(I, 4,3)=(3, * K D 4(I G, J)-5 . * K C 5(I G, J)+2 * * K D 6(I G, J))$

$X \quad * H R(J) /(P H 7(2, J) * P H(1, J) * D H(1, J))$

$x+(-K 01(1 G, K)+2 . * K D 2(I G, K)+2 . * K D 3(I G, K)$

SYNT 0325

SYNT 0326

SYNT 0327

SYNT 0328

SYNT 0329

SYNT 0330

SYNT 0331

SYNT 0332

SYNT 0.333

SYNT 0334

SYNT 0335

SYNT 0336

SYNT 0337

SYNT 0338

SYNT 0339

SYNT 0340

SYNT 0.341

SYNT 0342

SYNT 0343

SYNT 0344

SYNT0345

SYNT 0346

SYNT 0347

SYNT 0348

SYNT 0349

SYNT 0350

SYNT 0351

SYNT 0352

SYNT 0353

SYNT 0354

SYNT0355

SYNT 0356

SYNT 0357

SYNTO358

SYNT 0359

SYNT0360

PAGE 299 
$x \quad-8 . * K D 4(I G, K)+7 . * K D 5(I G, K)-2 . * K D 6(I G, K)) * H R(K)$

$X \quad /(P O 7(2, K) * P O(1, K) * D O(1, K))$

$F T(I, 5,3)=(3, * K D 2(I G, K)-2 . * K C 3(I G, K)-9 . * K D 4(I G, K)+12 . * K D 5(I G, K)$ $-4 . * K D 6(16, K)) /(P 07(2, K) * P H(1, K))$

$F T(I, 6,3)=(K D 2(I G, K)-K D 3(I G, K)-3 . * K D 4(I G, K)+5 . * K D 5(I G, K)$ $-2 . * K 06(1 G, K)) * H R(K) /(P O 7(2, K) * P H(1, K) * D H(1, K))$

$F T(1,1,4)=(3 . * K C 2(I G, J)-2, * K C 3(I G, J)-9 . * K C 4(I G, J)+12 . * K C 5(I G, J)$

$X \quad-4 . * K C 6(1 G, J)) /(P H 7(1, J) * P(2, J))$

$F T(I, 2,4)=(-3, * K C 3(I G, J)+8 . * K C 4(I G, J)-7 . * K C 5(I G, J)+2 . * K C 6(I G, J))$

$X \quad * H R(J) /(P H 7(1, J) * P O(2, J) * D O(2, J))$

$F T(I, 3,4)=(9, * K C 4(I G, J)-12 . * K C 5(I G, J)+4 . * K C 6(I G, J))$

$X \quad /(P H 7(1, J) * P H(2, J))$

$x+(K C O(I G, K)-6, * K C 2(I G, K)+4 . * K C 3(1 G, K)+9 . * K C 4(I G, K)$

$X \quad-12 . * K C 5(I G, K)+4 . * K C 6(I G, K)) /(P O 7(1, K) * P O(2, K))$

$F T(I, 4,4)=(3, * K C 4(I G, J)-5, * K C 5(I G, J)+2 . * K C 6(I G, J))$

$X \quad * H R(J) /(P H 7(1, J) * P H(2, J) * D H(2, J))$

$x+1-K C 1(I G, K)+2, * K C 2(I G, K)+2, * K C 3(I G, K)$

$x \quad-8 . * K C 4(1 G, K)+7 . * K C 5(I G, K)-2 . * K C 6(I G, K)) * H R(K)$

$\mathrm{X} \quad(\mathrm{POT}(1, K) * \mathrm{PO}(2, K) * D O(2, K))$

$F T(I, 5,4)=(3 . * K C 2(I G, K)-2 . * K C 3(I G, K)-9 . * K C 4(I G, K)+12 * * K C 5(I G, K)$

$X \quad-4 . * K C 6(1 G, K)) /(P O T(1, K) * P H(2, K))$

$F T(I, 6,4)=(K C 2(I G, K)-K C 3(I G, K)-3 . * K C 4(I G, K)+5 . * K C 5(I G, K)$ $57^{X} I=I+1$ $-2 . * K C G(1 G, K)) * H R(K) /(P O T(1, K) * P H(2, K) * D H(2, K))$

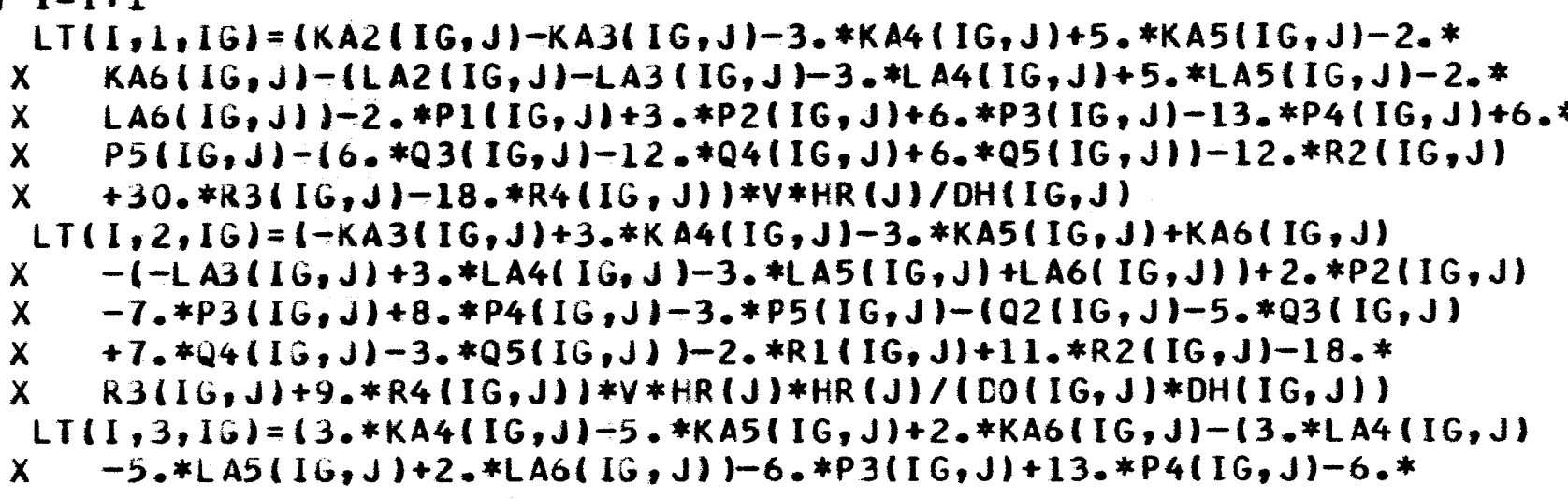

SYNT 0361

SYNT 0362

SYNT 0363

SYNT 0364

SYNT 0365

SYNT 0366

SYNT 0367

SYNT 0368

SYNT0 369

SYNT 0370

SYNT0371

SYNT 0372

SYNT 0373

SYNT 0374

SYNT 0375

SYNT 0376

SYNT 0377

SYNT0378

SYNT0379

SYNT 0380

SYNT 0381

SYNT 0382

SYNT 0383

SYNT 0384

SYNT0385

SYNTO386

SYNT 0387

SYNT 0388

SYNT 0389

SYNT 0390

SYNT 0391

SYNT 0392

SYNT 0393

SYNT 0394

SYNT 0395

SYNT 0396

PAGE 300 


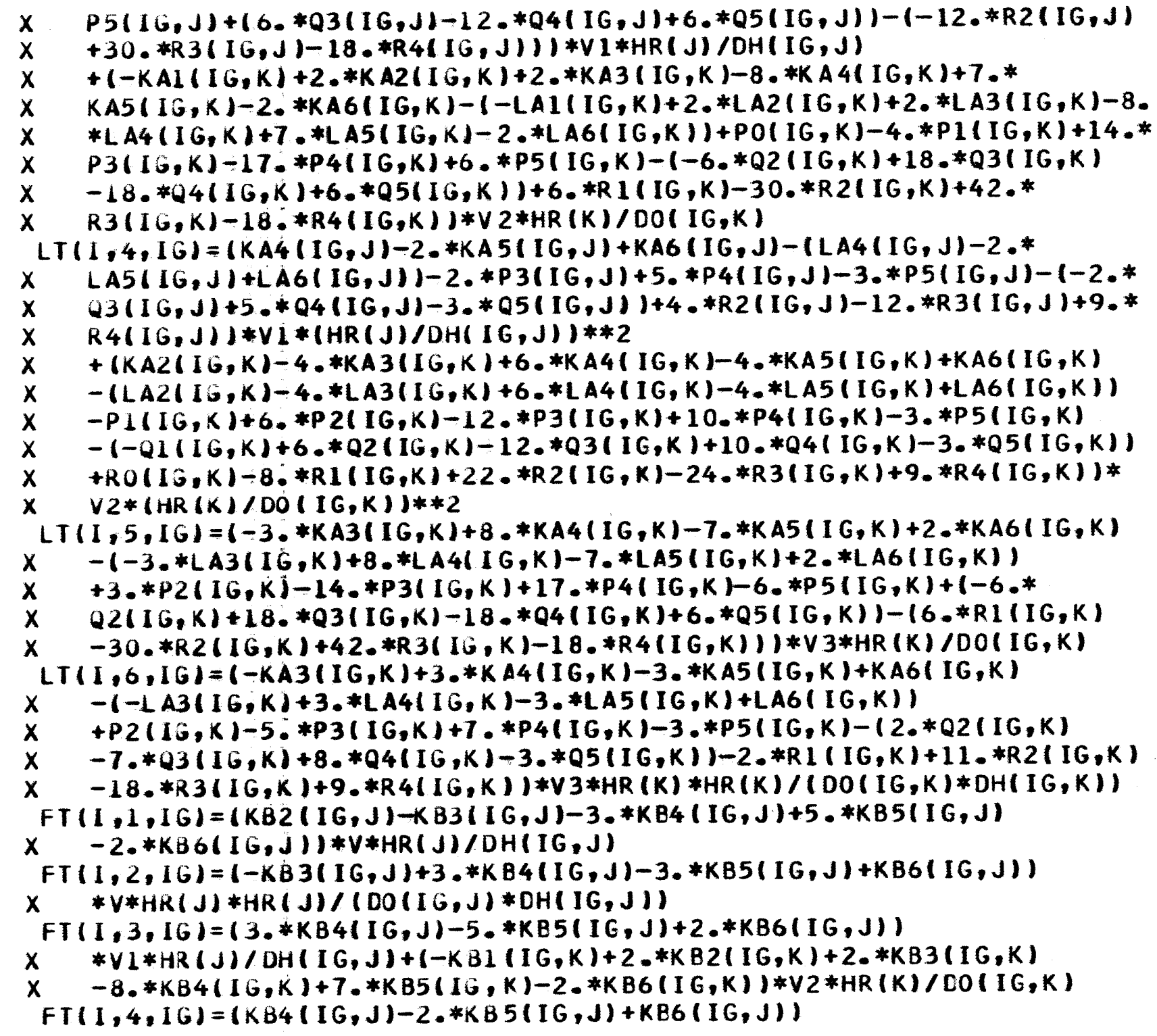

SYNT 0397 SYNT 0398 SYNT0399 SYNT 0400 SYNT 0401 SYNT 0402 SYNT0403 SYNT 0404 SYNT0405 SYNT 0406 SYNT 0407 SYNT 0408 SYNT 0409 SYNT 0410 SYNT 0411 SYNT 0412 SYNT 0413 SYNT 0414 SYNTO 415 SYNT 0416 SYNT0417 SYNT 0418 SYNT 0419 SYNT 0420 SYNT 0421 SYNT 0422 SYNT 0423 SYNT 0424 SYNT 0425 SYNT 0426 SYNT 0427 SYNT 0428 SYNT 0429 SYNT 0430 SYNT0 431 SYNT 0432 PAGE 301 
$X \quad * V I *(H R(J) / O H(I G, J)) * * 2+(K E 2(I G, K)-4, * K B 3(I G, K)+6 . * K B 4(I G, K)$

$X \quad-4 . * K B 5(I G, K)+K B 6(I G, K)) * V 2 *(H R(K) / C O(I G, K)) * * 2$

$F T(I, 5, I G)=(-3, * K B 3(I G, K)+8 . * K B 4(I G, K)-7 . * K B 5(I G, K)+2 . * K B 6(I G, K))$

$X \quad * V 3 * H R(K) / D O(I G, K)$

$F T(I, 6, I G)=(-K B 3(I G, K)+3 . * K B 4(I G, K)-3 . * K B 5(I G, K)+K B 6(I G, K))$

$X$ $* V 3 * H R(K) * H R(K) /(D O(I G, K) * C H(I G, K))$

IF (IG.EQ.2) GO TO 60

$T(I, 1)=(\operatorname{SR} 2(I G, J)-\operatorname{SR} 3(1 G, J)-3 . * \operatorname{SR} 4(I G, J)+5 . * S R 5(I G, J)$

$X \quad-2 . * \operatorname{SR} 6(I G, J)) * H R(J) /(P H 7(2, J) * P((1, J) * D H(2, J))$

$I(I, 2)=(-\operatorname{SR} 3(I G, J)+3 . * S R 4(I G, J)-3 . * \operatorname{SR}(I G, J)+\operatorname{SRG}(I G, J))$

$X \quad * H R(J) * * 2 /(P H 7(2, J) * P O(1, J) * D O(1, J) * D H(2, J))$

$T(I, 3)=(3 . * \operatorname{SR} 4(I G, J)-5 . * \operatorname{SR} 5(I G, J)+2 * * \operatorname{SRG}(I G, J))$

$X \quad * H R(J) /(P H 7(2, J) * P H(1, J) * D H(2, J))$

$X+(-S R I(I G, K)+2 . * S R 2(I G, K)+2 . * S R 3(I G, K)$

$X \quad-8 . * S R 4(I G, K)+7 . * \operatorname{SR} 5(I G, K)-2 . * \operatorname{SR} 6(I G, K)) * H R(K)$

$X \quad /(P O 7(2, K) * P O(1, K) * D O(2, K))$

$T(I, 4)=(\operatorname{SR} 4(I G, J)-2 . * \operatorname{SR} 5(I G, J)+\operatorname{SR6}(I G, J))$

$X \quad * H R(J) * * 2 /(P H 7(2, J) * P H(1, J) * D H(2, J) * D H(1, J))$

$x+(S R 2(I G, K)-4 . * S R 3(I G, K)$

$X+6 . * S R 4(I G, K)-4, * \operatorname{SR} 5(I G, K)+\operatorname{SR} 6(I G, K))$

$X \quad * H R(K) * * 21(P O 7(2, K) * P O(1, K) * D O(2, K) * D O(1, K))$

$I(I, 5)=(-3 . * S R 3(I G, K)+8 . * S R 4(I G, K)-7 . * S R 5(I G, K)+2 . * S R 6(I G, K))$

$X \quad * H R(K) /(P O 7(2, K) * P H(1, K) * D O(2, K))$

$T(I, 6)=(-S R 3(I G, K)+3 . * S R 4(I G, K)-3 . * S R 5(I G, K)+S R 6(I G, K))$

$X \quad * H R(K) * 2 /(P O 7(2, K) * P H(1, K) * D O(2, K) * D H(1, K))$

$F T(I, 1,3)=(K D 2(I G, J)-K D 3(I G, J)-3 . * K D 4(I G, J)+5 . * K D 5(I G, J)$

$x-2 . * K D G(I G, J)) * H R(J) /(P H 7(2, J) * P C(1, J) * D H(2, J))$

$F T(I, 2,3)=(-K D 3(I G, J)+3, * K D 4(I G, J)-3 . * K D 5(I G, J)+K D 6(I G, J))$

$X \quad * H R(J) * * 2 /(P H 7(2, J) * P O(1, J) * D O(1, J) * D H(2, J))$

$F T(1,3,3)=(3 . * K D 4(I G, J)-5 . * K C 5(I G, J)+2 . * K D 6(I G, J))$

$X \quad * H R(J) /(P H 7(2, J) * P H(1, J) * D H(2, J))$

$x+(-K D 1(I G, K)+2, * K D 2(I G, K)+2, * K D 3(I G, K)$

$x \quad-8 . * K D 4(I G, K)+7 . * K D 5(I G, K)-2 . * K D 6(I G, K)) * H R(K)$

$X \quad /(P J 7(2, K) * P O(1, K) * D O(2, K))$

$F T(1,4,3)=(K D 4(I G, J)-2, * K D 5(I G, J)+K D 6(I G, J))$

$X \quad * H R(J) * 2 /(P H T(2, J) * P H(1, J) * D H(2, J) * D H(1, J))$

SYNT 0433

SYNT 0434

SYNT 0435

SYNT 0436

SYNT 0437

SYNT 0438

SYNT 0439

SYNT 0440

SYNT 0441

SYNT 0442

SYNT 0443

SYNT 0444

SYNT 0445

SYNT 0446

SYNT 0447

SYNT 0448

SYNT 0449

SYNT 0450

SYNT0451

SYNT 0452

SYNT 0453

SYNT 0454

SYNT 0455

SYNT 0456

SYNT 0457

SYNT 0458

SYNT 0459

SYNT 0460

SYNT 0461

SYNT 0462

SYNT 0463

SYNT 0464

SYNT 0465

SYNT 0466

SYNT 0467

SYNT 0468

PAGE 302 
$X \quad+(K 02(I G, K)-4 . * K D 3(I G, K)+6 . * K D 4(1 G, K)$
$X \quad-4 * * K 05(I G, K)+K D 6(I G, K)) * H R(K) * * 2$

$X \quad-4, * K D 5(16, K)+K D 6(I G, K)) * H R(K) * * 2$

$X \quad /(P O 7(2, K) * P O(1, K) * D 0(2, K) * D 0(1, K))$

$F T(I, 5,3)=(-3, * K D 3(I G, K)+8 . * K D 4(I G, K)-7 . * K D 5(I G, K)+2, * K D 6(I G, K))$

$F T(I, 6,3)=(-K D 3(I G, K)+3 * * K C 4(I G, K)-3 . * K D 5(I G, K)+K D 6(I G, K))$

$X$ $* H R(K) * 2 /(P 07(2, K) * P H(1, K) * D O(2, K) * D H(1, K))$

$F T(I, 1,4)=(K C 2(I G, J)-K C 3(I G, J)-3 . * K C 4(I G, J)+5 . * K C 5(I G, J)$ $-2 . * K C 6(1 G, J)) * H R(J) /(P H 7(1, J) * P C(2, J) * D H(1, J))$

FT $(I, 2,4)=(-K C 3(I G, J)+3 * k C 4(I G, J)-3 . * K C 5(I G, J)+K C 6(I G, J))$ *HR $(J) * 2 /(P H 7(1, J) * P O(2, J) * D O(2, J) * D H(1, J))$

FT $(1,3,4)=(3, * K C 4(I G, J)-5, * K C 5(I G, J)+2, * K C 6(I G, J))$

$X \quad * H R(J) /(P H 7(1, J) * P H(2, J) * D H(1, J))$

$X+(-K C L(I G, K)+2 * * K C 2(I G, K)+2 * * K C 3(I G, K)$

$X \quad-8 . * K C 4(I G, K)+7 . * K C 5(I G, K)-2 . * K C 6(I G, K)) * H R(K)$

$X \quad /(P 07(1, K) * P 0(2, K) * 00(1, K))$

$F T(I, 4,4)=(K C 4(I G, J)-2, * K C 5(I G, J)+K C 6(I G, J))$

$x \quad * H R(J) * 2 /(\operatorname{PH} 7(1, J) * \operatorname{PH}(2, J) * D H(1, J) * D H(2, J))$

$x+(K C 2(I G, K)-4 . * K C 3(I G, K)+6 . * K C 4(I G, K)$

$X \quad-4, * K C 5(1 G, K)+K C 6(1 G, K)) * H R(K) * * 2$

$X \quad /(P O 7(1, K) * P O(2, K) * D O(1, K) * D O(2, K))$

$F T(I, 5,4)=(-3, * K C 3(I G, K)+8, * K C 4(I G, K)-7, * K C 5(I G, K)+2, * K C 6(I G, K))$

$X \quad * H R(K) /(P O T(1, K) * P H(2, K) * D O(1, K))$

FT $(1,6,4)=(-K C 3(I G, K)+3, * K C 4(I G, K)-3, * K C 5(I G, K)+K C 6(I G, K))$

$X \quad * H R(K) * * 2 /(P 07(1, K) * P H(2, K) * D O(1, K) * D H(2, K))$

60 CONT INUE

IF $(I B C \cdot E Q .4) \quad G 0$ TO 63 ZERO FLUX COEFFICIENTS ON THE LEFT:

DO $61 \quad I G=1,2$

$V 2=1 . /(P O 7(I G, 1) * P O(I G, 1))$

$V 3=1 . /(P \cup 7(I G, 1) * P H(I G, 1))$

$L T(1,4, I G)=(K A 2(I G, 1)-4$.*KA $3(I G, 1)+6, * K A 4(I G, 1)-4, * K A 5(I G, 1)$

$X \quad+K A G(I G, 1)-(L A 2(I G, 1)-4 . * L A 3(I G, 1)+6 . * L A 4(I G, 1)-4, * L A 5(I G, 1)$

$x \quad+L A G(I G, 1))-P 1(I G, 1)+6, * P 2(I G, 1)-12 . * P 3(I G, 1)+10 . * P 4(I G, 1)$

$X \quad-3 . * P 5(I G, 1)-(-01(I G, 1)+6 . * 02(I G, 1)-12, * 03(I G, 1)+10 . * 04(I G, 1)$

$X \quad-3 . * 25(I G, 1))+R 0(I G, 1)-8 . * R I(I G, 1)+22 . * R 2(I G, 1)-24 . * R 3(I G, 1)$

SYNTO 469

SYNT 0470

SYNT 0471

SYNT 0472

SYNT 0473

SYNT 0474

SYNT 0475

SYNT 0476

SYNT 0477

SYNT 0478

SYNT 0479

SYNT 0480

SYNT 0481

SYNT 0482

SYNT 0483

SYNT 0484

SYNT 0485

SYNT 0486

SYNT0487

SYNT 0488

SYNT 0489

SYNT 0490

SYNT 0491

SYNT 0492

SYNT 0493

SYNT 0494

SYNT0495

SYNT 0496

SYNT 0497

SYNT 0498

SYNT 0499

SYNT 0500

SYNT 0501

SYNT 0502

SYNT 0503

SYNT 0504

PAGE 303 
$X+9 . * R 4(1 G, 1)) * V 2 *(H R(1) / D O(I G, 1)) * 2$

$L T(1,5,16)=(-3, * K A 3(I G, 1)+8 . * K A 4(I G, 1)-7, * K A 5(I G, 1)+2, * K A 6(I G, 1)$

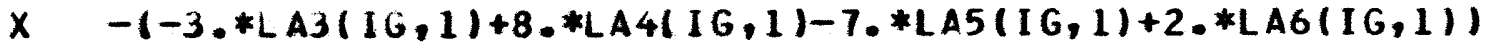

$X+3 . * P 2(1 G, 1)-14 . * P 3(16,1)+17 . * F 4(16,1)-6 . * P 5(1 G, 1)+(-6 . *$

$X \quad Q 2(I G, 1)+18, * 03(I G, 1)-18, * 04(I G, 1)+6 . * 05(I G, 1))-(6, * R 1(I G, 1)$

$X \quad-30 . * R 2(I G, 1)+42, * R 3(I G, 1)-18, * R 4(I G, 1))) * V 3 * H R(1) / D 0(I G, 1)$

$L T(1,6, I G)=(-K A 3(I G, 1)+3 . * K A 4(I G, 1)-3 . * K A 5(I G, 1)+K A 6(I G, 1)$

$X \quad-(-L A 3(I G, 1)+3 . * L A 4(I G, 1)-3 . * L A 5(I G, 1)+L A 6(I G, 1)+P 2(I G, 1)$

$X \quad-5 . * P 3(1 G, 1)+7 . * P 4(I G, 1)-3 . * P 5(I G, 1)-(2, * Q 2(I G, 1)-7 . * Q 3(I G, 1)$

$X \quad+8 . * 44(16,1)=3 . * 05(I G, 1))-2 . * R 1(I G, 1)+11 . * R 2(I G, 1)-18, * R 3(I G, 1)$

$X+9 . * R 4(I G, 1)) * V 3 * H R(1) * H R(1) /(D O(I G, 1) * D H(I G, 1))$

$F T(1,4, I G)=(K B 2(I G, 1)-4 . * K B 3(I G, 1)+6 . * K B 4(I G, 1)-4 . * K B 5(I G, 1)$

$X \quad+K B \circ(I G, 1)) * V 2 *(H R(1) / 00(I G, 1)) * 2$

FT $(1,5, I G)=(-3, * K B 3(I G, 1)+8 * k B 4(I G, 1)-7, * K B 5(I G, 1)+2, * K B 6(I G, 1))$

$X \quad * V 3 * H R(1) / 00(16,1)$

$F T(1,6, I G)=(-K B 3(I G, 1)+3 . * K B 4(I G, 1)-3 . * K B 5(I G, 1)+K B 6(I G, 1))$

$X \quad * V 3 * H R(1) * H R(1) /(D 0(I G, 1) * D H(1 G, 1))$

IF $(1 \mathrm{G} . \mathrm{EQ} .2)$ GO TO 61

$\mathrm{T}(1,4)=(\operatorname{SR} 2(\mathrm{IG}, 1)-4, * \operatorname{SR} 3(\mathrm{IG}, 1)+6, * \operatorname{SR} 4(\mathrm{IG}, 1)-4 . * \operatorname{SR} 5(\mathrm{IG}, 1)$

$X+\operatorname{SRG}(I G, 1)) * H R(1) * 2 /(P 07(2,1) * P C(1,1) * D O(2,1) * D 0(1,1))$

$T(1,5)=(-3 . * S R 3(I G, 1)+8 . * S R 4(I G, 1)-7 . * \operatorname{SR} 5(\operatorname{IG}, 1)+2 *$ I $(\mathrm{SR} 6(\mathrm{IG}, 1))$

$X \quad * H R(1) /(P 07(2,1) * P H(1,1) * 00(2,1))$

$T(1,6)=(-\operatorname{SR} 3(I G, 1)+3 . * S R 4(I G, 1)-3 . * \operatorname{SR} 5(I G, 1)+S R 6(I G, 1))$

$\mathrm{X}$ $* H R(1) * 2 /(P 07(2,1) * P H(1,1) * 00(2,1) * D H(1,1))$

FT $(1,4,3)=(K D 2(I G, 1)-4$ *KD $3(I G, 1)+6 . * K D 4(I G, 1)-4 . * K D 5(I G, 1)$

$X \quad+K D 6(1 G, 1)) * \operatorname{HR}(1) * 2 /(P 07(2,1) * \operatorname{PO}(1,1) * D 0(2,1) * D 0(1,1))$

FT $(1,5,3)=(-3, * K D 3(I G, 1)+8 . * K D 4(I G, 1)-7 . * K D 5(I G, 1)+2, * K D 6(I G, 1))$

$X \quad * H R(1) /(P 07(2,1) * P H(1,1) * D 0(2,1))$

$F T(1,6,3)=(-K D 3(I G, 1)+3, * K D 4(I G, 1)-3 . * K D 5(I G, 1)+K D 6(I G, 1))$ $* H R(1) * 2 /(P 07(2,1) * P H(1,1) * D 0(2,1) * D H(1,1))$

FT $(1,4,4)=(K C 2(I G, 1)-4 * * K C 3(I G, 1)+6, * K C 4(I G, 1)-4 * * K C 5(I G, 1)$

$X \quad+K C 6(I G, 1)) * H R(1) * * 2 /(P 07(1,1) * \operatorname{PO}(2,1) * D 0(1,1) * D 0(2,1))$

$\mathrm{FT}(1,5,4)=(-3, * K C 3(\mathrm{IG}, 1)+8, * K C 4(\mathrm{IG}, 1)-7 . * K C 5(I \mathrm{G}, 1)+2 * k \mathrm{C} 6(\mathrm{IG}, 1))$

$X \quad * H R(1) /(P O 7(1,1) * P H(2,1) * D O(1,1))$

FT $(1,6,4)=(-K C 3(I G, 1)+3, * K C 4(I G, 1)-3, * K C 5(I G, 1)+K C 6(I G, 1))$

$X \quad * H R(1) * 2 /(\operatorname{PO} 7(1,1) * \operatorname{PH}(2,1) * D O(1,1) * D H(2,1))$

SYNTO505

SYNT 0506

SYNT 0507

SYNT 0508

SYNT0509

SYNT 0510

SYNT0511

SYNT 0512

SYNT 0513

SYNT 0514

SYNT 0515

SYNT 0516

SYNT 0517

SYNT0518

SYNT 0519

SYNT 0520

SYNT 0521

SYNT 0522

SYNT0523

SYNTO5 24

SYNT 0525

SYNT 0526

SYNT 0527

SYNT 0528

SYNT 0529

SYNT 0530

SYNT 0531

SYNT 0532

SYNT0533

SYNT 0534

SYNT0535

SYNT 0536

SYNTO537

SYNT 0538

SYNTO539

SYNT 0540

PAGE 304 
SYNT 0541 SYNT 0542 SYNT 0543 SYNT0544 SYNT 0545 SYNT 0546 SYNT 0547 SYNT 0548 SYNT 0549 SYNT 0550 SYNT 0551 SYNT 0552 SYNT0553 SYNT 0554 SYNT 0555 SYNT 0556 SYNT 0557 SYNT 0558 SYNT 0559 SYNT 0560 SYNT 0561 SYNT 0562 SYNT 0563 SYNT 0564 SYNT 0565 SYNT 0566 SYNT 0567 SYNT 0568 SYNT0569 SYNT 0570 SYNT0571 SYNT 0572 SYNT 0573 SYNT 0574 SYNT0575 SYNT 0576 PAGE 305 
$T(I, 5)=(3 . * S R 2(I G, K)-2 * * S R 3(I G, K)-9 . * S R 4(I G, K)+12 . * S R 5(I G, K)$

IF (IBC.EQ.2.OR,IBC.EQ.4) GO TO 74

SYNT 0598

SYNT 0599

SYNT 0600

SYNT0601

SYNT 0602

SYNT 0603

SYNT 0604

SYNT 0605

SYNT 0606

SYNT 0607

SYNT0608

SYNT0609

SYNT0610

SYNT 0611

SYNT 0612 
$V 1=1 . /(P H 7(I G, J) * P H(I G, J))$

SYNT 0613

SYNT0614

$L T(I, 1, I G)=(K A 2(I G, J)-K A 3(I G, J)-3 . * K A 4(I G, J)+5 . * K A 5(I G, J)-2 . *$ $K A 6(I G, J)-(L A 2(I G, J)-L A 3(I G, J)-3 . * L A 4(I G, J)+5 . * L A 5(I G, J)-2 . *$ $L A G(I G, J))-2 . * P 1(I G, J)+3 . * P 2(I G, J)+6 . * P 3(I G, J)-13 . * P 4(I G, J)+6 . *$ $P 5(I G, J)-(6, * Q 3(I G, J)-12 * * Q 4(I G, J)+6 . * Q 5(I G, J))-12 * * R 2(I G, J)$ $+30 . * R 3(I G, J)-18, * R 4(I G, J)) * V * H R(J) / D H(I G, J)$

$L T(I, 2, I G)=(-K A 3(I G, J)+3 . * K A 4(I G, J)-3 . * K A 5(I G, J)+K A 6(I G, J)$ $-(-L A 3(I G, J)+3 . * L A 4(I G, J)-3 . * L A 5(I G, J)+L A 6(I G, J))+2 . * P 2(I G, J)$ $-7 . * P 3(I G, J)+8 . * P 4(I G, J)-3 . * P 5(I G, J)-(Q 2(I G, J)-5 . * Q 3(I G, J)$ $+7 . * Q 4(I G, J)-3 . * Q 5(I G, J))-2 . * R I(I G, J)+11 . * R 2(I G, J)-18$.* $R 3([G, J)+9, * R 4(I G, J)) * V * H R(J) * H R(J) /(D O(I G, J) * D H(I G, J))$

$L T(I, 3, I G)=(K A 4(I G, J)-2$.*KA $5(I G, J)+K A 6(I G, J)-(L A 4(I G, J)-2$.*

$X \quad L A 5(I G, J)+L A 6(I G, J))-2 . * P 3(I G, J)+5 . * P 4(I G, J)-3 . * P 5(I G, J)-(-2 . *$

$X \quad Q 3(I G, J)+5 . * Q 4(I G, J)-3 . * 05(I G, J))+4 . * R 2(I G, J)-12 . * R 3(I G, J)+9 . *$

$X \quad R 4(I G, J)) * V i *(H R(J) / D H(I G, J)) * * 2$

$F T(I, 1, I G)=(K B 2(I G, J)-K B 3(I G, J)-3 . * K B 4(I G, J)+5 . * K B 5(I G, J)$

$X \quad-2 . * K B 6(I G, J)) * V * H R(J) / D H(I G, J)$

$F T(I, 2, I G)=(-K B 3(I G, J)+3 . * K B 4(I G, J)-3 . * K B 5(I G, J)+K B 6(I G, J))$

$X * V * H R(J) * H R(J) /(D O(I G, J) * C H(I G, J))$

$F T(I, 3, I G)=(K B 4(I G, J)-2 * * K B 5(I G, J)+K B 6(I G, J))$

$X \quad * V I *(A R(J) / D H(I G, J)) * * 2$

IF (IG.EQ.2) GO TO 72

$T(I, 1)=(\operatorname{SR} 2(I G, J)-\operatorname{SR} 3(I G, J)-3 . * \operatorname{SR} 4(I G, J)+5 . * \operatorname{SR} 5(I G, J)$ $-2 . * S R 6(16, j)) * H R(J) /(P H 7(2, J) * P(1, J) * D H(2, J))$

$T(I, 2)=(-S R 3(I G, J)+3, * S R 4(I G, J)-3 . * S R 5(I G, J)+S R 6(I G, J))$ $* H R(J) * * 2 /(P H 7(2, J) * P O(1, J) * D H(2, J) * D O(1, J))$

$T(I, 3)=(S R *(I G, J)-2 * * \operatorname{SR} 5(I G, J)+\operatorname{SRG}(I G, J))$

$X \quad * H R(J) * 2 /(P H 7(2, J) * P H(1, J) * D H(2, J) * D H(1, J))$

$F T(I, 1,3)=(K D 2(I G, J)-K D 3(I G, J)-3 . * K D 4(I G, J)+5 . * K D 5(I G, J)$

$X \quad-2 . * K D 6(I G, J)) * H R(J) /(P H 7(2, J) * P((1, J) * D H(2, J))$

$F T(I, 2,3)=(-K D 3(I G, J)+3 . * K D 4(I G, J)-3 . * K D 5(I G, J)+K D 6(I G, J))$ $* H R(J) * 2 /(P H 7(2, J) * P O(1, J) * D H(2, J) * D O(1, J))$

$F T(I, 3,3)=(K D 4(I G, J)-2 * * K D 5(I G, J)+K D 6(I G, J))$

$X \quad * H R(J) * * 2 /(P H 7(2, J) * P H(1, J) * D H(2, J) * D H(1, J))$

$F T(I, 1,4)=(K C 2(I G, J)-K C 3(I G, J)-3 . * K C 4(I G, J)+5 . * K C 5(I G, J)$

$x-2 . * K C G(I G, J)) * H R(J) /(P H 7(1, J) * P C(2, J) * D H(1, J))$

SYNTO615

SYNT 0616

SYNT 0617

SYNTO618

SYNT0619

SYNT 0620

SYNT 0621

SYNT 0622

SYNT 0623

SYNT 0624

SYNT 0625

SYNT 0626

SYNT 0627

SYNT 0628

SYNT 0629

SYNT 0630

SYNT 0631

SYNT 0632

SYNT 0633

SYNT0634

SYNT0635

SYNT 0636

SYNT 0637

SYNT0638

SYNT 0639

SYNT 0640

SYNT 0641

SYNT 0642

SYNT0643

SYNT 0644

SYNT 0645

SYNT 0646

SYNT 0647

SYNT 0648 
$F T(I, 2,4)=(-K C 3(I G, J)+3 . * K C 4(I G, J)-3 . * K C 5(I G, J)+K C 6(I G, J))$

SYNT0649

$X \quad * H R(J) * 2 J(P H 7(1, J) * P O(2, J) * D H(1, J) * D O(2, J))$

$F T(I, 3,4)=(K C 4(I G, J)-2 * * K C 5(I G, J)+K C 6(I G, J))$

$X \quad * H R(J) * 2 /(P H 7(1, J) * P H(2, J) * D H(1, J) * D H(2, J))$

72 CONT INUE

FIX JP THE LAST TWO COLUMNS TO MATCH $G(K+1)$ (NOT $F(K+1))$ :

$I 1=2 * K R-2$

$12=11+1$

DO $73 \quad 16=1.2$

DO $73 \quad I=I 1, I 2$

$\operatorname{LT}(I, 5, I G)=\operatorname{LT}(I, 6, I G)$

$F T(I, 5, I G)=F T(I, 6,1 G)$

IF (IG.EQ.2) GO TO 73

$T(1,5)=T(1,6)$

$F T(1,5,3)=F T(1,6,3)$

$F T(1,5,4)=F T(I, 6,4)$

73 CONTINUE

GO TO 80

SYNT0650

SYNT0651

SYNT 0652

SYNT 0653

SYNT 0654

SYNT 0655

SYNT0656

SYNT0657

SYNT0658

SYNT 0659

SYNT0660

SYNT 0661

SYNT0662

SYNT0663

SYNTO664

SYNT0665

SYNT0666

SYNT 0667

SYNT0668

SYNT 0669

SYNT 0670

SYNT 0671

SYNT 0672

SYNT0673

SYNT0674

SYNT 0675

SYNT 0676

SYNT 0677

SYNT0678

SYNT0679

SYNT 0680

SYNT 0681

SYNT 0682

SYNT 0683

SYNT0684

PAGE 308 


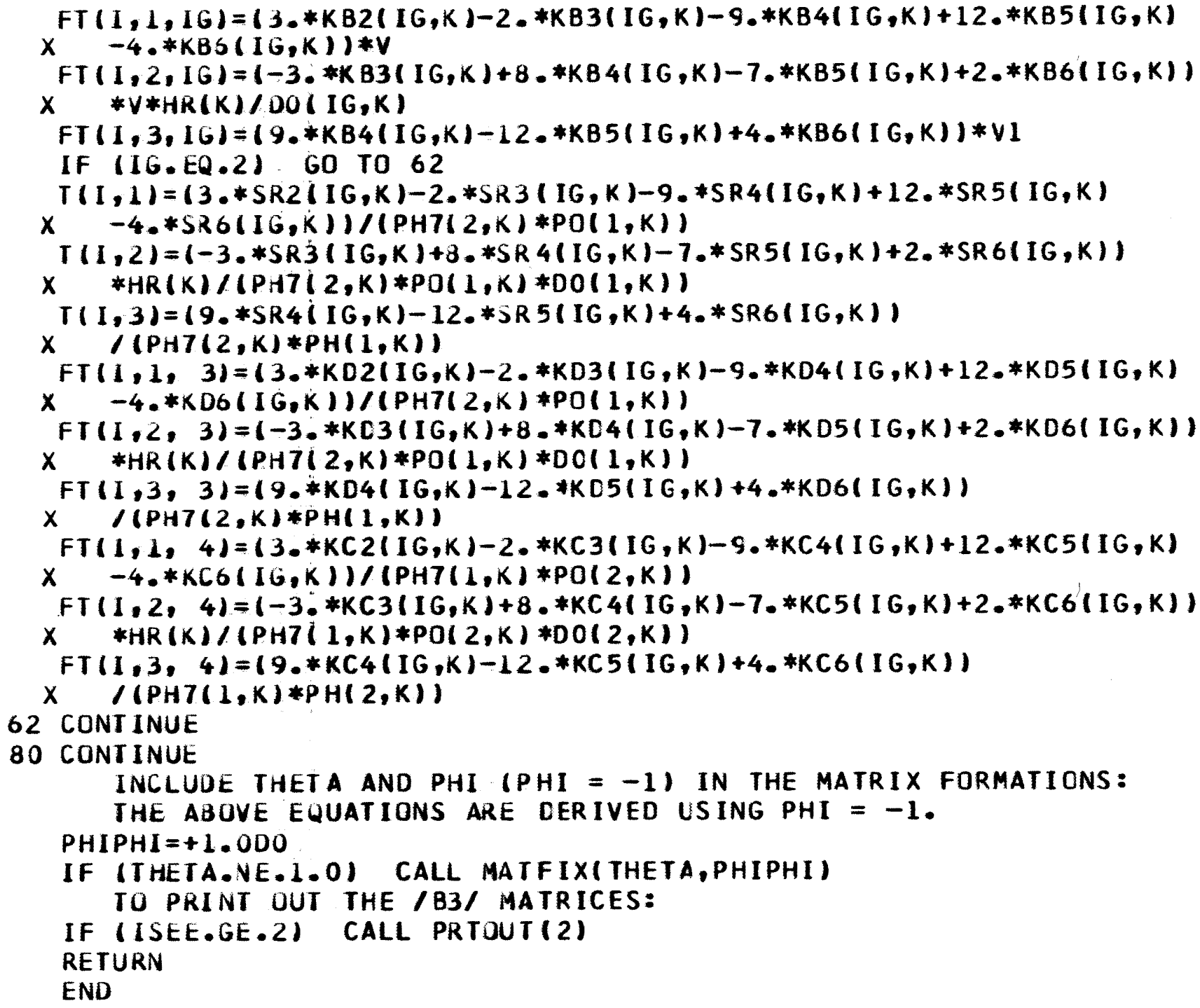

SYNT 0685

SYNT 0686

SYNT 0687

SYNT0688

SYNT 0689

SYNT 0690

SYNT 0691

SYNT 0692

SYNT 0693

SYNT0694

SYNT 0695

SYNT 0696

SYNT 0697

SYNT0698

SYNT0699

SYNT 0700

SYNT 0701

SYNT 0702

SYNT 0703

SYNT 0704

SYNT0705

SYNT 0706

SYNT 0707

SYNT 0708

SYNT 0709

SYNT 0710

SYNT0711

SYNT 0712

SYNT0713

SYNT 0714

SYNT0715

SYNT 0716

SYNT 0717

SYNTO718 
SUBROUT INE ERROR $(I, J)$

ANNOUNCES INPUT ERRORS AND TERMINATES PROGRAM EXECUTION:

ERR00001

ERROO002

GO TO $(1,2,3,4,5,6,7,8,9), I$

1 WRITE $(6,101)$

GO TO 10

2 WRITE $(6,102) \mathrm{J}$

GO TO 10

3 WRITE $(6,103) \mathrm{J}$

GO TO 10

4 WRITE $(6,104) \mathrm{J}$

GO TO 10

5 WRITE $(6,105) \mathrm{J}$

GO TO 10

6 WRITE $(6,106) \mathrm{J}$

GO TO 10

7 CONT INUE

8 CONTINUE

9 CONTINUE

10 WRITE $(6,110)$

101 FORMAT ('IMUST HAVE $>2$ SUBREGICNS FOR ZERO FLUX B.C.S. INVALID.")

102 FORMAT ('INUMBER OF SUBREGICNS $=\cdot, 13,0^{\circ}>25$. INVALID.")

103 FORMAT ('ISUBREGION NUMBER',I3," HAS > 100 SECTIONS. INVALID.')

104 FORMAT ('IINPUT ERROR IN REGICN SEQUENCING AT REGION', I5,, 1$)$

105 FORMAT $\left(\cdot 12(1)=0\right.$. IN REG ICN I $=0^{\circ}, 13, \cdots$ INVALID.')

106 FORMAT ('1THE TOLERANCE: EPS 111, IS $<1.0 E-16$. INVALID.')

107 FORMAT ('IBOUNDRY CONDITION OPTICN $=9,12, "\langle 1$ OR > 4. INVALID.")

110 FORMAT (IHO, PROBLEM TERMINATED.')

CALL EXIT

RETURN

ERRO0003

ERRO0004

ERR00005

ERRO0006

ERRO0007

ERR00008

ERR00009

ERRO0010

ERRO0011

ERRO0012

ERRO0013

ERRO0014

ERRO0015

ERRO0016

ERRO0017

ERRO0018

ERRO0019

ERRO0020

ERRO0021

ERRO0022

ERRO0023

ERRO0024

ERRO0025

ERRO0026

ERRO0027

ERRO0028

ERRO0029

END

ERRO0030 
SUBROUTINE REPEAT (K,L)

C SETS THE /B5/ ARRAYS (K) EQUAL TO PAST STORED ARRAYS (L): IMPLICIT REAL *8 (A-Z)

COMMON /BS/

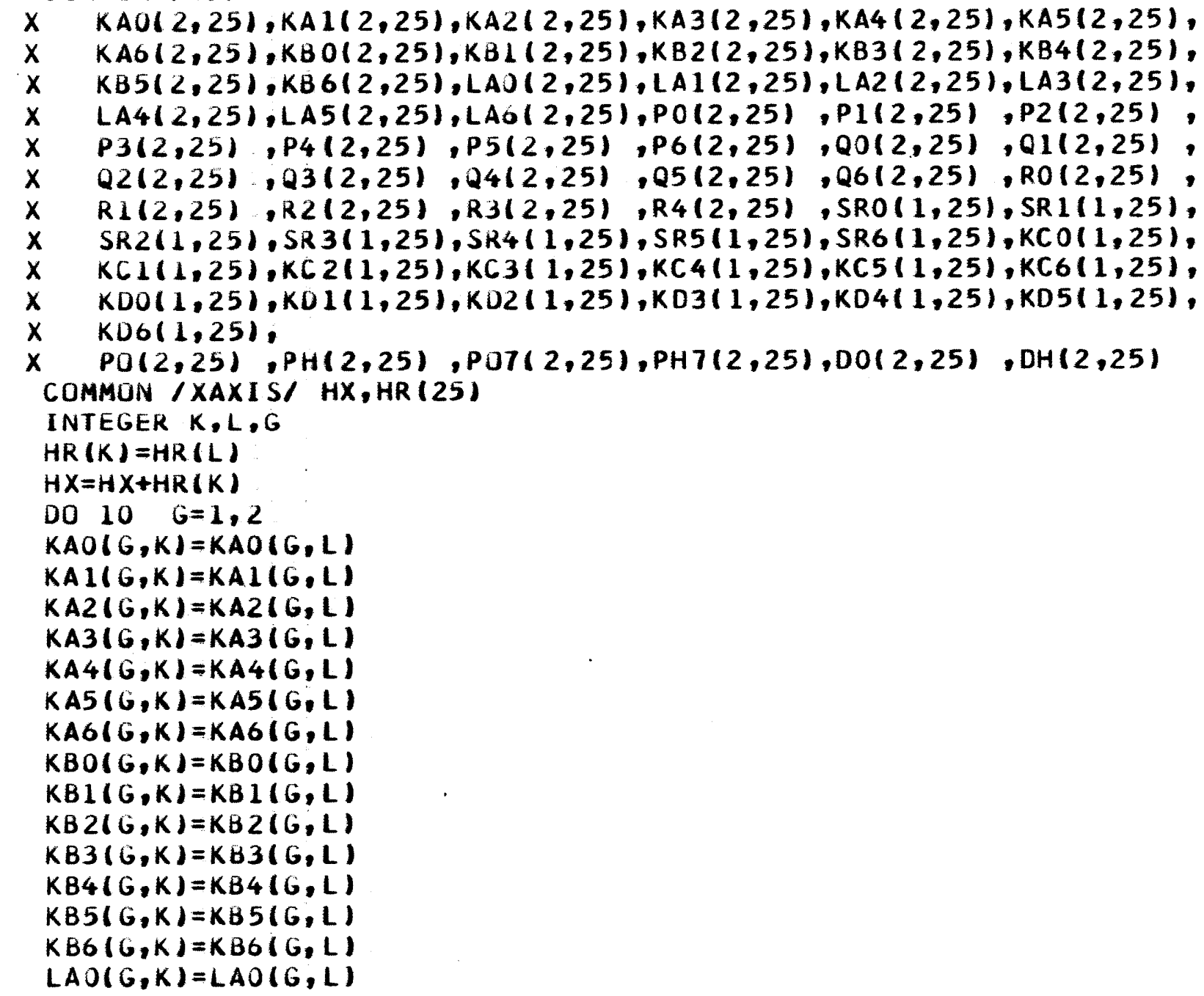

REPE0001

REPE0002

REPE0003

REPE 0004

REPE0005

REPE 0006

REPE 0007

REPE0008

REPE 0009

REPE0010

REPE0011

REPE 0012

REPEO013

REPE0014

REPE0015

REPE0016

REPEO017

REPE0018

REPE0019

REPE 0020

REPE0021

REPE 0022

REPE 0023

REPE0024

REPE0025

REPE0026

REPE 0027

REPE 0028

REPE0029

REPE0030

REPE 0031

REPE0032

REPE 0033

REPE 0034

REPE 0035

REPE0036

PAGE 311 


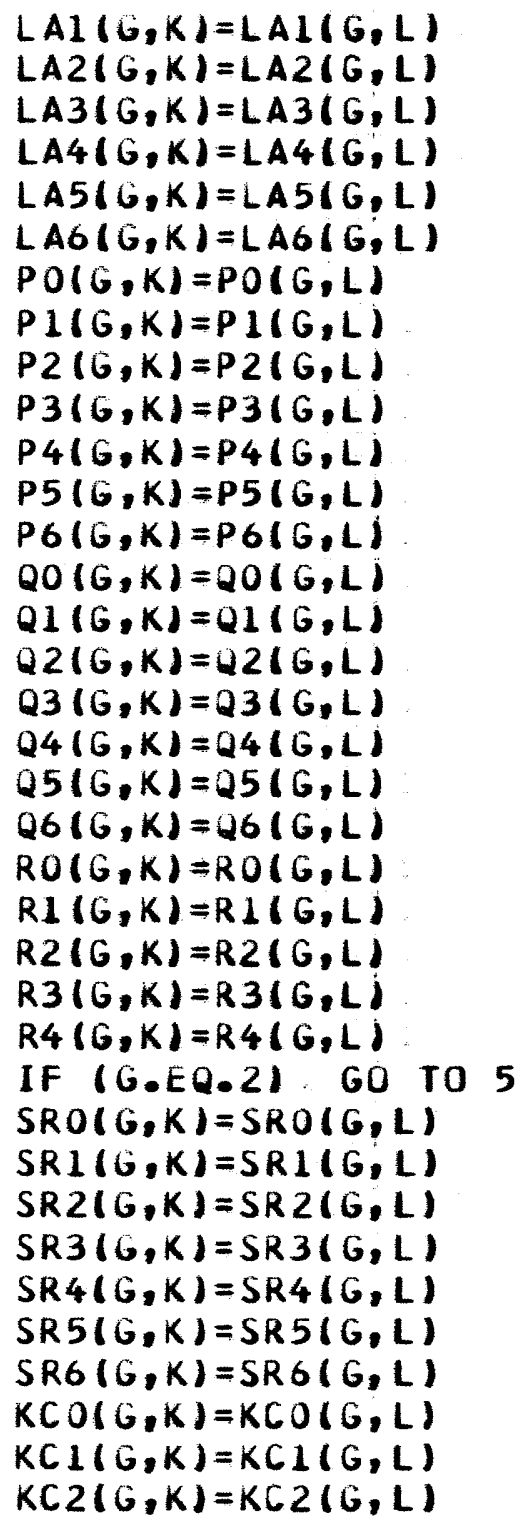

REPE0037

REPE0038

REPE0039

REPE0040

REPE 0041

REPE0042

REPE 0043

REPE0044

REPE 0045

REPE 0046

REPE0047

REPE 0048

REPE0049

REPE0050

REPE 0051

REPE0052

REPE 0053

REPE 0054

REPE0055

REPE 0056

REPE0057

REPE0058

REPE0059

REPE0060

REPE0061

REPE 0062

REPE0063

REPE 0064

REPE0065

REPE0066

REPE0067

REPE0068

REPE0069

REPE0070

REPE0071

REPE 0072

PAGE 312 
$K C 3(G, K)=K C 3(G, L)$ $K C 4(G, K)=K C 4(G, L)$ $K C 5(G, K)=K C 5(G, L)$ $K C 6(G, K)=K C 6(G, L)$ $K D O(G, K)=K D O(G, L)$ $K D 1(G, K)=K D 1(G, L)$ $K D 2(G, K)=K D 2(G, L)$ $K D 3(G, K)=K D 3(G, L)$ $K D 4(G, K)=K D 4(G, L)$ $\operatorname{KD} 5(G, K)=\operatorname{KD} 5(G, L)$ $K D 6(G, K)=K D 6(G, L)$

5 CONT INUE

$P O(G, K)=P O(G, L)$

$P 07(G, K)=P 07(G, L)$

$P H(G, K)=P H(G, L)$

$\operatorname{PH} 7(G, K)=P H 7(G, L)$

$D O(G, K)=D O(G, L)$

$\operatorname{DH}(G, K)=\operatorname{DH}(G, L)$

10 CONTINUE

RETURN

END

REPE0073

REPE 0074

REPE0075

REPE0076

REPE 0077

REPE 0078

REPE0079

REPE 0080

REPE0081

REPE 0082

REPE0083

REPE0084

REPE 0085

REPE 0086

REPE 0087

REPE 0088

REPE0089

REPE 0090

REPE0091

REPE0092

REPE0093 
SUBROUTINE BHSET(K)

SETS UP THE /BH/ ARRAYS FOR GIF: IMPLICIT REAL*8 (A-H,L-Z)

COMMON /BH/ X(101), H(101), Z(101) DO $1 \quad I=1, K$

$1 Z(I)=X(I)-X(1)$ RETURN

END
BHSE0001

BHSE0002

BHSEOOO3

BHSE0004

BHSE0005

BHSE0006

BHSE0007

BHSE0008 
DOUBLE PREGISION FUNCTION GIFIN,G1,F,G $, C, G, K$, ITC)

$c$
$c$

* identical to subroutine gif previcusly listed in program linear. RETURN

END

GIF 0001

GIF 0002

GIF 0003

GIF 0004

GIF 0005

GIF 0006 
DOUBLE PRECISION FUNCTION FACT(N)

FACT 0001

FACT 0002

FACT 0003

FACTOO04

FACT 0005

FACT 0006

FACT0007

I FACT $=F A C T * D F L O A T$ (I)

RETURN

FACT 0008 
SUBRUUTINE MATFIX (THEATA,PHI)

C MODIFYS THE MATRIX ELEMENTS OF THE /B3/ MATRICES BY THEATA AND PHI.

MATF0001

MATF0002

MATF0003

PROPER CHOICE OF THEATA PROVIDES EASIER INVERSION OF THE MATRICES.

MATF0004 MATRIX SOLUTION SHOULD BE INDEPENDENT CF PHI. HOWEVER:

USE OF PHI > O RESULTS IN POSITIVE CEFINITE MATRICES.

COMMON /BI/ IBC

COMMON /B2/ KR, NN

COMMON /B3/ A(50,6,7)

REAL*8 A, THEATA, PHI, $X, Y, Z$

$N O=N N-1$

DO $10 L=1,7$

DO $5 \quad I=2$, NO

$X=$ THEATA

$Y=1.000$

IF $(\operatorname{MOD}(1,2), E Q .0)$ GO TO 1

$X=$ THEATA*PHI

$Y=P H I$

1 DO $2 M=2,6,2$

$2 A(I, M, L)=X * A(I, M, L)$

DO $3 M=1,5,2$

$3 A(I, M, L)=Y * A(I, M, L)$

5 CONT INUE

BUUNDARY CONDITION EQUATIONS:

$\mathrm{X}=1.000$

$Y=1.000$

$Z=T H E A T A$

IF $(I B C . G T .2)$ GO TO 6

$X=$ THEATA*PHI

$Y=P H I$

$\mathrm{Z}=\mathrm{X}$

$6 A(1,4, L)=X * A(1,4, L)$

$A(1,5, L)=Y * A(1,5, L)$

$A(1,6, L)=Z * A(1,6, L)$

$X=1.000$

$Y=$ THEATA

$Z=X$

(ATF0005

MATF 0006

MATF0007

MATF0008

MATF0009

MATF0010

MATF0011

MATF0012

MATF0013

MATF 0014

MATF0015

MATF 0016

MATF0017

MATF0018

MATF 0019

MATF 0020

MATF0021

MATF 0022

MATF0023

MATF0024

MA TF 0025

MATF 0026

MATF0027

MATF 0028

MATF0029

MATF 0030

MATF0031

MATF 0032

MATF0033

MATF0034

MATF0035

MATF 0036

PAGE 317 
IF (IBC.EQ.2.OR . IBC.EQ.4) GO TO 7

MATF0037

MATF0038

MATF0039

$Y=T H E A T A * P H I$

$Z=Y$

$7 A(N N, 1, L)=X * A(N N, 1, L)$

$A(N N, 2, L)=Y * A(N N, 2, L)$

$A(N N, 3, L)=Z * A(N N, 3, L)$

10 CONT INUE

RETURN

MATF 0040

MATF 0041

MATF 0042

MATF 0043

MATF0044

MATF0045

END

MATF 0046 


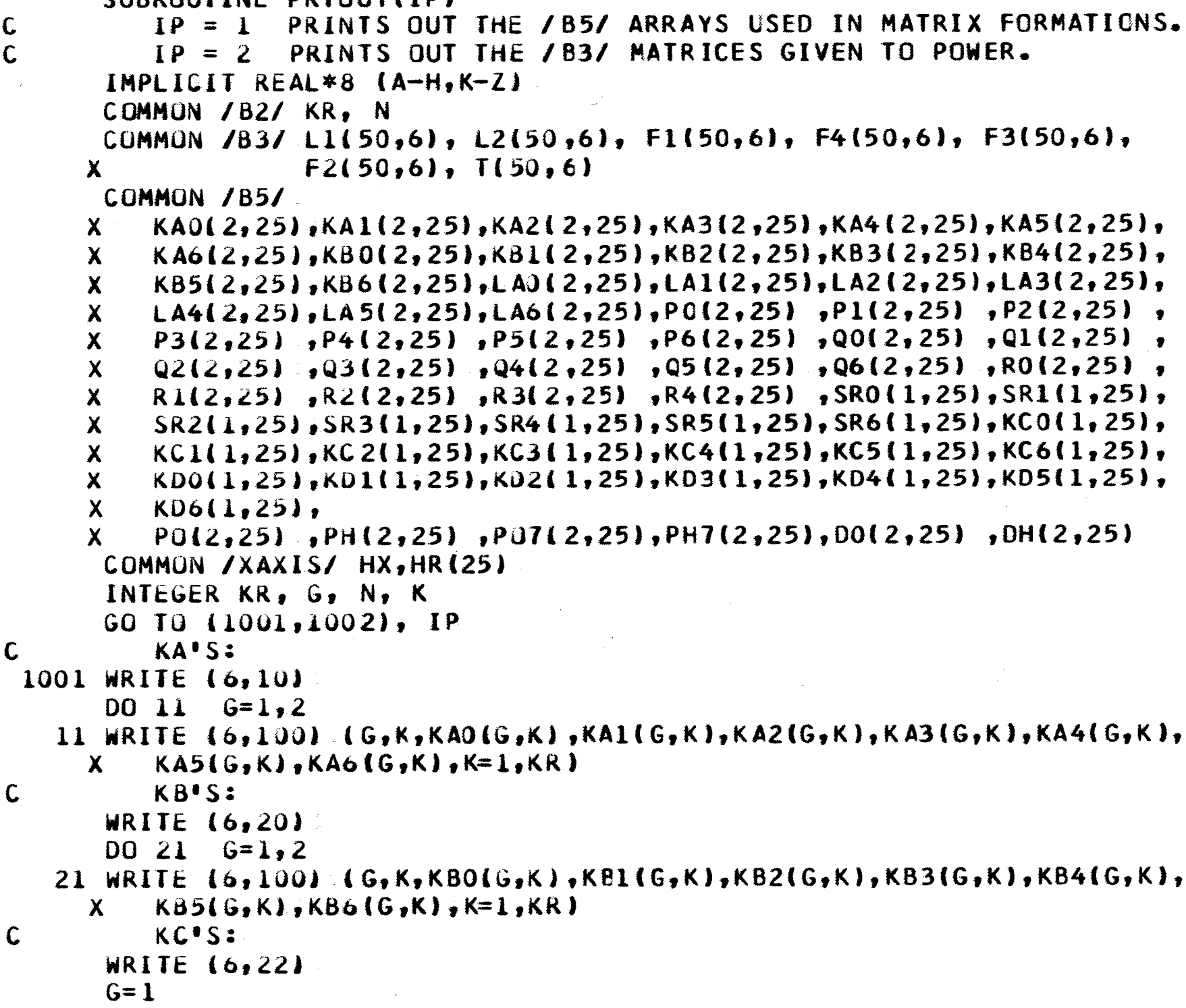

PRTO0001 PRTO0002 PRTO0003 PRTO0004 PRTO0005 PRTO0006 PRTO0007 PRTO0008 PRTO0009 PRTO0010 PR TO0011 PRTO0012 PRTO0013 PRTO0014 PRTO0015 PRTO0016 PRTO0017 PRTO0018 PRTO0019 PRTO0020

PRTO0021 PRTO0022 PRTO0023 PRTO0024 PRTO0025 PRTO0026

PRTO0027 PRTO0028

PRTO0029

PRTO0030

PRTO0031

PRTO0032

PRTO0033

PRTO0034

PRTO0035

PRTO0036

PAGE 319 
23 WRITE $(6,100)(G, K, K C O(G, K), K C 1(G, K), K C 2(G, K), K C 3(G, K), K C 4(G, K)$, $X \quad K C 5(6, K), K C 6(G, K), K=1, K R)$

C $K D \cdot S:$

WRITE $(6,24)$

$G=1$

25 WRITE $(6,100)(G, K, K D 0(G, K), K D 1(G, K), K D 2(G, K), K D 3(G, K), K D 4(G, K)$, $X \quad K U 5(G, K), K D 6(G, K), K=1, K R)$

C $L A \cdot S:$

WRITE $(6,30)$

DO $31 G=1,2$

31 WRITE $(6,100)(G, K, L A 0(G, K), L A 1(G, K), L A 2(G, K), L A 3(G, K), L A 4(G, K)$, $X \quad L A 5(G, K), L A G(G, K), K=1, K R)$

C. $S R \cdot S:$

WRITE $(6,40)$

$G=1$

41 WRITE $(6,100)(G, K, S R O(G, K), S R 1(G, K), S R 2(G, K), S R 3(G, K), S R 4(G, K)$,

C

$X \quad S R 5(G, K), S R 6(G, K), K=1, K R)$

POS:

WRITE $(6,50)$

DO $51 G=1,2$

51 WRITE $(6,100)(G, K, P O(G, K), P 1(G, K), P 2(G, K), P 3(G, K), P 4(G, K)$,

$X \quad P 5(G, K), P 6(G, K), K=1, K R)$

C Q'S:

WRITE $(6,60)$

DO $61 G=1,2$

61 WRITE $(6,100)(G, K, Q 0(G, K), Q 1(G, K), Q 2(G, K), Q 3(G, K), Q 4(G, K)$,

$X \quad Q 5(G, K), Q 6(G, K), K=1, K R)$

C $R \cdot S$ :

WRITE $(6,70)$

DO $71 \quad G=1,2$

71 WRITE $(6,101)(G, K, R O(G, K), R 1(G, K), R 2(G, K), R 3(G, K), R 4(G, K), K=1, K R)$ BOUNDARY VALUES:

C WRITE $(6,80)$

DO $81 G=1,2$

81 WRITE $(6,100)(G, K, P O(G, K), P H(G, K), P O 7(G, K), P H 7(G, K), D O(G, K)$, $X \quad D H(G, K), H R(K), K=1, K R)$

PR T00037

PRTO0038

PRTO0039

PRTO0040

PRTO0041

PR 100042

PRT00043

PRT00044

PR T00045

PRT00046

PRT00047

PRT 00048

PRTO0049

PR TO0050

PRTO0051

PRTO0052

PR T00053

PRT00054

PRT00055

PRT00056

PRTO0057

PR TO0058

PR TO0059

PRTO0060

PR T00061

PRTO0062

PRT00063

PRT00064

PRTO0065

PRT00066

PRTO0067

PRT00068

PRTO0069

PRT00070

PRT00071

PRTO0072

PAGE 320 
WRITE $(6,91)$

WRITE $(6,110),((L 2(I, J), J=1,6), I=1, N)$

PRTO0075

WRITE $(6,92)$

WRITE $(6,110) \quad((F 1(I, J), J=1,6), I=1, N)$

PR T00076

PRT T00077

WRITE $(6,93)$

WRITE $(6,110) \quad((F 2(I, J), J=1,6), I=1, N)$

WRITE $(6,94)$

WRITE $(6,110) \quad((F 3(I, J), J=1,6), I=1, N)$

WRITE $(6,95)$

WRITE $(6,110) \quad((F 4(I, J), J=1,6), I=1, N)$

WRITE $(6,96)$

WRITE $(6,110)((T(I, J), J=1,6), I=1, A)$

10 FORMAT $(11$ G',4X,'K',7X,'KAO(G,K)',7X,'KA1(G,K)',7X,'KA2(G,K)',

$\left.X \quad 7 X, \cdot K A 3(G, K) \cdot, 7 X,{ }^{\circ} K A 4(G, K) \cdot, 7 X, \cdot K A 5(G, K) \cdot, 7 X, \cdot K A 6(G, K) \cdot, 1\right)$

20 FORMAT $1{ }^{\circ} 1 G^{\circ}, 4 X,{ }^{\circ} K, 7 X,{ }^{\circ} K B O(G, K), 7 X,{ }^{\circ} K B 1(G, K) \cdot, 7 X,{ }^{\prime} K B 2(G, K)^{\prime}$,

$\left.X \quad 7 X,{ }^{\circ} K B 3(G, K) \cdot, 7 X,{ }^{\circ} K B 4(G, K) \cdot, 7 X,{ }^{\circ} K B 5(G, K) \cdot, 7 X,{ }^{\circ} K B 6(G, K) \cdot, /\right)$

22 FORMAT $1^{\prime} 1 \quad G \cdot, 4 X,{ }^{\circ} K,, 7 X,{ }^{\circ} K C O(G, K) \cdot, 7 X,{ }^{\circ} K C 1(G, K) \cdot, 7 X,{ }^{\circ} K C 2(G, K) !$,

$\left.X \quad 7 X,{ }^{\circ} K C 3(G, K) !, 7 X,{ }^{\circ} K C 4(G, K) \cdot, 7 X, \cdot K C 5(G, K) \cdot, 7 X,{ }^{\circ} K C 6(G, K) \cdot, /\right)$

24 FORMAT $1^{\prime} 1$ G',4X, $K \cdot, 7 X,{ }^{\circ} K D O(G, K) \cdot, 7 X,{ }^{\circ} K D 1(G, K),, 7 X,{ }^{\circ} K D 2(G, K)^{\prime}$,

$\left.X \quad 7 X, 0^{\prime} K D 3(G, K) \cdot, 7 X, 0^{\circ} K D 4(G, K) \cdot, 7 X,{ }^{\prime} K D 5(G, K) \cdot, 7 X,{ }^{\circ} K D 6(G, K) \cdot, /\right)$

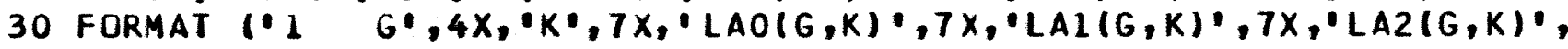

$\left.X \quad 7 X, \cdot L A 3(G, K) \cdot, 7 X,{ }^{\prime} L A 4(G, K) \cdot, 7 X, \cdot L A 5(G, K) \cdot, 7 X, \cdot L A 6(G, K) \cdot, /\right)$

40 FORMAT $\left(\cdot 1 \quad G \cdot, 4 X,{ }^{\prime} K \cdot, 7 X, \cdot S R O(G, K)^{\prime}, 7 X,{ }^{\prime} S R 1(G, K), 7 X,{ }^{\prime} S R 2(G, K)^{\prime}\right.$,

$\left.X \quad 7 X, \cdot S R 3(G, K) \cdot, 7 X, \cdot S R 4(G, K) \cdot, 7 X, \cdot S R 5(G, K) \cdot, 7 X,{ }^{\prime} S R 6(G, K) \cdot, 1\right)$

50 FORMAT $\left(\cdot 1 \quad G \cdot, 4 X,{ }^{\circ} K, 8 X,{ }^{\prime} P O(G, K) \cdot, 8 X, \cdot P\left(1(G, K) \cdot, 8 X, P^{\prime} P(G, K) \cdot, 8 X\right.\right.$,

$X \quad P 3(G, K) \cup, 8 X, \cdot P 4(G, K) \cdot, 8 X, \cdot P 5(G, K),, 8 X, \cdot P 6(G, K) \cdot, /)$

60 FORMAT $1^{\circ} 1 \quad G^{\prime}, 4 X, K^{\circ}, 8 X,{ }^{\circ}, 00(G, K)^{\prime}, 8 X,{ }^{\prime} Q 1(G, K) \cdot, 8 X,{ }^{\prime} Q 2(G, K) \cdot, 8 X$,

$X \quad Q 3(G, K) \cdot, 8 X, \cdot Q 4(G, K) \cdot, 8 X, \cdot Q 5(G, K) \cdot, 8 X, \cdot Q 6(G, K) \cdot, /)$

70 FORMAT $\left(\cdot 1 \quad G{ }^{\prime}, 4 X,{ }^{\prime} K \cdot, 8 X,{ }^{\prime} R O(G, K) \cdot, 8 X,{ }^{\prime} R(1, G, K)^{\prime}, 8 X,{ }^{\prime} R 2(G, K) \cdot, 8 X\right.$, $\left.X \quad R 3(G, K) \cdot, 8 X, R^{\prime}(G, K) \cdot, /\right)$

80 FORMAT $\left({ }^{\circ} 1 G^{\circ}, 4 X,{ }^{\prime} K \cdot, 8 X,{ }^{\prime} P O(G, K) \cdot, 8 X, P^{\prime} P H(G, K) \cdot, 7 X, P^{\prime} P O 7(G, K) \cdot, 7 X\right.$,

$\left.X \quad P H 7(G, K) \cdot, 8 X, 0^{\circ} D O(G, K) \cdot, 8 X, \cdot D H(G, K) \cdot, 10 X, \cdot H R(K) \cdot, /\right)$

PRT00078

PRTO0079

PRT00080

PRTO0081

PRTO0082

PRTO0083

PRTO0084

PRTO0085

PRT00086

PR T00087

PRTO0088

PRT00089

PRT00090

PRTO0091

PRTO0092

PRT00093

PRTO0094

PRTO0095

PRTO0096

PRTO0097

PRTO0098

PRTO0099

PRTO0100

PRTO0101

PRTO0102

PRTO0103

PRTO0104

PRTO0105

PRTO0106

PRTO0107

PRTO0108

100 FORMAT $(215,7015.7)$ 


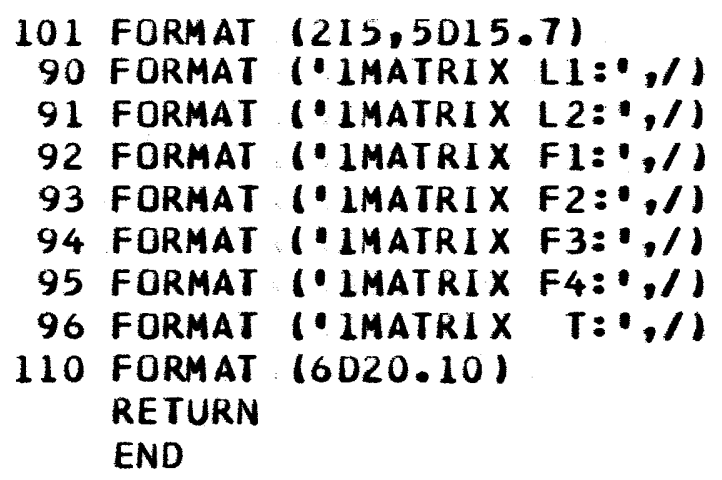

PRT00109

PRTO0110

PRTO0111

PR TO0112

PR TO0113

PRTO0114

PR TO0115

PRTO0116

PRTO0117

PRTO0118

PRTO0119 


\section{SUBROUTINE POWER}

C SOLVES THE $2 * N$ MULTIGROUP EQUATICNS: M*PHI $=(1 /$ LAMDA $) * F * P H I$

BY THE FISSION SOURCE PONER METHOD

USING SIMULTANEOUS OVERRELAXATION.

WHERE: M AND F ARE DOUBLE PRECISION 2N BY 2N BLOCK MATRICES;

AND:

PHI IS THE 2N FLUX (FAST AND THERMAL) VECTCR.

$L 1 * P H I 1=C H I 1 *(F 1 * P H I 1+F 2 * P H I 2)$

$-T * P H 11+L 2 * P H I 2=C H I 2 *(F 3 * P H I 1+F 4 * P H I 2)$

METHOD FOLLOWS WACHPRESS, PAGE 83. SOLUTION BY GROUP ITERATION.

IMPLICIT REAL*8 (A-H,L-Z)

COMMON /BI/ IBC,IPLOT, JPLOT, I PUNCH, ISEE

COMMON /B2/ KR,N

COMMON /B3/ L $1(50,6), L 2(50,6), F 1(50,6), F 4(50,6), F 3(50,6), F 2(50,6)$,

$x$ $T(50,6)$

COMMON /B4/ PHI $(2,52)$, PSI $(2,52)$, LAMDA, ICOUT

COMMON /B5/ S(52), ERROR $(2,52), Z(52), G(50,6), G T(50)$

COMMON /B6/ TE1 $(2,5), \operatorname{TE} 2(2,5), \operatorname{TE} 3(5)$, IN(5)

COMMON /CHIF/ CHI (2)

COMMON /XAXIS/ HX, HR (25)

COMMON /ER/ EPS 1, EPS 2,EPS3

COMMON /FSTR/ PHISTR $(2,26,6)$

COMMON /ESTR/ LAMSTR(300), EFSTR(2,300), EFMSTR $(2,300), \operatorname{ERLAM(300)}$ COMMON /TRJE/ TRULAM, TRUPHI(2,52), PHICON(2,300), LAMCON(300), IFT DIMENSION PSI 1(52), PSI $2(52)$, SO(2), DPHI (2), ERRMAX (2)

INTEGER N, NN

DEFAULT OPTICNS FOR THE TRUE EIGENVALUE AND FLUX-CURRENT VECTOR: TRULAM $=1.0$

DO $5 \quad$ I $G=1,2$

DO $4 . \quad I=1, N$

TRUPHI $(I G, I)=1.0$

IF (MOO(I, 2) EQ.1) TRUPHI (IG,I)=0.0

4 CONTINUE

IF $(I B C, E Q .1) \quad$ TRUPHI $(I G, N)=0.0$

IF (IBC.EQ.4) TRUPHI $(I G, 1)=1.0$

5 CONT INUE DEFAULT OPTIONS FOR POWER PARAMETERS:

POWE0001

POWE0002

POWE 0003

POWE0004

POWE0005

POWE0006

POWE0007

POWE 0008

POWE0009

POWE0010

POWE0011

POWE0012

POWE0013

POWEO014

POWE0015

POWE 0016

POWE0017

POWEOO1B

POWE0019

POWE0020

POWE0021

POWE0022

POWE0023

POWE 0024

POWE0025

POWE0026

POWE 0027

POWE0028

POWE0029

POWE0030

POWE0031

POWE0032

POWE0033

POWE0034

POWE0035

POWE0036

PAGE 323 
PHI $(I G, 1)=1.0$

GO TO 555

$551 X=3.1415926 / H X$

IF (IBC.NE.1) $X=X / 2.0$

POWE0043

POWE 0044

POWE0045

POWE 0046

POWE0047

POWE0048

PHI $(1 G, 1)=-X$

POWE0049

SUM $1=0.0$

DO $552 K=2, K R$

$I=2 * K-2$

$\mathrm{J}=\mathrm{I}+1$

SUMI $=$ SUM $L+H R(K-1)$

PHI (IG,I) $=$ DSIN( SUM $1 * X)$

$552 \operatorname{PHI}(I G, J)=-X * D C O S(S U M 1 * X)$

$\operatorname{PHI}(I G, N)=1.0$

IF (IBC.EQ.1) PHI(IG,N) =X

C

555 CONTINUE I $F T=0$

READ $(5,500, E N D=501)$ TRULAM, (TRUPHI $(1, I), I=1, N)$

POWE0050

POWE0051

POWE 0052

POWE0053

POWE 0054

POWE0055

POWE0056

POWE0057

POWE0058

POWE0059

POWE0060

POWE0061

POWE 0062

POWE 0063 IF $T=1$

500 FORMAT (E25.14,/, (4E20.10))

C READ IN: OVERRELAXATION

C

501 READ $(5,506, E N U=510)$ ALPHA

INITIAL GUESS AT EIGENVALUE:

(OUTER ITERATION)

POWE0064

POWE 0065

POWE 0066

POWE 0067

POWE 0068

POWE 0069

POWE0070

READ $(5,502, E N D=510)$ LAMOA

$\operatorname{READ}(5,503)$ (PHI $(1, I), I=1, N)$

$\operatorname{READ}(5,503) \quad(\operatorname{PHI}(2, I), I=1, N)$

POWE0071

POWE0072 


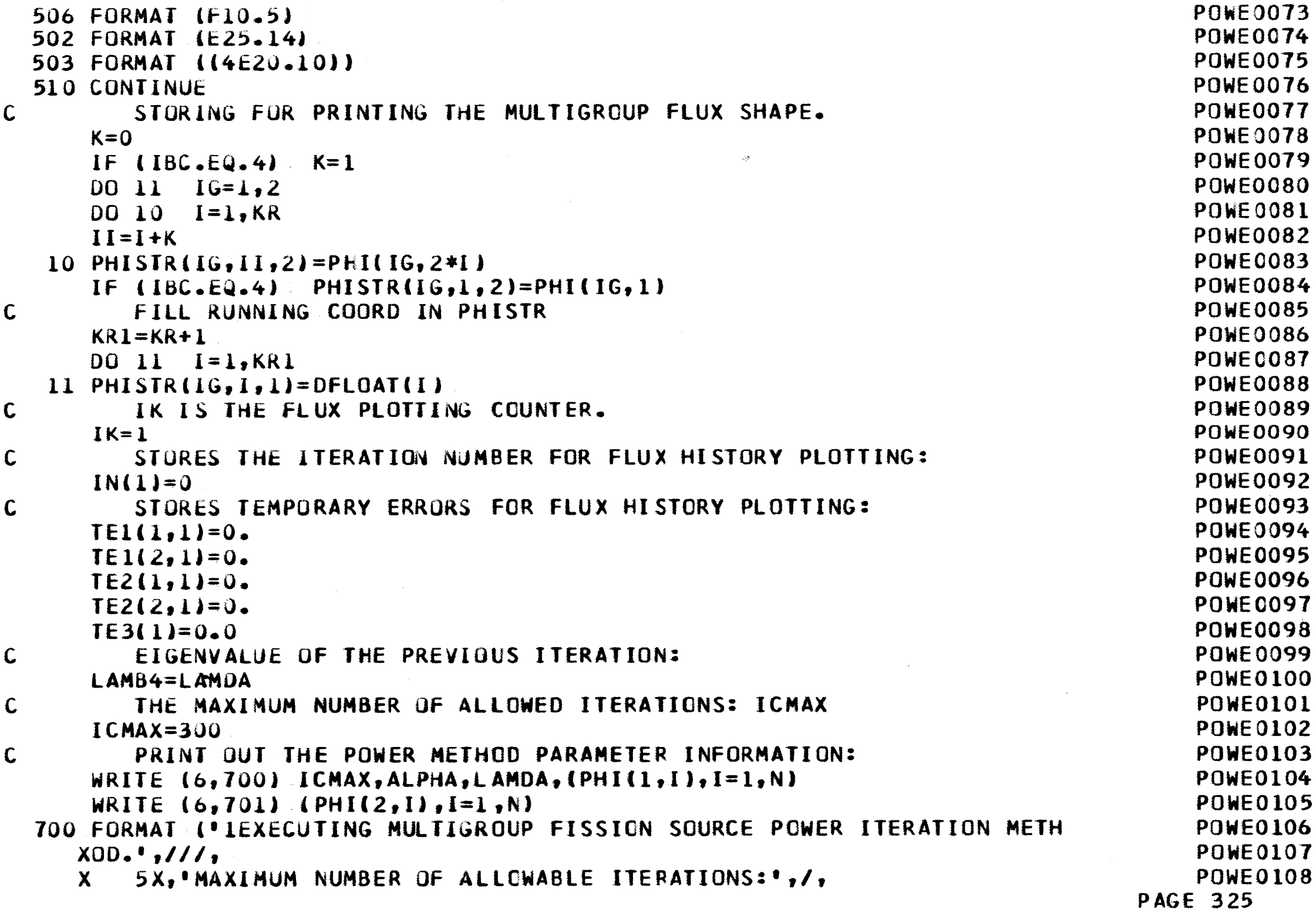

700 FORMAT 1 'IEXECUTING MULTIGROUP FISSION SOURCE POWER ITERATION METH $X O D \cdot, / / /$,

$X \quad 5 X$, MAXIMUM NUMBER OF ALLCWABLE ITERATIONS:, 1,

PONE0073

POWE 0074

POWE0075

POWE 0076

POWE0077

POWE0078

POWE 0079

POWEO080

POWE 0081

POWE0082

POWE0083

POWE0084

POWE0085

POWE0086

POWE C087

POWE0088

POWE0089

POWE 0090

POWE0091

POWE 0092

POWE0093

POWE0094

POWE0095

POWE0096

POWE 0097

POWE0098

POWE 0099

POWEO 100

POWE0101

POWE 0102

POWE0 103

POWE0104

POWE0105

POWEO106

POWE 0107

POWE0 108

PAGE 325 


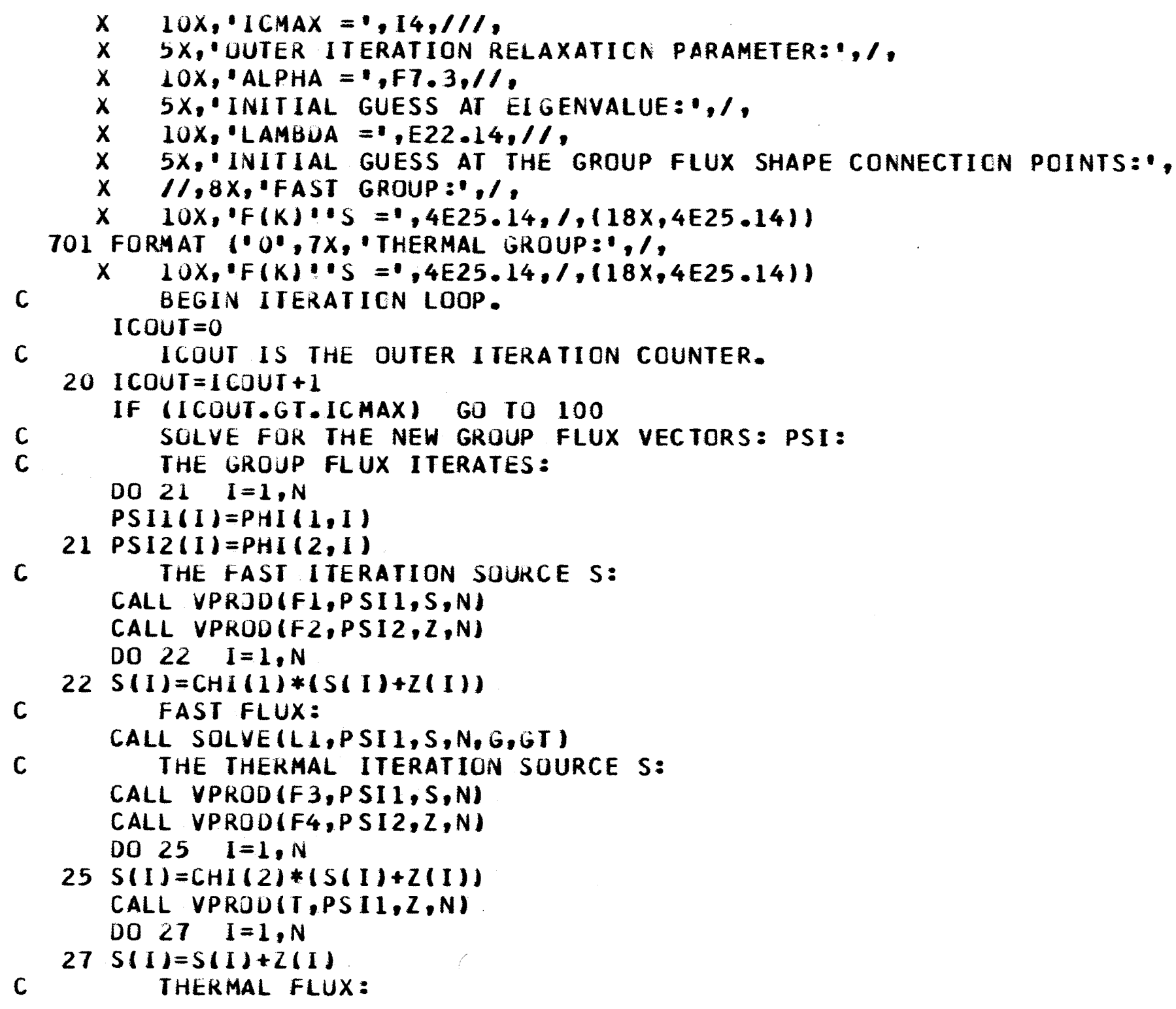

POWE0109

POWE 0110

POWEOI11

POWEO112

POWE0113

POWE0114

POWE 0115

POWE0116

POWE0117

POWEO118

POWE0119

POWEO120

POWE 0121

POWE0122

POWE 0123

POWE0 124

POWE0125

POWE 0126

POWE0127

POWE0128

POWE0129

POWEO 130

POWE 0131

POWE 0132

POWE0133

POWEO 134

POWE0135

POWE 0136

POWE 0137

POWE0138

POWEO139

POWE0140

POWE 0141

POWE 0142

POWE0143

POWE 0144 
POWE0152

POWE0153

POWE 0154

POWE0155

POWE0156

POWE 0157

POWE0158

POWE0159

POWE 0160

PSI $(1, I)=P S I I(I)$

$30 \operatorname{PSI}(2,1)=\operatorname{PSI}(2(1)$

PUINT BY POINT SIMULTANEOUS RELAXATION FLUX ITERATION:

POWE0161

POWE 0162 $X=A L P H A$

C DO NOT RELAX DURING THE FIRST THREE ITERATIONS:

IF (ICOUT.LE.3) $\quad X=1.0$

C CALCULATE THE NEW GROUP FLUX ITERATES AND GROUP ERRORS:

DO $40 \quad I G=1,2$

DO $40 \quad I=1, N$

PSI I IG,I) $=P H I(I G, I)+X *(P S I(I G, I) / L A M D A-P H I(I G, I))$

40 CONT INUE

CALL NORMAL (PSI,N)

$N N=N$

IF (IBC.EQ.1) $\mathrm{NN}=\mathrm{N}-2$

NURHALIZED ERRORS OF THE FLUX CNLY:

DO $39 \quad 1 G=1,2$

$\operatorname{ERRMAX}(I G)=0.0$

$S Q(I G)=0.0$

IF (IBC.NE.4) GO TO 37

ERROR $(I G, 1)=$ DABS( $(\operatorname{PSI}(I G, 1)-\operatorname{PHI}(I G, 1)) / \operatorname{PSI}(I G, 1))$

POWE 0163

POWE0 164

POWE 0165

POWE0166

POWE 0167

POWE0 168

POWE0169

POWE0170

POWE0171

POWE 0172

POWE0173

POWE 0174

POWE0175

POWE0176

POWE0177

POWE0178

POWE0179

POWE0180

PAGE 327 
$\operatorname{ERRMAX}(I G)=\operatorname{ERROR}(I G, 1)$

POWE0181

$S Q(I G)=E R R O R(I G, 1) * * 2$

POWE0182

POWE0183

POWE0184

ERROR (IG,I)=DA\&S( (PSI (IG,I)-PHI (IG,I))/PSI(IG,I) )

POWE 0185

POWE0 186

POWE0187

POWE0188

POWE0189

POWE0190

POWE 0191

POWE0192

POWE 0193

POWEO 194

POWE0195

POWE0196

POWE 0197

POWE0198

POWE 0199

POWE0200

POWE 0201

POWE0202

POWE0203

POWE 0204

POWE 0205

POWE 0206

POWE 0207

POWE0208

POWE0209

POWE0210

POWE0211

POWE 0212

POWE0213

POWE0214

IF $(I K \cdot E Q .0) \quad J K=5$

DO 42 I $G=1,2$

DO $42 I=1, K B C$

POWE 0215

POWE0216

PAGE 328 


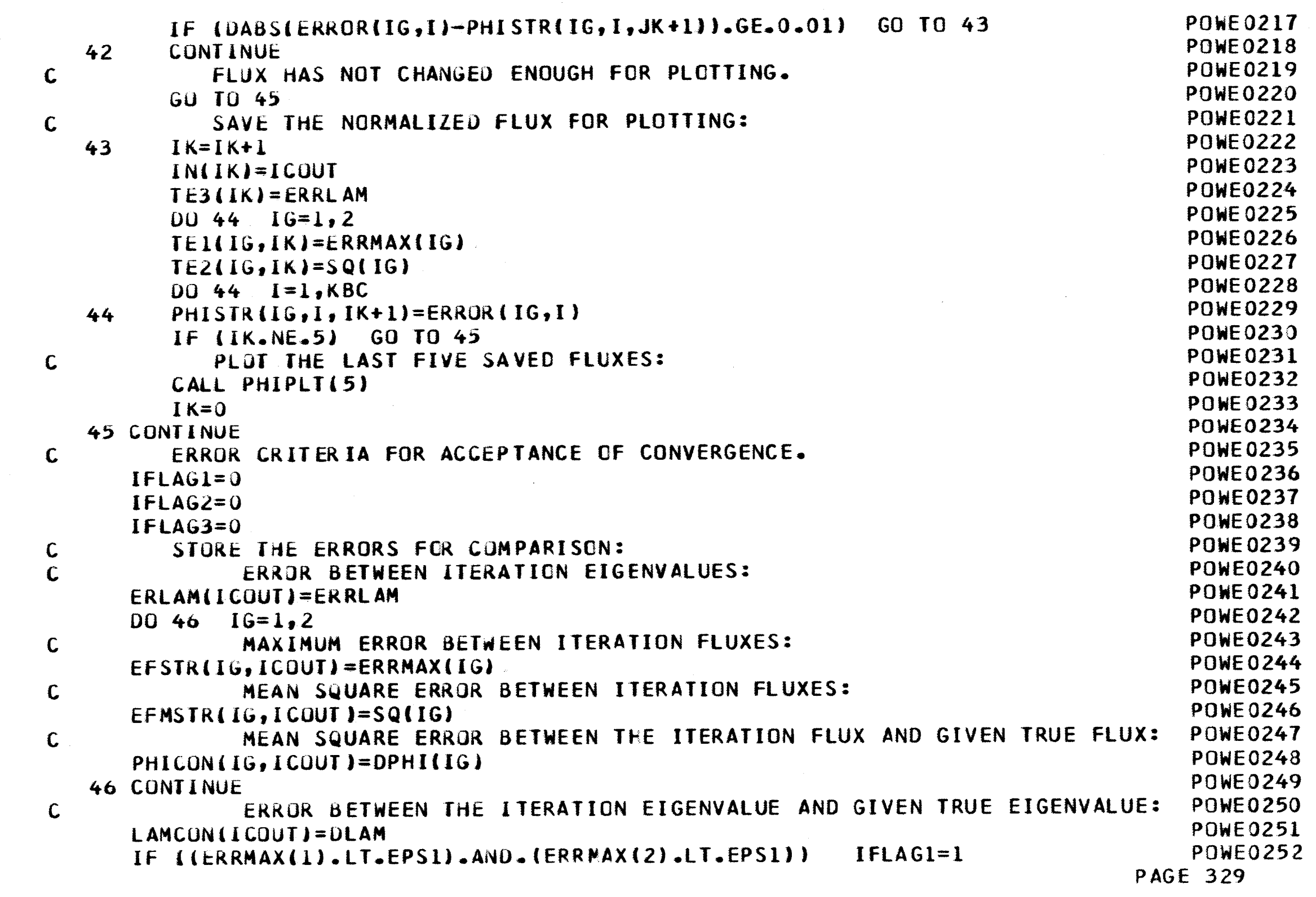




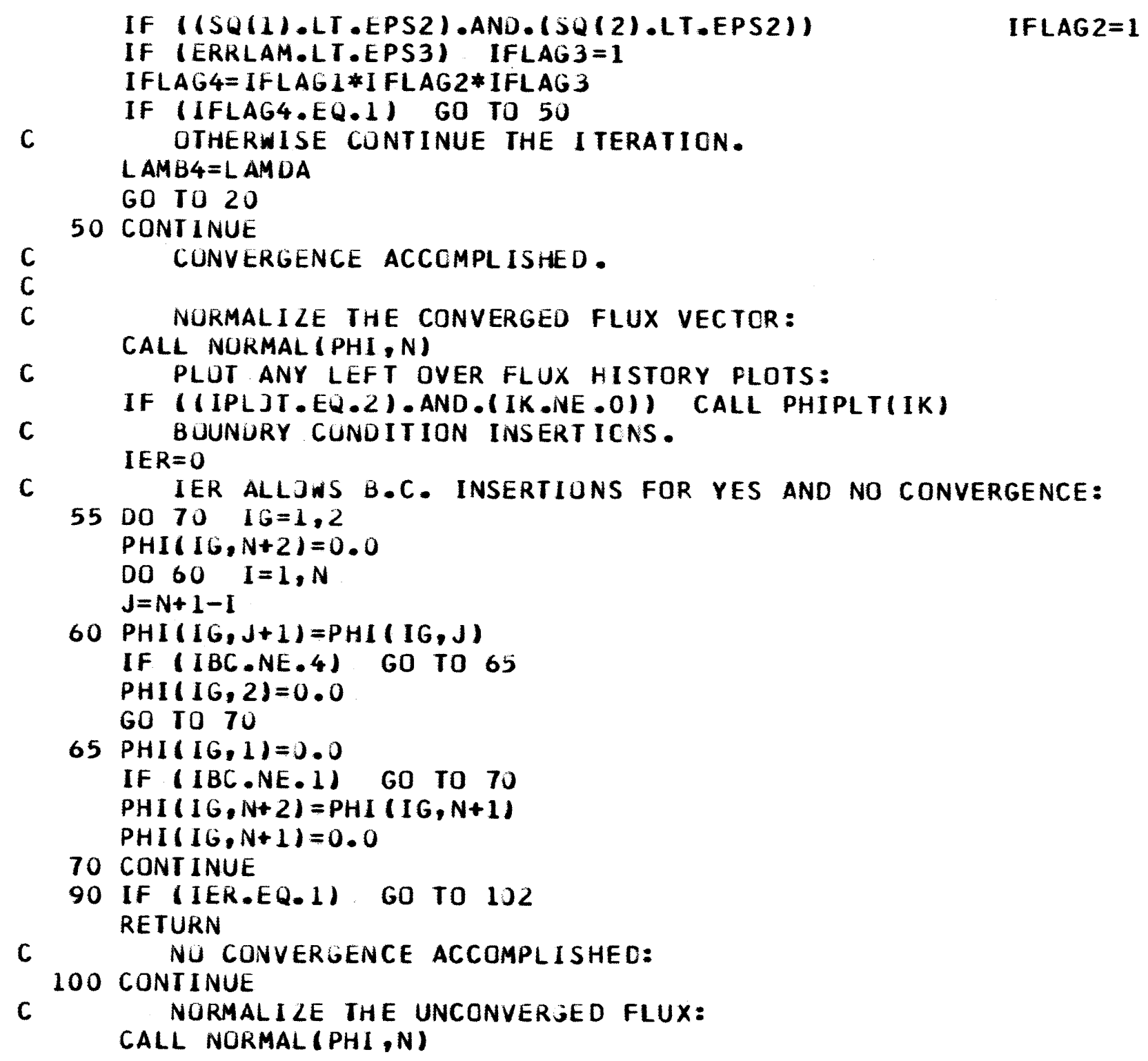


ICOUT $=I$ COUT -1

WRITE $(6,101)$ I COUT

101 FORMAT I $1 \mathrm{HL}$, 'POWER METHOD DID NOT CCNVERGE FOR THIS CASE AFTER',

$\mathrm{X}$ I4," ITERATIONS,,$/ /, 1 X$, 'EXECUTION TERMINATED $'$

IER $=1$

GO TO 55

C

102 CONT INUE

FUR PRINIING OUT THE EIGENVALUE history AND THE FINAL FLUX SHAPE: I PLOT $=1$

JPLUT $=1$

RETURN

END

POWE0289

POWE 029 ]

POWE0291

POWE0292

POWE 0293

POWE0294

POWE0295

POWE0296

POWE0297

POWE 0298

POWE 0299

POWE0300 
SUBROUTINE VPROD $(A, X, S, N)$

FURMS THE VECTOR $S=$ PRODUCT OF NXN MATRIX A AND VECTOR $X$ :

VPRO0001 WHERE A IS THE CUBIC HERMITE $(1,6)$ STORAGE MATRIX. REAL*8. A $(50,6), X(50), S(50)$

DO $1 \quad I=1, N$

$S(I)=0.0$

DO $1 \quad M=1,6$

$K=2 *(1 / 2)+M-3$

IF $((K \cdot L T \cdot 1) \cdot O R \cdot(K \cdot G T \cdot N))$ GO TO 1

$S(I)=S(I)+A(I, M) * X(K)$

1 CONTINUE

C

RETURN

END

VPRO0002

YPRO0003

VPRO0004

VPRO0005

VPRO0006

VPRO0007

VPRO0008

VPROO009

VPRO0010

VPRO0011

VPRO0012

VPRO0013

VPROO014 
SUBROUTINE SULVE(A,X,Y,N,G,Z)

$C$ SOLVES A*X $=Y$ USING CHOLESKY'S METHOD OF FACTORAZATION FOR POSITIVE DEFINITE REAL AND SYMMETRIC MATRICES A.

REFERENCE: FORSYTHE \& MOLER.

$G$ AND $Z$ ARE TEMPORARY WORK AREAS.

MODIFIEU FOR THE CRAZY CUBIC HERMITE $(1,6)$ MATRICES:

IMPLICIT REAL *8 (A-H,O-Z)

DIMENSI ON $A(50,6), G(50,6), X(50), Y(50), Z(50)$
FURM IHE MATRIX FACTORAZATION TO $G$ :

CALL FORMG $(A, G, N)$

SOLVE: $G * Z=Y$ :

CALL LOWTRI $(G, Z, Y, N)$

FORM G AS SYMMETRIC MATRIX:

CALL SYMG $(G, N)$

SOLVE: G-TRANSPOSE*X $=Z$ :

CALL UPPTRI $(G, X, Z, N)$

RETURN

END

SOLV0001

SOLV 0002

SOLV0003

SOLV0004

SOLV0005

SOLV0006

SOLV0007

SOLV0008

SOLV0009

SOLV0010

SOLV0011

SOLV0012

SOLVOO13

SOLV0014

SOLV0015

SOLV0016

SOLV 0017

SOLV0018 
$L O=1$

IF (J.LE.3) $L O=2$

IF $(J \cdot E Q \cdot 1) \quad L O=4$

SUM $=0.0$

IF (LO.GT.KO) GO TO 2

DO $1 L=L O, K O$

$1 S U M=S U M+G(J, L) * * 2$

$2 S U M=A(J, K)-S \cup M$

IF (SUM.LT.0.0) GO TO 100

$G(J, K)=D S Q R T$ (SUM)

IF (J.EQ.N) 60 TO 20

$11=\mathrm{J}+1$

$12=J+6-K$

IF $(12.6 T \cdot N) \quad$ I $2=N$

$M=2$

IF $(K \cdot E Q \cdot 3), M=3$

DO $10 . I=I 1, I 2$

SUM $=0.0$

$L O=1$

IF (I.LE.3) LO=2

$M O=M-1$

IF (LO.GT.MO) GO TO 7

DO $5 L=L O, M O$

$\mathrm{JL}=\mathrm{L}$

IF $(M . E Q \cdot 2) \quad J L=3$

5 SUM $=S U M+G(I, L) * G(J, J L)$

$7 G(I, M)=(A(I, M)-S U M) / G(J, K)$

If (M.EQ.3) $M=1$

FORMO003

FORMO004

FCRMO005

FORMOOO6

FORM0007

FORMOO08

FORMO009

FORMO010

FORMO011

FORMO012

FORMO013

FORMO014

FORM0015

FORMO016

FORM 0017

FORMO018

FORMO019

FORMO020

FORMO021

FORM 0022

FORM 0023

FORMO024

FORMO025

FORMO026

FORMO027

FORM 0028

FORMO029

FORMO0 30

FORMO031

FORMO032

FORMO033

FORM 0034

FORMO035

FORM 0036

10 CONTINUE 
20 CONT I NUE RETURN

100 WRITE $(6,101) \mathrm{J}, \mathrm{K}$

101 FORMAT 1 OERROR IN FORMG:, $1 /$,

$\left.X \quad 5 X, \cdot A 1 \cdot, 120^{\circ}, 1,12,0\right)<0.0^{\circ}, 1 /$,

$X \quad 5 X, 1$ CHOLESKY METHOD HAS FAILED., $1 /$,

$X$ 5X, MATRIX A MAY NOT BE PCSITIVE DEFINITE OR SYMMETRIC, $1 /$,

$X$ OEXECUTION TERMINATED 1

CALL EXIT

RETURN

END

FORMO037

FORMO038

FORMO039

FORM0040

FORMO041

FORMO042

FORM 0043

FORM0044

FORM 0045

FORMO046

FORM0047 
SUBROUT INE SYMG $(G, N)$

FORMS SYMMETRIC G FROM G LCWER TRIANGULAR:

SYMGO001

SYMG0002 REAL *8 G(50,6)

$\mathrm{N} 2=\mathrm{N}-2$

C

FILL THE UPPER PORTION OF SYMMETRIC $G$ :

10 DO $20 \quad I=1, N 2$

IF (MUD $(1,2), E Q \cdot 1)$ GO TO 15

I IS EVEN:

$G(1,4)=G(I+1,3)$

$G(I, 5)=G(I+2,1)$

$G(1,6)=G(1+3,1)$

GO TO 20

I IS ODO:

C

$15 G(I, 5)=G(I+1,2)$

$G(1,6)=G(1+2,2)$

20 CONTINUE

$G(N-1,5)=G(N, 2)$

RETURN

END

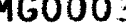

SYMG0004

SYMG0005

SYMG0006

SYMG0007

SYMG0008

SYMG0009

SYMG0010

SYMG0011

SYMG 0012

SYMG0013

SYMG0014

SYMG0015

SYMG0016

SYMG0017

SYMG0018

SYMG0019 


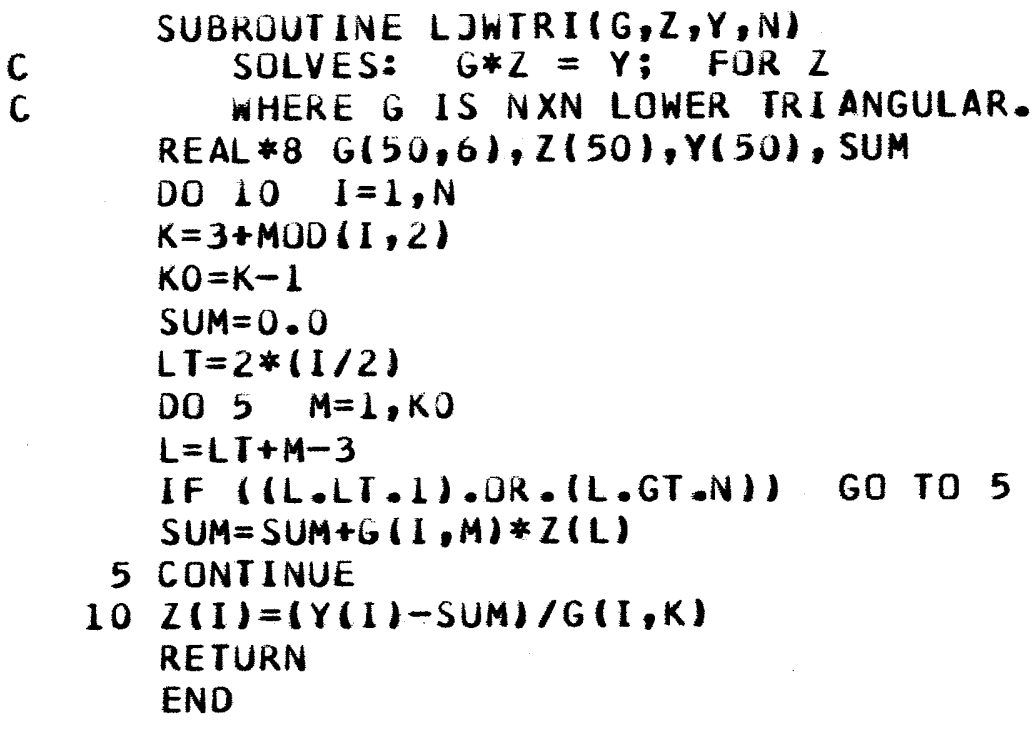

LOWT 0001

LOWTO002

LOWTO003

LOWT 0004

LOWT0005

LOWT 0006

LOWTO007

LOWT0008

LOWT 0009

LOWTO010

LOWT 0011

LOWT 0012

LOWT 0013

LOWT 0014

LOWT0015

LOWT 0016

LOWT0017 
SUBRUUTINE UPPTRI $(G, X, Z, N)$

$C$ SULVES: $G * X=Z$; FOR $X$

$C$ WHERE G IS NXN UPPER TRI ANULAR. REAL*8 G(50,6),X(50),Z(50), SUM

DO $10 \mathrm{~J}=1, \mathrm{~N}$

$\mathrm{I}=\mathrm{N}+\mathrm{I}-\mathrm{J}$

$K=3+\operatorname{MOD}(I, 2)$

$K 1=K+1$

SUM $=0.0$

$L T=2 *(1 / 2)$

DO $5 \quad M=K 1,6$

$L=L T+M-3$

IF ((L.LT.1).OR.(L.GT.N)) GO TO 5

$S U M=S U M+G(I, M) * X(L)$

5 CONTINUE

$10 X(I)=(Z(I)-S U M) / G(I, K)$

RETURN

END

UPPT 0001

UPPT 0002

UP P T0003

UPPT 0004

UPPT 0005

UPPT 0006

UPPT 0007

UPPT 0008

UPPT 0009

UPPT 0010

UPP T0011

UPPT 0012

UPPT 0013

UPPT 0014

UPPT 0015

UPPT 0016

UPPT 0017

UPPT 0018 
SUBROUT INE NORMAL(PHI,N)

NORMALI LES THE GROUP FLUXES AND CURRENTS BY THE LARGEST FLUX VALUE:

NORLOOO1

NORL 0002

COMMON /BI/ IBC

REAL*8 PHI $(2,52), A$

$N N=N$

IF $(I B C \cdot E Q \cdot 1) \quad N N=N-2$

$A=0.0$

IF (IBC.NE.4) GO TO 5

$A=D A B S(P H I(1,1))$

IF $(D A B S(P H I(2,1)), G T . A) \quad A=\operatorname{CABS}(\operatorname{PHI}(2,1))$

5 DO $1 \quad I G=1,2$

DO $1 \quad I=2, N N, 2$

IF (DABS(PHI(IG,I)).GT.A) A=DABS(PHI(IG,I))

1 CONTINUE

DO 2 I $G=1,2$

DO $2 \quad I=1, N$

$2 \operatorname{PHI}(I G, I)=\operatorname{PHI}(I G, I) / A$

RETURN

END

NORL 0004

NORL 0005

NORL 0006

NORL 0007

NORL 0008

NORLO009

NORL 0010

NORL 0011

NORL 0012

NORL 0013

NORL0014

NORL 0015

NORL 0016

NORLO017

NORL 0018

NORL 0019 
SUBROUTINE PHIPLT(L)

PLUTS THE GROUP F

PHI P0001

PHIP0O02

PHIP0O03

THERMAL GROUP FLUXES ARE PLOTTED SEPERATELY.

PHIP0O04

$L$ IS THE NUMBER OF FLUXES TO BE PLOTTED.

$L$ IS BETWEEN 1 AND 5 .

IMPLICIT REAL *8 (A-H,O-Z)

COMMON /BI/ IBC

COMMUN /B2/ KR,N

COMMON /B5/ $A(26,6), B(26,6)$

COMMUN /B6/ TE1 $(2,5), \operatorname{TE} 2(2,5), \operatorname{TE} 3(5), \operatorname{IN}(5)$

COMMON /ER/ EPS 1, EPS2,EPS3

COMMUN /FSTR/ PHISTR $(2,26,6)$

DIMENSI ON SYMBUL (5)

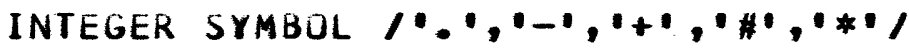

$K R 1=K R+1$

C

$$
\text { SET UP B.C. CONDITIONS }
$$

IF (IBC.EQ.4) GO TO 5

IF $(I B C . E Q .3)$ GO TO 3

DO $2 \quad I G=1,2$

DO $2 K=1, L$

DO $1 \quad I=1, K R$

$J=K R+1-I$

$1 \operatorname{PHISTR}(I G, J+1, K+1)=\operatorname{PHISTR}(I G, J, K+1)$

PHISTR $(I G, 1, K+1)=0$.

3 IF $(I B C . E Q .2)$ GO TO 5

DO $4 \quad 1 G=1,2$

DO $4 K=1, L$

4 PHISTR (IG,KR $1, K+1)=0$.

5 CONTINUE

FLUXES IN PHISTR HAVE BEEN NORMALIZED IN POWER. $L 1=L+1$

PUT THE FAST FLUX IN A, AND THE THERMAL FLUX IN B:

PHIP0005

PHIP0006

PHIP 0007

PHIP0008

PHIP0009

PHIPOO 10

PHIP0011

PHIP0012

PHIP0013

PHIP0014

PHIP0015

PHIP0016

PHIP0017

PHIPOO 18

PHIP 0019

PHIP0020

PHIP0021

PHIP0022

PHIP 0023

PHIP0024

PHIPO025

PHIP0026

PHIP0027

PHIP0028

PHIP0029

PHIP0030

PHIP 0031

PHIP0032

DO $10 K=1, L 1$

DO $10 \quad I=1, K R I$

$A(I, K)=\operatorname{PHISTR}(1, I, K)$

PHIP0033

PHIP 0034

PHIP 0035

PHIP0036

$10 B(I, K)=\operatorname{PHISTR}(2, I, K)$ 
20 FORMAT $1 \%$, OFAST FLUX ITERATION HISTORY PLOT. , /)

PHIP 0039 WRITE $(6,30)$

30 FORMAT I

$X$ 'OKEY:',5X,'SYMBOL',5X,'ITERATICN NUMBER:',7X,'ERRCR CRITERIA',

$X \quad 11 X$, 'ERROR', $13 X$, 'TOLERANCE')

DO $35 \quad I=1, L$

35 WRITE $(6,40) \operatorname{SYMBOL}(1), \operatorname{IN}(1), T E 1(1, I), \operatorname{EPS} 1, \operatorname{TE} 2(1, I), \operatorname{EPS} 2$, $X$ TE $3(I), E P S 3$

40 FORMAT $1 /, 12 X, A 1,15 X, 13,16 x$, 'FLUX, 14X,1PD15.5, 5X,1PD15,5,/,

$X \quad 47 X, M E A N$ SQ. FLUX',5X,1 PD15.5,5X,1PD15.5,1,

$X \quad 47 X$, 'EIGENVALUE', 8X,1PD15.5.5X,1PD15.51

PLOT THE L THERMAL FLUX SHAPES ON THE OTHER GRAPH:

CALL PRTPLT $(0, B, K R 1, L 1, K R 1,0,26,6,2)$

WRITE $(6,50)$

50 FORMAT (/, O OTHERMAL FLUX ITERATICN PLOT.',/)

WRITE $(0,30)$

DO $55 \quad I=1, L$

55 WRITE $(6,40)$ SYMBOL (I),IN(I),TE1 (2, I), EPS 1,TE2 (2,I), EPS2,

$X$

TE3(I), EPS3

RETURN

PHIP 0040

PHIP 0041

PHIP 0042

PHIP 0043

PHIP0044

PHIP0045

PHIP0046

PHIP0047

PHIP0048

PHIP0049

PHIP0050

PHIP0051

PHIP0052

PHIP0053

PHIP 0054

PHIP0055

PHIP0056

PHIP 0057

PHIP0058

PHIP0059

END

PHIP 0060 
SUBROUTINE CURENT

FORMS THE SEPERATE FLUX AND CURRENT VECTORS

CURE0001

CURE 0002

FROM THE COMBINED ELEMENTS OF PHI.

CURE 0003

THEN: FLUX $=$ PHI; CURRENT $=$ CUR.

CURE0004

CURE0005

CURE 0006

CURE0007

CURE 0008

CURE0009

CURE0010

CURE0011

CURE0012

CURE0013

CURE 0014

CURE0015 
PRINTS THE RESULTS OF THE METHOD.

OUTPOOO1

DUTP0002

OUTP0003

IMPLICIT REAL *8 (A-H,L-Z)

COMMUN /BI/ IBC, IPLOT,JPLOT, I PUNCH

COMMON /B2/ KR, N

COMMON /B4/ PHI $(2,52), \operatorname{CUR}(2,52)$, LAMDA, ICOUT

DUTP0004

OUTP 0005

OUTP0006

OUTP 0007

COMMON /B5/ PSI $(2,26)$

COMMON /ER/ EPS 1, EPS 2,EPS 3

COMMON /ESTR/ LAMSTR(300), EFSTR $(2,300), \operatorname{EFMSTR}(2,300), \operatorname{ERLAM}(300)$

COMMON /TRUE/ TRULAM, TRUPHI $(2,52)$, PHICCN $(2,300)$, LAMCON(300), IFT

INTEGER N

$K R O=K R-1$

$K R 1=K R+1$

WRITE $(6,1)$

1 FORMAT ('IRESULTS OF THE MULTIGRCUP METHOD:')

WRITE $(6,10)$ ICOUT

10 FURMAT $1 / 1, \circ$ PROBLEM TERMINATED AFTER, 15 ,

$X$ - outer (PONER) iterations To: :)

WRITE $(0,20)$ LAMDA

20 FORMAT $(1,10 X, \cdot$ LAMDA $=,, 1$ PE 21.14$)$

C PRINT UUT EI GENVALUES.

CALL PLOT

WRITE $(6,30)$

30 FORMAT ('IRESULTS AFTER PROBLEM TERMINATION:,$/$,

$X$ ' OINDEX',8X, THERMAL FLUX',11X,'FAST FLUX',5X,

$X$ 'THERMAL CURRENT', $8 X$, 'FAST CURRENT', $/ 1$

WRITE $(6,50)(K, P H I(2, K), \operatorname{PHI}(1, K), \operatorname{CUR}(2, K), \operatorname{CUR}(1, K), K=1, K R 1)$

50 FORMAT $(16,4020.7)$

IF (IPUNCH.EQ.1) CALL PUNCH PRINT OUT GROUP NORMALI $Z$ ED RESULTS:

DO $52 \quad I G=1,2$

$A=0.0$

DO $51 \quad I=1, K R 1$

IF $($ PHI $(1 G, I), . G T . A) \quad A=P H I(I G, I)$

OUTP0008

OUTP 0009

OUTP0010

OUTP0011

OUTP 0012

DUTP0O13

OUTP 0014

OUTP0015

DUTP0016

DUTP0017

DUTP0018

OUTP0019

DUTP 0020

DUTP0021

OUTP0022

DUTP 0023

DUTP 0024

OUTP 0025

OUTP0026

OUTP0027

DUTP0028

OUTP0029

DUTP 0030

OUTP0031

OUTP0032

OUTP0033

OUTP0034

OUTP0035

DUTP 0036

DO $52 \quad I=1, K R I$ 
PHI (IG,I)=PHI (IG,I)/A

$52 \operatorname{CUR}(I G, I)=C U R(I G, I) / A$

WRITE $(6,55)(K, \operatorname{PHI}(2, K), \operatorname{PHI}(1, K), \operatorname{CUR}(2, K), \operatorname{CUR}(1, K), K=1, K R 1)$

C

55 FORMAT $(/ /,$, OGROUP NORMALI I EC RESULTS:,,$/ /,(16,4020.7))$

$K 1=1$ CALCULATE THE FINAL TO EXPECTED FLUX RATIOS:

$K 2=K R 1$

IF (IBC.LE. 2$) \quad K 1=2$

IF $((I B C \cdot E Q \cdot 1), O R \cdot(I B C, E Q .3)) \quad K 2=K R$

DO $60 \quad I G=1,2$

IF $(I B C, L E \cdot 2) \quad P S I(I G, 1)=1.0$

IF $((I B C . E Q .1) . O R \cdot(I B C . E Q .3)) \quad P S I(I G, K R I)=1.0$ $I=0$

DO $60 \mathrm{~K}=\mathrm{K} 1, \mathrm{~K} 2$

$I=I+2$

60 PSI $(I G, K)=P H I(I G, K) / T R U P H I(I G, I)$

WRITE $(6,70)$ (I, PSI $(2, I), P S I(1, I), I=1, K R 1)$

70 FORMAT IIRATIOS DF THE TERMINATED GROUP FLUX TO THE EXPECTED GROU XP FLUX: :, //,

$X$ IOX, - AN INCICATION JF THE ACCURACY OF THE CONVERGENCE -, $1 / 1$, $X \quad K \cdot, 12 X, \cdot$ THERMAL RATIO',15X,'FAST RATIO',//,(I5,2E25.10) $)$ PRINT OUT THE STORED ITERATICN ERRORS:

WRITE $(6,110)$ EPSI, (EFSTR $(2,1), I=1$, ICOUT)

WRITE $(6,111)$ EPS $1,(E F S T R(1,1), I=1$, ICOUT)

WRITE $(6,112)$ EPS2, (EFMSTR $(2,1), I=1$, ICOUT)

WRITE $(6,113)$ EPS3, (EFMSTR $(1,1), I=1, I C C U T)$

WRITE $(6,114)$ EPS3, (ERLAMI I), I =1, I COUT)

110 FORMAT IIMAXIMUM NORMALIZED ERRORS BETWEEN THE THERMAL FLUX ITERA XTIONS:",

$X \quad 25 X$, TULERANCE USED $=1,1$ PE 12.4. $/ 1,(1 P 5 E 20.5))$

111 FORMAT 1 IMAXIMUM NORMALIZED ERRCRS BETHEEN THE FAST FLUX ITERATIO $X N S: \bullet$,

$X \quad 25 X, \cdot T$ OLERANCE USED $=1,1$ PE12.4, $/ 1,(1$ P5E 20.5)

112 FORMAT IMEAN SQUARE NORMALIZED ERRCR BETWEEN THE THERMAL FLUX IT XERATIONS: ,

X 18X,'TOLerance USED $=1,1$ PE12.4./1, (1P5E20.5)

OUTP0037

OUTP0038

DUTP0039

DUTP0040

OUTP 0041

OUTP0042

OUTP0043

OUTP0044

OUTP0045

OUTP0046

OUTP0047

OU TP 0048

OUTP 0049

OUTP0050

DUTP0051

OUTP0052

DUTP0053

OUTP 0054

OUTP0055

DUTP 0056

DUTP0057

OUTP0058

OUTP0059

OUTP0060

DUTP 0061

OUTP0062

DUTP 0063

OUTP0064

OUTP 0065

OUTP0066

OUTP 0067

OUTP0068

OUTP0069

OUTP0070

OUTP0071

OUTP 0072

PAGE 344 
113 FORMAT 'IMEAN SQUARE NORMALIZED ERROR BETWEEN THE FAST FLUX ITERA XTIONS: :

$X \quad 18 X$, TOLERANCE USED $=1,1$ PE12.4, $/ 1,(1 P 5 E 20.5))$

114 FORYAT (IERROR BETWEEN THE ITERATICN EIGENVALUES:", $X \quad 28 X$, TOLERANCE USED $=1,1$ PE12.4,1/, (1P5E20.5))

C IF (IFT.EQ.O) RETURN PRINT DUT THE GIVEN TRUE EIGENVALUE AND FLUX: WRITE $(6,115)$ TRULAM, ( (TRUPHI $(3-\mathrm{J}, I), J=1,2), I=1, N)$

115 FORMAT 1 'ITHE GIVEN TRUE EIGENVALUE:", $1 /, 15 X$,

$X$ 'TRULAM = ', E22.14,1/1,

$x$ - OTHE GIVEN MULTIGROUP fLUXes:, $1 /$,

$X$ 13X,'THERMAL',16X,'FAST , //, (2020.10))

C PRINT OUT THE STORED CONVERGENCE ERRORS: WRITE $(6,120)$ (PHICON $(2, I), I=1, I C C U T)$ WRITE $(6,121)$ (PHICON $(1,1), I=1, I$ COUT) WRITE $(6,122)$ (LAMCCN (I), I = 1, ICCUT)

120 FORMAT ('IMEAN SQUARE ERROR BETWEEN THE THERMAL ITERATION FLUX AND $X$ THE GIVEN TRUE THERMAL FLUX:1,1/, (1P5E20.5))

121 FORMAT ('IMEAN SQUARE ERROR BETWEEN THE FAST ITERATICN FLUX AND TH XE GIVEN TRUE FAST FLUX:,,$/ /,(1$ P 5E20.5))

122 FORMAT 'IERROR BETHEEN THE ITERATICN EIGENVALUES AND THE GIVEN TR C XUE EIGENVALUE:,,$/ /,($ IP5E20.5))

RETURN

END

OUTPO073

DUTP0074

OUTP0075

DUTP 0076

OUTP 0077

OUTP0078

OUTP 0079

DUTP0080

OUTP0081

OUTPOOB2

OUTP0083

OUTP0084

OUTP0085

OUTP0086

DUTP0087

DUTP0088

OUTP0089

DUTP0090

OUTP 0091

OUTP0092

OUTP 0093

DUTP0094

OUTP0095

OUTP0096

OUTP0097 
SUBROUTINE PLOT

$\begin{array}{ll}\text { C } & \text { PLOTS OUT THE EIGENVALUE HISTORY AS A TABLE AND A GRAPH, } \\ \text { C } & \text { AS WELL AS PLOTTING OUT THE FINAL MULTIGROUP FLUX SHAPES. }\end{array}$

PLOTO001

PLOTO002 IMPLICIT REAL*8 (A-H,L-Z)

COMMON /BI/ IBC, IPLOT,JPLOT, IPUNCH

COMMON /B2/ KR

COMMUN /B4/ PHI $(2,52)$, PSI $(2,52)$, LAMDA, ICOUT

COMMON /B5/ B $(300,2)$

COMMUN /ESTR/ LAMSTR(300)

DIMENSION C $(26,3)$

C

IN ORDER TO SAVE SOME SPACE:

EQUI VALENCE (B(1), $(1))$

WRITE $(0,1)$ (LAMSTR (I), I =1, I COUT)

1 FORMAT ('UTABLE OF EIgENVALUES DURING THE POWER ITERATION:", $1 /$ (1P5E25.14)1)

IF (JPLOT.EQ.0) GO TO 20

DO $10 \quad I=1, I$ COUT

$B(I, 1)=I$

$10 B(I, 2)=L A M S T R(I)$

CALL PRTPLT $(1, B$, ICOUT, 2, ICOUT $, 0,300,2,1)$

WRITE $(6,11)$

11 FORMAT ('OPLOT OF THE EIGENVALUE HISTORY THROUGH THE ITERATIONS.")

20 IF (IPLOT.EQ.0) RETURN

$K R 1=K R+1$

DO $30 \quad I=1, K R I$

$C(1,1)=1$

$C(I, 2)=P H I(1, I)$

$30 \mathrm{C}(1,3)=\mathrm{PHI}(2,1)$

CALL PRTPLT $(2, C, K R 1,3, K R 1,0,26,3,2)$

WRITE $(6,31)$

31 FORMAT ( OFINAL CONVERGED CCNNECTING FLUX POINTS; $F(K), \cdots / /$,

$X$ 5X, $F A S T$ FLJX: $\because, 1,5 X$, THERMAL FLUX: -1$)$

RETURN

END

PLOT0004

PLOT 0005

PLOT0006

PLOT 0007

PLOT0008

PLOT0009

PLOT0010

PLOT 0011

PLOT 0012

PLOT0013

PLOT 0014

PLOT 0015

PLOT0016

PLOT 0017

PLOT0018

PLOT 0019

PLOT0020

PLOT 0021

PLOT 0022

PLOT 0023

PLOT 0024

PLOT 0025

PLOT0026

PLOT 0027

PLOT0028

PLOT0029

PLOT0030

PLOT 0031

PLOT 0032

PLOT 0033

PLOT 0034 

REAL $* 8$ F, C COMMON /B2/ KR

COMMUN /B4/ $\mathrm{F}(2,52), \mathrm{C}(2,52)$

$K R 1=K R+1$

WRITE $(7,1) K R,(F(1, I), C(1, I), F(2, I), C(2, I), I=1, K R 1)$

1 FORMAT $(15,1,(4020.7))$

WRITE $(6,100)$

100 FORMAT $(\% / \%$. THE OUTPUT HAS BEEN PUNCHED OUT ONTO CARDS $\because$ RETURN

END

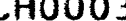

PNCHOOO4

PNCH0O05

PNCHOOO6

PNCH0007

PNCHOOO

PNCHOOO9

PNCHOOLO

PNCHOO11

PNCHOO12 
SUBKOUTINE NORM2(PSI, TRUPHI, N)

NORMALIZES BOTH ENERGY GROUP FLUXES IN PSI TO 1.0:

NOR20001

NOR20002

NOR 20003

NOR20004

COMMON /BI/ IBC

REAL*8 PSI $(2,52)$, TRUPHI $(2,52), A(2)$

DATA K /O/

$K=K+1$

$\mathrm{NN}=\mathrm{N}$

IF (IBC.EQ.1). NN=NN-2

DO 1 I $G=1,2$

$A(I G)=0.0$

IF (IBC.NE.4), GO TO 7

$A(I G)=D A B S(P S I(I G, 1))$

7 DO $1 \quad I=2, N N, 2$

IF (DABS(PSI(IG,I)),GT.A(IG)) A(IG)=DABS(PSI(IG,I))

NOR20005

NOR 20006

NOR20007

NOR2 0008

NOR20009

NOR20010

NOR20011

NOR20012

NOR20013

NOR20014

NOR20015

NOR20016

NOR20017

NOR20018

NOR20019

NOR 20020

NOR20021

NOR 20022

NOR20023

NOR2 0024

NOR 20025

NOR20026

NOR20027

NOR20028

NOR20029

NOR20030

NOR20031

NOR2 0032

NOR 20033

NOR2 0034

NOR20035

NOR20036

6 CONT INUE

END

PAGE 348 


\section{SUBROUTINE PRTPLT (NO, B, N,M,NL,NS, KX,JX, ISP)}

PRTP0001

PRTP 0002

PRTP0003

* identical to subroutine prtplt previously listed in program ref2g.

PRTP0004

RETUKN

END

作

PRTP0006 
F.4. SOURCE LISTING of Program ANALYZE 


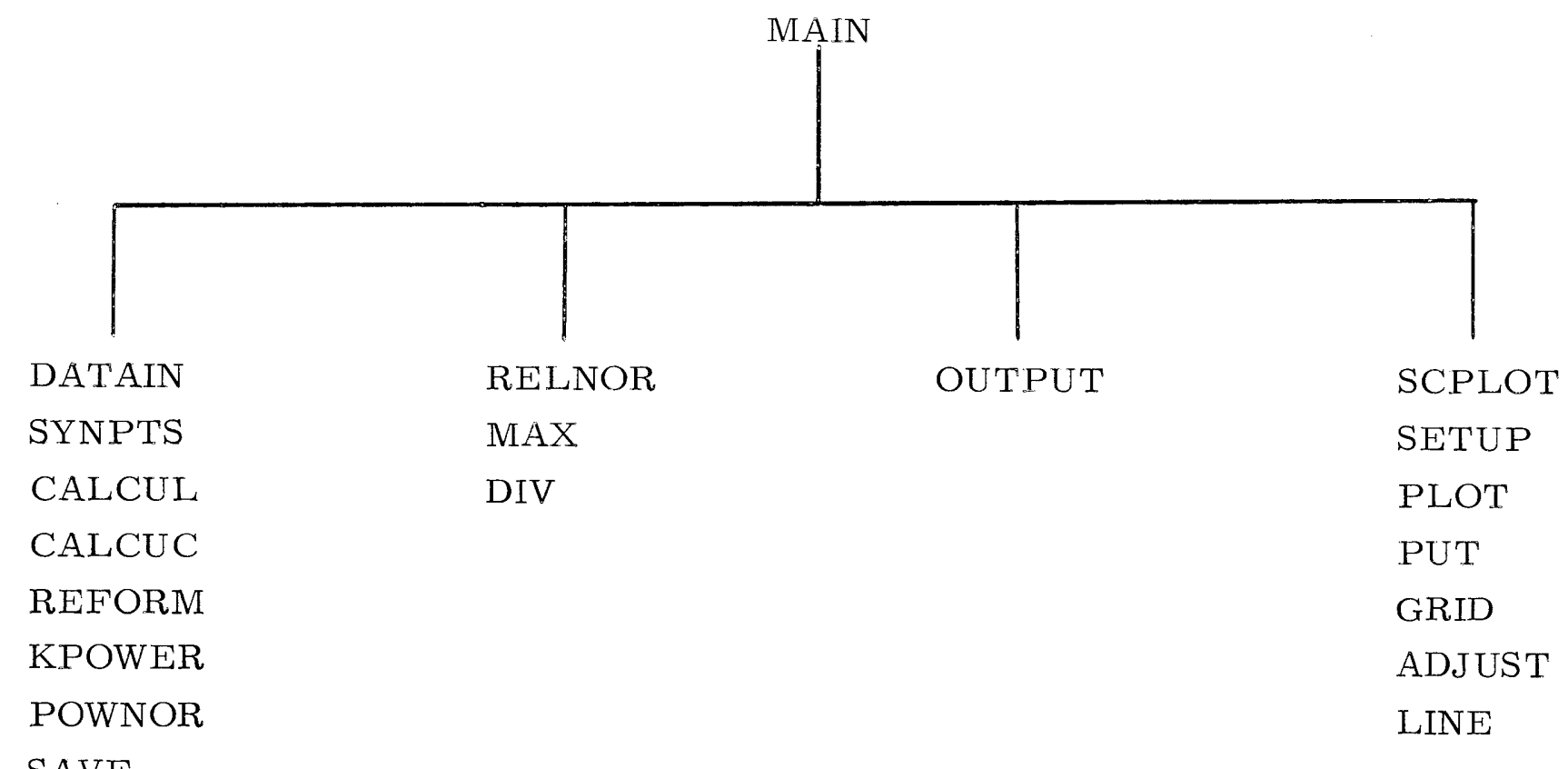

Figure F.4. Structure of Program ANALYZE.

Not including the M.I.T.SC-4020 Subroutine Package. ${ }^{54}$ 
C PROGRAM ANALYZE:

ANALO001

DATA ANALYSIS AND COMPARISON PRCGRAM:

ANAL 0002

DIMENSION NP(200), CHI $(2), R(9,26), F(2,201), G(2,201), H(1000)$,

AN AL 0003

$x$

$x$

C

$\mathrm{C}$

C

C $X(1001), P H I(2,1001), S F(2,1000), D(2,1000)$. $U(2,1001), X B(1001), U B(2,1001), X C(1001), U C(2,1001)$, $X S(1001), N S R(3,26), N R N K(25)$

THETA IS DEF INED SUCH THAT THE CURRENT $(0)=$ THETA * G, FOR EACH $K$. DO FOR THE 3 FLUX DATA BLOCKS:

DO 10 I T $=1,3$ READ IN THE MATERIALS INPUT DATA BLCCK:

CALL DATAINI I T, METHOD, NK, NR, NRNK, NP, N, XS, PHI, SF, D, CHI, THETA, NAP, H) READ IN THE CONVERGED FLUX PCINTS DATA BLOCK:

CALL SYNPTSIIT, METHOD,NK,NR,F,G)

CALCULATE THE DETAILED FLUX SHAPES:

IF (METHOD.EQ.2) GO TO 4

CALL CALCULIIT, METHOD,NK,NR,NRNK,NP,N,X,U,XS,PHI, SF, D,CHI, $X$

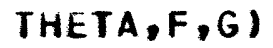

4 CALL CAL:UCII T, METHOD, NK, NR, NRNK, NP, $N, X, U, X S, P H I, S F, D, C H I$, $x$ THETA, F, G, NAPI

C

TRANSFORM IHE FLUX POINTS INTC NK DISTINCT REGIONS:

C

5 CALL REFORM (IT, METHOD, NK, NR, NRNK, NP, N, $X, U, S F, D$ )

- CALCULATE THE POWER IN EACH OF THE NK REgIONS FOR EACH FLUX:

CALL KPOWER I I T, METHOD,NK,NP, N, X, U, SF, D, CHI, THETA, NAP, F, G, H,

$\mathrm{X}$ $N S R, R, T P O W E R$ I

C NORMALIZE THE REgION POWERS AND THE FLUX POINTS BY TPOWER)

CALL POWNOR (I T, METHOD,NK, NP, $N, X, U, R, T P O W E R$ ) SAVE THESE RESULTS FOR PLOTTING:

$C A L L$ SAVE(IT,NK,N,X,U,NB,XB,UB,NC,XC,UC)

10 CONT INUE

C NORMALIZE THE FLUX RESULTS TOGETHER FOR EACH GROUP TO 1.0:

CALL RELNOR(NK, $N, X, U, N B, X B, U B, N C, X C, U C)$ PRINT DUT THE POWER AND ARRAY RESULTS:

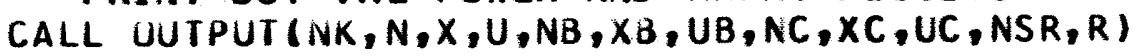

READ IN IHE PLOTTING INFORMATION AND PLOT THE RESULTS ON THE SC 4020:

CALL SCPLOT (NK, N, X,U,NB,XB,UB,NC,XC,UC)

ANAL 0004

ANAL 0005

AN AL 0006

ANAL 0007

ANAL 0008

ANAL 0009

ANAL 0010

AN AL 0011

ANALO012

ANAL 0013

AN AL 0014

ANAL 0015

ANAL 0016

ANAL 0017

ANAL 0018

AN AL 0019

ANAL 0020

ANAL 0021

AN AL 0022

ANAL 0023

ANAL 0024

ANAL 0025

ANAL 0026

ANAL 0027

ANAL 0028

ANAL 0029

AN AL 0030

ANAL 0031

ANAL 0032

ANALO033

ANAL 0034

AN AL 0035

ANAL 0036

PAGE 352. 
WRITE $(6,20)$

20 FORMAT $(\%$, ONORMAL PROGRAM TERMINATION.")

STOP

ANAL 0037

ANAL 0038

ANAL 0039

AN AL 0040 
SUBROUT INE DATA INIIT, METHOD, NK, NR, NRNK, NP, $N, X, P H I, S F, C, C H I, T H E T A$,

DATA0001

DATA0002

DATA0003

DATA0004

DATA0005

DATA0006

DATA0007

DATA0008

DATA0009

DATA0010

DATA0011

DATAO012

DATA0013

DATA0014

DATA0015

DA TA0016

DATA0017

DATA0018

DATA0019

DATA0020

DATA0021

DATA0022

DATA0023

DATA0024

DATA0025

DATA0026

DATA0027

DATA0028

DATA0029

DATA0030

CATA0031

DATA 0032

DATA0033

DATA0034

DATA0035

DATA0036

$S F(2, J), D(2, J), J=K 0, L 2)$

$\operatorname{READ}(I T, L 5) \times(L 1), X(L O), H(L 1), S F(1, L 1), D(1, L 1), S F(2, L 1), D(2, L 1)$

PAGE 354 
XSTART $=$ XSTART $-X(K O)$

DATA0037

DO $1 J=K O, L O$

$1 x(J)=x(J)+x S T A R T$

$X S T A R T=X(L O)$

IF (IT.EQ.3) GO TO 10

READ (IT, 16) (PHI $(1, J), J=K O, L O)$

READ (IT, 16) (PHI $(2, J), J=K O, L O)$

GO TO 10

OLO REPEATED REGION DATA:

C

$2 M=I T F(I)$

$M=K T F(M)$

$N P(I)=N P(M)$

$K(I)=K(I-I)+N P(I-1)$

$L(I)=L(I-1)+N P(I)$

$K O=K(I)$

$L O=L(I)$

$L 1=L O-1$

$K M=K(M)$

$I B=K O-K M$

XSTART $=X S T A R T-X(K M)$

DO $5 J=K O, L O$

$N J=J-I B$

$X(J)=X(N J)+X S T A R T$

$H(J)=H(N J)$

DO $5 \quad I G=1,2$

$D(I G, J)=D(I G, N J)$

$\operatorname{SF}(1 \mathrm{G}, J)=\operatorname{SF}(I G, N J)$

$\operatorname{PHI}(I G, J)=P H I(I G, N J)$

5 CONT INUE

$X S T A R T=X(L O)$

10 CONT INUE

11 FORMAT $(/, 15)$

12 FORMAT (25I2)

13 FORMAT (5X,15)

14 FORMAT (F10.5,10X,F10.5, 10X,2E10.3, /,40X,2E 10.3)

15 FORMAT (3F10.5,10X,2E10.3,/,40X,2E10.3)

DATA0038

DATA0039

DATA0040

DATA0041

DATA0042

DATA0043

DATA0044

DATA0045

CATA0046

CATA0047

DATA0048

DATA0049

DATA0050

DATA0051

DATA0052

DATA 0053

DATA0054

DATA0055

DATA0056

CATA0057

DATA0058

DATA0059

DATA0060

DATA0061

DATA0062

DATA0063

DATA0064

DATA0065

DATA 0066

DATA0067

DATA0068

DATA0069

DATA0070

DATA0071

DATA0072

PAGE 355 
16 FORMAT (E20.7)

DATA0073

7 FORMAT (3F10.5)

DATA0074

$N=L$ (NR)

IF (IT.NE.3) RETURN

DATA0075

DO $50 \quad I=1, N$

DO $50 \quad I G=1,2$

$50 \operatorname{PHI}(I G, I)=1.0$

RETURN

DATA0076

DATA0077

DATA0078

DATA0079

CATA0080

END

DATA0081

PAGE 356 
SUBROUTINE SYNPIS IIT, METHOD,NK, NR,F,G)

SYNP 0001

SYNP0002

SYNP 0003

DIMENSION $F(2,201), G(2,201)$

$J T=I T+10$

READ (JT, 1) NR

SYNP0004

SYNP0005

SYNP0006

I FORMAT (15)

$N R 1=N R+1$

IF (METHOD.NE.1) GO TO 10

FOR LINEAR SYNTHESIS:

C

IF (IT,NE.3) READ (JT,2) (F(1,I),F(2,I),I=1,NR1)

IF (IT,EQ.3) REAC (JT,3) ( F $(1, I), I=1, N R I),(F(2, I), I=1, N R 1)$

SYNPO006

SYNP0008

SYNPOOO9

SYNP0010

SYNP0011

SYNP 0012

2 FORMAT (2E20.7)

3 FORMAT (E20.7)

60 TO 20

$10 \operatorname{READ}(J T, 11),(F(1, I), G(1, I), F(2, I), G(2, I), I=1, N R 1)$

11 FORMAT (4E20.7)

20 RETURN

END

SYNP0013

SYNP 0014

SYNPO015

SYNP 0016

SYNP0017

SYNP0018 
C $x$

SUBROUT INE CALCULIIT, METHOD, NK, NR, NRNK, NP,NU,XU,U,XS,PHI, SF, D, CHI, THETA,F,G)

CALCULATES THE FLUX TRIAL FUNCTICN U FOR LINEAR SYNTHESIS:

DIMENSI ON NP(200), XU(10)1),U(2,1001), PHI $(2,1001), S F(2,1000)$,

$x$

I SYNTH $=20$

C

FOR EACH K'TH REGION:

$N=0$

$L L=0$

DO $20 K=1$, NR

$L=L L+1$

$L L=L L+N P(K)$

DO 7 IG $=1,2$

IF $(P H I(I G, L), E Q .0 .0) \quad P H I(I G, L)=1.0 E-6$

IF (PHI (IG,LL).EQ.0.0) PHI $(I G, L L)=1.0 E-6$

7 CONTINUE

C

FOR ALL POINTS IN THIS REGION:

DO $10 \quad I=L, L L$

$\mathrm{N}=\mathrm{N}+1$

$X U(N)=X S(1)$

$X=(X U(N)-X S(L)) /(X S(L L)-X S(L))$

IF ( (K.EQ.1).AND.(ISYNTH.EQ.21.OR.ISYNTH.EQ.24)) $X=1.0$

IF ( (K.EQ.NR).AND. (ISYNTH.EQ.22.CR. ISYNTH.EQ.24) $\quad X=0.0$

DO 1 I $G=1,2$

$1 U(I G, N)=\operatorname{PHI}(I G, I) *(F(I G, K) *(1,-X) / P F I(I G, L)$

$\mathrm{x}$

$+F(I G, K+1) * X / P H I(I G, L L))$

10 CONTINUE

20 CONTINUE

$N U=N$

RETURN

END
CALLOOOL

CALL 0002

CALLO003

CALL 0004

CALL 0005

CALLOO06

CALL0007

CALL0008

CALL0009

CALL 0010

CALLO011

CALL 0012

CALLOD13

CALLOO14

CALL 0015

CALL0016

CALLOO17

CALL0018

CALLOO19

CALL0020

CALL 0021

CALLO022

CALL 0023

CALL 0024

CALL 0025

CALL 0026

CALL 0027

CALL 0028

CALL 0029

CALL0030

CALL0031 
SUBROUT INE CALCUCIIT, METHOD, NK, NR, NRNK, NP,NU, XU,U,XS,PHI, SF, D,CHI,

$X$ THETA,F,G,NAPT)

C CALCULATES THE FLUX TRIAL FUNCTICN U FOR CUBIC HERMITE SYNTHESIS: NAP = \# UF ADDITIONAL POINTS TO BE CALCULATED IN EACH REGION

AND IS VEGATIVE IF NAP APPLIES CNLY TO THE FIRST REGION.

DIMENSION NP(200),XU(1001),U(2,1001), PHI $(2,1001), S F(2,1000)$,

$X$

NAP $=$ NAPT $D(2,1000), C H I(2), F(2,201), G(2,201), X S(1001), N R N K(25)$

$N L=0$

DO $50 \quad K=1, N R$

C

$50 N L=N L+N P(K)$ $N=0$

FOR EACH K'TH REGION:

$L L=0$

DO $20 K=1, N R$

$L=L L+1$

$L L=L L+N P(K)$

$H=X S(L L)-X S(L)$

DO $7 \quad I G=1,2$

IF (PHI $(I G, L) . E Q .0 .0)$ PHI $(I G, L)=1.0 E-6$

IF $(P H I(I G, L L), E Q .0 .0) \quad P H I(I G, L L)=1.0 E-6$

7 CONTINUE

C

FOR ALL POINTS IN THIS REGION:

DO $10 \quad I=L, L L$

$\mathrm{N}=\mathrm{N}+1$

$X U(N)=X S(I)$

$X=(X U(N)-X S(L)) /(X S(L L)-X S(L))$

DO $1 \quad I G=1,2$

$1 U(I G, N)=\operatorname{PHI}(I G, I) *(F(I G, K) *(1,-3 . * X * * 2+2 . * X * * 3) / P H I(I G, L)$

$X \quad+F(I G, K+1) *(3, * X * * 2-2, * X * * 3) / P H I(I G, L L)$

$x \quad+H *(G(I G, K) /(C(I G, L) * P H I(I G, L)) *(-X+2 * * X * * 2-X * * 3)$

$X \quad * G(I G, K+1) /(D(I G, L L-1) * P H I(I G, L L)) *(X * * 2-X * * 3)) *$ THETA $)$ $N A P P=I A B S(N A P)$

IF (NAP.EQ.0) GO TO 10

IF (I.EQ.LL) GO TO 10

$D X=(X S(I+1)-X S(I)) / F L O A T(N A P P+1)$

CALCOOOL

CALCOOO2

CALC 0003

CALC 0004

CALC0005

CALC0006

CALC0007

CALC 0008

CALC0009

CALC0010

CALC0011

CALC 0012

CALC 0013

CALCOO14

CALC0015

CALC0016

CALCO017

CALCOO18

CALC 0019

CALC 0020

CALC0021

CALC 0022

CALCOO23

CALC0024

CALC0025

CALC0026

CALC 0027

CALC0028

CALC 0029

CALC 0030

CALC0031

CALC 0032

CALC 0033

CALC0034

CALC0035

CALC0036

PAGE 359 
DO 5 I NP=1, NAPP

$\mathrm{N}=\mathrm{N}+1$

$X U(N)=X U(N-1)+D X$

$X=(X U(N)-X S(L)) /(X S(L L)-X S(L))$

DO $5 \quad I G=1,2$

5 U(IG,N)=PHI $(I G, I) *(F(1 G, K) *(1 .-3 . * X * * 2+2 * * X * * 3) / P H I(I G, L)$

$X \quad+F(I G, K+1) *(3, * X * * 2-2 * * X * * 3) / P H I(I G, L L)$

$X \quad+H *(G(I G, K) /(D(I G, L) * P H I(I G, L)) *(-X+2 * * X * * 2-X * * 3)$

$X \quad+G(I G, K+1) /(D(I G, L L-1) * P H I(I G, L L)) *(X * * 2-X * * 3)) *$ THETA $)$

10 CONTINUE

IF (NAPP.EQ.0) GO TO 15

IF (IT.EQ.1) GO TO 15

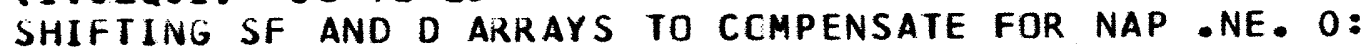

$N N P=N S * N A P P$

$N P S=0$

$K 1=K+1$

IF $(K 1 . G T . N R)$, GO TO 60

DO $51 \quad I=K 1, N K$

$51 N P S=N P S+N P(I)$

DO $55 J=1$, NPS

$I=N L+I-J$

$I I=I+N N P$

DO $55 \quad I G=1,2$

$\operatorname{SF}(I G, I I)=\operatorname{SF}(I G, I)$

$55 D(I G, I I)=D(I G, I)$

$60 \mathrm{NL}=\mathrm{NL}+N \mathrm{NP}$

$L=N L-N P S$

$L I=L-N N P-N P(K)$

DO $70 \quad I=1$, NS

$I I=N S+I-I+L I$

NAPP $1=N A P P+1$

DO $65 \mathrm{~J}=1$, NAPP 1

$L=L-1$

DO $65 \quad I G=1,2$

$S F(I G, L)=S F(I G, I I)$

CALC 0037

CALC 0038

CALC 0039

CALC 0040

CALC0041

CALC 0042

CALC0043

CALC 0044

CALC 0045

CALC0046

CALC0047

CALC 0048

CALC0049

CALC 0050

CALC0051

CALC0052

CALC 0053

CALC0054

CALC0055

CALC0056

CALC0057

CALC0058

CALC0059

CALC0060

CALC 0061

CALC0062

CALC0063

CALC 0064

CALC0065

CALC0066

CALC0067

CALC0068

CALC 0069

CALC 0070

CALC 0071

CALCO072

PAGE 360 
$65 D(I G, L)=0(I G, I I)$

CALCO073

CALC 0074

70 CONTINUE

$15 N P(K)=N P(K)+N A P P$

CALC0075

IF (NAP.LT.O) NAP $=0$

CALC 0076

CALC CO77

CALC0078

$N U=N$

RETURN

CALC 0079

END

CALC0080

PAGE 361 
SUBROUT IVE REFORMI IT, METHOD, NK, NR, NRNK, NP, $N, X, U, S F, D$ )

REFORMS THE NR GIVEN REGIONS INTC NK DESIRED REGIONS:

DIMENSION NP(200),X(1001),U(2,1001),SF(2,1000),D(2,1000),NRNK(25)

IF (NK.EQ.NR) RETURN

C IT SHOULD BE 3 ONLY.

C. DELETE ALL DOUBLE ENTRIES HITHIN EACH NK DATA BLOCK: $M=-1$

$I=0$

DO $5 \quad K=1$, NK

$L=N R N K(K)$

DO $4 J=1, L$

$\mathrm{I}=\mathrm{I}+1$

$M=M+2$

$X(I)=X(M)$

DO $4 \quad I G=1,2$

$U(I G, I)=U(I G, M)$

$S F(I G, I)=\operatorname{SF}(I G, M)$

$4 D(I G, I)=D(I G, M)$

$I=I+1$

$X(I)=X(M+1)$

DO $5 \quad I G=1,2$

$5 U(I G, I)=U(1 G, M+1)$

$\mathrm{N}=\mathrm{I}$

NP(K) = \# UF FLUX POINTS IN REGICN $K=$ \# NS SUBREGIONS + 1: DO $10 \quad K=1, N K$

$10 N P(K)=N P(K)+N R N K(K)-1$

$N R=N K$

RETURN

END

REF00001

REFO0002

REF00003

REF00004

REF00005

REF00006

REF00007

REF00008

REF00009

REFO0010

REF00011

REF 00012

REFO0013

REFO0014

REFO0015

REF00016

REF00017

REFO0018

REF00019

REF 00020

REFO0021

REFO0022

REFO0023

REFO0024

REF00025

REFO0026

REFO0027

REF00028

REF00029 
SUBROUT INE KPOWER I IT, METHOD, NK, NP, N, X, U, SF, D, CHI, THET A, NAP, F, G,

$X$ $H, N S R, R, T$ POWER I

C CALCULATES THE POWER IN EACH REGICN BY INTEGRATIONS:

DIMENSION NP $(200), X(1001), U(2,1001), S F(2,1000), D(2,1000), C H I(2)$, $F(2,201), G(2,201), H(1000), R(9,26), N S R(3,26)$

TPOWER $=0.0$

IF (IT.NE. I) GO TO 20

FOR THE HOMOGENEOUS CASE:

C ASSUMING THAT NP IS ALWAYS 2 AND THUS D'S ARE CONSTANT. DO $10 \mathrm{~K}=1$, NK

$M=2 * K-1$

$N S R(I T, K)=1$

$R(I T, K)=0.0$

DO $8 \quad 1 \mathrm{G}=1,2$

IF (METHOD.NE.1) GO TO 5

C

LINEAR FLUX:

$R(I T, K)=(F(I G, K)+F(I G, K+1)) * H(M) * S F(I G, M) / 2,0+R(I T, K)$

GO TO 8

CUBIC FLUX:

C

$5 R(I T, K)=((F(I G, K)+F(I G, K+1)) * H(M) / 2,0+\operatorname{THETA*}(-G(I G, K)$

$X \quad / D(I G, M)+G(I G, K+1) / D(I G, M)) * H(M) * * 2 / 12.0) * S F(I G, M)+R(I T, K)$

8 CONTINUE

10 TPOWER $=\operatorname{IPOWER}+R(I T, K)$

RETURN

C $20 \mathrm{M}=-1$

SYNTHESIS OR REFERENCE DETAILED FLUX CASE:

DO $50 \quad K=1$, NK

$M=M+1$

$N S=N P(K)-1$

$N S R(I T, K)=N S$

$R(I T, K)=0.0$

DO $40 \quad J=1$, NS

$M=M+1$

DO $40 \quad I G=1,2$

$40 R(I T, K)=(U(I G, M)+U(I G, M+1)) *(X(M+1)-X(M)) * S F(I G, M) / 2.0+R(I T, K)$

50 TPOWER $=$ TPUWER+R $(I T, K)$

KPCWO001

KPOW 0002

KPCW 0003

KPOW0O04

KPOW0005

KPOW0006

KPOW 0007

KP OW0008

KPCW0009

KP OW 0010

KPOW0011

KPCWO012

KPOWO013

KPCWO014

KPOW 0015

KPOWOO16

KPOW 0017

KPOWOO18

KPOW0019

KPOWO020

KPOWO021

KPCW0022

KPCW 0023

KPOW0024

KPOW0025

KPOW 0026

KPCW0027

KPOW0028

KPOW0029

KPOW 0030

KPOW0031

KPOW0032

KPOW0033

KPOW0034

KPOW 0035

KPOW0036

PAGE 363 
RE TURN

END

KPOW 0037

KPOW 0038

PAGE 364 
SUBRUUT INE PUWNOR (IT, METHOO, NK, NP, $N, X, U, R, T P O H E R$ )

NORMALI LES THE FLUXES AND REGION POWERS BY TPOWER:

POWN0001

DIMENSIUN NP(200),X(1001),U(2,1001),R(9,26)

DO $1 K=1$, NK

$1 R(I T+3, K)=R(I T, K) / T P O H E R$

DO 2 I $G=1,2$

DO $2 K=1, N$

$2 U(I G, K)=U(I G, K) / T$ POWER

RETURN

END

POWNOOO2

POWN0003

POWN0004

POWN0005

POWN0006

POWN0007

POWN0008

POWNO009

POWNOO1O 
SUBROUTINE SAVE (IT,NK,N,X,U,NB,XB,UE,NC,XC,UC)

SAVEO0O 1

SAVES THE FLUX DISTRIBUTIONS IN THE IT LOOP BY PLACING:

IT $=1$ : HOMOGENEOUS RESULTS IN UC;

IT $=2$ : SYNTHESIS RESULTS IN UB;

IT = 3: REFERENCE RESULTS IN U.

DIMENSIUN $X(1001), U(2,1001), X B(1001), U B(2,1001)$,

$X$ $X C(1001), U C(2,1001)$

C

IF (IT.NE.1) GO TO 2

HOMUGENEOUS RESULTS:

DO $1 \quad I=1, N$

$X C(1)=X(1)$

DO $1 \quad I G=1,2$

$1 U C(I G, I)=U(I G, I)$

$N C=N$

RETURN

2 IF (IT.EQ.3) RETURN

SYNTHESIS RE SULTS:

DO $3 \quad 1=1, N$

$X B(1)=X(1)$

$D O 3 \quad$ I $G=1,2$

SAVE0002

SAVE0003

SAVE0004

SAVE0005

SAVE0006

SAVE0007

SAVE0008

SAVE0009

SAVE0010

SAVE0011

SAVE 0012

SAVE0013

SA VE0014

SAVE0015

SAVE0016

SAVE0017

SAVEOO 18

SAVE0019

SAVE 0020

SAVE0021

SAVE0022

SAVE0023

SAVE0024

END 


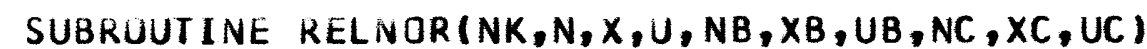

DIMENSION A(2), E(2), C(2),Z(2,3)

CALL MAX $(N, U, A)$

CALL MAX (NB,UB,B)

CALL MAX $(N C, U C, C)$

$D O \perp I G=1,2$

$Z(I G, 1)=A(I G)$

$Z(I G, 2)=B(I G)$

$1 Z(I G, 3)=C(I G)$

CALL $\operatorname{MAX}(3, Z, A)$

CALL DIV $N, U, A)$

CALL DIV(NB,UB,A)

CALL DIVINC,UC, A)

RETURN

RELN0003

RELNOOO4

RELN0005

RELN0006

RELNO007

RELNO008

RELNO009

RELNOO10

RELNO011

RELNOO12

RELNOO13

RELNO014

RELNO015

RELN0016

RELN0017

END

RELNOO18 
SUBROUTINE MAXIN,U, A)

FINDS THE MAXIMUM POSITIVE ELEMENT CF U FOR EACH GROUP:

$\operatorname{MAX} 0001$

DIMENSION U(2,1001),A(2)

DO 1 I $G=1,2$

$A(I G)=0$.

DO $1 \quad I=1, N$

IF $(U(16, I), G T . A(I G)) \quad A(I G)=U(1 G, I)$

1 CONT INUE

RETURN

END

$\operatorname{MAX} 0002$

$\operatorname{MAX} 0003$

MAX 0004

MAX 0005

MAX 0006

MAX 0007

MAX 0008

$\operatorname{MAX} 0009$

MAX 0010 
SUBROUTINE DIV $(N, U, A)$

DIVIDES A INTO U FOR EACH GRCUP:

DIMENSION U $(2,1001), A(2)$

DO 1 I G $=1,2$

DO $1 I=1, N$

$1 U(I G, I)=U(I G, I) / A(I G)$

RE TURN

DIV 0001

DIV 0002

DIV 0003

DIV 0004

DIV 0005

DIV 0006

DIV 0007

END

DIV 0008 
SUBRUUT INE OUTPUT (NK, $N, X, U, N B, X B, U B, N C, X C, U C, N S R, R)$

OUTP0001 PRINTS OUT THE ANALYSIS RESULTS:

DIMENSION $X(1001), U(2,1001), X B(1001), U B(2,1001)$, $X C(1001), U C(2,1001), R(9,26), N S R(3,26)$

OUTP0002

DUTP0003

DUTP0004

OUTP 0005

DUTP0006 DO 5 I T $=1,6$

IF (IT.LE.3). NSR $(I T, 26)=0$

$R(I), 26)=0.0$

DO $5 \quad K=1, N K$

IF $(1 T, L E .3)$ NSR $(I T, 26)=N S R(I T, 26)+N S R(I T, K)$

OUTP0007

OUTP0008

OUTPOOO9

OUTP0010

OUTP0011

$5 R(I T, 26)=R(I T, 26)+R(I T, K)$

THE PERCENT NORMALIZED DIFFERENCES OF THE REGION FRACTIONAL POWERS:

DO $60 \quad K=1, N K$

DO $50 \quad$ IT $=7,9$

$50 R(I T, K)=0.0$

If $(R(6, K) \cdot E Q .0 .0)$ GO TO 60

$R(7, K)=(R(6, K)-R(4, K)) * 100 \cdot / R(6, K)$

$R(8, K)=(R(6, K)-R(5, K)) * 100, / R(6, K)$

IF $(R(5, K) \cdot E Q \cdot 0.0)$ GO TO 60

$R(9, K)=(R(5, K)-R(4, K)) * 100, / R(5, K)$

60 CONTINUE

THE PERCENT NORMALIZEO DIFFERENCES OF THE TOTAL RAW POWER PRODUCED:

OUTP 0012

OUTP 0013

OUTP 0014

OUTPOO15

OUTP0016

OUTP0017

DUTPOO18

DUTP0019

OUTP 0020

OUTP 0021

OUTP0022

DUTP 0023

$R(7,26)=(R(3,26)-R(1,26)) * 100 . / R(3,26)$

$R(8,26)=(R(3,26)-R(2,26)) * 100 . / R(3,26)$

$R(9,26)=(R(2,26)-R(1,26)) * 100 . / R(2,26)$

WRITE $(6,10), N K,(K,(N S R(I T, K), R(I T, K), I T=1,3), K=1, N K)$

10 FORMAT IRESULTS OF THE INTEGRATED POHER IN EACH OF THE', I3,

$X$ - REGIONS:,$/ / /$,

$X$ OCALCULATEd POWER LEVEls, AND NUMBER OF SUBREgiONS PER REg ION:

DUTP0024

OU TP0025

OUTP 0026

OUTP 0027

OUTP 0028

OUTP0029

$\because, /, 3 X, \cdot$ REG ION: $, 10 X, "$ HOMOGENI ZED RESULTS: $", 5 X$,

- SYNTHESIZED RESULTS:",7X,"REFERENCE RESULTS:",/1,

$(I 10,10 X, 13, E 17,7,18, E 17,7,110, E 15.7))$

WRITE $(6,12)$ (NSR (IT, 26),R (IT, 26), I T=1,3)

12 FORMAT $(/, 3 \mathrm{X}$, 'TOTALS: $, 10 \mathrm{X}, 13, \mathrm{E} 17,7,18, \mathrm{E} 17,7,110, \mathrm{E} 15.7)$

WRITE $(6,20) \quad(K,(R(I T, K), I T=4,6), K=1, N K)$

20 FORMAT $1 / 1, \cdot$ JFRACTIONAL POWER LEVELS: $, 1 /, 3 X, '$ REGION:, $10 X$,

OUTP0030

OUTP0031

OUTP 0032

OUTPOO33

OUTP 0034

OUTP0035

DUTP 0036

PAGE 370 


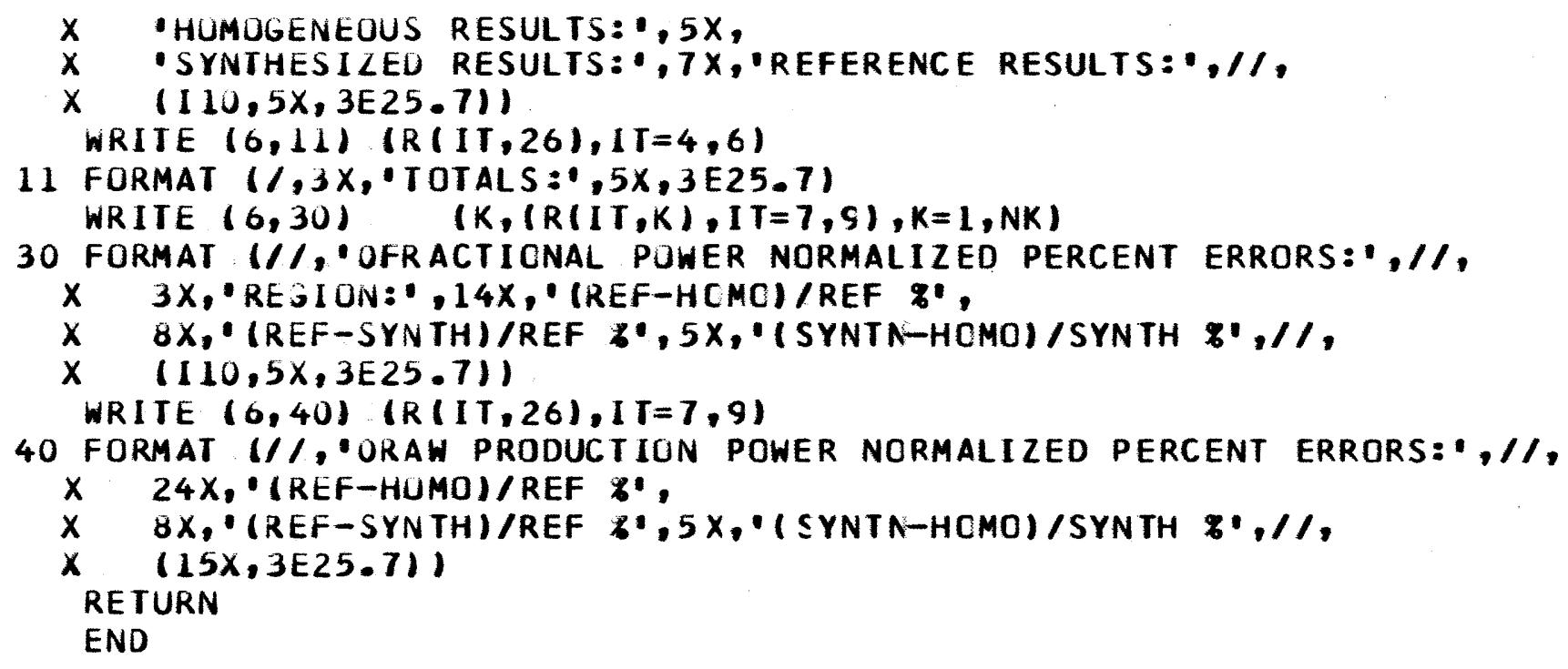

OUTP0037

OUTP0038

OUTP0039

OUTP0040

OUTP0041

OUTP0042

DUTP0043

DUTP0044

OUTP0045

OUTP0046

OUTP0047

OUTP0048

DUTP0049

OUTP0050

OUTPO051

OUTPO052

OUTP0053 
SUBROUT INE SCPLOT (NK,N,X,U,NE,XB,UB,NC,XC,UC)

READS IN PLJTTING INFORMATICN AND PLOTS THE FLUX COMPARISCNS:

SCPLO001

COMMON /SC/ TITLE(20),XINCH, YINCH, NCELL,WCELL, NLL, XL(100)

SCPLO002

COMMON /MAX/ XMIN, XMAX, YMIN, YMAX

COMMON /RASTER/ IXS, IYS, IXE, IYE, IXT, IYT, IXLX, IYLX,

$x$ IXLY,IYLY ( 3$), L H, L W, I S, I R$

COMMUN /MARGIN/ ML,MR,MB,MT

DIMENSION X(1001),U(2,1001),XB(1001),UB(2,1001),XC(1001), $X$ UC $(2,1001)$

READ $(5,1, E N D=20)$ TITLE

READ $(5,4, E N D=20) \times I N C H, Y I N C H$ NEGATIVE NCELL SPECIFIES THAT THE LAST CELL IS A HALF CELL:

READ $(5,3, E N D=20)$ NCELL, WCELL

READ $(5,3, E N D=5) \quad N L L,(X L(I), I=1,7)$

IF (NLL.GT.7) READ (5,4) (XLII),I=8,NLL)

1 FORMAT (20A4)

3 FORMAT (110,7F10.5)

4 FORMAT (8F 10.5$)$

GO TO 10

$5 \mathrm{NLL}=0$

10 WRITE $(6,11)$ TI TLE

11 FORMAT ('LEXECUTING general ANALYSIS AND FluX PlotTING PROGRAM:" $X \quad 1 /, 5 X,{ }^{\prime}$ TITLE OF PLOTIING RUN IS: $1,, 20 A 4,{ }^{\circ} 1,1$ $X M I N=0.0$

$X M A X=W C E L L * F L O A T$ (NCELL)

IF (NCELL.LT.O) XMAX=WCELL*(FLOAT(-NCELL)-0.5)

$Y M I N=0$.

YMAX $=1.0$

WRITE $(6,13)$ NCELL, WCELL, XMIN, XMAX, YMIN, YMAX, NLL

13 FORMAT 1 OREACTOR GEOMETRY PARAMETERS: $, 1,5 \mathrm{X},{ }^{\prime}$ NCELL $=1,15,1$,

$X \quad 5 X,{ }^{\prime} W C E L L=,, F 10.5, /, 5 X,{ }^{\circ} X M I N={ }^{\prime}, F 10.5, /, 5 X,{ }^{\prime} X M A X=1, F 10.5, /$,

$X \quad 5 X, \cdot Y M I V=0, F 10.5,1,5 X, \cdot Y M A X=1, F 10.5,1$,

$X \quad 5 X,{ }^{\circ} \mathrm{NLL}=1,151$

IF (NLL.GT.0) WRITE $(6,14) \quad(X L(I), I=1, N L L)$

14 FORMAT $(5 X, \cdot(X L(I), I=1, N L L)=0,1,(10 X, 10 F 10.5))$

CALL SETUP

SCPL 0004

SCPLO005

SCPL 0006

SCPL 0007

SCPL 0008

SCPL 0009

SCPL 0010

SCPLO011

SCPL 0012

SCPL 0013

SCPLO014

SCPL 0015

SCPLO016

SCPLO017

SCPL 0018

SCPLO019

SCPL 0020

SCPL 0021

SCPL 0022

SCPL 0023

SCPL 0024

SCPL 0025

SCPL 0026

SCPL0027

SCPL 0028

SCPL 0029

SCPL 0030

SCPL 0031

SCPLO032

SCPL 0033

SCPL 0034

SCPL 0035

SCPL 0036

PAGE 372 
CALL PLOT $(N, X, U, N B, X B, U B, N C, X C, U C)$

SCPLO037

SCPL 0038

20 RETURN

END

SCPL 0039 
SUBROUTINE SETUP
C FINDS THE RASTER

FINDS THE RASTER LOCATIONS FOR THE GRID CORNERS, LABELS, AND TITLE

SETU0001

SETU 0002

ENTIRE PLOTIING AREA IINCLUDING LABELS) IS XINCH X YINCH.

SETU0003

COMMON /SC/ TITLE(20), XINCH, YINCH, NCELL, WCELL, NLL, XL (100)

COMMON /RASTER/ IXS, IYS, IXE, IYE, IXT, IYT, IXLX, IYLX,

$\mathrm{x}$

IXLY,IYLY $(3), L H, L H, I S, I R$

C

COMMON /MARGIN/ML,MR,MB,MT SET THE LETIER SIZE FOR THE LABELS AND TITLES: $\mathrm{LH}=2$

$L W=2$

I $S=5 * L W+3$

$I R=7 * L H+5$

C PLOTTING AREA:

C ONE INCH $=137$ RASTERS. PLCTTING AREA IS 1023 RASTERS SQUARE. $I X E=X I N C H * 137 .+0.5$

$I Y E=Y I N C H * 137 .+0.5$

IF (IXE.GT.1020) IXE $=1020$

IF (IYE.GT.1020) IYE $=1020$

$I X S=(1023-I X E) / 2 \cdot+1$

$I Y S=(1023-I Y E) / 2+1$

$I X E=I X S+I X E-1$

$I Y E=I Y S+I Y E-1$

C COORDS FOR THE LETTERS ARE FOR THEIR CENTERS: TITLE (UPPER LEFT CORNER):

$I X T=8$

$I Y T=1014$

C $X$ AXIS LABEL

$I X L X=(I X S+60+I X E-6 * I S) / 2+1+8$

$I Y L X=I Y S+10$

$Y$ AXIS LABELS (FOR EACH FLUX PLOT TYPE):

C

$I X L Y=I X S+10$

I $Y L Y(1)=(I Y S+43+I Y E-20 * I S) / 2+1+4$

$I Y L Y(2)=(I Y S+43+I Y E-23 * I S) / 2+1+4$

C

$I Y L Y(3)=(I Y S+43+I Y E-26 * I S) / 2+1+4$

SET MARJIN SPACING FOR GRID:

$M L=I X S+2 * 18-1$

SETU0004

SETU0005

SETU0006

SETU 0007

SETU0008

SETU0009

SE TUOO10

SETU0011

SETU0012

SE TUO013

SETU0014

SETU0015

SETU0016

SETU0017

SETU0018

SETU0019

SETU 0020

SETU0021

SETU0022

SETU 0023

SETU0024

SETU0025

SETU0026

SETU0027

SETU0028

SETU0029

SETU0030

SETU0031

SETU0032

SETU0033

SETUO034

SETU0035

SETU 0036

PAGE 374 
$M R=1023-I X E$

SETU0037

SETU0038

SE TU0039

$M B=I Y S+2 * 18-1$
$M T=1023-1 Y E$

RETURN

SETU0040

END

SETU0041 
SUBRUUT INE PLOT (NA, XA,UA, NB, XB, UB, NC, XC,UC)

C USES MIT-IPC'S SC-4020 SUBROUTINE PLOTTING PACKAGE.

PLOT 0001

PLOT 0002

PLOT 0003

PLOTS THE COMPARISON FLUX DISTRIBUTIONS:

KEY: HOMOGENEOUS - DASHED LINE.

SYNTHESIS - SOLID LINE.

PLOT 0004

PLCT0005

REFERENCE - COTTED LINE.

PLOT0006

COMMON /RASTER/ IXS, IYS, IXE, IYE, IXT, IYT, IXLX, IYLX,

$\mathrm{X}$ IXLY, IYLY (3)

DIMENSIUN XA(1001), UA $(2,1001), X B(1 \mathrm{CO}), \mathrm{UB}(2,1001)$, $X C(1001), U C(2,1001), X(1001), T(1001)$

C HARDCOPY INI TIALIZATION:

CALL STUIDV $(\cdot M 7788-6571 \cdot, 9,2)$

C DO FOR EACH ENERGY GROUP:

DO $10 \quad I G=1,2$

NTH $=1$

CALL GRID

IF (IG.EQ.1) CALL RITE2V(IXLY,IYLY(1),1024,90,1,20,NTH, - NORMALILED FAST FLUX',N)

IF (IG.EQ.2) CALL RITE2VIIXLY,IYLY(2),1024,90,1,23,NTH,

$X$ 'NORMAL ILED THERMAL FLUX・,N)

C HOMUGENEOUS RESULTS:

CALL PUT (IG,NC, XC,UC,N,X,T)

CALL ADJUST $(N, X, T, 1)$

CALL LINE $(N, X, T, 1)$

SYNTHESIS RESULTS:

C

CALL PUT (IG,NB,XB,UB,N,X,T)

CALL ADJUST $(N, X, T, O)$

CALL LINE $(N, X, T, 0)$

REFERENCE RESULTS:

C

CALL PUT (IG,NA, XA,UA,N, $X, T)$

CALL ADJUST $(N, X, T, 2)$

CALL LINE $(N, X, T, 2)$

PLOT0007

PLOT0008

PLOT 0009

PLOTO010

PLOT 0011

PLOT 0012

PLOT0013

PLOT0014

PLOT0015

PLOT 0016

PLOT 0017

PLOTO018

PLOT 0019

PLOT 0020

PLOT 0021

PLOT 0022

PLCT 0023

PLOT 0024

PLOT 0025

PLOT0026

PLOT0027

PLOT 0028

PLOT0029

PLOT 0030

PLOT 0031

PLOT 0032

PLOT 0033

PLOT 0034

CALL PLTND(NTH)

PLOT 0035

WRITE $(0,20)$ NTH

20 FORMAT $1:, 15$, SC 4020 PLCTS HAVE BEEN PLOTTED. $)$

PLOT 0036 
RETURN

PLOT 0037

END

PLOT 0038

PAGE 377 
SUBROUT INE PUT( IG,NA, XA, UA, $N, X, T$ )

TRANSFERS NA,XA,UA OF IG INTC $N, X, T$ :

DIMENSION XA(1001),UA(2,1001),X(1001),T(1001)

$N=N A$

DO $1 \quad I=1, N$

$X(I)=X A(I)$

$1 \mathrm{~T}(I)=U A(I G, I)$

RETURN

END

PUT 0001

PUT 0002

PUT 0003

PUT 0004

PUT 0005

PUT 0006

PUT 0007

PUT 0008

PUT 0009 
SUBROUTINE GRIO

GR IDO001

GR ID0002

GR ID0003

GRID0004

GR ID0005

GR ID0006

GR ID0007

GR ID0008

GRID0009

GR ID0010

GR ID0011

GR ID0012

GR I D0013

GR ID0014

GR ID0015

GRID0016

GRIDO017

GR ID0018

GRID0019

GR ID0020

GR I D0021

GR ID 0022

GR ID0023

GR ID0024

GR ID0025

GR ID0026

I CALL LINEVIIX, I Y 1, IX, IY 2$)$

5 RETURN

END

GR ID0027 
SUBROUTINE ADJUST $(N, X, Y, L)$

ADJU0001

ADJUSTS THE $X$ AND $Y$ ARRAYS BY DELETING ANY $(X, Y)$ POINTS

ADJU0002

WITHIN OR EQUAL TC NR RASTERS DISTANCE OF EACH OTHER. HOPEFULLY EL IMINATES DARK SPCTS CN THE PLOTS.

$L=0:$ SOLID LINE PLOTTING: NR $=2$.

$L=1:$ DASHED LINE PLOTTING: NR $=2$;

INCRV $(10,5)$.

$L=2:$ DOTTED LINE PLOTTING: NR $=4 ;$ INCRVI 2,2$)$.

OIMENSION $X(1), Y(1)$

$N R=2$

IF $(L \cdot E Q \cdot 2) \quad N R=4$

NR $=4$
RE TURN

If

$K=1$

$I X I=I X V(X(1))$

$I Y 1=1 Y V(Y(1))$

DO $1 \quad I=2, N$

$I \times 2=I \times V(X(I))$

$I Y 2=I Y V(Y(I))$

I $D=S Q R T(F L J A T((I X 2-I X 1) * * 2+(I Y 2-I Y 1) * * 2))+0.5$

IF (ID.LE.NR) GO TO 1

$K=K+1$

$X(K)=X(I)$

$Y(K)=Y(I)$

$I X 1=I X 2$

I $Y 1=1 Y 2$

1 CONTINUE

$N=K$

RETURN

ADJU0003

ADJU0004

AD JU0005

ADJU0006

ADJU0007

ADJU0008

ADJU0009

ADJU0010

ADJU0011

ADJU0012

ADJU0013

ADJU0014

ADJU0015

ADJU0016

ADJU0017

ADJU0018

ADJU0019

ADJU0020

ADJU0021

ADJU0022

ADJU0023

ADJU0024

ADJU0025

AD JU0026

ADJU0027

END

ADJU 0028 
SUBROUT INE LINE $(N, X, Y, K)$ $C$
$C$
THROUGH $Y(X)$ DATA POINTS.

LINE 0001 DIMENSI ON $X(1), Y(1)$

$I X I=I X V(X(1))$

$I Y I=I Y V(Y(1))$

DO $10 \quad I=2, N$

$I \times 2=I X V(X(I))$

$I Y 2=I Y V(Y(I))$

IF $(K . N E .0)$ GO TO 1

SOLID LINE PLOT:

C

CALL LINEV I IXI, IY $1, I X 2, I Y 2)$

GO TO 5

DOTTED OR DASHED LINE PLOT:

C

1 IF $(K . E Q .1)$ CALL INCRV $(10,5)$

IF (K.EQ.2) CALL INCRV $(2,2)$

CALL DOTLNV(IXI,IY1,IX2,IY2)

5 I $X 1=I X 2$

$10 \quad I Y 1=I Y 2$

RETURN

END

LINE0002

LI INE0003

LINE0004

LINE0005

LI NE0006

LI NE0007

LINE0008

LINE0009

LINE 0010

LINE0011

LI NE 0012

LINE0013

LINE0014

LI NE 0015

LINE0016

LI NE0017

LINE00 18

LI NE0019

LI NE 0020

LINE0021 